$a y^{2}=n^{2}$ 


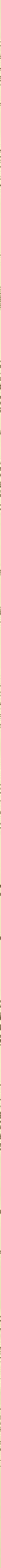




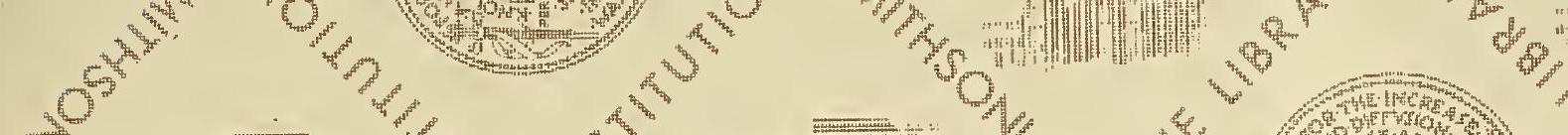

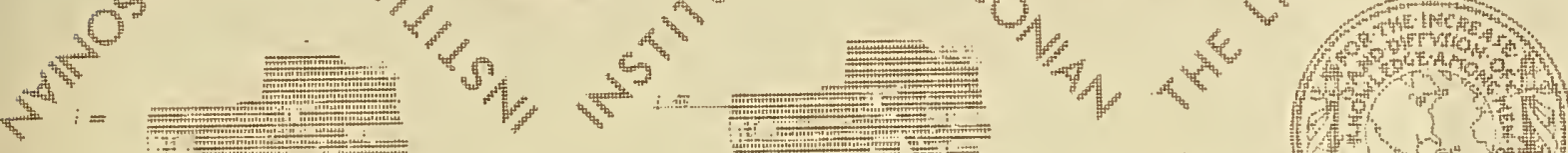

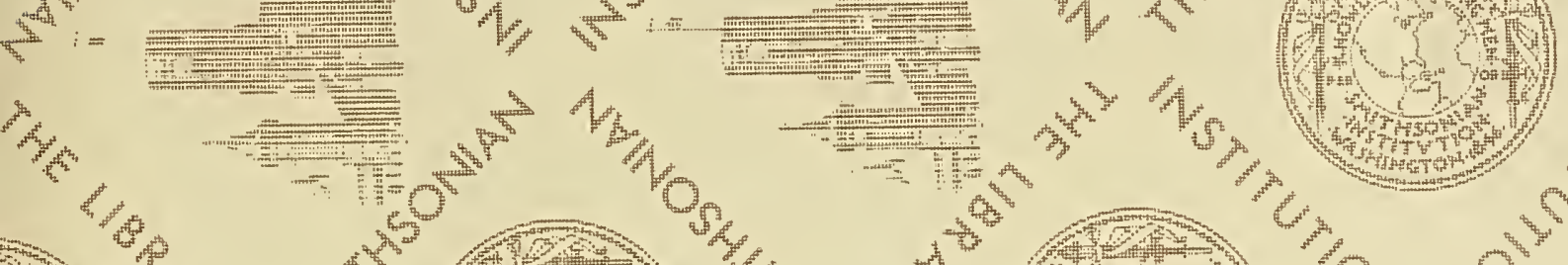

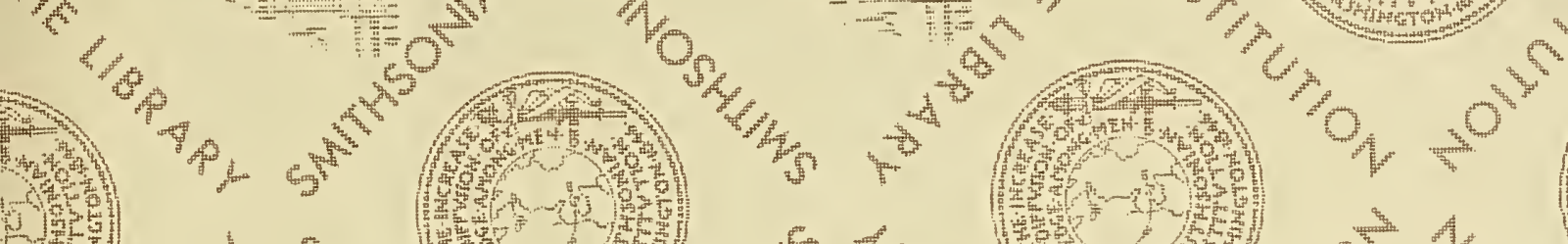

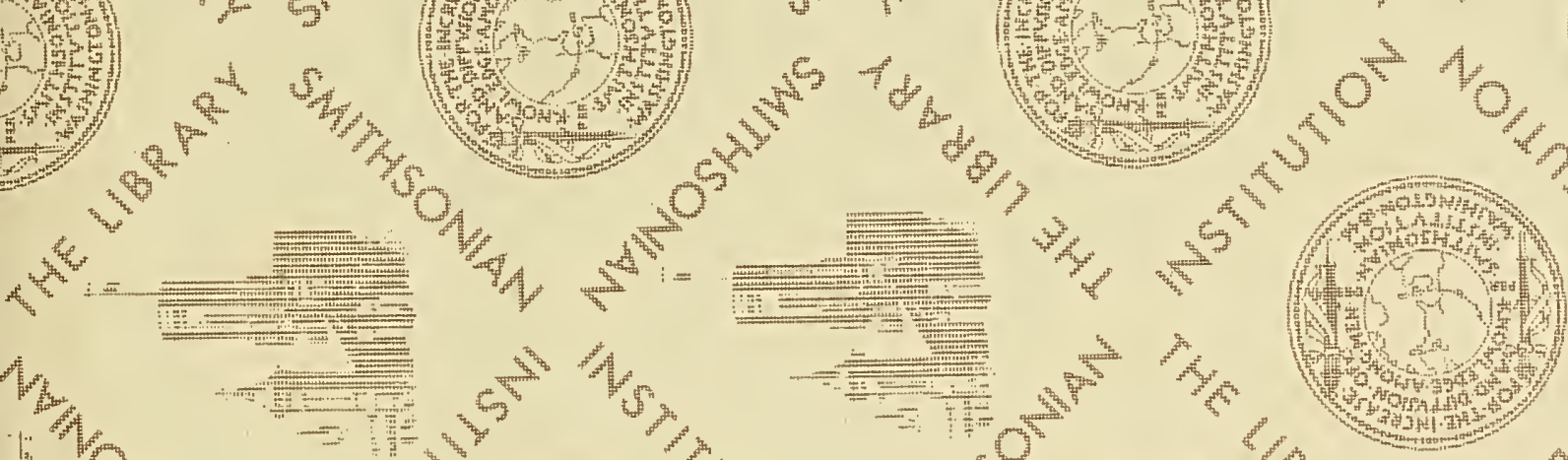

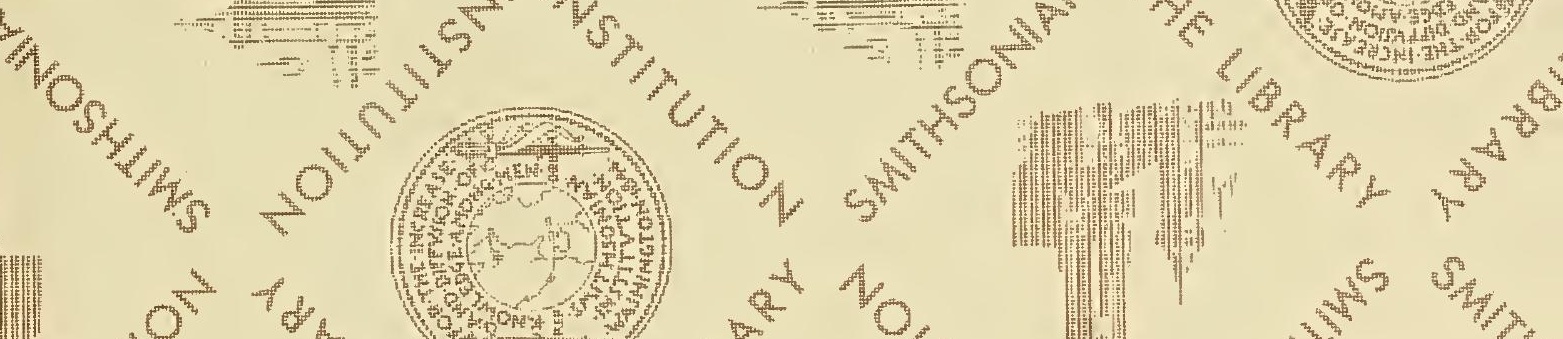

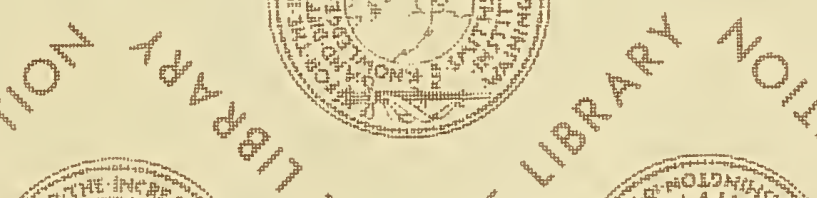

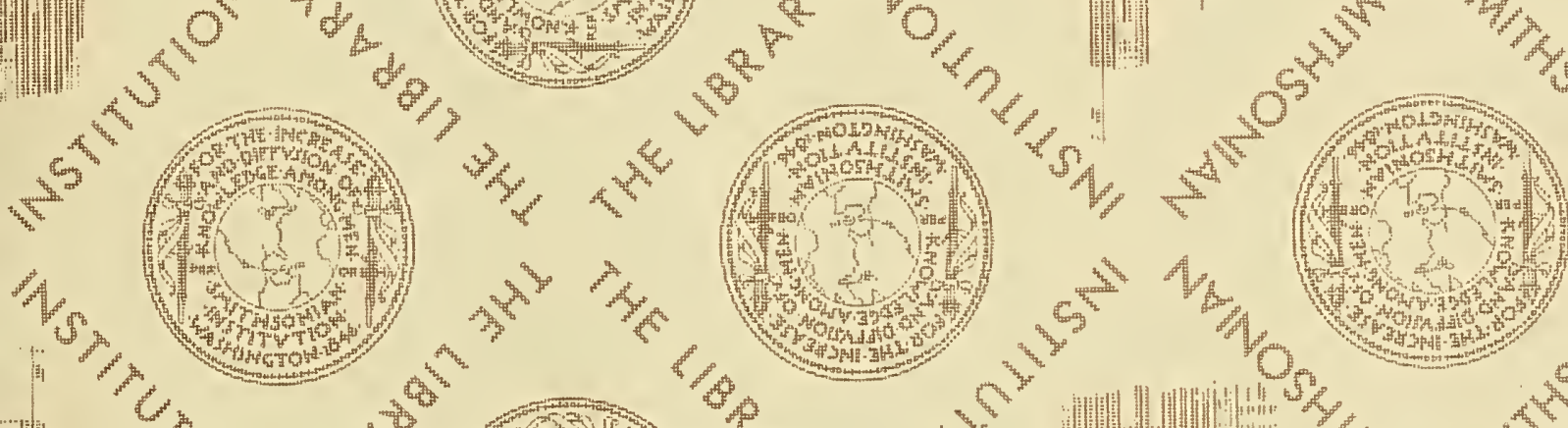

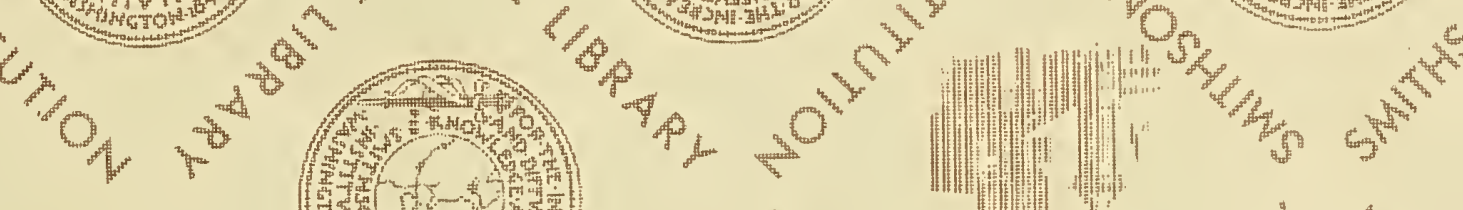

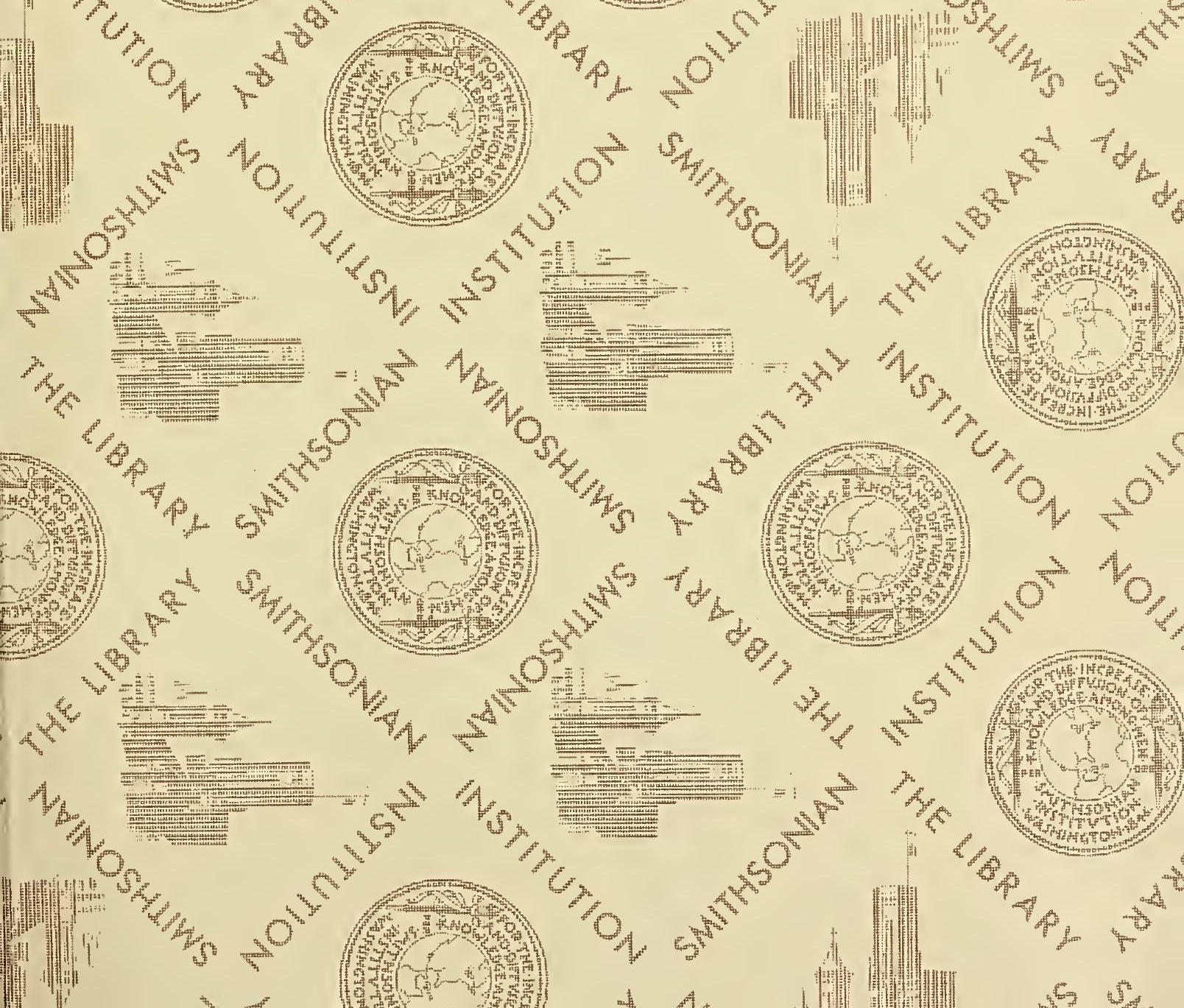





Bible, O.T.

\section{THE BOOKS}

$$
\text { OF }
$$

\section{GENESIS, EXODUS, LEVITICUS, NUMBERS,}

\section{AND DEUTERONOMY,}

TRANSLATED TNTO

THE CHOCTAW LANGUAGE.

CHENESIS, EKSOTUS, LEFITIKLS, NUMBAS,

\section{MICHA TUTELONOMI HOLISSO}

AIENA KUT TOSHOWUT

CHAHTA ANUMPA TOBA HOKE.

\section{NEW YORK:}

A M ERICA N B I BLE SOCIETY,

INSTITUTED IN THE YEAR MDCĆCXVI. 


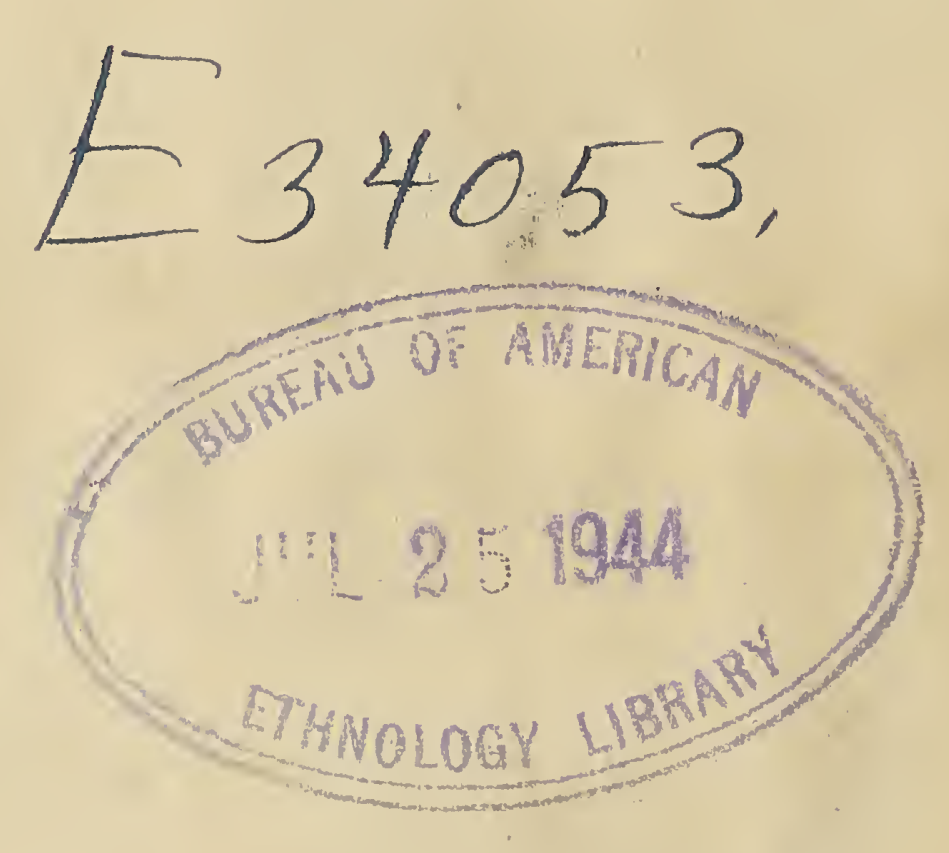




$$
\begin{aligned}
& B S \\
& 345 \\
& C 623 \\
& 1507 \\
& S O A
\end{aligned}
$$

CHENESIS HOLISSO. 



\section{$6346-13$}

\section{MOSES I HOLISSO VMMONA KVT}

\section{CHENESIS HOHCHIFO HOKE.}

\section{CHAPTA I.}

YMONA ka Chitokaka yut vba, micha yakni aiena ka ikbi tok.

2. Mihma yakni vt shahahbi bieka, micha nana kvt vlhtaiyaha iksho bieka tok; mihma okhlili hot oka hofobi chito paknaka ya onvtoyula tok: mihma Chitokaka imi Shilombish vt oka pataiya paknaka ai o kanahanli tok.

3. Mihma Chitokaka yot, Tohwikelashke, achi tok: mihma tohwikeli tok.

4. Mihma Chitokaka yut towikeli hut achukma ho pisa tok: mihmut Chitokaka yut tohwikeli ash okhlilika ai iti filummichi tok.

5. Micha Chitokaka yot tohwikeli ash, Nitak hochifo tok, mihmvt okhlilika, Ninak hochifo tok: mihma opia kvt, micha onnahinli kvt nitak vmmona hatok.

6. Mihma Chitokaka yut, Oka pataiya ka itintakla ya shutik osh talaiashke; micha mihi yosh oka ya oka bika iti filummichashke, achi tok.

7. Mihma Chitokaka yut shutik a ikbi cha, shutik nutaka oka aiasha kạ, micha shutik paknaka oka aiasha ka itị filummichi tok: mihma yumohmi tolk.

8. Mihma Chitokaka yụt shutik a Vba hochifo tok: mihma opia kvt, micha onnahinli kvt nitak atukla hatok.

9. Mihma Chitokaka yot, Oka pataiya shutik nutaka aiasha kvt itiba fohkvt atalaia achufa ho ai itunahashke, mikma a shila yut haiakashke, achi tok: mihma yumohmi tok.

10. Mihma Chitokaka yut ashila yash Yakni hochifo tok; 
mihmvt oka itiba fohkvt itunaha yash, Okhvta hochifo tok: mihma yummut achnkma ho Chitokaka yut pisa tok.

11. Mihma Chitokaka yvt, Yakni vt hushuk, haiyukpulo vt nihi waya, micha iti vni vt ilap aiachvfa vnit, nihi mot ilap akinli ho foka ho, offochi na yakni paknaka ya aiashashke, achi tok: mihma yumohmi tok.

12. Mihma yakni vt hưshuk, micha haiyukpulo ilap aiachvfa nihi waya, micha iti vni ilap aiachvfa vni, nihi mot ilap akinli ho foka ho, offochi tok: mihma yummut achukma ho Chitokaka yut pisa tok.

13. Mihma opia kvt, micha onnahinli kvt nitak atuchina hatok.

14. Mihma Chitokaka yvt, Nitak a ninak iti filummicha chi kvt tohwikelichi hvt vba shutik a hiohmayashke : micha nana isht vlhpisa kvt kocha ilaiyuka nitak, micha afummi aiena ka isht vlhpisashke.

15. Micha yvmmak osh tohwikelichi yosh vba shutik a ataloha cha, yakni ya on tohwikelichashke, achi tok: mihma yumohmi tok.

16. Micha Chitokaka yvt tohwikelichi chito tuklo ho, tohwikelichi $\underline{\mathrm{i}}$ shahli kut nitak ak o ayakma, tohwikelichi ik lauwo hokvto ninak ak o aya hi yo ikbi tok: mihmut fochik puta ka aieninchit ikbi tok.

17. Micha Chitokaka yut yakni ya on tohwikelicha he yo,

18. Micha nitak ma aya he, micha ninak ma aya he, micha tohwikeli kash okhlileka iti filvmmicha he yo, vba shutik a talohli tok: mihma yummut achukma ho Chitokaka yut pisa tok.

19. Mihma opia kvt, micha onnahinli kvt nitak ai ushta hatok.

20. Mihma Chitokaka yut, Oka puta kvt nana ilhkohonli im ilhfiopak asha ya lauachit ikbashke; mikma hushi vt yakni paknaka vba shutik takla ya helit itvnowashke, achi tok.

21. Micha Chitokaka yvt nuni hochito, micha nan okchaya ilhkohonli ilap aiachufa aiyukali oka yvt lauachit ikbi ya moma, micha hushi sanahchi assha puta ilap aiachvfa aiyukali 
ka ikbi tok: micha yummut achukma ho Chitokaka yut pisa tok.

22. Mihma Chitokaka yvt yukpalit, Chelit hush apakna cha, okhvta puta oka ya hush alotashke; mikma hushi vt yakni ya apaknashke, achi tok.

23. Mihma opia kvt, micha onnahinli kvt nitak ishit tahlapi hatok.

24. Mihmvt Chitokaka yvt, Yakni vt nan okchaya puta ilap aiachvfa aiyukali, micha nan vlhpoa, micha nana aka balvlli, micha nampoa yakni paknaka asha ilap aiachvfa aiyukali ka tobachashke, achi tok: mihma yumohmi tok.

25. Micha Chitokaka yot nampoa puta ilap aiachvfa aiyukali, micha nan vlhpoa puta ilap aiachvfa aiyukali, micha nana hosh yakni paknaka om balulli puta ilap aiachvfa aiyukali ka ikbi tok: mihma'yummut achukma ho Chitokaka yot pisa tok.

26. Mihmut Chitokaka yvt, Hatak a ila hobachit pishno pi holba ho kiloh ikbí : mikma nuni okhvta aiasha, micha hushi vba itunowa, micha nan vlhpoa puta, micha yakni moma, micha nana balvlli puta yakni ya om balvlli ya a mominchi hosh $\underline{\mathrm{i}}$ shahlashke, achi tok.

27. Yohmi cha Chitokaka yut ilap akinli holba ho hatak a ikbi tok; Chitokaka holba ho ikbi tok; nakni, micha tek itatuklo ho ikbi tok.

28. Micha Chitokaka yvt yukpali tok, micha Chitokaka yvt, Wayvt hvsh apaknashke, micha yakni ya hush alotowa cha, hush imaiyachashke : michạ nuni okhuta aiasha, micha hushi vba itunowa, micha nan okchaya puta yakni paknaka itunowa moma ka hush $\underline{\underline{i}}$ shahli hashke.

29. Micha Chitokaka yvt, Yakeh! haiyukpulo puta nihi waya yakni paknaka moma aiasha, micha iti puta iti nan vni nihi waya moma ka hvchim issa lishke: hvchim ilhpak ahi oke.

30. Mikmvt nampoa puta yakni paknaka aiasha ka, micha vba hushi puta, micha nana puta yakni paknaka om balvlli

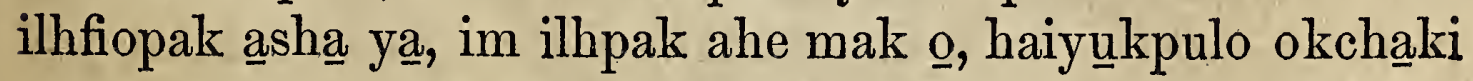
moma ka im issa lishke, achi tok : mihma yumohmi tok. 
31. Mihma Chitokaka yot nana moma ikbi tuk vt pisa ma, yakeh! achukma fehna tok. Mihma opia kut micha onnahinli kut nitak ishit hannali tok.

\section{CHAPTA II.}

YAKOHMI hosh vba moma, micha yakni, mikmvt nana

1 ai i puta kut momut vlhtaiyaha tok.

2. Mihma nitak ont isht untuklo ma Chitokaka yot natoksuli ikbi tuk vt atahli tuk atok: micha nitak ont ishit untuklo ma natoksvli moma isht ahanta tuk vt foha tok.:

3. Micha Chitokaka yvt nitak ont isht untuklo kak o, Chitokaka nana isht ikba chi hosh ikbi tuk toksvli puta kvt yummak o issut a foha hatuk okvt, holitoblichit hullochi tok.

4. Ilvppa puta kak osh Chihowah Chitokaka yut yakni, micha vba moma aiena ikbi tok, nitak a vba moma, micha yakni aiena kvt toba tok a isht annoa hoke;

5. Chihowah Chitokaka yut yakni, micha vba moma jkbi tok nitak a, haiaka itupushi moma kvt yakni ya ik ai iksho ma, haiaka haiyukppulo moma kut ik ai offo kisha tok: Chihowah Chitokaka yut yakni paknaka ik on umbacho

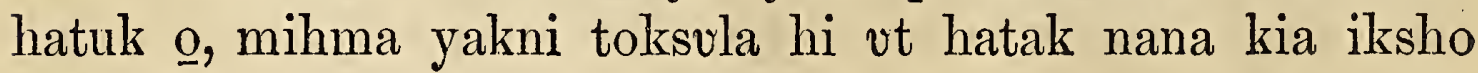
tok.

6. Yohmi tuk kia yakni ya a kifillit vba ia cha, yakni paknaka moma ka shummichi tok.

7. Mihma Chihowah Chitokaka yut yakni ya lukfi ak o ai ishi cha, hatak ikbi mvt, ilhfiopak okchaya ya ibishakni chiluk ak o fioput fohki tok; mihma hatak ash osh shilombish okchaya toba tok.

8. Mihma Chihowah Chitokaka hot hushi akochaka imma yo Eten a osapushi ya ahokchi tok; micha yummak o hatak a ikbi tuk vt bohli tok.

9. Micha Chihowah Chitokaka yut iti pisa achukma puta, micha vpa achukma aiena, iti aiokchaya, micha iti nana achukma, micha nana ik achukmo aiena isht ai ithuna yak kia osapushi iklunna hikia kạ, yakni ya ai offochi tok. 
10. Mihma okhina yvt osapushi yash shummicha chi kut Eten a ai atehli cha, yumma ont ia mut, iti filamohli cha, okhina ushta toba tok.

11. Vmmona hohchifo kvt Pison oke : yummak osh Hafilah yakni moma, yumma toli lakna vt aiasha ka apakfoyupa mak oke.

12. Micha yakni yumma tuli lakna asha kut achukma hoke: yumma betellium micha oniks tvli vt asha hoke.

13. Mikma okhina atukla hohchifo kvt Kihon oke: yummak osh Ehliopia yakni moma ka apakfoyupa mak oke.

14. Mikma okhina atuchina hohchifo kvt Hittekel oke: yummak osh Assilia $\underline{i}$ hushi akochaka imma pit yanulli mak oke. Mikma okhina ai ushta kvt Euflates ak oke.

15. Mihmà Chihowah Chitokaka yut hatak ash ishi cha, Eten osapushi ya a toksulit atona chi ho bohli tok.

16. Micha Chihowah Chitokaka yot hatak ash im apesvt, Osapushi ya iti aiasha puta ka pilla ish ai vpashke.

17. Amba nana achukma, micha nana ik achukmo aiena isht ai ithuna iti ak okuno chik ai vpo kashke: yummak o jsh ai vpa hokvto, i nitak makinli ho chilli pulla hi okvt, achi tok.

18. Mihmut Chihowah"Chitokaka yvt, Hatak vt ilap banot ahanta kvt ik achukmoshke: apelvchi ilap a im vlhpiesa ho im ikbi lashke, achi tok.

19. Micha Chihowah Chitokaka yot nampoa yakni haiaka aiasha moma ka yakni ya ai isht ikbi tuk atok; micha hushi vba itunowa moma aiena ka ikbi tuk vt Alam vt nana ho hochifo na pisa chi kvt pehlichit isht im ona tok; atuk $\underline{o}$ nan okchaya puta ka nana ho Alam vt hochifokma, yvmmak osh hohchifo hatok.

20. Mihma Alam vt nanvlhpoa moma, micha vba hushi puta, micha nampoa haiaka aiasha puta aiena ka hohchifo imvt tahli tok: yohmi kia Alam ak okvno apelvchi ilap a im vlhpiesa kvt iksho tok.

21. Mihma Chihowah Chitokaka yot Alam a nusi kvilo ho nusechi na, nusi tok: mihma naksi foni ya achufalit ishi cha, yưmmak atuk a nipi ya it afummichi tok. 
22. Micha Chihowah Chitokaka yot hatak ash naksi foni im ishi tuk vt ohoyo ho ikbi cha, ilauelit hatak ash isht im ona tok.

23. Mihma Alam vt, Himak a sa foni ya ai achvfa foni yoke, micha sa nipi ya ai achufa nipi yoke : hatak o ai ishi tuk oka, Ohoyo hobchifashke, achi tok.

24. Yvmohmi hoka hatak vt iki, micha ishki ya issa cha, tekchi ak $\underline{0}$ asitia hi oke: mikmvt itatuklo kvt nipi achvfa hi oke.

25. Mihma hatak ash osh micha tekchi itatuklo kut nipi bano hosh ahashwa cha, hofahya iksho tok.

\section{CHA PTA III.}

A TUK o sinti ak osh nahaksich imponna kvt nampoa A haiaka itunowa Chihowah Chitokaka yvet ikbi tuk a moma i shahli tok. Atuk osh ohoyo hash, Yohmi muhli cho? Iti puta osapushi anuka aiasha ka huch ik ai apo kashke, Chitokaka yvt achi tuk oh cho? im achi tok.

2. Mihma ohoyo hash osh sinti ya aiasha ka vni ya il ai vpa hinla hoke:

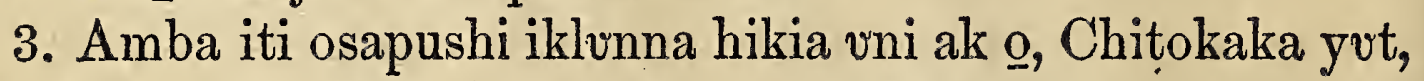
Hvsh ai vpa he keyu, micha hush potola he keyu, yohmi cha hvoch illi ná, achi tuk oke, achi tok.

4. Mihma sinti yash osh, ohoyo ha Huch illi pulla he keyu hoke.

5. Yumma hush ai vpakmvt $\underline{\mathrm{i}}$ nitak mak inli ho, hvechi nishkin vt fatuma hi yo Chitokaka yvt ithana hoka; mikmvt Chitokaka chohmit, nana achukma, micha nana ik achukmo aiena ka hush ithana hi oke, im achi tok.

6. Mihma ohoyo hash osh iti ya vpa achukma ho, micha nishkin ishit pisa ka achukma ho, micha hopoyuksula hi ya iti ya bunna he vlhpesa ho pisa mvt, yummak ash o vni ya ishi cha, vpa tok: mikmvt $\underline{i}$ hatak ash kia aieninchit ipeta na, vpa tok.

7. Mihma itatuklo kvt nishkin vt fatuma cha, nipi bano 
hatuk vt akostininchi tok; mihmut fik hishi yo it achonli cha tikba takali ikbit isht il atahlit okla tok.

8. Atuk osh nitak vt kapussuchit taha ho, Chihowah Chitokaka yut osapushi aya na, im anumpa ha haklot okla tok; mihmot Alam, micha tekchi itatuklo kvt Chihowah Chitokaka ya osapushi iti hiohmaya ka ai in lumbout okla tok.

9. Mihma Chihowah Chitokaka yut Alam a pit $\underline{\mathrm{i}}$ howvt, Katima ak o ish anta cho? im achi tok.

10. Mihma Osapushi ya chim anumpa ha a haiyaklo li mot, sa nipi bano hatuk osh, sa nukshopa cha, luma li tuk oke, achi tok.

11. Mihma, Kvta hosh chi nipi bano hoka, chim anoli tuk o? Ish ai upa he keyu chim apesa li kash o iti ya ish ai upa tuk $\underline{\text { ? }}$ ? achi tok.

12. Mihma hatak as l osh; Si awant ahanta chi ho ohoyo is suma kash osh, iti ya ai ishit vma na vpa li tuk oke, achi tok.

13. Mihma Chihowah Chitokaka yut ohoyo hash, Ilvpput nanta mak o ish yumihchi tuk oh cho? achi tok. Mihma - ohoyo hash osh, Sinti ak osh sa haksichi na vpa li tuk oke, achi tok.

14. Mihma Chihowah Chitokaka yot sinti yash, Iluppa ish yumihchi tuk okvt, kalakshi kvt nanvlhpoa moma, micha nampoa moma yakni haiaka aiasha ka ish $\underline{i}$ shahlishke : chikfoka ak o ishit shalvllit ish aya cha;, chi okchaya takla nitak moma kut lukfi ak o ish pa hi oke.

15. Mikma chishno, micha ohoyo hash micha chishtaiunchololi micha ohoyo ishtaiunchololi aiena ka hvch itin tanampichi la chi hoke: yummak olvut chi noshkobo ha litolikma, chishna mut iỵikotoba ya ish litola hi oke, im achi tok.

16. Micha ohoyo hano, Chakalit isht chilbusha hi ya chim apaknvchi li fehna chi hoke; ilbaiyusha hosh vlla ish esha hi oke; mikmot nana ish ahni kvt chi hatak ak o ish anukchietokma, yummak osh chi shahlit chilauela hi oke; im achi tok. 
17. Micha Alam ano, Chi tekchi im anumpa ha ish $\underline{i}$ haponaklo cha yummak oka chim apesut, Ish ai vpa he keyu hoke, achi li tuk iti ya ish ai vpa tuk oka, chishno ak atuk pulla kak o yakni vt kalakshi hoke ; chi okchaya nitak moma kwt nukhaklo hosh ish ai vpa hi oke.

18. Nana halupa puta, micha shumo aiena ka chi wayvcha he; mikma osapa haiyukpulo aiasha kak o ish ai vpa hi oke.

19. Chi nashuka yut laiyaksha hosh pvska ilhishpa tuk osh falamut lukfi ish toba hi oke; yvmmak o a chishi tuk oka: lukfi chia hokvt, falamvt lukfi ish toba hi oke, im achi tok.

20. Mihma Alam vt tekchi ya Ef hochifo tok: yummak osh okchaya moma ka ishki yoka.

21. Mihma Alam micha tekchi aiena ka Chihowah Chitokaka yot na hakshup o nafohka isht im ikbi cha, fohkvchechi tok.

22. Mihmot Chihowah Chitokaka yot Yakeh! hatak ash osh hupishno akinli chohmi ho tobvt, nana achukma, micha nana ik achukmo aiena ka ithanvshke: yohmi kvt himak a ibbak a pit weli cha, iti aiokchaya ak kia ai ishit vpa cha, okchaya na bilia yoba ná, achi tok.

23. Yohmi hatuk osh Chihowah Chitokaka yut yakni yummak o ai ishi tuk a atoksula chi ho osapushi Eten a a kochi tok.

24. Yvmohmi hosh hatak ash kocha pit chvffichi tok: mihmut osapushi Eten hushi akochaka ont vhli ka Chelubim vhleha, micha iti aiokchaya aiona ya atoni yosh bushpo falaia lihimbi, kanima moma pit bihliblit fullota aiena ho hilechi tok.

\section{CHAPTA IV.}

IHMA Alam vt tekchi Ef a ithana tok; mihma yum11 mak ash osh chakali tuk osh Ken a eshi mvt, Chihowah vt ahni ho hatak o eșhi lishke, achi tok.

2. Atuk osh anonti nakfish Abel a eshi tok. Atuk o 
Abel ak osh chukfulhpoa apesvchi yokma, Ken ak osh osapa toksvli atok.

3. Atuk osh nitak vt ont ai vlhpiesa yut vla ma, yakohmi tok, Ken vt yakni nana awaya ya nan im issa yo Chihowah ya isht im ona tok.

4. Mihma Abel yummak kia nan im vlhpoa chipinta chelahpi puta, micha $\underline{i}$ nia aiena ka isht ona tok. Mihma Chihowah vt Abel micha nan im issa aiena ka im aiokpanchi tok:

5. Amba Ken, micha nan im issa aiena ka ik im ahnincho tok. Mihma Ken vt nukhobela fehna cha, nashuka vt ilapissa tok.

6. Mihma Chihowah vt Ken a Nanta katiohmi ho chi nukhobela clıo? micha nanta katiohmi ho chi nashuka vt ilapissa cho?

7. Ai vlhpiesa ho ish yumohmikma chim aiokpuchi kak a hetuk keyu cho? micha aivlhpiesa ho ish yumhichi keyu hokma nanashvchi vt okhissa yakinli ho itoyula hoke. Mikma Abel nana ahni kvt chishno ak o chi pihissakma, ish $\underline{\mathrm{i}}$ shahla hi oke, im achi tok.

8. Atuk o Ken vt itibapishi Abel a im anumpuli tok; atuk o yakohmi tok, yakni haiaka asshwa ma, Ken vt itibapishi Abel a $\underline{i}$ sanalit wakaya cha, vbi tok.

9. Mihma Chihowah vt Ken a, Chitibapishi Abel muto? im achi tok. Mihma, Ak ithano; vno ak osh itibapishi li ha apistikeli sia hoh cho? achi tok.

10. Mihma, Nanta mak o ish katiohmi tuk oh cho? chitibapishi im issish vt anumpulit aka yạ, auet ai a pahayushke.

11. Yohmi ka himak a yakni ya yummak okvt itakha ya wakummi cha, chitibapishi im issish a chibbak a ai ishi tuk ash a kalakshi hosh ish ayashke.

12. Yakni ya ish a toksuli hokma, himak pilla ka im $\underline{\mathrm{i}}$ hlampko hosh chi waya he keyu hoke: i yakni kohcha yosh yakni paknaka ya fullokahanchit aya hatak chia ha chi hoke, im achi tok.

13. Mihma Ken vt Chihowah yạ, Nan ashvchi lish nan isht vm a palummi vt chito kvt vm atampa fehnushke. 
14. Yakeh! himak nitak a yakni paknaka a kohchit is sa chuffichishke: yohmi na chi nashuka yatukma ai in luma la chishke; micha hatak kunia, micha yakni paknaka ya fullokahanchit aya hatak sia ha chi hoke; atukma yakohma chi hoke, kuna hosh si ahayuchikmut, suba chi hoke, im achi tok.

15. Mihma Chihowah vt, Yvmohmi hoka kvna hosh Ken a vbikma napalvmmi vt mihinlohmi untuklo hosh onvtola chi hoke, im achi tok. Mihmut kuna hosh ahayuchikmvt, ik bo ka hi yo, Chihowah vt Ken a inchuwa ka lapalichi tok.

16. Mihma Ken vt Chihowah itikba yash a kohchvt ia cha, Eten $\underline{i}$ hvshi akochaka Not yakni ak o ont vtta tok.

17. Micha Ken vt tekchi ya ithana tok: atuk o chakali tuk osh Enok a eshi tok; mihma tumaha holihta ya ikbi mut, tumaha holihta yash ushi hohchifo ha a hochifochit Enok hochifo tok.

18. Atuk o Enok a Ilat vt im vtta tok: atuk o Ilat vt Mehuchael a tobachi tok: atuk o Mehuchael vt Mehlusael a tobachi tok : atuk o Mehlusael vt Lamek a tobachi tok.

19. Atuk o Lamek vt ohoyo tuklo ho ishit itauwaya tok: achvfa yvt hohchifo kvt Atah yokma, achvfa yvt hohchifo kvt Sillah atok.

20. Mihma Atah vt Chabal a eshi tok: yummak okvt vlhtipo anuka asht aya, micha nan vlhpoa im asha chohmi kak o iki atok.

21. Mihma itibapishi hohchifo kvt Chubal atok: yummak okvt hap, micha vhlepa chito olachi chohmi kak $\underline{0}$ iki atok.

22. Atuk o Sillah vt yummak kia Tubal-ken asonak lakna, micha tuli pilesa puta ithunanchi ya eshi tok: mihma Tubalken in tek vt Naamah atok.

23. Atuk o Lamek vt tekchi tuklo Atah, micha Sillah yash, Vm anumpa ha ho hakló ; anumpuli li ka oh a haponakló Lamek tekchi hvchia ma: Uno ishit sa hotopa chi mak osh hatak a, micha ishit sa hotopa chi mak osh hatak himita ya vbi li tuk oka. 
24. Ken a vlhtoba kvt mihinlohmi untukla hetuk okma, Lamek a mihinlohmi pokoli untuklo akucha untukla he vilhpesushke, im achi tok.

25. Atuk o anonti Alam vt tekchi ya ithana tok: mihma vlla nakni yo eshi cha, hohchifo ha Selh hochifo tok: Ken vt vbi tuk Ebel ash o vlhtomba ho Chitokaka yut vlla inla ho auet vma hoka, achi tok.

26. Atuk o Selh yummak kia ushi ut im vtta tok: mihma hohchifo ha Enos hochifo tok: yohmi ma hatak puta kut Chihowah hohchifo ha i payvt wakaya tok.

\section{CHAPTA V.}

LUPPAK osh Alam isht-atiaka isht annoa holisso hoke. 1 Chitokaka yut hatak a ikbi tuk nitak fehna ka Chitokaka hak o holba ho ikbi tok.

2. Nakni, micha tek itatuklo ho ikbi tok; mihmvt yuk-. pali cha, tobvt okla nitak makinli ho hohchifo ha Alam hochifo tok.

3. Atuk o Alam vt okchayvt ahanta tok afummi tahlepa achufa cha, pokoli tuchina mut, vlla nakni ilap chohmi, micha ilap holba ho tobachi tok; micha hohchifo ha Selh hochifo tok.

4. Atuk osh Selh a tobachi hayvt nitak ahanta kvt afummi tahlepa untuchina tok; micha ushi vhleha, micha ushetik vhleha ha tobachi tok.

5. Mihma nitak moma Alam vt okchayvt ahanta kvt afummi tahlepa chakkali cha, pokoli tuchina tok; atuk osh illi tok.

6. Atuk o Selh vt okchayvt ahanta tok afummi tahlepa achvfa cha tahlapi mut Enos a tobachi tok.

7. Atuk osh Selh vt Enos a tobachi hayvt afummi tahlepa untuchina cha untuklo ho okchayvt ahanta cha, nshi vhleha, micha ushetik vhleha ha tobachi tok.

8. Mihma nitak moma Selh vt ahanta kvt afummi tahlepa chakkali cha, auah tuklo tok; atuk osh illi tok. 
9. Atuk o Enos vt okchayvt ahanta tok afummi pokoli chakkali mut Kainan a tobachi tok.

10. Atuk osh Enos vt Kainan a tobachi hayvt afummi tahlepa untuchina cha auah tahlapi ho okchayvt ahanta cha, ushi vhleha, micha ushetik vhleha ha tobachi tok.

11. Mihma nitak moma Enos vt ahanta kvt afummi tahlepa chakkali cha tahlapi tok; atuk osh illi tok.

12. Atuk o Kainan vt okchayvt ahanta tok afưmmi pokoli untuklo mvt, Mahalaleel a tobachi tok.

13. Atuk osh Kainan vt Mahalaleel a tobachi hayvt afưmmi tahlepa untuchina cha pokoli ushta ho okchayut ahanta cha ushi vlheha micha ushetik vhleha ha tobachi tok.

14. Mihma nitak moma Kainan vt ahanta kut afưmmi tahlepa chakkali cha pokoli tok; atuk osh illi tok.

15. Atuk o Mahalaleel vt okchayvt ahanta tok afummi pokoli hannali akucha tahlapi mvt, Chalet a tobachi tok.

16. Atuk osh Mahalaleel vt Chalet a tobachi hayvt afummi tahlepa untuchina cha pokoli tuchina ho okchayvt ahanta cha, ushi vhleha micha ushetik vhleha ha tobachi tok.

17. Mihma nitak moma Mahalaleel vt ahanta kvt afummi tahlepa untuchina cha pokoli chakkali akucha tahlapi tok; atuk osh illi tok.

18. Atuk o Chalet vt okchayvt ahanta tok afummi tahlepa achvfa cha, pokoli hannali akucha tuklo mvt Enok a tobachi tok.

19. Atuk osh Chalet vt Enok a tobachi hayut afummi tahlepa untuchina ho okchayvt ahanta cha ushi vhleha micha ushetik whleha ha tobachi tok.

20. Mihma nitak moma Chalet vt ahanta kvt afummi tahlepa chakkali cha, pokoli hannali akucha tuklo tok atuk osh illi tok.

21. Atuk o Enok vt okchaygut ahanta tok afummi pokoli hannali akucha tahlapi mut Mehluselah ya tobachi tok.

22. Atuk osh Enok vt Mehluselah ya tobachi hayut Chitokaka ya ibanowvt ahanta na afummi tahlepa tuchina cha, ushi vhleha, micha ushetik vhleha ha tobachi tok. 
23. Mihma nitak moma Enok vt ahanta kut afummi tahlepa tuchina cha pokoli hannali akucha tahlapi tok.

24. Micha Enok vt Chitokaka ya ibanohowa tok, atuk osh iksho tok: Chitokaka yot ishi tuk'oka.

25. Atuk o Mehluselah vt okchayvt ahanta tok afvmmi tahlepa achofa cha, pokoli untuchina akucha untuklo mot Lamek a tobachi tok.

26. Atuk osh Mehluselah vt Lamek a tobachi hayvt afummi tahlepa untuklo cha, pokoli untuchina akucha tuklo ho okchayvt ahanta cha, ushi vhleha micha ushetik vhleha ha tobachi tok.

27. Mihma nitak moma Mehluselah yot ahanta kvt afummi tahlepa chakkali cha, pokoli hannali akucha chakkali tok: atuk osh illi tok.

28. Atuk o Lamek vt okchayvt ahanta tok afummi tahlepa achofa cha pokoli untuchina akucha tuklo mot vlla nakni yo tobachi tok.

29. Micha hohchifo ha Noah hochifot, Iluppak osh Chihowah yut yakni ya kalakshichi tuk ak o, pibbak na toksuli a pilbusha pulla ka pi hopohlula hi oke, achi tok.

30. Atnk osh Lamek vt Noah ya tobachi ha yut afummi tahlepa tahlapi cha pokoli chakkali akucha tahlapi ho okchayvt ahanta cha, ushi vhleha, micha ushetik vhleha ha tobachi tok.

31. Mihma nitak moma Lamek vt ahanta kut afưmmi tahlepa untuklo cha, pokoli untuklo akucha untuklo tok: atuk osh illi tok.

32. Mihma Noah hot afummi tahlepa tahlapi tok: mihmot Noah hut Shem, Ham, micha Chafelh a tobachi tok.

\section{CHAPTA VI.}

A TUK o yakohmi tok, hatak vt yakni paknaka ya ai A apaknut ishtia cha, ushetik puta kvt im asha ma,

2. Chitokaka ushi vhleha hot hatak ushetik vhleha hut 
aiokli ho pisa tok: mihmvt aiahni tuk aiyukali kvt ishi cha, itauwaya tok.

3. Mihma Chihowah yut, (Hatak vt nanashvchit ahanta ka,) Umi Shilombish vt hatak takla ahanta na, bilia he keyu hoke, hatak vt nipi akinli hoka: yohmi kia i nitak vt afummi tahlepa achvfa cha, pokoli tuklashke, achi tok.

4. Nitak yumma foka ka hatak hofaloha yut yakni paknaka ya aiasha tok; atuk o i himmak ak kia Chitokaka ushi vhleha hvt hatak ushetik vhleha ha itauwayvt maya na, vlla ya okla im eshi tok $\underline{0}$, yummak ash osh hatak hlampko chilita cha chạshpo ka annohowa fehna tok.

5. Atuk o hatak vt yakni paknaka ya a haksi kvt atampa fehna mvt, chukvsh im anukfila nana isht ahni puta kvt peh ik achukmo banot nitak bilia ho Chitokaka yut pihisa tok.

6. Mihmot Chihowah yưt hatak a ikbit yakni paknaka ya o hilechi tuk vt nukhakklot chukvsh hotopa tok.

7. Micha Chihowah yot, Hatak a ikbi le tuk vt yakni paknaka ya a mosholechit, hatak, micha narnpoa, micha nana aka balulli, micha hushi vba itunowa aiena ka yumihchi lashke; ikbi le tuk vt sa nukhakklo hokvt, achi tok.

-8. Yohmi kia Noah hut Chihowah itikba ya isht $\underline{i}$ kana ya ai ahayuchi tok.

9. Ilvppak osh Noah ya anoli holisso hoke: Noah hut hatak aivlhpiesa, micha ai itishali yumma aiahli hosh ahanta tok; micha Noah hut Chitokaka ya iba nohowa tok.

10. Micha Noah hvt ushi tuchina Shem, Ham, micha Chafelh a tobachi tok.

11. Yakni ak kia Chitokaka itikba ya okpulo tok: micha yakni ya nan ik vlhpeso bano kak osh alotowa tok.

12. Atuk o Ckitokaka yut yakni ya et pisa tok, mihma yakeh! okpulo tok; nipi moma kut yakni paknaka ya ai il aiokpunit tahli hatuk oka.

13. Mihmvt Chitokaka yvt Noah ya Nana nipi moyuma kut sa tikba ya ant aivhli hoke; Iluppa pulla hatuk mak o yakni paknaka ya nan ik vlhpeso bano kak osh alotowa 
hoka: yohmi hoka yakeh! yummak atukma mosholichit yakni ya ai okpune la he akinli hoke.

14. Kofi iti yo chi peni chito ish ikbashke : micha peni chito anukaka ya abohushi puta ish ai ikbashke; micha anukaka, micha kocha aiena ka tiak nia isht ish apoluslashke.

15. Micha yakohmichit ish ikbashke: Peni chito yut falaia kvt kubit tahlepa tuchina, pvtha kvt kubit pokoli tahlapi, micha chaha kvt kubit pokoli tuchinashke.

16. Peni chito ya atohwikeli ya ish ai ikbi cha kubit achvfa ho paknaka ya işh ai atahlashke : micha peni chito okhowata yak o okhissa ya ish hilechashke: micha aka ishtaiyopi, atukla, micha ont atuchina patulhpo yo ish ikbashke.

17. Atuk o yakeh! vno, vno fehna kvt nipi puta ilhfiopak okchaya a foyuka ka mominchit shutik nutaka ya a mosholichi la chi kut oka falama yo yakni paknaka ya isht aya la chi hoke: mikma nana puta yakni paknaka aiasha kvt illa chi hoke.

18. Amba chishno yano kvllochit na chitim apesa la chi hoke: mikma chishno vt, micha chiso vhleha, micha chi tekchi, micha chiso vhleha tekchi aiena kvt chi apiha ho peni chito ya ish chukowa hi oke.

19. Mikmvt nan okchaya nipi puta moma tuklo bieka ho pehlinchit peni chito ya ish chukowa na, chibai okchayashke ; nakni, micha tek ashke.

20. Hushi puta kvt ilap ai achvfa aiyukali, micha nampoa puta kvt ilap ai achvfa aiyukali, micha nana aka balvlli yakni paknaka aiasha moma ilap ai achvfa tuklo aiyukali hosh okchaya chi hosh chim ai via hi oke.

21. Micha nan ilhpak vpa chatuk a mominchit chishno inli osh hoyot ish itunahlashke; mikma yummak osh chishno, micha yummak ash aiena kvt huchim ilhpak ashke, in achi tok.

22. Iluppak o Noah hut yumihchi tok: Chitokaka yut im apesa tuk mak o moma yumihchi tok. 


\section{CHAPTA VII.}

MIHMA Chihowah vt Noah ya I chuka achufa moma aienvt peni chito ya ant hush chukowashke: aiitishali achvfa iluppa chishno ak osh aivlhpiesa kvt sa tikba ya ish ahanta na chi pihisa li hoke.

2. Nampoa kashofa puta kvt nakni, micha tek itichapa hosh untuklo bieka, micha nampoa kashofa keyu vt nakni, micha tek itichapa hosh tuklo bieka,

3. Micha hushi vba itunowa nakni, micha tek itichapa hosh untuklo bieka ho ish ishi cha, ish okchalinchi na, ishtai unchuloli vt yakni paknaka moma ka aiashashke.

4. Nitak untuklo momakma, nitak pokoli ushta, micha ninak pokoli ushta ho yakni paknaka ya on umbvche la chị hoke; micha nan okchaya puta ikbi li tuk vt yakni paknaka ya a mosholichi la chi hoke, im achi tok.

5. Mihma Chihowah yut ịm apesa tuk mak o Noah hut mominchit yumihchi tok.

6. Mihma Noah hvt afummi tahlepa hannali ho oka falama vt yakni paknaka ya onvtola tok.

7. Mihma oka falama yut aya chi pulla kak $\underline{\text {, Noah hot }}$ ushi vhleha, micha tekchi, micha ushi vhleha ha tekchi vhleha aiena kvt apiha ho peni chito ya chukowa tok.

8. Nampoa kashofa, micha nampoa kashofa keyu, micha hushi puta, micha nana hosh yakni paknaka ya om balulli chatuk moma mot,

9. Chitokaka yut Noah ya im apesa tuk mak o, tuklo aiyukali hosh nakni, micha tek ita tuklo hosh Noah ya im onvt peni chito ya chukowa tok.

10. Atuk o yakohmi tok, nitak untuklo ha ya , oka falama yut yakni paknaka ya onvtoyula tok.

11. Noah hvt vtta tok a afummi tahlepa ishit hannali hosh hushi atukla, hushi yash nitak ont isht auah untuklo, mih nitak fehna ho oka hofobi chito oka akohcha puta kvt momvt mitahli tok, mikma vba shutik okhissushi puta kvt tiwa tok. 
12. Mihma nitak pokoli ushta, micha ninak pokoli ushta ho yakni paknaka ya ai on umba tok.

13. Nitak yumma fehna akinli ho Noah, micha Șhem, micha Ham, micha Chafelh Noah ushi vhleha hash, micha Noah tekchi, micha ushi vhleha tekchi tuchina aiena kvt apiha ho, peni chito yash chukowa tok.

14. Yvmma, micha nampoa puta ilap ai achufa aiyukali, micha nan vlhpoa moma ilap ai achvfa aiyukali, micha nana balvlli puta yakni paknaka om balvlli ilap ai achvfa aiyukali, micha hushi puta ilap ai achvfa aiyukali, hushi chipinta ai il aiyukali puta aiena tok.

15. Micha nanipi asha puta kvt ilhfiopak okchaya mut atakali hatuk vt momvt tuklo bieka hosh Noah ya im onvt, peni chito yash ibachukowa tok.

16. Micha chukowa kvt, Chihowah yvt im apesa tuk

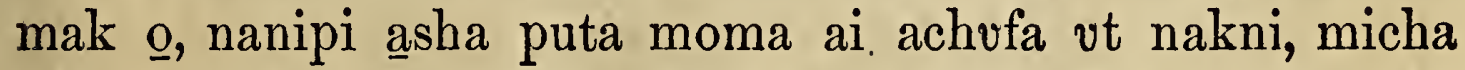
tek aiena kut chukowa tok: mihma Chihowah yut im okhishta tok.

17. Mihma oka falama yvt nitak pokoli ushta ho yakni paknaka ya onvtoyula tok: mihma oka yvt i shahlit mahaya mut, peni chito yash shalit wakeli na yakni ya ai vba takali tok.

18. Mihma oka yut yakni paknaka ya okchito kvt $\underline{i}$ shahlit mahaya fehna tok; mihma peni chito vt oka paknaka ya takalit aya tok.

19. Mihma oka yut yakni paknaka ya okchito atampa fehna tok; mihma nunih chaha moma, shutik, nutaka moma aiasha tuk vt ompoholmo tok.

20. Oka chito isht $\underline{i}$ shahlit vba ia kvt kubit auah tahlapi ho isht vbanubli tok; mihma nunih chaha puta kvt ompoholmo tok.

21. Mihma nana nipi moma yakni paknaka ya ai itunowa tuk, hushi, micha nanulhpoa, micha nampoa, micha nana balvlli puta yakni paknaka om balvlli tuk, mikmut hatak puta aiena kvt illi tok:

22. Ilhfiopak okchaya vt ibishakni ya foyuka hatuk moma yakni shila aiasha tuk vt momvt illi tok. 
23. Micha nan okchaya moma yakni paknaka ya aiasha tuk, hatak, micha nan vlhpoa, micha nana balvlli puta, micha hushi vba itunowa aiena kvt illit taha tok; yummak osh yakni paknaka ya ai illit taha tok: mihma Noah, micha apihut peni chito ya aiasha tuk ak illa hosh okchaya tok.

24. Mihma oka yut yakni paknakka ya ai okchito kut onvtoyula na nitak tahlepa achufa cha pokoli tahlapi tok.

\section{CHAPTA VIII.}

WIHMA Chitokaka yut Noah yạ, micha nan okchaya 1 moma, micha nampoa moma apihut peni chito ya alhto tuk ash ithaiyana tok: mihma Chitokaka yot yakni paknaka ya mahli hlopullichi ma, oka yut halata tok.

2. Mihma oka hofobi chito oka akohcha puta, micha shutik okhissushi puta aiena kut vlhkuma ma, umba chito vba minti kvt oktvpa tok.

3. Mihma oka yut yakni "paknaka ya a falamut yanahanli na bilia tok: atuk osh nitak tahlepa achufa cha pokoli tahlapi kut ont taha ha ya oka yvt shipa tok.

4. Mihma hushi isht untuklo, hvshi yummvt nitak ont isht auah untuklo kak $\underline{0}$, peni chito yvt nunih chaha Alalat paknaka ya ontula tck.

5. Mihma oka yvt tahvt mahaya na hushi ont ishit pokoli tok: hushi ont ishit pokoli hushi yvmmut nitak vmmona ma, nunih chaha puta paknaka yot haiaka tok.

6. Atuk o yakohmi tok, nitak pokoli ushta kvt ont taha ma, Noah hvt peni chito ikbi tok ash okhissushi ya tiwi tok:

7. Micha fula chito yo kohchechi na, kohcha mut il afalamạhowa na, yakni paknaka ya oka yot a shiput taha tok.

8. Micha oka yut yakni paknaka ya a shiput tahakmá, nana tukma akostinincha chi hosh puchi yoshuba ak kia kohchechi tok.

9. Yohmi tuk kia pvchi yoshoba yvt iyi yvt ai o hikivt foha hi vt ik ahayucho mot, falamvt im vla cha, peni chito 
yash chukowa tok: oka yut yakni paknaka moma ka onvtoyula hatuk oka: yohmi ma ibbak a pit weli cha, ishit peni chito et il iba fohki tok.

10. Atuk osh anta na nitak untuklo akinli ma, anonti pvchi yoshuba yash peni chito ya kohchechi tok.

11. Atuk o opia ma pvchi yoshuba ash osh falamvt im vla mvt, yakeh! itih ha alif hishi okchaki vt foyuka tok. Yohmi ho yakni paknaka ya oka yut a shiput taha tuk a Noah hut akostininchi tok.

12. Atuk osh anonti nitak untuklo ho anta tuk osh, puchi yoshuba yash a kohchechi tok: mihma yummak osh himakma falamut ik im alo tok.

13. Atuk o yakohmi tok, afummi tahlepa hannali cha achvfut aiena, hvshi vmmona, micha hvshi yummut nitak vmmona ma, oka yvt yakni paknaka ya a shiput taha tok: mihma Noah hut peni chito isht ompoholmo ash kanvllichi cha, hopokoyo ma, yakeh! yakni paknaka yvt shila atok.

14. Atuk osh hvshi atukla, hushi yvmmut nitak ont ishit pokoli tuklo akucha untuklo ma, yakni vt shilvt taha tok.

15. Mihma Chitokaka yut Noah a im anumpulit,

16. Chishno, micha chi tekchi, micha chiso vhleha, micha chiso vhleha tekchi puta aiena ka pehliechit peni chito ya ish a kohchashke.

17. Nan okchaya nipi ilaiyukali puta moma chi apehvt maya tuk, hushi, micha nan vlhpoa, micha nana balulli yakni paknaka om balvlli aiena ka pehliechit ish kohchashke; yohmi na yakni paknaka ya alauachi fehnvt chelashke, micha yakni paknaka ya a wayut apaknashke, achi tok.

18. Mihma Noah hvt ushi vhleha, micha tekchi, micha. ushi vhleha tekchi puta itvpehvt kohcha tok.

19. Nan okchaya puta, nana balvlli puta, micha hushi puta, micha nana hosh yakni paknaka om balulli, ilap aiachvfa aiyukali momvt peni chito ya kocha wiha tok.

20. Mihma Noah hot Chihowah ya alta im ikbi cha, nan vlhpoa kashofa puta, micha hushi kashofa puta ai ishi cha, alta yash onochit hukmit isht aiokpvehi ya im issa tok. 
21. Mihma Chihowah yut na balama achukma ka huwa tok; micha Chihowah yut ilap chukvsh akinli ka, Hatak

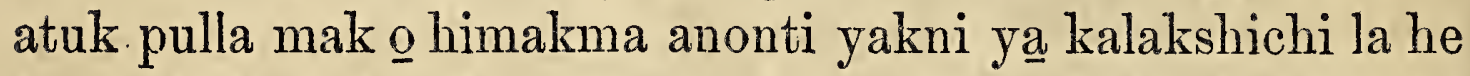
keyu hoke; hatak chukvsh im anukfila hot vlla tomba makinli ho ik achukmo hoka: micha yumihchi li tuk a anonti himakma chohmichit nan okchaya moma ka issot kanchi la he keyu hoke.

22. Yakni vt talaia moma ka, nahokchi nitak, micha nawaya vmo, micha kapussa, micha lushpa, micha toffa, micha hushtula, micha nitak, micha ninak aiena kvt hokofa he keyu hoke, achi tok.

\section{CHAPTA IX.}

MIHMUT Chitokaka yut Noah ya micha ushi vhleha 1 aiena ka yukpalit, Hush wayut apakna cha, yakni paknaka ya hvsh alotowashke.

2. Yohmikma isht a hvchi nukshopa, micha isht a hvchi komota aiena kut nampoa puta yakni paknaka aiasha ka moma, micha hushi puta vba itvnowa moma, micha nana hosh yakni paknaka aya moma, micha nuni okhvta aiasha ka moma onvtoyula hi oke; hvch ibbak a fohka hoke.

3. Nan okchaya itunowa moma kvt hvchim ilhpak achi hoke: haiyukkpulo okchaki atok akinli ho ai itilauechit nan okluha ka hvchim issa li hoke.

4. Amba nipi vt isht okchaya yummot im issish ak oka aieninchit hush pa ná,

5. Atukma hvchim ilhfiopak hvchim issish ak o a hoyo li pulla hi oke: nan okchaya moma ka im a hoyo likmvt, hatak ibbak a a hoyo la hi oke; hatak a itibapishi puta hatukma ibbak a hatak im ilhfiopak a a hoyo la hi oke.

6. Kvna hosh hatak im issish a hlatublikma, ilap im issish ma hatak osh hlatubla hi oke: Chitokaka ya holba ho hatak a ikbi tok oka.

7. Yohmi ka hvchishno vt wayut hush apaknashke; yakni paknaka ya a waya fehnvt ai apaknvt hush aiashashke, im achi tok. 
8. Micha Chitokaka yvt Noah, micha ushi vhleha aiena ka im anumpulit,

9. Uno, yakeh! vno vt huchishno, micha hvchim ishtatiaka chi akaiya aiena,

10. Micha nan okchaya puta hvoch ibafoyuka kạ, hushi, nan vlhpoa, micha nampoa puta yakni paknaka aiasha hvch iba foyuka kot peni chito yash kohcha tuk, nampoa puta yakni paknaka aiasha ka kvllochit nana itim apesa lishke.

11. Micha kvllochit nana hvchitim apesa la chi hoke: mikma himakma nanipi moma kvt oka falama isht illit taha he keyu hoke; micha himakma oka falama vt aya cha, yakni ya okpuna he keyu hoke, achi tok.

12. Micha Chitokaka yvt, Ilvppak osh vno, micha hvchishno ya micha nan okchaya moma hvchi apeha ka ai itishali bilia ka nana itim apesa itim ikbi li ka isht atokowa yoke.

13. Uno vt a hinakbitepuli ya hoshonti anukaka ya takalichi li hoke; mikma yummak osh vno vt yakni ya nana itim apesa li tuk isht atokowa yosh talaia achi hoke.

14. Atukma yakohma hi oke, hoshonti ha yakni paknaka isht aya likma, hinakbitepuli hot hoshonti ha takalit haiaka hi oke.

15. Mikma vno, micha hvchishno, micha nanokchaya nipi âsha moma aiena ka nana itim apesa vt a pitin takla takali ka ithaiyana la hi oke; mikma himakma oka yut oka falama toba cha, nana nipi asha moma ka oka tumola he keyu ka hi oke.

16. Mikma hinakbitepuli hut hoshonti ha takala hi oke; mikma nana itim apesa ont ataha iksho vt Chitokaka, micha nan okchaya nipi asha moma yakni paknaka aiasha aiena kvt im ai itin takla takali ka ithaiyana la chi kvt pihisa la hi oke, achi tok.

17. Micha Chitokaka yut Noah yạ, Iluppak osh nana itim apesa vno vt nan okchaya nipi asha moma yakni pakna$\mathrm{ka}$ aiasha ka kullochit itim apesa li tuk a isht atokowa yoke, im achi tok.

18. Mihma Noah ushi vhleha peni chito ya asht kocha 
wiha kvt Shem, micha Ham, micha Chafelh ak atok: atuk o Ham ak osh Kenan a iki atok.

19. Iluppak osh Noah ushi vhleha tuchina mak oke; atuk o yummak atok ak $\underline{0}$ yakni moma ka ai ita fimimpa tok.

20. Mihma Noah hut yakni a toksvlit wakaya cha, osapushi pakupi ahollokchi ya ikbi tok.

21. Atuk osh oka pạki ya ishko cha haksi tok; mihmut i shahbi hosh im vlhtipo anukaka ya itonla tok.

22. Mihma Kenan iki Ham vt iki vt nipi bano ka pisa mot, itibapishi tuklo kocha ashwa tuk a im anoli tok.

23. Mihma Shem, micha Chafelh ita tuklo kak osh anchi yo ishi cha, tahchi ha onochit bikka cha, olbulh-pelvt ia hosh iki nipi bano tuk a ont ompohomo tok: micha nashuka vt i filvmmi hatuk osh iki nipi bano ka ik pesot okla tok.

24. Atuk @ Noah hvt oka paki ishkot haksi tuk vt kostini mvt, ushi nakfish ak osh nana im akaniohmi tuk a akostininchi tok.

25. Micha, Kenan vt kalakshashke; tishu vhleha in tishu

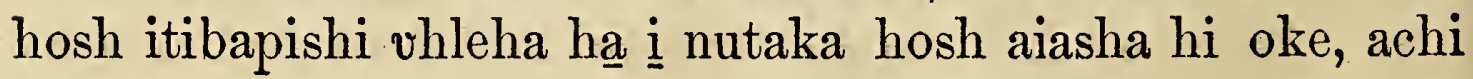
tok.

26. Mihmut Shem in Chitokaka Chihowah yvt holitopashke: mikma Kenan vt in tishu ha hi oke.

27. Chitokaka yvt Chafelh a im auatvchikma, Shem im vlhtipo puta ka vttut aya hi oke ; mikma Kenan vt in tishu ha hi oke, achi tok.

28. Atuk osh Noah hvt, oka falama vt aya ha ya okchayot ahanta na, afummi tahlepa tuchina cha, pokoli tahlapi tok.

29. Mihma Noah hut nitak moma ahanta kut afummi tahlepa chakkali cha, pokoli tahlapi tok: atuk osh illi tok.

\section{CHAPTA X.}

TLUPPAK osh Noah ushi vhleha, Shem, Ham, micha 1 Chafelh aiena ka isht annoa holisso hoke: yohmi ka 
yummak okvt oka falama vt aya ha yak o ushi vt im aiasha tok.

2. Chafelh ushi vhleha hvt, Kome, micha Makok, micha Matai, micha Chafan, micha Tubal, micha Meshek, micha Tilas ak atok.

3. Mikma Kome ushi vhleha hvt, Ashkenas, micha Lifalh, micha Tokamah ak atok.

4. Mikma Chafan ushi vhleha hot, Elishah, micha Tashish, Kittim, micha Totanim ak atok.

5. Yakohmi kak osh i yakni Chentail i yakni tashaiyi puta ka ilap aiokla achvfa im anumpa achvfa cha, in chuka achufa ishtatiaka aiyukalit ita kushkoa tok.

6. Mikma Ham ushi vhleha hvt Kush, micha Mislaim, micha Fut, micha Kenan ak atok.

7. Mikma Kush ushi vhleha hvt Seba, micha Hafilah, micha Sabtah, micha Laamah, micha Sabteka ak atok: mikma Laamah ushi vhleha hvt Sheba micha Tetan ak atok.

8. Mihma Kush vt Nimlot a tobachi tok: atok o yummak osh hatak palummi tobut ishit mahaya tok.

9. Chihowah itikba ya hatak owvtta fehna yosh ahanta tok: yohmi tok mak o hatak owvtta fehna mak osh Chihowah itikba hikia Nimlot atok mak o chohmi hoke, achi beka tok.

10. Yohmi ka im apehlichika ishtia vmmona kvt Shina yakni taloha Babel, micha Elek, micha Akkat, micha Kalneh ak atok.

11. Yakni yummak o Asha vt kohchvt ia hosh Ninefeh, micha tumaha holihta Lehobolh, micha Kalah ya ikbi cha,

12. Ninefeh, micha Kalah iti takla ak o Lesen a ai ikbi tok: mih mak osh tumaha holihta chito atok.

13. Mihma Mislaim vt Lutim, micha Anamim, micha Lehabim, micha Naftuhim,

14. Micha Pahlusim, micha Kasluhim (yummak o Filistim okla hut a kohcha tok,) micha Kaftolim aiena ka tobachi tok.

15. Mikma Kenan vt ushi akni fehna Siton, micha Helh, 16. Micha Chebusait, micha Amolait, micha Kikasait, 
17. Micha Hifait, micha Akait, micha Sinait,

18. Micha Afatait, micha Semalait, micha Hamahlait aiena ka tobachi tok: atuk o $\underline{\mathrm{i}}$ himmakma Kenanait okla in chuka achvfa vhleha hot ita fimimpa tok.

19. Micha Kenanait okla im aivhli vt Siton a vttvt ia hosh Kela isht ona ka maya hosh Kasa ya ona tok: Sotom, micha Komolloh, micha Atmah, micha Seboim ish ia ka maya hosh Lasha ya ont vhli tok.

20. Ilvppa puta kak osh Ham ushi vhleha ilap in chuka achvfa ishtatiaka aiyukali, anumpa aiachvfa, okla talohvt ilap i yakni aiyukali aiasha mak oke.

21. Mihma Ebe im vlla vhleha moma ka iki, akni Chafelh a itibapishi Shem ak kia vlla vt im aiasha tok.

22. Shem im vlla vhleha hot Elam, micha Asshu, micha Afaksat, micha Lut, micha Alam ak atok.

23. Mikma Alam im vlla vhleha hvt Us, micha Hul, micha Kehle, micha Mash ak atok.

24. Mihma Afaksat vt Salah ya tobachi tok: mihma Salah hut Ebe ya tobachi tok.

25. Atuk o Ebe vt ushi tuklo hosh im ashwa tok: achvfa kvt hohchifo kut Pelek atok: yvmma i nitak ak o yakni vt itakushkoa tok: mikma itibapishi hohchifo kvt Choktan atok.

26. Mihmvt Choktan vt Almotat, micha Shelef, micha Hasamafelh, micha Chelah,

27. Micha Hatolam, micha Usal, micha Tiklah,

28. Micha Obal, micha Abimael, micha Sheba,

29. Micha Ofa, micha Hafilah, micha Chobab aiena ka tobachi tok: ilvppa puta kak osh momvt Choktan a ushi vhleha hatok.

30. Mihmvt abinili kvt Mesha ak o aivhlit ia hosh, hvshi akochaka nunih Sefa ak o ont ai vhli tok.

31. Iluppa puta kak osh Shem ushi vhleha ilap in chuka achvfa ishtatiaka aiyukali, anumpa aiachvfa okla talohvt ilap i yakni aiyukali aiasha mak oke.

32. Ilvppa puta kak osh Noah im vlla vhleha in chuka achvfa puta ilap ishtatiaka aiyukali okla taloha mak oke: 
micha oka falama vt aya ha ya iluppa puta tok ak o oklushi puta kvt yakni ya itakvshkoa tok.

\section{CHAPTA XI.}

IHMA yakni moma kvt itih, micha anumpa aiena kvt 1 achufa tok.

2. Atuk o yakohmi tok, hushi akochaka imma ya ashvt itvnowvt mihinti mot, yakni patali vt Shina yakni ya talaya na, ant ahayuchi tok: mihmvt yvmmak $\underline{0}$ okla aiasha tok.

3. Mihmvt, Inta! lukfi nuna kiloh ikbí, micha achukmalit eho hukmashke, itim achi tok. Mihmvt tvli a hetuk $\underline{a}$ lukfi nuna yokma, anonti isht vlhpolosa ya na shinvshbi yosh okla im aiasha tok.

4. Mihmvt, Inta! hupimmi hachi ka tumaha holihta micha chuka chaha ai atoni paknaka yut shutik a ona ho iloh ikbashke; micha pi hohchifo iloh ikbashke; keyukmvt ita fimimput yakni paknaka moma ka il itunowa yoba ná, okla achi tok.

5. Mihma Chihowah vt hatak im vlla vhleha hvt tvmaha holihta, micha chuka chaha ai atoni ikbi ka pisa chị hosh akowvt vla tok.

6. Micha Chihowah vt, Yakeh! okla hvt achvfa, micha moma kvt im anumpa hot achufushke: atuk osh ilvppa yumihchit isht ivshke: yohmi ka himak a yumihcha chi im anukfila tuk a nana hosh im ai ataklumma hi vt ikshoshke.

7. Inta! pit kil akowa cha, im anumpa ha kil im it aiokomi na, ilap bika yvt im anumpa ha ik itim akostinincho, kashke, achi tok.

8. 'Yumohmi ho Chihowah vt yumma a kanvllichit yakni paknaka moma ka ita fimibli tok: mihma tumaha holihta ikbi tuk vt issa tok.

9. Yumohmi hatuk o yummvt Babel hohchifo tok: yưmmak o Chihowah vt yakni moma im anumpa ha ai it- 
im aiokomi tok oka: micha yumma Chihowah vt akanullichit yakni paknaka moma ka ita fimibli tok.

10. Ilvppa puta kak osh Shem isht ai unchololi hoke: Shem vt afummi tahlepa achufa hosh oka falama vt aya ha ya afummi tuklo ma, Afaksat a tobachi tok.

11. Atuk osh Shem vt Afaksat a tobachi ha yvt afummi tahlepa tahlapi ho okchayvt ahanta cha, ushi vhleha, micha ushetik vhleha ha tobachi tok.

12. Atuk o Afaksat vt okchayvt ahanta tok afummi pokoli tuchina akucha tahlapi hosh Salah ya tobachi tok.

13. Atuk osh Afaksat vt Salah ya tobachi ha yut afummi tahlepa ushta cha tuchina ho okchayvt ahanta cha, ushi vhleha, micha ushetik vhleha ha tobachi tok.

14. Atuk o Salah vt okchayvt ahanta tok afưmmi pokoli tuchina hosh Ebe ya tobachi tok.

15. Atuk osh Salah vt Ebe ya tobachi ha yut afvmmi tahlepa ushta cha tuchina ho okchayvt ahanta cha ushi vhleha, micha ushetik vhleha ha tobachi tok.

16. Atuk o Ebe yut okchayut ahanta tok afummi pokoli tuchina akucha ushta hosh Pelek a tobachi tok.

17. Atuk osh Ebe yot Pelek a tobachi ha yot afummi tahlepa ushta cha, pokoli tuchina ho okchayut ahanta cha ushi vhleha, micha ushetik vhleha ha tobachi tok.

18. Atuk o Pelek vt okchayvt ahanta tok afummi pokoli tuchina hosh Leu ya tobachi tok.

19. Atuk osh Pelek vt Leu ya tobachi ha yut afummi tahlepa tuklo cha chakkali ho okchayvt ahanta cha, ushi vhleha, micha ushetik vhleha ha tobachi tok.

20. Atuk o Leu vt okchayvt ahanta tok afummi pokoli tuchina akucha tuklo hosh Seluk a tobachi tok.

21. Atuk osh Leu vt Seluk a tobachi ha yot afummi tahlepa tuklo cha, untuklo ho okchayvt ahanta cha, ushi vhleha, micha ushetik vhleha ha tobachi tok.

22. Atuk o Seluk vt okchayvt ahanta tok afummi pokoli tuchina hosh Naho ya tobachi tok.

23. Atuk osh Seluk vt Naho ya tobachi ha yut afưmmi 
tahlepa tuklo ho okchayvt ahanta cha, ushi vhleha, micha ushetik vhleha ha tobachi tok.

24. Atnk $\underline{0}$ Naho vt okchayut ahanta tok afummi pokoli tuklo akucha chakkali hosh Telah ya tobachi tok.

25. Atuk osh Naho ut Telah ya tobachi ha yut afummi tahlepa achufa cha ahbichakkali ho okchayut ahanta cha, ushi vhleha, micha ushetik vhleha ha tobachi tok.

26. Atuk o Telah vt okchayvt ahanta tok afvmmi pokoli untuklo ho Eblam, Naho, micha Halan aiena ka tobachi tok.

27. Yohmi ka iluppa puta kak osh Telah isht aiunchololi hoke: Telah vt Eblam, Naho, micha Halan aiena ka tobachi tok: atuk 0 Halan vt Lot a tobachi tok.

28. Mihmut Halan vt iki Telah ya tikba, yakni aivtta tok Kaltea talaia Uh ai illi tok.

29. Atuk o Eblam, micha Naho ita tuklo kvt ohoyo itauwayut okla tok: Eblam tekchi hohchifo kut SSalai atok; mikma Naho tekchi hohchifo kut Milkah atok, Milkah ya iki, micha Iskah ya i iki Halan a ushetik atok.

30. Yohmi kia Salai vt ushi iksho yatuk oṣh vlla ik im iksho tok.

31. Mihma Telah vt ushi Eblam, micha Halan ushi Lot, ilap ushi akinli ushi, micha ushi Eblam tekchi ipok Salai aiena ka pehlichi cha Kenan yakni ya ona chi hosh Kaltea yakni talaia Uh ha kohchut ja tok: atuk osh Halan a ona cha, yummak o aiasha tok.

32. Mihma Telah i nitak puta kvt afummi tahlepa tuklo cha tahlapi tok; atuk osh Telah vt Halan ak o ai illi tok.

\section{CHAPTA XII.}

YOHMI ka Chihowah vt Eblam a, Chi yakni chi kanomi 1 vhleha micha chiki in chuka achvfa aiena ka $\underline{i}$ filvmmit a kohchvt ayvt yakni chi pesvehi la chị ka ish onashke :

2. Mikma okla chito fehna a chikbi la he, mikmvt chi 
yukpali likmvt, anonti chi hohchifo ha holitoblichi la hi oke; mikma ishit na chi yukpa yachi hoke.

3. Atukma kuna hosh anumpa yukpali chi onochikma yukpali la hi oke; mikmut kuna hosh anumpa kalakshichi chi onochikma, kalakshichi la hi oke : mikma chuka achvfa puta yakni paknaka aiasha kvt chi aiyukpa hi oke, achi tuk atok.

4. Yohmi ma Chihowah yut im achi tuk mak o, Eblam vt ia tok, mihma Lot vt awant ia tok: mihma Eblam vt Halan a kohchut ia mvt, afummi pokoli untuklo akucha tahlapi tok.

5. Micha Eblam vt tekchi Salai, micha itibapishi ushi Lot, micha i nanvlhpoa ahayuchi tuk vt molna, micha Halan a tishu okla ai ahayuchi tuk vt pehlichi cha, Kenan yakni ya okla ona chi hosh kohchut ia tok; atuk osh Kenan yakni ya okla ona tok.

6. Mihma Eblam vt yakni yash hlopullit ayvt Moleh i baiyi hieli Sikem a ona tok. Yohmi ma Kenan okla hot yakni ya aiasha hatok.

7. Mihma Chihowah yout Eblam a i haiaka cha, Yakni iluppa chim ishtatiaka ya ima la hi oke, im achi tok: mihma yummak o Chihowah yut $\underline{i}$ haiaka tuk ash alta ya im ikbi tok.

8. Atuk osh yumma vttut kanvllit ia mut, Behlel $\underline{i}$ hvshi akochaka imma nunih chaha hak o ona cha, Behlel vt hvshi ai okatula pilla talaya ho, micha Hai vt hưshi akochaka pilla ma, talaya ho im vlhtipo ya hilechi tok: mihmvt yummakma Chihowah ya alta im ikbi cha, Chihowah ya hochifot i pahaya tok.

9. Mihmut, Eblam vt ivt oka mahli pilla ho mạhaya tuk vt moma hosh aya tok.

10. Atuk o yakni yash hohchvfo hot asha tok: mihma Eblam vt Echip yummak o anta chi hosh ia tok; yakni yash hohchufo kvt palvmmi fehna hatuk oka.

11. Atuk osh yakohmi tok, Echip a chukowa chi mak osh bilikasi ona mut, tekchi Salai yạ, Pisa ka ohoyo chi aiokli ka himak a ithana lishke: 
12. Yưmohmi hoka yakohma chi hoke, Echip okla hut chi pisakmvt, Ilvppak osh tekchi oke, acha chi hoke; mikmvt vno ya subi cha, chishno yano chi okchalincha chi hoke.

13. Chishno vt, In tek sia hoke, ish achashke, chim asilhha lishke: yvmohmi ho chishno ak atuk pulla mak o nana isht vm achukmashke: micha chishno atuk pulla mak o vmi shilombish vt okchayashke, im achi tok.

14. Atuk o yakohmi tok, Eblam vt Echip a ont chukowa ma, Echip okla hvt ohoyo hut aiokli fehna ho pisa tok.

15. Falaoh $\underline{i}$ holitopa vhleha hak kia pisa mut ahnichit Falaoh itikba ya anohonli tok; mihma ohoyo hash ilauelit Falaoh in chuka ya isht ona tok.

16. Mihma yummak atuk pulla mak o Falaoh vt Eblam a i kana fehna tok: mihma chukfulhpoa, micha wak, micha issuba nashoba nakni, micha hatak tishu vhleha, micha ohoyo tishu vhleha, micha issuba nashoba tek, micha kamel puta aiena kvt im asha hatok.

17. Mihma Chihowah yot Eblam tekchi Salai ak atuk pulla mak o Faloah micha in chuka achufa aiena ka napalummi chinto ka onochi tok.

18. Mihma Falaoh vt Eblam a hoyo cha, Iluppak osh nanta mak o is sa yumihchi tuk oh cho? nanta katiohmi ho chi tekchi atuk a chik sum anolo túk oh cho?

19. Nanta katiohmi ho, Ilupput an tek oke, ish achi tuk o? yohmi na, ishit sa tekchi ikbit itanwaya la he tuk oka; yohmi hoka chi tekchi ya ish ilaueli cha, ish iashke, achi tok.

20. Micha Falaoh vt in tushka vhleha ha yummak ash nana ish, $\underline{i}$ miha na, yummak ash micha tekchi, micha nana im asha tuk moma aiena ka ik iá, okla im ahni tok.

\section{CHAPTA XIII.}

MIHMA Eblam vt ilap, micha tekchi, micha nana im 1 asha tuk moma aiena kvt Echip a kohchvt mintit, micha Lot vt ibafohka ho oka mahli ka ai vla tok. 
2. Micha Eblam vt nanvlhpoa, micha tvli hvta, micha tvli lakna aiena kvt in laua fehna hatok.

3. Atuk osh oka mahli ya vttut falamvt mintit Behlel, yvmmut Behlel micha Hai iti takla yakni talaia ho vmmona ka im vlhtipo vt a hikia tok atok;

4. Ummona ka alta ai ikbi tok yakni talaia yo vla tok: micha yummak o Eblam vt Chihowah ya hochifot ai i pahaya tok.

5. Mihma Eblam a awant ia tuk vt Lot ak kia nanvlhpoa chipinta, micha nan vlhpoa hochito, micha vlhtipo puta aiena kvt im asha hatok.

6. Mihma yakni vt ik im ono hatuk osh, itupehvt a hashwa he keyu tok: imilayak chinto hatuk okvt, itupehut ahashwa he keyu tok.

7. Atuk o Eblam $\underline{i}$ nan vlhpoa apistikeli vhleha, micha Lot i nanvlhpoa apistikeli vhleha aiena kut itachowa tok: micha mihmuno Kenanait okla, micha Pelissait okla aiena kvt yakni yumma asha hatok.

8. Mihma Eblam vt Lot a Uno, micha chishno piti takla, micha a nan vlhpoa apesvchi vhleha, micha chi nan vlhpoa apesvchi vhleha aiena iti takla ya itachowa kvt iksho kashke; chim asilhha lishke, itibapishi vhleha pia hokvt.

9. Yakni vt moma hosh chi tikba itoyula keyu cho? Is sa filvmmashke, chim asilhha lishke: vlhfubek imma hak $\underline{o}$ ish ia hokma, ishtimpak imma hak $\underline{o}$ ia lashke; keyukmut ishtimpak imma hak o ish ia hokma, vlhfubek imma hak o ia lashke, im achi tok.

10. Mihma Lot vt akachakali mvt, Chatan yakni patali moma kvt kanima moma kia oka laua achukma kvt asha; Chihowah yut Sotom, micha Komollah ya okpvnit ik tahlo kisha ma, Chihowah im osapushi ak o chohmi cha, Soa ish chukowa Echip yakni ak o chiyuhmi tok a pihisa tok.

11. Yohmi mvt Lot vt Chatan yakni patali moma ilap immi achị hosh atokoli mvt, Lot vt hushi akochaka pilla ho ia tok: yohmi ma iti filummit okla tok.

12. Eblam vt Kenan yakni ak $\underline{o}$ ai ahanta tok; mikma 
Lot vt yakni patali tưmaha holihta taloha kak o ai vtta cha, Sotom pilla hak o im vlhtipo ya hilechi tok.

13. Yohmi kia Sotom hatak vhleha hvt haksi fehna cha, Chihowah itikba ya nanashvchi atampa fehna tok.

14. Atuk o Chihowah yvt Eblam a Lot vt i filimmi ha ya Himak a akachakali cha, ish aivtta ka hikivt falvmmi imma, micha oka mahli imma, micha hushi akochaka imma, micha hushi ai okatula imma aiena ka pit ish hopokoyashke:

15. Yakni ish pisa ka mominchit chishno, micha chim isht aiunchololi aiena ka immi bilia he mak o ima la chị hoke.

16. Mikma chim isht aiunchololi ya lukfi pushi yakni paknaka aiasha kak $\underline{o}$ chiyuhmichi la hi oke: yohmikma hatak nana hosh lukfi pushi yakni paknaka aiasha ka hotihnvt tahla hinla hok mak $\underline{\mathrm{g}}$, chim isht aiunchololi ak kia holhtina hi oke.

17. Wakaya cha yakni afalaiaka, micha apvthaka nowvt hlopulli, chima la chi hoka, im achi tok.

18. Yohmi ma Eblam vt im vlhtipo ya kanvllichit Mamli i baiyi Heblon yakni talaia hieli ka ont ai vtta tok: micha yumma Chihowah ya alta im ikbi. tok.

\section{CHAPTA XIV.}

TUK o yakohmi tok, Shina i miko Amlafel, Ellasa $\underline{i}$ A miko Aliok, Elam i milko Chetolaoma, micha oklushi puta i miko Tital aiena kvt aiasha nitak ma ;

2. Ilvppa puta kvt Sotom $\underline{i}$ milko Bela, micha Komollah $\underline{i}$ miko Besha, Atmah $\underline{i}$ miko Shinab, micha Seboim i miko Shemebe, micha Bela yummut Soa ya $\underline{\mathbf{i}}$ milko aiena ka in tanampi tok.

3. Iluppa moma kak osh okfa Sittim, yummut hupi okhota ha ai itahobut aiasha tok.

4. Afummi auah tuklo ho Chetolaoma ya i nutaka tok; atuk osh afummi ont isht auah tuchina ho i filummit $\underline{i}$ sanali tok: 
5. Atuk o afummi ont isht auah ushta ma, Chetolaoma yvt, micha miko vhleha yumma apeha kash aiena kvt vla cha, Lefaim okla Ashtelolh Kanaim aiasha, micha Susim okla Ham aiasha, micha Emim okla Shafeh Kiliahlaim aiasha tuk aiena ka issot kanchi mut,

6. Micha Holim okla $\underline{\underline{i}}$ nunih Sei aiasha tuk aiena ka ai issot kanchit El-palan yummut yakni chukushmi bilika talaia ka ont vhlichi tok.

7. Mihmvt falamvt En-mishpat, yummvt Katesh a ai vla cha, Amalek okla i yakni moma kạ, micha Amolait vhleha yummot Haseson-tama asha tuk a issot kanchi tok.

8. Mihma Sotom i miko, micha Komollah i miko, micha Atmah i miko, micha Seboim i miko, micha Bela yummut

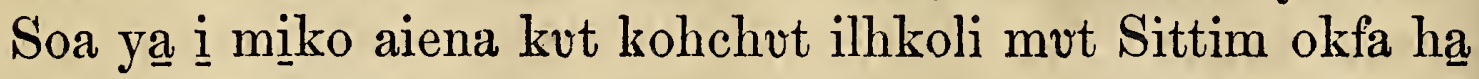
ont ai itafamvt,

9. Elam i miko Chetolaoma, micha oklushi puta $\underline{\underline{i}}$ miko Tital, micha Shina i miko Amlafel, micha Ellasa $\underline{i}$ miko Aliok aiena kash itiba chi kvt il atahli tok: miko ushta hosh tahlapi ho itafama tok.

10. Mihma Sittim okfa hvt bitumen aiasha yakni mitahli yosh alotowa tok: atuk o. Sotom i miko, micha Komollah $\underline{i}$ miko vt tihlaya tuk osh yummak o ai akakoha tok: mihma hlakoffi tuk vt yihlepvt nunih chaha ya ona tok.

11. Mihma Sotom, micha Komollah i na chukushpa, micha im ilhpak moma ka ishi cha ilhkoli tok.

12. Micha Eblam itibapishi ushi Sotom vtta hatuk Lot, micha im vlhpoyak aiena ka ishi cha ilhkoli tok.

13. Atuk o hlakoffi tuk achufa kvt Heblu hatak Eblam a ont im anoli tok: Eshkol itibapishi, micha Ane itibapishi, Amolait hatak Mamli $\underline{i}$ baiyi hieli ka bilika ya vtta yatuk oka: micha yummut Eblam a anumpa itibai achvfut itapela hatok.

14. Mihma Eblam vt itibapishi ha yukachit ishi tuk $\underline{o}$ haklo mvt, ilap in chuka akinli ho ai vttvt, nan im vlbvchit hofantit aiasha ya tahlepa tuchina cha auah untuchina ho nahalupa im atahlit pehlichi cha, hliolit Tan a ona tok.

15. Mihma ilap, micha in tishu vhleha aiena kvt itakvsh- 
koa cha, ninak o issot kanchi mut, hliolit isht ayvt Tamaskus im vlhfubekimma talaia Hobah ya ona tok.

16. Micha vlhpoyak moma ka falvmmichit isht vla mut, anonti itibapishi Lot ash kia, micha im vlhpoyak aiena, mikmvt ohoyo puta, micha okla hash aiena ka falvmmichit isht vla tok.

17. Mihma (Eblam vt Chetolaoma micha apehvt aya tok, miko vhleha ha vbi cha, falamvt aya ha ma) Sotom i miko vt Shafeh yummut miko im okfa ha aiafama chi hosh kohchvt ia tok.

18. Mihma Salem i miko Melkisetek vt puska, micha oka paki aieninchit i kohchechi tok; micha yummut vba chaha moma i shahli Chitokaka ya naholitompa isht im vtta yatok.

19. Micha anumpa yukpali Eblam a onochit, Urba chaha moma i shahli, vba, micha yakni aiena ikbi Chitokaka ya Eblam vt i holitopashke.

20. Mikma vba chaha moma 1 shahli Chitokaka, yummak osh chin tunup vhleha ha chibbak fohki tuk vt holitopashke, achi tok. Mihma moma ka ishit pokoli aiyukali ho ai ishit Melkisetek ash ima tok.

21. Mihma Sotom i miko vt Eblam a , Hatak-vhleha hano wno ak o is sama cha, nachukushpa ya chishno akinli hosh ish ishashke, im achi tok.

22. Mihma Eblam vt Sotom i miko yạ, Chihowah vba chaha moma i -shahli Chitokaka, vba, micha yakni aiena ka ikbi ya subbak a vba pit $\underline{i}$ wieli li kvt,

23. Ponola honnula achvfa kia, shulush isht talakchi peh nana kia ishi la he keyu, micha nana hosh chimmi atuk a ishi la he keyu, miha li tuk oke; yummak keyukma Eblam a vno ak osh nan in laua ikbi lishke, ish acha hinla hoke.

24. Peh hatak himithoa hvt vpa tuk ak illa, micha hatak si apehvt aya tuk ai isha he vlhpesa kak illa Ane, Eshkol, micha Mamli aiena kak osh ai isha he vlhpesa kut ishashke, achi tok. 


\section{CHAPTA XV.}

TLUPPA puta kut yưmohmi ha yạ, Eblam vt na pisa ho 1 Chihowah im anumpa hvt im aya cha, Chi nukshopa ná, Eblam ma, chin telihpa, micha nan isht chim vlhtoba chito atampa fehna sia hoke, im achi tok.

2. Mihma Eblam vt, Nanta ho is suma chị cho? Chihowah Chitokaka ma, vlla ik im iksho yosh aya li hoka, mikma an chuka achofa apesvchi vt Tamaskus hatak Eliese iluppak oke, achi tok.

3. Mihmvt Eblam vt, Yakeh, vno ya vlla nana kia chik samoshke : yohmi ka an chuka ai vtta tuk achvfa hosh vmmi hash ai isha he vlhpesa kak oke, achi tok.

4. Mihma yakeh, Chihowah im anumpa hut im aya mut, Ilvppoto chimmi ha ai isha he vlhpesa ya he keyu; amba chikfoka akinli a kohcha he ak osh chimmi ha ai isha he vlhpesa ya hi oke.

5. Mihmvt ilauelit kocha isht ona mvt, v́ba pilla ha pit ish hopokoyo cha, fochik puta ka ish hotihna hinlakmvt, ish anolashke, achi tok: Chim isht aiunchololi hot yummak o chiyuhma hi oke, im achi tok.

6. Mihma Chihowah ya $\underline{\mathrm{j}}$ yimmi tok: mihma yummut aivlhpiesa im ai itilauechi tok.

7. Mihma, Uno ak osh yakni ilupput chimmi hachị mak

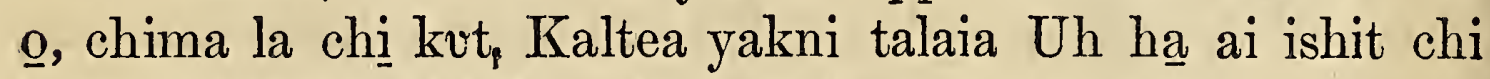
kohchi li tok vt Chihowah sia hoke, im achi tok.

8. Mihma, Chihowah Chitokaka ma, yumma ishi la hi vt nanta ho isht ithana la chi cho? achi tok.

9. Mihma, Wak tek afummi tuchina, micha issi kosoma tek afummi tuchina, micha chukfulhpoa nakni afummi tuchina, micha pvchi yoshuba achufa, micha pvchi himita achvfa aiena ka is sa hoyashke, im achi tok.

10. Mihma iluppa moma kash ishit im ona cha, iklunna ita pahlulli mut, pahlata aiyukali ka iti chablit kahpuli tok: yohmi kia hushi ano ik ita pahlalo tok. 
11. Atuk o hushi puta kvt nipi yash ant o fohopa ma, Eblam vt helihinchi tok.

12. Atuk o hushi vt okatulvt ishtia ma, nusi kvllo kvt Eblam a onvtola tok ; mihma yakeh, okhlili chito nukshoblichi fehna hosh onvtola tók.

13. Mihma Eblam a, Chim ishtatiaka yut okla inla yosh i yakni keyu ka aiasha cha, i yuka hosh a hashwa he ya ish ithana pullashke: mikma afưmmi tahlepa ushta ho ilbushahancha hi oke.

14. Atukma i yuka hosh a hashwa he oklushi yưmma nana im apesa la hi oke : atukma i himmakma vlhpoyak chito fehna ho isht kohcha hi oke.

15. Micha chishno yokvto nnktaiyalvt chiki vhleha hash o ish im ona hi oke: chi sipoknit taiyaha hosh chi hollohpa hi oke.

16. Yohmi kia aiitishali ont aiushta kvto anonti ilvppa ai vla hi oke: Amolait okla nan ai ashahanchi kvt ik aloto kisha hoka, im achi tok.

17. Atuk o yakohmi tok, hvshi vt okatula na okhlili ma, yakeh, shuti shobulli, micha pula libbi aiena kut nipi pahlahli, kanohmi kash iti takla ya ant ia tok.

18. Mih nitak fehna Chihowah yut Eblam a nana itim apesut, Echip okhina yak o aivhlit ia hosh okhina chito Euflates okhina ont vhli ;

19. Kenait okla, micha Kenissait okla, micha Katmonait okla,

20. Micha Hittait okla, micha Pelissait okla, micha Lefaim okla,

21. Micha Amolait okla, micha Kenanait okla, micha Kikashait okla, micha Chebusait okla aputa aiasha yakni iluppa chim ishtatiaka ya ima lashke; achi tok.

\section{CHAPTA XVI.}

YOHMI ma Eblam tekchi Salai vt vlla ik im esho tok: 1 mihmut Echip ohoyo, tishu ohoyo hosh hohchifo kut Haka yosh im anta tok. 
2. Atuk osh Salai vt Eblam a, Yakeh himak a Chihowah yvt vno vlla eshi la he tuk a vm oktublishke : an tishu ohoyo

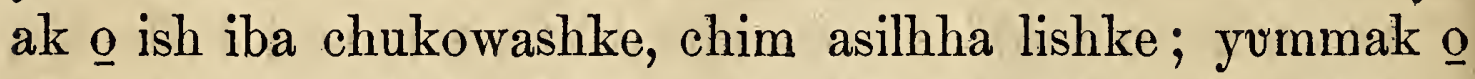
vlla isht vm aiasha hinla yoba ká, im achi tok. Mihma Eblam vt Salai im anumpa ha $\underline{i}$ haponaklo tok.

3. Mihma Eblam vt Kenan yakni ya ahanta tok afummi pokoli ma, Eblam tekchi Salai vt in tishu ohoyo Echip ohoyo Haka yash eshi cha $\underline{i}$ hatak Eblam a tekchi ya chi ho i bohli tok.

4. Mihma Haka ya ibachukowa ma, chakali tok; micha chakali hatuk vt akostininchi mvt, $\underline{i}$ nahullo ohoyo yash kanimanchi tok.

5. Mihma Salai vt Eblam a, Nana ik sum achukmo kvt chishno ak atuk pulla kak o yvmohmishke: an tishu ohoyo hatuk a chi shakba fohki li tuk oke : atuk o chakali kvt il akostininchi mut, sa kanimanchishke; Chihowah yot vno; micha chishno pim itin takla nana ai apesashke, im achi tok.

6. Yohmi kia Eblam vt Salai ya, Yakeh, chin tishu ohoyo hut chibbak a foka hoke; nana hosh chim vlhpesakmvt, ish yumihchashke, im achi tok. Atuk o Salai vt $\underline{i}$ kvllo ma itikba ya i maleli tok.

7. Atuk o chukushmi foka oka kvli, Shu hina oka kvli hikia ka asha na, Chihowah im enchel vt ahayuchi tok.

8. Micha Haka Salai in tishu ohoyo ma, katima ish minti cho? micha katima ish ia chi cho? achi tok. Mihna A nahullo ohoyo Salai itikba yak o $\underline{i}$ maleli hosh aya lishke, achi tok.

9. Mihma Chihowah im enchel ash osh, Falamvt chi nahullo ohoyo ha ish im ona cha, im antia achukma hosh ibbak nutaka ma ish ahantashke im achi tok.

10. Micha Chihowah im enchel ash osh chisht aiunchololi ka apaknvchit atamblichi la hi oke, yohmi na apakna kvt a holhtina hi vt iksho ka hi oke, im achi tok.

11. Micha Chihowah im enchel ash osh, Yakeh, chi chakalishke, yohmi kvt vlla nakni yo ish esha hi oke: micha chilbusha ka Chihowah yut haklo tuk oka, hohchifo ha Ishmael ish hochifa hi oke. 
12. Atukma hatak kostina he keyu ya hi oke: ibbak vt

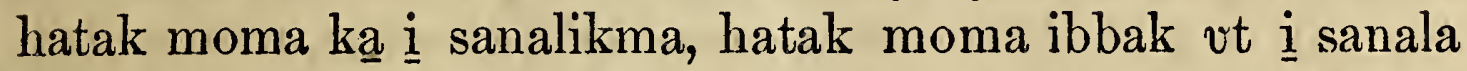
hi oke; mikma itibapishi vhleha moma ka itin takla yo ahanta hi oke, im achi tok.

13. Mihma Chihowah yot im anumpohonli tuk ash hochifot Chitokaka chia hot is sa pihisa yoke, achi tok: mihmvt, Uno vt ilvppa pisa li ha yak kia hopokoyo li ha cha? achi tok.

14. Yohmi hatuk o kuli hofobi yash osh Bee-lahai-loi hohchifo tok; Yakeh Katesh, micha Belet iti takla ya hikia hoke.

15. Atuk o Haka vt Eblam a vlla nakni yo im eshi tọk: mihma Eblam vt ushi, Haka vt im eshi tuk ash hohchifo ha Ishmael hochifo tok:

16. Mihma Eblam vt afummi pokoli untuchina akucha hannali ho, Eblam a Haka vt Ishmael a im eshi tok.

\section{CHAPTA XVII.}

A TUK o Eblam vt afummi pokoli chakkali akuchá chakA kali ma, Chihowah yvt Eblam a i haiaka cha, Chitokaka Nan isht im aiahli ataha iksho sia hoke: sa tikba ya ish anohowa cha, ish aiahlashke.

2. Mikma vno vt chishno ya nana chitim apesa li pullashke; micha chi apaknvchit atambliche lashke, im achi tok.

3. Mihma Eblam vt hlipivt itoyula tok; yohmi ma Chitokaka yvt im anumpulit,

4. Vno yokvto yakeh, nana chitim apesa li vt itoyulushke; yohmi ka oklushi lawwa ho iki chia ha chị hoke.

5. Micha himak pilla hoka chi hohchifo hot Eblam ah he keyu, amba chi hohchifo hot Eblaham ah hi oke: oklushi lawa ho iki ya chị ho chikbi li hoka.

6. Micha chi wayachi li fehnvt, oklushi puta chi ai ikbi likma, miko puta kvt chi akohoncha hi oke.

7. Micha vno vt chishno, micha chim ishtatiaka et chi- 
akaiya aiena kut ai itishalit mihinti ka nana itim apesa bilia mak o, kullochit chitim apesa lish, chishno, micha chim ishtatiaka et chiakaiya aiena ka in Chitokaka sia ha chị hoke.

8. Mikmvt Kenan yakni vt moma, okla inla yosh ish ai ahanta yakni ya chishno, micha chim ishtatiaka et chiakaiya aiena kut huchị yakni bilia chị ho hvch ima la chị hoke: mikmut in Chitokaka sia ha hi oke, achi tok.

9. Micha Chitokaka yot Eblaham a, Yvmohmi hoka a nan itim apesa ya chishno, micha chim ishtatiaka et chiakaiyot ai iti shalit mihinti aiena kvt hush holitoblashke.

10. Chishno, micha chim ishtatiaka et chiakaiya aiena kut hush holitobla he a nan itim apesa pitin takla takali kut iluppak oke: Vlla nakni yosh hvch iti takla aiasha kvt momvt hakshup tvpashke.

11. Micha hvchi tikba hakshup nipi ya hvsh tvblashke; mikma yummak osh nan itim apesa yash osh vno, micha hvchishno hupitin takla ya itoyula ka isht atokowa ya hi oke.

12. Mikma hvchim ai itishali puta ka vlla nakni yosh nitak untuchina ho hvchitin takla ya aiasha kvt momvt hakshup tvpashke; chuka ai vtta tuk, keyukmot okla inla, chim ishtatiaka keyu puta ho chin tvli holisso ha isht vlhtoba tuk.

13. Chin chuka ai vtta tuk, micha chin tvli holisso ha isht vlhtoba tuk aiena kvt hakshup tupa pullashke: mikma a nan itim apesa yvt hvchi nipi ya nana itim apesa bilia mako foyuka hi oke.

14. Atukma vlla nakni hakshup ik tapo, tikba hakshup nipi ut ik tapokmvt, shilombish yummut im okla achvfa ai iti takla ya, fiopa ut tupa hi oke: a nan itim apesa ya kobuffi hoke, im achi tok.

15. Mikma Chitokaka yvt Eblaham a, Chi tekchi Salai ak okvno Salai ish hochifa he keyu, amba hohchifo hot Salah yashke.

16. Mikmvt yukpali la chi hoke, micha yummak kia vlla nakni yo a chima la hi oke : ahli yukpali la chị hoke; mik- 
ma oklushi lana ho ishki ya chi hoke: okla i mịko putạ kvt a tohomba hi oke, im achi tok.

17. Yohmi ma Eblaham vt hlipia cha, yukpa mvt, chunkvsh anukaka, Afvmmi tahlepa achufa kvt vlla im asha chi cho? mikma Salah vt afummi pokoli chakkali kvt vlla esha chi cho? achi tok.

18. Micha Eblaham vt, Chitokaka ya Ishmael vt chi tikba ya okchayut ahantakbano, im achi tok.

19. Mihma Chitokaka yvt, Chi tekchi Salah vt vlla nakni yo chim eshi pulla chi hoke; mikma Aisak ish hochifa chi hoke: mikma nan itim apesa bilia mak o yumrna, micha im ishtatiaka et iakaiya aiena ka kullochit nan itim apesa la hi oke.

20. Ishmael ak okuno chi haponaklo lishke: yakeh, yukpali lishke, wayvchit apaknvehi kvt atamblichi la chi hoke; miko auah tuklo ho tobacha chi hoke; micha oklushi chito ho ikbi la chị hoke.

21. Yohmi kia Aisak yummak oka afưmmi inla nitak vlhpisa vt ont vlhpesa himak a chohmikma, chi tekchi Sa-

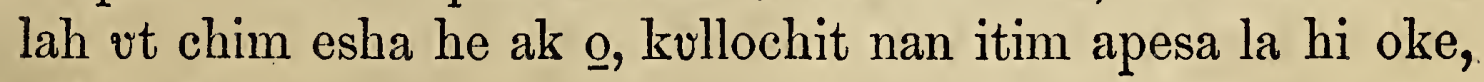
achi tok.

22. Mihmut im anumpuli kvt issa cha, Chitokaka yut Eblaham a i filummit ia tok.

23. Mihma Eblaham vt ushi Ishmael ishi cha, in chuka achvfa ai vtta tuk moma, micha in tuli holisso isht vlhtoba tuk moma, micha Eblaham in chuka achvfa hatak nakni puta moma, Chitokaka yot miha tuk mak $\underline{o}$, mih nitak akinli ho tikba hakshup nipi ya in tuptuli tok.

24. Micha Eblaham vt afummi pokoli chakkali akucha chakkali mot, tikba hakshup nipi vt in tvpa tok.

25. Mihma ushi Ishmael vt afummi auah tuchina mut, tikba hakshup nipi vt in topa tok.

26. Mih nitak akinli ho, Eblaham vt tikba hakshup tupa ma, ushi Ishmael vt yohmi tok;

27. In chuka achufa hatak puta moma chuka ai vtta tuk, micha okla inla ai achvfa tvli holisso isht vlhtoba tuk aiena kut tikba hakshup tupa tok. 


\section{CHAPTA XVIII.}

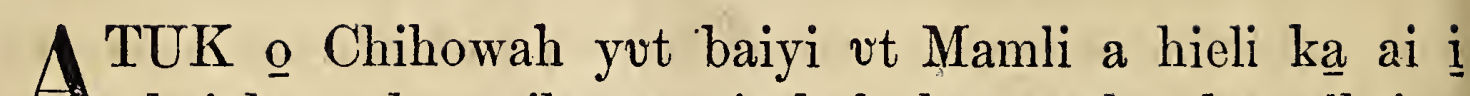
Al haiaka tok: mihmot nitak lushpvt taha ho vlhtipo okhissa ya bininli tok.

2. Atuk osh akachakali cha, hopokoyo ma, yakeh, hatak tuchina kvt bilika hieli tok: atuk o pisa mvt, vlhtipo okhissa yash hikivt malelit ont afama cha, aka pit hlipia mut,

3. A shahli ma, himak a holhponayo hanta ha chi tikba ai ahayuchi likma, chin tishu ha i filummit chik aiyo kashke, chim asilhha lishke:

4. Oka kanomosi ka isht vla na, hvch iyi ya hvsh achifa cha, iti nuta ya chieyut hush fohashke, huchim asilhha lishke :

5. Mikma pvska vpa achvfa isht vla likma, hvchi chulkvsh a hush fihoblashke; yohmi ha yosh hvsh ayashke: yumohma chị kak o, hvchin tishu ha hush im vla tuk okvt, achi tok. Mihma, Ish achi tuk mak osh ish yumohmashke, okla achi tok.

6. Mihma Eblaham vt tushpvt vlhtipo ya ont chukowvt, Salah ya im ona cha, Tushpvt atahlit bota tohbi lepihbi vlhpisa tuchina ho yamusli cha, luak ituksita puska lumboa ya ai apushli, achi tok.

7. Mihmvt Eblaham vt malelit wak aiasha ka ona cha; wak ushi nipi ik kvllo achukma ho ont isht vla cha, hatak himita yo ima tok; mihma tushpvt atahli tok.

8. Mihmot pishukchi nia, micha pishukchi, micha wak ushi atahli kash aiena ka ishi cha, itikba talohli tok: micha bilika iti nuta ya hikia ma, okla impa tok.

9. Mihmut, Chi tekchi Salah moto? okla im achi tok. Mihma, Yakeh, vlhtipo ya ashushke, achi tok.

10. Mihma, Aiokchaya he nitak vt takanlik mak o falamut chim aya li pulla hi oke; mikma yakeh, Salah chi tekchi vt vlla nakni yo esha hi oke, achi tok. Mihma vlhtipo ok-

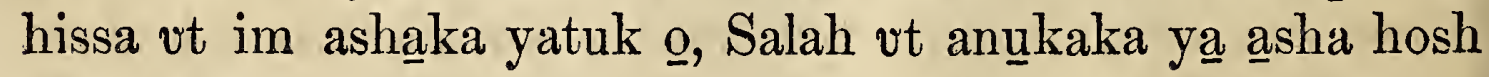
haklo tok. 
11. Yohmi ka Eblaham, micha Salah vt sipoknit nitak in lauvt taiyaha hatok ; mikma Salah vt ohoyo nana im a kaniohmi vt issa tok atok.

12. Yvmohmi hatuk osh Salah vt ilap inlit yukpvt, Sa sipokni hay vt vm achukma hinla? a shahli hvt sipokni akinli ka, achi tok.

13. Mihma Chihowah yut Eblaham a , Nanta katiohmi ho Salah vt, Sa sipokni kvt vila eshi li pulla chị ho, achit yukpa cho?

14. Nana kia Chihowah ya i kullo kvt im atampa hinla cho? Nitak vlhpisa yut ont ai vlhpiesa ka aiokchaya he nitak vt takanlikmak o falamvt chim aya li pulla hi oke, mikma Salah vt vlla nakni yo esha hi oke, im achi tok.

15. Yohmi ma Salah vt holabit, Yukpa li tuk keyu, achi tok; nukshopa hatuk okvt. Mihma, Aha, yohmi kia ish yukpa tuk oke, achi tok.

16. Mihma hatak ash osh yumma chieya tuk vt wakaya mvt, Sotom pilla ho pit hopokoyo tok: mihma Eblaham vt hina fohkit pila chi hosh awant ia tok.

17. Mihma Chihowah yut, Nana yumihchi la chi kvt Eblaham a in lubmi la chi cho?

18. Eblaham vt oklushi chito, micha kvllo hosh toba pullakma, oklushi puta moma yakni paknaka aiasha kvt yummak o a yukpa hi oka.

19. Yvmmak ash osh im vlla vhleha ha, micha in chuka achufa et iakaiya ka im apihisakma, Chihowah $\underline{i}$ hina ya holitoblit. aivlhpiesa ai atokowa kak o yumihcha hi ya ithana lishke; yvmohmi ho Chihowah yvt Eblaham a isht anumpuli tuk vt im aiahlicha hi oke, achi tok.

20. Mihmvt Chihowah yut Sotom, micha Komollah ya ishit pahaya kvt chito, micha $\underline{1}$ nanashvchi vt weki fehna hatuk $\underline{0}$.

21. Himak a akowa lish, ishit pahaya na vm ahanla tuk mak osh a hli bieka yomohmi tuk okmá pisa lashke; amba keyu hokmá ithuna lashke, achi tok.

22. Mihma hatak ash osh yumma hielit nashuka ya filimmi mvt, Sotom pilla ha itiachi tok: amba Eblaham vt Chihowah itikba ya hikia moma tok. 
23. Mihmvt Eblaham vt bilika ona cha, Aivlhpiesa ak kia hatak haksi aieninchit ishit ish kancha chi cho?

24. Tumaha holihta anukaka ya aivlhpiesa vt pokoli tahlapi hosh asha yoba hokmuno; aivlhpiesa pokoli tahlapi kvt

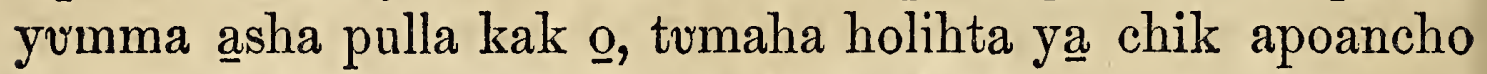
hosh ishit ish kanchi hak inla hinla cho?

25. Aivlhpiesa ya hatak haksi aieninchit vbi, yumma chohmi ka chi ahalaia he' keyu hoke; micha aivlhpiesa vt hatak haksi ya itilaua he, yummak okvt chi ahalaia he keyushke: yakni moma Nan im apesa yut aivlhpiesa kak o yumihcha he keyu cho? achi tok.

26. Mihma Chihowah yut Sotom a tumacha holihta anukaka ya hatak aivlhpiesa pokoli tahlapi ho ai ahayuchi likmvt, yummak atuk pulla mak o tumaha holihta ya mominchit apoanchi la chi hoke, achi tok.

27. Mihma Eblaham vt afalamichit, Yakeh, himak a lukfi. pushi, micha hituk chubbi ak o sia hakinli kia Chihowah ya im anumpulit pisa lishke:

28. Aivlhpiesa pokoli tahlapi kash tahlapi hosh isht ik ono yoba hokmuno, táhlapi hosh ik ono ma, tumaha holihta ya mominchit okpunit ish kancha hinla cho? achi tok. Mikma Pokoli ushta akucha tahlapi ho yumma ai ahayuchi likmut, okpunit kanchi la he keyushke, achi tok.

29. Mihma anonti im anumpulit, Pokoli ushta hosh yum-. ma asha yoba hokmvno, achi tok. Mihma, Pokoli ushta hatuk pulla mak o yumihchi la he keyu hoke, achi tok.

30. Ikikkeh, Chitokaka yvt ik a nukhobelo kashke, mikma anumpuli lashke: Pokoli tuchina hosh yumma asha yoba hokmuno, achi tok. Mihma, Pokoli tuchina ho yumma ai ahayuchi likmot, yumihchi la he keyu hoke, achi tok.

31. Mihma, Yakeh, himak a Chihowah ya im anumpulit pisa lishke : Pokoli tuklo hosh yumma asha yoba hokmuno, achi tok. Mihma, Pokoli tuklo hatuk pulla mak o okpunit kanchi la he keyushke, achi tok.

32. Mihma, Ikikkeh, Chitokaka yvt ik a nukhobelo kashke, mikma himakma himonna illa ho anumpuli lashke: Pokoli 
hosh yumma asha yoba hokmuno, achi tok. Mihma, Pokoli hatuk pulla mak o okpunit kanchi la he keyushke, achi tok.

33. Mihmvt Chihowah yvt Eblaham a itim anumpulit issa mut ia tok: mihma Eblaham vt falamvt aivtta ya ona tok.

\section{CHAPTA XIX.}

A TUK o opia ma, enchel tuklo kash osh Sotom a ona A tok; mihma Lot vt Sotom holihta okhissa ya bininli tok; mihmvt Lot vt pisa mut, wakayvt afamvt ia tok: micha nashuka ya isht akachunolit aka hlipia tok.

2. Mihmvt, Yakeh, a shahli ma! himak a fullotvt hvchin tishu im aboha ya hush chukowa cha, hush ashwa na onnashke ; micha hvch iyi ya hush achefashke ; atuk mut onnahinli fehna hush wakaya cha, hvchi hina ya hush ayashke, achi tok. Mihma, Ahá, amba aiitunowa ak o il ashwa na onna chi hoke, achit okla tok.

3. Mihma im achunanchi fehna tok: mihma im a fullota cha, im aboha ya chukowa tok; mihma impuchi im ikbi cha, pvska ik shatvmmo i nunachi ma, impvt okla tok.

4. Yohmi kia okla ik tushko kisha ho, tumaha holihta hatak vhleha, Sotom hatak vhleha fehna kvt sipokni, micha himita aiena okla moma kanima moma ka minti hosh aboha yash apakfoyubli tok.

5. Micha Lot $\underline{a} \underline{\mathrm{i}}$ howa mut, Himak ninak a hatak vt chim vla tuk muto? kohchit isht ish pim vla na, il ithunashke, okla im achi tok.

6. Mihma Lot vt okhissa ya kohchvt im ona cha, okhissa isht vlhkvma yash okhishtvt kohcha mvt,

7. Itibapishi li vhleha ma! nanashvchi iluppa chohmi ka hvch ik yumohmo kashke, hvchim asilhha lishke.

8. Yakeh, himak a svso tek tuklo kvt hatak haleli keyu hosh vm ashwvshke; hvchim asilhha lishke, isht ak hvchi kohchi na, kaniohmi kak osh hvchim vlhpiesakmvt hush yvmihchashke : peh hatak iluppak okvno nana huchik kanioh- 
mecho kashke; yvmohmi kak o vm aboha ishitholmo a hoshonti ka nuta ya vla hatuk oka, achi tok.

9. Mihma, Misha hikiá, okla achi tok. Micha anonti, Hatak achvfa iluppot cheki itintakla pi takla anta chị hosh vla tuk vt nanapesa ya chi hotuma? Himak a chishno ak $\underline{0}$ yumma e yumilhcha hetuk $\underline{i}$ shahlichi hosh e chi yumihcha chi hoke, okla achi tok. Mihmvt hatak Lot ash okla i kvllo fehna tok; micha okhissa ya tivbla chị hosh bilika ai vla tok.

10. Yohmi tuk kia hatak ash osh ilap ibbak a pit weli cha, Lot a halvllit aboha ya fohki cha, okhissa yash okhishta tok.

11. Micha hatak okhissa aiasha kash hochito, micha chipunta moma ka nishkin a lublichit okla tok: yohmi ho okhissa ya ahayuchi bunnot okla ile tikambichi tok.

12. Mihma hatak ash osh Lot a, Iluppa inla kvt chim asha moma cho? chiyup, micha chiso vhleha, micha chisotek vhleha, micha nana hosh tumaha holihta ya chim ashakmvt, ilvppa isht ish kohchashke.

13. Ishit pahaya kvt Chihowah itikba ya chito fehnvt taha hatuk o, tumaha holihta iluppa okpunit e kancha chi hoke; mikma okpunit e kancha chi ho, Chihowah yot et pi pila tuk oke, im achi tok.

14. Mihma Lot vt kohchvt ia cha, iyup vhleha hvt ushetik vhleha ha itauwaya tuk a ont im anumpulit, Ho wakayá, iluppa hush kohchashke; Chihowah yut tumaha holihta ilvppa okpunit tahla chi hoke, achi tok: yohmi kia yopula nana ho iyup vhleha hut im itilaui tok.

15. Atuk o onnvt minti ma, enchel ash osh Lot a tushpalechit, Wakaya cha, chi tekchi, micha chisotek tuklo iluppa ashwa hokuno pehlichí, yohmi hosh tumaha holihta nanashvchika iba takkla luvt ik hvchi tamoo kashke, achi okla tok.

16. Atuk o sulaha ma, hatak ash osh ibbak a halvlli mut, tekchi ibbak a halvlli mut, ushetik tuklo ibbak a halvllit okla tok: Chihowa yut i nukhaklo hatuk o ; micha tumaha holihta ya ishit kohchit hilechit okla tok.

17. Atuk o kocha ya isht ona ma yakohmi tok, Malelit chi okchayashke : chim ashạka ya filemut ish pisa ná, micha 
yakni patali kanima chik anto kashke; malelit nưnih ha ish onashke, luvt chi taha ná, achi tok.

18. Mihma Lot vt, Ikikkeh, ik yohmo kashke, an Chitokaka ma!

19. Yakeh, himak a chin tishu hut chi tikba ya chi holhponayo hanta ai ahayuchishke, micha vm ilhfiopak a ish okchalinchi, yumma ish yumihchi kvt nan ish i nukhaklo vt ish chitolichishke: atuk o malelit nvnih ha ona la he keyushke, nan ik achnkmo kia si onvtola cha, sulli yoba na.

20. Himak a yakeh, tumaha holihta iluppot olanlosi, malelit ona hinla hoke; micha iskitinesishke: ikikkeh, yummak o malelit ona lashke, (iskitinesi keyu cho?) mikma umi shilombish vt okchaya chị hoke, im achi tok.

21. Mihma, Yakeh, nana iluppa chim aiokpanchi li ak inli hokvt, isht ish anumpuli tuk tumaha holihta iluppa okpunit ak kancho ka chi hoke.

22. Tushpá, malelit yummak $\mathrm{o}$ ish onashke : yưmma chik ono kisha ka nana kaniohmichi la he keyu hoka, im achi tok: yumohmi hoka tumaha holihta yummut Soa hohchifo tok.

23. Lot vt Soa ya ont chukowa ma, hushi vt kohchvt yakni ya on tommi tuk atok.

24. Yohmi ma Chihowah yut hituk lakna micha luak Chihowah ya a minti Sotom micha Komollah aiena ka vba et ai on umbvchi tok.

25. Micha tvmaha holihta yumma puta kash, micha yakni patali moma, micha tumaha holihta yumma puta kash okla moma, micha yakni ai offo aiena ka okpunit kanchi tok.

26. Amba tekchi vto im ashaka ayvt olbvl pit hopokoyo cha, hupi hikia tobut hikia tok.

27. Mihma Eblaham vt onnahinli fehna ho wakaya cha, Chihowah itikba ya hikia tuk ash osh ona tok.

28. Mihmvt Sotom pilla, micha Komollah pilla, yakni patali moma pilla ka pit hopokoyut pisa ma, yakeh, yakni yash shobulli kvt toli abila șobulli chiyuhmit vba mahaya tok.

29. Atuk o yakohmi tok, Chitokaka yot yakni patali twmaha holihta puta ka okpvnit tahli mvt, Eblaham a jithaiyana tok; micha tumaha holihta puta Lot vt ai vtta yash ok4 
punit tahli mut, nana okpulot taha kash im itin takla ya ai ishit Lot a kohchi tok.

30. Mihma Lot vt Soa ya kohchvt ushetik tuklo ka pehlinchit nvnih ha ont ai vtta tok: Soa ya vtta kvt nukshopa hatuk okvt; micha ushetik tuklo kash aienvt hichukbi yo asha tok.

31. Atuk o akni ash osh nakfish a, Piki vt sipoknishke, mikma yakni moma a yumohmi chatuk a yumohmit piba tvshka hi vt hatak nana kia yakni paknaka ya ikshoske.

32. Inta piki ya oka paki il ipeta cha, il iba tushkashke, yumohmi hosh piki im ishtatiaka ya il apoanchashke, im achi tok.

33. Micha mih ninak a iki ya oka paki ipetvt okla tok; micha akni hut iki ya iba chukowa cha, iba tushki tok; mihma itola tuk osh tani ka ik akostinincho tok.

34. Atuk $\underline{o}$ ont onna ma yakohmi tok, akni hvt nakfish $\underline{a}$, Yakeh, pilashash okhlili ma aki ya iba tushki li tuk oke: himak ninak ak kia oka paki il jpetashke; mikma ish iba chukowa cha, ish iba tushkashke, yumohmi hosh piki im ishtatiaka ya il apoanchashke, im achi tok.

35. Micha ninak yummak kia iki ya oka paki ipetut okla tok; mihma nakfish ash osh wakayut ont iba tushki tok; mihma itola tuk osh tani ka ik akostinincho tok.

36. Yakohmi hosh Lot ushetik ita tuklo kvt i iki ya vlla i shalit okla tok.

37. Atuk osh akni hut vlla nakni yo sholi cha, hohchifo ka Moab achi tok: mihi yosh Moabait okla ha iki yosh himak nitak a ant ai vhli hoke.

38. Mihma nakfish yưmmak kia vlla nakni yo sholi cha, hohchifo ka Ben-ammi achi tok: yummak osh Ammon im vlla vhleha ha iki yosh himak nitak a ant vhli hoke.

\section{CHAPTA XX.}

MIHMA Eblaham vt yumma vttut apoyua cha toshowvt 1 oka mahli pilla yakni yo ia mut, Katesh micha Shu 
itin takla ak o vttut, cheki itin takla Kela ak o ai vtta tok.

2. Mihmvt Eblaham vt tekchi Salah ya , An tek oke, achi tok. Mihma Kela i miko Abimelek vt Salah ya pit hoyo cha, ishi tok.

3. Yohmi tuk kia Abimelek vt ninak a holhpokuna ho, Chitokaka yut im ona cha, Yakeh! ohoyo yumma ish ishi tuk pulla kak osh hatak illi chia hoke : hatak tekchi yoka, im achi tok.

4. Yohmi kia Abimelek vt i bilika ik ono ke tuk atok: mihmot Chitokaka ma, oklushi aivlhpiesa yak kia ish ba chi cho?

5. An tek oke, vm achi tuk keyu cho? mihma ilap fehna ak kia, A nakfi oke, achi tuk oke; sa chukvish vt aivlhpiesa hosh, micha subbak vt nana okpvni keyu hosh ilvppa yvmihchi li tuk oke, achi tok.

6. Mihma Chitokaka yvt holhpokuna ho, Ahli, chi chukvsh vt aivlhpiesa hosh ish yumihchi tuk a ithana lishke; chik sa yoshubo ka chi ka, vno ak kia chi halanli li tuk oka: yumohmi kak o ik potolí, ak chim ahno tuk oke.

7. Yumohmi hoka himak a hatak ash tekchi ya ish i falummichashke; hopaii yoka, mikma anumpa ilbusha ishit chi anumpulikma, chi okchayashke; atuk o chik i falummechokmvt, chishno, micha nana hosh chimmi moma ka aienvt chilli pulla chi kvt ish ithaiyanashke, im achi tok.

8. Yumohmi na Abimelek vt onnahinli fehna ho wakaya cha, in tishu vhleha moma ka hoyo cha, nana ilvppa moma ka im anolit haklochi tok: mihma hatak vhleha hash osh nukshopa fehna tok.

9. Yohmi ma Abimelek vt Eblaham a hoyo cha, Nanta mak o ish pi yumihchi tuk oh cho? micha nanta ho chim ashvchi li tuk $\underline{o}$, vno, micha vm apehlichika ya nanashvchi chinto ish pi onochi tuk oh cho? nana kut a yumohma he vlhpesa keyu ho is si yumihchishke, im achi tok.

10. Micha Abimelek vt Eblaham a, Nanta ho ish pisa hosh nana iluppa ish yumihchi tuk oh cho? im achi tok.

11. Mihma Eblaham vt, Yakni iluppa Chitokaka ya i 
nukwia kvt iksho pullushke; micha sa tekchi pulla kak o sv ba chi hoke, ahni li kak atuk oke.

12. Atuk o yohmi kia an tek o muhlishke; aki yo ushetik, yohmi kia hushki ya ushetik keyu hoke: atuk osh sa tekchi toba tok.

13. Atuk o yakohmi tok, Chitokaka yvt aki in chuka ya issuchit fullohlihinchit susht aya ma, lluppak o is sa kana kvt ish otunichashke; yakni moma kanima yo il onakma, vno ya, A nakfi yoke, is si achashle, im achi li tuk oke, achi tok.

14. Mihma Abimelek vt chukfulhpoa micha wak, micha hatak tishu, micha ohoyo tishu aiena ka ishi cha, Eblaham a ima mvt tekchi Salah ya $\underline{i}$ falummichi tok.

15. Micha Abimelek vt, Yakeh, a yakni vt chi tikba itoyulvshke: kanima yo chim vlhpiesakmvt, ish ai ahantashke, achi tok.

16. Micha Salah yạ, Yakeh! chị nakfi ya tuli hvta tushahli tahlepa sipokni achvfa ho ima lishke: yakeh, chi apeha moma, micha inla aiena moma ka yummut nishkin ishit chim ompoholmo ya chi hoke, im achi tok: yumihchi ho Salah vt nan ashvchi tuk vt nukfohka tok.

17. Yvmohmi ma Eblaham vt Chitokaka ya im ilbvshvt isht anumpuli tok: mihma Chitokaka yvt Abimelek, micha tekchi, micha in tishu ohoyo moma ka hlakofichi tok: mihma vila okla eshi tok.

18. Chihowah yut Eblaham tekchi ak atuk pulla kak o Abimelek in chuka achvfa im oshvtto moma ka katalichit im okshita tuk oka.

\section{CHAPTA XXI.}

TUK o Chihowah yut achi tuk mak osh Salah ya $\underline{\mathrm{i}}$ A nowa tok: micha Chihowah yvt anumpuli tuk mak osh Salah ya i yumihchi tok.

2. Eblaham vt sipoknit taiyaha ho nitak vlhpisa Chihowah yvt im anoli tuk a ont vlhpiesa ma, Salah vt chakali cha, vila nakni yo im eshi tok oka. 
3. Mihma Eblaham vt vlla nakni im vtta kash Salah vt im eshi kash hohchifo ha Aisak achi tok.

4. Mihmvt Eblaham vt, Chitokaka yvt $\underline{i}$ miha tuk mak $\underline{\text {, }}$ ushi Aisak vt nitak untuchina ho hakshup in tubli tok.

5. Yohmi ka Eblaham vt afummi tahlepa achvfa ho, ushi Aisak vt im vtta tok.

6. Mihma Salah vt, Chitokaka yut sai yukpvchi tuk oke; yohmi ka kuna hosh haklo moma kut subai yukpa chi hoke, achi tok.

7. Micha, Kvta hosh Eblaham a Salah vt vlla pishecha chi hoke, im acha hinla tuk oh cho! sipoknit taiyaha ho vlla nakni yo im eshi li hoka, achi tok.

8. Mihma vlla yash osh hofantit ivt, pishit issa tok: atuk o pishi ka issuchi nitak makinli ma Eblaham vt impvchi chito ikbi tok.

9. Atuk o Echip ohoyo Haka ushi Eblaham im eshi tok vt ishit yopulvt anta na Salah vt pisa tok.

10. Yohmi kak o Eblaham a, Ohoyo yuka iluppa, micha ushi itatuklo ka ish kampilashke: ohoyo yuka iluppa ushi vt aiisha he vlhpesa ya suso Aisak fehna ka ibai isha he keyu hoke, achi tok.

11. Mihma ushi ak atuk pulla mak o nana ka Eblaham vt ik im vlhpeso fehna tok.

12. Mihma Chitokaka yut Eblaham a, vlla ak atuk pulla mak $\underline{o}$, micha chi yuka ohoyo ak atuk pulla mak o isht ik chim vlhpeso kashke: Salah vt nana chim achi tuk mak $\underline{o}$ moma ka im anumpa ha ish $\underline{\mathrm{i}}$ haponaklashke: Aisak ak $\underline{\mathrm{o}}$ chim ai unchololi hut iba holhtinvt hohchifa hi oka.

13. Mikma ohoyo yuka ushi ak kia chim ai unchololi yoka, oklushi ikbi la hi oke, im achi tok.

14. Atuk o Eblaham vt onnahinli fehna ho wakaya chă, ilhpak, micha oka kotoba allhto ho Haka ya imvt tahchi ya abanvli hosh vlla yash ima cha, chvffichi tok: mihma Haka vt ia tuk osh Bee-sheba yakni haiaka ya fullokahanchi tok.

15. Atuk osh oka kotoba alhto tuk vt taha ma, vlla yash itupushi achvfa ho nuta pila tok.

16. Micha ivt mishema oski naki a pila hotuk ohmi ho 
ona cha, et ichapaka yo binili tok: vlla yvt illi ka ak peso kashke, achi hatuk okvt. Mihmot et ichapaka ya binili cha chitolit yaiya tok.

17. Mihma Chitokaka yvt vlla ilbushvt paya ka haklo tok: mihma Chitokaka im enchel vt Haka ya vba et ai $\underline{\mathbf{i}}$ howvt, Nanta chi katiohmi cho? Haka ma, Ik chi komunto kashke; Chitokaka yut vlla yut ai itolut ilbushut paya ka haklo hoka.

18. Wakayá, vlla yash wakayachi cha, chibbak a isht ish ieshashke; oklushi chinto ikbi la hi oka, im achi tok.

19. Micha Chitokaka yut nishkin i fatummi na, kvli hofobi oka ya pisa tok: mihmut ivt kotoba yash oka alotoli cha, vlla yash ishkochechi tok.

20. Mihma Chitokaka yvt vlla ya iba foyuka tok; atuk o hofantit taha mvt, yakni haiaka vtta cha, ititanampo eshi imponna toba tok.

21. Micha Palan yakni haiaka ak o vtta tok: mihma ishki vt Echip yakni ak o ohoyo ha ai ishit ima tok.

22. Atuk o nitak yumma foka ma yakohmi tok, Abimelek, micha Fikol in tushka chipota pehlichi ita tuklo kvt Eblaham a im anumpulit, Nana ho ish a kaniohmi moma ka Chitokaka yot chiba foyuka hoke;

23. Yvmohmi hoka himak a Chitokaka hochifot anumpa kvllo a mihvt vno, micha svso, micha suso ha ushi aiena ka nana kia haksichit chik pi yumihcho kashke; amba chi kana hosh chi yumihich li tok a choyuhmit vno, micha okla inla yosh yakni ish ai ahanta tuk vt i yumihcha he osh (achit) is sum il onochashke, achit okla tok.

24. Mihma Eblaham vt, Anumpa kvllo il onochi lashke, achi tok.

25. Mihma Eblaham vt Abimelek in tishu vhleha hot kvli hofobi ya afout im ishi tuk ak o, Abimelek a nukfohkichi tok.

26. Mihma Abimelek vt Kuna hosh nana iluppa yumihchi tuk a ithana li tuk keyu: micha is sum anoli tuk keyu ho himak nitak illa ho haklo lishke, achi tok.

27. Mihma Eblaham vt chukfulhpoa, micha wak aiena ka 
ishi cha, Abimelek a ima tok: mihma ita tuklo kvt nan itim apesa tok.

28. Mihma Eblaham vt chukfulhpoa ya ai ishit chukfulhpoa ushi tek untuklo ho ila hiohlichi tok.

29. Mihma Abimelek vt Eblaham a, Chukfulhpoa ushi tek untuklo iluppak o nanta ho ish miha hosh ila ish hiohlichi cho? achi tok.

30. Mihma, Chukfulhpoa ushi tek untuklo iluppa subbak ish ai isha chi hoke; yumohmi ho kvli hofobi iluppa kulli li tuk a nan isht vm atokowa ya chi hoke, achi tok.

31. Yumohmi hatuk o yakni yumma Bee-sheba hochifo tok; yummak o ita tuklo kvt anumpa kvllo ai il onochi tuk okvt.

32. Yakohmi hosh Bee-sheba ya nana ai itim apesvt okla tok: yohmi ma Abimelek, micha in tushka chipota pehlichi Fikol ash ita tuklo kvt wakaya cha, falamvt Filistin okla i yakni ya onvt okla tok.

33. Mihma Eblaham vt Bee-sheba ak o iti lokoli hilechi cha, yưmmak o Chihowah Chitokaka bilia hohchifo ha ai $\underline{i}$ pahaya tok.

34. Mihmut Eblaham ut Filistin okla i yakni ya okla inla yosh ahanta na nitak laua tok.

\section{CHAPTA XXII.}

A TUK o ilúppa puta kut yumohmi ha ya yakohmi tok, A Chitokaka yut Eblaham a imomaka pisvt, Eblaham ma, achi tok. Mihma, Yakeh, yak asha lishke, achi tok.

2. Mihma, Himak a chiso hash, chiso yummak bieka Aisak ash ish holitobli chatuk vt ishi cha, Moliah yakni ya ish onashke; micha nunih achvfalit chim anoli la chi ka paknaka ya hukmit im issa mak o ikbit ont ai is sum issashke, achi tok.

3. Mihma Eblaham vt onnahinli fehna ho wakaya cha, im issuba haksobish falaia ya umpatali mut, hatak himithoa im asha tuk ash tuklo ho, micha ushi Aisak aiena ka pehlichi 
cha, ishit hukmit im issa iti ya puhli mut, wakaya cha Chitokaka vt im anoli tuk mak o ai ona tok.

4. Yvmohmi tuk osh nitak ont atuchina ma Eblaham vt akachakalit hopokoyo mot, ushi ai im issa chi nunih hvt hopaki ho et hikia na pisa tok.

5. Mihmvt Eblaham vt hatak himithoa yash, Ilvppa issuba haksobish falaia ya awant hush ai âshwa na vno, micha vlla ya il ita tuklo kvt yumma ont il ai aiokpvchi cha, anonti e huchim vlashke, im achi tok.

6. Mihmvt Eblaham vt ishit hukmit im issa iti ya ishi cha, ushi Aisak a shalichi tok; mihmut luak, micha bushpo aiena ka ilap ibbak a isht ai ishi cha, itatuklot ia tok.

7. Mihma Aisak vt iki Eblaham a im anumpulit, Akki, achi tok. Mihma, Yak aya lishke, svso ma, achi tok. Mihma, Yakeh! luak, micha iti okvto yak ash vshke, amba hukmit im issa ckukfulhpoa ushi muto? achi tok.

8. Mihma Eblaham vt, Suso ma, Chitokaka yut ilap akinli hosh hukmit isht aiokpvchi chukfulhpoa ushi ya ahayucha chi hoke, achi tok : yohmi ma ita tuklot ia tok.

9. Atuk osh Chitokaka yvt im anoli tuk nunih ha onvt okla tok: mihma Eblaham vt yummak o alta ikbi cha, iti ya achukmalit bohlit tahli tok; mihmvt ushi Aisak a takchi cha, alta paknaka iti on asha tuk a onochi tok.

10. Mihmut Eblaham vt ushi ya vba chị hosh ibbak a pit welit bushpo a ishi tok.

11. Mihma Chihowah im enchel vt vba ya et $\underline{i}$ howvt, Eblaham ma! Eblaham ma! ahanchi tok. Mihma, Yak hikia lishke, achi tok.

12. Mihma, Chibbak a vlla nakni yash ish onochi ná, micha nana kia chik $\underline{i}$ kaniohmecho kashke : himak a chiso hash, chiso yummak illa kash chik sum $\underline{i}$ halvllo hokvt, Chitokaka ya ish $\underline{i}$ nukshohompa ka ithana li hoka, achi tok.

13. Mihma Eblaham vt akachakalit hopokoyo ma, yakeh, im ashaka ya chukfulhpoa nakni vt abohli ho lapish isht talakchi hosh hikia tok: mihmvt Eblaham vt ivt chukfvlhpoa nakni ash ont ishi cha, ushi ya atobbichit hukmit isht aiokpuchi mak o im issa tok. 
14. Mihma Eblaham vt yvmma Chihowah-chileh hochifo tok: yummak atuk mak o, Chihowah $\underline{\mathrm{i}}$ nunih ha nana kut aivlhtaiya hoke, himak nitak a achi moma hoke.

15. Mihma Chihowah im enchel vt atukla ma, Eblaham a vba et ai i howa tok.

16. Micha Chihowah vt, Uno ak o vno akinli ho hochifot anumpa kullo il onochit, Nana iluppa ish yumihchi hatuk oka, micha chiso hash, chiso yummak illa kash chik sum $\underline{\underline{i}}$ halvllo hatuk oka;

17. Nayukpahanlit chi yukpali la hi oke, micha chim ishtatiaka ya apaknvchi hokvno apaknahanchit vba fochik $\underline{a}$ choyuhmichi likmot, okhvta vhli shinuk aiasha kak o choyuhmichi la hi oke; mikma chim ishtatiaka yut in tunup $\underline{i}$ holihta okhissa ya iesha hi oke.

18. Mikma vm anumpa ha ish im antia hoka, chim ishtatiaka ak o oklushi moma yakni paknaka aiasha kvt nan isht aiyukpa hi oke, achi li tuk oke, achi tok.

19. Yohmi ma Eblaham vt hatak himithoa im aiasha kash osh falamvt im ona tok; micha okla wakaya mut itapehut Bee-sheba ya ona tok: mihma Eblaham vt Beesheba ak o vtta tok.

20. Atuk o iluppa puta kvt yưmohmi ha ya yakohmi tok, Eblaham a im anolit, Yakeh! Milkah yummak kia chitibapishi Naho ya vlla im ehishishke;

21. Ushi vtta ahpi Hus, micha itibapishi Bus, micha Alam iki Kemuel,

22. Micha Cheset, micha Haso, micha Piltash, micha Chitlaf, micha Behluel.

23. Micha Behluel vt Lebekah ya tobachi tok : untuchina iluppak o Milkah vt Eblaham itibapishi Naho ya im eshi tok.

24. Mihma im itaiena yvt yvmma hohchifo kvt Leumah atuk osh yommak kia Tebah, micha Kaham, micha Hlahash, micha Maakah aiena ka im eshi tok, achi tok. 


\section{CHAPTA XXIII.}

MIHMA Salah vt afummi tahlepa achufa cha pokoli tuklo I 1 akucha unfuklo tok: ilvppak osh Salah vt okchayvt ahanta tok afummi holhtina hoke.

2. Atuk osh Salah vt Kichalh-alba ak o ai illi tok; yummot Kenan yakni talaia Heblon mak oke. Atuk o Eblaham vt Salah ya ishit nukhaklot yaiyvt aya hosh vla tok.

3. Mihmot Eblaham vt wakayvt im illi yash $\underline{\underline{i}}$ filvmmit hikia cha, Helh ushi vhleha ha im anumpulit,

4. Okla inla micha himo wihvt aya sia hosh hvchi takla ahanta lishke: hvchi itin takla ya ahollohpi yakni yosh an talaia chị ho micha vm illi ya a hohpit sa tikba ya a luhmi la chi ka hvs sumashke, achi tok.

5. Mihma Helh im vlla vhleha hot Eblaham a im afalaminchit,

6. Ish pi haponaklashke, a shahli ma, Chitokaka i holitopa yosh pi takla ahanta chia hoke: pim ahollohpi achukma i shahli kak o chim illi ya ish a hohpashke: e kanima kia pim ahollohpi ya e chi halvlla he keyu hoke, yohmi na chim illi ya chik a hohpo ka he keyu hoke, im achi tok.

7. Mihma Eblaham vt wakayut hikia cha, yakni yash okla hạ, Helh im vlla vhleha kash im akachunolit aka hlipia tok.

8. Mihmvt itim anumpulit, Um illi ya hohpit sa tikba ya a luhmi la hi ya hush ahnikmvt, hvs sa haponaklo cha, Soha ushi Eflon a hus sum ai im asilhhashke.

9. Yohmi na Makpelah hichukbi, yummvt im osapa ont vhli in talaia kvt vmashke; hvchitin takla ya ahollohpi yakni yosh an talaia chi ho tvli holisso ho aivlli vlhpiesakma vmashke, achi tok.

10. Yohmi ka Eflon vt Helh im vlla vhleha takla vtta hatok. Mihmvt Eflon Hittait hatak ash osh Eblaham a im afalaminchit, Helh im vila vhleha, in tvmaha holihta okhissa ya ont chukowa moma kvt hakklo ho,

11. Ahă $\underline{a}$ shahli ma, is sa haponaklashke: osapa ya chima li hoke, micha hichukbi vt anukaka talaia ka chima li 
hoke: vm okla hạ ushi vhleha itikba ya a chima lishke: chim illi ya ish a hohpashke, achi tok.

12. Mihma Eblaham vt yakni yash okla itikba ya akachunolit aka hlipia tok.

13. Micha yakni yash okla vhleha hot hạkklo họ, Eflon a im anumpulit, Yohmi kia is suma chi hokmvt, is sa haponaklashke, chim asilhha lishke: osapa ya tuli holisso ya chima lashke: is sum ishikma, vm illi ya a hohpi lashke, achi tok.

14. Mihma Eflon vt Eblaham a im afalaminchit.

15. A shahli ma, is sa haponaklashke; yakni vt toli hvta shekel tahlepa ushta ai iti vlli vlhpesushke; yummut pim itin takla ya nanta hoh cho? chim illi ya ish a hohpashke, im achi tok.

16. Mihma Eblaham vt Eflon a i haponaklo tok; micha Helh ushi vhleha hvt haklo ho, Eflon vt miha tuk mak o, Eblaham vt tuli huta ya $\underline{\mathbf{i}}$ wekichi tok, nan itatoba vhleha hot itim ishi chatuk mak osh toli hota shekel tahlepa ushta hatok.

17. Mihma Eflon im osapa yummut Makpelah ya talaia Mamle itikba talaia kash osapa, micha anukaka hichukbi talaia aiena ka , micha iti moma osapa ya hiohmaya tuk, micha aivhli fullota ka hiohmaya tuk $\underline{0}$,

18. Eblaham i yakni ya chị ho Helh im vlla vhleha itikba ya , micha in tumaha holihta chukowa moma ka itikba ya ai im aiahlichi tok.

19. Mihma $\underline{i}$ himmak ak o Eblaham vt Mamle ya itikba talaia Makpelah osapa hichukbi hikia ak o tekchi Salah ya a hohpi tok: yvmmut Heblon Kenan yakni talaia kak oke. 20. Mihma osapa, micha hichukbi anukaka talaia kvt ahollohpi yakni ya chị ho, Helh ushi vhleha hvt Eblaham a im aiahlichi tok.

\section{CHAPTA XXIV.}

A TUK o Eblaham vt sipoknit nitak lauvt in taiyaha haA tok: mihma Chihowah yut Eblaham a nana moma ka isht yukpali tok. 
2. Atuk o Eblaham vt in chuka achvfa tishu sipokni i shahli, yummvt nana im asha tnk moma ka pehlichi yo, Chibbak a saiyubi nuta ya isht ish potolashke, chim asilhha lishke:

3. Mikma Kenan okla takla ahanta li ka ushetik vhleha ha ohoyo ai ishit suso ha chik emo ka he yạ, Chihowah, vba in Chitokaka, micha yakni in Chitokaka ya hochifot anumpa kullo chil onochechi lashké.

4. Amba a yakni yạ, a kanomi $\underline{1}$ yakni ya ish ona cha, ohoyo ha ai ishit suso Aisak a ish imashke, im achi tok.

5. Mihma tishu hash osh, Ohoyo hut yakni iluppa si awant vla he keyu hokmuno, chiso ha yakni aivttut ish minti tok a anonti isht ona la hi pulla hoh cho? im achi tok.

6. Mihma Eblaham vt, Ahah, ish ahni cha, suso ha yumma isht chik ono kashke,

7. Chihowah, vba in Chitokaka yut, yummak osh aki in chuka, micha a kanomi $\underline{i}$ yakni ya a sushi tok vt, yummak osh vm anumpuli cha, anumpa kvllo vm ilonochit, Chim ishtatiaka ya yakni iluppa ima la hi oke, vm achi tuk vt yummak osh im enchel a et pila na, chi tikba ayakma, yummak o ohoyo ha ai ishit suso ha ish ima hi oke.

8. Atuk o ohoyo hvt chi awant minta he keyukma, anumpa kvllo iluppak ash ish $\underline{\mathrm{i}}$ mokofa hi oke; peh suso ha anonti yumma isht chik ono kashke, im achi tok.

9. Mihma tishu hash osh $\underline{\underline{i}}$ shahli Eblaham iyubi nuta ya ibbak ishit potoli cha, nana yumma isht anumpa kullo im il onochit anumpuli tok.

10. Micha tishu hash osh i shahli hvt kamel im aiasha kash kamel pokoli ho ishi cha ia tok: (i shahli imilayak moma kut ibbak ak o foyuka hatuk okvt;) micha wakaya mut, Mesopotamia yakni ya onvt, Naho in tumaha holihta ya ona tok.

11. Atuk osh opivt taha ma, ohoyo hut kohchvt oka ochi chatuk vt ont ai vlhpesa ma kvli hofobi tvmaha holihta ko. cha ya i kamel a huchukbilhkvchi tok.

12. Mihmvt, Chihowah, a shahli Eblaham in Chitokaka 
ma, nana ahni li ka himak nitak a is sưm aiahlichi cha, $\underline{a}$ shahli Eblaham a ish $\underline{i}$ kanashke, chim asilhha lishke.

13. Yakeh, oka kuli hofobi bilika iloppa hikia lishke; micha tvmaha holihta hatak a ushetik vhleha hvt oka ocha chi hosh et kohchushke:

14. Atukma yakohmashke, ohoyo himita nana ho, Chim isht ochi ya akvchi na, ak ishkó, chim asilhha lishke, achi likma, Ishkó, micha chị kamel ak kia oka ya ipeta lashke, achikmvt, yummak osh chin tishu Aisak a ish im atokoli tuk mak ashke; mikma yummak $\underline{o} \underline{a}$ shahli ha ish $\underline{\mathrm{i}}$ kana tuk a isht ithuna lashke, achi tok.

15. Atuk o yakohmi tok, anumpulit ont ik tahlo moma ho, yakeh, Lebekah vt, yummut Eblaham itibapishi Naho tekchi Milkah ya ushi Behluel im vtta tok vt im isht-ochi vt tahchi ha on talaia ho kohcha tok.

16. Micha ohoyo himita yvt hatak haleli keyu, hatak nana kia ithana tok keyu yosh pisaka aiokli fehna hatok: atuk osh kuli ya ona cha im isht-ochi yash alotoli cha, vla tok.

17. Mihma tishu hash osh malelit ont afama cha, Oka chim isht-ochi allhto ka kanomosi ka ak ai ishkó, chim asilhha lishke, achi tok.

18. Mihma, ohoyo himita yash osh, Ishkó, a shahli ma, achi tok: mihmvt tushpot im isht-ochi ya akvechit ibbak isht ishi cha, oka ya ipeta tok.

19. Atuk osh oka ya ipetvt tahli mot, Chi kamel ak kia oka ochit ipeta li, ishkot im vlhpesashke, achi tok.

20. Mihmvt tushpvot im isht-ochi ya tosholit oka aiipeta peni ya pit vni cha, oka ocha chi hosh anonti malelit kuli hofobi ya ona cha, i kamel moma ka oka im ochi tok.

21. Mihma hatak ash osh ohoyo himita yash aiokchifelit pisa mut, nana ahnit aya li tuk a Chihowah vt vm aiahlichikmá, keyukmá, nanta tuk oh chishba? ahnit ithuna bunna mut, nana ik acho tok.

22. Atuk osh yakohmi tok, kamel vt oka ishkot issa ma hatak ash osh toli lakna ibichilu takali weki kvt shekel ik- 
luna ho, micha ibbak vlbeha tuklo kvt weki kvt tuli lakna shekel pokoli ho ishi tok.

23. Micha, Kvta ushetik chia hoh cho? vm anolí, chim asilhha lishke: chiki in chuka ya il anusi vt asha cho? achi tok.

24. Mihma, Milkah ushi yummak oka Naho im eshi tok Behluel ushetik sia hoke, im achi tok.

25. Micha achakalinchi mut, Onush vpi, micha nanvlhpoa vpa aiena kvt pin laua achukma, micha anusi vt asha hoke, im achi tok.

26. Mihma hatak ash osh akachunoli cha, aka hlipivt Chihowah ya aiokpuchi tok.

27. Chihowah a shahli Eblaham a in Chitokaka yut a shahli hạ isht $\underline{i}$ nukhạklo, micha im anumpa ahli ya ik im ai esho kvt holitopashke: hina aya li ma, Chihowah yvt susht ayvt a shahli itibapishi vhleha ha in chuka ya susht vlushke, achi tok.

28. Mihma ohoyo himita yash osh malelit nana iluppa puta ka ishki in chuka ai achufa ya ont im anoli tok.

29. Yohmi ka Lebekah vt i nakfi vt anta cha, hohchifo kvt Laban atok: mihma Laban vt kohchvt malelit hatak ash osh kuli hofobi ya bilika hikia ka ona tok.

30. Atuk o yakohmi tok, ibichilu takali, micha ibbak vlbeha aiena kvt in tek ibbak a vlbiha na pisa mvt, micha in tek Lebekah im anumpa, Hatak vt yak vm achishke, achi na haklo mot, hatak ash im ona tok; mihma yakeh, kamel vt kvli hofobi aiasha na, hatak ash osh bilika hikia tok.

31. Atuk osh, Ant chukowá, Chihowah i holitopa chia ma! nanta katiohmi ho kocha ish hikia cho? aboha ya atahli li, micha kamel a aiasha im atahli li hoka, achi tok.

32. Mihma hatak ash osh chuka ya ont chukowa tok: mihma i kamel ash ikfoka isht asheha ya shoeli cha, onush vpi, micha nanvlhpoa vpa aiena ka ipeta mvt, iyi, micha hatak apehvt aya tuk iyi aiena ka isht achifa chi ka oka ya ima tok.

33. Mihma vpa chi ka ilhpak vt itikba taloha tok: yohmi tuk kia, Nana aiahnit aya li tuk vt ak anolo kisha kvt 
impa la he keyu hoke, achi tok. Mihma, Anumpuli', achi tok.

34. Mihma, Eblaham in tishu sia hoke.

35. Atuk o Chihowah yut a shahli ha nayukpali fehna, na holitopa fehnut tahushke: micha nanvlhpoa chipinta, micha nanvlhpoa hochito, micha tvli huta, micha tvli lakna, micha hatak tishu, micha ohoyo tishu, micha kamel, micha issuba haksobish falaia aiena ka ima tuk oke.

36. Mihma a shahli ha tekchi Salah vt sipoknit taiyaha hosh a shahli ha vlla nakni yo im eshi tok oke: atuk o yummak o nana im asha tuk vt mominchit ima tuk oke.

37. Atuk osh a shahli hvt anumpa kvllo si ilonochechit Kenan okla $\underline{\mathrm{i}}$ yakni ahanta li tuk $\underline{a}$ ushetik vhleha ha ai ishit suso ha ohoyo ish ima he keyu:

38. Amba ivt a ki in chuka, micha a kanomi i yakni ya ish ona cha, ohoyo ai ishit suso ha ish imashke, vm achi tuk oke:

39. Mihma a shahli ha, Ohoyo hut siakaiya he keyu yoba hokmuno, im achi li tuk oke.

40. Mihma, Chihowah yummak o itikba nohowa li chatuk vt im enchel a chi apehvchit pila cha, nana ahnit ish aya ka chim aiahlicha hi oke; mikma a kanomi yo, micha aki in chuka achufa yo ai ishit sưso hạ ohoyo ish ima hi oke.

41. Yohmikma a kanomi ya ish im onakmvt, vm anumpa kullo iluppa ish i mokofa hi oke; atuk o achvfa ka ik chemokma, um anumpa kullo iluppa ish I mokofa hi oke, vm achi tuk oke.

42. Atuk o himak nitak a kvli hofobi ya vla li mvt, Chihowah, a shahli in Chitokaka ma, himak a nana ahnit aya li tuk $\underline{a}$ is sum aiahlichi hokma:

43. Yakeh, oka kvli hofobi bilika hikia lishke; atuk o yakohmashke, ohoyo himita hatak haleli keyu yosh et kohchut oka ochi na, Oka chim isht-ochi alhto ka kanomosi ka ak ai ishkó, chim asilhha lishke, achi likma,

44. Yohmikma, Ishkó, mikma chi kamel ak kia im ochi lashke; vm achikmvt, Yohmikmvt ohoyo yummak o Chi- 
howah yot a shahli ushi ya im atokoli tuk mak ashke, achi li tuk oke.

45. Atuk o sa chụkvsh anukaka anumpuli li kvt ont ak tahlo kisha ho, yakeh, Lebekah vt im isht-ochi yvt tahchi ha on talaia ho et kohcha tuk oke; micha kuli hofobi ya okvtaha cha, oka ochi tuk oke; mihma, Ak ishkó, chim asilhha lishke im achi li tuk oke.

46. Mihma tushput im isht-ochi yut tahchi ha on talaia ko akvchi mvt, Ishkó, mikma chị kamel ak kia oka ipeta lashke, achi tuk oke: yohmi na ishko li ma, kamel ak kia oka ipeta tuk oke.

47. Mihma i ponaklot, Kvta ushetik chia hoh cho? achi li ma, Naho ushi yummak oka Milkah vt im eshi tok Behluel ushetik sia hoke, achi tuk oke: mihma ibichilu takali ibichilu ya takalichi li mvt, ibbak vlbeha aiena ka ibbak abehli li tuk oke.

48. Mihmut akachunolit aka hlipivt Chihowah ya aiokpuchi li tuk oke; micha Chihowah a shahli Eblaham in Chitokaka, yummak osh a shahli Eblaham itibapishi ushetik $\underline{0}$ ishit ushi ya ima la chị ho hina aivlhpiesa ka súsht aya tuk a holitoblichi li tuk oke.

49. Yohmi ka himak a a shahli ha $\underline{a}$ kanvt hvsh im aia $\underline{a}-$ la chi hokmut, hus sum anolashke: keyukmut hus sum anolashke: yumohmi ho ishtimpak imma, keyukmut vlhfubekimma pit fullota lashke, achi tok.

50. Yohmi ma Laban, micha Behluel vt afalaminchit, Nana ka Chihowah ak osh ahni tuk pullushke: okpulo hokma, achukma hokma nana kia e chi miha he keyushke.

51. Yakeh, Lebekah vt chi tikba ashushke, ishi cha iá, mikma Chihowah yot anumpuli tuk mak o chi shahli ushi ya tekchi yashke, achit okla tok.

52. Atuk o yakohmi tok, Eblaham in tishu hvt im anumpa ha haklo mut, aka hlipia cha, Chihowah ya aiokpvichi tok.

53. Mihmot tishu hash osh tuli huta ishit shema, micha tvli lakna ishit shema, micha nafohka ishit kohchi cha, Lebekah ya ima tok. Micha Lebekah i nakfi, micha ishki itatuklo ka naholitopa puta ka ima tok. 
54. Mihma yummak ash, micha hatak apehvt aya tuk aiena kut okla imput nan ishko tok, micha asha na onna tok: atuk osh ont onna ma wakaya tok: mihma tishu hash osh A shahli ha pit hus sa pelashke, achi tok.

55. Mihma i nakfi, micha ishki yash osh, Ohoyo himita vt nitak holhtina, peh nitak pokoli hokvno pi takla ahantashke; yohmi ha yosh iashke, achit okla tok.

56. Mihma, Hưs sưm ataklummi ná, nana ahnit aya li tuk a Chihowah yot vm aiahlichi hatuk oka: huss sa pila na a shahli ha im ona lashke, im achi tok.

57. Mihma, Ohoyo himita ako e hoyo cha, ilap itih akinli ho ai il im aponaklashke, achit okla tok.

58. Mihma Lebekah ya hoyot okla cha, Hatak iluppa awant ish ia chi cho? im achi ma, Ia la chi hoke, achi tok. .

59. Mihma in tek Lebekah, micha ilaueli yatok, micha Eblaham in tishu, micha hatak apehvt aya tuk aiena kash okla pila tok.

-60. Micha Lebekah ya nayukpalit, Pin tek chia hut; milyun tahlepa sipokni puta hoh kia ishki chia hashke; mikma chim ishtatiaka yut $\underline{\mathbf{i}}$ nukkilli puta $\underline{\mathbf{i}}$ holihta okhissa ya ieshashke, im achi tok.

61. Mihma Lebekah, micha ohoyo himita im asha aiena kvt wakaya cha, kamel ash on chieya mut, hatak ash iakaiya tok: mihma tishu hash osh Lebekah ya ishi cha, ia tok.

62. Mihma Aisak vt kvli hofobi Lahai-loi pilla ha minti tok: oka mahli yakni ak o vtta hatuk okvt.

63. Atuk osh opivt taha ma, Aisak vt osapa ya nan anukfillit hikikị chi hosh kohchvt ia tok: atuk osh akachakali cha pisa ma, yakeh, kamel ash osh minti na pisa tok.

64. Mihma Lebekah vt akachakali cha, Aisak a pisa mot, kamel a om binili tuk vt akowa tok:

65. Tishu hash, Hatak iluppot kuta hosh osapa nohowvt pi afamvt minti cho? im achi na tishu hash osh, A shahli yoke, achi tuk atuk oka; yumohmi ma nashuka isht ompoholmo ishi cha il ompohomo tok.

66. Mihma tishu hash osh nana kaniohmi tuk vt moma Aisak a im anoli tok. 
67. Mihma Aisak vt ishki Selah im vlhtipo ak o isht ona tok, micha Lebekah ya ishi na tekchi toba tok: mihma holitobli tok: micha Aisak vt ishki illi tuk a hopohla tok.

\section{CHAPTA XXV.}

UMOHMI tuk o anonti Eblaham vt ohoyo ishi tok;

1 mihma hohchifo hut Ketulah atok.

2. Mihmvt Simlam, micha Chokshan, micha Metan, micha Mitian, micha Ishbak, micha Shuah aiena kak o im eshi tok.

3. Atuk o Chokshan vt Sheba, micha Tetan a tobachi tok. Mihma Tetan ushi vhleha hut Ashulim, micha Letushim, micha Leummim aiena kak atok.

4. Mikma Mitian ushi vhleha hut Efah, micha Efe, micha Hanok, micha Abitah, micha Eltàah aiena kak atok. Ilvppa puta kak osh Ketulah im vlla vhleha hatok.

5. Mihma Eblaham vt nana im asha tuk vt moma Aisak ak o ima tok.

6. Amba im itaiena ushi vhleha ha Eblaham vt im asha tuk vt Eblaham vt nahvlbina ima cha, okchaya moma kak osh ushi Aisak a i filummichit hushi akochaka pilla, hushi akochaka yakni ak o pila tok.

7. Atuk o Eblaham vt okchayvt ahanta afvmmi puta ya nitak puta okchayut ahanta kut iluppak oke, afummi tahlepa achvfa cha pokoli untuklo akucha tahlapi tok.

8. Yohmi mut Eblaham vt fiopissa tok, micha sipokni achukma hatak sipokni, micha afummi laua hosh illi tok; micha im okla ha iba holhtina tok.

9. Mihma ushi Aisak, micha Ishmael vt Hittait hatak Soha ushi Eflon im osapa hikia hichukbi Makpelah, yvmmut Mamle itikba ya hikia kak o a hohpi tok.

10. Osapa ya Eblaham vt Helh ushi vhleha ha in chumpa tok ak atok: yummak o Eblaham micha tekchi Salah vt a hollohpi tok.

11. Atuk o yakohmi tok, Eblaham vt illi ha ya, ushi Ai- 
sak a Chitokaka yot yukpali tok: mihma Aisak vt kvli hofobi Lahai-loi ak o ai vtta tok.

12. Yohmi ka iloppa puta kak osh Echip ohoyo, Salah in tishu ohoyo Haka vt Eblaham a im eshi tok, Ishmael Eblaham ushi a im ishtatiaka yoke.

13. Ilvppa puta kak osh Ishmael ushi vhleha hohchifo hosh in chuka achvfa puta kvt vlhpisvt hohchifo hoke: Ishmael a im vttahpi Nebayolh: micha Keta, micha Atbeel, micha Mibsam,

14. Micha Mishma, micha Tumah, micha Massa,

15. Hata, micha Tema, Chetu, Nafish, micha Ketemah :

16. Iloppa puta kak osh Ishmael ushi vhleha hoke, micha iluppa puta kak osh in tumaha vlhpisvt, micha i holihta vlhpisut hohchifo hoke; apehlichi vlhpisvt pehlichi auah tuklo hoke.

17. Atuk o iluppa puta kak osh Ishmael vt okchayvt ahanta afummi puta kvt afvmmi tahlepa achvfa cha, pokoli tuchina akucha untuklo tok: atok osh fiopissut illi cha, im okla ha ibaholhtina tok.

18. Mihma Hafilah ya vttut ia hosh Assilia pilla ish ia ka Shu Echip itikba talaia kak o okla aiasha tok: mihma itibapishi moma itikba ya Ishmael vt ai illi tok.

19. Mihma ilvppak osh Eblaham ushi Aisak a in chuka achvfa anoli holisso hoke: Eblaham vt ushi Aisak a tobachi tok:

20. Mihma Aisak vt afvmmi pokoli ushta hosh Silian hatak Patan-alam vtta Behluel ushetik, Silian hatak Laban in tek Lebekah ya ishit itauwaya tok.

21. Atuk osh tekchi vt vlla ik im iksho yatuk o, tekchi ya isht anumpulit Chihowah ya im asihihla tok: mihma Chihowah yut vlhpesa im ahni na, tekchi Lebekah vt chakali tok. 22. Atuk o vlla yash osh ikfoka alhto kvt itvboa tok: mihma, Yohmi hokma, nanta katiohmi ho si yakohmi cho? achi mvt, Chihowah ya ont im a ponaklo tok.

23. Mihma Chihowah yvt, Oklushi tuklo hosh chim oshvtto ya alhto hoke; micha okla nana a kaniohmi kvt tuklo hosh chikfoka ya a kohcha chị hoke: mikma okla achvfa 
kvt kvllo kvt okla achvfa ya i shahla hi oke, mikma akni ak osh nakfish a i nutaka hi oke; im achi tok.

24. Atuk o vlla esha he vlhpesa nitak vt vla ma, yakeh, haiyup vt im oshvtto ya alhto tuk atok.

25. Atuk o tikba kohcha kvt hummut nipi moma kvt nafohka hishi asha chohmi tok: mihma Esau, okla hochifo tok.

26. Atuk o i himmak o itibapishi mvt kohcha mvt, Esau iyikatoba ya ibbak ishit hoklit ieshi tok: mihma hochifo kvt Chekob achi tok: mihma yvmma Lebekah vt eshi ma, Aisak vt afummi pokoli hannali hatok.

27. Mihma vlla yash osh hofantit taha tok; atuk osh Esau vt owvtta imponna, kowi aya hatak atok: micha Chekob vt hatak aivlhpiesa yosh vlhtipo ahanta tok.

28. Mihma Aisak vt Esau im issi nipi vpa kak osh holitobli tok: amba Lebekah vt Chekob a holitobli tok.

29. Mihma Chekob vt tubi ya honi tok: atuk o Esau vt kowi aya tuk osh vla mot, hoyvbli tok.

30. Mihma Esau vt Chekob a, Chim asilhha lishke, nahonni humma yưmma fehna ka supetá, sa hoyubli hoka, im achi tok: yumohmi kak o hochifo kut Etom achi tok.

31. Mihma Chekob vt, Himak nitak a vttahpi ish ai isha he vlhpesa ya is sa kanchashke, achi tok.

32. Mihma Esau vt, Yakeh, svlla hosishke, mikma vttahpi ai isha he vlhpesa ilupput nanta isht vm achukma hinla cho? achi tok.

33. Mihma Chekob vt Himak nitak a, anumpa kvllo il onochit a mihá, achi tok: mihma anumpa kvllo im il onochit miha tok: mihmvt vttahpi ai isha he vlhpesa yash Chekob a $\underline{i}$ kanchi tok.

34. Yohmi ma Chekob vt Esau ya puska, micha tubi honni aiena ka ima tok ; milima impa cha, ishkot issa mvt, wakaya cha ia tok: yakohmit Esau vt vttahpi ai isha he vlhpesa ya shitilema tok. 


\section{CHAPTA XXVI.}

A TUK o yakni ya Eblaham ahanta nitak ma, hohchufo A chito vmmona tok vt asha na, anonti hohchvfo chito tok. Mihma Aisak vt Filistin okla i miko Abimelek im onvt, Kela ya ona tok.

2. Mihma Chihowah yut i haiaka cha, Echip a chik aiyo kashke: yakni chim anoli la chị kak o ish ahantashke.

3. Yakni iluppak o nowvt aya yosh ish ahantashke, mikma chibafoyuka lish, chi yukpali lashke; chishno, micha chi aiunchololi yak o yakni ịluppa moma ka ima la chi hoka, micha anumpa kvilo yumma anumpulit chiki Eblaham a im il onochi li tok vt aiahlichi la chi hoke.

4. Micha chim ishtatiaka ya apaknvchit vba fochik choyuhmichi la chi hoke; mikmvt yakni iluppa moma ka chim ishtatiaka ya ima la chi hoke: mikma chim ishtatiaka yak o oklushi moma yakni paknaka aiasha kvt ai yukpa hi oke.

5. Eblaham vt vm anumpa ha im antivt, atokolit i miha li, micha vm anumpa vlhpisa, micha a nanvlhpisa a puta aiena ka holitobli tok oka, achi tok.

6. Mihma Aisak vt Kela ya ahanta tok.

7. Atuk o yakni yumma hatak vhleha hut tekchi ya im a ponaklo tok; mihma, An tek oke, achi tok: Sa tekchi yoke, achi kut nukshopa hokvt; yakni yash hatak vhleha hut Lebekah ak atuk pulla mak o subi yoba na, achi hatok; pisaka aiokli hatuk oka.

8. Atuk o yakohmi tok, yumma ahanta tuk a hopakit taiyaha ma, Filistin okla i miko Abimelek vt okhissushi pit a hopokoyot pisa ma, yakeh, Aisak vt tekchi Lebekah ya itiba ihlauvlli hosh ashwa na pisa tok.

9. Mihma Abimelek vt Aisak a hoyo cha, Yakeh, chi tekchi pullushke: yohmi hokak o nanta katiohmi ho, An tek oke, ish achi tuk oh cho? achi tok. Mihma Aisak vt, Ishit svlli yoba na, achi li hatuk oke, im achi tok.

10. Mihma Abimelek vt, Iluppak osh nanta ho ish pi yumihchi cho? okla hash achufa kut chi tekchi ya yummak 
fokalichit iba tushka hinla tuk oke; yohmi tuk ma nanashvchi ish pi onocha hetuk oke, achi tok.

11. Mihmvt Abimelek vt im okla moma ka atokolit $\underline{i}$ mihut, Kuna hosh hatak ilvppa, keyukmut tekchi ya potolikmvt, illi pullashke, achi tok.

12. Yohmi ma Aisak vt yakni yumma a hokchi cha, afummi mih makinli ka inlohmi tahlepa achvfa ho ishi tok: mihma Chihowah yot yukpali tok.

13. Mihma hatak ash osh holitopa cha, tikba mahayut holitopa kvt i shahlit mahaya cha, holitopa fehnut taha tok.

14. Imilayak vlhpoa chipinta, micha imilayak vlhpoba hochito, micha tishu laua fehna aiena kvt im aiasha tok: mihma Filistin vhleha hut isht i potunno tok.

15. Iki Eblaham ahanta nitak a iki in tishu vhleha hot kvli hofobi moma ka kulli tok a Filistin vhleha hot $\underline{i}$ kamalit lukfi a isht alotoli tok oka.

16. Mihma Abimelek vt Aisak a Ish pi filvmmashke: kvllo kvt ish pi shahli fehna hokvt, im achi tok.

17. Mihma Aisak vt yumma vttut ia mut, Kelaokfa ak $\underline{0}$ vlhtipo ha hilechi cha, yummak o ahanta tok.

18. Micha iki Eblaham vt ahanta nitak mvt, kvli hofobi oka okla kulli tok ash Aisak vt anonti kulli tok: Eblaham vt illi ha ya Filistin vhleha hut i kamali tok oka: micha hohchifo ha iki vt hochifo tok akinli ho ita hochifo tok.

19. Atuk o Aisak in tishu vhleha hvt okfa ha a kulli mot oka tobohonli kvli ho ahayuchi tok.

20. Atuk o Kela ya nan vlhpoa apistikeli vhleha hvt Aisak $\underline{i}$ nan vlhpoa apistikeli vhleha ha im afovt, Oka vt pimmi hoke, achi tok: mihma kuli hofobi yash hohchifo ha Esek achi tok; im afoa tuk ak atok.

21. Atuk o kuli hofobi inla ho okla kulli tok; atuk $\underline{o}$ yummak kia afoa tok: mihma hohchifo ha Sitnah achi tok.

22. Mihma yumma vtta tuk vt kanvlli cha, kvli hofobi inla ho kulli tok; mihma yummuno ik im afowo tok: mihma hohchifo ha Lehobolh achi tok: micha, Himak ano Chihowah yut aiasha pin chitoli hoke; yohmi ho yakni ya waya achukmot il aiasha chị hoke, achi tok. 
23. Atuk osh yumma vtta tuk vt ivt Bee-sheba ya ona tok.

24. Atuk o mih nitak akinli ka Chihowah yot $\underline{i}$ haiaka cha, Chiki Eblaham a in Chitokaka sia hoke: chi nukshopa ná, an tishu Eblaham ak atuk pulla kak o chibafoyuka lish chi yukpalit chim ishtatiaka ya apaknvchi la chi hoke, achi tok.

25. Mihma yumma alta ai ikbi cha, Chihowah hochifut $\underline{\mathrm{i}}$ pahaya tok; micha yummak o im vlhtipo ya a hilechi tok: mihma yummak o Aisak in tishu vhleha hvt kvli hofobi ya a kulli tok.

26.Atuk o Abimelek, micha i kana vhleha ha achvfa Ahussalh, micha in tushka chipota $\mathrm{i}$ kupituni chito Fikol aiena kvt Kela asha tuk vt Aisak a im ona tok.

27. Mihma Aisak vt, Hus sa nukkilli cha, hvochi filummichit hus sa chvffichi tuk okvt, nanta katiohmi ho hus sum vla cho? im achi tok.

28. Mihma Chihowah yut chibafoyuka pulla ka e pisa tuk oke: mihmvt himak a anumpa kvllo vt pitin takla itonlashke, pishno, micha chishno pulla ka pitin takla itonlashke; yohmi kvt chishno ya nan e chitim apesashke.

29. Yumohmi ho nana e chi kaniohmichi tuk keyu kă, micha nana kvt achukma bieka ho e chi yumihchi tuk, micha achnkma ho ayashke, e chim ai ahni tuk oka, chiyuhmit chik pi hotopalo kashke: himak a Chihowah yut yukpali chia hoke, okla achi tok.

30. Mihma Aisak vt impvchi im ikbi na, imput nan ishkot okla aiasha tok.

31. Atuk osh onnahinli fehna ho okla wakaya cha, anumpa kullo itim il onochi tok: mihma Aisak vt ik iá ahni na, filvmmit iti kana achukma hosh ilhkoli tok.

32. Atuk o mih nitak akinli ka yakohmi tok, Aisak in tishu vhleha hvt kvli hofobi kulli tuk vt ant im anolit, Oka il ahayuchishke, achi tok.

33. Mihma Sheba hochifo tok: yumohmi tok o himak nitak a tumaha holihta yut hohchifo kut Bee-sheba moma hoke.

34. Atuk o Esau vt afummi pokoli ushta hosh Hittait ha- 
tak Beeli ushetik Chutilh $\underline{\text { a }}$, micha Hittait hatak Elon ushetik Bashemalh a itauwaya tok.

35. Yvmmak oka Aisak micha Lebekah vt ishit nukhakklo beka tok.

\section{CHAPTA XXVII.}

TUK o yakohmi tok, Aisak vt sipoknit taiyaha cha, nish A kin vt illi tok; yohmi cha, pisa he keyu mot, ushi ak ni fehna Esau ya $\underline{i}$ howa cha, Suso ma, im achi tok; mihma, Yakeh, yak hikia lishke, im achi tok.

2. Mihma, Yakeh, sa sipoknit taiyahushke, nitak a sulla he vt ithana li keyushke:

3. Yvmohmi hoka himak a chị nan owvttut ishtaya, chim oski naki aivlhto, micha chim ititanampo aiena ka ishi cha, kowi haiaka ya ivt issi nipi hoyot isht is svm vlashke,

4. Micha nipi champuli an champuli chatuk a chohmi ho is sum ikbi cha, isht is sum vla na vpa lashke: yohmikma ik svllo kisha kak o vmi shilombish vt chi yukpalashke, im achi tok.

5. Atuk o Aisak vt ushi Esau ya im anumpuli ma, Lebekah vt haklo tok: mihma Esau vt issi nipi hoyot isht vla chị hosh kowi haiaka ya ia tok.

6. Mihma Lebekah vt ushi Chekob a im anumpulit, Yakeh, chiki vt chitibapishi Esau ya im anumpulit,

7. Issi nipi isht vm vla cha, nipi champuli vm ikbi na ak pá, micha ik svllo kissha kak osh Chihowah itikba ya chi yukpali lashke, achi ho haklo li tuk oke,

8. Yvmohmi hoka himak a suso ma, um anumpa nana chi miha li ka is sum antiashke.

9. Himak a nan vlhpoa chipinta aiasha ka ia cha, issi kosoma ushi achukma tuklo ho ont ai ishit vm vlá; mikma chịki ya nipi champuli in champuli chatuk a chohmi ho im ikbi lashke;

10. Mikma chịki ya isht ish im ona na vpashke, micha ik illo kisha kak osh chi yukpalashke, im achi tok.

11. Mihma Chekob vt ishki Lebekah ya Yakeh, itibapi- 
shi li Esau vt hatuk hishi chito yokma, vno vt hatak hishi iksho sia hoke:

12. Aki vt sa pashohli yoba hokmvno, yohmikma nahaksichi im i chohmi la chi hoke; yohmikma ishit kalakshi ak o isht il onochi la chi hoke, micha nan isht aiyukpa achi keyu ka chi hoke, im achi tok.

13. Mihma ishki vt, Uno ak o nan ishit chi kalakshi vt si onvtoyulashke, suso ma, peh vm anumpa hak o ish im antia cha, yumma ont isht is sum vlashke, im achi tok.

14. Mihma ivt ont hokli cha, ishki ya isht im vla tok: mihma ishki vt nipi champuli, iki in champuli chatnk a chohmi ho ikbi tok.

15. Micha Lebekah vt ushi akni fehna Esau i nafohka holitopa yosh Lebekah vt aboha im asha tuk a ishi cha, ushi Chekob nakfish ak $\mathrm{a}$ fohkvichi tok:

16. Mihmot issi kosoma ushi yash hakshup a ibbak a isht ompohomo mot, micha ikunla, hishi ik ai iksho aiena ka isht ompohomo tok.

17. Mihmot nipi champuli, micha puska atahli tuk vt ushi Chekob a imvt ibbak fohki tok.

18. Mihma îki ya im ona cha, Aki ma, achi tok. Mihma, Yak asha lishke; kuta chia hoh cho? suso ma, achi tok.

19. Mihma Chekob vt iki ya , Chim vttahpi Esau sia hoke; is sa miha tuk mak o yvmihchi lishke: chim asilhha lishke, ish wakaya cha, binilit vm issi nipi ya ish pashke, yvmohmi hosh chimi shilombish ut si yukpalashke, im achi tok.

20. Mihma Aisak vt ushi yash, Nanta katiohmi ho chekosi ish ahayuchi tuk oh cho? suso ma, im achi tok. Mihma, Chihowah chin Chitokaka ak osh isht vm vla tuk oke, achi tok.

21. Mihma Aisak vt Chekob a , Bilika mintí, chim asilhha lishke, suso ma, chi pashohli lashke, suso Esau chia fehna hokmá, keyukmá, nana hokmá, achi tok.

22. Mihma Chekob vt iki Aisak a bilika ona tok; mihma pashohlit pisa mvt, Anumpa huto Chekob im anumpa, amba ibbak vto Esau ibbak oke, achi tok. 
23. Mihmvt ibbak vt hishi chito itibapishi Esau ibbak chohmi hatuk o ik ithano tok: yohmi mot yukpali tok.

24. Mihmvt, Suso Esau fehna chia hoh cho? achi tok. Mihma, Mih sia hoke, achi tok.

25. Mihma, A bilika isht ish lashke, mikma suso im iss nipi ya vpa lish, vmi shilombish vt chi yukpalashke, achi tok. Mihma i bilika isht ona na vpa tok: mihmot oka paki ya isht im ona na ishko tok.

26. Mihmvt iki Aisak vt, Inta, bilika mintí, micha is sa suksowashke, suso ma, im achi tok.

27. Mihma bilika ona cha, $\underline{i}$ suksowa tok: mihma $\underline{\underline{i}}$ nai fohka balama ka huwa mot, yukpalit, Yakeh, svso balama kut osapa yo Chihowah yut achukmali tuk a balama kalk $\underline{0}$ chohmishke.

28. Yvmohmi hoka Chitokaka yut vba fichak, micha yakni a nia, micha onush, micha oka paki aiena laua ho chimashke.

29. Okla hut chim antiashke, micha oklushi puta lkvt chim akachunolit aka hlipkaiyachashke: chitibapishi vhleha ha ish ishahlashke: mikma chishki ushi vhleha hot chim akachunolit aka hlipkaiyachashke: kuna hosh chi kalakshichikmut kalakshashke, mikma kuna hosh chi yukpalikmvt nayukpashke, aghi tok.

30. Atuk o yakohmi tok, Aisak vt Chekob a yukpali kvt ont tahli makinli ho, atuk o Chekob vt íki Aisak itikba ya i filummit peh kohcha makinli ho, itibapishi Esau vt owvttot aya tuk vt vla tok.

31. Micha yưmmak kia nipi champuli ikbi tuk atok; atuk osh ㅌki ya isht im vla cha, îki yash, Alki vt ik wakaya cha, ushi im issi nipi ya ik pá, micha chimi shilombish vt sa yukpalashke, im achi tok.

32. Mihma iki Aisak vt, Kvta chia hoh cho?' im achi tok. Mihma, Chim vttahpi Esau, chiso sia hoke, achi tok.

33. Mihma Aisak vt wunihinchi kvt atampa fehna tok; micha, Kvta hosh himak a chik lo kisha ma, issi nipi ishit isht vm vla na moma ka ai ishit apa lish, yukpali li tuk mvto? ahli, yohmi na nayukpa hi oke, achi tok. 
34. Mihma Esau vt iki im anumpa ha haklo mvt, yaiya ilbusha atampa fehna ho yaiya tok ; micha ilki ya , Is sa yukpalashke, vno ak kia, aki ma, im achi tok.

35. Mihma, Chitibapishi hut sa haksichit vla cha, nan isht chi yukpa ya ishi tuk oke, achi tok.

36. Mihma, Aivlhpiesa ho Chekob hohchifo keyu cho? ilupput ont atukla mak o sa haksichi hokvt: vttahpi ai isha he vlhpesa ya vm ishi tok; atuk osh himak a, yakeh, nan ishit sai yukpa ya um ishishke, achi tok. Mihmơt, Nan ishit yukpali ya chik sum ilatombo tuk oh cho? achi tok.

37. Mihma Aisak vt afalaminchit Esau yạ, Yakeh, chi shahli ikbi li tuk oke, micha itibapishi vhleha moma kut in tishu vhleha ha chị ho ima li tuk oke; micha onush, micha oka paki yo ishit hlampkochi lishke: yohmi ka suso ma, himak a nanta ho chi katiohmichi la hinla cho? im achi tok.

38. Mihma Esau vt ikki ya Alki ma, nan isht yukpali vt chim achvfa illa cho? is sa yukpalashke, vno ak kiá, akki ma, im achi tok. Mihmut Esau vt chitoli hosh yaiya tok.

39. Mihma iki Aisak vt afalaminchit, Yakeh, ish aiahanta yut yakni a nia, micha vba fichak vba a minti ak ahi oke.

40. Micha chi bushpo falaia ak $\underline{0}$ ish ieshi kak osh ish ahanta cha, chitibapishi, ha ish $\underline{\mathrm{i}}$ nutaka hi oke: atukma yakohma hi oke, ish imaiyakmot, im ikunla abana chikunla abana tuk a ish kobvffa hi oke, im achi tok.

41. Mihma nan ishit yukpali iki vt ishit yukpali tuk ak

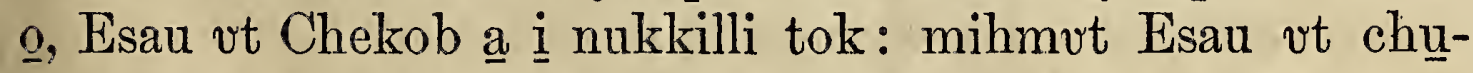
kv'sh anukaka, Aki ishit yaiya nitak vt v]a hosishke, yohmikma itibapishi li Chekob a vbe la hi oke, achi tok.

42. Mihma ushi akni Esau im anumpa ilvppa puta kvt Lebekah ya im annoa tok: mihma ushi nakfish Chekob a pit hoyot $\underline{i}$ howa cha, Yakeh, chitibapishi Esau vt chishno ya chiba chi ka ahni cha, ile nuktalalit ahantushke.

43. Yvmohmi hoka inta, suso ma, vm anumpa ha ish im antiashke; micha wakayvt malelit a nakfi Laban a im onvt, Halan a ish onashke. 
44. Micha nitak ik lauo ho yummak o takla ish ahanta na, chitibapishi nukhobela kvt nuktula ha yashke:

45. Chitibapishi nukhobela tuk vt issa cha, nana ish $\underline{\mathrm{i}}$ kaniohmichi tuk a imahaksi ha yashke: yohmikma pit chi hoyo lish, ont a chisht vla la hi oke. Nanta katiohmi ho hvchishno ak kia nitak achufa ho itatuklot hus san tamoa hinla cho? im achi tok.

46. Mihma Lebekah vt Aisak a, Helh ushetik vhleha pulla kak. $\underline{a}$ nitak a isht an takobit tahushke: Chekob vt ilvppakinli chohmi yakni ya ushetik vhleha, Helh ushetik vhleha ho ohoyo ai ishikma, a nitak a nanta ho isht vm achukma hinla cho? im achi tok.

\section{CHAPTA XXVIII.}

IIHMA Aisak vt Chekob a hoyo cha, yukpali mut, ato11 kolit i mihut, Kenan okla ushetik vhleha ho ohoyo ha ish ai isha he keyu hoke.

2. Wakaya cha, Patan-alam a onvt, chishki ịki Behluel in chuka ya ish onashke; micha chishki ya itibapishi Laban ushetik vhleha yummak o ohoyo ha ont ish ai isha hi oke.

3. Mikma Chitokaka Nan isht im aiahli ataha iksho hvt chi yukpalashke; micha chi wayvchit chi apaknuchashke, yumohmi ho okla laua chia hashke;

4. Micha Eblaham isht-aiyukpa ya chishno, micha chi aiunchololi aiena ka chimashke: yumohmi ho yakni ya Chitokaka yut Eblaham ima tok a okla inla chia hosh ish ai ahanta kvt ish ishashke, im achi tok.

5. Micha Aisak vt Chekob a pila tok: mihma Patanalam a onvt, Silian hatak Behluel ushi Chekob micha Esau ya ishki Lebekah y $\underline{a}$ i nakfi Laban a im ona tok.

6. Aisak vt Chekob a yukpali cha, Patan-alam yummak o ohoyo ont ai isha chị ho pila hatok, micha yukpali mot, atokolit $\underline{\mathrm{i}}$ mihvt, Kena ushetik vhleha ha ohoyo ish ai isha he keyu, achi tuk; 
7. Mihma Chekob vt îki, micha ishki ya im antia cha, Patan-alam a ia tuk a Esau vt pisa tok.

8. Micha Kenan ushetik vhleha ha iki Aisak vt vlhpesa ik ahno ho Esau vt pisa mut;

9. Yohmi mut Esau vt Ishmael a im ona cha, Eblaham ushi Ishmael ushetik, Nebayolh in tek Mahalalh a tekchi ya chị ho tekchi im ashwa kash ibafohkit ishi tok.

10. Mihma Chekob vt Bee-sheba ya akohchut Halan pilla ho ia tok.

11. Atuk osh yakni kaniohmi ho ona cha, hushi vt okatula hatuk $\underline{o}$, itonla na onna tok: micha toli ya yumma ai ishi cha, vlhpisha chị hosh bohli mut, yummak o tushkit nusi tok.

12. Atuk osh holhpokna tok, mihma yakeh, atuya yut yakni ya $\underline{o}$ hikia mut, paknaka yvt shutik a ont bikeli tok: mihma yakeh, Chitokaka im enchel vhleha hvt ai atuhuyvt, micha ai akohowa tok.

13. Mihma yakeh, Chihowah yut paknaka ya hikia cha, Chiki Eblaham in Chitokaka Chihowah sia hoke, micha Aisak in Chitokaka sia hoke: yakni ish ai on itoyula ka chishno, micha chim ishtatiaka ya ima la hi oke.

14. Mikma chim ishtatiaka yut yakni lukfi pushi ak $\underline{o}$ choyuhma hi oke; micha fimimpa cha, hushi ai okatula, micha hushi akochaka, micha falummi, micha oka mahli aiena ka pit ish pataiya chi hoke: mikma chishno, micha

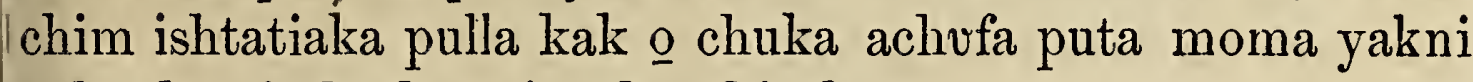
paknaka aiasha kvt ai yukpa hi oke.

15. Micha yakeh, chibafoyuka lishke, micha kanima moma ish onakma, chi halulli la chi hoke, atuk osh anonti yakni iluppa chisht vla la hi oke: anumpuli li tuk vt ak yomihcho kisha kvt chissa la he keyu hokvt, achi tok.

16. Mihma Chekob vt nusi tuk vt okcha tok; mihmvt, Chihowah yot iluppa anta pulla ho ak ithano ke tushke, achi tok.

17. Micha nukshopa tok; mihmut, Ilvpput yakni holitopa hatushke; iluppvt inla keyu, amba Chitokaka in chuka, micha iluppot uba yakni holihta okhissa yoke, achi tok. 
18. Atuk osh onnahinli fehna ho Chekob vt wakaya cha, toli vlhpishi bohli kash ishi cha, nan-isht-ithana he a hilechi mot, paknaka ya bila on hlatubli tok.

19. Mihma vt yakni yumma Behlel hochifo tok: yohmi kia vmmona ka tumaha holihta yumma hohchifo kvt Lus atok.

20. Mihma Chekob vt anumpa il onochit, Chitokaka yvt subafoyukvt iluppa pilla ia li ka sa halulli cha, vpa la chị ka pvska, micha fohka la chi ka nafohka vma,

21. Yvmohmi ho achukmakvot aki in chuka ya anonti vka li hokma, yohmikma Chihowah ak osh an Chitokaka yashke.

22. Mikma tvli iluppa nan isht-ithana mak o hilechi li tuk vt Chitokaka in chuka ya hi oke: micha nana is suma moma ishit pokoli ka chima li pulla hi oke, achi tok.

\section{CHAPTA XXIX.}

YUMOHMI mut Chekob vt nowvt aya tuk vt ia tok;

1 atuk osh hushi akochaka okla $\underline{i}$ yakni ya ona tok.

2. Atuk osh hopokoyo ma, yakeh, osapa ya kuli hofobi vt hikịa na yakeh, chukfulhpoa aya tuchina kvt bilika kahvt aiasha tok: kvli hofobi yummak $\underline{o}$ nan vlhpoa chipinta ya oka ai ipeta chatuk oka: mihma tuli chito yosh kuli hofobi paknaka ya onvtoyula tok.

3. Micha yummak o nanvlhpoa moma ka ai itunahli chatuk atok: mikmut kvli hofobi paknaka ya tuli chito vt onvtonla kash tonolichit kanvllichi cha chukfulhpoa ya oka ipeta mut, anonti tuli yash ai itola kuli hofobi paknaka ya onochi chatuk atok.

4. Atuk o Chekob vt, Itibapishi li vhleha ma, katima hush minti cho? im achi tok. Mihma Halan minti pia hoke, achi tok.

5. Mihma, Naho ushi Laban a hush ithana cho? im achi tok. Mihma, Il ithanushke, achi tok.

6. Mihma Chekob vt, Im achukmaka cho? im achi tok. 
Mihma, Im achukmakvshke; atuk o yakeh, ushetik Lechel vt chukfulhpoa pehlichit mintishke, achi tok.

7. Mihma Chekob vt, Yakeh, hushi vt chaha moma, micha nanvlhpoa yot itahoba he ai ona keyushke: chukfulhpoa ya oka hush ipeta cha, pehlichit ont hush impvchashke, achi tok.

8. Mihma, Nanvlhpoa chipinta moma kvt itahobvt taha na kuli hofobi paknaka ya tuli vt itonla ka tonolichit ik kanullicho kisha ka e yumihcha he keyu hoke; yohmikmak o chukfulhpoa ya oka il ipeta chatuk oke, achi tok.

9. Atuk o im anumpulit isht anta moma ho, Lechel vt iki in chukfulhpoa pehliechit vla.tok: yummak osh pehliechi yatuk okvt.

10. Atuk o yakohmi tok, Chekob vt ishki i nakfi. Laban ushetik Lechel a micha ishki $\underline{\mathbf{i}}$ nakfi Laban in chukfulhpoa aiena ka pisa mut, Chekob vt bilika onvt, kvli hofobi paknaka ya tvli v.t onvtonla kash tonolichit kanullichit ishki i nakfi Laban $\underline{\mathrm{i}}$ nanulhpoa chipinta ya oka ipeta tok.

11. Micha Chekob vt Lechel a $\underline{\mathrm{i}}$ suksowa mvt, chitoli hosh yaiya tok.

12. Micha Chekob vt Lechel a iki ya itibapishi, micha Lebekah ya ushi yatuk vt.im anoli tok; mihma Lechel vt malelit iut i $\mathrm{k} i \mathrm{y}$ a ont im anoli tok.

13. Atuk o yakohmi tok, Laban vt in tek ushi Chekob a anoli ka haklo mvt, malelit ivt afama cha, shakba ont afohomi mvt, $\underline{\mathbf{i}}$ suksowa cha, in chuka isht ona tok. Mihma nana iluppa puta ka Laban a im anoli tok.

14. Mihma Laban vt, Sa foni, micha sa nipi chia pullushke im achi tok: mihma hvshi achvfa ho Chekob vt takla ahanta tok.

15. Mihma Laban vt Chekob a, Svtibapishi chia hatuk ak osh peh pilla ho is san toksvla hetuk oh cho? chim vlhtoba kvt nanta chi cho? vm anolí, im achi tok.

16. Mihma Laban vt ushetik tuklo hosh im ashwa tok: akni hohchifogkvt Leah yokma, nakfish hohchifo kut Lechel atok. 
17. Leah vt nishkin okwilonli yokma, Lechel vto aiokli, micha nipi pisa achukma tok.

18. Mihma Chekob vt Lechel ak o anushkunna tok; mihmut, Chiso tek nakfish Lechel ak o afummi untuklo ho chin toksvli lashke, achi tok.

19. Mihma Laban vt, Chishno ak o chima li kak osh achukma kvt hatak inla ima la hetuk a $\underline{\mathrm{i}}$ shahlishke: sa takla ish ahantashke, achi tok.

20. Mihma Chekob vt Lechel ak atuk pulla mak o, afưmmi untuklo ho toksvli tok: mihmvt anushkunna fehna hatuk osh peh nitak kanomosi in chohmi tok.

21. Mihmvt Chekob vt Laban a , Sa tekchi ya vmá, (nitak a holhtina tuk vt taha hoka,) yohmi na iba chukowa lashke, im achi tok.

22. Mihma Laban vt chuka alokoli yumma hatak vhleha moma ka hoyot itunahli cha, impuchi ikbi tok.

23. Atuk osh yakohmi tok, opivt taha ma, ushetik Leah ak o ilauelit isht im ona tok: mihma iba chukowa tok.

24. Mihma Laban vt ushetik Leah in tishu ha chi ho in tislu ohoyo Silpah ya ima tok.

25. Atuk o yakohmi tok onnahinli ma, yakeh, Leah ak atuk atok: mihma Laban a, Nanta mak o iluppa is si yumihchi cho? Lechel ak achị ho chiba toksuli li tuk keyu cho? yuhmohmi hokak o nanta katiohmi ho is sa haksichi cho? im achi tok.

26. Mihma Laban vt, Nakfish ak o akni hạ tikkba hatak i bohli kvt pi yakni ya a yumohma he vlhpesa keyu hoke.

27. Leah $\underline{\mathrm{i}}$ nitak untuklo ka ish onachashke: mikma afưmi untuklo inla ka is san toksula chi kak o iluppak kia e chimashke, achi tok.

28. Mihma Chekob ut yumohmi cha, i nitak untuklo ka onachi tok: mihma tekchi yachị ho ushetik Lechel ak kia ima tok.

29. Micha Laban vt ushetik Lechel a in tishu ha chị ho in tishu ohoyo Bilhah ya ima tok.

30. Mihma Lechel ak kia iba chukowa tok; micha Le- 
chel a $\underline{\mathrm{i}}$ hullo kvt Leah ya $\underline{\mathrm{i}}$ shahlichi tok; mihmut afummi untuklo inla ho iba toksvli tok.

31. Atuk o Leah ano ik ahnincho ho Chihowah yut pisa mot, im oshvtto ya $\underline{\mathrm{i}}$ wakvmmichechi tok: amba Lechel vto vlla ik im iksho atok.

32. Mihma Leah vt chakali tok; atuk osh vlla nakni yo eshi cha, Luben hochifo tok: Sulbusha ka Chihowah yut sa pisa tuk pullushke; himak ano a hatak vt a hulla chịshke, achi tuk okvt.

33. Atuk osh anonti chakali cha, vlla nakni yo eshi mvt, Ik si ahnincho ka Chihowah yot haklo tuk; atuk osh vlla nakni iluppak kia vmushke, achi tok: mihmut Simeon hochifo tok.

34. Atuk osh anonti chakali cha, vlla nakni yo eshi mvt, Himak ano a hatak vt si asitia chi hoke, vlla nakni tuchina ho im eshi li tuk oka; yumohmi hatuk osh Lefai hochifo tok.

35. Atuk osh anonti chakali cha, vlla nakni yo eshi tok: mihmut, Himak a Clihowah ya aiokpuchi lashke : yumohmi hatuk osh Chutah hochifo tok; mihmvt vlla eshit issa tok.

\section{CHAPTA XXX.}

TUK o Lechel vt Chekob a vlla ik im esho kut pisa Il mut, Lechel vt akni hash i potunno tok; micha Chekob â, Villa vmá, keyukma sulla chi hoke, im achi tok.

2. Mihma Chekob vt Lechel a $\underline{\mathbf{i}}$ sanalit isht im ai $\underline{\mathrm{i}}$ nukkilli vt libbit, Chitokaka vlhtobvt hikia li cho? yummak osh chim oshutto atoba ka chị katubli tuk oke, achi tok.

3. Mihma, Yakeh, an tishu ohoyo Bilhah ya ish iba chukowashke; yohmi na vlla vm eshit saiyikalaha paknaka ya bohlashke: yohmi na vno ak kia yummak a tuk ak. o vlla yot vm ashashke, achi tok.

4. Mihmvt tekchi ya chị ho in tishu ohoyo. Bilhah ya ima tok: mihma Chekob vt iba chukowa tok.

5. Atuk o Bilhah hut chakali cha, Chekob a vlla nakni yo im eshi tok. 
6. Mihma Lechel vt, Chitokaka yvt si apehpoushke, micha vm anumpa hạ haklo cha, vlla nakni yo vmushke, achi tok : yumohmi hatuk osh Tan hochifo tok.

7. Atuk osh Lechel in tishu ohoyo Bilhah hvt anonti chakali cha, atukla ma Chekob a vlla nakni yo im eshi tok.

8. Mihma Lechel vt, Umuni ya iti kvllo fehnvt iti kvllo li hosh imaiya lishke achi tok: mihmot Naftali hochifo tok.

9. Atuk o Leah vt vlla eshit issa tuk vt pisa mvt, in tishu ohoyo Silpah ya ishi cha, tekchi ya chi ho Chekob a ima tok.

10. Atuk o Leah in tishu ohoyo Silpah vt Chekob a vlla nakni yo im eshi tok.

11. Mihma Leah vt, Laua hosh mintishke, achi tok: mihmvt hohchifo ha Kat achi tok.

12. Atuk o Leah in tishu ohoyo Silpah vt Chekob a atukla ma vlla nakni yo im eshi tok.

13. Mihma Leah vt Sai yukpushke, ohoyo vhleha hvt nayukpa si acha chi hoke: achi tok, mihmot hohchifo ha Ashe achi tok.

14. Atuk o onush vmo nitak o Luben vt osapa ya ia tuk osh okchak holba ahayuchi cha, ishki Leah ya isht im ona tok. Mihma Lechel vt Leah ya, Chiso im okchak holba kaniohmi ka is sumashke, chim asilhha lishke, im achi tok.

15. Mihma, A hatak a is sum ishi ka nan ik ahobo cho? yohmi mut suso im okchak holba ak kia ish isha chi cho? im achi tok. Mihma Lechel vt, Yumohmi hoka chiso im okchak holba atuk mak o himak ninak a chiba nusashke, achi tok.

16. Atuk o Chekob vt opia ma osapa vttut minti ma, Leah hvt kohchvt ivt afama cha, Is swba nusashke: svso im okchak holba ishit chi tohno li tuk vt ahli hoka, achi tok. Atuk o mih ninak ma iba nusi tok.

17. Atuk o Chitokaka yut Leah ya $\underline{\mathrm{i}}$ haponaklo na chakali cha, vlla nakni ishit tahlapi ka Chekob a im eshi tok.

18. Mihmut Leah vt, Vm vlhtoba ya Chitokaka yvt vmushke, an tishu ohoyo yash a hatak a ima li tuk oka, achi tok: mihmot hohchifo ba Issaka achi tok. 
19. Atuk osh Leah vt anonti chakali cha vlla nakni ishit hannali ka Chekob a im eshi tok.

20. Micha Leah vt, Nahulbina achukma ho Chitokaka yut sa habenvchishke; himak ano a hatak vt sa takla ahanta chi hoke, vlla nakni hannali ho im eshi li hoka, achi tok: mihmvt hohchifo ha Sebulun achi tok.

21. Atuk osh $\underline{i}$ himmakma ushetik $\underline{o}$ eshi cha, hohchifo ha Tinah achi tok.

22. Mihma Chitokaka yut Lechel $\underline{a}$ anukfilli cha $\underline{\mathrm{i}}$ haponaklo mut, im oshutto ya $\underline{\mathbf{i}}$ wakummichechi tok.

23. Mihma chakali cha, vlla nakni yo eshi tok; micha, Chitokaka yut nan ishit sa hofahya ya ishit a kanchishke, achi tok.

24. Micha hohchifo ha Chosef achi tok: mihmvt, Chihowah yut vlla nakni inla ya vm ibafohka chi hoke, achi tok.

25. Atuk o yakohmi tok, Lechel vt Chosef a eshi ma, Chekob vt Laban a, Ik iá is sum ahni na aivtta li, micha a yakni ya ona lashke.

26. Sa tekchi vhleha, micha vm vlla vhleha yummak oka chin toksuli li tuk a is suma na ia lashke; natoksuli chim atahli li tuk $\underline{a}$ ish ithana hokvt, im achi tok.

27. Mihma Laban vt, Holhponayo hanta ya chi tikba ai ahayuchi li tuk okma, ish antashke, chim asilhha lishke: chishno ak atuk pulla kak o Chihowah yvt sai yukpali hoka, akostininchi lishke, ịm achi tok.

28. Micha, Chim vlhtoba ya is sum apesashke, mikma chima lashke, achi tok.

29. Mihma Chekob vt, Kaniohmit chin toksahanli li tuk, micha chị nanvlhpoa vt kaniohmit si apehvt aiasha tuk a ish ithanushke:

30. Ak lo kisha kano kanomosi hosh chim aiasha tuk osh himak ano wayvt apaknvt taha hoka: mihma Chihowah yvt vla li tuk takla ka chi yukpalishke: yohmi ka himak a katiohmik mak o an chuka achvfa yak kia nan im atahlit ahanta la chi cho? im achi tok.

31. Mihma Laban vt, Nanta ho chima la chị cho? achi tok. Mihma Chekob vt, Nana is suma he keyu: ilvppak o 
is sa yumihchikma, anonti chị nanulhpoa chipinta ya pehliechit impuchi lashke.

32. Himak nitak a chi nanvlhpoa chipinta moma ka iba chukowvt, ahlopullit chikchiki moma, micha bakowa moma ka ila filvmmichit, micha lusbi moma chukfulhpoa ya ibalhto ka , micha chikchiki, micha bakowa issi kosoma ibalhto ka ila filummichi lashke: mikma yumma chohmi kak osh vm vlhtoba yashke.

33. Yohmi ho nitak inla minti ka asitubi li tuk vt hoyo likmut, aivlhpiesa ho yumihchi li tuk vt chi tikba si ai atokolashke: chikchiki, micha bakowa keyu yosh issi kosoma ya ibafoka, micha lusbi keyu yosh chukfulhpoa ya ibafokak mvt, moyuma kvt hukopa li tuk osh vm anta vlhpisashke, achi tok.

34. Mihma Laban vt, yakeh, chim anumpa hatuk mak o yumohmik bano ahni lishke, achi tok.

35. Mihmut nitak yummak inli ka issi kosoma nakni tohbit afohoma, micha bakowa, micha issi kosoma tek chikchiki, micha bakowa, moma micha tohbi kaniohmi kvt laponli, moma micha lusbi yosh chukfulhpoa ya ibafoka moma ka ila filummichit ushi vhleha ha ibbak fohki tok.

36. Micha ilap, micha Chekob itatuklo ka iti filvmmichit nitak tuchina anowa yo ashachi tok: mihma Laban $\underline{\mathrm{i}}$ nanvlhpoa inla ka Chekob vt pehliechit impvchi tok.

37. Micha Chekub vt sipsi okchaki, michà hasel, micha oti upi tuptua aiena ka ishi cha hlohlit busolichit tohbichi cha, iti tuptua atohbi kash haiakvchi tok.

38. Micha nanvlhpoa chipinta yut ushi shala chi ho iti tuptua hlohli kash osh nan vlhpoa chipinta yot ishka chi hosh vlak ma itikba oka a yanvlli, micha oka ai ipeta peni ya a hiohlichi tok.

39. Atuk o nanvlhpoa chipinta yvt iti tuptua yash itikba ya ushi a shali beka tok; micha tohbit afohoma, chikchiki micha bakowa aiena ka cheli tok.

40. Mihma Chekob vt chukfulhpoa ushi yash ila filvmmichi mvt, nanvlhpoa chipinta nashuka ya Laban $\underline{\underline{i}}$ nanvlhpoa chipinta yash tohbit afohoma, micha lusbi moma aiena 
ka pit apissulechi beka tok; mihmvt ilap $\underline{i}$ nanvlhpoa chipinta ya ila ashachi cha, Laban $\underline{\mathbf{i}}$ nanvlhpoa chipinta yano ik ibafohko tok.

41. Atuk o yakohmi tok, nanvlhpoa kvllo i shahli vt ushi shalikma no Chekob ut iti tuptua ai itin takla ya ushi shala chị ho nanvlhpoa nishkin itikba oka ayanvlli ya iti tuptua yash ashachi tok.

42. Amba nanulhpoa hliposhikmuno, ik ashacho beka tok: yumohmi ho hliposhi ak osh Laban immi hokma, kvllo i shahli yak osh Chekob immi hatok.

43. Micha hatak ash osh $\underline{\mathrm{i}}$ shahlit mahaya fehna cha, nanvlhpoa, micha ohoyo tishu, micha hatak tishu, micha kamel, micha issuba haksobish falaia puta aiena kvt in laua fehna hatok.

\section{CHAPTA XXXI.}

A TUK osh Laban ushi vhleha hvt anumpulit, Chekob vt A piki nana immi hatuk a ishit tahlishke; micha, piki immi hatuk a nan-isht-holitopa iluppa moma ai ishishke, achi na haklo tok.

2. Atuk osh Chekob vt Laban nashuka ya pisa ma, yakeh, tikba chatuk a in chohmi keyu tok.

3. Mihma Chihowah yvt Chekob a, Falamvt chiki vhleha i yakni onvt, micha chi kanomi vhleha ish im onashke; mikma chibafoyuka lashke, im achi tok.

4. Mihma Chekob vt Lechel, micha Leah ya hoyot $\underline{\underline{i}}$ nanulhpoa aiasha yakni haiaka ya isht ona cha,

5. Hvch iki nashuka yut tikba chatuk vt an chohmi keyu ka pisa lishke : yohmi kia aki in Chitokaka yưt subafoyuka hoke.

6. Micha nan isht aiahhlika vm aiokluhha hosh hvch ịki ya in toksuli li tuk a hush ithanvshke.

7. Mihma huch iki vt sa haksichi cha, nan isht vm vlhtoba ya pokoli ha vm inlvchi tuk oke : yohmi kia Chitokaka yut ik hotopalí, ik ahno tuk oke.

8. Yakohmikma, Chikchiki ak osh ishit chim vlhtobash- 
ke, achi tuk ma, yohmikma nanvlhpoa moma kvt chikchiki yo cheli chatuk: mihma, Tohbit afohoma ak oṣ isht chim vlhtobashke, achi tuk ma, yohmikma nanvlhpoa moma kut tohbit afohoma ho cheli chatuk oke.

9. Yakohmichi hosh Chitokaka yut huch iki i nanvlhpoa ya im ishi cha, vma tuk oke.

10. Atuk o yakohmi tok, nanulhpoa yut ushi shali nitak a sa holhpokna cha, hopokoyot pisa li ma, yakeh, nakni vt nanvlhpoa ya oiya kut tohbit afohoma, chikchiki, micha bakowa aiena tok.

11. Mihma Chitokaka im enchel vt, sa holhpokna ho vm anumpulit, Chekob ma, wm achi tok: mihma, Yak itonla lishke, achi li tok.

12. Mihma, Akachakali cha pisá, nakni moma kvt nanulhpoa ya oiya kut tohbit afohoma, chikchiki, micha bakowa aiena hoke: Laban vt nana moma chi kaniohmichi ka pisa li tuk oka.

13. Behlel yumma nan isht-ithana tuli ya nan ish ahvmmi tok, micha yumma anumpa kullo is sum il ai onochi tok

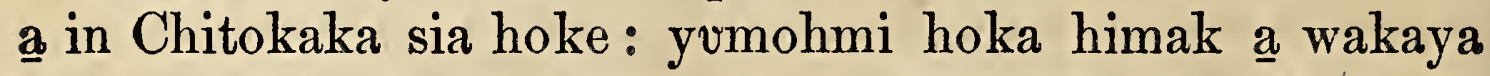
cha, yakni ilvppa ish kohchashke, micha falamvt chi kanomi i yakni ya ish onashke, vm achi tuk oke, im achi tok.

14. Mihma Lechel, micha Leah vt afalaminchit, Piki in chuka achvfa ya akashapa, keyukmvt il ai isha he vlhpesa yot asha moma cho?

15. Okla inla yak o yummut pit apesa keyu cho? pi kanchi tok osh pin tuli holisso ak kia tahli tuk oka.

16. Imilayak moma Chitokaka yot piki ya im ishi tuk vt pimmi, micha pim vlla vhleha aiena kak o immi hoka: yvmohmi hoka himak a Chitokaka yut nana ho chị mihakma, ish yumihchashke, im achit okla tok.

17. Yohmi ma Chekob vt wakaya mvt, ushi vhleha, micha tekchi vhleha aiena ka kamel a om binohlichi tok.

18. Mihmut îki Aisak aivtta Kenan yakni ya ia chi hosh nanvlhpoa moma, micha vlhpoyak ahayuchi tuk vt moma, Patan-alam a nanvlhpoa ya ai ahayuchi tuk vt isht ia tok. 19. Mihma Laban ut in chukfulhpoa hishi vmot ia tuk 
atok: mihma Lechel vt ịki i naholbvt toba ya i hukopa tọk atok.

20. Atuk o Chekob vt kunia mvt, ik im anolo hatuk osh Silian hatak Laban a haksichi tok.

21. Yohmi hosh nana moma im asha tuk vt ishi cha kvnia tok: micha wakaya cha okhina ya tanublit, nunih Kileat pilla ha nashuka ya pit talali tok.

22. Atuk o nitak ont atuchina kak o Chekob vt kunia tuk a Laban a im anoli tok.

23. Mihma Laban vt itibapishi vhleha pehlichi cha, hliolit, nitak untuklo anowa yo ona tok; micha nunih Kileat ak $\underline{\mathrm{o}}$ ont a sakki tok.

24. Atuk o Silian hatak Laban vt ninak ma holhpokna na Chitokaka yot im vlvt, Ahah, ish ahni cha, Chekob a achukma hokma, okpulo hokma nana kia chik im anumpulo kashke, im achi tok.

25. Yohmi ma Laban vt Chekob a sakki tok. Yohmi ma Chekob vt nunih ak o im vlhtipo ya a hilechi tok: atuk o Laban vt itibapishi vhleha aiena kvt Kileat nunih ak o a binachi tok.

26. Atuk osh Laban vt Chekob a, Nanta mak o ish yvmihchi cho? is sa haksichi cha, bushpo falaia ishit yukachi tuk chohmichit susotek vhleha ha pehlichit ish kunia tuk oke.

27. Nanta katiohmi ho lumvt malelit is sa haksichi cha chik sum anolo na nayukpa, micha ilhtvlwak, micha vhlepa, micha hap aiena olahanchi hosh ik ia ak chim ahno tuk oh cho?

28. Micha suso vhleha, micha susotek vhleha áiena ka ik i suksowa, chik sum ahno tuk oh cho? himak a im anukfila iksho chohmit ish yumihchi tuk oke.

29. Chi hotopali la hi vt subbak a foyuka hoke: yohmi kia chiki ya in Chitokaka yut pilashash ninak a vm anumpulit, Ahah, ish ahni cha achukma hokma, okpulo hokma nana kia Chekob a chik im anumpulo kashke, vm achi tuk oke.

30. Atuk o himak a ia hosh ish ayushke, chiki in chuka 
ya ish i palata fehna hokvt: yohmi kia nanta katiohmi ho a naholbut toba ya is sa hulkopa tuk oh cho? im achi tok.

31. Mihma Chekob vt afalaminchit Laban a , Sa nukshopa hatuk oke: Chisotek vhleha ha afovt is svm isha hinla yoba ká, achi li tuk oke.

32. Yumohmi hoka kuna hosh chi naholbvt toba ya ishi ka ish ahayuchikma, yummot ik okchayo kashke : pitibapishi vhleha itikba nana hosh chimmi hosh subafoyuka tukma, ish, ithana cha, ish ishashke im achi tok: Lechel vt hukopa tuk a Chekob vt ik ithano tuk okvt.

33. Mihma Laban vt Chekob im vlhtipo ya ont chukowa mot, Leah im vlhtipo ma ont chukowa cha, tishu ohoyo tuklo im vlhtipo ya ont chukowa tok: yohmi kia ik ahayucho tok. Yohmi mut Leah im vlhtipo ya a kohcha mut, Lechel im vlhtipo ya ont chukowa tok.

34. Yohmi ka naholbut toba ya Lechel ak osh ishi tuk osh kamel i nachukushpa ibafohki cha, om binili tuk atok. Mihma Laban vt vlhtipo ya a mominchit hoyo kia, ik ahayucho tok.

35. Mihma Lechel vt ịki ya, chi tikba ya ak wakayo ka $\underline{a}$ shahli hut ik nuklibisho kashke; ohoyo nana im akaniohmi vt si onvtonla hoka, im achi tok. Mihma hoyo kia naholbut toba ya ik ahayucho tok.

36. Mihma Chekob vt nukhobela cha, Laban a itachowa tok: micha Chekob vt afalaminchit Laban a, Nanta ho vbanubli li tuk oh cho? micha nanashvchi li vt nanta hatuk o achunanchit is sa hlioli kvt chi yumohmi tuk oh cho?

37. A nachukushpa ya moma ish a hoyo hókvt nana achvfa nanta ho chin chuka achvfa nachukushpa yatuk a ish ahayuchi cho? svtibapishi vhleha, micha chitibapishi vhleha aiena itikba iluppa talalí, yohmi na pim itin takla ya nana a pim apesashke.

38. Afummi pokoli tuklo iluppa chi takla ahanta li chatuk; chin chukfulhpoa tek, micha chim issi kosoma tek aiena kvt ushi im akvma chatuk keyu; micha chi nanvlhpoa ya ai ishit chukfulhpoa nakni ya vpa li chatuk keyu hoke. 
39. Nampoa okpulo yosh vbi tuk a ishit chim ona li chatuk keyu; vno ak osh a kunia chatuk; nitak o hukopa tuk okmá, keyukmot ninak o hukopa tuk okmá nana kia subbak ak o is sa hoyo chatuk oke.

40. Si yakohmi chatuk oke; nitak ma lushpa, micha ninak ma hotonti hosh sa tahli chatuk oke; mihma nusi hot sa nishkin a i filummi chatuk oke.

41. Yakohmi hosh afummi pokoli tuklo ho chin chuka ahanta li tuk oke: chisotek tuklo ka afummi auah ushta ho chin toksuli li tuk oke, micha chi nanvlhpoa ya afummi hannali ho chin toksvli li tuk oke : micha nan isht vm vlhtoba ya pokoli ha is sum inluchi tuk oke.

42. Aki in Chitokaka, Eblaham in Chitokaka, micha Aisak vt I nukshopa vt subafoyuka hatuk keyukma, nana iksho ho is sa chuffichi pulla hetuk oke. Chitökaka yvt a sulbusha, micha subbak toksahanli ka pisa tuk osh pilashash ninak ma na chi miha tuk oke, im achi tck.

43. Mihma Laban vt afalaminchit Chekob a, ushetik ilvpput susotek vhleha hoke, mikma vlla vhleha iluppvt vm vlla vhleha hoke, micha nanvlhpoa ilvppvt a nanvlhpoa yoke, micha nana ish pisa moma kvt vmmi hoke; yohmi ka himak nitak a svsotek vhleha iluppa, micha eshi tok vlla vhleha ha nanta ho katiohmichi la hinla cho?

44. Yvmohmi hoka himak a inta, vno, micha chishno il ita tuklo kvt nan il itim apesashke : mikma nan atokoli yosh pim itin takla ya hikiashke, im achi tok.

45. Mihma Chekob vt toli ishi cha, nan-isht-ithana ikbit hilechi tok.

46. Mihmut Chekob vt itibapishi vhleha hạ, Tvli oh aiowá, im achi tok; mihma tuli aiowa cha, itunahli tok: mihmvt itunaha ya paknaka okla ai impa tok.

47. Mihma Laban vt, Cheka-sahatuhla hochifo ma, amba Chekob vt Kaleet hochifo tok.

48. Mihma Laban vt, Itvnaha ilupput himak nitak a vno micha chishno nan atokoli yosh pim itin takla ya hikia hoke, achi tok. Yumohmi hatuk o hohchifo ha Kaleet; achi tok. 
49. Micha Mispeh achi tok: 'Il itifilummit il ayakma, Chihowah yut pim itin takla ya a pi apesahanchashke.

50. Svsotek vhleha ha ish ilbvshalikma, keyukmvt susotek vhleha ha aieninchit ohoyo inla ho ish ishikma, hatak nana hosh piba fokvt pi pisa keyushke: Chitokaka ak osh vno, micha chishno, pim itin takla ya pim atokoli yosh hikia hoke, achi hatuk okvt.

51. Micha Laban vt Chekob a, Yakeh, itunaha iloppa, micha yakeh, nan isht-ithana iluppa uno, micha chishno pim itintakla ya hilechi lishke.

52. Itunaha ilvppot, micha nan isht-ithana iluppot nan okpulo aiahnit, itunahahchi iluppa ant iot ak chim ono ka he, mikma itunahahchi iluppa, micha nan isht-ithana iluppa ant ivt chik sum ono ka he atokoli yashke.

53. Eblaham in Chitokaka, micha Naho in Chitokaka, iki ya in Chitokaka yak osh pim itin takla ya nana ai apesashke, im achi tok. Mihma Chekob vt iki Aisak vt I nukshopa ak o hochifot anumpa kullo il onochi tok.

54. Yohmi mut Chekob vt i nanvlhpoa ya ai ishit nunih hash ai vbi cha, puska vpa chi ho itibapishi vhleha ha hoyo tok: mihma puska okla vpa cha, nunih ha asha na onna tok.

55. Atuk o onna kvt onnahinli fehna ho Laban vt wakaya cha, ushi vhleha, micha ushetik vhleha ha $\underline{i}$ suksowa mut, anumpa yukpali onochi tok: micha Laban vt falamut iut aivtta ya ona tok.

\section{CHAPTA XXXII.}

IHMA Chekob vt aya tok; atuk o Chitokaka im en-
chel vhleha hvt afama tok.

2. Atuk o Chekob vt pisa mut, Ilupput Chitokaka im vlbina hoke achi tok: micha yakni yumma hohchifo ha Mahanaim achi tok.

3. Mihmvt Chekob vt anumpa shali tikba tihlelit, Sei yakni, Etom apehlichika, itibapishi Esau aiasha ya pit im pila tok. 
4. Micha i miha mvt, Yakohmichi hosh a shahli Esau ya hvsh im anumpulashke; Chin tishu Chekob vt yak achishke, Nowvt aya yosh Laban ak o takla ahanta li chatuk, micha yummak o ahanta li himak a hoke:

5. Micha wak, micha issuba haksobish falaia, micha nanvlhpoa chipinta, micha hatak tishu vhleha, micha ohoyo tishu vhleha aicna ka pehliechi lishke: atuk osh a shahli ha pit im anolit, holhponayo hanta ya chi tikba ai ahayuchi la chi hosh pit chi pila lishke, achishke, hvsh im achashke, achi tok.

6. Atuk o anumpa shali ash osh falamvt, Chekob a im vla mvt, Ish itibapishi Esau ya il im ona tuk oke; mihma chi afamvt minti akinlishle, mihma hatak tahlepa ushta hosh apiha hoke, achi tok.

7. Mihma Chekob vt nukshopa cha komota fehna tok: mihmvt okla apehvt aya tuk a, rnicha nanvlhpoa chipinta, micha nanvilhpoa hochito, micha kamel puta aiena ka ita kvshkolit aya tuklochi tok.

8. Micha Esau vt aya achvfa ka ant afama cha, issot kanchikma, yohmikma aya achvfa kuto yihlepvt hlakoffashke, achi tok.

9. Mihmut .Chekob vt, Aki Eblaham a in Chitokaka, micha aki Aisak a in Chitokaka, Chihowah, Falamot chi yakni, micha chi kanomi ya ish im onashke, mikma achukma ho chi yumihchi lashke, is sum ahanchi tuk chia ma,

10. Nan isht ish i nukhaklo moma ik lauwo ka, micha chim anumpa ahli moma chin tishu ha ish pisachi chatuk a si ahalaia he vlhpesa keyushke: an tvbi ya ieshi hosh Chatan iluppa hlopulli li tok osh, himak ano aya tuklo toba li hokvt.

11. Itibapishi li ibbak, Esau ibbak a im ai ishit is sa hlakofichashke, chim asilhha lishke, $\underline{i}$ sa nukshopa hoka: vlut issot sa kanchikmvt, ishki, micha vlla vhleha ma issot kanchi yoba ná.

12. Achukma ho chi yumihchi li pulla chị hoke; micha chim ishtatiaka ya laua kvt holhtina he keyu, shinuk okhvta aiasha kak o choyuhmichi la hi oke, ish achi tuk oke, achi tok. 
13. Micha mih ninak ma yummak inli anta tok; micha itibapishi Esau ya habenveha chi kvt nana hosh ibbak ant fohkakmvt, ishi tok.

14. Issi kosoma tek tahlepa tuklo, micha issi kosoma nakni pokoli tuklo, chukfulhpoa tek tahlepa tuklo, micha chukfulhpoa nakni pokoli tuklo,

15. Kamel i bishahchi pokoli tuchina, ushi aiena, wak tek pokoli ushta, micha wak nakni pokoli, issuba haksobish falaia tek pokoli tuklo, micha ushi pokoli aiena hatok.

16. Micha ilap aya achvfa aiyukali ho in tishu vhleha ibbak fohki tok: micha in tishu vhleha hạ, Sa tikba hvsh tanvbli cha, aya achvfa aiyúkali kvt iti mishemanchi hosh hush ayashke, im achi tok.

17. Micha tikba fehna aya ka, Itibapishi li Esau vt afama cha chi ponaklot, Kvta immi chia hoh cho? micha katima ish ia cho? micha chi tikba aya ilupput kuta immi hoh cho? achikma:

18. Yohmikma, Chin tishu Chekob a immi hoke: nahvlbina yo a shahli Esau ya et i pila tuk: atuk o yakeh, olbvl minti akinli hoke, ish achashke, achit $\underline{\underline{i} \text { miha tok. }}$

19. Micha yumihchi hosh atukla, micha atuchina, micha

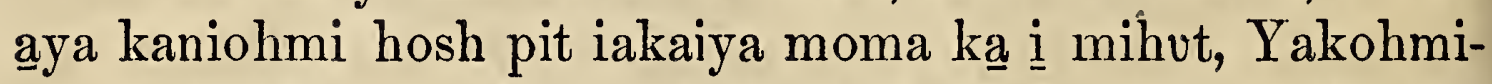
chi hosh Esau ya hush pisakmut, hush im anumpulashke, ahanchi tok.

20. Micha achakalinchit, Yakeh, chin tishu Chekob vt olbvl minti akinlishke, hvsh achashke, achi tok. Nahulbina sa tikba ia kak o ishit nuktalali lashke; yohmi ha yosh nashuka ya pisa lashke : a kanvt $v m$ aiokpvcha hinla yoba ká, achi hatuk okvt.

21. Yohmi ma nahulbina yut tikba ia tok; mihma ilap vto mih ninak a abinanchi ya anta tok.

22. Atuk osh mih ninak a wakaya mvt, tekchi tuklo, micha in tishu ohoyo tuklo, micha ushi auah achvfa aiena ka ishi cha, Chabbok okhina kohcha ya pit hlopulli tok.

23. Mihmot ishi cha, okhina yash pit hlopullichi mot, moma ishi tuk vt pit hlopullichi tok. 
24. Micha ilap bano hosh anta tok; atuk o hatak osh ont itihishi na onnvt minti tok.

25. Atuk osh imaiya he keyu kvt pisa mot, Chekob iyubi itachosholi kolukbi ya potoli tok: mihma itishi mut, Chekob vt iyubi ai itachasholi vt shufa tok.

26. Mihma, Onnut minti hoka, ak iá, achi tok. Mihma, Chik sai yukpalokma, ik iá, chim ahni la he keyu, achi tok.

27. Mihma, Chi hohchifo but nanta hoh cho? im achi ma, Chekob sia hoke, achi tok.

28. Mihma, Himak pilla ma chi hohchifo ha Chekob acha he keyu, amba Islael ahi oke: hatak holitopa chia hosh Chitokaka ya ish $\underline{\mathbf{i}}$ kvllo bokvt, hatak vhleha ha ish imaiya chi hoke, achi tok.

29. Mihma Chekob vt i ponaklot, Chi hohchifo ha vm anoli, chim asilhha lishke, achi tok: mihma Nanta katiohmi ho sa hohchifo ha ish ponaklo cho? achi tok. Mihmut yummak o a yukpali tok.

30. Mihma Chekob vt yakni yumma hohchifo ha Penuel achi tok: Chitokaka ya nashuka iti sanalit pisa lish si okchaya moma hokvt, achi hatok.

31. Atuk osh Penuel a hlopullit ia ma, hushi vt kohchvt on tommi tok; mihma iyubi ya ishit shaiiksheli hosh nowa tok.

32. Yvmohmi kak o Islael im vlla vhleha hvt akshish yvmmut iyubi itachosholi kolukbi o hikia kvt shulla tok ak o ik po chatuk osh himak nitak a moma hoke: Chekob iyubi itachosholi kolukbi ya potoli na akshish vt shulla tuk atok.

\section{CHAPTA XXXIII.}

A TUK osh Chekob vt akachakalit pisa ma, yakeh, Esau A vt hatak tahlepa ushta hosh apiha ho minti tok. Mihmut vlla vhleha ha ila hiohlichit Leah, micha Lechel, micha tishu ohoyo tuklo aiena ka ima tok.

2. Micha tishu ohoyo tuklo, micha im vlla vhleha aiena kak o tikba fehna hiohlichi mut, Leah micha im vlla vhleha 
aiena ka iakaiyvchi mut, Lechel micha Chosef a olbul fehna hilechi tok.

3. Mihmvt tikba ia tok; micha untuklo ha akachunolit aka hlipivt mạhaya mvt, itibapishi ha bilika ona tok.

4. Mihma Esau vt malelit ont afama mvt, shoyulit ikunla ya on hlipia cha, i suksowa tok: mihma yaiyvt okla tok.

5. Atuk osh akachakali cha, ohoyo, micha vlla vhleha aiena ka pisa mvt, Yvmmvt kuta hosh chi apiha cho? achi tok. Mihma, Chin tishu ha Chitokaka yut i nukhakklot ima tuk vlla vhleha hoke, achi tok.

6. Yohmi ma tishu ohoyo tuklo, im vlla vhleha aiena kvt bilika vlut akachunolit aka hlipkvchi tok.

7. Mihma Leah ak kia im vlla vhleha aiena kvt bilika vlvt akachunolit aka hlipkvchi tok: mihma iakaiya kvt Chosef, micha ishki itatuklo kvt bilika vlvt akachunolit aka hlipkvchi tok.

8. Mihma, Pehlichit isht aya na afama li tuk moma iluppak osh nanta chi katiohmi hoh cho? achi tok. Mihma, A shahli itikba ya holhponayo hanta ya ai ahayuchi la chintuk mak oke, achi tok.

9. Mihma Esau vt, Um vlhpesa akinli hoke, itibapishi li ma, nana ish ishi tuk vt ish ieshashke, achi tok.

10. Mihma Chekob vt, Ahạ, himak a holhponayo hanta ya chi tikba ai ahayuchi li hokma, a nahvlbina subbak foyuka ka ish ishashke, chim asilhha lishke: yumohma he ak o chi nashuka ya Chitokaka i nashuka chohmi ho pisa li ma, is sa hulhpasha hoka.

11. Nan ishit yukpali li ishit chim vla tuk a ishí, chim asilhha lishke; Chitokaka yvt a kana tuk oka, micha vm vlhpesa tuk oka, achi tok: mihmot im achiba ma, ishi tok.

12. Mihmvt, Wakayvt il iashke, mikma chi tikba ia lash$\mathrm{ke}$, achi tok.

13. Mihma, vlla vhleha hut ik kvllo, micha nanvlhpoa chipinta, micha nanvlhpoa hochito vt ushi pehlichi hosh si apihvishke; atuk o hatak vt nitak achvfa ho atablit tihlelikma, nanulhpoa moma kvt illit taha hinla ka a shahli hvt ithanvshke. 


\section{CHAPTA XXXIV.}

14. A shabli hut in tishu ha itikba iashke, chim asilhha lishke: mikma nanvlhpoa yvt sa tikba aya kak osh kaniohmit ayakma, micha vlla vhleha hak osh kaniohmit ayakma, lumvt mahaya li tuk osh a shahli hot Sei ai vtta ka im ona lashke, im achi tok.

15. Mihma Esau vt, Yohmi ka hatak kaniohmi ka si apiha ka ak chim ashachí, achi tok: mihma, Nanta katiohma chi ho ? A shahli itikba ya holhponayo hanta ya ai ahayuchi lashke, achi tok.

16. Yohmi ho mih nitak a Esau vt falamut $\underline{i}$ hina fohkvt Sei pilla ha ia tok.

17. Mihmvt Chekob vt aya mut, Sukkolh a ona cha, in chuka ikbi cha, $\underline{i}$ nanvlhpoa ya hoshontika im ikbi tok: yumohmi kak o yakni yưmma hohchifo ha Sukkolh achi tok.

18. Atuk o Chekob vt Patan-alam vttvt vla mot, Shalem, yummot Shekem in tumaha holihta yosh Kenan yakni talaia ka vla tok: micha tvmaha holihta itikba ak o im vlhtipo ya hilechi tok.

19. Micha im vlhtipo a a hilechi tuk vt Shekem iki Hamo ya im vlla vhleha ha tvli hota tushahli tahlepa achvfa ho osapa kashapa yo chumpa tok.

20. Micha yumma alta hilechi cha, El-elohe-Islael hochifo tok.

\section{CHAPTA XXXIV.}

MIHMA Leah ushetik Tinah Chekob a im eshi tok vt I yakni yumma ohoyo vhleha ha kohchvt pisut ia tok.

2. Atuk o yakni yưma i miko Hifait Hamo ya ushi Shekem vt pisa mot, hokli cha iba tushkit litehli tok.

3. Atuk osh Chekob ushetik Tinah ya chukvsh vt asitia tok; micha ohoyo himita ya anushkunna mvt, ohoyo himita yash i kanvt im anumpuli tok.

4. Micha Shekem vt. iki Hamo ya im anumpulit, Ohoyo himita iluppa sa tekchi ya chi ho ishit is sumashke, achi tok.

5. Atuk o Chekob vt, ushetik Tinah ya litehli tuk a haklo tok: (yohmi ka ushi vhleha hot i nanvlhpoa pehlichit 
yakni haiaka ya a asha tok: mihmut ik lo kisha kạ, Chekob vt nana ik acho tok.)

6. Mihma Shekem iki Hamo vt kohchvt iut Chekob a ont itim anumpuli tok.

7. Atuk o Chekob ushi vhleha hot haklo mot, yakni haiaka ya kohchvt vla tok: micha Chekob ushetik a iba toshkit, Islael a nana ik achukmo, yummvt yưmohma he vllhpesa

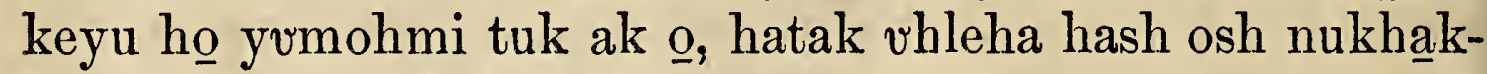
lo mvt, okla nukhobela fehna tok.

8. Mihma Hamo vt itim anumpulit, Suso Shekem chukvsh vt hvch isotek a ahni fehnushke : tekchi ya chị ho hush i bohlashke, hvchim asilhha lishke.

9. Micha hush pim itauwaya cha, hvch isotek vhleha ha hush pimakmvt, pisotek vhleha ha hush ishashke.

10. Micha pi takla hush aiashashke: mikma yakni vt hvchi tikba talaia chi hoke; ai itatobut hush aiasha cha, hvch imilayak a hush ai ikbashke, achi tok.

11. Mikma Shekem vt ohoyo himita hash îki ya micha i nakfi vhleha hă , Isht $\underline{i}$ kana ya hvchi tikha ya ai ahayuchi lashke; mikma nana ho hus sa mihakma, bohli lashke.

12. Ohoyo isht vlhtoba, micha nahulbina laua kvt kaniohmi kia hus sum apesashke; mikma nana ho hus sa mihakma bohli lashke: ik kaniohmi kia satekchi ya chi ho ohoyo himita yash hvs sumashke, im achi tok.

13. Mihma Chekob ushi vhleha hvt, in tek Tinah ya litehli tuk ak $\underline{\text { }}$, Shekem, micha ilki Hamo itatuklo ka haksichi hosh afalaminchit,

14. Nana iluppa e yumihcha he keyu, kuna hosh hakshup tupa keyu ka pin tek a il ima he keyu hoke: yvmmak okvt ishit pi hofahya hinla hoka.

15. Amba iluppak okmuno vlhpesa e chim ahna hinla; Pishno akinli chohmit, nakni hvchia moma kvt hakshup hvchi tvpa hokma,

16. Yohmikma pisotek vhleha ha e huch ima cha, hvch isotek vhleha ha il isha hi oke; yohmi kvt hvchi takla il ai asha cha, okla achufa eho tobashke.

17. Amba huchi tupa hi vt hush pi haponakla he keyuk- 
ma, yohmikma pisotek a il ishikmut, il ia cḥi hoke, im achi tok.

18. Mihma im anumpa ha Hamo, micha Hamo ushi Shekem itatuklo kvt im aiokpanchi tok.

19. Mihma hatak himita yash osh Chekob ushetik a anushkunna hatuk osh nana ka ik ahchibalo hosh yumihchi tok: micha holitopa kvt ikki in chuka achvfa ya moma i shahli hatok.

20. Mihma Hamo, micha ushi Shekem itatuklo kvt in tvmaha holihta okhissa ya ona cha, in tvmaha holihta hatak whleha ha itim anumpulit,

21. Hatak iluppa puta kut pit i kana achukmushke; yumohmi hokvt yakni ya aiasha cha, ai itatobashke; yakeh, yakni vt chito kvt im vlhpesa hoka: ushetik vhleha ha ishit il itauwayakmvt, pisotek vhleha ha il imashke.

22. Peh yummut hakshup tupa ka chohmit nakni hupia moma iloh aiasha kvt hakshup hupi tvpa hok mak o, ilvppak okmuno hatak vhleha hash osh vlhpesa pim ahni cha, pi takla aiasha na okla achufa eho toba hinla hoke.

23. I nanulhpoa chipinta, micha imilayak, micha i nanulhpoa hochito puta moma kvt pimmi ha chi keyu cho? peh vlhpesa il im ahni hok mak o, pi takla aiasha hinla hoke, achit okla tok.

24. Mihma in tumaha holihta kohchvt ia puta moma kvt Hamo, micha Hamo ushi Shekem $\underline{a} \underline{\mathrm{i}}$ haponaklo tok: micha in tumaha holihta ya kohchvt ia moma nakni puta moma kvt hakshup topa tok.

25. Atuk o nitak ont atuchina ma yakohmi tok, hotopvt taha ma, Chekob ushi tuklo, Simeon micha Lefai, Tinah i nakfi vt nukshopa iksho hosh $\mathrm{i}$ bushpo falaia ieshi bika cha, tumaha holihta ya vla cha, nakni moma ka vbit tahlit okla tok.

26. Micha Hamo, micha Hamo ushi Shekem itatuklo ka bushpo falaia halupa isht vbi tok; mihmvt Tinah ya Shekem in chuka ai ishit kohchi cha, kohchvt ia tok.

27. In tek a litehli tuk ak o, Chekob ushi vhleha hvt illi aiasha ka ont iba chukowa cha, tumaha holihta ya wehpuli tok. 
28. In chukfulhpoa, micha $\underline{\mathbf{i}}$ wak, micha im issuba haksobish falaia, micha nana tumaha holihta ya assha tuk, wicha osapa ya asha tuk a ishi tok.

29. Micha imilayak moma ka ishi cha, im vlla chipunta moma, micha ohoyo vhleha aiena ka yukachit ishi tok: micha aboha nana aiasha tuk aiena ka wehpuli tok.

30. Mihma Chekob vt Simeon micha Lefai ya , Hvs si ataklummi cha, yakni ya okla Kenanait, micha Pelissait a im itin takla ya huss sa shuwuchishke: holhtina kut ik sa lauwo hatuk $\underline{o}$, iti hoyot itunaha cha, a sanali cha, suba chi hoke, mikma vno, micha an chuka achvfa aiena kvt illit sv taha chi hoke, im achi tok.

31. Mihma, Pin tek a ohoyo haui yo chohmicha hinla cho? achi tok.

\section{CHAPTA XXXV.}

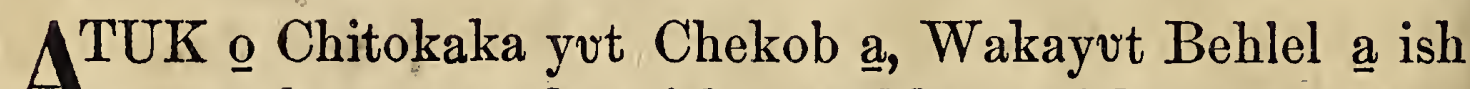
A ona cha, yummak o ish vttashke: micha yummak o chitibapishi Esau itikba ya ish maleli ma: Chitokaka yvt a chi haiaka tok a alta ya ish im ai ikbashke, im achi tok.

2. Yohmi ma Chekob vt in chuka achvfa ya, micha apiha tuk moma ka, Naholbvt toba puta hvch itin takla asha ka hush kanchashke; micha huchi kashofa cha, nafohka inla ishit hush fohkashke.

3. Micha wakayut Behlel ak o il iashke; mikma yummak o Chitokaka ya yummak osh a sulbusha nitak ma a haponaklo tok, micha kanima aya li ma subafoyuka tuk a alta im ai ikbi lashke, im achi tok.

4. Mihma naholbvt toba puta ibbak foka tuk vt, micha haksobish takali haksobish in takohmaya tuk vt Chekob a okla ima tok: mihma Chekob vt baiyi vt Shekem bilika hikia tuk o nutaka luhmi tok.

5. Micha bina ha kanvllichi cha ilhkoli tok: mihma tvmaha holihta apakfoyupvt aiasha ka Chitokaka i nukshopa kvt onvtonla hatuk o, Chekob ushi vhleha ha ik hliolo tok. 
6. Micha Chekob, micha okla moma apehvt aya tuk vt Lus, (yvmmvt Behlel) Kenan yakni talaia ka ona tok.

7. Micha yumma alta ai ikbi mut, yumma El-behlel

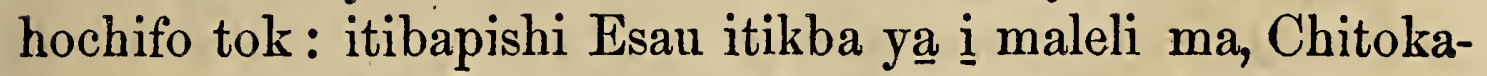
ka yut yummak o ai i haiaka tok oka.

8. Atuk o Lebekah ya sholi atok Tebolah vt illi cha, Behlel nutaka baiyi hikia ho nutaka yo hollohpi tok: mihma hohchifo ha Baiyi a yaiya, achi tok.

9. Atuk o Chekob vt Patan-alam a kohchvt minti ha ma, Chitokaka yut anonti i haiaka cha, yukpali tok.

10. Micha Chitokaka yvt, Chi hohchifo hvt, Chekob oke; himak pilla ma chi hohchifo ha Chekob acha he keyu, amba Islael chi hohchifa hi oke, im achi tok; micha hohchifo ha Islael, achi tok.

11. Micha Chitokaka yut, Uno ak osh Chitokaka Nan isht im aiahli im ataha he iksho sia hoke: wayut ish - apaknashke; oklushi, micha oklushi kanohmi kia chi atoba hi oke, micha miko puta kvt chi haknip a a kohcha hi oke.

12. Micha yakni ya Eblaham micha Aisak ima li tok vt chishno ak o chima la chi hoke; micha chim isht atiaka et chiakaiya kak o yakni ya ima la hi oke, im achi tok.

13. Mihmut Chitokaka yut ai im anumpulit hikia tuk vt ai i filvmmit vba ia tok.

14. Mihma Chekob vt ai im anumpuli tuk a nan isht-ithana, nan isht-ithana tuli yo hilechi tok: micha nan-ishko im issa yo on hlatubli mut, bila yo on hlatubli tok.

15. Micha Chitokaka yvt ai im anumpuli tuk yumma hohchifo ha Chekob vt Behlel, achi tok.

16. Mihma Behlel a a ashut bina ha kanullichi cha, ilhkoli tok: atuk o Eflalh $\underline{a}$ ona chi ka ik hopako tok; atuk o Lechel vt vlla esha chi hosh nukhummi cha i palummi fehna tok.

17. Atuk osh yakohmi tok vlla esha chi hosh 1 palvimmi fehna ma, vlleshi ka apeluchi ohoyo hvt, Chi nukshopa ná, iluppak kia vlla nakni yo ish esha chi hoke, im achi tok.

18. Atuk osh yakohmi tok im ilhfiopak vt kohchvt ia 
mut, (illi tok okut,) hohchifo ha Benoni achi tok: yohmi kia iki vto Benchamin hochifo tok.

19. Mihma Lechel vt illi cha, Eflalh hina takla, yummut Behlehem ak o a hollohpi tok.

20. Mihma Chekob vt im ahollohpi paknaka ya nan ishtithana hilechi tok: yummvt Lechel im ahollohpi nan ishtithana atok osh himak nitak a hikia hoke.

21. Mihma Islael vt bina ha kanvllichi cha, aya mvt Eta holihta chaha ai atoni ya misha yo im vlhtipo ya ont a hilechi tok.

22. Atuk o yakohmi tok, Islael vt yakni yumma anta ma, Luben vt ia cha, iki im itaiena Bilhah ya iba nusi tok: atuk o Islael vt haklo tok. Yohmi ka . Chekob ushi vhleha hut auahtuklo tok.

23. Leah ushi vhleha hvt Luben Chekob ushi vttahpi, micha Simeon, micha Lefai, micha Chutah, micha Issaka, micha Sebulun ak atok:

24. Lechel ushi vhleha hvt Chosef, micha Benchamin ak atok.

25. Mikma Lechel in tishu ohoyo Bilhah ya ushi tuklo kvt Tan, micha Naftali ak atok.

26. Mikma Leah in tishu ohoyo Silpah ya ushi tuklo kvt Kat, micha Ashe ak atok. Ilvppa puta kak osh Chekob ushi vhleha hosh Patan-alam a ai im asha tok.

27. Mihma Chekob vt iki Aisak vt aivtta Mamle, tumaha holihta Abah (yvmmvt Heblon oke,) yvmmak o Eblaham, micha Aisak vt ai ashwa yatuk o ona tok.

28. Mihma Aisák i nitak puta kvt afummi tahlepa achvfa cha pokoli untuchina tok.

29. Atuk o Aisak vt sipokni, micha nitak lawa hosh fiopissut illi cha, im okla ha iba holhtina tok: mihma ushi Esau, micha Chekob vt hohpit okla tok.

\section{CHAPTA XXXVI.}

OHMI ka iluppak osh Esau, yummut Etom in chuka 1 achufa ya anoli holisso yoke. 
2. Esau vt Kenan ushetik vhleha ho ohoyo ha ai ishi tok: Hittait hatak Elon ushetik Atah, micha Hifait hatak Sikeon ushetik Anah ya ushetik Aholibamah;

3. Micha Ishmael ushetik, Nebacholh in tek Bashemalh ak atok.

4. Mihma Atah vt Elifas a Esau im eshi tok; mihma Bashemalh vt Luel a im eshi tok.

5. Mikma Aholibamah vt Cheush, micha Chaalam, micha Kolah ya eshi tok: iluppa puta kak osh Esau ushi vhleha hosh Kenan yakni ya im aiasha tok.

6. Atuk o Esau vt tekchi vhleha, micha ushi vhleha, micha ushetik vhleha, micha nana hosh in chuka achvfa aiasha tukk moma, micha i nanvlhpoa chipinta, micha $\underline{i}$ nanvlhpoa hochito, micha imilayak moma Kenan yakni ai ahayuchi tuk vt ishi cha, itibapishi Chekob itikba ya i filummit yakni inla ya ia tok.

7. Imilayak laua kak osh itibai ashwa he keyu hokvt: micha i nanvlhpoa pulla kak o okla inla yosh yakni ai ahashwa tuk vt ai impa yot taha hinla kak atok.

8. Yumohmi ho Esau vt Sei nunih hak o ahanta tok: Esau vt Etom oke.

9. Micha iluppa puta kak osh Etomait vhleha ha iki Esau ya in chuka achufa yosh Sei nunih ha aiasha tok a holisso anoli hoke,

10. Hohchifo iluppa puta kak osh Esau ushi vhleha hohchifo hoke: Esau tekchi Atah ya ushi Elifas: Esau tekchi Bashemalh a ushi Luel ak atok.

11. Mikma Elifas ushi vhleha hut Teman, Oma, Sefo, Katam, micha Kenas ak atok.

12. Mihma Timna vt Esau ushi Elifas a im itaiena atok: atuk osh Elifas a Amalek im eshi tok: iluppa puta kak osh Esau tekchi Atah ya ushi vhleha hatok.

13. Mikma iluppa puta kak osh Luel a ushi vhleha hatok: Nahalh, micha Selah, Shammah, micha Missah: iluppa puta kak osh Esau tekchi Bashemalh a ushi vhleha hatok.

14. Mikma iluppa puta kak osh Sibeon ushetik Anah ya ushetik Esau tekchi Áholibamah ya ushi vhleha hatok: mi- 
cha Esau ya Cheush, micha Chaalam, micha Kolah aiena ka im eshi tok.

15. Ilvppa puta kak osh Esau ushi hatak pehlichi vhleha hatok: Esau ushi vttahpi Elifas a ushi vhleha hvt hatak pehlichi Teman, hatak pehlichi Oma, hatak pehlichi Sefo, hatak pehlichi Kenas.

16. Hatak pehlichi Kolah, hatak pehlichi Katam, micha hatak pehlichi Amalek ak atok; iluppa puta kak osh hatak pehlichi vhleha hosh Elifas a akohcha tok osh Etom yakni ya aiasha tok: iluppa puta kak osh Atah ya ushi vhleha hatok.

17. Mikma ilvppa puta kak osh Esau ushi Leuel a ushi vhleha hatok: hatak pehlichi Nahalh, hatak pehlichi Selah; hatak pehlichi Shammah, hatak pehlichi Missah, ak atok: iluppa puta kak osh hatak pehlichi vhleha hosh Leuel a akohcha tuk osh Etom yakni ya aiasha tok: iluppa puta kak osh Esau tekchi Bashemalh a ushi vhleha hatok.

18. Mikma iluppa puta kak osh Esau tekchi Aholibamah ya ushi vhleha hatok; hatak pehlichi Cheush, hatak pehlichi Chaalam, hatak pehlichi Kolah ak atok: iluppa puta kak osh hatak pehlichi vhleha hosh Anah ushetik Esau tekchi Aholibamah ya akohcha tok.

19. Iluppa puta kak osh Esau (yummut Etom oka) ushi vhleha hatok: micha iluppa puta kak osh $\underline{\mathrm{i}}$ hatak pehlichi vhleha hatok.

20. Iluppa puta kak osh Holait hatak Sei ya ushi vhleha hosh yakni yumma asha tok: Lotan, micha Shobal, micha Sibeon, micha Anah,

21. Micha Tishon, micha Ese, micha Tishan : iluppa puta kak osh Holait okla hatak pehlichi vhleha hosh Sei im vlla vhleha hosh Etom yakni ya aiasha tok.

22. Mikma Lotan im vlla vhleha hvt Holi, micha Heman ak atok: mikma Lotan in tek vt Timna ak atok.

23. Mikma Shobal im vlla vhleha hvt iluppa puta kak atok: Alfan, micha Manahalh, micha Ebal, Shefo, micha Onam ak atok.

24. Mikma iluppa puta kak osh Sibeon im vlla vhleha 
hatok: Akah, micha Anah itatuklo tok: Anah iluppak osh iki Sibeon im issuba haksobish falaia impvchi hosh yakni haiaka anta mut, kuli lushpa ka ai ahayuchi tok.

25. Mikma Anah im vlla vhleha hut iluppa puta kak atok: Tishon, micha Anah ushetik Aholibamah ak atok.

26. Mikma iluppa puta kak osh Tishon im vlla vhleha hatok; Hemtan, micha Eshban, micha Ilhlan, micha Chelan ak atok.

27. Ese im vlla vhleha hvt iluppa puta kak atok: Bilhan, micha Saafan, micha Akan ak atok.

28. Tishan im vlla vhleha hvt iluppa puta kak atok: Us, micha Alan ak atok.

29. Hatak pehlichi iluppa puta kak osh Holait okla a kohcha tok; hatak pehlichi Lotan, hatak pehlichi Shobal, hatak pehlichi Sibeon, micha hatak pehlichi Anah.

30. Hatak pehlichi Tishon, hatak pehlichi Ese, hatak pehlichi Tishan : iluppa puta kak osh hatak pehlichi vhleha hut Holi ya a kohchvt, hatak i pehlichi vhleha ha iba holhtina yosh Sei yakni ya aiasha tok.

31. Miko iluppa puta kak osh, Islael ushi vhleha ha i miko vt ik i miko kisha ma, Etom yakni ya a miko tok.

32. Mikma Beo ushi Bela vt Etom a a miko tok: micha in tumaha holihta hohchifo hvt Tinhabah atok.

33. Atuk osh Bela vt illi tok; mihma Boslah vtta Selah ushi Chobab ak osh vlhtobvt miko tok.

34. Atuk osh Chobab vt illi tok: mihma Temani yakni aivtta Husham ak osh vlhtobvt miko tok.

35. Atuk osh Husham vt illi tok; mihma Betat ushi Hatat (yummak osh Mitian okla ha Moab i yakni ya ai issot kanchi tok ak osh,) vlhtobut miko tok: micha in tumaha holihta hohchifo hvt Afilh atok.

36. Atuk osh Hatat vt illi tok: mihma Maslekah aivtta Samlah ak osh vlhtobvt miko tok.

37. Atuk osh Samlah vt illi tok; mihma okhina bilika talaia Lehobolh aivtta Sal ak osh vlhtobut miko tok.

38. Atuk osh Sal vt illi tok: mihma Akbo ushi Baal-hanan ak osh vlhtobut miko tok. 
39. Atuk osh Akbo ushi Baal-hanan vt illi tok; mihma Hatat ak osh vlhtobvt miko tok: micha in tvmaha holihta hohchifo hvt Pau atok; micha tekchi hohchifo kvt Mehetabel osh Matlet ushetik a Mesahab ushetik atok.

40. Mikma hohchifo iluppa puta kak osh hatak pehlichi vhleha Esau a kohcha tok vt in chuka achvfa vlhpisvt, $\underline{\mathrm{i}}$ yakni ya vlhpisvt hohchifo hatok: hatak pehlichi Timnah, hatak pehlichi Alfah, hatak pehlichi Chehlelo,

41. Hatak pehlichi Aholibamah, hatak pehlichi Elah, hatak pehlichi Pinon,

42. Hatak pehlichi Kenas, hatak pehlichi Teman, hatak pehlichi Mibsa,

43. Hatak pehlichi Maktiel, hatak pehlichi Ilam, iluppa

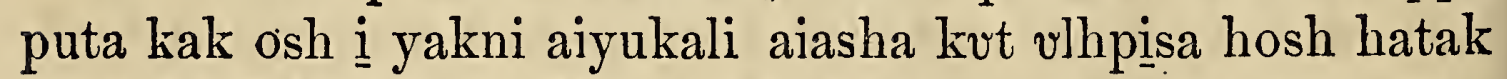
pehlichi vhleha hosh Etom a aiasha tok: yummut Esau Etomait vhleha ha iki atok.

\section{CHAPTA XXXVII.}

M.IKMA iki vt okla inla yosh yakni aiahanta tok; 1 Kenan yakni ak o Chekob vt ai ahanta tok.

2. Ilvppak osh Chekob in chuka achvfa ya anoli holisso yoke: Chosef vt afummi auah untuklo hosh itibapishi vhleha ha takla nanvlhpoa chipinta impvchi hosh ahanta tok, micha vlla yut iki tekchi vhleha Bilhah ushi vhleha micha Silpah ushi vhleha hak o takla ahanta tok; atuk osh Chosef vt nana ik achukmo yumohmi tuk a iki ya ont im anoli tok.

3. Sipoknit taiyaha hosh ushi im vtta tok, atuk osh Islael vt Chosef a holitobli kvt im vlla vhleha moma ka 1 shahlichi tok: micha nafohka pisa il aiyukali yo im ikbi tok.

4. Atuk $\mathrm{g}$ iki vt itibapishi vhleha moma ka $\underline{\mathrm{i}}$ shahlichit holitobli na itibapishi vhleha hut pisa mut, okla i nukkilli cha, i kanvt im anumpula he keyu tok.

5. Atuk o Chosef vt naholhpokna ho holhpokna cha, itibapishi vhleha hạ im anoli tok: mihma $\underline{\underline{i}}$ shahlichit $\underline{\mathrm{i}}$ nukkilli tok. 
6. Micha, Naholhpokna iluppa sa holhpokna tuk a ho hakló, hvchim asilhha lishke.

7. Yakeh, osapa anulkaka ya onush takchi hosh il asha tuk $\underline{0}$, yakeh, a sita hut wakaya cha apissvt hikia tuk oke; mihma yakeh, hvchi sita hot a sitha ha hiohlit apakfoyupa cha, im akachunoli tuk oke, im achi tok.

8. Mihma itibapishi vhleha hvt, Ish pi mịka chi ho muhli cho? micha ish pi shahla chi ho mvhli cho? im achi tok: micha holhpokna, micha im anumpa aiena atuk pulla kak $\underline{0}$ i shahlichit i nukkilli tok.

9. Atuk osh himakma naholhpokna ho holhpokna cha, itibapishi ha im anolit, Yakeh, himak a naholhpokna ho sa holhpoknvshke: mihma yakeh, hushi, micha hushi ninak aya, micha fochik auahachvfa hash osh vm akachunoli tuk oke, achi tok.

10. Mihmvt ikki, micha itibapishi vhleha ha im anoli tok: mihma iki vt im alvmmit, Ilvppak osh holhpokna nanta mak o chi holhpokna tuk oh cho? Uno, micha chishki, micha chitibapishi vhleha il aiena kvt ant akachunolit aka pit $\mathrm{e}$ chin hlipkvcha chi ho mvhli cho? im achi tok.

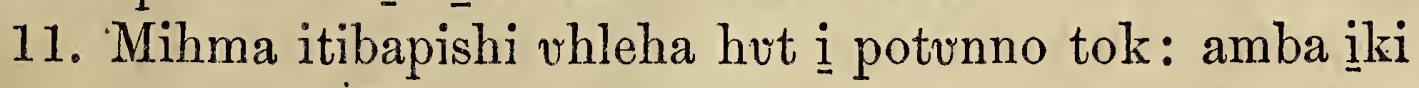
vto anumpa ha ithaiyanvt aya tok.

12. Atuk o itibapishi vhleha hut $\underline{i k i} \underline{i}$ nanvlhpoa chipinta ya impvcha chị hosh Shekem a ilhkoli tok.

13. Mihma Islael vt Chosef a, Chitibapishi vhleha hvt Shekem a nanvlhpca chipinta ya ai impvchi keyu cho? Inta, pit $\underline{i}$ chi chvffichi lashke, im achi tok. Mihma, yak hikia lishke, im achi tok.

14. Mihma, Iá, chim asilhha lishke, chitibapishi vhleha hut achukma hosh ashakma, micha nanvlhpoa chipinta vt achukma hosh ashakmá, nanakmá, ont pisa cha, anonti anumpa isht is svm vlashke, im achi tok. Yohmi mot Heblon okfa ha a chuffichi na, Shekem a ona tok.

15. Atuk o hatak osh ahayuchi ma, Yakeh, yakni haiaka ya fullokahanchi tok: mihma hatak ash osh $\underline{\mathrm{i}}$ ponaklot, Nanta ish hoyo cho? achi tok.

16. Mihma Itibapishi li vhleha ha hoyo li hoke : kanima 
yo i nanvlhpoa ya ai impvchikma vm anolí, chim asilhha lishke, achi tok.

17. Mihma hatak ash osh, Iluppa asha tuk vt ilhkoli tuk oke: Tohlan ak o kiloh iá, achi ho hakklo li tuk oka, achi tok. Mihma Chosef vt iakaiyvt ia tuk osh itibapishi vhleha ha Tohlan a ont ai ahayuchi tok.

18. Atuk o hopaki ho minti na, bilika ik lo kisha ho pisa mut, haksichi hosh vba chi kut itim apesa tok.

19. Micha ilap bika, itim anumpulit, Yakeh, ilvppot holhpokna shahli ash osh mintishke.

20. Yvmohmi hoka inta, himak a iloh vbashke, micha hichukbi kanima pit eho kanchashke: micha, Nampoa okpulo hosh vbit vpvt tahli tuk oke, iloh achashke; mikma holhpokna tuk vt kaniohmi na eho pisashke itim achi tok.

21. Mihma Luben vt haklo cha, ibbak a a kohchit hlakofichi tok; micha kiloh abo kashke, achi tok.

22. Micha Luben vt, Issish a hvch ik hlatablo kashke, amba yakni haiaka hichukbi hikia ilvppak o pit hush kanchi cha, hvch ibbak hvch ik onocho kashke, im achi tok, ibbak a a kohchit hlakofichi cha, anonti iki ya falaminchit pit ima chi hosh yưmohmi tok.

23. Atuk o yakohmi tok, Chosef vt itibapishi vhleha ha im ona ma, Chosef $\underline{a} \underline{i}$ nafohka, $\underline{i}$ nafohka pisa ilaiyukali fohka kash i shufi tok.

24. Micha okla ishi cha, hichukbi yo pit fohki tok: mihma hichukbi ya nana iksho, oka vt iksho tok.

25. Micha okla binilit puska vpa tok; atuk osh akachakalit hopokoyo ma, yakeh, Ishmaelait hatak kanohmi kvt Kileat minti hosh i kamel vt nabalama, micha balsam, micha hlitili muh shali ho, Echip isht ona chi hosh aya tok.

26. Mihma Chutah vt itibapishi vhleha ha , Pitibapishi ha e bi cha, im issish a e luhmi kak osh nanta ho ishit pim ai achukma hinla cho?

27. Inta, Ishmaelait vhleha hak o iloh $\underline{i}$ kanchi cha, pibbak vt ik onvtoyulo kashke; pitibapishi, micha pi nipi yoka, im achi tok: mihma itibapishi vhleha hvt vlhpesa, ahni tok. 
28. Yohmi ma Mitianait nan-itatoba vhleha hvt ant aya tok; mihma Chosef a halvllit vba takalichit, hichukbi ya a kohchi cha, Chosef a tuli huta tushahli pokoli tuklo ho Ishmaelait hatak vhleha hash $\underline{i}$ kanchi tok: mihma Chosef a Echip a isht ona tok.

29. Atuk o Luben vt falamvt hichukbi ya ona tok: mihma yakeh, Chosef vt hichukbi ya iksho tok: mihma i nafohka ya hliluffi tok.

30. Mihmvt itibapishi vhleha ha falamvt im ona cha, Vlla yash osh ikshoshke: yohmi ka vno vto katima yak o ia la chi cho? achi tok.

31. Atuk o Chosef $\underline{i}$ nafohka yash ishi cha, issi kosomushi ya vbi mvt, nafohka yash issish a okvchi tok.

32. Mihmut nafohka pisa ilaiyukali yash okla pila na iki ya isht im ona tok; micha, Iluppa il ahayuchi tuk oke: yohmi hoka chiso i nafohka yokmá, keyukmá nanakmá himak a ish ithwnashke, achi tok.

33. Mihma ithana cha, Suso i nafohka yoke; nampoa okpulo vt vput tahli tuk oke: Chosef vt nipvt taha pullushke, achi tok.

34. Mihmut Chekob vt i nafohka ya hliluffi cha, na hishi tunna isht vskofuchi mut, nitak laua ho ushi ya ishit yahaya tok.

35. Mihma ushi vhleha moma, micha ushetik vhleha moma aiena kvt wakayut hopohluli bunna tok: yohmi kia hopohla ik bunno tok; micha, Yahayut ahollohpi ya sus o hash im ona la chi hokvt, achi tok. Yvmohmit iki vt ishit yahaya tok.

36. Mihma Mitianait vhleha hash osh Echip a isht ona cha, Falaoh in tishu, micha nan atoni i kupituni Potifa ya $\underline{\underline{i}}$ kanchi tok.

\section{CHAPTA XXXVIII.}

A TUK o nitak yumma yakohmi tok, Chutah vt itibapishi A vhleha ha i filummit Atullamait hatak osh hohchifo kvt Hilah ya i fullotvt ont iba chukowa tok. 
2. Micha Chutah vt yumma Kenanait hatak, yumma hohchifo kut Shuah ushetik ó pisa tok; mihmut ishi cha, iba chukowa tok.

3. Mihma chakali cha, vlla nakni yo eshi tok; mihma hohchifo ha El achi tok.

4. Atuk o anonti chakali cha, vlla nakni yo eshi tok; micha hohchifo ha Onan achi tok.

5. Atuk osh himakma anonti chakali cha, vlla nakni yo eshi tok; micha hohchifo ha Shelah achi tok: mihmvt eshi ma, Chutah vt Chesib ak o anta tok.

6. Atuk osh Chutah vt ushi vttahpi El a tekchi ya chi ho - ohoyo yumma hohchifo kut Tama yo isht ima tok.

7. Atuk o El Chutah im vttahpi hut Chihowah itikba ya haksi hatuk o Chihowah vt vbi tok.

8. Atuk o Chutah vt Onan a, Chitibapishi tekchi ya iba chukowvt ish itauwaya cha, chitibapishi ha aiunchololi a ișh i hilechashke, im achi tok.

9. Mihma Onan vt ishtatiaka yvt immi ha he keyu kvt ithana tok: atuk osh yakohmi tok, itibapishi tekchi ya iba chukowa mut, itibapishi ishtatiaka ik emo ka chi kvt yakni a on hlatubli tok.

10. Nana yumihchi tuk vt Chihowah ya ik im vlhpesacho tok: yohmi hatuk o yummak kia vbi tok.

11. Yohmi ma Chutah vt ipok Tama ya , Ohoyo i hatak illi yosh chiki in chuka ish ahanta tuk o, suso Shelah vt hofantit tahak mak ashke, im achi tok: (yummak kia itibapishi vhleha hakinli chohmit illi yoba ná achi hatuk okvt:) mihma Tama yut ilki in chuka ont ahanta tok.

12. Atuk o nitak hopakit taha ma, Shuah ushetik Chutah tekchi vt illi tok; atuk osh Chutah vt hopohla cha, in chukfulhpoa hishi vmo puta kvt Timnalh a asha tuk o $\underline{i}$ kana Atullamait hatak Hilah yut apiha ho im ona tok..

13. Atuk o Tama ya im anolit, Yakeh; chimvfo vt in chukfulhpoa hishi vmot Timnalh a iushke, achi tok.

14. Mihma ohoyo $\underline{\mathrm{i}}$ hatak illi i nafohka ya shufi mut, nashuka isht ompoholmo ya isht il ompohomo cha, il apakfohli mut, ahikia yưmmvt Timnalh hina kvli tuklo ai ia yo 
binili tok: Shelah vt hofantit taha hokak o ishit tekchi ya chi ho ik $\underline{i}$ bohlo ka, pisa hokvt.

15. Chutah vt pisa mut, ohoyo haui yoke, ahni tok: nashuka ya ompohomo hatuk oka,

16. Atuk o hina bilika binili ka $\underline{i}$ fullotvt, Inta, ak chiba chukowa, chim asilhha lishke, achi tok: jpok atuk a ik ithano hatuk okvt: mihma, Is suba chukowa chi kvt nanta ho is suma hinla cho? achi tok.

17. Issi kosoma ushi yo nanvlhpoa chipinta ya ai ishit et chi pila lashke, achi tok: mihma, Isht is sum aiahlichi ya is suma tuk osh et is sa pila hinla cho? achi tok.

18: Mihma, Isht-aiahlichi nanta ho chima la hinla cho? achi ma, Chibbak ushi fohka nanisht in chunli, micha napuna chi shakba vlbeha, micha chin tubi chibbak foka aiena ka, achi tok: mihma ima cha, iba chukowa tok; atuk o vlla in chakali tok.

19. Micha wakaya cha, ia mvt, nashuka isht ompoholmo ya shufit kanima bohli cha, ohoyo ị hatak illi i nafohka ya ishit fohka tok.

20. Atuk o Chutah vt i kana Atullamait hatak a issi kosoma ushi yash ibbak fohki cha, $\underline{i}$ nan-isht aiahlichi yash ohoyo hash ibbak fohka tuk a im ai isha chi hosh pit i pila tok, yohmi tuk kia ik ahayucho tok.

21. Mihmut okla yumma asha kash i ponaklot, Ohoyo haui vt haiakvt hina lapalika kvli tuklo ai ia ya asha tuk mvto? achi tok. Mihma, Iluppa haui vt iksho ke uk oke, olkla achi tok.

22. Mihma falamut Chutah ya im ona cha, Ahayuchi la he keyushke; micha yumma hatak vhleha hot, Ilvppa haui vt iksho ke tuk, achi akinli tuk oke, achi tok.

23. Mihma Chutah vt, ohoyo hash osh ik ishí, ishit pi hofahya yoba ná; Yakeh, issi kosoma iluppa pit i pila li tuk o, chik ahayuchoshke, achi tok.

24. Atuk o yakohmi tok, $\underline{\mathrm{i}}$ himmak hushi tuchina foka ma, Chutah ya im anolit, Chipok Tama vt haui tobvshke; micha yakeh, hauit chakalishke, achi tok. Mihma Chutah vt, Kocha isht hush ona na luashke, achi tok. 


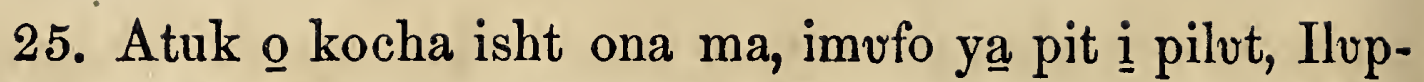
pvt immi, hatak yummak o vlla i shali lishke: micha ibbak ushi fohka nan isht in chunli, micha napuna shakba vlbeha, micha tubi ut kuna immi hokma ithuná, chim asilhha lishke, achi tok.

26. Mihma Chutah vt ithana mvt, Aivlhpiesa kvt vno ya a shahlishke; suso Shelah ya ak i bohlo tuk okvt, achi tok: micha himmakma anonti ik ithano tok.

27. Atuk o yakohmi tok, vlla esha chi hosh nukhvmmi itanitak ma, yakeh, haiyup osh im oshvtto ya alhto tok.

28. Atuk osh vlla esha chi hosh nukhummi ma, yakeh, achvfa kvt ibbak kocha pit weli tok: mihma vlleshi ka apelvchi ohoyo hot ponola tishepa ishi cha, ibbak a ishit ita takchi cha, Iluppak osh tikkba kohcha hoke, achi tok.

29. Atuk o ibbak a falvmminchit ishi ma, yakeh, itibapishi hvt kohcha tok; mihma, Nanta Katiohmi ho tivblit ish kohcha cho? tivbli ilupput chishno akinli hosh chi onvtonlashke, achi tok: yumohmi hatuk o hohchifo ha Fales, achi tok.

30. Atuk o yohmi ha yak o itibapishi ibbak ponola tishepa ishit talakchi yash osh kohcha tok ; mihma hohchifo ka Salah achi tok.

\section{CHAPTA XXXIX.}

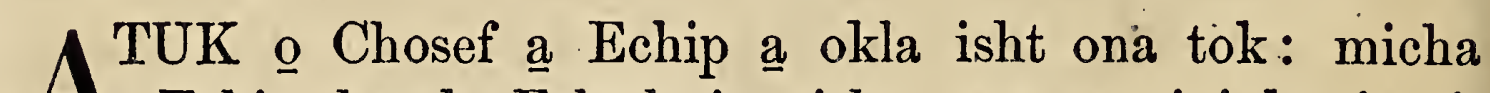
A Echip hatak, Falaoh in tishu, nan atoni i kvpituni, Potifa ak osh yummaisht ona tuk; Ishmaelait hatak vhleha hash in chumput im ishi tok.

2. Atuk o Chihowah yot Chosef a ibafoyuka na hatak nana amosholit, im aiahli achukma atok: mihma $\underline{i}$ shahli Echip hatak in chuka ya ahanta tok.

3. Mihma Chihowah yvt ibafoyuka, micha nana moma ka ibbak isht ieshikma, Chihowah yvt im aiahhlichi ka $\underline{\mathbf{i}}$ shahli hut pisa tok.

4. Mihma Chosef vt itikba ya holhponayo hanta ya ai ahayuchi cha, in toksvli tok; mihma in chuka achvfa ya 
apesuchi ikbi mvt, nana im asha tuk vt moma ibbak fohki tok.

5. Atuk o yakohmi tok, in chuka achvfa, micha nana moma im asha tuk vt apesuchi ikbi tok nitak itin takla ma, Chihowah yut Chosef ak atuk pulla mak o, Echip hatak ash in chuka achufa ya yukpali tok: micha Chihowah nan ishit yukpali vt nana moma chuka aiasha, micha osapa aiasha ka onvtoyula tok.

6. Mihma nana im asha tuk moma kut Chosef ibbak a fohki tok: nana im asha tuk vt achvfa kia ithana keyu, amba puska upa ak illa beka tok: mihma Chosef vt hatak aiokli nipi pisa achukma atok.

7. Atuk o ilvppa puta kash $\underline{i}$ himmakma yakohmi tok, $\underline{\mathbf{i}}$ shahli ha tekchi vt Chosef a nishkin a onochi tok; micha, Suba tushkí, achi tok.

8. Yohmi kia vlhpesa ik ahno tok; micha i shahli tekchi ya , Yakeh a shahli hut subbak foyuka tuk osh chuka nana aiasha ka ithana keyu, micha nana im aiasha tuk vt moma subbak fohki tuk oke.

9. Chuka iluppa achvfa kia vno ya a shahli kvt ikshoshke: micha nana kia a halanli tuk keyu, amba chishno ak illa tuk, tekchi chia hoka, yohmi ka nanta katiohmi ho nana ik achukmo chinto iluppa yumihchit Chitokaka ya i yoshuba la hinla cho? im achi tok.

10. Atuk o yakohmi tok, onna hosh onnak ma Chosef a im anumpuli kia, ik i haponaklot iba tushka he keyu, micha iba takla ahanta he keyu tok.

11. Atuk o nitak yumma foka ma Chosef vt im atoksvli isht vtta chi hosh chuka ya chukowa tok; mihma chuka yumma hatak asha chatuk achvfa kia anukaka iksho tok.

12. Mihma yummak o i nafohka ya hoklit ai ishi cha, Svba tushkí, achi tok: mihma i nafohka vt ibbak foka na kanchi cha, malelit kohcha tok.

13. Atuk o yakohmi tok, i nafohka yut ibbak foka na kanchi cha, malelit kohcha na pisa mvt,

14. In chuka hatak vhleha ha hoyo cha, im anumpulit, Heblu hatak vt ishit pi yopula chị ho ishit pim via tuk a ho 
pesá; suba tushka chị hosh suba chukowa ma, chitoli hosh paya li tuk oke.

15. Atuk osh yakohmi tuk oke, chitoli hosh paya li ka haklo mvt, i nafohka yot an tonla na, kanchi cha malelit kohcha tuk oke achi tok.

16. Mihma i nafohka yash achukmalit bohli na, itonla tuk

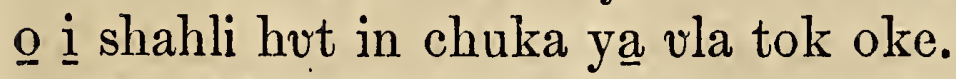

17. Mihmot anumpa ilvppa chohmi kak o im anumpulit, Heblu tishu isht ish pim vla tuk vt ishit si yopoma chi hosh suba chukowa tuk oke.

18. Atuk osh yakohmi tuk oke, chitoli hosh paya li ma, i nafohka yot an tonla na, kanchi cha malelit kohcha tuk oke, achi tok.

19. Atuk o yakohmi tok, $\underline{i}$ shahli hash osh tekchi im anumpa ha im anumpulit, Chin tishu hvt si yakohmichi tuk oke, achi na haklo mvt, im ai i nukkilli vt libbi tok.

20. Mihma Chosef a $\underline{\mathrm{i}}$ shahli yash osh ishi cha, aboha kvllo, miko i yuka hut talakchit aiasha ya fohki tok: mihma aboha kullo yummak o foyuka tok.

21. Yohmi kia Chihowah yut Chosef a iba foyukvt i nukhạklo cha, aboha kullo apistikeli yvt i kana hi ya nukfohoki tok.

22. Mihma aboha kvllo apistikeli vt yuka aboha kvllo aiasha moma ka Chosef jbbak a fohki tok: mihma nana hosh yumma a yumohmikma, yummak osh yumihchi atok.

23. Nana hosh yumma ibbak foyukak ma, aboha kullo apistikeli vt ik apesacho tok: Chihowah yot ibafoyuka hatuk oka, micha nana yumihchikma, Chihowah yot im aiahlichi beka tok.

\section{CHAPTA XL。}

TUK o iluppa puta kut yumohmi ha ya yakohmi tok, A Echip i milko aiishko sholi, micha pvska ikbi itatuklo kvt i shahli Echip i miko ya nan im ashvchi tok.

2. Atuk o Falaoh vt in tishu tuklo, aiishko sholi i shahli, micha puska ikbi i shahli ya $\underline{\underline{i}}$ nukhobela tok. . 


\section{CHAPTA XL.}

3. Micha aboha kullo yà , nan atoni i kupituni in chuka, aboha kullo Chosef vt a talakchi tuk a apitta tok.

4. Mihma nan atoni $\underline{i}$ kupituni vt Chosef ibbak fohki na, apesuchit nana hoyot ima tok: mihma nitak hopaki ho aboha kvllo ya ai alhto tok.

5. Atuk o itatukklot naholhpokna ho holhpokna mvt, hatak achufa ila bika ninak achvfa holhpokna tok; Echip i miko aiishko i sholi, micha puska im ikbi talakchit aboha kvllo alhto tuk vt naholhpokna toshowa inla bika ho holhpokna tok.

6. Atuk o Chosef vt onnahinli ma ont iba chukowa cha, pisa ma, Yakeh, ilapissa tok.

7. Mihma Falaoh in tishu tuklo kvt $\underline{\mathrm{i}}$ shahli in chuka

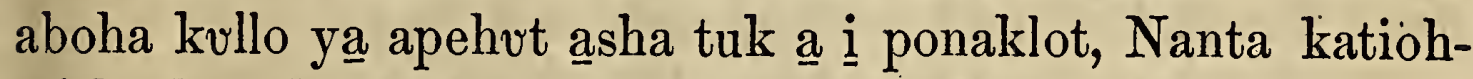
mi ho himak nitak a hush ilapissa cho? achi tok.

8. Naholhpokna ho pi holhpokna tuk $\underline{o}$, tosholi. vt ikshoshke, im achit okla tok. Mihma Chosef vt, Tosholi kvt Chitokaka ak osh immi keyu cho? Vm anolí, hvchim asilhha lishke, im achi tok.

9. Mihma miko aiishko sholi $\underline{i}$ shahli vt holhpokna tuk vt Chosef a im anoli mut, Sa holhpokna ma pakvpi vt sa tikba ya hikia tuk oke.

10. Mihma pakupi ya naksish tuchina hosh hieli tuk oke: micha bokubli ahoba mut, pakanlit kohcha tuk oke: mihmot lukussa yash osh paki nuna yo toba tuk oke.

11. Mihma Falaoh im isht-ishko vt subbak foka tuk osh paki yash ishi li mvt, bushlit Falaoh im isht-ishko ya uni li mut, isht-ishko yash Falaoh ibbak a fohki li tuk oke, im achi tok.

12. Mihma Chosef vt, Toshowa kvt ilvppak oke: Naksish tuchina kvt nitak tuchina hoke :

13. Nitak tuchina takla ma Falaoh vt chi noshkobo ha akachakalichi cha, ish ahikia ya falumminchit chi hilecha chi hoke : mikma Falaoh im isht-ishko ya aiishko sholi chia moma mut, ish yumihchi beka tok vt chohmit Falaoh ibbak a ísh fohka chi hoke.

14. Yohmi kia chim achukmakmvt, is suthanvt is sa ka8 
nashke, chim asilhha lishke; micha Falaoh ya is si anoli cha, aboha kvllo iluppa is sa kohchichashke;

15. Heblu yakni ya a hukopvt susht vla tok o muhli hoka: micha iluppak kia nana ho yumihchi li tuk o aboha kvllo iluppa sa fohka he vlhpesa kut iksho akinli hoke, im achi tok.

16. Mihma puska ikbi i shahli hvt toshowa kut achukma na pisa mut, Chosef a, V̌no ak kia sa holhpokna tuk oke; mihma yakeh, sa noshkobo ha tvpak yauwahut tunna tuchina hosh on taloha tuk oke.

17. Mihma tupak paknaka fehna talaia ka napaluska ilaiyukali hosh Falaoh im alhto tuk oke : mihma hushi puta kvt tupak ut sa noshkobo on talaia ka ai vpa tuk oke, im achi tok.

18. Mihma Chosef vt afalaminchit, Toshowa kvt iluppak oke: Tupak tuchina kut nitak tuchina hoke:

19. Nitak tuchina taklakma, Falaoh vt chi noshkobo ha akachakalichit chim ai ishi cha, iti yo on chi takalicha chị hoke: mikma hushi puta kut chi nipi ya chim ai ishit vpa chi hoke, achi tok.

20. Atuk o nitak ont atuchina, yummvt Falaoh vt aivtta tok nitak atuk o yakohmi tok, in tishu vhleha moma ka impvchi im ikbi tok: mihmvt aiishko sholi i shahli, micha puska ikbi i shahli ya noshkobo ha akachakalichit in tishu vhleha takla hiohlichi tok.

21. Micha aiishko sholi i shahli yash falumminchit aiishko sholi ya anonti hilechi tok: mihma isht-ishko ya Falaoh ibbak a fohki beka tok.

22. Amba Chosef vt in tosholi tuk mak o, pvska ikbi i shahli ya iti yo on takalichi tok oke.

23. Yohmi tuk kia aiishko sholi $\underline{\mathrm{i}}$ shahli hvt Chosef a ik ithano hosh, amba im ahaksi tok.

\section{CHAPTA XLI.}

A TUK o yakohmi tok, afummi tuklo vlhpiesa kvt ont taA ha ma, Falaoh vt holhpokna tok: mihmot yakeh, okhina ontalaka hikia tok. 
2. Mihma yakeh, wak tek pisa achukma, micha nipi nia achukma untuklo hosh okhina et kohcha tok; micha okhina ontalaka ya ai impa tok.

3. Atuk o yakeh, wak tek inla pisa ik achukmo, micha nipi chunna untuklo hosh et iakaiyut okhina ya et kohcha tok: micha wak tek inla yash bilika okhina ontalaka ya hieli tok.

4. Mihmot wak tek pisa ik achukmo; micha nipi chunna yash osh wak tek pisa achukma, micha nipi nia achukma yash vput tahli tok. Yohmi ma Falaoh vt okcha tok.

5. Atuk osh nusi mut, atukla ma holhpokna tok: mihma yakeh, onush vpi achvfa ka noshkobo untuklo hochito, micha achukma hosh kohcha tok.

6. Mihma yakeh, noshkobo chipinta, hushi akochaka mahli ishit bushshi untuklo hosh et iakaiyut kohcha tok.

7. Mihmvt noshkobo chipinta untuklo kash osh noshkobo hochito alota achukma untuklo kash nanvblit tahli tok. Mihma Falaoh ut okcha tok; mihma yakeh, holhpokna hatuk atok.

8. Atuk osh onnahinli ma yakohmi tok, chukvush vt ik im vlhpeso fehna tok; mihmut natosholi vhleha moma micha hatak hopoyuksa vhleha moma Echip aiasha ka pit hoyo tok: micha Falaoh vt holhpokna tuk vt im anoli tok; yohmi kia achufa kia Falaoh ya in toshola hinla kvt iksho tok.

9. Yohmi ma aiishko sholi i shahli hut Falaoh ha im anumpulit, Nana ashvchi li tuk vt himak nitak fehna ithaiyana lishke:

10. Falaoh hut in tishu ha i nukhobela cha, ai atoni, nan atoni i kupituni in chuka hikia kă, vno, micha puska ikbi i shahli ya il itatuklo ka pi fohki tok.

11. Atuk o ninak achufa ho vno, micha yưmma il itatuklot naholhpokna yo pi holhpokna tok: na holhpokna toshowa kvt ila bikka ho pi holhpokna tok. .

12. Mihma Heblu hatak himita vt nanatoni $\mathbf{i}$ kvpituni in tishu hosh pibafohka tok; atuk o il im anoli ma, pi holhpokna tuk a pin tosholi tok: ila pi holhpokna bika tuk mak o tosholi tok. 
13. Atuk o yakohmi tok, pin tosholi tuk mak o yumohmi tok: vno ano ahikia li ya falvmminchit sa hilechi mvt, yummuno uba takalechi tok, achi tok.

14. Yohmi ma Falaoh hut Chosef a pit hoyo tok; mihma tushpvt aboha kvllo ya okla kohchit isht-vla tok: mihma ile shạfi mot, nafohka inla ishit fohka cha, Falaoh ha ont iba chukowa tok.

15. Mihma Falaoh hut Chosef $\underline{a}$, Naholhpokna sa holhpokna tuk o kuna hosh an toshola hinla kvt ikshoshke: atuk o chishno ak osh naholhpokna ha ithanvt ish toshola hinla achi ho chi haklo li tuk oke, im achi tok.

16. Mihma Chosef vt Falaoh ha im afalaminchit, Uno ak keyu hoke, Chitokaka ak osh Falaoh ha anumpa falama achukma ho ima chi hoke, achi tok.

17. Mihma Falaoh hut Chosef a, Sa holhpokna mut, yakeh, okhina ontalaka hikia li tuk oke.

18. Mihma yakeh, wak tek untuklo nipi nia achukma, micha pisa achukma hosh okhina et kohcha tuk: micha okhina ontalaka ai impa tuk oke.

19. Mihma yakeh, wak tek inla untuklo hliposhi, pisaik achukmo, nipi chunna hosh okpulo kvt yvmma chohmi ka Echip yakni moma ka pisa li chatuk keyu ho et iakaiyut kohcha tuk oke.

20. Mihmot wak tek chunna, pisa ik achukmo yash osh wak tek nia achukma untuklo vmmona kash vpvt tahli tuk oke.

21. Atuk osh vpvt tahli mut vpvt tahli tuk a akostinincha he keyu tuk; amba pisa ik achukmo kvt vmmona tuk vt imoma tuk oke. Yohmi ho sai okcha tuk oke.

22. Atuk osh sa holhpokna cha, pisa li ma, yakeh, vpi achufa ka noshkobo untuklo hosh alota, micha achukma hosh kohcha tuk oke.

23. Mihma yakeh, noshkobo untuklo hosh shila, chipinta, micha hushi akochaka mahli ishit bushshi yosh iakaiyut kohcha tuk oke.

24. Mihma noshkobo chipinta yash osh noshkobo achukma untuklo kash nanublit tahli tuk oke: atuk o natosholi 


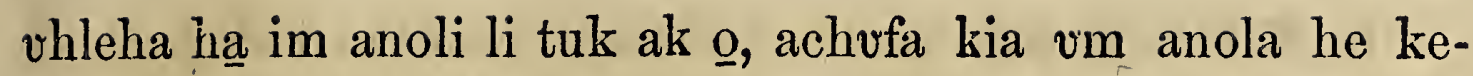
yushke, im achi tok.

25. Mihma Chosef vt Falaoh ha, Falaoh holhpokna tuk vt achufa hoke: Chitokaka yut nana kaniohma chi kvt Falaoh ha im otunichi hoke.

26. Wak tek achukma untuklo kash osh afummi untuklo hoke: micha onush noshkobo achukma untuklo kash osh afummi untuklo hoke: naholhpokna hot achofa hoke.

27. Mikma wak tek untuklo chunna, micha pisa ik achukmo hosh et iakaiyvt kohcha tuk ash osh afummi untiklo hoke; mikma onush noshkoho chipinta, micha hushi akochaka mahli ishit bushshi untuklo kash osh afummi untuklo hohchvfo ha chị hoke.

28. Nana Iluppak o Falaoh ha im anumpuli li tuk oke; Chitokaka yut nana kaniohma chi kut Falaoh ha im otunichi hoke.

29. Yakeh, Echip yakni moma ka nana waya chinto fehna afưmmi untuklo hosh minti hoke.

30. Atukma afummi untuklo hohchufo hosh et iakaiya chi hoke : mikma nawaya laua achukma tuk moma ka Echip yakni ya ai im ahaksa chị hoke: mikma hohchufọ kvt yakni ya tahla chi hoke.

31. Hohchufo yummut et iakaiya kak o nawaya laua achukma tuk a yakni yumma ai ithana chi keyu hoke: palummi fehna chi hoka.

32. Micha hitukla ha Falaoh ha naholhpokna in tuklochi tuk vt Chitokaka yut nana ka kvllochit apesa cha chekosikma Chitokaka yut aiahhlichia chi kak atuk oke.

33. Yvmohmi hoka himak a Falaoh hvt hatak vt nana ka ahah, ahni cha hopoyuksa yo hoyot ik ahayuchi cha, Echip yakni ya pehlichi ya chi ho hilechashke.

34. Falaoh hot iluppak o ik yumihchi, micha yakni ya apesvchi vhleha atokolit hilechi cha, Echip yakni ya afummi untuklo nawaya laua achukmakma ishit tahlapi ka okla ishashke.

35. Mikma afummi achukma yummut vlakma, ilhpak moma ka itunahlashke, mikmut Falaoh ha ibbak fohkit bohli cha, tomaha holihta puta ka ilhpak ashachit ieshashke. 
36. Mikma ilhpak yummut afummi untuklo hohchvfo ut Echip yakni ya a asha chị takkla ka yakni ya im ilhpak osh aiashashke; yumohmi ho yakni vt hohchufo kak o ik mosholo kashke, im achi tok.

37. Mihma nana ka Falaoh micha in tishu vhleha moma aiena kvt achukma, ahni tok.

38. Mihmut Falaoh hut in tishu vhleha ha, Chitokaka imi shilombish vt ibafoyuka hatak iluppa chohmi ka il ahayucha hinla cho? im achi tok.

39. Micha Falaoh vt Chosef a, Chitokaka yvt ilvppa moma ka chim otunichi tuk oka, achufa kia nana ka ahah, ahni, micha hopoyuksa kvt chishno chohmi kvt ikshoshke:

40. An chuka achvfa ya ish pehliecha chi hoke: micha chim anumpa pulla ho vm okla moma kut iakaiya chi hoke : miko ịm aiombinili holitompa ak illa ho chị shahli la chị hoke, im achi tok.

41. Micha Falaoh hvt Chosef a, Yakeh, Echip yakni moma ka pehlichi ya chị ho chi atokoli lishke, im achi tok.

42. Micha Falaoh hot ibbak ushi fohka ya shüf cha, Chosef ibbak a fohki mvt, linen tapuski nafohkvchi mvt, tuli lakna itatakuli ikunla afohommi tok.

43. Micha itichanvlli atukla $\underline{i}$ hikia tuk vt ombinilichi tok: mihma tikba okla payvt ayvt, Hvsh huchukbihlepashke, ahanchi tok: micha Echip yakni moma ka pehlichi ikbi tok.

44. Micha Falaoh hut Chosef a, Falaoh sia hoke, chishno ak o keyu ka Echip yakni moma ka hatak nana kia ibbak okmá, iyi yokmá, nana ka uba wiela he keyushke, im achi tok.

45. Micha Falaoh hvt Chosef hohchifo ha Safnalh-paaneah achi tok; micha naholitompa isht vtta On vtta Poti-felah ya ushetik Asenalh, tekchi ya chị ho ima tok: mihma Chosef vt Echip yakni moma ka hlopullit aya tok.

46. Micha Chosef vt Echip $\underline{1}$ milko ya itikba hikia mut, afummi pokoli tuchina tok: mihmot Chosef vt Falaoh itikba hikia tuk vt kohcha mut, Echip yakni moma ka hlopullit aya tok. 
47. Atuk o afummi untuklo hana waya achukma yot vla na, yakni ut ibbak alota ho waya tok.

48. Mihma afummi untuklo ilhpak moma Echip yakni aiasha tuk a itunahli cha, tumaha holihta puta ilhpak a ai ashachi tok: tvmaha holihta puta apakfoyupa ka ilhpak osapa aiasha tuk a mih makinli ho ai ashachi tok.

49. Micha Chosef vt onush itunahli ka laua fehna kvt okhuta shinuk aiasha kak o choyumichit hotihna kvt issa tok: a holhtina iksho hatuk oka.

50. Afummi hohchufo vt ik lo kisha ho Chosef vt ushi tuklot asha tok: yumma On naholitompa isht vtta ushetik Asenalh vt im eshi tuk atok. .

51. Mihma Chosef vt vttahpi hohchifo ha Manasseh achi tok; nan-jsht-svlbvsha moma, micha aki in chuka achvfa moma ka Chitokaka yvt vmi haksichi hoka, achi tok.

52. Micha atukla hohchifo ha Eflaim achi tok: yakni a sulbusha ya Chitokaka yut sa wayuchi hoka, achi tok.

53. Atuk o Echip yakni ya afưmmi untuklo nawaya laua yut ont taha tok.

54. Mihma Chosef vt achi tok mak o afummi untuklo hohchvfo vlut ishtia tok: micha hohchvfo kvt yakni moma tok: yohmi kia Echip yakni moma ka puska vt asha tok.

55. Atuk o Echip yakni moma ka hohchvfo mot, okla hut Falaoh ha pvska im asilhha ma, Falaoh hut Echip okla moma ka Chosef ak o oh im oná; nana ho huchim achikma, hush yumohmashke, im achi tok.

56. Mihma hohchvfo kvt yakni paknaka moma ka palvmmi fehna tok: mihma Chosef vt ilhpak ai itola moma ka tiwi cha, Echip okla ha i kanchi tok: mihma Echip yakni ya hohchvfo palummi kvt i shahlit mahaya tok.

57. Mihma yakni inla moma kvt onush chumpa chi hosh Echip a onvt Chosef a im ona beka tok: yakni moma ka hohchufo kvt palummi hatuk oka. 


\section{CHAPTA XLII.}

ATUK o Echip a onush vt asha ho Chékob vt akostininA chi mvt, Chekob vt ushi vhleha ha, Nanta katiohmi ho filemout hush iti pisa cho? im achi tok.

2. Micha yakeh, Echip o onush vt asha ho haklolishke :

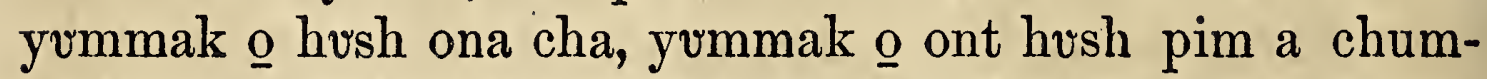
pashke; yumohmi ho pi okchaya cha, ik pillo ka chi hoke, achi tok.

3. Mihma Chosef itibapishi pokoli kvt Echip a onush a chumpa chi hosh ilhkoli tok. .

4. Amba Chosef itibapishi Benchamin ano Chekob vt, Nan ik achukmo nana kia onvtola yoba ná, achi hatuk osh itibapishi vhleha takkla ik pelo tok.

5. Islael ushi vhleha hvt onush chumpvt ai ona ka takla ona tok: Kenan yakni ya hohchvfo kvt asha hatuk oka.

6. Mihma Chosef ut yakni ya pehlichi atok; micha yummak osh yakni yumma okla moma ka $\underline{i}$ kanchi tok: atuk $\underline{0}$ Chosef itibapishi vhleha hvt ona mvt, itikba ya im akachunolit aka hlipkvchi tok.

7. Mihma Chosef vt itibapishi vhleha ha pisa mut ithana tok; yohmi kia inla im il ahobit, ilhpash ikshot im anumpuli tok; micha, Katima hush minti cho? im achi tok. Mihma Kenan yakni e minti hosh ilhpak chumput il aya hoke, okla achi tok.

8. Mihma Chosef vt itibapishi vhleha ha ithana tok, yohmi kia Chosef ano okla ik ithano tok.

9. Mihma Chosef vt naholhpokna ishit holhpokna tok vt ithaiyana tok; micha, Yakni pisvt aya hvchia hoke; yakni vt ashaiyahbi ka pisut hush aya hoke, im achi tok.

10. Mihma, Keyú, a shahli ma, peh ilhpak chumpvt aya hosh chin tishu vhleha hvt vla hoke.

11. E moma kvt hatak achvfa ushi vhleha pia hoke: hatak aiahli vhleha pia hoke; chin tishu vhleha hot yakni pisut aya keyu hoke, okla im achi tok. 
12. Mihma, Keyú, peh yakni vt ashaiyahbi ka pisvt aya hosh hush la hoke, im achi tok.

13. Mihma, Hatak achvfa Kenan yakni ya vtta ho ushi vhleha hosh chin tishu vhleha hut itibapishi auahtuklo hoke: atuk o yakeh, nakfish ishtaiyopi vt himak nitak a piki takla ahantakma, achvfa kvt iksho hoke, achi tok.

14. Mihma Chosef vt, 'Yakni pisut aya huchia hoke, anumpulit achi li tuk vt yummak oke.

15. Iluppak o isht hush otvna chi hoke: Falaoh hot okchaya pulla ka hvchi nakfish ishtaiyopi vt iluppa ik lo ka iluppa kohchut hush ilhkola he keyu hoke.

16. Hush achufa kanima ka hush pila na hvch itibapishi ha ont ishtvlashke, mikma talakchit aboha kvllo hush aiashashke, yohmi ho nana kia hush ahlikma hvch im anumpa hvt isht otvnashke : keyukmvt Falaoh hot okchaya pulla ka yakni pisut aya hvchia hoke, im achi tok.

17. Mihmut mominchit itiba fuhkit nitak tuchina ho aboha kvllo apitta tok.

18. Mihmut Chosef vt nitak ont atuchina ma, Ilvppak $\underline{0}$ hush yumihchi cha, hush okchayashke: Chitokaka ya $\underline{\text { i sa }}$ nukshopa hoka.

19. Hatak aiahli hvchia hokma, hvch itibapishi achvfa hosh talakchit huchim aboha kvllo yash fokashke: mikma ivt hvchin chuka achvfa hohchvfo ka onush isht hvsh im onashke.

20. Yohmi kia hvchi nakfish ishtaiyopi ya ont ishit hvs sum vlashke; yohmi ho hvchim anumpa hvt âhla chi hoke, mikma ik hvch illo ka chi hoke, im achi tok. Mihma okla yumohmi tok.'

21. Mihmvt ilap bikka, Pitibapishi pulla ka chukvush komunta ka e pisa, micha pim ilbusha ma e hakla he keyu kvt nana il im ashvchi fehna tok vt pi onvtoyula mubli hoke: yumohmi kak o nan ik achukmo ilupput pi onvtola hoka, itim achit hieli tok.

22. Mihma Luben vt afalaminchit, Hvchim anumpulit, vlla ya hush i yoshuba ná, achi li tok keyu cho? mihma hush haponakla he keyu tok: yumohmi tok ak o yakeh, im issish a pi a hoyo akinlishke, im achi tok. 
23. Micha Chosef vt hakklo ka okla ik ithano tok: anumpa tosholi yo isht itim anumpuli hatuk oka.

24. Mihmvt i filummi cha, nishkin okchi mihinti tok; atuk osh anonti falamut im ona cha, itim anumpuli mut, Simeon a im ishi cha, okla pisa ho takchi tok.

25. Yohmi mut i bahta ya onush alotoli cha, hatak in tvli holisso aiyukali falumminchit $\underline{i}$ bahta ya apittakmut, hina takla pinak ima chị ka Chosef vt apesa tok: micha yummak o yumihchi tok.

26. Mihma im issuba haksobish falaia ya onush ash shapolichi mvt, ilhkoli tok.

27. Atuk o anusi aboha ya okla ona ma, achvfa kvt im issuba haksobish falaia ya nanupa ipeta chi hosh $\underline{i}$ bahta ya hotofi mut in tuli holisso ya pisa tok: yakeh, $\underline{\mathrm{i}}$ bahta ya foka tuk oka.

28. Mihma itibapishi vhleha hạ, An tvli holisso hvt a falamvshke; micha yakeh, a bahta fehna ka fokvshke, im achi tok: mihma chukvush kunia cha, nukshoput okla wvnihinchi mut, Ilvppak osh nanta mak o Chitokaka yvt pi yumihchi cho? itim achi tok.

29. Atuk osh Kenan yakni ya onvt, iki Chekob a im ona mut, nana im a kaniohmi tuk vt moma im anolit,

30. Hatak vt yakni yumma i shahli yosh ahanta kvt ilhpash ikshot pim anumpuli cha, yakni pisut aya pi ahni tuk oke.

31. Mihma, Hatak aiahli pia hoke: yakni pisvt aya pia keyu hoke.

32. Hatak achvfa piki ushi vhleha pia hosh itibapishi auahtuklo pia hoke: achvfa kvt iksho, mikma nakfish ishtaiyopi vt himak nitak a piki takla Kenan yakni ya anta hoke, il im achi tuk oke.

33. Mihma hatak yakni yumma i shahli yash osh, Iluppak o hatak aiahli hvchia ka isht ithuna la chi hoke: huch itibapishi achvfa ka hus sa kanchi cha, hvchin chuka achvfa hohchufo aiasha ka ilhpak oh im ishi cha oh ilhkolí:

34. Micha huvchi nakfish ishtaiyopi ya ishit hus sum vlashke: yohmikmak o yakni pisvt aya keyu, amba hatak 
aiahli hvchia ka ithuna la chi hoke : yohmikma huch itibapishi ha huch ima likma, yakni ya hush ai itatobashke, pim achi tuk oke, im ahanchi tok.

35. Atuk o yakohmi tok, $\underline{i}$ bahta ya okla tosholi ma, yakeh, in tvli holisso bonunta aiyukali kvt i bahta ya foke bano tok: mihma yvmmvt micha iki aiena kvt tuli holisso bonunta ya okla pisa rnvt, nukshopa tok.

36. Mihma ịki Chekob vt, Uno ya vlla huss sa wehpulishke: Chosef vt iksho, Simeon vt iksho, mihma Benchamin a ishit hush ia chishke: ilvppa puta kut si onvtonla hoke, im achi tok.

37. Mihma Luben ut ikki ya im anumpulit, Ishit ak chim vlokma, suso tuklo ka ish bashke: subbak a ish fohki tukma, anonti ishit chim vla la chi hoke, achi tok.

38. Mihma, Suso hot hvchi awant ia he keyu; itibapishi

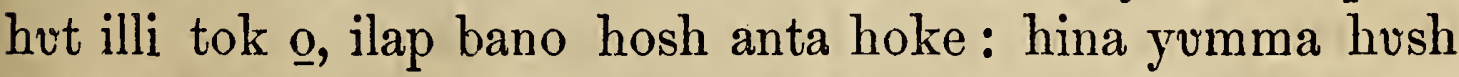
ia ka nana ik achukmo kia onvtolakma, yohmikma sa pashi tohbi ya ilbushalit ahollohpi ya hus susht ona chi hoke, achi tok.

\section{CHAPTA XLIII.}

TUK o yakni ya hohchvfo kvt palummi tok. A 2. Atuk o yakohmi tok, onush a Echip a ont ai ishtvla tuk vt vpvt tahli ma, iki vt, Anonti hvsh ia cha, ilhpak kanomosi ka ont hush pin chumpashke, im achi tok.

3. Mikma Chutah vt im anumpulit, Hatak vt anumpa kullo il onochit, Hvch itibapishi hvt hvchi awant aya keyukma, sa nashuka ya hush pisa he keyu, pim achi tok.

4. Pitibapishi ha pi takla ish pila hokmuno, il ia cha, ilhpak a ont e chin chumpashke;

5. Amba ish pila he keyu hokmuno, il ia he keyu hoke: Hvch itibapishi hot hvchi awant aya keyukma, sa nashuka ya hush pisa he keyu, hatak ash osh pim achi tuk oka, achi" tok.

6. Mihma Islael vt, Nanta katiohmi ho nan ik achukmo ka si yumihchit, himakma hvch itibapishi hvt anta ka, hvsh im anoli tuk oh cho? achi tok. 
7. Mihma, Hatak ash osh kaniohmit il aiasha chatuk a, micha pi kanomi aiena ka atokolit pim a ponaklot, Huch iki vt okchaya moma ho? hvch itibapishi inla yot anta ho ? achi tok; mihma anumpa iluppa chohmi kak o il im anoli tok : Huch itibapishi ha ont isht oh vlá, acha hinla ka il ithana hinla pulla tuk $\underline{0}$ ? achi tok.

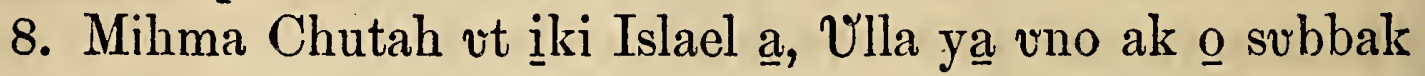
fohkit ish pilakma, e wakaya cha, il iashke, yumohmi ho pishno, micha chishno, micha pim vlla vhleha aiena kvt pi okchaya cha, ik pillo kashke.

9. Uno ak osh aiahlichi sia hashke; subbak ak $\underline{o}$ ish a hoyashke: ishit chim vlvt chi tikba ak hilechokmvt, vno ak osh anumpa si onvtoyula na biliashke.

10. Kil ahchibalo tuk mut, himak $\underline{o}$ atukla mak $\underline{o}$, ont falamvt e la he tuk pulla hoka, im achi tok.

11. Mihma îki Islael vt, Himak a yumohma he vlhpesakma, iluppak o hush yumihchashke: yakni iluppa nawaya achukma $\underline{i}$ shahli chim aivlhto fohkit hush ishi cha, hatak ash, hlitili balsam kanomosi, micha foibila kanomosi, nabalama, micha hlitili mvh, tiak holba vni, micha almont aiena kvt i na hvlbina chi ho ishit hush im onashke.

12. Micha tuli holisso yash inlohmi tuklo ho chibbak a hush ieshashke; micha tuli holisso vt hvchị bahta foka na anonti ishit hush la tuk a ishit hvsh iashke; yohmi, ik ahno ketuk yoba ká:

13. Micha huch itibapishi ha hush ishi cha, wakayut anonti hatak ash hush im onashke:

14. Mikma Chitokaka Nan isht im aiahli ataha iksho vt hatak ash itikba ya a hvehi nukhaklo na, hvch itibapishi achvfa, micha Benchamin a et pilashke : vlla yut sa wehpoa hokmvt, sa wehpoa hoke, im achi tok.

15. Mihma hatak vhleha hash osh nahvlbina yummak ash, micha tuli holisso yash inlohmi tuklo ho, ibbak foka micha Benchamin a ishi tok; micha wakaya mơt, Echip a ilhkoli tuk osh Chosef itikba ya ont hieli tok.

16. Atuk o Chosef vt Benchamin vt takla aya na pisa mot, in chuka achufa pehlichi yạ, Hatak vhleha ilvppa chuka 
isht ish ona cha, nan vbit atahlí ; hatak vhleha ilvpput tvbokolikma, subaiimpa chi hoke, im achi tok.

17. Mihma Chosef vt miha tuk mak o hatak ash osh yumihchi tok: micha hatak ash osh hatak vhleha hash $\underline{o}$ Chosef in chuka isht ona tok.

18. Mihma hatak vhleha hash osh, Chosef in chuka isht

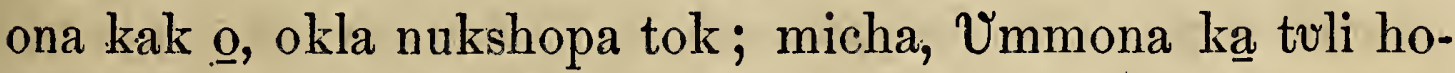
lisso vt pi bahta fokvt falama tok ak $\underline{o}$ nana isht a mihvt pishicha, yuka pikbi mut, pim issuba haksobish falaia aiena ka isha chi hosh pisht, vla hoke, okla achi tok.

19. Mihmvt Chosef in chuka achvfa apesvchi yash bilika ona cha, aboha okhissa ya ai itim anumpuli tok.

20. Mihmvt, Ikikkeh, a shahli ma, vmmona ka ilhpak chumput il aya tok vt ahli hoke.

21. Atuk o yakohmi tok, aboha anusi ya il ona mot, pi bahta ya e hotofi ma, yakeh, pin tuli holisso yash osh pin tvli holisso weki hatuk vt moma hosh pi bahta aiyukali ka foka tuk oke: atuk o anonti pibbak foka ho ishit e lvshke.

22. Micha ilhpak isht e chumpa chị kut tuli holisso inla pibbak foka ha ishit e lushke : kưna hosh pin tuli holisso ya pi bahta fohki tuk a kil ithanoshke, achi tok.

23. Mihma, Hvchi nuktaiyalashke, ik hvchi nukshopo kashke: hvchin Chitokaka, micha hvch iki in Chitokaka ak osh tvli holisso huch imvt, hvchi bahta ya fohki tuk oke: huchin tuli holisso ya ishi li tuk oke, achi tok. Mihmut Simeon a kohchit isht im ona tok.

24. Mihmut hatak ash osh hatak vhleha hash 0 Chosef in chuka isht ona cha, oka ima na iyi ya okla achifa tok; mihma im issuba haksobish falaia ya nanupa ipeta tok.

25. Mihma Chosef vt tobokolikma vla chi itin takla ka nahulbina yash atahli tok: yummak o okla ai impa chi kut haklo tuk okvt.

26. Atuk o Chosef vt chuka vla ma, nahvlbina ishit vla tuk vt aboha okla isht im vla cha, im akachunolit aka pit hlipkvchi tok.

27. Mihma im achukmakka ka i ponaklot, Hvch iki, hatak 
sipokni hus sum anoli chash osh im achukmaka cho? okchaya moma cho? achi tok.

28. Mihma afalaminchit, Chin tishu piki vt im achukmakushke, okchaya momushke; achi tok: mihmut akachunoli cha, aka pit hlipkuchi tok.

29. Mihma akachakali cha, ishki ya ushi itibapishi Benchamin a pisa mut, Ilvppak osh hvchi nakfish ishtaiyopi hus sum anoli tuk mak oh cho? achi tok. Mihmut, Suso ma, Chitokaka yot chi kanashke, achi tok.

30. Mihmvt Chosef vt itibapishi ha chulkvsh ishit nukhaklo hatuk osh tushput a yaiya chị kvt hoyo tok: mihmvt in chuka anukaka ont chukowa cha, a yaiya tok.

31. Atuk osh okami cha, kohcha mut il oktambli cha, Puska ho talalí, achi tok.

32. Mihma Chosef a ila in talali, anonti yumma ila in talali mot, Echip okla hot takkla impa ka ila in talali tok: Echip okla hot Heblu okla ha itibai impa he keyu chatuk; yommot Echip okla hot nan isht im ayuwala yatuk oka.

33. Mihma vttahpi yvt ai isha he vlhpesa yak o vlhpisa hosh, micha ont ishtaiyopi yano himita kak o vlhpisa hosh itikba okla binohli tok: mihmot hatak vhleha hash osh okokko, it ahni tok.

34. Mihma ilap itikba ai ishi cha, kushkoa ho pit i pila tok: yohmi kia Benchamin a $\underline{\mathbf{i}}$ kvshkoa hvto inla ka immi ha inlohmi tahlapi tok. Mihma okla nanishko cha, ibai yukpa tok.

\section{CHAPTA XLIV.}

A TUK $\underline{o}$ Chosef vt in chuka achvfa pehlichi ya i mihvt, A Isht aya he vlhpesa ho hatak vhleha hash i bahta ya ilhpak hush aluli cha, hatak moma ka in tuli holisso aiyukali ka i bahta ya hush apittashke.

2. Micha vm ishtishko, ishtishko tvli hvta ya micha im onush ailli tvli holisso ya nakfish ishtaiyopi i bahta ya ish fohkashke achi tok: mihma anumpa Chosef vt anumpuli tuk mak o yumihchi tok. 
3. Onnahinli kvt tohwikeli makinli ho hatak vhleha hash, micha im issuba haksobish falaia aiena ka pit tihleli tok.

4. Atuk osh tumaha holihta ya kohchut ik hopakecho kisha ho Chosef vt in chuka achvfa pehlichi yạ, Wakayá, hatak vhleha hash hliolí; atuk osh ont ish sakkikmvt, Nanta katiohmi ho nan achukma ka nan ik achukmo ho hush atobbi cho?

5. A shahli hvt ai ishko chatuk, micha nan isht ithvna chatuk muhli iluppak keyu cho? iluppa hush yvmihchi kut ik achukmo ho hush yumihchishke, ish im achashke, im achi tok.

6. Atuk o ont sakki cha, anumpa iluppak inli ho im anumpuli tok.

7. Mihma, Nanta katiohmi ho anumpa iluppa a shahli hut achi cho? chin tishu vhleha hvt nana ilvppa chohmi ka yumihchi ka Chitokaka yut im olvbashke.

8. Yakeh, tvli holisso hvt pi bahta ya foka na il ahayuchi tuk vto anonti Kenan yakni ya ai isht e la tuk oke: yohmi tuk vt katiohmi kak o tvli hvta, keyukmvt tvli holisso lakna. kia hukopvt a shahli in chuka ya isht e kohcha hinla cho?

9. Chin tishu vhleha kanima kak osh ishi tuk o haiakakma, yưmmut illashke, mikma pishno ak kia a shahli ha $\underline{\text { i yu- }}$ ka vhleha pia hashke, im achi tok.

10. Mihma, Himak akinli ka hvchim anumpa pulla hatuk mak o yumohmashke: kuna hosh ishi tuk o haiakma, yummak osh an tishu ha chi hoke; mikma hvchishno vto nana ik hvchi kaniohmo ka chi hoke, achi tok.

11. Yohmi ma tushpvt hatak i bahta aiyukali kvt akvchit aka bohli tok: micha hatak i bahta aiyukali kut hotofi tok.

12. Mihma hoyo tok, micha akni fehna ak o vttut ai ishtia hosh nakfish ishtaiyopi ya isht ona tok: mihma isht ishko vt Benchamin $\underline{i}$ bahta ak o fokvt haiaka tok.

13. Yohmi ma i nafohka ya okla hliluffi cha, hatak im issuba haksobish falaia aiyukali kvt shapolechi mut, falamvt tomaha holihta yash ona tok.

14. Mihma Chutah, micha itibapishi vhleha aiena kvt 
Chosef in chuka ya ona tok; (yumma anta moma hatuk oka,) micha itikba ya okla hlipkvchi tok.

15. Mihma Chosef vt, Ilvpput nanta mak o hush yumihchi tuk oh cho? hatak uno chohmi kvt nan ithuna la hinlá pulla ka hvch ik ithano tuk oh cho? im achi tok.

16. Mihma Chutah vt, $\underline{\mathrm{A}}$ shahli ha nanta ho il im acha chi cho? nanta ho il im anumpula chi cho? e katiohmi kak osh il il aivlhpiesvecha chị cho? Chitokaka yut chin tishu vhleha nanashvchi yo ahayuchishke: yakeh, a shahli in tishu vhleha pia hoke, pishno, micha isht ishko ya ishi tuk o haiaka tuk aiena kvt, achi tok.

17. Mihma, Yohmi la he ya Chitokaka yvt olvbashke: amba isht ishko vt ibbak fokvt haiaka tuk hatak yummak osh an tishu ha chị hoke: amba hvchishno vto hvch ịki ya achukmakvt hush im onashke, achi tok.

18. Yohmi ma Chutah vt i bilika ona cha, $\underline{A}$ shahli ma! chim asilhha lishke, a shahli hut hakklo ho, chin tishu hut anumpa achvfa anumpuli lashke; mikma chim ai i nukkilli hut chin tishu ha $\underline{i}$ sanalit ik libbo kashke: Falaoh hak $\underline{0}$ ish chohmi hokvt.

19. A shahli hut in tishu vhleha hạ i ponaklot, Hưch iki vt asha? keyukmvt hvch itibapishi hvt anta hoh cho? achi tok.

20. Mihma a shahli hạ, Piki vt anta, hatak sipokni, micha sipoknit taha ho vlla im vtta tok osh, iskitini yosh anta: mikma itibapishi hut illi tok, ishki achufa yut yummak illa hosh okchaya ho, iki vt holitobli hoke, il im achi tok oke.

21. Mihma chin tishu vhleha ha, Isht hus sum vla na a holhponayo onochit pisa lashke, ish im achi tok oke.

22. Mihma pishno vt a shahli hạ, vlla yut ịki a $\underline{\mathrm{i}}$ filummit aya he keya; ikki a i filummit aya hokma, ikki vt illa hi oka il im achi tok oke.

23. Mihma chin tishu vhleha hạ, Hvchi nakfish ishtaiyopi vt huchi awant vla keyukma, himakma sa nashuka ya hush pisa chi keyu hoke, ish im achi tok oke.

24. Atuk o yakchmi tok, piki chin tishu ha il im ona mut, a shahli im anumpa ha il im anoli tok oke. 
25. Atuk o piki vt, Anonti hvsh ia cha, ilhpak kanomosi ont hush pin chumpashke, achi tuk oke.

26. Mihma, Il ia he keyu hoke; pi nakfish ishtaiyopi vt pi takla ia hokmuno, il ia hinla hoke: pi nakfish ishtaiyopi vt pi takla aya keyukma, hatak ash nashuka ya e pisa he keyu hokvt, il achi tuk oke.

27. Mihma chin tishu piki vt, Sa tekchi vt vlla nakni tuklo ho vm eshi tok a hush ithanushke:

28. Atuk o achufa kvt a filummit kohchut ia tuk $\underline{o}$, Niput taha tuk pullushke, achi li tok; yummak atok itin takla ma ak peso chatuk oke.

29. Atuk o iluppak kia isht hvsh ia tuk o; nana ik achukmo kia onvtolakma, sa pashi tohbi ya ilbushalit ahollohpi ya hush susht ona chi hoke, pim achi tuk oke.

30. Yvmohmi hoka himak a chin tishu aki ya jm ona likma, vlla yvt ik piba fokokma, (im ilhfiopak vt vlla im ilhfiopak ak o ita talakchi hatuk okvt;)

31. Yakohma chi hoke; vlla yut pi takla ik ayo ho pisakmvt, illa chi hoke: mikma piki pashi tohbi ya hvchin tishu pia hot ilbvshalit ahollohpi ya isht il ona chi hoke.

32. Chin tishu hut vlla yash ilki ya isht im ona la hi vt aiahlichi tobvt, Isht akk chim vlokma, aki vt anumpa si onochi na biliashke, achi li tuk oke.

33. Yvmohmi hoka himak a chin tishu hak osh vlla yash vlhtobvt a shahli ha $\underline{\mathrm{i}}$ yuka tobvt antashke; chim asilhha lishke: mikma vlla yut itibapishi vhleha ha takla iashke.

34. Katiohmi kak osh vlla yvt sa takla aya keyu ka a ki ya im ona la hinla cho? keyukma nana ik achukmo kut alki ya onvtola chị ka pisa la chị yoba ká, achi tok.

\section{CHAPTA XLV.}

VOHMI ma Chosef vt bilika hieli tuk ash moma itikba 1 ya il oktambla he keyu tok; mihmot chitoli hosh, $\mathrm{Ha}$; tak moma ka a filummichit hush kocha wehlashke, achi tok: 
mihma Chosef vt itibapishi vhleha im il anoli takkla ka hatak nana kia apehvt hinli kvt iksho tok.

2. Mihma Chosef vt chitolit yaiya tok; mihma Echip okla, micha Falaoh in chuka achvfa aiena kvt haklo tok.

3. Mihma Chosef vt itibapishi vhleha ha, Chosef sia hoke; aki vt okchaya moma cho? im achi tok. Mihma itibapishi vhleha hvt im afalamicha he keyu tok; nashuka pisa kvt nukhlakancha hatuk okvt.

4. Mihma Chosef vt itibapishi vhleha hạ, $\underline{\mathrm{A}}$ bilika ho mintí, hvchim asilhha lishke, im achi tok: mihma bilika okla ona tok: Mihma, Hvch itibapishi Echip et hvsh kanchi tok Chosef sia hoke, achi tok.

5. Yvmohmi hoka himak a iluppa et hus sa kanchi tok vt ik huchi nukhaklo cha, hvch ik il i nukkillo kashke: Chitokaka ak osh hatak okchalinchi la chị ho huchi tikba et sa pila tok oka.

6. Iluppot afummi tuklo mak o hohchvfo yvt yakni ya itoyula tuk oke: mikma yohmi hokak o afummi tahlapi moma ho yakni patuffi, micha nan vmo nana kia iksho ka chi hoke.

7. Yohmi ka hvchim ishtatiaka okchalinchi lí; yakni paknaka aiasha chị ho, micha ahlakofi chinto ho isht hvchi okchalinchi la chị ho, hvch itikba Chitokaka yot sa pila tuk oke.

8. Yohmi ka huchishno ak osh iluppa et hus sa pila tuk keyu, amba Chitokaka ak atok oke: micha Falaoh ha iki; micha in chuka achvfa ya $\underline{\mathbf{i}}$ shahli, micha Echip yakni moma ka pehlichi ya sa hilechi tuk oke.

9. Hush tusshpashke, micha aki ya hưsh im ona cha, Chiso Chosef vt yak achishke, Chitokaka yvt Echip moma ka $\underline{i}$ shahli ha sa hilechi tuk oke: is sum vlashke, ish svlaha ná ;

10. Micha Koshen yakni ak o, is sa bilika chi, chishno, micha chim vlla vhleha, micha chim vlla vhleha ha im vlla vhleha, micha chi nanvlhpoa chipinta, micha chi nanvlhpoa hochito, micha nana ohim asha moma kvt ish aiashashke.

11. Mikma yummak o a huchi ipeta la chi hoke, (hoh- 
chvfo hot afummi tahlapi kvt asha moma hoka ;) yohmi ho chishno, micha chin chuka achvfa, micha nana chim asha moma kut hohchufot chi taha yoba ná, achishke, hush im achashke;

12. Micha huchi nishkin, micha itibapishi li Benchamin nishkin vt pisa ka sutih hak osh hvchim anumpulelishke.

13. Micha Echip a nana sa holitopa moma, micha nana hush pisa tuk a mominchit hush im anolashke : micha tushpot aki ya iluppa isht hush lashke, im achi tok.

14. Micha itibapishi Benchamin ikunla ya on hlipia cha yahaya tok: mihma Benchamin vt yumma ikunla ya on hlipia cha yahaya tok.

15. Micha itibapishi vhleha moma ka $\underline{\mathrm{i}}$ suksowa cha, on hlipivt yahaya tok: mihma yohmi ha yak o, itibapishi vhleha hvt itim anumpuli tok.

16. Atuk o Chosef itibapishi vhleha hot vlvshke, achi na annoa ka Falaoh in chuka achufa yvt haklo tok: mihma Falaoh, micha in tishu vhleha aiena kvt im achukma fehna tok.

17. Micha Falaoh hvt Chosef a , Chitibapishi vhleha ha iluppa ish im achashke, Iluppak o hush yumihchashke; hvchim issuba haksobish falaia ya hush shapolechi cha, ivt Kenan yakni ya hush onashke;

18. Micha hvch iki, micha húchin chuka achufa ya hush ishi cha, hus sum vlashke : mikina Echip yakni ai achukma kak o hvch ima likmá, yakni nia kak o hvsh ai vpashke, ish im achashke.

19. Himak a chi miha lishke, iluppak o hush yumohmashke; hvchim vlla vhleha, micha hvchi tekchi vhleha aiena kvt ai vlbeha chi ka, Echip yakni ya itichanvlli puta ai ishit hush ona cha, hvch iki ya isht hush minti cha, hush lashke.

20. Micha hvchi nachukushpa ya huch ik anukfillo kashke: Echip yakni ai achukma moma kvt hvch immi hokvt, im achi tok.

21. Mihma Islael im vlla vhleha hut yumihchi tok: mihma Falaoh hot miha tuk mak o, Chosef vt itichanvlli puta ima cha, hina takla pinak aiena ka ima tok. 
22. Moma ka nafohka fohka tuklo aiyukali ho ima tok: amba Benchamin ano toli hota tushahli tahlepa tuchina ho, micha nafohka fohka tahlapi ho ima tok.

23. Micha îki ya yưmmak inli chohmi ho pit i pila tok; micha issuba haksobish falaia yut Echip nana achukma puta shali pokoli, micha issuba haksobish falaia tek pokoli hosh, hina takla iki vt upa chị ho onush, micha puska, micha pinak shali ho pila tok.

24. Yohmi mut itibapishi vhleha ha pila na ilhkoli tok: mihma, Ahah, hush ahni cha, hina takla hvch ik itim okpulo kashke, im achi tok.

25. Mihma Echip a kohchvt ia mut, Kenan yakni ya onvt, iki Chekob a im ona tok;

26. Micha im anolit, Chosef vt okchaya moma cha, Echip yakni moma ka pehlichi yoke, achi tok. Mihma Chekob chukvsh vt shimoha tok, ik yimmo hatuk okvt.

27. Mihma Chosef im anumpa nana im achi tuk a moma im anoli tok: micha fohkit ishtia chị ka itichanvlli puta Chosef vt et i pila tuk a pisa mut, iki Chekob imi shilombish vt okchvt taha tok:

28. Mihma Islael vt, vlhpesvshke, svso Chosef vt okchaya momushke; ik sullo takla kak osh ivt ont pisa lashke, achi tok.

\section{CHAPTA XLVI.}

MIHMA Islael vt nana im asha tuk vt moma ishi cha ia 11 mut Bee-sheba ona cha îki Aisak in Chitokaka ya nanvbit isht aiokpvchit im issa tok.

2. Atuk o ninak a Islael vt na pisa ho, Chitokaka yut (Islael a) il im otunichit im anumpuli cha, Chekob ma, Chekob ma, ahanchi tok. Mihma, Yak itonla lishke, achi tok.

3. Mihma, Uno vt Chitokaka, chịki in Chitokaka sia hoke: Echip ia ka ik chi nukshopo kashke; yummak o okla chito a chihịkbi la chị hoka.

4. Chi awant Echip a ona la chi hoke : atuk osh anonti 
ishit chi kohchi li pulla hi oke; mikma Chosef vt chi nishkin a ibbak isht umbitepa hi oke, achi tok.

5. Mihma Chekob vt Bee-sheba ya vttut wakaya tok: mihma Islael ushi vhleha hot iki Chekob, micha im vlla vhleha, micha tekchi vhleha ai vlbeha chi ho itichanvlli puta Falaoh hut i pila tuk a apitta cha, ishtia tok.

6. Micha i nanvlhpoa, micha im ilayak puta Kenan yakni ai ahayuchi tuk vt ishi cha, Chekob, micha im aiunchololi moma kvt apiha ho Echip a ona tok.

7. Ushi vhleha, micha ushi vhleha ha ushi vhleha hvt apiha ho, micha ushetik vhleha, micha ushi vhleha ha ushetik vhleha hvt apiha ho, im aiunchololi moma ka pehliechit Echip a isht ona tok.

8. Hohchifo iluppa puta kak osh Islael im vila vhleha, Chekob, micha ushi vbleha aiena hosh Echip a ona tok: Chekob ushi vttahpi Luben ak atok.

9. Mihma Luben ushi vhleha hvt Hanok micha Fallu, micha Heslon, micha Kami ak atok.

10. Mikma Simeon ushi vhleha hvt Chemuel, micha Chamin, micha Ohat, micha Chakin, micha Soha, micha Kenan ohoyo ho ushi Shaul aiena kak atok.

11. Mikma Lefai ushi vhleha hot Keshon Kohalh, micha Melali ak atok.

12. Mikma Chutah ushi ühleha hvt El, micha Onan, micha Shelah, micha Fales, micha Salah ak atok: yohmi kia El, micha Onan itatuklo kvt Kenan yakni ya ai illi tok. Mihma Fales ushi vhleha hot Heslon, micha Hamul ak atok.

13. Mikma Issaka ushi vhleha hut, Tola, micha Fufah, micha Chob, micha Shimlon ak atok.

14. Mikma Sebulun ushi vileha hvt Selet, micha Elon, micha Chahleel ak atok.

15. Iluppa puta kak osh Leah ushi vhleha ha yvmma, micha ushetik Tinah aiena ka Chekob a Patan-alam a ai im eshi tok: ushi vhleha, micha ushetik vhleha imi shilombish moma kvt pokoli tuchina akucha tuchina tok.

16. Mikma Kat ushi vhleha hvt Sifion, micha Hakki, Shuni, micha Esbon, Eli, micha Aloti, micha Aleli ak atok. 
17. Mikma Asha ushi vhleha hvt Chimnah, micha Ishuah; micha Isui, micha Beliah, micha in tek Selah ak atok. Mikma Beliah ushi vhleha hut Hebe, micha Malkiel ak atok.

18. Iluppa puta kak osh Laban vt ushetik Leah ima tok Silpah ya ushi vhleha hatok: mihma ilvppa puta kak o Chekob a im eshi tok, shilombish anahhannali hatok.

19. Chekob tekchi Lechel ushi vhleha hvt Chosef, micha Benchamin ak atok.

20. Mikma Chosef a On a naholitompa isht vtta Potifelah ushetik Asenalh im eshi tok a Manasseh, micha Eflaim ak osh Echip yakni ya im ai ashwa tok.

21. Mikma Benchamin ushi vhleha hut Belah, micha Beke, micha Ashbel, Kela, micha Naaman, Ehi, micha Losh, Muppim, micha Huppim, micha Alt ak atok.

22. Iluppa puta kak osh Lechel a ushi vhleha hosh Chekob a im ashwa tok, shilombish moma kvt auah ushtahatok.

23. Mikma Tan ushi vhleha hut Hushim ak atok.

24. Mikma Naftali ushi vhleha hvt Chaseel, micha Kuni, micha Chese, micha Shillim ak atok.

25. Iluppa puta kak osh Laban vt ushetik Lechel ima tok Bilhah ya ushi vhleha hatok; micha iluppa puta kak o Chekob a im eshi tok: shilombish moma kvt untuklo tok.

26. Chekob ushi vhleha ha tekchi vhleha hvto asha na haknip a toba tok shilombish moma hosh, Chekob a apihut Echip a ona kvt, shilombish moma kut pokoli hannali akucha hannali tok.

27. Mikma Chosef ushi vhleha Echip a im ai ashwa tok vt shilombish tuklo tok: Chekob in chuka achufa shilombish moma kvt Echip a ona tok vt pokoli untuklo tok.

28. Mihma Chosef ot Koshen ak o apissalit ont ai afama chị ho, Chutah ya itikba pit $\underline{i}$ pila tok: mihmot Koshen yakni ya okla ona tok.

29. Mihma Chosef vt im itichanvlli ya atahli cha, iki Islael a Koshen a ai afamvt ia tuk osh itikba ont im ile haiakvchi tok: micha ikunla on hlipia cha, ikunla ya onvtoyulut yahaya na hopaki tok. 
30. Mihma Islael vt Chosef a, Inta, ik svlli, chi nashuka ya pisa li hokvt, chi okchaya moma hoka, im achi tok.

31. Mihma Chosef vt itibapishi vhleha, micha iki in chuka achufa yạ, Ia lish, Falaoh ha ont im anolit, Itibapishi li vhleha, micha aki in chuka achvfa Kenan yakni aiasha tuk vt um vlushke:

32. Micha hatak okvt nanvlhpoa apesvchi vhleha hoke; nan isht aiasha yvt nanvlhpoa impvchi ak oh chatuk okvt:

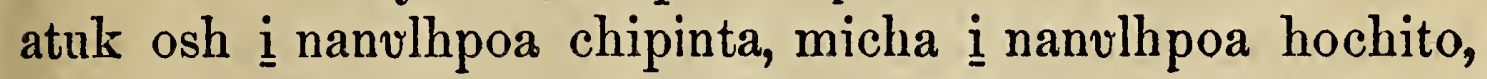
micha nana im asha tuk vt moma isht vlvshke, im achi lashke.

33. Atukma yakohma chi hoke, Falaoh hut hvchi hoyo cha, Nanta ho ishit hushai asha chatuk oh cho? achikma,

34. Yohmikma, Chin tishu vhleha nan isht aiasha yvt vlla pi moma hakinli tok osh himak a ant vhli kvt, pishno, micha piki vhleha aiena kvt nanvlhpoa apesuchi ak oh chatuk oke, hush achashke: yohmi hosh Koshen yakni ak $\underline{0}$ hvsh aiashashke; nanvlhpoa apesvchi vt Echip okla hvt nana isht ayuwala yoka, im achi tok.

\section{CHAPTA XLVII.}

YOHMI mut Chosef vt ivt Falaoh hạ ont im anolit, Aki, 1 micha itibapishi li vhleha, micha i nanvlhpoa chipinta, micha i nanvlhpoa hochito, micha nana im asha tuk moma kvt Kenan yakni ya kohchvt vlushke; atuk osh yakeh, Koshen yakni ya aiashushke, achi tok.

2. Mihmvt itibapishi kanimi, hatak tahlapi ho ishi cha Falaoh itikba ya hilechi tok.

3. Mihma itibapishi vhleha hạ Falaoh hvt, Nanta ho ishit hush asha chatuk $\underline{0}$ ? im achi tok. Mihma Falaoh hạ, Pishno, micha piki vhleha il aiena, chin tishu vhleha hvt nanvlhpoa apesvchi pia hoke, im achi tok.

4. Micha Falaoh ha, Cheki itin takla ya yakni ya il aiasha chi hosh e la hoke: Kenan yakni ya hohchufo hut palummi fehna hatuk o chin tishu vhleha hut $\underline{i}$ nanvlhpoa ai impa yot 
ik im iksho hoka: yumohmi hoka inta, chin tishu vbleha hut Koshen yakni ak o aiashashke, e chim asilhhvshke, im achi tok.

5. Mihma Falaoh hvt Chosef a im anumpulit, Chilki, micha chitibapishi vhleha hut chim vlushke:

6. Echip yakni vt chi tikba talaivshke; yakni ai achukma $\underline{i}$ shahli kak $\underline{o}$ chiki, micha chitibapishi vhleha ha ish $\underline{a}$ binohlechashke, Koshen yakni ak o aiashashke; mikma hatak kuna hosh tushpa achukma kvt iba foka ho ish ithanakmvt, a nanvlhpoa pehlichi ish hiohlechashke, im achi tok.

7. Mihma Chosef vt iki Chekob a ishit chukowa cha, Falaoh itikba ya hilechi tok; mihma Chekob vt Falaoh ha yukpali tok.

8. Mihma Falaoh hut Chekob a, Nitak okchayvt ish ahanta afummi moma kvt katohmi cho? im achi tok.

9. Mihma Chekob vt Falaoh hạ, Nitak okchayvt ahanta li tuk vt afummi tahlepa achvfa cha pokoli tuchina hoke: nitak okchayvt ahanta li afummi puta.kvt kanomosi cha, ik achukmo tuk oke; micha aki vhleha hvt nitak okchayvt ahanta tok afummi puta ka ik ono hoke, im achi tok.

10. Mihma Chekob ut Falaoh ha yukpali cha, Falaoh itikba ya vttut kohchvt ia tok.

11. Mihma Chosef vt iki, micha itibapishi vhleha aiena ka Echip yakni binohlechi cha, Falaoh hvt miha tuk mak o, yakni ai achukma i shahli Lameses yakni ya aiasha chi ho ima tok.

12. Micha Chosef vt iki yạ, micha itibapishi vhleha ha, micha iki in chuka achvfa moma ka chuka achvfa vlhpisa ho puska ipeta tok.

13. Mihma yakni yưmma moma ka puska vt iksho tok: hohchvfo hut palvmmi hatuk oka; yohmi ma Echip yakni, micha Kenan yakni moma kvt hohchufo kak osh kotvt taha tok.

14. Atuk o Chosef vt onush ishit chumpa ka Echip yakni, micha Kenan yakni ya tvli holisso vt asha tuk a moma itvnahlit ishit tahli tok: mihma Chosef vt tuli holisso yash Falaoh ha in chuka ya isht ona tok. 
15. Atuk o Echip yakni, micha Kenan yakni aiena ka tvli holisso vt taha ma, Echip okla moma kvt Chosef a im ona cha, Puska pi ipetá; nanta katiohmi ho chi tikba a pilla chi cho?. tuli holisso vt taha hoka, achi tok.

16. Mihma Chosef vt, Hvchi nanvlhpoa ak o hush imashke; micha tvli holisso hut taha hokma, nanvlhpoa yak okmuno hvch ima la chi hoke, achi tok.

17. Mihma Echip okla hot i nanvlhpoa ya Chosef a isht im ona tok: mihma Chosef ut issuba, micha nanulhpoa chipinta, micha nanvlhpoa hochito, micha issuba haksobish falaia aiena a puta kak o itatobvt puska ima tok ; micha afummi yumma $\underline{\mathrm{i}}$ nanvlhpoa moma ak atuk mak o puska ipeta tok.

18. Afummi yummut ont taha ma, afvmmi atukla ka im onvt, Pin tvli holisso hvt taha ka $\underline{a}$ shahli ha il in luhma he keyu hoke; a shahli hot pi nanvlhpoa hochito ya isshi akinli hoke: a shahli itikba ya nana hlakofi kut ikshoshke, amba pi haknip, micha pi yakni ak illushke.

19. Nanta katiohmi ho chi tikba ya pishno, micha pi yakni aiena kvt pilla chi cho? pishno, micha pi yakni aiena ka pvska isht ish pi chumpashke, mikma pishno, micha pi yakni aiena kvt Falaoh ha in tishu vhleha pia hashke: micha pehna ya ish pima na pi okchaya cha, ik pillo kashke, yvmohmi ho yakni vt ik chukilisso kashke, im achi tok.

20. Mihma Chosef vt Echip yakni moma ka Falaoh hạ im in chumpa tok; Echip okla hvt hohchvfo kvt i palvmmi hatuk o im osapa aiyukali ka kanchi tok: yohmi ho yakni vt Falaoh immi toba tok.

21. Mihma okla hano Echip aivhli vttut ia hosh in tvnnup fullota ont vhli ka kanvllichit tumaha holihta inla puta ka ai ashachi tok.

22. Naholitompa isht asha i yakni ak illa ho ik chumpo tok; naholitompa isht asha hano Falaoh hak osh kushkoa im apesa na, Falaoh hut ima tuk mak o $\underline{i}$ kvshkoa ya vpa hatuk oka; yumohmi hatuk osh i yakni ya ik kancho tok.

23. Yohmi ma Chosef vt okla hạ, Yakeh, himak nitak a Falaoh ha hvchishno, micha hvchi yakni aiena ka $\underline{\underline{i}}$ hvchi 
chumpa lishke: yakeh, pehna hvt yak hvchim aiashvshke; yohmi ka yakni ya hush a hokchashke.

24. Atukma yakohma hi oke, wayakma kushkoa ishit tahlapi kak o Falaoh ha hush imashke, mikma kushkoa ushta kak osh hvch immi ya chi hoke, micha osapa a hollokcha chi pehna, micha hvchishno, micha hvchin chuka achvfa im ilhpak, micha im vlla vhleha im ilhpak achị hoke, im achi tok.

25. Mihma; Ish pi okchalinchi tuk oke: a shahli itikba ya holhponayo hanta ya il ai ahayuchashke; mikma Falaoh in tishu vhleha pia hashke, okla achi tok.

26. Yumohmi ho Chosef vt Echip yakni ya nana im apesa ka, Falaoh hvt kushkoa ishit tahlapi kak o isha hi atok osh himak nitak a yumohmi hoke; amba naholitompa isht asha i yakni ak illa hosh" Falaoh immi ik tobo tok.

27. Mikma Islael vt Echip yakni, Koshen yakni ak $\underline{o}$ ahanta tok ; micha yumma osapa, micha im jlayak vt im aiasha cha, okla apakna kvt atampa fehna tok.

28. Mihma Chekob vt afummi auahuntuklo ho Echip yakni ya ahanta tok: yumohmi hokvt Chekob vt okchayvt ahanta nitak puta moma kvt afummi tahlepa achufa cha, pokoli ushta akucha untuklo tok.

29. Atuk o Islael vt ai illa he nitak vlhpisa kvt atikonofvt mihinti tok: mihmut ushi Chosef a hoyo cha, Himak a chi tikba holhponayo hanta ya ai ahayuchi likma, saiyubi nuta ya chibbak isht ish potolashke, chim asilhha lishke; micha a kana cha, is sum ahli achukmashke : Echip a chik si a hohpokashke, chim asilhha lishke:

30. Amba aki vhleha hak o takkla itoyula la hi oke: yohmi ka Echip a sushit ish kohcha cha, im ahollohpi ak o ont is sa hohpa hi oke, im achi tok. Mihma, Ish achi tuk mak o yumihchi la hi oke, im achi tok.

31. Mihma Islael vt, Anumpa kvllo vm il onochí, achi tok: mihma anumpa kullo im il onochi tok: Mihma Islael vt in topa noshkobo ya on hlipia cha, Chihowa ya aiokpvchi tok. 


\section{CHAPTA XLVIII.}

A TUK osh iluppa i himmakma yakohmi tok, Chosef a im A anolit, Yakeh, chiki vt abekushke, achi tok: mihma ushi tuklo Manasseh, micha Eflaim a pehliechi cha ia tok.

2. Atuk o Chekob a im anolit, Yakeh, chiso Chosef vt chim vlushke, achi tok: mihma Islael vt ile hlampkochi cha, topa paknaka om binili tok.

3. Mihma Chekob vt Chosef a, Chitokaka, Nan isht im aiahli ataha iksho hut Kenan yakni Lus a vm a haiaka cha, sai yukpali tok,

4. Micha, Yakeh, chi wayvchit apaknvchi lish, okla laua chikbi la chi hoke; micha yakni iluppot immi bilia chi ho, chim isht atiaka chiakaiya ka ima la hi oke, vm achi tok.

5. Yohmi ka himak a chiso tuklo Eflaim, micha Manasseh itatuklo Echip yakni ya chim vlut, Echip a ak lo kisha ma chim ashwa tok vt vmmi hoke: Luben, micha Simeon ak o chohmit vmmi ha chi hoke.

6. Atukma chim isht atiaka $\underline{\underline{i}}$ himmak a ish tobachi ak osh chimmi cha, itibapishi vhleha hohchifo hak o a hohchifot yakni ya ibai isha hi oke.

7. Uno ak okvto Patan a vttut minti li ma, hina takla Kenan yakni ya Eflalh ona chi ka ik hopako moma ho, Lechel vt vm ai illi tok; mihma Eflalh hina takla ya a hohpi li tok, yummut Behlehem mak oke, im achi tok.

8. Atuk osh Islael vt Chosef ushi tuklo ka pisa mut, Ilıpput kuta ho ? achi tok.

9. Mihma Chosef ut ịki ya , Suso tuklo ka Chitokaka yut iluppa ai uma tok oke, im achi tok. Mihma, A bilika isht ish mintashke, chim asilhha lishke, mikma yukpali lashke, achi tok.

10. (Yohmi ka Islael nishkin vt sipokni kak osh elli cha, nana pisa he keyu tok:) mihma Chosef vt $\underline{i}$ bilika isht ona ma, i suksowa cha shoyuli tok.

11. Mihmot Islael vt Chosef a, Chi nashuka ya pisa la he 
ak ahno tok: atuk ak o yakeh, Chitokaka yot chim isht atiaka yak kia sa pisachishke, im achi tok.

12. Mihma Chosef vt iki im iỵikalaha ititakla ya a kohchit kanvllichi tok; mihmvt akachunolit aka hlipia tok.

13. Mihmut Chosef vt ita tuklonchit ishi mvt, Eflaim vt ibbak isht impak imma ak o fohka ho Islael ibbak vlhfubekimma ak o pit apissullechi tok, micha Manasseh vt ibbak vlhfubek imma ak o fohka ho Islael ibbak ishtimpak imma ak o apissullechi cha, bilika isht im ona tok.

14. Mihma Islael vt ibbak ishtimpak imma pit weli mvt, Eflaim, yummak osh nakfish atuk $\underline{0}$, noshkobo ha onochi nuvt, ibbak vlhfubekimma ya Manasseh noshkobo ha onochi tok, ithana hosh ibbak a itai opitummichi tok; Manasseh ak osh akni hatuk oka.

15. Mihmvt Chosef a yukpalit, Chitokaka yummak o aki vhleha Eblaham, micha Aisak vt itikba a nohowa tok, Chitokaka yummak osh si okchaya takla ka supihinta na, himak nitak a ant vhli chatuk vt,

16. Enchel yummak osh nana ik achukmo moma ka a sa hlakofichi chatuk vt vlla nakni ash yukpalashke; mikma sa hóhchifo hut, micha aki vhleha Eblaham, micha Aisak hohchifo hvt onvtoyulashke: micha yakni paknaka iklunna ya a' wayut apakna tobashke, achi tok.

17. Atuk o iki vt ibbak ishtimpak imma ya Eflaim noshkobo ha onochi na Chosef vt pisa mvt, ik im vlhpeso tok: micha Eflaím noshkobo ha ishit kanvllichit Manasseh noshkobo ha onocha chi hosh, iki ibbak a wakeli tok.

18. Micha Chosef vt ịki ya, Yvmmak keyu, aki ma, iluppak osh vttahpi hoka; chibbak ishtimpak imma ya iluppa noshkobo hak o ish onochashke, im achi tok.

19. Mihma iki vt vlhpesa ik ahno hosh, Ithana li, suso ma, ithana li, yummak kia okla toba chi hoke, micha yummak kia chitot holitopa chi hoke: yohmi kia nakfish ak osh okla chito kut yumma i shahla chi hoke, micha im ishtatiaka yut oklushi apakna puta toba hi oke, achi tok.

20. Micha nitak yumma yukpalit, Islael vt yukpali cha, Chi hohchifot, Chitokaka yut Eflaim, micha Manasseh chi 
chohmichashke, acha hi oke, achi tok: mihmvt Eflaim a Manasseh itikba hilechi tok.

21. Mieha Islael vt Chosef a, Yakeh, svlla chịshke; yohmi kia Chitokaka yvt chiba foyuka cha, anonti hvch iki vhleha i yakni ya chisht ona hi oke.

22. Micha kvshkoa achvfa chitibapishi vhleha ha $\underline{i}$ shahlichit, yvmma a bushpo falaia, micha vm ititanampo ak $\underline{o}$ Amolait hatak ibbak a a kohchit isht im ishi li tok vt chima lishke, im achi tok.

\section{CHAPTA XLIX.}

MIHMUT Chekob vt ushi vhleha hạ hoyo cha, Hưsh I itvnaha na, nitak himmak a pilakma nana hvchim a kaniohma hi ya hvchim anoli lashke.

2. Hush itvnaha cha, hvsh haklashke, Chekob ushi vhleha huchia ma, micha Islaẹl hvch iki ya hush i haponaklashke, achi tok.

3. Luben ma, vm vttahpi, a sa kullo, micha nan isht anhlampko vmmona ka atoba holitopa i shahli, micha nan isht aiahli i shahli chia hoke;

4. Abikeli iksho oka ak o chohmit ish imaiya chi keyu hoke; chiki in topa ha ish oiya tok okvt; yohmi mut ish litehli tok oke: an topa ha oiya tok.

5. Simeon, micha Lefai vt itibapishi hoke; i bushpo falaia puta kvt nan isht ilbushali yoke.

6. Vmi shilombish ma, lumvt itim anumpulit a chieya ka, chik iba chukowo kashke; ishit sa holitopa hut itunahut chieya ka ik ibafoyuko kashke: nokowa mut hatak vbi tok oka; micha ilapunla hosh tvmaha holihta chaha ya kullit akvchi tok oke.

7. Nokowa kut libbi tok okvt, micha im ai i nukkilli et ilbushali tok okvt kalakshashke: Chekob a ai ita kushkoli la hi oke, micha Islael a ai ita fimibli la hi oke.

8. Chutah ma, chishno ak o chitibapishi vhleha hot holitoblicha he chia mak oke; chibbak vt chin tunup vhleha 
ikunla ya onvtoyula hi oke: chiki im vlla vhleha hut chi tikba ya akachunolit aka hlipkaiyvcha hi oke.

9. Chutah vt koi chito ushi yoke; suso ma, niblit vbit ish oiyushke: bikotushke, koi chito micha koi chito sipokni yo chohmit hlipiushke: kuta hosh wakayvcha hinla cho?

10. Shiloh hvt ik lo kisha kạ, Chutah ya mikko in tubi vt micha nanapesa yut iyi itin takla ya $\underline{\mathrm{i}}$ filvmmit ia he keyu hoke: mikma okla im antia kut yummak a hi oke.

11. Im issuba haksobish falaia himita ya pạkupi yo, micha im issuba haksobish falaia ushi ya pakvpi achukma $\underline{\mathrm{i}}$ shahli yo a takchechi hoke; i nafohka ya aka paki ya ai achefa, micha $\underline{i}$ nafohka ya paki issish $\underline{o}$ ai achefa hoke:

12. Nishkin vt okapaki yo ishit humma hi oke, micha noti vt pishukchi yo ishit tohba hi oke.

13. Sebulun vto okhvta vhli yạ, micha peni ai ataiya yo ai ahanta hi oke : micha im ai vhli vt Siton ak a hi oke.

14. Issaka vto issuba haksobish falaia kvllo yosh shapoh tuklo ititakla a hlipia hoke.

15. Atuk osh afoha hut achukma ho, micha yakni vt achukma ho pisa mvt, shala chi hosh tahchi ya akachunolichi cha, tishu tobut noshkobo atobbi tok.

16. Tan vto Islael apehlichi achvfa chohmit im okla ha nan im apesa hi oke.

17. Tan vt sinti yosh hina takla itonla hi oke, sinti lapish asha yosh hina takla itonla tuk osh issuba iyi ya kopoli na om binili tuk vt obvlhpilvt ivt ont itula hi oke.

18. Chim aiokchaya ya hoyo lishke, Chihowah ma.

19. Kat ano nahalupa im vlhtaha okla hưt imaiya hi oke: yohmi tuk kia ont isht aiyopikma imaiya hi oke.

20. Asha yano a kohcha kvt i puska yut nia hi oke, micha miko im ilhpak kvshaha ya waya hi oke.

21. Naftali vto issi holba yosh $\underline{\underline{i}}$ hotofa hoke: anumpa achukma yo anumpuli hoke.

22. Chosef vto naksish waya achukma yoke, naksish waya achukma yosh kuli hofobi bilika hikia cha, naksish ash filamohlichi kut holihta chaha om balvlli yoke. 
23. Ititanampo ieshi yut nukhaklochi fehna tuk oke, micha husa tok oke, micha i nukkilli tok oke.

24. Yohmi kia im ititanampo vt kvllo kvt moma tok oke ; micha Chekob in Chitokaka kvllo ibbak, micha Islael in Chukfulhpoa apesvchi, Islael in Tuli,

25. Chiki in Chitokaka, yummak osh chi apelahancha chi kă, micha Nan im aiahhli ataha iksho, yummak osh vba nan-isht-aiyukpa, micha ahofobi nan-isht-aiyukpa nutaka aiasha yo, micha ipishik nan-isht-aiyukpa, micha im oshvtto nan-isht-aiyukpa ishit chi yukpala chi ka ibbak shakba yut ishit kullo tuk oke.

26. Chiki nan-isht-aiyukpa yvt an tikba vhleha nan-ishtaiyukpa ya imaiyvt nunih hikia na bilia puta vt ont vhli fehna ka ont vhlishke; iluppa puta kak osh Chosef noshkobo ha onvtoyula chi hoke, micha itibapishi vhleha ha $\underline{i}$ filummi tok noshkobo tubokaka ya onvtoyula chi hoke.

27. Benchamin vto niblit vbi kvt nashoba chohma hi oke; onnahinlikma nipvt illi ya tahlikmut, opiakma niput illi ya ita kushkoa hi oke, ahanchi tok.

28. Iluppa moma kak osh Islael apehlichi anahtuklo hoke : milhma iki vt ilvppak o im anumpuli cha, isht yukpali tok mak oke; nan-isht-aiyukpa aiyukali ho ishit yukpali tok.

29. Micha atokolit im anumpulit, Um okla ha iba holhtina la chí hoke: akki vhleha ha takla Hittait hatak Eflon im osapa hichukbi talaia kak $\underline{\mathrm{o}}$

30. Yakni ahollohpi vt in talaia chi hosh Eblaham vt osapa aieninchit Hittait hatak Eflon a in chumpa tok a, yummut Mamle itikba Kenan yakni talaia ka Makpelah osapa ya hichukbi hikía kak o hvs si a hohpashke.

31. Yummak o Eblaham, micha tekchi Selah ya okla a hohpi tok; yummak o Aisak, micha tekchi Lebekah ya okla a hohpi tok; mikma yummak o Leah ya a hohpi li tok oke.

32. Osapa, micha hichukbi vt anukkaka hikịa ka in chumpa kvt Helh im vlla vhleha hak atok, achi tok.

33. Atuk osh Chekob vt ushi vhleha ha im anumpuli kvt 
ont tahli mot, iyi ya aiowvt topa pit kahpuli tok; milhmvt fiopissa cha, im okla iba holhtina tok oke.

\section{CHAPTA L。}

M IHMA Chosef vt iki nashuka ya on hlipivt yahaya cha Di i suksowa tok.

2. Mihmvt Chosef vt in tishu vhleha, alikchi vhleha ha iki Islael a nabalama ahumma chi ka im apesa tok: mihma alikchi vhleha hvt Islael a nabalama ahvmmi tok.

3. Atuk $\mathrm{o}$ i nitak pokoli ushta kut ont taha tok; nabalama ahama kvt yohmi hosh i nitak vt ont taha chatuk oka: mihma Echip okla hot nitak pokoli untuklo ho ishit yahaya tok.

4. Atuk osh ishit yaiya nitak vt ont taha ma, Chosef vt Falaoh in chuka achvfa ya im anumpulit, Himak a hvch itikba ya holhponayo hanta ya ai ahayuchi likmvt, hvehim asilhha lishke, Falaoh ha haklochit hush im anumpulit,

5. Aki vt anumpa kullo si il onochechit, Yakeh, sulla chishke, vm ahollohpi Kenan yakni a kulli li tuk ak o is si a hohpashke achi tok. Yumohmi hoka chim asilhha lishke, ak ia cha, aki ya ont ak hohpí; atuk osh anonti vla lashke, hush achashke, achi tok.

6. Mihma Falaoh hot, Anumpa kvllo chi il onochechi tuk mak o ish ia cha, chiki ya ont ish hohpashke, achi tok.

7. Mihma Chosef vt iki ya hohpa chị hosh ia tok; mihma Falaoh in tishu vhleha moma, micha in chuka achvfa im asunonchi vhleha, micha Echip yakni im asunonchi vhleha moma,

8. Micha Chosef in chuka achvfa moma, micha itibapishi vhleha, micha iki in chuka achvfa apihvt ia tok: peh im vlla vhleha, micha i nanvlhpoa chipinta, micha i nanvlhpoa hochito aiena kak illa ho Koshen yakni ya ashachi hatok.

9. Mihma itichanvlli puta, micha hatak issuba omibinili puta aiena hosh apihut ia tok: mihma okla chito fehna hatok.

10. Atuk osh Atat onush anihechi, yommvt Chatan 
misha talaia ka ona tok; micha yumma a yaiya kut yaiya ilbusha chinto fehna hatok; micha iki ya nitak untuklo ho ishit yaiya tok.

11. Mihma yakni yumma okla, Kenanait vhleha hot Atat anihechi a yaiya na pisa mvt, Echip okla hosh iluppot yaiya ilbusha fehna hoke, achi tok: yumohmi hatuk o yummut Abel-mislaim hohchifo tok osh, yummut Chatan misha talaia hoke.

12. Micha $\underline{i}$ miha tuk mak o ushi vhleha hot yumihchi tok.

13. Ushi vhleha hot Kenan yakni isht ona cha, hichukbi Makpelah osapa anukaka hikia Mamle itikba talaia ka yumma yakni ahollohpi ya chi hosh osapa aieninchit Eblaham vt Hittait hatak Eflon a in chumpa tok a a hohpi tok.

14. Mihma Chosef vt iki ya hohpit tahli ha yvt, yummut, micha itibapishi vhleha, micha iki ya hohpit ia ma, apihvt ia tuk moma kvt falamvt Echip a ona tok.

15. Atuk o Chosef itibapishi vhleha hot iki vt illi na pisa mut, Chosef vt pi nukkilla chi yoba ká; yohmikmut nana. ik achukmo moma e yumihchi tok a pi falvmmicha chi pulla hoke, achi tok.

16. Micha Chosef a anumpa shali ha pit tihlelit, Chịki vt ik illo kisha hosh apesa mut,

17. Chosef a yak hush im achashke, Chim asilhha lishke, himak a chitibapishi vhleha vbanvbli, micha nanashvchi ya ish $\underline{i}$ kashofashke, nan ik achukmo ho chi yumihchi tok oka, achi tok; yumohmi hoka himak a chiki in Chitokaka in tishu vhleha vbanubli ya ish $\underline{\underline{i}}$ kashofashke, e chim asilhhushke, achi tok. Mihma okla im anumpuli ma, Chosef vt nishkin okchi· mihinti tok.

18. Mihma itibapishi vhleha yak kia itikba ya ont hlipkvchi tok: micha, Yakeh, chin tishu pia hoke, achi tok.

19. Mihma Chosef vt, Ik hvchi nukshopo kashke: Chitokaka ya vlhtobut hikia li hoh cho?

20. Amba hvchishno yuto ik achukmo ho hưs si anukfilli tok; yohmi kia Chitokaka yvto achukma kak o ont aiahli- 
chit okla laua ho okchalincha he ahni tok o himak nitak a yohmi hoke.

21. Yumohmi hoka himak a ik hvchi nukshopo kashke: hvchishno, micha hvchim vlla vhleha aiena ka hvch ipeta la chi hoke, im achi tok. Micha hopohlvlit i kanvt im anumpuli tok.

22. Mihmvt Chosef vt, micha iki in chuka achvfa aiena kvt Echip a aiasha tok: micha Chosef vt afummi tahlepa achufa cha pokoli ho okchayvt ahanta tok.

23. Micha Chosef vt Eflaim im vlla vhleha aiitishali ont atuchina ho pisa tok: micha Manasseh ushi Maki im vlla vhleha aiena ka Chosef vt shoyuli tok.

24. Atuk osh Chosef vt itibapishi vhleha hạ, Svlla chịshke; atukma Chitokaka yvt hvchim aya cha, pehlichit yakni ilvppa hvchi kohchi cha, anumpa il onochit Eblaham, micha Aisak, micha Chekob a yakni im issa tok vt huch isht ona pulla hi oke, im achi tok.

25. Micha Chosef vt Islael im vlla vhleha anumpa kvllo im il onochechit, Chitokaka yvt hvchim aya pulla hi oke; mikma sa foni ha iluppa ai ishit isht hush ia hi oke, im achi tok.

26. Yohmi mut Chosef vt afummi tahlepa achufa cha pokoli hosh illi tok: mihma nabalama ahvmmi na, Echip a itombi ya fohki tok. 


\section{EKSOTUS HOLISSO.}



MOSES I HOLISSO ATUKLA KUT

\section{EKSOTUS HOHCHIFO HOKE.}

\section{CHAPTA I.}

YHMI ka hohchifo iluppa puta kak osh Islael im vlla 1 vhleha hosh Echip a vla tok. Hatak puta kvt in chuka achvfa aiena kvt Chekob a apihvt vla tok,

2. Luben, Simeon, Lefai, micha Chutah,

3. Issaka, Sebulun, micha Benchamin,

4. Tan, micha Naftali, Kat, micha Asha ak a tok.

5. Chekob haknip a a toba tok shilombish moma kut shilombish pokoli untuklo tok: Chosef vt Echip a anta hatok oka.

6. Atuk o Chosef vt micha itibapishi vhleha moma kvt, micha aiitishali yumma moma kvt illi tok.

7. Mihma Islael im vlla vhleha hvt waya achukma mot, isht $\underline{\mathrm{i}}$ shahlit, lauvt isht mahayvt, apakna cha, kvllot atamput mahaya tok; micha yakni ya alota tok.

8. Yohmi tuk o miko himona vt Chosef a ik itháno hosh Echip a mikot hikia tok.

9. Micha im okla hạ, Yakeh, Islael im vlla vhleha okla hut laua, micha kullo kvt pi shahli hoke.

10. Inta, iloh i kostinashke, keyukma lauat taiyaha tukmot, yakohma hi oke, tunup thana kia ashakma, pin tunup vhleha ha ibvlhto cha pitibikmvt, yakni ya kocha hi oke, im achi tok.

11. Yumohmi hatuk osh toksuli kullo onochit isht ilbushala chị ka, toksvli apesuchi vhleha ha im apesa tok. Mihma vlhpoyak aiasha tvmaha holihta Pihlom, micha Lameses a Falaoh a im ikbi tok.

12. Yohmi kia ilbushali kvt i shahlechik mak o, apakna 
kvt, isht $\underline{i}$ shahlit ia tok. Mikma Islael im vlla vhleha hatuk mak o okla komunta tok.

13. Micha Echip okla hut Islael im vlla vhleha ha toksvlechi kvt i kvllochi tok.

14. Micha yuka kvllo, lukfi yamuska, micha lukfi nuna, micha osapa natoksvli ilaiyukali moma toksvlechit i nitak a * hominchi tok; natoksvli moma toksvlechi kvt i kvllochi ha tok.

15. Mihma Echip i miko vt vlla eshikma apestikeli Heblu ohoyo vhleha ha im anumpulit, (yumma achufa hohchifo kvt Shiplah, mikma achvfa hohchifo kut Puah ya tok.)

16. Vlla eshi apesvchi isht-ai-vtta ya Heblu ohoyo ha hush i yumihchi tuk osh, vlla eshi na, hush pisakmot, vlla nakni yokma hushbashke, amba vlla tek okmvt okchayashke, achi tok.

17. Yohmi tuk kia ohoyo vlla eshi apesvchi vhleha hut Chitokaka ya i nukshopa cha, Echip i mikko vt im apesa tuk a ik yumihcho hosh, amba vlla nakni ya okchalinchi tok.

18. Mihma Echip i miko vt ohoyo vlla eshi apesvchi vhleha hash hoyo cha, Nanta katiohmi ho iluppa hush yumihchi cha, vlla nakni yo hush okchalinchi cho? im achi tok.

19. Mihma vlla eshi ka apesvchi ohoyo hash osh Falaoh hạ, Heblu ohoyo vhleha hvt Echip ohoyo vhleha hạ chohmi keyu hoka; tushpa hatuk osh vlla eshi ka apesvchi ohoyo vhleha hovt ik im ono kisha ho, vlla eshi hoke, im achi tok.

20. Yumohmi hoka Chitokaka yvt vlla eshi ka apesvchi ohoyo vhleha hash achukma kak o $\underline{i}$ yumihchi tok: mihma okla hut apaknut kullo fehnut ishit mahaya tok.

21. Atuk o yakohmi tok, vlla eshi ka apesvchi ohoyo whleha hash osh Chitokaka ya $\underline{\mathbf{i}}$ nukshopa hatuk $\underline{\text {, }}$, chuka achvfa puta im ikbi tok.

22. Mihma Falaoh hot im okla moma ka atokolit, vila nakni vtta puta ka okhina ya pit hush okvchashke; micha vlla tek ano hush okchalinchashke, im achi tok. 


\section{CHAPTA II.}

A TUK o Lefai in chuka achufa aiachufa hatak osh ivt, LeA fai ushetik o ishi tok.

2. Atuk o ohoyo hash osh chakali tuk osh, vlla nakni yo eshi tok; micha vlla achukma ho pisa mut, hushi tuchina ho luhmi tok.

3. Atuk osh himak ano luhma he keyu mut, hashuk holba kishushi yo eshi cha, nashinusbi, micha tiak nia aiena ho isht apolusli mvt vlla yash fohki tok; micha okhina lapalika hashuk chaha foka talali tok.

4. Mihma kaniohma chị ka, akostinincha chị hosh, in tek vt hopaki ho hikia tok.

5. Atuk o Falaoh ushetik vt okhina ya a yupa chi hosh ona tok; mihma in tishu ohoyo whleha hut okhina lapalika ya itunohowa tok; atuk o kishushi yash hashuk chaha foka ka pisa mot, in tishu ohoyo hut ont isht vla chi ka. pila tok.

6. Atuk osh wakummi ma, vlla yash pisa tok, mihma, yakeh, vlla yash osh yaiya tok. Mihma i nukhakklo tok, micha, Ilupput Heblu aiachufa vlla yoke, achi tok.

7. Mihma in tek ash osh Falaoh ushetik a, Ia lish vlla pishechi vt Heblu ohoyo hosh chi pishecha chi ka , hoyo la chi cho? im achi tok.

8. Mihma Falaoh ushetik vt, Iá, im achi tok; mihma ohoyo himita yash osh vlla yash ishki ak o ont hoyo tok.

9. Mihma Falaoh ushetik vt, vlla iluppa ishtia cha, a pishechi, mikma chim vlhtoba ya chima lashke, im achi tok: mihma ohoyo hash osh vlla yash ishi cha, pishechi tok.

10. Atuk osh vlla vt hofantit taha ma, Falaoh ushetik a isht im ona na, ushi toba tok, mihma hohchifo ha Moses achi tok: micha oka ya halvllit kohchi li tok okvt, achi tok.

11. Atuk o nitak yumma yakohmi tok, Moses vt hofantit taha mot, kohchvt, itibapishi vhleha ha im ona cha, nan isht i weki puta ka pisa tok; mihmvt Echip hatak vt itibapishi vhleha ha ai achvfa Heblu yo isso na, pisa tok.

12. Mihmot iluppa imma, micha yưmma imma aiena ka 
pit hopokoyo cha, hatak nana kia iksho, pisa mot, Echip hatak a vbi cha, shinak anukaka ya a luhmi tok.

13. Atuk osh nitak atukla ka kohchvt ia ma, yakeh, Heblu hatak tuklo kvt itachowvt ashwa tok; mihma ashvchi tuk a yummak o, Nantihmi ho chishno bika ya ish isso cho? im achi tok.

14. Mihma, Kvta hosh pi shahli, micha na pim apesa chikbi tuk oh cho? Echip hatak ishbi tuk vt chohmichit suba chi ho ish ahni cho? achi tok. Mihma Moses vt nukshopa cha, Nana iluppot otuni tuk pullushke, achi tok.

15. Atuk o Falaoh vt nana iluppa haklo mơt, Moses a ubi bunna tok. Yohmi kia Moses vt Falaoh itikba ya vttut maleli cha, Mitian yakni ya ont vtta tok, micha kvli hofobi bilika binili tok.

16. Atuk o naholitompa isht vtta Mitian vtta yvt ushetik untuklo hosh asha tok; atuk osh iki i nanvlhpoa chipunta ya oka ̣̣peta chị hosh vla cha, oka ochit peni puta ka alotoli tok.

17. Atuk o chukfi apesvchi vhleha hvt vla cha, kanima pit tihleli tok. Yohmi tuk kia Moses vt wakayvt hikia cha, apelvchit 1 nanvlhpoa chipunta ya oka ipeta tok.

18. Atuk o ikki. Luel a im ona ma, Nantihmi ho himak nitak ano chekosi hush la cho? achi tok.

19. Mihma Echip hatak osh chukfi apesvchi vhleha ibbak a apihlakofichi mvt, vlhpesa ho oka ya pim ochi cha, nanvlhpoa chipunta ya oka ipeta tok, achi tok,

20. Mihma ushetik vhleha ha, Atuk osh katima anta cho? nantihmi ho hatak a issut hush minti tuk oh cho? oh im anoli na, puska ik pá, im achi tok.

21. Mihma Moses vt im vlhpesa cha, hatak a takla ahanta tok; mihma ushetik Sippola ya Moses a $\underline{i}$ bohli tok.

22. Atuk o vlla nakni yo im eshi ma, Moses vt hohchifo ha Keshom achi tok; okla inla sia hosh yakni inla yo aya li hokvt, achi ha tok.

23. Atuk o nitak kaniohmi ma, yakohmi tok. Echip i miko vt illi tok; mihma yuka kak osh Islael im vlla vhleha 
hvt kifahahha cha, yahaya tok; micha yuka kak osh yạhaya kvt Chitokaka ya im ona tok.

24. Mihma kifahaha ka Chitokaka yut haklo tok: micha Chitokaka yut Eblaham Aisak micha Chekob aiena ka nana itim apesa tok vt ithana tok.

25. Micha Chitokaka yut Islael im vlla vhleha ha pihisa tok, micha Chitokaka yut ithaiyana tok.

\section{CHAPTA III.}

JOHMI ka Moses vt ipochi naholitompa isht vtta Mitian 1 vtta Chehlo $\underline{i}$ nan vlhpoa chipunta ya im apesuchi tok; atuk osh nan vlhpoa chipinta ya pehlichit isht ayvt, yakni haiaka ya ont ashakah ishtona mut, Chitokaka in nunih chaha Holeb a ona tok.

2. Mihma Chihowah im enchil vt luak libbi afoyukvt bvfaha iklunna et ai i haiaka tok; atuk osh pisa ma, yakeh, bufaha hvt luak isht lua, mihmot bufaha hot ik taho tok.

3. Mihma Moses vt, Fullota lish na pisa chinto ilupput nanihmi ho bvfaha hvt ik luokma pisa lashke, achi tok.

4. Atuk osh pisa chi hosh fullota ma, Chihowah yut pisa mut, Chitokaka yut bufaha iklunna et ai i howvt Moses, Moses, achi tok, Mihma yak hikia lishke, achi tok.

5. Mihma, Bilika chik minto kashke, chiyi, shulush isht ish hoyolo kvt ish shufashke, ish ahikia yakni vt holitopa hoka, achi tok.

6. Micha achakalinchit, Chiki in Chitokaka, Eblaham in Chitokaka, Aisak in Chitokaka, micha Chekob in Chitokaka sia hoke, achi tok. Mihma Moses vt nashuka ya luhmi tok; Chitokaka ya pisa kvt nukshopa tuk okvt.

7. Mihma Chihowah yvt, Um okla Echip aiasha ka ilbusha ka pihisa li kut a hlishke, toksuli ka apistikeli pulla kak o yahaya ka hạaklo lishke, nan isht im a nukhaklo ka ithana li hokvt.

8. Atuk osh Echip okla ibbak a kohchit hlakofichit, yakni yumma kohchit, yakni achukma, micha chito, pishuk- 
chi, micha foebila aiena kvt ayanahanli, Kananait, micha Hittait, micha Amolait micha Pelissait, micha Hifait, micha Chebusait, aiasha yak o isht ona la chi hosh vla lishke.

9. Yumohmi hoka himak a yakeh, Islael im vlla vhleha ya aya kut vm vlushke; micha Echip okla hut isht ilbushali na, ilbusha aiena ka pihisa le tuk oke.

10. Yumohmi hoka, inta, himak a Falaoh ya pit in chi pila lashke; yumohmikma vm okla Islael im vila vhleha ha pehlichit Echip a isht ish kohchashke, achi tok.

11. Mihma Moses vt Chitokaka yạ, Kvta sia hatuk osh, ivt Falaoh ya im ona la hinla cho? micha Islael im vlla vhleha ha Echip a apehlichit kohchi la hinla cho? im achi tok.

12. Mihma, Chiba foyuka li pulla chi hoke, mikma chipila li tuk a iluppak osh chim isht atokowa ya chi hoke; okla ha pehlichit Echip a ish kohchikmvt, nunih paknaka iluppa Chitokaka hush aiokpvcha hi oke, achi tok.

13. Mihma Moses vt Chitokaka yạ, Yakeh, Islael im vlla vhleha ha im ona lish, Hvchiki vhleha in Chitokaka yvt et hvchi sa pila tuk oke, im achi likma, mikma, Hohchifo hvt nanta ho? vm achikma, nanta ho im achi la he oh cho? im achi tok.

14. Mihma Chitokaka yut Moses a, Sia кuт mir Sia ha Cнг ноке, im achi tok, micha Islael im vlla vhleha ha yak ish im achashke, mih Sia нaкоsн hvchi sa pila tuk vt, ish im achashke, achi tok.

15. Micha achakalinchit Chitokaka yut Moses a, Islael im vlla vhleha hă, yak ish im achashke, Huchiki vhleha in Chitokaka Chihowah, Eblaham in Chitokaka, micha Aisak in Chitokaka, micha Chekob in Chitokaka ak osh et hvchi sa pila tuk oke; iluppak osh sa hohchifo bilia yoke, mikmvt iluppak osh himak a pilla aiitishali moma aiasha he vt ishit svthaiyana hi oke.

16. Iá, micha, Islael im asunonchi vhleha ha ish itunahli cha, Huchiki vhleha in Chitokaka Chihowah, Eblaham, micha Aisak, micha Chekob in Chitokaka yut a haiaka cha, 
Huchim aya li pulla cha, Echip a nana a hvchị kaniohmihinchi ka, pihisa le tuk oke.

17. Micha Echip aiilbvsha ka hvchi kohchi lish, Kananait, micha Hittait, micha Amolait, micha Pelissait, micha Hifait, micha Chebusait aiena i yakni, pishukchi, micha foebila a yanahanli yakni a chishtona lashke, achi le tuk oke, achishke, ish im achashke.

18. Mikma chim anumpa ha hakla chi hoke; mikma chishno, micha Islael im asunonchi vhleha aienvt Echip i miko ya hush im ona cha, Heblu in Chitokaka Chihowah yvt pi afama tuk oke; Yohmi ka himak a chim asilhhvshke, yakni haiaka nitak tuchina anowa yo il ona cha, Chihowah pin Chitokaka ya vbit isht aiokpuchi il im issashke, hush im achashke.

19. Mikma Echip i miko vt ik iá hvchim ahna chi kieyuka ithana lishke, keyushke, ibbak kvllo mak osh ik onvtoyula hoh kia.

20. Mihma subbak a wieli lish a nafehna moma im itin takla ya ayumihchi la chị kvt, Echip a isht issot kanchi la chi hoke; atuk o yohmi ha yokmuno ik ia hvchim ahna chi hoke.

21. Mikma okla ilupput Echip okla itikba ya holhponayo hanta ya ai ahayucha chị ho ima la chị hoke; atukma yakohma hi oke; hush iakmut nan iksho ho hush ia he keyu hoke.

22. Amba ohoyo puta kvt chuka abilika ya, micha nowvt aya yosh in chuka ahanta tuk aiena ka, tvli huta ishtshema, micha tuli lakna ishtshema, micha nafohka aiena ka im a potashke, micha huchiso, micha bvchiso tek vhleha aiena ka hush fohkuchashke; micha Echip okla ha hush wehpula chi hoke, hush im achashke, achi tok.

\section{CHAPTA IV.}

MIHMA Moses vt afalumminchit, Yohmi kia yaki, $\underline{a}$. $V_{\text {yimma he keyu, micha vm anumpa ha hakla he keyu }}$ 
hoke; Chihowah yut chi haiaka tuk keyu, acha chị hoke, achi tok.

2. Mihma Chihowah yvt, Yummut nanta hosh chibbak a foka cho? im achi ma, Tubi yoke, im achi tok.

3. Mihma, Aka pilah, achi tok; mihma aka pila ma, sinti o toba tok; mihma Moses ut i filummit maleli tok.

4. Mihma Chihowah yut Moses a, Chibbak a pit weli cha hasimbish a ishih, im achi tok. Mihma ibbak a weli cha, hokli ma, tubi toba cha, ibbak foka tok.

5. Yvmohmi ho iki vhleha in Chitokaka Chihowah, Eblaham in Chitokaka, Aisak in Chitokaka, micha Chekob in Chitokaka vt chi haiaka tuk a yimmashke, achi tok.

6. Micha Chihowah yot achakalinchit, Himak a chibbak a chikkishi pit fohkih, im achi tok. Mihma ibbak a ikkishi pit fohki tok; atuk osh kohchi ma, yakeh, ibbak vt leplosi vbit okti chiyuhmi tok.

7. Mihma, Anonti chibbak a chikkishi pit fohki, achi tok mihma ibbak a anonti ikkishi pit fohki tok ; atuk osh ikkishi ha kohchi ma, yakeh, falamut haknip inla ya chohmi tok.

8. Atukma yakohma hi oke, chi yimma he keyu, micha nanisht-atokowa vmmona ka hakla he keyu tukmvt, nanishtatokowa ishtaiopi yokuno yimma hi oke.

9. Atukma yakohma hi oke, nanishtatokowa tuklo iluppa ik yimmo micha chim anumpa ha hakla he keyukma, okhina oka ya ish ishi cha, yakni shila ya ish onhlatublashke, mihma okhina oka ish ai ishi kash osh issish tobvt, yakni shila yash o aiasha hi oke, im achi tok.

10. Mihma Moses vt Chihowah ya $\underline{\mathbf{a}}$ A shahli ma, Anumpuli imponna sia keyu, chashpo ka si yumohmi chatuk osh, is sum anumpuli taiyakla kia, si yumohmi keyushke; amba anumpa sulaha, micha isunlush svlaha sia hoke, im achi tok.

11. Mihma Chihowah yut, Kuta hosh hatak itvkha ikbi tok oh cho? micha kvta hak osh ik anumpulo, micha ik haponaklo, micha pisa, micha ik hopokoyo ikbi hoh cho? Chihowah sia hoh keyu cho?

12. Yvmohmi hoka inta, iah, mikma chitih ha iba foyuka lish, nana ish acha chi ka chim abvche lashke, im achi tok. 
13. Mihma Moses vt, $\underline{\mathrm{A}}$ shahli ma, chim asilhha lishke, kuna ho ish ahnikmvt ish tohnashke, achi tok.

14. Mihma Chihowah im ai $\underline{i}$ nukkilli vt Moses $\underline{a} \underline{i}$ sanalit libbi mvt, Lefait Alon vt chitibapishi keyu cho? anumpuli imponna hinla ka ithana li hoke: micha yakeh, chi afamut minti ak inli hoke. Atuk osh chi pesakmut, chukvsh yukpa chi hak inli hoke.

15. Mikma ish im anumpuli cha, itih ha anumpa ish fohka chi hoke: mikma Uno vt chitih ha, micha yumma itih ha iba foyuka la chi hoke; micha nana ish a kaniohma chi ka hưchim abvehi la hi oke.

16. Micha okla im anumpula chị hosh, chim anumpa eshi ya chi hoke, micha yummak osh, yumma fehna kak osh itih ha he tuk, chim vthtobakma, Chitokaka yak a he tuk a chishno ak achi hoke.

17. Micha tubi iluppa ish ieshi tuk osh, nan isht atokowa ya isht ish yumihchashke, achi tok.

18. Mihma Moses vt falamut ipochi Chehlo ya im ona cha, Chim asilhha lishke, falamvt ak ia cha, itibapishi li vhleha Echip aiasha ka ak im ona cha, okchaya momakma, ont ak pesa, im achi tok, Mihma Chehlo vt Moses a Achukmakvut ish ayashke, im achi tok.

19. Mihma Chihowah yut Moses a Mitian a , Iut falamut Echip $\underline{a}$ ish onashke, ilhfiopak chim ishi bunna tok hatak moma kvt illit taha hoke, ai im achi tok.

20. Mihma Moses vt tekchi, micha ushi vhleha aiena ka -ishit, issuba nashoba om binilichit pehliechi cha, falamot Echip yakni ya ona tok. Micha Moses vt Chitokaka in tubi ya ishit ibbak fohki tok.

21. Mihma Chihowah yvt Moses a , Falamut ivt Echip $\underline{a}$ ish onakmut, yohmi ish ahni cha, nafehna moma chibbak fohki le tuk a, Falaoh itikba ish a yumihchashke, yohmi kia chukvush a kullochi likma, okla ha ik iah, ik ahno ka chị hoke.

22. Micha Falaoh ya Chihowah yut yak achishke, Islael vt suso hoke, vm vttahpi fehna yoke.

23. Atuk o suso hvt ik iah, yumohmi cha vm antiashke, chim achi lishke, atuk o ik iah chik ahnokma, yakeh, chiso 
ha chim vttahpi fehna ka vbe la chị hoke, achishke, ish im achashke, im achi tok.

24. Atuk o yakohmi tok, hina takla chuka afoha ona ma, Chihowah yut afama cha, vbi bunna tok.

25. Yohmi ma Sippola hvt tvli halupa eshi cha, ushi yash tikba hakshup a in tubli tok; micha iyi imma pit pila mut, Issish im vlhpesa a hatak chia ho muhlishke, achi tok.

26. Yohmi ho ik iah ahni tok: yohmi ma, Hakshup tupa pulla kak o issish im ai vlhpesa a hatak chia hoke, achi tok.

27. Mihma Chihowah yut Alon a, Yakni haiaka ya Moses afamot iah, im achi tok. Mihma ia tuk osh, Chitokaka i nưnih chaha ya ont ai afama cha, i sukssowa tok.

28. Mihma Moses vt Alon a Chihowah yummak osh et pila tuk a im anumpa moma, micha nan isht atokowa moma im apesa tuk $\underline{a}$, im anoli tok.

29. Mihma Moses, micha Alon vt ia cha, Islael im asunochi moma ka hoyot itunahli tok.

30. Mihma anumpa moma Chihowah yut Mọses a im anumpuli tuk a Alon vt anumpuli cha, okla itikba ya nanishtatokowa ya a yumihchi tok.

31. Mihma okla hut yimmi tok; micha Chihowah yut Islael im vlla vhleha ha im aya tuk, micha ilbusha ka pisa tuk a haklo mut, noshkobo ha akachunoli cha, okla aiokpuchi tok.

\section{CHAPTA}

AUK osh yohmi ha yak osh, Moses, micha Alon vt iba A chukowvt, Falaoh ya im anolit, Islael in Chitokaka Chihowah yot yak achishke, Um okla hut ik ia cha, yakni haiaka ya impvchi isht ik sum aiashah, achishke, achi tok.

2. Mihma Falaoh vt Chihowah hak osh Kvta ha tuk o im anumpa ha im antia lish, Islael a ik iah, ahni la hinla cho? Chihowah ya ithana li keyu hoke, micha Islael a ik iah ahni la he keyu hoke, achi tok.

3. Mihma Heblu in Chitokaka yut pi afama tuk oke; e chim asilhhushke, ivt nitak tuchina anowa yakni haiaka 
ya il ona cha, pin Chitokaka Chihowah ya vbit aiokpvehi il im issashke; ililli okpulo micha bushpo falaia aiena ishit pi fạhama yoba na: achi tok.

4. Mihma Echip i miko yut, Nantihmi ho Moses, micha Alon hush ita tuklo kvt okla toksuli ka hush ataklummi cho? hush a toksuli oh iah, im achi tok.

5. Micha Falaoh vt, Yakeh, himak a okla yakni aiasha kut laua ho, toksula he tuk a hush fohachishke, achi tok.

6. Mihma Falaoh vt mih nitak akinli ho okla toksvli apeswchi vhleha, micha i nanvlhtoka vhleha ha im apesvt,

7. Himakma chashpo chatuk a chohmit, lukfi nuna ikba chi ka okla ha onush vpi hush ima he keyu, ilap ak inli hosh ivt onush vpi a ik aiowa cha.

8. Micha chạshpo ka lukfi nuna holhtina kaniohmi ho ikbi chatuk a hush im apesashke, achufa kia hush ola taklvcha he keyu hoke, peh okla aiasha hoke; Yohmi kak osh pahayvt, Ki lia cha pin Chitokaka ya vbit isht aiokpvchi il im issashke, achi hoke.

9. Hatak ut toksuli hut i shahli hosh onvtolashke, yumohmi cha toksvlashke, micha anumpa ik ahobo ya yohmi, ik ahno kashke, achi tok.

10. Mihma okla toksvli ha apesvchi, micha i nanvlhtoka aiena kvt kohchvt ia mut, okla ha im anumpulit, Falaoh vt yak achishke, onush vpi ya hvchima la he keyu.

11. Hvchishno ak osh kohchut oh ia cha, kanima moma ka hvsh ai ahayucha hinlakmvt hvsh aiowashke, yohmi kia hush toksuli kvt achvfa kia ola takla he keyu hoke, achi tok.

12. Yohmi ho okla hut Echip yakni moma ka ita fimimput onush vpi ya he tuk a, nanvpi ya aiowvt itunowa tok.

13. Mihma toksvli apesvchi vt atushpalechit, onush vpi vt asha tok a chohmit ish toksula he tuk a nitak osh nitakma hush atahlashke, achi tok.

14. Atuk o Falaoh ya toksvli im apesvchi vt Islael im vlla vhleha nanvlhtoka atokolit i hiohlichi tuk ash osh boa tok, mihmot ponaklot, Nantihmi ho chashpo chatuk a chohmit, pilashash lukfi nuna ikbi hvchim vlhpisa tuk vt hvch ik onacho tuk osh himak nitak a yohmi akinli cho? achi tok. 
15. Yohmi ma Islael im vlla vhleha ha vlhtoka yvt vlut, Falaoh ya im ilbushut, Nantihmi ho chin tishu vhleha ha ish yakohmichi cho? achi tok.

16. Chin tishu vhleha ha onush vpi ik emoshke, mikmvt, Lukfi nuna oh ikbi, pim achishke, mihma yakeh, chin tishu vhleha hut bovshke. Yohmi kia ashvchi kvt chim okla hatuk oke, achi tok.

17. Yohmi kia, Peh hush aiasha hoke, peh hvsh aiasha hoke, yumohmi kak osh, Il ia cha, Chihowah ya vbit isht aiokpuchi ya il í yumihchashke, hush achi hoke.

18. Yumohmi hokvt, Oh ia cha, himak a ho toksvli, onush vpi ya huch ema chị keyu; yohmi kia lukfi nuna holhtina hano hush ima chi hoke, achi tok.

19. Mihma Islael im vlla vhleha nanvlhtoka vhleha hvt, Nitak achufakma hush atahla he tuk lukfi nuna achvfa kia, hush ola taklucha he keyu, achi ha ya ik achukmo hosh aiasha ka pisa tok.

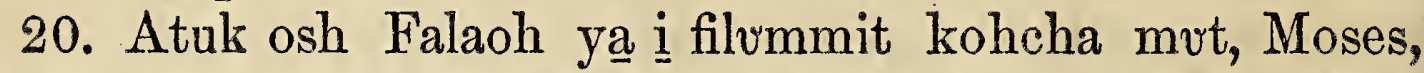
micha Alon ita tuklo kvt hina takla hinli tuk $\underline{0}$, afama tok.

21. Micha Chihowah yut huchi pisa cha, hvchim apesashke; pi balama yash Falaoh itikba, micha in tishu vhleha itikba ya hưsh yuwalvchi; micha ishit piba chị ka bushpo falaia ibbak a hush fohki hoke, im achi tok.

22. Mihma Moses vt falamvt Chihowah ya im ona cha, Chihowah ma, nantihmi ho okla iluppa nana ik achukmo ka ish yumihchi cho? nantihmi ho et is sa pila tuk oh cho?

23. Chi hohchifo pulla kak o Falaoh ya im anumpule la chi hosh, vla li tuk takla ka, okla iluppa ik achukmo ho yvmihinchi hoke; mihma chim okla ha ish hlakofichi keyu kumohmishke, achi tok.

\section{CHAPTA VI.}

YOHMI ma Chihowah yut Moses a, Himak a Falaoh ya 1 nana i kaniohmichi la chi ka ish pisa chi hoke; ibbak 
kvllo ho ik iá, ahna chi micha ibbak kvllo ho isht tihlelit $\underline{\text { i }}$ yakni ya kohcha chi hoke, im achi tok.

2. Mihmut Chitokaka yut Moses a im anumpulit, Chihowah sia hoke.

3. Micha Chitokaka nan isht im aiahli ataha iksho hohchifo hosh Eblaham, Aisak, micha Chekob aiena ka i haiaka li tok; amba CHIHOWAH sa hohchifo hokuno, yummak ash osh suthana tok keyu hoke.

4. Micha Kenan yakni, yakni aiitunowa atok, okla inla yosh aiasha tok a ima la chi hosh, kullochit itim apesa li tok.

5. Atuk osh Echip okla hut Islael im vlla vhleha ha yukachit ieshi na, kifahaha ka haklo li akinlishke, micha nana itim apesa li vt ithaiyana lishke.

6. Yvmohmi hoka Islael im vlla vhleha hạ, Chihowah sia hoke, micha Echip okla hvt naweki hvchi onochi tuk a a hvchi kohchi la chi hoke, micha hvchi yukachi tuk a hvchi hlakoffichi la chi hoke; micha shakba wieli hosh, micha napalummi chinto onochi hosh hvchi kohchit isht huchi hlakoffichi la chi hoke.

7. Micha vm okla huchia ha chi ho hvch ishe la chi hoke: micha hvchin Chitokaka sia ha chi hoke: micha vno ak osh Chihowah hvchin Chitokaka sia hosh Echip i naweki nutaka ya hvchi kohchi le tuk a , hvsh ithana chi hoke.

8. Mikma yakni yumma Eblaham, Aisak, micha Chekob a ima la chi hosh, anumpa kvilo il onochi li tok vt, hvchisht ona la chi hoke; mikma hush aiasha he vlhpesa yakni ya chị ho, huchima la chị hoke; Chihowah siahoke, im achi tok.

9. Mihma Moses vt Islael im vlla vhleha ha yumihchit im anumpuli tok; yohmi kia shilombish nukhahummit, micha yukut ilbusha kak osh Moses a $\underline{\mathrm{i}}$ haponaklo tok keyu tok.

10. Mihma Chihowah yut Moses a im anumpulit,

11. Ont chukowa cha, Echip i miko Falaoh ya im anumpuli na, i yakni ya Islael im vlla vhleha hvt kochvt ik ia ik im ahni, im achi tok.

12. Mihma Moses vt Chihowah itikba im anumpulit, Ya11 
keh. Islael im vlla vhleha hvt ik sa haponakloshke; yohmi ka Katiohmi kăk o itivlbi hakshup ik tapo sia ha Falaoh vt a haponakla hinla cho? achi tok.

13. Mihma Chihowah yot Moses micha Alon a im anumpulit, Islael im vlla vhleha ha pehlichit Echip yakni a kohcha chi kvt, Islael im vlla vhleha, micha Falaoh ya anumpa ima chi ka atokolit i miha tok.

14. Iluppa puta kak osh iki vhleha in chuka achvfa puta ka i noshkoboka yoke, Islael a im vttahpi Luben ushi vhleha hut; Hanok, micha Pallu, Heslon, micha Kami. Iluppa puta kak osh Luben in chuka achvfa vhleha hoke.

15. Mikma Simeon ushi vhleha hoke, Chemuel, micha Chamin, micha Ohat, micha Chakin, micha Soha, micha Kenan ohoyo ushi Shaul ak atok. Ilvppak osh Simeon in chuka achufa vhleha hoke.

16. Mikma iluppa puta kak osh im ai iti shali vlhpisa hosh Lefai ushi vhleha hohchifo ha tok: Keshon, micha Kohalh, micha Melali ak atok. Mihma Lefai vt okchayvt ahanta afummi moma kut afummi tahlepa achvfa cha, pokoli tuchina akucha untuklo tok.

17. Keshon ushi vhleha hvt in chuka achvfa puta kvt vlhpisa hosh Libni, micha Shimi ak atok.

18. Mikma Kohalh ushi vhleha hvt; Amlam, micha Isha, micha Heblon, micha Ussiel ak atok. Mihma Kohalh vt okchayut ahanta afummi moma kut, afummi tahlepa achufa cha pokoli tuchina akucha tuchina tok.

19. Mikma Melali ushi vhleha hut Mahali, micha Mushi ak atok. Ilvppa puta kak osh im ai iti shali vlhpisa kvt Lefai in chuka vhleha hatok.

20. Atuk o Amlam vt iki in tek Chokebet a ishit itauwaya tok; atuk o Alon, micha Moses im eshi tok, Mihma Amlam vt okchayut ahanta afummi moma kut afummi tahlepa achvfa cha pokoli tuchina akucha untuklo tok.

21. Mikma Isha ushi vhleha hvt; Kolah, micha Nefek, micha Sikli ak atok.

22. Mikma Ussiel ushi vhleha hvt; Mishael, micha Elsafan, micha Silhli ak atok. 
23. Mihma Alon vt Aminnitab ushetik Naashon in tek Elishiba ya ishit itauwaya tok; atuk o Natab, micha Abihu, Eliasa, micha Ihlama im eshi tok.

24. Mihma Kolah ushi vhleha hvt Assi, micha Elkanah, micha Abiesaf ak atok. Ilvppa puta kak osh Kolait in chuka achvfa vhleha hoke.

25. Atuk o Alon ushi Eliasa vt Putiel ushetik vhleha achufa ha eshi cha itauwaya tok; atuk o Finihas a im eshi tok; iluppa puta kak osh Lefait vhleha hot in chuka achvfa vlhpisa hosh iki vhleha i noshkoboka a tok.

26. Apehlichi vlhpisa ho Chihowah yut Alon, micha Moses a iluppak o Islael im vlla vhleha ha pehlichit Echip yakni ya hush kohchashke, im achi tok mak oke.

27. Iluppak osh Islael im vlla vhleha ha Echip a apehlichit kohcha chi hosh Echip i mikno ya im anumpuli tok mak oke: iluppak osh Moses, micha Alon mak oke.

28. Atuk o yakohmi tok, Chihowah yut Moses a Echip yakni ya ai im anumpuli nitak vt,

29. Chihowah yot Moses a im anumpulit, Chihowah sia hoke, Echip i miko Falaoh ya nana chim achi li tuk a moma ish im anumpulashke, im achi tok.

30. Mihma Moses vt Chihowah itikba ya , Yakeh, itivlbi hakshup ik tapo sia hoke: yohmi ka katiohmi kak o Falaoh vt a haponakla hinla cho? achi tok.

\section{CHAPTA VII.}

MIHMA Chihowah yut Moses a, Yakeh, Falaoh ya in I Chitokaka chikbi lishke, mikma chitibapishi Alon vt chi hopaii ya chị hoke.

2. Nana chim apesa li tuk a moma ish anumpulashke; mikma chitibapishi Alon vt Falaoh ya im anumpuli na, Islael im vlla vhleha ha i yakni ya kohchashke.

3. Mihma Falaoh chukvsh a kvllochi likmvt, Echip yakni ya nan vm isht atokowa, micha a na fehna aiena ka ai apaknuche la chi hoke. 
4. Yohmi kja Falaoh hvt hvchi haponakla he keyu hoke. Yohmi ho Echip a subbak onochit, napalummi chinto yumihchit, vm apehlichi puta ka, micha vm okla Islael im vlla vhleha ha Echip yakni ya pehlichit kohchi la chi hoke.

5. Mihma subbak a Echip a onochi lish, Islael im vlla vhleha ha im ai itin takla ya apehlichit kohcha likma, Chihowah sia ka Echip okla hot ithaiyana chi hoke, im achi tok.

6. Mihma Moses, micha Alon vt yumohmi tok; Chihowah yut im apesa tuk mak o yumihchi tok.

7. Mihmvt Falaoh ha im anumpulit okla mut, Moses vt afummi pokoli untuchinakma. Alon vt afummi pokoli untuchina akucha tuchina ha tok.

8. Mihma Chihowah yut Moses, micha Alon a im anumpulit,

9. Falaoh hvt, chishno ak osh, na fehna haiakvchi, hvchim achikma, Alon a Chin tubi ya eshi cha Falaoh itikba pila, ish im achashke mikma, sinti o toba hi oke, achi tok.

10. Mihma Moses, micha Alon vt Falaoh ya ibachukowa cha, Chihowah yut im apesa tuk mak o yumihchi tok; mihma Alon vt in tubi ya Falaoh itikba, micha in tushka vhleha itikba aka pit pila ma, sinti toba tok.

11. Yohmi ma Falaoh ak kia hatak hopoyuksa vhleha, micha yushpakummi vhleha aiena ka pit hoyo tok; yohmi ma yushpakummi vhleha Echip asha yatuk vt nan im isht yushpakummi ya yumma chohmi yumihchi ak inli tok.

12. In tubi aiyukali ka aka pila ma, sinti toba tok oka; - yohmi kia Alon in tubi vt yumma in tubi puta kash ishit nanvbli tok.

13. Mihma Chihowah hut achi tuk mak osh, Falaoh chukvsh a kvllochi na, ik i haponaklo tok.

14. Mihma Chihowah yvt Moses a, Falaoh chukkvsh vt kvllashke, okla ha ik ia; ik ahnoshke.

15. Onnahinlikma Falaoh ya ish im onashke; yakeh oka aiasha ka ia hoke; vla tạkla ka okhiná lapalika ya ish hikiashke, micha sinti toba tuk tubi yash ish ishashke.

16. Micha Heblu vbleha in Chitokaka Chihowah yot chi 
sa pela cha, Ưm okla hut ik ia, yakni haiaka ont ashut ik

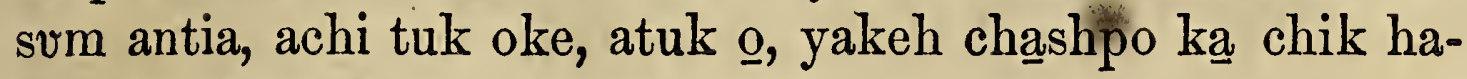
ponaklo tuk oke.

17. Chihowah yut yak achishke, Chihowah sia ka iluppak $\underline{o}$ isht ish ithaiyana chi hoke, yakeh, okhina oka aiasha ka tubi ishi li kvt, ishit fahama likma, issish toba chi hoke.

18. Mikma nuni vt okhina aiasha tuk vt illa chi hoke; mikma okhina vt shua chi hoke: mikma Echip okla hut okhina oka ishko kut yuwala chi hoke, im achi tok.

19. Mihma Chihowah yut Moses a im anumpulit, Alon a, Chin tubi ya eshi cha, Echip oka puta, oka i yanvili puta, im okhina puta, $\underline{\mathrm{i}}$ haiyip puta, micha im oka taloha moma ka chibbak a ish o wielashke; yưmohmi na, issish tobashke; micha yumohmi na, Echip yakni moma hlopulli ka , iti nan aivlhto, micha tuli nan aivlhto a puta kvt issish biekashke, ish im achashke, achi tok.

20. Mihma Moses, micha Alon vt Chihowah im apesa

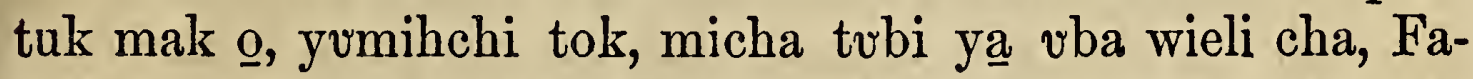
laoh hut pisa ho micha in tishu vhleha hut pisa ho oka okhina asha tuk a isso tok; mihma oka moma okhina asha tuk vt issish toba tok.

21. Mihma nuni okhina aiasha tuk vt illi tok; mihma okhina vt shua na, Echip okla hvt okhina oka ya ishka he keyu tok; mihma Echip yakni moma ka issish vt aiasha tok.

22. Mihma Echip yushpakummi vhleha hot nan im isht yushpakummi ya ishit yumihchi tok; mihma Chihowah yut achi tuk mak $\underline{0}$, Falaoh chukvush vt kvllo cha $\underline{i}$ haponakla he keyu tok.

23. Micha Falaoh hut falama cha, in chuka ont chukowa cha, ilvppak kia chukvsh onochit ik anukfillo tok.

24. Mihma Echip okla moma kvt oka ishka chi kvt, okhina bilika ka kullit iitunowa tok; okhina oka ya ishka he keyu hokvt.

25. Atuk o Chihowah yut okhina ya isso ha ya nitak untuklo kvt ont taha tok. 


\section{CHAPTA VIII.}

MIHMA Chihowah yot Moses a im anumpulit, Falaoh II ha ish im ona cha, Chihowah yout yak achishke, Vm okla hut ik ia cha, ik sum antia, achishke.

2. Atuk o, vlhpesa chik sum ahnokma, yakeh, chim aivhli moma ka shukutti yo ishit fahama la chị hoke.

3. Mihma okhina hvt shukvtti a lauachit ikbi na, yummot oiyvt chim aboha, micha ish a nusi aboha iba chukowvt, chin topa oiyvt, chin tishu vhleha im aboha iba chukowvt, chim okla ha atuyot, micha chim apalvska micha ta pushi chim ayamuska puta ka chukowa chi hoke.

4. Mihma shukvtti vt chishno, micha chim okla, micha chin tishu moma aiena ka atuya chị hoke, ish im achashke, achi tok.

5. Micha Chihowah yut Moses a im anumpulit, Alon a Chin tubi ya ishit oka yanulli puta, okhina puta, micha haiyip puta ka chibbak a ish $\underline{0}$ wieli cha, shukvtti ya kohchichit Echip yakni ya ish onochashke, ish im achashka, achi tok.

6. Mihma Alon vt Echip oka puta ka ibbak a o wieli tok: mihma shukvtti vt kohcha cha, Echip yakni ya ompohomo tok.

7. Mihma yushpakummi vhleha hut $\underline{i}$ nan isht yushpakvmmi ya yumihchi cha, Echip yakni ya shakvtti i kohchi tok.

8. Yohmi ma, Falaoh hot Moses micha Alon a hoyo cha, Chihowah ya ish im asilhha na, shukvtti yạ, Uno, micha vm okla aiena ka i kanvllichit ishit kanchashke : mikma okla ha ik iá ahni li, Chihowah ya vbit isht aiokpvehi ya im issashke, achi tok.

9. Mihma Moses vt Falaoh hạ, Is sum apesashke; katiohmikma chishno, micha chin tishu vhleha, micha chim okla ha isht anumpule li, shukvtti ya chishno, micha chin chuka puta kanvllichit ishit kanchi na, okhina ak illa ho asha chi cho? im achi tok. 
10. Mihma onnaha yah achi tok, Mihma chim anumpa hatuk mak o yumohmashke. Yumohmi ho pin Chitokaka Chihowah chohmi kvt iksho ka, ish ithaiyanashke.

11. Mikma shukvtti ut chishno, micha chin chuka puta, micha chin tishu vhleha, micha chim okla vhleha ha $\underline{i}$ filummit ia chị hoke, okhina ak illa ho asha chi hoke, achi tok.

12. Mihma Moses, micha Alon vt Falaoh ha $\underline{\underline{i}}$ filummit ia tok: mihmut Falaoh ya $\underline{\mathbf{i}}$ sanalit shukhutti ya kohchi tuk ak $\underline{0}$, Moses vt Chihowah ya $\underline{i}$ paya tok.

13. Mihma Chihowah yut Moses im anumpa ha tuk mak o yumihchi tok: mihma shukvtti vt chuka puta, micha chuka alokoli, micha osapa a puta ka ai illit taha tok.

14. Mihmal hoyot itunahlit ashachi tok; micha yakni vt shua tok.

15. Yohmi kia ik yumohmo ho Falaoh, vt pisa mut, Chihowah yot achi tuk mak $\underline{0}$, chukvsh a kullochi cha, ik $\underline{i}$ haponaklo tok.

16. Mihma Chihowah yot Moses a $\underline{\text {, Alon }}$ a, Chin tubi ya ish weli cha, yakni pushi ya ish isso na, Echip yakni hlopulli moma ka issup tobashke, ish im achashke, im achi tok.

17. Mihma yumihchit okla tok, Alon vt in tubi ya eshi cha, ibbak wieli cha, yakni pushi ya isso tok, mihma issup toba cha, hatak micha nan ulhpoba aiena ka a toba tok: mihma Echip yakni moma ka yakni pushi moma kut issup toba tok.

18. Mihma yushpakummi vhleha hvt issup kohcha chi hosh im isht yushpakummi ya yumihchi tuk osh kohcha he keyu tok; yumohmi ho issup vt hatak, micha nanvlhpoba aiena ka on aiasha tok.

19. Mihma yushpakvmmi vhleha hvt Falaoh ya , Ilvpput Chitokaka ibbak ushi yoke, im achi tok: mihma Chihowah yot achi tuk mak $\underline{0}$, Falaoh chulkvsh vt kullo tuk osh ik $\underline{\mathbf{i}}$ haponaklo tok.

20. Atuk o Chihowah yut Moses a , Onnahinli fehna mak o tani cha, Falaoh ya itikba ish hikiashke, (yakeh oka aiasha ya minti hoke,) micha, Chihowah yut yak achishke, Um okla hut ik ia cha, ik sum antiá. 
21. Keyukma vm okla ha ik iá ish ahna he keyukma, yakeh, chishno, micha chin tishu vhleha, micha chim okla vhleha, micha chin chuka anukaka puta ka shushi laua ho chi onochi la chi hoke; yohmikma Echip okla in chuka puta, micha oka aiasha aiena kvt shushi laua hosh alota chj hoke.

22. Mikma nitak yưmma um okla aiasha, Koshen yakni ya filummichi likma, shushi laua nana kia yumma iksho ka hi oke, yohmi ho yakni ai iklunnaka vno ak osh Chihowah sia ka ishit ithaiyana chi kak oke.

23. Micha vm okla ha micha chim okla iti filummichi la chi hoke, onnakma nan isht atokowa ilupput asha chi hoke, ish im achashke, im achi tok.

24. Atuk osh Chihowah yut yumihchi tok, mihma shushi laua ik achukmo fehna hosh vlvt, Falaoh in chuka, micha in tishu vhleha in chuka puta micha Echip yakni moma ka iba chukowa tok: shushi laua pulla kak o yakni vt okpulo tok.

25. Mihma Falaoh vt Moses, micha Alon a hoyo cha, Oh ia cha, yakni iluppak inli ho hvchin Chitokaka ya vbit hush ai okpvchashke, achi tok.

26. Mihma Moses vt, Yvmohma he vlhpesa keyu hoke, Echip okla nan isht a yuwala yo pin Chitokaka Chihowah ya nan vbit ishit il aiokpucha hinla hokvt: yakeh, Echip okla itikba ya i nan isht yuwala ya isht il aiokpvcha hinla cho? Yohmikma tuli ishit pi bola hinla keyu cho?

27. Yakni haiaka nitak tuchina anowa yo il ona cha, kaniohmichit, pim apesakma, pin Chitokaka Chihowah ya nan vbit isht il aiokpucha chi hoke, achi tok.

28. Mihma Falaoh hvt, Ik ia hvchim ahni li, yakni haiaka ya hvchin Chitokaka Chihowah ya nan vbit isht hush aiokpuchashke, peh hopaki fehna ho huchilk aiyo kashke: isht is si anumpulashke, achi tok.

29. Mihma Moses vt, Yakeh, chi filummit ia lishke, micha shushi laua vt onnakma, Falaoh ha, micha in tishu vhleha, micha im okla vhleha ha i filvmma chị ho, Chihowah ya im asilhha la chi hoke. Yohmi kia Falaoh hvt himakma ik 
ahlot, okla hut ivt Chihowah ya nan vbit ik aiokpuchi, ik ahno tuk vt ik yohmo kashke, achi tok.

30. Mihmut Moses vt Falaoh ya $\underline{i}$ filvmmit kohchut ia cha, Chihowah ya im asilhha tok.

31 Mihma Chihowah yvt Moses im anumpa hatuk mak $\underline{0}$ yumihchi tok: micha shushi lana yash Falaoh, micha in tishu vhleha, micha im okla vhleha aiena ka i filvmmichi tok; achvfa kia iksho tok.

32. Mihma himak ak kia Falaoh hvt chukvsh a kullochi cha, okla ik ia, ik ahno tok.

\section{CHAPTA IX.}

YOHMI ma Chihowah yut Moses a Falaoh hạ ont iba 1 chukowa cha, im anolit; Heblu in Chitokaka Chihowah yot yak achishke, Um okla hot ik ia cha ik svm antiá.

2. Ik iá chik ahno hosh, ish halvlli kvt momkama,

3. Yakeh, Chihowah ibbak vt chi nan vlhpoa osapa aiasha ka, issuba puta, issuba nashoba puta, kamel puta, wak toksuli puta, micha chukfi vlhpoa puta aiena ka onvtoyula chi hoke; issish ililli okpulo fehna hosh asha chi hoke.

4. Mikma Chihowah yot Islael i nanvlhpoa, micha Echip okla i nanvlhpoa iti filummicha chị hoke; mikma nana Islael im vlla vhleha ha immi hokvto illa chi keyu hoke, ish im achashke, im achi tok.

5. Mihma Chihowah yvt nitak vlhpisa ka apesvt, Onnakma Chihowah yut yakni ya nana iluppa a yumihcha chi hoke, achi tok.

6. Mihmut onna ma nana iluppa Chihowah yut yvmihchi tok; mihma Echip i nanvlhpoa moma kut illi tok; yohmi kia Islael im vlla vhleha ha i nanvlhpoa vt achvfa kia ik illo tok.

7. Yohmi ka Falaoh hut pit pila tok: mihma yakeh, Issael vhleha $\underline{i}$ nanvlhpoa vt achvfona kia illi tok keyu. Mihma Falaoh chukvush vt kvllo cha, okla hạ ik iá, ik ahno tok.

8. Mihma Chihowah yut Moses, micha Alon a , Tuli abi- 
la yo hituk chubbi ya ibbak alota ish eshi cha, Falaoh hut pisa ho Moses vt vba pilla pit fimmashke.

9. Mikma Echip yakni moma ka lukfi pushi chipuntasi toba cha, Echip yakni moma ka hatak, micha nanvlhpoa aiena ka onvtula cha, hichi hlachowa toba chi hoke, im achi tok.

10. Mihma tvli abila ya hituk chubbi ya eshit okla cha, Falaoh ha itikba hieli tok; mihma Moses vt vba pilla pit fimmi tok; mihma hichi hlachowa toba cha, hatak, micha nanvlhpoa aiena ka onvtoyula tok.

11. Mihma yushpakvmmi vhleha hvt hichi ak atuk mak o, Moses itikba ya hiela he keyu tok; hichi vt yushpakummi vhleha, micha Echip okla moma ka onvtoyula tok oka.

12. Mihma Chihowah yvt Falaoh chukvsh a kvllochi na, Chihowah yut Moses a im anumpuli tuk mak o; ik $\underline{\mathrm{i}}$ haponaklo tok.

13. Mihma Chihowah yut Moses a , Onnahinli fehna wakaya cha, Falaoh itikba hikia cha, Heblu in Chitokaka Chihowah yout yak achishke, Um okla hut ik"ia cha, ik sum antiá.

14. Himak ano a nanililli okpulo puta ka chi chukkush, micha chin tishu vhleha, micha chim okla ha onochi la chi hoke; yumohmi ho yakni moma ka uno chohmi kvt iksho ka ish ithaiyana chi hoke.

15. Himak ano chishno, micha chim oklá aiena ka ililli okpulo ishit chi fahama la chi hosh, svbbak a chi o weli lash. ke ; mihna yakni ya ai ishit chi kunia chi hoke.

16. Micha ilvppak achi ho muhli fehnak o, nan isht vm aiahli yut chi ai otuninchi la chi hosh, chi hilechi li tuk oke; micha sa hohchifo hokvt yakni moma ka hlopullit ai annohowa hi oke.

17. Ile chahvchit vm okla hạ ish i sanali moma cha, ik iá ish ahna he keyu hoke.

18. Yakeh, onnaha himak a chohmi fokakma, hatafo ik achukmo fehna, Echip vt talaia tok mihintit himak ant vhli kă, a yumohmi chatuk keyu ho huchi on umbuche la chi hoke.

19. Yvmohmi hoka himak a chị nanvlhpoa, micha nana 
hosh osapa chim aiasha moma ka hoyot ish itunahlashke; hatak, micha nanvlhpoa chuka isht ik lo tuk a osapa asha moma hatafo vt onvtola na, illa chin tuk, achishke, ish im achashke, achi tok.

20. Falaoh in tishu vhleha hvt Chihowah im anumpa ha nukwia kvt, in tishu vhleha, micha i nanvlhpoa ya tihlelit aboha ha apitta tok.

21. Mihma Chihowah im anumpa ha ik yimmo hokvto in tishu vhleha, micha i nanvlhpoa osapa ya ashachi tok.

22. Mihma Chihowah yut Moses a chibbak a vba pit ișh wielashke, yvmohmi na, hatafo vt Echip yakni moma ka itolot, hatak, micha nanvlhpoa, micha haiyukpulo puta osapa asha ka Echip yakni hlopulli ka onvtoyulashke.

23. Mihma Moses vt in tubi a vba pit wieli tok; mihma Chihowah yut hiloha, micha hatafo aiena ka et pila ma, luak vt yakni paknaka ya aya tok: micha Chihowah yut Echip yakni ya hatafo et on umbvchi tok.

24. Yohmi ho hatafo, micha luak vt hatafo yash itai okoma hosh ik achukmo fehna, Echip vt okla toba tok itin takla yakni moma ka yohmi chatulk keyu ha tok.

25. Mihma hatafo vt Echip yakni moma ka osapa asha tuk moma, hatak, nanulhpoa aiena ka issot kanchi tok; micha hatafo vt haiyukpulo puta osapa aiasha issot kanchi mut, iti puta osapa aiasha ka kobalichit tahli tok.

26. Koshen yakni, Islael im vlla vhleha hvt aiasha ak illa ho hatafo vt iksho tok.

27. Mihma Falaoh hut Moses, micha Alon a pit hoyo cha, Himak a sa yoshubvshke, Chihowah yut ai vlhpiesakma, vno, micha vm okla aiena kvt yoshoba pia hoke.

28. Vlhpesa hoke, Chihowah ya ish im asilhhashke, mihma himakma hiloha, micha hatafo vt iksho kashke; mikma ik iá hvchim ahni likma, himakma hvchik asho kashke, im achi tok.

29. Mihma Moses vt, Tumaha holihta ya kohcha li makinli hosh, Chihowah ya subbak a pit isht im ashatupa la chị hoke; mikma hiloha kvt issa chị hoke, micha himak- 
ma hatafo vt asha chi keyu hoke; Yohmi ma yakni vt Chihowah ak $\mathrm{o}$ immi ka ish ithaiyana chi hoke.

30. Yohmi kia chishno, micha chin tishu vhleha hoto Chihowah Chitokaka ya hush $\underline{i}$ nukwia chị keyu moma ka ithana lishke.

31. Micha nuchi, micha bali aiena ka issot kanchi tok; bali vt nihi tobakma, micha nuchi vt yushkobolichi hatuk oka.

32. Yohmi kia onush micha onush issuba upa ano issot ik kancho tok, chahvt ik taho kisha hatuk oka.

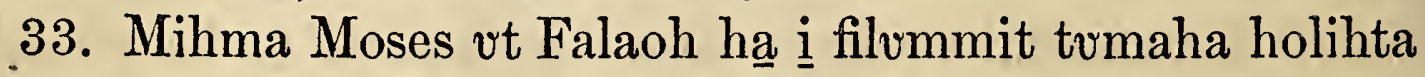
ya kohcha mut, Chihowah ya ibbak isht im ashatupa tok; mihma hiloha, micha hatafo aiena kvt issa ma, umba kvt yakni ya et ik o fohopo tok.

34. Atuk o umba, micha hatafo, micha hiloha aiena kvt issa na, Falaoh hut pisa mot, himakma yoshuba cha, yumma, micha in tishu vhleha aiena kvt chukvsh a kvllochi tok.

35. Mihma Chihowah yot Moses a tohnot anumpuli tuk mak o Falaoh chukvsh vt kullo cha, Islael im vlla vhleha ha ik iá, ik ahno tok.

\section{CHAPTA X.}

MIHMA Chihowah yvt Moses a, Falaoh ya ish iba chukotunichi la chi hosh, chukvush a micha in tishu vhleha chúkvsh a kvllochi li tuk oka.

2. Micha Echip a nana ayumihchi li tok, micha nan isht vm atokowa ya im itin takla ayumihchi li tuk a, chiso ha im anolit, ish haklocha he, mikma chiso hvt ushi ya haklocha he ak oke; yumohmi ka vno ak osh Chihowah sia ka isht hush ithana he ak oke.

3. Mihma Moses, micha Alon vt Falaoh ya im ona cha, Heblu in Chitokaka Chihowah yut yak achishke, Katiohmi foka ho sa tikba ya chi chukvsh ish akanlusecha he keyu cho? Um okla hvt ik ia cha, vm antiashke. 
4. Keyukma, vm okla ha ik ia ish ahna he keyukma, yakeh, onnakma hawa ya chim aivhli ya isht vla la chị hoke.

5. Mikma yakni paknaka ya ompoholmokma, kuna hosh yakni ya pisa he keyu ka chi hoke : mikmut hlakofi tuk osh asha moma hatafo vt chim ashanchi tuk a vpa chi hoke, micha iti puta osapa chim ai offo ya vpa chi hoke.

6. Micha chim aboha puta, micha chin tishu vhleha moma im aboha puta, micha Echip okla moma im aboha puta ka alota chi hoke, yumma chohmi ka chịki vhleha, micha chiki inki vhleha hut yakni paknaka ya asha tok nitak a vttut minti hosh, himak nitak ant vhli ka pisa chatuk keyu ka chi hoke, achishke, achi tok: mibmvt fullotvt Falaoh ya filummit kohcha tok.

7. Mihma Falaoh in tishu vhleha hot, Katiohmi foka ho hatak ilupput ishit pim ahleka chi cho? ik ia cha, in Chitokaka Chihowah ya ik aiokpvchi, Echip vt okpulot taha kah, chik ithano kisha cho? im achi tok.

8. Mihma Moses, micha Alon a anonti Falaoh itikba isht ona tok, mihma, Oh ia cha, hvchin Chitokaka Chihowah ya oh im antia. Yohmi kia kvta hosh ia chị ho? im achi tok.

9. Mihma Moses vt, $\mathrm{P} i$ himithoa, micha pi sipokni, piso vhleha, micha piso tek whleha pi nanvlhpoa chipinta, micha pi nanvlhpoa hochito a puta pehliechit il ia chi hoke: Chihowah ya impvchi isht il im asha chi hoke, achi tok.

10. Mihma Falaoh hvt, Ik ia hvchim ahni li ka chohmit Chihowah yot hvchishno, micha hvchin chipunta puta ka huchiba foyukashke: yohmi hosh ahnashke, nan ik achukmo kvt hvchi tikba itoyula hoka.

11. Yohma he keyu, himak a hatak hvchia hokvt, Chihowah ya hvsh im antiashke, yvmmak o hvchi bunna tuk okvt, im achi tok; mihma Falaoh itikba hieli tuk a tihlelit kohcha wehli tok.

12. Mihma Chihowah yut Moses a, Hawa yut vla chi ka chibbak a Echip yakni paknaka ya ish o wielashke; yumohmi na, vlut yakni ya onvtoyula cha, hatafo vt ashanchi tuk moma haiyúkpulo puta yakni aiasha ka vput tahlashke, im achi tok. 
13. Mihma Moses vt Echip yakni paknaka ya in tubi ya o wieli ma, Chihowah yut nitak yumma moma kă, micha ninak yumma moma ka hushi akochaka mahli ha isht vlut yakni ya onochi tok: atuk o onnahinli ma, hvshi akochaka, mahli hut hawa ya isht vla tok.

14. Mihma hawa yvt Echip yakni moma ka ona mvt, Echip im aivhli moma ka aka foha ha tok: ik achukmo fehna ha tok; chasshpo ka hawa yut yohmi chatuk keyu, micha himakma yohma he keyu ha tok.

15. Yakni paknaka moma ka alotowa tok oke. Yohmi hosh yakni ya okhilichi micha yakni ya haiyukpulo puta' micha iti vni puta hatafo vt ashahanchi tuk a vpvt tahli tok; micha Echip yakni hlopulli moma ka iti puta ka, micha haiyukpulo osapa aiasha puta ka nana okchạki nana kia iksho tok.

16. Yohmi ma Falaoh hot tushpvt Moses, micha Alon a hoyo tok; micha, Hvchin Chitokaka Chihowah, micha huvchishno ya hvchi sa yoshobvshke.

17. Yumohmi hoka himak a himonasi ya iluppa nan ashuchi li ya huss sa kashofashke, huvchim asilhha lishke, micha hvchin Chitokaka Chihowah ya hush im assilhhashke; yumohmi na nan illi iluppa illa ho isht a kanullichashke, achi tok.

18. Mihma Falaoh ya i filummit ia cha, Chihowah ya im asilhha tok.

19. Mihma Chihowah yvt hushi okatula mahli kvllo fehna ho fullolichi na, hawa ya ishtivt okhvta humma ya oka tamoli tok; Echip ai vhli fullota moma ka hawa achvfa kia iksho tok.

20. Yohmi kia Chihowah yut Falaoh chúkush a kullochi na, Islael im vlla vhleha ha ik iá, ik ahno tok.

21. Mihma Chihowah yvt Moses a Chibbak a vba pit ish wielashke; yohmi na okhlili hot okhlili ha yakohmi, ahna he vlhpesa hosh Echip yakni paknaka ya onvtoyulashke, im achi tok.

22. Mihma Moses vt ibbak a vba pit wieli tok; mihma nitak tuchina ho okhlili sukko hosh Echip yakni moma ka onvtoyula tok. 
23. Nitak tuchina ka iti pesa keyu, micha aiasha tuk vt ik kanvllo tok. Yohmi kia Islael im vlla vhleha moma im aboha ya atohwekili ha tok.

24. Mihma Falaoh hut Moses a hoyo cha, Oh ia cha, Chihowah oh aiokpvchi peh hvchi nanvlhpoa chipinta, micha hvchi nanvlhpoa hochito ak illa hosh aiashashke, hvchim vlla vhleha hut hvchi apihvt iashke, achi tok.

25. Mihma Moses vt, Nanubit isht aiokpvchi, micha na hukmit isht aiokpvchi aiena ka ish pimashke, Yvmohmi ho pin Chitokaka Chihowah ya nan vbit isht il aiokpuchashke.

26. Pi nanvlhpoa vt pi apehut ia chi hoke: jyakchush achvfa kia olbut anta chi keyu hoke: yumma aieshi cha, Chihowah pin Chitokaka ya isht il aiokpvcha chi hoka; micha yumma kil ono kisha kut nana ho Chihowah isht il jm antia chi kvt kil ithano hokut, achi tok.

27. Yohmi kia Chihowah yut Falaoh chukvush a kullochi na, ik ia, ik ahno tok.

28. Mihma Falaoh hut, 1 filvmmit iá, ahah ish il ahnashke; himakma sa nashuka ya ish pisa na; nitak nana ho sa nashuka ya ish pisakmvt chilla chi hoke, im achi tok.

29. Mihma Moses vt, vllhpesa ho ish mihvshke, himakma anonti chi nashuka ya pisa la chi keyu hoke, achi tok.

\section{CHAPTA XI.}

MIHMA Chihowah yvt Moses a, Himakma nan ik achukI mo achufa ho Falaoh micha Echip a onochi la chi hoke, Yohmi ha yokmuno iluppa ashwvt ik ia, hvchim ahna chi hoke; ik ia, hvchim ahnikmut ahli hosh kocha pit hvchi tobla chi bano hoke.

2. Himak a okla im anumpulit, ish haklochashke, mikma hatak puta kvt in chuka a bilika, mikma ohoyo puta kvt in chuka abilika ya tvli huta isht ilakshema, micha tvli holisso lakna isht ilakshema i potashke, im achi tok.

3. Mihma Chihowah yut Echip okla ha okla hash i kanuchi tok. Micha hatak Moses vt Echip yakni ya Falaoh 
in tushka vhleha itikba, micha okla hash itikba aiena ka holitopa fehna tok.

4. Mihma Moses vt, Chihowah yvt yak achishke, Ninak iklunna fokakma, kohchut Echip im iti takla ona la chi hoke.

5. Mikma vttahpi moma Echip yakni aiasha kvt, Falaoh im vttahpi yosh, im aiombinili holitompa om binili ka vttut ia hosh, ohoyo tishu im vttahpi yosh afotoha ashaka hikia ka ont vhli kut illa chi hoke; micha nanulhpoa vttahpi moma chi hoke.

6. Mikma Echip yakni hlopulli moma ka yaiya chinto fehna, yumma chohmi kvt iksho, micha himakma yumma chohmi kut iksho ka chi hoke.

7. Amba Islael im vlla vhleha hokuno, hatak okmá, keyukmut nan vlhpoa yokmá, nana ofi vt i sanalit isunlush $\underline{a}$ ilhkolicha he keyu ka chi hoke: yumohmi ho Chihowah yut Echip okla, micha Islael a itim inlvchi ka , hush ithaiyana chi hoke.

8. Mikma chin tishu vhleha iluppa moma kvt vm vlvt, vm akachunolit, kohchut iá, micha okla huchiakaiya moma ká, acha chị hoke; mikma yohmi ha yak o kohchvt ia la chị hoke, achi tok, Mihmot nokowa fehna hosh Falaoh ha itikba hikivt kohchut ia tok.

9. Mihma Chihowah yut Moses a, Falaoh hvt hvchi haponakla chị keyu hoke; Yohmi na, a na fehna puta ka lauachi la chi kak oke, im achi tok.

10. Mihma Moses, micha Alon vt na fehna iluppa moma ka Falaoh itikba ya a yumihchi tok; mihma Chihowah yut Falaoh chukkvsh a kvllochi na, i yakni ya a kohchut Islael im vlla vhleha ha ik ia, ik ahno tok.

\section{CHAPTA XII.}

MIMMA Chihowah yut Moses, micha Alon a Echip yak2. Hushi ilvppak osh hvshi talohvt hvchim ishtia vmmona 
ya chị hoke: afummi ishtia ka huchị hushi vmmona chị hoke.

3. Islael okla moma ka im anumpulit, Hushi iluppa nitak ont ishit pokolikma, hatak moma kvt chukfushi ya îki vhleha in chuka achvfa vlhpisa hosh ishashke, chuka achvfakma chukfushi achvfashke.

4. Atuk o chuka achvfa yut ik lauwo fehna hatuk $\underline{o}$, chukfushi vt atampakma, chuka abilika et apanta ak o itatuklot shilombish holhtina kak o vlhpisa hosh ishashke, hatak moma kut chukfushi ya vpa vlhpesa ho hush hotenashke.

5. Hvchin chukfushi vt ik ai ono iksho, nakni yosh afummi ummona hashke, chukfi ha keyukmut, issi kosoma fllummichit hush ishashke.

6. Micha hvchim anta na, hvshi mih mak inli kvt nitak ont isht auah ushtashke; atukma Islael okla moma hosh opiakma vbashke.

7. Mihmvt issish ishi cha, aboha yumma anukaka ai upa chi kvt, okhissa tahlaklakka lapali tuklo ka, micha okhissa paknaka lapali aiena ka isht issashke.

8. Mikmut ninak yumma nipi ya luak isht apushli cha, puska ik shatummo aiena vpashke, micha haiyukpulo homi aieninchit vpashke.

9. Okchaki ka huchikpo kashke, micha oka ishit honni keyu kumohmi; amba luak isht vlhpusha hashke; noshkobo, iyi aiena, micha chukvsh, salakha, micha shilukpa aiena hashke.

10. Micha kaniohmi kia hvchin tonla na onnaha he keyu hoke : kaniohmit itonla na onnakma luak ishit hush hukma hi oke, ish achashke.

11. Micha yakohmi hosh hushpa hi oke, vskofuchi ya isht hush il vskofaiyachi cha, hvchi shulush a hush hoyulo, micha hvchin tubi vt hvchibbak foyukashke; micha anukwaya hosh hushpa hi oke, Chihowah im vbanvblit ont ia yoke.

12. Himak ninak a Echip yakni ya hlopulli lish vttahpi moma, hatak micha nanvlhpoa aiena Echip yakni aiasha ka 
issot kanchi la chị hoke; micha Echip $\underline{\underline{i}}$ naholba puta $\underline{i}$ sanalit na palummi onochi la chi hoke. Chihowah sia hoke.

13. Mikma issish vt aboha hush ai asha ka a lapoht maya kvt huchi nan isht vlhpisa chi hoke; atuk o issish a pisa likmvt, hvchi vbanvbli la chi hoke, mikmut Echip yakni ya issot kanchi likma, ililli okpulo vt hvchi onvtolvt ishit hvchi kancha he keyu hoke.

14. Mikma himak nitak a hush ithana bilia yosh hvchin takala chi hoke, mikmvt hvchim aiitishali puta hlopulli ka holitoblit Chihowah impvchi isht hush aiasha hi oke; nạ vlhpisa bilia yo impvchi isht hvsh aiasha hi oke.

15. Nitak untuklo ho puska ik shatummo ya hush pa hi oke; nitak vmmona mak inli ho shatummi ya hvchim aboha puta ka hush kohchashke; kuna hosh nitak vmmona vttut ia hosh nitak ont isht untuklo ont vhli takla ka pvska shatummi ya vpakmvt, shilombish yvmmvt Islael a akohchvt fiopa topa hi oke.

16. Micha nitak vmmonakma itunaha holitopa aiasha hi oke, atuk osh nitak ont isht untuklokma, itunaha hvchi holitopa kut aiasha hi oke, yummak oka toksuli kaniohmi kia vlhtaha he keyu, peh hatak vt nana vpa vlhpesa ho, yummak illa hush atahla hi oke.

17. Micha puska ik shatummo impvchi ya isht hush aiashashke; mih nitak fehna ak o hvchim apelichi puta ka Echip yakni ya apehlichit, hvchi kohchi li tuk oka. Yvmohmi hoka nitak ilvppa hvchim aiitishali puta kvt nanvlhpisa bilia yo hush holitobla hi oke.

18. Hushi vmmona, hushi vt nitak ont isht auahushtakma opiakma, puska ik shatvmmo vput isht hush ia tuk osh, hushi vt nitak ont ishit pokoli tuklo akucha achvfa opia ka, ont hush vhlicha hi oke.

19. Nitak untuklo ho shatummi vt hvchim aboha puta ka iksho ka hi oke ; Kuna hosh okla inla yokmá, keyukmut yakni ya ai itola tok okmá, nana hosh puska shatummi ya vpakmvt, shilombish yvmmut Islael okla moma ya akohchvt fiopa tupa hi oke.

20. Nana kia shatummi ya hush pa he keyu hoke; hvsh 
aiasha moma ka puska ik shatummo yo hush pa hi oke, achi tok.

21. Yvmohmi ma Moses vt Islael im asvnonchi moma ka hoyo cha, hvchin chuká achvfa vlhpisa hosh chukfushi achvfa ya ont ishit hush kohchi cha vbanublit ont ia ya hush bashke.

22. Micha hissop pukussa ho hưsh eshi cha, issish ampushi alhto ka hush okvchi cha, issish ampushi vlhto kash okhissa paknaka lapat, micha tahlalakka lapali tuklo ka isht hush issashke : mikma hvsh achvfa kia' chim aboha okhissa ya huchik kohcho ho onna chi hoke.

23. Chihowah yut Echip okla ha issot kancha chi hosh hlopullit aya chi hoke: atuk osh issish vt okhissa paknaka lapali, micha tahlaklakka lapali tuklo ka issish asha na, pisakmut okhissa ya vbanubli cha, nanvbi ya huchim aboha puta ka iba chukowvt issot ik kanchi, im ahna chi keyu hoke.

24. Mikma nana iluppak osh chishno, micha hvchiso vhleha aiena ka i nan vlhpisa bilia yo hvsh holitobla hi oke.

25. Atukma yakohma hi oke, Chihowah yut achit hvchim issa tok mak osh hvchima na, yakni ya hush onakmut, nan nan isht asha ilvppa isht hush ahsa hi oke.

26. Atukma yakohma hi oke, hvchim vlla vhleha hvt, Nan isht asha ilupput, nanta ho miha ho ? huchim achikma.

27. Chihowah im vbanublit ont ia nan vbit isht aiokpvchi yoke; yummut Echip okla ha issot kanchi mut, Islael im vlla vhleha im aboha puta ka vbanublit ont ia cha, pin chuka achufa puta Echip talaia ka pi hlakofichi tok oke, hush acha hi oke, im achi tok. Mihma okla hut noshkoboka isht akachunoli cha, aiokpvchi tok.

28. Micha Islael im vlla vhleha hvt ia mvt, Chihowah yut Moses, micha Alon a im apesa tuk mak o yumohmi tok, yohmi tok.

29. Atuk o yakohmi tok, ninak iklunna ma, Chihowah yut Echip yakni ya vttahpi moma ka issot kanchi tok, Falaoh im vttahpi yosh im aiombinili holitopa binili tuk a vttut ia hosh yuka hosh aboha kvllo ya itonla ka ont vhli, micha nanvlhpoa vttahpi puta moma tok. 
30. Mihma ninak a Falaoh, micha in tishu vhleha moma, micha Echip okla moma kvt tani tok; mihma Echip a a yaiya chito fehna tok; aboha ya ik ai illo kvt iksho hatuk oka.

31. Mihma ninak a Moses, micha Alon a hoyo cha, Ho wakaya, micha vm okla ai iti takla ya hvchishno, micha Islael im vlla vhleha aienvt kohchvt hush iashke, micha hvsh ia cha, hush achi tuk okvt, Chihowah ya hush im antiashke.

32. Micha hvchi nanvlhpoa chipinta, micha hvchị nanvlhpoa hochito aiena hush achi tuk okvt, hush eshi cha, oh iá, micha uno ak kia anumpa yukpali hus si onochi akinlashke, achi tok.

33. Mihma Echip okla hvt anukwayvt yakni kohcha chi ka okla ha im acheba fehna tok; momut illit pi taha chishke, achi hatuk okvt.

34. Mihma okla hut tansh yamuska ik shatummo kisha ho, micha a yamuska aiena ka $\underline{\underline{i}}$ na fohka abonunta ho cshit iabanali tok:

35. Micha Islael im vlla vhleha hovt Moses im anumpa hatuk mak o yumihchi tok: micha Echip okla ha tvli hvta isht ilakshema, micha tuli holisso lakna isht ilakshema, micha nafohka aiena ka im a pota tok.

36. Mihma Chihowah yvt okla hạ Echip okla itikba ya hollhpanayo hanta ya ima tok; yohmi ho nana ho bunnakma im pota tok: mihma Echip okla ha wehpuli tok.

37. Mihma Islael im vlla vhleha hut Lameses ashut ia mot, Sukkolh a ona tok, hatak akanowa yvt, vlla yut aholhtina keyu ka tahlepa sipokni tahlepa hannali foka tok.

38. Mihma okla lawa itunohowa ak inli hosh apehvt ia tok: mihma nanvlhpoa chipinta, micha nanvlhpoa hocheto, nanvlhpoa laua fehna, hatok.

39. Mihma tansh yamuska Echip ai ishit kohcha ka ik shatummo hatuk oka, puska luboa ik shatummo nunachi tok; Echip a kocha pit tihleli, mihma asha he keyu hatuk okvt, micha ilhpak kia ik ila tahlo tuk atok.

40. Yohmi ka Islael im vlla vhleha hut Echip aiasha kvt afommi tahlepa ushta cha pokoli tuchina hatok.

41. Atuk o yakohmi tok, afummi tahlepa ushta cha pok- 
vlhpoa yokmá, nana im oshvtto ya tiwi ka is sa hullochashke, vmmi hoke, achi tok.

3. Mihma Moses vt okla hạ, Himak nitak ak o, Echip, micha aboha ayuka hvsh kucha tok vt hush ithaiyanashke; ibbak hlompko hosh Chihowah yot iluppa a huchi kuchi tuk oke, puska shatummi ya upa he keyu hoke.

4. Himak nitak hushi Abib ak o hush kuchushke, im achi tok.

5. Atukma yakohma hi oke, Kenanait, micha Hittait, micha Amolait, micha Hifait, micha Chebusait $\underline{i}$ yakni, pishukchi, micha foe-bila a yanahanli yakni a hvchima chị hosh, hvchiki vhleha ha anumpa kvllo il im onohonchi tok a, Chihowah yut hvch ishtonakma, nan isht asha ilvppa hushi ilvp-

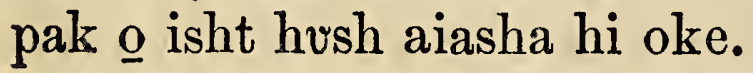

6. Nitak untuklo ho puska ik shatummo hush pa hi oke; atukma nitak untuklo kvt Chihowah impvchi ya hi oke.

7. Puska ik shatummo nitak untuklo ho vpa hi oka, mikma pvska shatummi yvt hvchin toyula na, pisa he keyu hoke, micha hush abinat moma ka shatummi vt huchin toyula na, pisa he keyu hoke.

8. Atukma nitak yumma chiso ha im anolit, Iluppa yakohmi kvt Echip a kohcha li ma, Chihowah yot nana sa kaniohmichi tok ak oke, ish achashke.

9. Mikma nan ishtatokowa yosh chibbak a lapala chi, micha ishit hvsh ithana bilia yosh hvchi nishkin ititakla ya takala chi hoke; yumohmi ho Chihowah $\underline{\mathrm{i}}$ nanvlhpisa hut huch itih ha foyuka chi hoke; Chihowah yut ibbak hlampko hosh pehlichit Echip a huchi kuchi tuk oke.

10. Yumohmi hoka nanvlhpisa iluppa ont aivlhpesakma, afummi hosh afummikma isht hush aiasha hi oke.

11. Atukma yakohma hi oke, Chihowah yot, chishno, micha chịki vhleha ha anumpa kvllo ya im il onochi tok mak osh, Kenanait i yakni chishtona cha, chimakma,

12. Vttahpi puta im oshvtto tiwi moma ka Chihowah ish i hullocha hi oke, mikma nanvlhpoa chim asha puta ka vttahpi moma nakni puta kvt Chihowah immi ha hi oke. 
13. Issuba nashoba vttahpi puta ka chukfushi yo falumminchit isht ish chumpa hi oke, micha falvmminchit ish chumpa he keyukmvt, ikunla ya ish kotvffa hi oke: micha chim vlla itin takla ya hatak osh vttahpi moma ka falvmmichit ish chumpa hi oke.

14. Atukma yakohma hi oke, Nitak himak minti ka chiso hut chim ponaklot, Ilupput nanta hoh cho? achikma, Chihowah yot ibbak hlampko hosh pehlichit Echip yakni, micha aboha ayuka ha pi kohchi tok oke.

15. Micha yakohmi tok. Falaoh vt ik ia, kvllo hosh pi ahna he keyu ma, Chihowah yot Echip yakni vttahpi moma ka, hatak vttahpi, micha nanvlhpoa vttahpi aiena ka issot kanchi tok; yumohmi tok ak o nakni yosh im oshvtto tiwi moma ka vbit Chihowah isht aiokpvcheli hoke; amba vm vlla vttahpi moma ka falumminchit chumpa li hoke, ish im achashke.

16. Atuk o nanisht atokowa yosh chibbak a lapala he, micha itikba takali yosh chi nishkin iti takla ya takala hi oke; ibbak hlampko hosh Chihowah yut pehlichit Echip a pi kohchi tok oke.

17. Atuk o yakohmi tok, Falaoh vt okla ha ik ia im ahni ma, Chitokaka yut pehlichit hina Filistin i yakni hlopullichit isht ik aiyo tok; yummak osh ik hopako ak inli kia; okla hut tunvp a pisakmut nukhaklo cha, falamvt Echip a ona yoba ná, Chitokaka yot achi hatuk okvt.

18. Amba Chitokaka yut okla fullolihinchit okhuta humma yakni haiaka ak o hlopullichit isht aya tok ; mihma Islael im vlla vhleha hot nahalupa im vlhtaha hosh Echip yakni a kohchvt ia tok.

19. Mihma Moses vt Chosef foni ya isht ia tok; Islael im vlla vhleha ha atokolit anumpa kvllo im il onochechit, Chitokaka yut huchim aya pulla hi oke; mikma sa foni ya iluppa ai ishit hush ia hi oke, achi tok oka.

20. Mihmvt Sukkolh a asht ia mvt Ehlam a yakni haiaka vhli ya a binachi tok.

21. Mihma Chihowah yut itikba ivt in tikba heka kvt nitak ma, tonik hoshonti hosh hikia beka tok; atuk osh 
ninak ma in tohwikela chi kut, tonik luak osh hikia beka tok; nitak, micha ninak ma, aya chi hatok.

22. Nitak ma hoshonti tonik atuk osh ninak ma tonik luak vt okla itikba hikia tuk a kanvllichit ik im esho tok.

\section{CHAPTA XIV.}

MIHMA Chihowah yvt Moses a im anumpulit,

IV 2. Islael im vlla vhleha ha ish im anumpoli na, fullotut Pihahilolh itikba Miktol micha okhvta iti takla, BaalSifon i chapaka yak o binachashke.

3. Falaoh vt Islael im vlla vhleha hạ, Yakni ya ai ia ikithano hoke, yakni haiaka yut im okshitushke, acha hi oke.

4. Mihma Falaoh ya chukvush a kvllochi likma, iakaiya chi hoke, mikma Falaoh, micha in tushka moma ka ishit sa holitopa chị hoke. Yvmohmi ho Echip moma kvt Chihowah sia ka ithana chi hoke, achi tok. Mihma yumohmi tok.

5. Atuk o Echip $\underline{\mathbf{i}}$ miko ya , Okla hut tihlaia ka im anoli tok: mihma Falaoh chukush, micha in tushka vhleha chukvsh aiena kut okla hash $\underline{i}$ sanalit, shanaia cha, Nantihmi ho iluppa e yumihchit, Islael vhleha hut pin toksula he tuk a ik ia il im ahni tuk oh cho? achi tok.

6. Mihmut tunvp im itichanulli ha atahli mut, im okla aiena ka ishtia tok.

7. Micha aiowa hosh itichanvlli tahlepa hannali, micha Echip itichanulli moma, micha 1 kupituni moma ka ishi tok.

8. Mihma Chihowah yut Echip i miko Falaoh ya chukush a kvllochi na, Islael im vlla vhleha ha iakaiyvt hliohli tok; mihma Islael im vlla vhleha hvt ibbak kvllo hosh kohchut ia tok.

9. Yvmohmi tuk kia Echip okla hvt jakaiya tok, (Falaoh ya im issuba, micha tunup im itichanvlli moma, im issuba om binit, micha in tushka chipota aiena hosh) atuk osh okhvta lapali ka Pihahilolh ichapaka Baal-Sefon itikba ak o binachit aiasha na, ont sakki tok.

10. Atuk osh Falaoh vt bilika mihinti ma, Islael im vlla 
vhleha hot akachakali ma, yakeh, Echip okla hot iakaiyut minti tok, mihma nukshopa fehna tok, mihmot Islael im vlla vhleha hut chitolit Chihowah ya im paya tok.

11. Mihmvt Moses a Echip a ahollohpi iksho kak o yakni haiaka a pilla chi ka jloppa pisht ish la tuk oh cho? Nantihmi ho ish pi yumihchit, Echip yakni ya ish pi kohchi tuk oh cho?

12. Echip a anumpa iluppak o e chim anolit, Ik ashá, ish pim ahni na, Echip okla ilin toksulashke, e chim achi tok keyu cho? Echip okla ha il im antia kak osh achukma kvt haiaka ai illit pi taha chi $\mathrm{ka}$ i shali hokah, im achi tok.

13. Mihma Moses vt okla hạ, Huvchi nukshopa ná, lumvt hieli cha, Chihowah im ai okchaya himak nitak a hvchi pisacha chị ka hush pisashke: Echip okla ha himak nitak a hush pisa tuk vt, himąkma anonti huvchik peso ho bilia chi hoke.

14. Chihowah yut hvchim itiba chi hoke; mihma luhmut hush hiela chị hoke, im achi tok.

15. Mihma Chihowah yot Moses a, Nantihmi ho is sa paya cho? Islael im vila vhleha ha ish im anumpuli na, tikba iashke.

16. Yohmi kia chin tubi ya vba ish wieli cha, chibbak a okhvta paknaka ya ish wieli cha, ish iti filummichashke, mikma Islael im vlla vhleha hut yakni shila yo okhvta iklonna hlopulla chi hoke.

17. Mikma Uno, yakeh, vno vt Echip okla chukvush a kvllochi likma, iakaiya chi hoke, mikma Falaoh, micha in tushka moma, micha tunvp im itichanvlli, micha im issuba ombinili puta ka holitopa isht ishe la chi hoke.

18. Mihma Echip okla hvt, Falaoh tunvp im itichanvlli, micha im issuba ombinili puta ka holitopa isht ishe likma, vno ak osh Chihowah sia ka ithana hi oke, im achi tok.

19. Mihma Chitokaka im enchil vt Islael $\underline{i}$ bina ha tikba aya tuk vt kanvllit asha ka ona tok, mihma tonik hoshonti hut tikba ia tuk vt asha ka ont hikia tok.

20. Mihma Echip okla i bina, micha Islael i bina iti takla ona tok; mihmut yummuno i hoshonti okhlili mut, amba 
iluppuno ninak o in tohwikeli tok; yohmi ho achufut ont $\underline{i}$ bilinchi keyu ho ninak vt ont hlopulli tok.

21. Mihma Moses vt ibbak a okhuta paknaka pit wieli tok; mihma Chihowah yut hushi akochaka mahli kullo ho ishtulut ninak yummak o hlopullichi cha, oka ya falummichi mut, okhuta ha yakni shila ikbi tok, mihma oka yut iti filvmmi tok.

22. Mihma Islael im vlla vhleha hut yakni shila yo okhvta iklvnna ona tok; mihma oka yut holihta tobvt isht impakimma, micha vlhfubekimma hikia tok.

23. Mihma Echip okla hvt iakaiyvt mahaya mot, Falaoh im issuba moma tunup im itichanvlli, micha im issuba ombinili puta aiena kvt okhvta iklvnna ona tok.

24. Atuk o yakohmi tok, onnahinli atoni ya , Chihowah yot tonik luak, micha hoshonti ha hlopullichit pisa cha, Echip okla tushka chipota ya komuntuchi tok.

25. Micha tunup im itichanưlli chunaha ya shụfi na, shalvllit weki tok; yohmi ma Echip okla hvt Islael vhleha i filvmmit eho tihlaiyashke, Chihowah yot Echip okla ha $\underline{\mathbf{i}}$ sanalit im itibi hoka, achi tok.

26. Mihma Chihowah yot Moses a, Chibbak a okhvta paknaka ish o welashke; yohmi na oka yot anonti vla cha, Echip okla hạ, micha tunup im itichanvlli, micha im issuba ombinili puta ompohomashke, im achi tok.

27. Mihma Moses vt ibbak a okhvta paknaka o weli tok: atuk o onnahinli ma, okhvta hut kvllo hosh falamvt tikba yatuk a chohmi tok: mihma Echip okla hot yihleput ai isso tok: mihma Chihowah yot Echip okla okhvta iklunna ho oka tưmoli tok.

28. Mihma oka vt falama cha, tunvp itichanvlli, micha issuba ombinili puta, micha Falaoh in tushka vhleha moma Islael a iakaiyvt okhvta ha et chukowa tok, moma ka ompohomo tok: achufa illa kia ik hlakoffo tok.

29. Amba Islael im vlla vhleha hokvto okhvta iklunna yvt yakni shila yo a nowa tok: mihma oka yut holihta yosh im ishtimpakimma, micha im vlhfubekimma yo $\underline{i}$ hikia tok.

30. Yakohmi hosh nitak yumma Chihowah yut Islael a 
Echip okla ibbak a kohchit okchalinchi tok: atuk o Echip okla illi vt okhvta vhli aiasha na, Islael vt pisa tok.

31. Micha Islael vt Chihowah yut nachinto fehna ho yumihchit Echip okla ha onochi ka pisa tok; mihmut okla hut Chihowah ya i nukshopa cha, Chihowah, micha Moses aiena ka i yimmi tok.

\section{CHAPTA XV.}

IHMA Moses micha Islael im vlla vhleha aiena kvt ta1 loa iluppa Chihowah ya in taloa cha, anumpulit, Chihowah ya in taloa la chi hoke, im aiyvt holitopa hoka, issuba micha im om binili kash okhuta ha oka kunivchi hoke.

2. Chihowah ak osh vmi hlampko, micha vm ilhtalowak oke, micha aiokchaya li tobushke; yummak osh an Chitokaka yoke; atuk o ai ahanta im atahli la chi hoke : aki in Chitokaka yoke, atuk o holitoblichi la chi hoke.

3. Chihowah yut hatak tunup oke, hohchifo kvt Chihowah yoke.

4. Falaoh im itichanvlli, micha in tushka chipota aiena ka okhvta pit kanchi tuk oke, in kvpetuni aiowa vhleha hash okhuta humma ya oka tumoli tuk oke.

5. Hofobi vt ompohomo tuk oke, tvli chohmit oklobushlit aka ona tuk oke.

6. Chihowah ma, chim ishtimpakimma yvt nan isht im aiahhli ya isht holitopushke, Chihowah ma chim ishtimpakimma yak osh tunvp a bushullichit kanchishke.

7. Micha chim aholitopa chinto kak osh chi sanalit hikia tuk a ish im aiyashke, chim ai $\underline{\mathbf{i}}$ nukkilli ya ish kanchi na, onush vpi chohmichit hukmit tahli tuk oke.

8. Chibishakni fiopa ma oka yot isht itunaha tuk oke: oka chito vt itvnaha chohmit wakayvt hikia tuk oke, okhvta iklunna ahofobi yut kalampi tuk oke.

9. Tunvp vt, Hlioli lashke, sakki lashke, nawehpoa ya itakushkoa lashke, sabunna tuk vt sa fihopashke, am bushpo falaia ya halullit shufi lashke, subbuk vt. ishit kanchashke, achi tuk oke: 
10. Chi mahli ha ish om mahlichi na, okhvta hut ompohomo tuk, naki chohmi kut oka chito ya a kunnia tuk oke.

11. Chihowah ma, naholbut toba puta im ai itin takla ya, kvta hak osh chishno chiyuhmi cho? nan ashvchi ik im iksho holitopa, holitopvt annohowa kvt chinto, nafehna yumihchi chia hoke: kuta hak osh chishno chiyuhmi cho?

12. Chibbak isht impakimma ya ish wieli ma, yakni vt ishit nanubli tuk oke.

13. Chishno vt ish i nukhaklo kak osh okla ha falumminchit ish chumpa tuk vt pehlichit ish kohcha tuk oke: chimi hlampko ak o ishit pehlichit ish aiahanta holitopa ya isht ish ona tuk oke.

14. Okla hut haklo cha, nukshopa hi oke; Palestina aiasha okla ha nukhâklo yosh chulkush a isha hi oke.

15. Yohmi ma Etom holitopa vhleha hvt nukhlakancha hi oke; Moab hatak kvllo vhleha hạ wunihinchi yosh onvtola hi oke, Kenan aiasha okla hut momut bilvt tumoa hi oke.

16. Nukshopa, micha komota hosh onvtola hi oke; chi shakba kvllo kak o lumvt tvli chohmi na, chim okla hut ont ia hi oke, Chihowah ma, okla ish chumpa tuk vt ont ia hoke.

17. Micha chi yakni, nunih ha ish ai vtta he yosh ish ikbi tok vt, ishtonvt ish a hilecha hi oke. Chihowah ma ; vlhtipo holitopa yummak oka chibbak osh kullochit hilechi tok vt, Chihowah ma.

18. Chihowah yưt mikko na bilivt bilia chị hoke, ahanchi tok.

19. Falaoh im issuba hot tunup im itichanvlli, micha issuba ombinili aiena kvt okhvta ha pit chukowa tuk; mihma Chihowah yut okhvta oka ya falumminchit, anonti ishtulvt onochi tuk; amba Islael im vlla vhleha hvto, yakni shila yo okhuta iklunna aya tok.

20. Mihma hopaii ohoyo Alon in tek Miliam vt timbil ishi tok; mihma ohoyo moma kvt iakaiyut timbil ieshi cha, hihihla hosh kohchut ia tok.

21. Mihma Miliam vt im afalumminchi mut, Chihowah ya 
hush in taloashke, holitopa hosh im aiya hoka; issuba, micha im ombinili kash aieninchit okhvta ha pit kanchishke, achi tok.

22. Yohmi ho Moses vt Islael a pehlichit okhvta humma ya ashut kohchi tok; mihma okla kohchut ivt, Shu yakni haiaka ya nitak tuchina anowa ona mot, oka ik ahayucho tok.

23. Atuk osh Malah ya ona mut Malah oka ya ai ishka he keyu tok; homi hatuk oka; yumohmi hatuk o hohchifo ka Malah achi tok.

24. Mihma okla hưt Moses a nanumachit, Nanta ho il ishka chi cho? achi tok.

25. Mihma Chihowah ya $\underline{\text { i }}$ paya tok; mihma Chihowah yot iti yo pisachi na, yummak ash oka pit okvchi ma, oka yut champulli toba tok; yummak o nanulhpisa bilia, micha nan isht apesa yo im ikbi tok, micha yummak o aiimoma ka pisa tok.

26. Micha Hvchin Chitokaka Chihowah im anumpa ha achunanchit hush $\underline{i}$ haponaklo cha, itikba ya ai vlhpiesa kak o yumihinchit, im anumpa vlhpisa ha hush $\underline{\mathrm{i}}$ haponaklot, $\underline{\mathrm{i}}$ nanvlhpisa puta ka hush holitoblikma ililli iluppa puta ka Echip okla ha onochi li tok vt hvchi onochi la he keyu hoke; Chihowah sia hosh hvchi hlakofichi li hoka, achi tok.

27. Atuk osh Elim, yumma kvli hofobi auahtuklo, micha pam iti pokoli untuklo hosh hiohmaya tok, mihmvt yummak o oka bilika binachi tok.

\section{CHAPTA XVI.}

A TUK osh Elim a ashtia tok: mihma Islael okla moma A kvt Sin yakni haiaka, yummut Elim micha Saina itin takla talaia kạ, Echip yakni ya kohcha tok vt hushi atukla, micha nitak ont isht auahtahlapi fehna ona tok.

2. Mihma Islael im vlla vhleha okla moma kvt yakni haiaka ya Moses, micha Alon a $\underline{\underline{i}}$ sanalit nanumachi tok.

3. Micha Islael im vlla vhleha hot, Chitokaka yvt ahni 
na, Echip yakni ya Chihowah ibbak ishit pilli tuk bano, nipi shuti yo il a chieya, micha puska vput pi kaiya ma, yakni haiaka iluppa pisht hushla, okla iluppa moma ho hohchufo isht ubit hush tahla chi hoka, im achi tok.

4. Yohmi ma Chihowah yut Moses a, Yakeh, vba ya puska et hvchi umbvche la chi hoke; mikma okla hvt kohchvt nitak moma vlhpisa kaniohmi ho aiowa bieka; yvmohmi $\underline{\mathbf{a}}$ nanvlhpisa ha anowa hinlakmá, keyukmá, nanakmá imomaka pisa la chi hoke.

5. Atuk osh yakohma hi oke, nitak ont isht hannalikma isht vla tuk vt atahla hi oke: mikma nitak moma ka aiowa beka tuk a inlohmi tukla hi oke, achi tok.

6. Mihma Moses micha Alon vt Islael im vlla vhleha moma ka , Opiakma Chihowah ak osh Echip yakni ya pehlichit a hvchi kohchi tuk a hvsh ithaiyana chi hoke.

7. Atuk osh onnahinlikma Chihowah a holitopa ya hvsh pisa chi hoke, Chihowah i sanalit hush nanumachi ka haklo hoka; mikma pishno vt nanta pia hatuk o nan ish pi nanumanchi cho? achi tok.

8. Micha Moses vt Iluppak achi hoke, Chihowah yut opiakma nipi vpa chị ho hvchima, atuk osh onnahinlikma pvska hvchimvt hvchi kaiyachikma, Chihowah yvt nanumachi ishtisanalit hush nanumachi ka haklo hoka, pishno vto nanta pia hoh cho? hush nanumachi kvt pishno ak o keyu; amba Chihowah ak oke, im achi tok,

9. Mihma Moses vt Alon a im anumpulit, Islael im vlla vhleha okla moma ka , Chihowah itikba hush lashke, hvsh nanumanchi ka haklo hoka, ish im achashke, im achi tok.

10. Atuk o yakohmi tok, Alon vt Islael im vlla vhleha okla moma ka im anumpuli ma, haiaka pilla okla pit hopokoyo ma, yakeh Chihowah isht a holitopa vt hoshonti ha fokvt haiaka tok.

11. Mihma Chihowah yvt Moses a im anumpulit,

12. Islael im vlla vhleha nanumahanchi ka haklo lishke, im anumpulit, opiakma nipi hush pa chi hoke; mikmvt onnahinlikma puska huchi kaiya chi hoke; micha vno huchin 
Chitokaka Chihowah sia ka hush ithaiyana chị hoke, ish achashke, achi tok.

13. A tuk o yakohmi tok, opia ma, kofi vt vla cha, abinanchi ka ompohomo tok; atuk o onnahinli ma fichak vt binah ha apakfopvt aiasha tok.

14. Atuk osh fichak itoyula kash osh vba ia ma, Yakeh yakni paknaka nana lumbo chipintasi, okti hvta chipintasi kut chohmi hosh aka yo asha tok.

15. Atuk o Islael im vlla vhleha hot pisa mot, ilap bika, Ilupput manna yoke, Ilvpput nanta hoh chọ? itim achit, nan ik ithano hatuk okvt; mihma Moses vt, Pvska iluppak o hush pa chi ho Chihowah yvt hvchima tuk oke.

16. Nana iluppak o Chihowah yưt apesa tuk oke, Hatak moma kvt vpa he vlhpesa kak osh aiowashke, hatak achvfa ka oma isht vlhpisa iskitini achvfat ishtia, hatak holhtina chi kaniohmi tuk a vlhpisashke hatak moma kvt binah aiasha ka im eshashke, im achi tok.

17. Mihma Islael im vlla vhleha hvt yvmihchi tok, micha kanimi kvt i shahli, kanimi kvt ik lauwo ho aiowa tok.

18. Atuk o omá isht apesa ma, laua aiowa tuk vt im atampa kut iksho ma, kanomosi aiowa tuk vt ik im ono kvt iksho tok, hatak moma kvt vpa he vlhpesa kak osh aiowa tok.

19. Mihma Moses vt A sha na kvna kia onnahinli ik im onno kashke, im achi tuk oke.

20. Yohmi kia Moses a ik i haponaklo tok; amba kanimi kvt ashanchi na onnahinli onna tok; atuk o shushi tobachi cha shua tok, mihma Moses vt $\underline{i}$ nukhobela tok.

21. Atuk o onnahinlikma hatak moma kvt vpa he vlhpesa kak osh aiowa beka tok; atuk o hushi vt lushpvt taha ma bila beka tok.

22. Atnk osh yakohmi tok, nitak ont isht hannali ma inlohmi tuklo puska hatak achvfakmvt oma tuklot ia ho aiowa tok, mihma okla moma ka pehlichi moma kut vlut, Moses a im anoli tok.

23. Mihma Iluppak o Chihowah yut achi tuk oke, Onnaha yut a foha Chihowah i nitak holitopa yoke; micha hush puska chi kvt himak nitak ak o hush puskashke; micha 
hvsh hona chi kvt hush honashke; micha hvchim atampa kvt hvchin talaia na onnashke.

24. Mihma Moses vt im apesa tuk mak o ashanchi na onna tok, mihma shua kvt iksho ma, shushi nana kia iksho tok.

25. Mihma Moses vt, Himak nitak a yvmmak o hushpashke, himak nitak vt Chihowah i nitak hullo hoke, himak nitak a haiaka ya asha na ish ahayucha he keyu kvt.

26. Nitak hannali ho hush aiowa chi hoke; amba nitak untuklo hokvto nitak holitopa hoke, yummak okvno hush ahayucha he keyu hoke, achi tok.

27. Atuk o yakohmi tok, nitak ishtuntuklo ka okla hash kanima kvt aiowa chi hosh kohchvt ia tuk osh ik ahayucho tok.

28. Mihma Chihowah yut Moses a, Katiohmi foka ho vm anumpa vlhpisa, micha a nan vlhpisa ka im antia ik hvchi bunno ka chi cho?

29. Yakeh, Chihowah yvt nitak hullo ya hvchima tuk oke; yohmi hatuk osh nitak isht hannali ka nitak tuklo puska ya huchima tuk oke; hatak hush moma kvt hush ai asha aiyukali hosh aiashashke; hatak achufa kia nitak ishtuntuklo ka aiasha tuk vt kohchvt ik aiyo kashke, im achi tok.

30. Yohmi ho okla hvt nitak isht untuklo ka foha tok.

31. Mihmut Islael in chuka achvfa yut hohchifo ha Manna, achi tok, micha tohbi kvt kolianta nihi chohmi tok; micha uput pisa ka puska lumbo foebila ishit toba chohmi tok.

32. Mihma Moses vt, Nana ka ilvppak o Chihowah yut apesa tok oke, Huchim ai itishali hvt in talaia chi ho, oma achufa ho alotolit hush talalashke; yưmohmi tuk o Echip yakni ya apehlichit huchi kohchi li mut, yakni haiaka ya puska ishit hvchipeta li tok a pisashke, achi tok.

33. Micha Moses vt Alon a, Shuti eshi cha, manna ya oma alota achvfa ho ish alotolashke, micha hvchim ai itishali vt in talaia chị ho Chihowah itikba ya ish talalashke, im achi tok.

34. Chihowah yut Moses a im apesa tuk mak $\underline{\text { g, talaia chi }}$ ho Alon vt itombi holitopa itikba talali tok. 
35. Mihma Islael im vlla vhleha hot manna vput itanowa na, afummi pokoli ushta hosh, okla aiasha yakni yo vla tok: manna vput itanowut Kenan yakni aivhli ya ona tok.

36. Yohmi ka oma vt efa kushkoa ishit pokoli hoke.

\section{CHAPTA XVII.}

WIHMA Islael im vlla vhleha okla moma kvt yakni haia1 ka Sin ashvt ia tok; micha Chihowah yvt im apesa

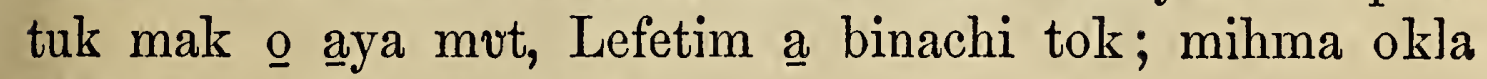
ishka he vt oka vt iksho tok.

2. Yvmohmi ho okla hut Moses a na mihachit, oka ish pima na, il ishkashke, achi tok, mihma Moses vt, Nantihmi ho na hus sa mihachi cho? Nantihmi ho Chihowah ya imomaka hush pisa cho? im achi tok.

3. Mihma okla hut yumma oka bunna tok; micha okla hut Moses a na mihachit, Katiohmi ho iluppa yumohmit pehlichit Echip a ish pi kohchi cha, pim vlla vhleha, micha pi nanvlhpoa aiena ka oka bunnuchit vbit ish pi tahla chi cho? achi tok.

4. Mihma Moses vt Chihowah ya $\underline{\text { i }}$ payut, Okla iluppa katiohmi chi la chi cho? tvli ishit sa bolahosi hoke, achi tok.

5. Milmma Chihowah yut Moses a, Islael im asunonchi whleha ha pehlichi cha, okla ha itikba ish iashke, micha chin tubi okhina ya isht ish isso tok vt ish eshi cha, ish iashke.

6. Yakeh, yumma chitikba Holeb a tuli chito paknaka hikia la chị hoke, mihma tuli ya ish issa chị hoke, mikma oka yut toli ya a kohcha na, okla hut oka ishka chi hoke, im achi tok. Mihma Islael im asunonchi vhleha hut pisa ho Moses vt yumihchi tok.

7. Mihma Islael im vlla vhleha hot na mihachi tuk ak o ; micha Chihowah ya imomaka pisut, Chihowah yut piba foka hokmá, keyukmá, nanta cho? achi tuk oka, yumma hohchifo ha Massah micha Melibah achi tok.

8. Mihma Amalek vt vla cha, Islael Lefitim a ai itibi tok. 
9. Mihma Moses vt Choshua ya, Hatak a ish atokoli cha, kohchut ivt Amalek a ish itibashke; onnakma Chihowah in tubi ya ieshi lish nunih paknaka ya hikia la chi hoke, im achi tok.

10. Yohmi ho Moses vt im achi tuk mak o Choshua vt yumihchi cha, Amalek a itibi tok, Mihma [Moses, Alon micha $\mathrm{Hu}$ aiena kut ivt nunih paknaka ya ona tok.

11. Atuk o yakohmi tok, Moses vt ibbak a vba wielikma, Islael im aiya tok; atuk osh ibbak a akvchikma, Amalek vt im aiya tok.

12. Yohmi kia Moses ibbak vt weki tok; mihma tvli eshi cha, nutaka i bohli na, om binili tok, micha Alon micha $\mathrm{Hu}$ vt achufut ibbak achufa ka vba wielichit im ieshi ma, achvfa kvt achvfa ya im eshi tok: mihma ibbak vt talaia achufa na, hvshi vt pit okatula tok.

13. Mihma Choshua vt Amalek, micha im okla aiena ka bushpo falaia halupa yo ishtimaiya tok.

14. Mihma Chihowah yut Moses a, Iluppa ithana bilia chị ho holisso ho aholissochit ish takalichashke; micha Choshua ya ish haklochashke, Amalek a ithana ya shutik nutaka ya amosholichit kanchi la chị hoke, im achi tok.

15. Mihma Moses vt alta ikbi cha, hohchifo ha CHrroWAH nissi, achi tok.

16. Chihowah yơt Amalek itim ai itishali itiakaiyvt mahaya ka itin tanampa chi hosh, Chihowah yot anumpa kvllo, il onochit anumpuli hoke, achi ha tok.

\section{CHAPTA XVIII.}

NAHOLITOMPA ishtvtta Mitian vtta Moses ipochi Che1 hlo vt Chitokaka yot Moses, micha im okla Islael aiena ka nana moma i kaniohmihinchi tuk, micha Chihowah yut Islael a pehlichit Echip a kohcha tuk a haklo mot,

2. Yohmi mut Moses ipochi Chehlo vt Sippolah Moses tekchi ya falummichit pila ha ya,

3. Micha ushi tuklo ka eshi tok, yumma ächvfa hohchifo 


\section{CHAPTA XVIII.}

kvt Keshom a tok, a yakni keyu hosh yakni inla yo ahanta li hoke, achi ha tok.

4. Mihma achvfa hohchifo kvt Eliesi atok, aki in Chito-

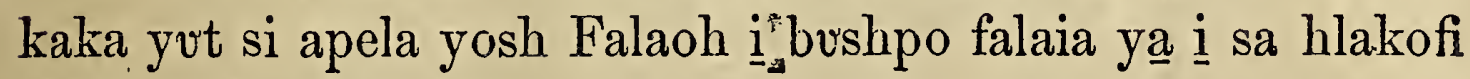
chi tuk oke, achi tok.

5. Mihmah Moses ipochi Chehlo vt Moses ushi vhleha, micha tekchi ya pehlichit yakni haiaka Chihowah i nunih ha binachit aiasha tuk im ona tok.

6. Micha Moses a Uno chipochi Chehlo sia hut, chi tekchi, micha ushi tuklo kut apeha ho pehlichit chim vla lishke, im achi tok.

7. Mihma Moses vt kohchut ivt ipochi yash ont afama cha, im akachunoli cha, $\underline{i}$ suksowa mvt, micha im achukma kvt iti ponaklo mut binah ha chukowvt okla tok.

8. Mihma Moses vt ipochi ya Islael pulla kak o Chihowah yut Falaoh, micha Echip okla aiena ka kaniohmichi tuk, micha hina takla ya na palummi kvt onvtola tuk, moma, mikma Chihowah yot hlakofichi tuk aiena tuk im anoli tok.

9. Mihma Chihowah yvt Islael a nana achukma ho yumihchit, yummak oka Echip okla ibbak? a kohchit hlakoffichi tuk aiena ka Chehlo vt nayukpa tok.

10. Micha Chehlo vt, Chihowah yummak osh Echip okla ibbak a kohchit micha Falaoh ibbak a kohchit huchi hlakoffichi tuk vt holitopashke; yummak osh okla hvt Echip okla ha ibbak nutakvt aiasha tuk a hlakoffichi tuk vt;

11. Himak a Chihowah ak osh naholba puta moma $\underline{\mathrm{i}}$ shahli ka ithana lishke; nana ka ilefehnvchi hosh yumohmi tuk a $\underline{\mathrm{i}}$ shahli hoka, achi tok.

12. Mihmot Moses ipochi Chehlo vt Chihowah ya hukmit im issa ya èshi cha, vbit im issa tok; mihma Alon, micha Islael im asunonchi moma kvt vla cha, Moses ipochi ya Chihowah itikba okla ibai impa tok.

13. Atuk osh onnakma yakohmi tok, Moses vt okla ha nan im apesut binili tok: mihma okla hut Moses a $\underline{\mathrm{i}}$ hinli na, onnahinli tuk vt opia tok.

14. Atuk o Moses ipochi vt okla ha kaniohmichi moma ka pisa mut, Nanta mak o okla ish yumihchi cho? Nantih- 
mi ho chishno ak bieka hosh ish binili na, okla moma kvt chi hieli na, onnahinli tuk vt opia hoh cho? im achi tok.

15. Mihma Moses vt ipochi ya Chitokaka ya $\underline{\text { i }}$ ponakla chi kvt, vm vla hoka, im achi tok,

16. Achvfa hosh inla ya nan iti kaniohmi cha, vm vlakma im itin takla ya nan im apesa li hoke; micha Chitokaka i nanvlhpisa, micha i nanvlhpisa bilia ya ithunanchi li hoke, im achi tok.

17. Mihma Moses ipochj vt, Nana ish yumihchi kvt achukma keyushke.

18. Chishno, micha okla iluppa chi apeha aiena kut hoyublit huchi taha chi hoke; nana ilupput chi weki fehna hoka, chishno ak bano kut ish atahla he keyu hoke.

19. Um anumpa is sa haponaklashke, na chi miha lashke, mikma Chitokaka yut chiba foyukashke; mikma chishno vt okla ha Chihowah isht ai anumpuli chia hashke : yumohmi na, nan im akaniohmi ka Chitokaka ya isht ish im onashke.

20. Micha nana akaniohma he nanvlhpisa, micha hina ataya he micha natoksuli ya yumihcha he aiena kak o ish im abvchashke.

21. Micha okla moma ka akohchvt hatak kvllo, Chitokaka i nukwiya, hatak ahli, nan anushkunna ya shitilema ak $\underline{0}$ ish ahayuchashke; micha yohmi kak o, tahlepa sipokni pehlichi, tahlepa pehlichi, pokoli tahlapi pehlichi, micha pokoli pehlichi ish $\underline{\mathrm{i}}$ hiohlechashke.

22. Mikma nitak moma okla ha nan im apesashke, atukma yakohmashke, nana chito moma ka isht chim vlashke: nana iskitini moma ka ilapinli hosh apesashke: yohmi ho chishno vt ik chị kullokma, na weki ka okla chiba shaiyalashke.

23. Iluppak o ish yumihchikma, micha Chitokaka yvt yumihchit chim apesakma, yohmikma ish hlopulla chi hoke; mikma okla iluppa moma kvt nuktaiyalut im aiasha ya onashke.

24. Yohmi ma Moses vt jpochi im anumpa i haponaklo cha, nana achi tuk a moma yumihchi tok.

25. Mihmovt Moses vt Islael moma ka hatak kvllo hạ ato- 
kolit okla ha i nushkoboka ikbi na, tahlepa sipokni pehlichi, tahlepa pehlichi, pokoli tahlapi pehlichi, micha pokoli pehlichi tok.

26. Mihma nitak moma ho okla nan im apesa tok; nana kullo kuno Moses a isht im ona cha, amba nana iskitini kailapak inli hosh apesa tok.

27. Mihma Moses vt ipochi ya ik ia ahni na, ia tok; mihma iut ilap $\underline{i}$ yakni ya ona tok.

\section{CHAPTA XIX.}

TSLAEL im vlla vhleha hut Echip yakni ya kohcha tok, 1 hushi ont isht atuchina mihi yo nitak fehna Sinai yakni haiaka ya ona tok.

2. Lefitim a asha tuk vt ja tuk osh Sinai chukushmi ya ona cha, yakni haiaka ya binachi tok; micha yummak $\underline{0}$ Islael vt nunih itikba ya abinachit aiasha tok.

3. Mihma Moses vt oiyut Chitokaka ya, im ona tok; mihma Chihowah yut nunih ha Moses a et ai $\underline{i}$ howut Chekob in chuka achvfa ya yak ish im achashke, micha lslael im vlla vhleha ha ish im anolashke.

4. Echip okla hạ nana ka kaniohmichi li ka hvsh pisa tuk oke; micha ossi sanahchi om binilichit shaiyalit a bilika hvch isht vla li tuk a hush pisa tuk oke.

5. Yvmohmi hoka himak a vm anumpa ha hush im asitia muhli cha, a nan itim apesa y $\underline{a}$ hvsh holitoblikmvt, yohmikmot okla moma 1 shahlit vmmi huchia ha chi hoke; yakni vt moma kvt vmmi hoka.

6. Mikma vm apehlichika na holitopa isht asha, micha okla holitopa, huchia ha chi hoke, ish im achashke. Anumpa iluppa puta kak o Islael im vlla vhlcha ha ish im anumpulashke, achi tok.

7. Mihma Moses vt vla cha, okla im asunonchi vhleha ha hoyo cha, anumpa ilvppa moma Chihowah yot im apesa tuk a itikba bohli tok.

8. Mihma okla moma kvt itibafokvt afalamichit Chihowah 
yout anumpuli tuk moma kak o e yumihcha chi hoke, achi tok. Mihma Moses vt okla im anumpa ha falamichit Chihowah ya isht im ona tok.

9. Mihma Chihowah yot Moses a, Yakeh, hoshonti sukko ho a foyukvt chim vla la chị hoke; yumohmi ho chitim anumpule likma okla hot haklo cha, chi yvmmi na bilia chi hoke, im achi tok. Mihma Moses vt okla im anumpa ha Chitokaka ya im anoli tok.

10. Micha Chihowah yut Moses a , Ivt okla hạ im ona cha, himak nitak, micha onnaha ya ish hullochashke, mikma $\underline{i}$ nafoka y $\underline{a}$ achefa cha,

11. Nitak atuchina chị ka im vlhtahashke; nitak ont atuchinakma okla moma kvt apisa ho Chihowah yvt akowut Sinai paknaka ya vla chi hoke.

12. Mikma ai vhli apakfoyupa ho okla ha im ikbit, Ahah hush ahni cha, nunih ha hvchik oiyo kashke, micha aivhli ya huch ik potolo kashke; kuna kia nunih ha potolikmut illi pulla hi oke.

13. Ibbak achvfa kia potola he keyu; amba yohmikmut tvli ishit boa he, keyukmot nvhlut hlopulla hi oke, vlhpoa yokmá is keyukmut hatak okmá nana kia okchaya he keyu hoke, ishtpufa vt ola kvt falaiakma nunih ha vla hi oke, ish achashke, im achi tok.

14. Mihma Moses vt nưnih ha akowvt okla ha im ona cha, okla hash hullochi tok, mihma i nafohka ya achefa tok.

15. Mihma okla hạ, Nitak atuchina chi ka hvchim vlhtahashke, hvchi tekchi vhleha ha bilika hvochik ono kashke, im achi tok.

16. Atuk o yakohmi tok, nitak ont atuchina onnahinli ma, hiloha, micha hushukmvlli, micha hoshonti sukko aiena kvt nunih ha onvtoyula ma, ishtpufa falaia ola kvt chitoli atampa tok: yohmi na okla moma binah asha tuk vt wunihinchi tok.

17. Mihma Chitokaka ya itafama chị ka Moses vt okla pehlichit binah ha kohchi tok; mihma nunih akishtvla ak $\underline{o}$ hieli tok.

18. Mihma Sinai nunih hut shobulli bieka tok, Chihowah 
yot luak afoyuka akowvt vla tuk oka, mihma shobulli vba ia kvt tuli abila shobulli kak a chiyuhmi ma, nunih hash osh momot winakahanchi fehna tok.

19. Atuk $\mathrm{o}$ ishtpufa falaia ola kvt falaivt chitoli kvt isht $\underline{\mathrm{i}}$ shahlit mahaya ma, Moses vt anumpuli ma Chitokaka yvt anumpa ha isht im afalaminchi tok.

20. Mihma Chihowah yot akowvt nunih paknaka Sinai nưnih paknaka ya vla tok; micha Chihowah yut Moses a hoyot nunih paknaka isht ona tok. Mihma Moses vt oiya tok.

21. Mihma Chihowah yut Moses a, Akowa cha, okla ha atokolit ish $\underline{\mathrm{i}}$ mihashke, vbanublit ont ia cha, Chihowah ya aiokchilefi cha, lauvt illi yoba ná.

22. Mikma naholitopa isht asha vhleha hut Chihowah bilika ona kvt ile hullochashke, keyukma Chihowah yut onvtola ná, im achi tok.

23. Mihma Moses vt Chihowah ya , okla hut nunih Sinai ha vla he keyu hoke; atokolit ish pi miha kvt, Nvnih apakfoyupa ka aivhli ya hush hilechi cha, hush hullochashke, ish achi tuk oka, im achi tok.

24. Mihma Chihowah yvt, Mahyá, akowá, atukmvt ish oiya chi hoke, chishno, micha Alon aienut: amba naholitompa isht asha, micha okla aiena kvto vbanvblit Chihowah ya ik im ono kashke. Chihowah yut onvtola ná, im achi tok.

25. Yohmi ma Moses vt akowvt okla im ona cha, im anumpuli tok.

\section{CHAPTA XX.}

\section{IHMA Chitokaka yot anumpa iluppa moma ka anum- 1 puli tok,}

2. U'no ak osh Chin Chitokaka Chihowah sia hosh Echip yakni, micha yuka aboha ya apehlichit chi kohchi li tuk oke.

3. Naholbat toba inla yot sa tikba chim aiasha he keyu hoke.

4. In chuwwt naholbat toba, keyukmvt nana kia shutik 
vba aiasha, keyukmut nutaka yakni aiasha, keyukmvt yakni nutaka oka ai asha holba ya ish ikba he keyu hoke.

5. Aka ish im akahchunoli, micha ish aiokpvcha he keyu hoke, Chin Chitokaka Chihowah sia hvt Chitokaka nuktahahla sia hosh iki vhleha nanashvchi tuk a im vlla vhleha ai itishali ont atuchina, micha ont ai ushta kia a nukkilli ka im aya li hoke.

6. Micha tahlepa sipokni kia sa holitobli cha, vm anumpa ha holitoblikma i nukhaklo li hoke.

7. Yummakfokalechit chin Chitokaka Chihowah hohchifo ha ish hochifa he keyu hoke; hohchifo ha yummakfokalechit hochifo tukma nan ik ashacho, Chihowah yot ahna he keyu hoke.

8. Nitak hullo nitak a ish ithaiyana cha, ish holitoblashke.

9. Nitak hanali ish toksuli cha, chin na toksuli moma ka ish atahlashke.

10. Amba nitak ont isht untuklo hokvto chin Chitokaka Chihowah $\underline{i}$ nitak hullo yoke, yummak ak o toksuli nana kia chishno, micha chisso, micha chisso-tek chin tishu nakni, micha chin tishu ohoyo, micha chi nanvlhpoa, micha okla inla yosh chị holihta okhissa anukaka chị hikia aiena kut ish yumihcha he keyu hoke.

11. Nitak hannali ho Chihowah yut vba, micha yakni, okhuta, micha nana yumma aiasha moma ka ikbi cha, nitak isht untuklo ka foha tok oke; yumohmi ha tuk osh Chihowah yot nitak hullo nitak a holitoblichi cha, hullochi tok oke.

12. Chiki, micha chishki ya ish holitoblashke; yumohmi hosh chi nitak vt falaia hosh yakni ya Chihowah chin Chitokaka yut chima tok a ish aiahantashke.

13. Kưna kia ish bi ná.

14. Hush itin lumąka ná.

15. Ish hụkopa ná.

16. Chin chuka a bilika hatak a $\underline{\mathrm{i}}$ sanalit anumpa ik anolit ish atokoli ná.

17. Chin chuka a bilika hatak th chuka ya ish anushkunna ná: chin chuka a bilika hatak tekchi, micha in tishu, micha in tishu ohoyo, i wak toksvli, micha im issuba haksobish 
falaia, micha nana hosh chin chuka a bilika hatak a immi hokma ish anushkunna ná, achi tok.

18. Mihma okla moma kvt hiloha, micha hushukmvlli, micha ishtpufa falaia vt ola, micha nvnih hvt shobulli aiena ka pisa tok; micha okla hut pisa mut, kanvllit hopaki ho hieli tok.

19. Micha Moses a, Chishno ak osh ish pim anumpulikma e haklashke; amba Chihowah yot ik pim anumpulo kashke, pilli yoba ná, im achi tok.

20. Mihma Moses vt okla hạ, Hưchi nukshopa ná, huchi tikba ya Chitokaka im anukshopa yvt takali na, ik huchi yoshobo ka chị ho imoma ka huchi pesvt vla hoke, im achi tok.

21. Mihma okla hvt hopaki ho hieli tok, mihma Moses vt okhlili sukko Chitokaka yut abinili ka bilika ia tok.

22. Mihma Chihowah yut Moses a, Yakohmichit Islael im vlla vhleha ha ish im achashke. Vba et a hvchim anumpule li ak inli ka hush pisa tuk oke.

23. Sa takla ka tvli hvta naholba hvsh ikba he keyu hoke; micha hưchimmi a he tuli lakna naholba hush ikba he keyu hoke.

24. Lukfi yo alta is sum ikbi cha, hukmit isht aiokpvchi, micha iti nanaiyvchi isht aiokpvchi, chin chukfi, micha chi wak aiena ka vbit ai onochit isht ish ai okpvchashke, $\mathrm{Ka}$ nima moma sa hohchifo ha a bohyuli likmut hvchim vla likmut huchi yukpali la hi oke.

25. Tuli yo alta is sưm ikba chi hokmut tuli tuhlahli yo isht ish ikba he keyu hoke; chim ishit tuhlachi ya ish o wielikmvt ish litehla chi hokvt.

26. Micha atuya yo iut vm alta ya chik ono kashke; yvmohmi ho o hikivt chi shahbi vt yvmmak o ik ahaiako kashke, im achi tok.

\section{CHAPTA XXI.}

VUMOHMI ka nan ishtapesa iluppa puta kak o itikba I ya ish in takali chashke. 
2. Heblu tishu ish chumpakma, afưmmi hanali ho chim antiashke; atukmut afummi isht untuklo ka nanvlhtoba keyu, pilla ho yuka issut kohchvt ia hi oke.

3. Ilap bano hosh vla tukmvt, ilap bano hosh kohcha hi oke; ohoyo im asha ya tukma, tekchi vt awant kohcha hi oke.

4. I shahli hut ohoyo ima tuk $\underline{\mathrm{o}}$, ushi vhleha, micha ushetik vhleha aiena ka im eshi tukma, tekchi yash micha im vlla vhleha aiena kuto $\underline{i}$ shahli ak $\underline{\mathrm{o}} \mathrm{immi}$ ha hi oke; mikma ilap bano hosh kohchet ia hi oke.

5. Atuk o tishu hash osh atokolit, $\underline{\mathrm{A}}$ shahli, sa tekchi micha vm vlla vhleha ha a holitopvshke, yuka issut kohchvt ia la he keyushke, achikma,

6. Yohmikma $\underline{i}$ shahli hvt nan apesa vhleha ha isht im ona hi oke; micha okhissa, keyukmvt okhissa lapali ya isht ona he ak inli hoke, mikmvt i shahli hut haksobish a chofak ishit bahlit hlumpla hi oke, mikma in toksanli na bilia hi oke.

7. Micha hatak osh ushetik vt tishu ohoyo ya chi ho kanchikma, hatak tishu chohmit kohchvt ia he keyu hoke.

8. I shahli ilapovchi tuk vt ik ahninchokmvt falvmmichit ik chumpá, ahna hi oke: oklushi inla i kanchi hokvto a yumohma he keyu ka hi oke, ik im ahlo hosh yumihchi tuk okvt.

9. Micha ushi ak o im apoachi tuk okmvt, ushetik vhleha akinli chohmicha hi oke.

10. Ohoyo inla ishikmvt im ilhpak, $\underline{\mathrm{i}}$ nafohka, micha itauwaya he aivlhpesa vt ik onancho ka he keyu hoke.

11. Tuchina ilvppa i yumihcha he keyukmvt, toli holisso vlhtoba keyu kia pilla ho kohchvt ia hi oke.

12. Kvna hosh hatak a isso na, illikĭha illi pulla hi oke.

13. Micha im aiehchi tuk keyu, amba Chitokaka ak osh ibbak a fohki tukma, malelit ai ona ya chim apesa la hi oke.

14. Amba hatak osh ilapunlvt haksichit chuka a bilika

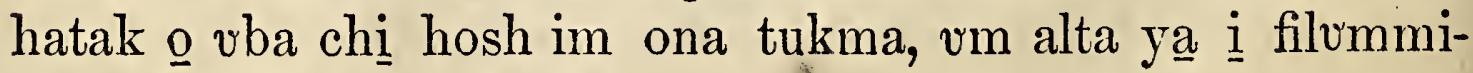
chit isht ish ia na, jllashke.

15. Mikma kuna hosh ilki micha ishki ya issokmvt illi pulla hi oke. 
16. Micha kuna hosh hatak a hukkopa cha, kanchi tuk, keyukmvt ibbak a fohkvt haiakakma illi pulla hi oke.

17. Mikma kuna hosh iki micha ishki ya , kalakshichit mihachikmvt illi pulla hi oke.

18. Mikma hatak vt iti kvllot ashwa tuk $\underline{o}$, achvfut tvli,

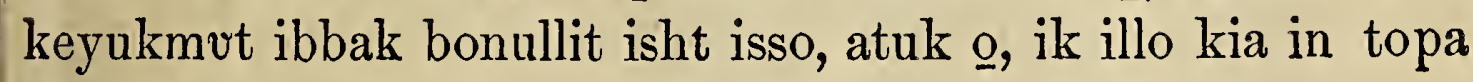
itoyulakma,

19. Atuk osh anonti wakaya cha, in tubi ieshit kohchvt hikikiakma, isso tuk vt ikashofa hi oke, peh nitak i kvnia tuk ak o atobba hi oke, micha hlakofichit vmohmicha hi oke.

20. Mikma hatak osh i yuka hatak, keyukmvt i yuka

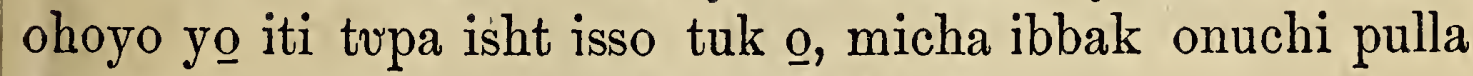
ho illikma, nahotopa onvtula pulla hi oke.

21. Yohmi kia itonla na, nitak achvfa, keyukmvt nitak tuklo ona tuk okmuno, nahotopa onvtula he keyu hoke, in twli holisso ya tuk oka.

22. Hatak vt iti kvillo cha, ohoyo vlla shali yo hotopali, yohmi na, vlla vt im akvma, yohmi kia achahaya kvt ik aiokpulokma, ohoyo $\underline{i}$ hatak ak osh apesvt onochi ho, nahotopa onvtula pulla hi oke, micha nan apesa vhleha hvt apesa ho atobba hi oke.

23. Amba achạka kvt aiokpulokma, fiopa hatuk a fiopa akinli ho hvsh abanalashke.

24. Nishkin okma nishkin, noti yokma noti, ibbak okma ibbak, iyi yokma iyi,

25. Hushmi yokma hushmi, nipi okpuni yokma nipi okpuni, fuma hokma fuma hashke.

26. Mikma hatak osh $\underline{\mathrm{i}}$ yuka hatak, keyukmvt $\underline{\mathrm{i}}$ yuka ohoyo yo nishkin a isso na, nishkin vt kvniakma, nishkin atuk mak o yuka issut ik ia, im ahnashke.

27... Micha $\underline{i}$ yuka hatak noti $\underline{\text { a, }}$ keyukmut $\underline{1}$ yuka ohoyo noti a issot kohchikma, noti atuk mak o kohchvt ik iá, im ahnashke.

28. Wak vt hatak o, keyukmvt ohoyo ho bahli na, illikma wak ash osh tvli ishit boa pulla hi oke, mikma nipi ya vpa he keyu hoke; amba wak immi vto $\underline{i}$ kashofa hi oke. 29. Amba wak vt chashpo ka lvpish isht bahli chatuk, 
mihma immi ya atokolit im anoli tok atok okma, atuk o ik im okhishto ho, hatak, keyukmvt ohoyo ho vbikmvt wak vt tvli ishit boa hi oke; mikma immi hvt illi ak inla hi oke.

30. Tuli holisso atobba he yo onochikma, im ilhfiopak vlhtoba ka kaniohmi ho onochi tukma atobba hi oke.

31. Ushi yo bahli tuk okma, keyukmut ushetik o bahli tuk okma, nan vlhpisa iluppak o ai iakaiya hosh $\underline{\mathrm{i}}$ yumihcha hi oke.

32. Wak vt hatak yuka ho, keyukmut ohoyo yuka bahlikma, í shahli ya tuli huta shekel pokoli tuchina ho imakma, wak ash osh tvli ishit boa hi oke.

33. Micha hatak osh hichukbi a tiwi, keyukmvt hichukbi ya kulli tuk osh ik ompohomo tuk $\underline{\underline{o}}$, wak, keyukmvt issuba nashoba yokma nana hosh aka itolvt fohakma,

34. Hichukbi immi atuk vt immi yatuk ash tvli holisso ima cha, atobba hi oke, micha nan vlhpoba illi ak osh immi hashke.

35. Micha hatak i wak ut inla immi ho okpuni na illikma, mikma wak okchaya ka kanchi cha, vlhtoba tuk tuli holisso yash ita kashapashke, mikma wak illi ak kia ita kashapashke.

36. Micha chashpo ka wak vt bahli chatuk a ithana atuk ak osh, immi vt ik im okhishto tukmvt; yohmikmvt wak a wak ak inli ho atobbashke; micha illi ak osh immi hashke.

\section{CHAPTA XXII.}

EATAK osh wak, keyukmvt chukfi yo hukkopa cha vbi, 1 keyukmot kanchikmot, wak atukma wak tahlapi, chukfi yatukma chukfi ushta ho falvmmichit atobbashke.

2. Hukupa ut tivbli hosh anta na, pisa cha isso tuk o illikma, yummak okvno vlhtobvt issish vt hlatapa he keyu hoke.

3. Hushi vt kohchut on tommi ha yokmvno vlhtobvt issish hlatapa hi oke, mominchit atobba he tuk oka; nana ik im ikshokma, hukupa tuk ak o kanchashke.

4. Nana hukkupa tuk vt okchaya moma hosh wak, keyuk- 
mut issuba nashoba, keyukmut chukfi yokmá nana hosh ibbak fohkvt haiaka ahlikma, inlohmi tuklo ho atobba hi oke.

5. Hatak osh osapa, keyukmut pakupi ahollokchi yo ai impuchi, micha $\underline{i}$ nanvlhpoa ya hatak inla im osapa yo fohkit ai impuchikmvt, im osapa achukma moma i shahli, micha palkvpi im ahollokchi achukma moma i shahli ho isht atobba hi oke.

6. Luak vt toba cha, kvti ha lapali na, onush talakchi, keyukmvt onush hikia vt, keyukmvt osapa vt luvt tahakma, luak tikelichi tuk vt atobbi pulla hi oke.

7. Hatak osh chuka a bilika hatak o tuli holisso, keyukmvt nachukushpa yo im îsha chi ka ibbak fohki tuk $\underline{\alpha}$, kvna hosh hukkopvt hatak ash in chuka ya ai ishit kohcha tuk osh hụkopa vt haiakakmvt inlohmi tuklo ho atobbashke.

8. Hukopa tuk vt ik haiakokma, chuka yash $\underline{i}$ shahli ya yummak osh in chuka abilika i nachukushpa ya potoli tuk oh chishba, ahnit nan apesa vhleha ha itikba isht onashke.

9. Anumpa kobuffi moma wak a tuk, issuba nashoba atuk okma, chukfi, nafohka, keyukmut nana kunia, nana kia inla hosh immi mihakma, anumpa ahalaia in tonnvp bika kvt nan apesa vhleha ha itikba onashke : atuk o kanima ho nan apesa vhleha hvt onochikma in chuka abilika hatak a inlohmi tuklo ho atobbashke.

10. Hatak osh in chuka abilika hatak osh im isha chị ho, im issuba nashoba, keyukmvt wak, keyukmvt chukfi, keyukmut nanvlhpoa nana kia ibbak fohki tuk $\underline{o}$, kuna hosh pisa keyu ho illi, keyukmut im omokpulo, keyukmvt tihlelit kanima isht ia tukma.

11. Yohmikma in chuka abilika hatak im vlhpoyak a ik potolo tuk vt Chihowah ya anumpa kvllo im il onochi ya ita tuklot ashwa ma anumpulashke : mikma immi yatuk vt vlhpesa ahni na, ik atobbo kashke.

12. Micha i hukopa tuk okmvt immi ya im atobba hi oke.

13. Nana hosh niblit tahli tuk okma, isht atokowa ya isht vlashke, mikma niput taha tuk ano atobba he keyu hoke. 
14. Micha hatak osh in chuka abilika hatak o nana ho im a pota tuk o immi hot awant aya keyu ho aiokpuloka, keyukmut illikma, atobbi pulla hi oke.

15. Amba immi vt awant aya hokma atobba he keyu hoke: nan ilhtohno yatuk okmut nana asitubachi ha tuk oke.

16. Mikma hatak osh ohoyo himita yo haksichi cha, iba tushki tukmut im atobbi cha, iba itauwayashke.

17. Iki vt ima he keyu kvt a hlikma, ohoyo himita im vlhtoba chatuk mak o tuli holisso im atobbashke.

18. Ishtahullo ohoyo hut ik okchayá, ish ahna he keyu hoke.

19. Kuna hosh nanvlhpoa ya iba tushkikmvt illi pulla hi oke.

20. Chihowah ak illa keyu, na holbut toba nana kia nan vbit isht aiokpvchikmut ishit kanchit vmohmicha hi oke,

21. Okla inla chukvsh okpunit ish ilbusha la he keyu hoke. Okla inla yosh Echip yakni hush aiasha tok okvt.

22. Ohoyo i hatak illi, micha vlla iki illi, kuna kia hush ilbushala he keyu hoke.

23. Kaniohmichit hush ilbushali na, et a pạhaya ka haklo li pulla hi oke.

24. Mikmvt um i nukkilli vt libbi fehnakmvt, bvshpo falaia ho ishit chibe la hi oke, mikma hvchi tekchi vhleha hvt i hatak illi yokma, hvchvm vlla vhleha hvt ilki illi ya hi oke.

25. Um okla ilbusha yut huchi bilika ahanta ka tvli holisso ish im pota hokmvt, im atablit chelichi a ish in chohma he keyu, micha chelichi ish onocha he keyu hoke.

26. Chin chuka abilika hatak i nafohka ya nan isht atoni jsh im eshi tuk mot, hvshi vt okatula takla ka ibbak a ish fohka hi oke.

27. Yummak illa ho anchi yatuk oke; haknip alata yatuk oke, nanta ho fohkvt nusa chi cho? atukma yakohma hi oke, a pahayakma, haklo la hi oke, nan ikana sia holvut.

28. Chitokaka vhleha ha ishit yopomot ish anumpula he keyu; micha chim okla i miko ya kalakshichit isht ish anumpula he keyu hoke.

29. Chi na waya ummona ya chim oka paki, micha alif 
10. Micha afummi hannali ho chi yakni ya ish hokchi cha, na waya ya ish ai vmashke.

11. Amba afummi ont ishtuntuklo hokvno, fohvt lumvt itoyulashke; yumohmi ho chim okla ilbvsha yvt ai vpashke; atuk o ashanchi na, nampoa oktak aiasha ak osh vpashke. Pakvpi chim ahollokchi, micha alif chim ahollokchi aiena yummak inli ish chohmichashke.

12. Nitak hannali ho chi pilesa ha ish yumihchashke; atuk osh nitak isht untuklo ka ish fohashke; yumohmi ho chi wak, micha chim issuba nashoba, micha chi yuka ohoyo ushi, micha okla inla aiena kvt fohashke.

13. Nana moma huchim ahanchi li tuk a , ahah, hvsh ai ahnashke; micha naholbvt toba hohchifo ha hvchik anolo kashke, micha chitukha a kohcha na ik haklo kashke.

14. Afummi achufakma, hituchina ha impvchi isht is sum asha hi oke.

15. Pvska ik shatummo impvchi isht hush asha hi oke, hushi Abib vlhpisa hi a chim apesa li tuk mak $\underline{o}$, nitak untuklo ho puska ik shatummo ish pa hi oke: hushi yumma Echip a ish kohcha tok okvt; achufa kia nan iksho ho sa tikba ya hikia he keyu hoke.

16. Micha hush toksuli osapa ya nana ish hokchi tukma nawaya vmmona vmo impvchi, micha nahoyo impvchi, afvmmi ont tahakma osapa ish atoksvli tuk vt na ish hoyokmvt isht hush asha hi oke.

17. Afvimmi achvfakma hituchina ha nakni yosh hwchim aiasha moma kvt Chithowah Chiokaka itikba ya hiela hi oke.

18. Vbit ishit vm aiokpvchi issish a pvska shatummi ya aieninchit is sum issa he keyu hoke; micha vbit isht vm aiokpvchi nia vt asha na onna he keyu hoke.

19. Chi yakni awaya vmmona tikba yo Chihowah chin Chitokaka im aboha ya isht ish ona hi oke. Issi kosoma ushi yo ishki i pishukchi a isht ish honna he keyu hoke.

20. Yakeh, ai atahli le tuk vt chisht ala la chi, hina chi fohkit chisht aya chi ka enchel o chitikba et hilechi li tok.

21. Ahah ish im ahni cha, im anumpa im antivt, chik 
nukowacho kashke, na hvchi yoshuba ya chi kashofa he keyı; sa hohchifo kvt iba foyuka hoka.

22. Amba im anumpa ha ish im antia cha, anumpohonli li moma ka ish yumihchi ho muhlikma, chin tvnvp vhleha ha in tunvp sia ha he; micha chi sanali vhleha ha $\underline{\mathrm{i}}$ sanali sia ha hi oke.

23. Um enchel vt chi tikba ia cha, Amolait, micha Hittait, micha Pelissait, micha Kananait, micha Hifait, micha Chebusait aiena aiasha ka chisht onakma, ai ishit kanchi la hi oke.

24. I naholbvt toba puta ish im akahchunola he keyu, micha ish im antia he keyu, micha nana atoksvli ya iakaiyvt ish yumihcha he keyu hoke; amba hush akvchit hush vmohmichi cha, $\underline{\mathrm{i}}$ nan in chuwa ya kinahlichit ish kaiyancha hi oke.

25. Micha Chihowah hvchin Chitokaka ya hvsh im antia hi oke; mikma hvchi pvska, micha hvchim oka hvchim apaknvcha hi oke; mikma vno vt hvchim itin takla ya abeka ha ai isht kanchi la hi oke.

26. Nana kia huchi yakni asha kut ushi kanchi, micha ushi iksho kvt iksho ka hi oke, chi nitak holhtina ka onanchi la hi oke.

27. An nukshopa ka chi tikba ya pila likmvt, okla moma ish im ona ka ishit kanchi la chi hoke; micha chin tunup moma ka chi filummichi la hi oke.

28. Micha fohkvt puta ka chitikba ya pila likma, Hifait, Kananait, micha Hittait chi tikba tihlela chi hoke.

29. Afummi achufa ho chi tikba tihlelit kohchi la he keyu; yohmi na yakni vt chukillissakma, nampoa hvt apaknvt chi sanala hinla hoke.

30. Kanomosi anonti kanomosi ho chi tikba tihlelit kohonchi likma, ish apaknvt yakni ya ish a binila hi oke.

31. Micha chim aivhli ya okhvta Humma vttut ia hosh Filistin im okhvta ont vhli, micha chokushmi vttvt ia hosh

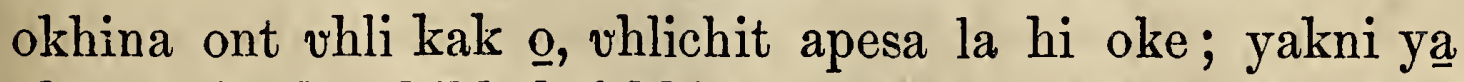
okla aiasha ka chibbak fohki la chị hoke; mikma chi tikba tihlelit ish kohcha hi oke. 
32. Yumma, micha i naholbut toba puta ka nana ish itim apesa he keyu hoke.

33. Chi yakni ya aiasha he keyu a, chi yoshublicha hinla hoka; i naholbut toba ya ish im antiakma ishit chi yoshuba chi pulla hoke im achi tok.

\section{CHAPTA XXIV.}

MICHA Moses a , Chishno micha Alon, Natab, micha 1 Abihu, micha Islael im asunonchi pokoli untuklo aienvt Chihowah ya oiyut hush im vlashke: micha hopaki ho hielit hvsh aiokpvchashke.

2. Mikma Moses ak illa hosh Chihowah ya bilika ona chi hoke: amba yummak okvto bilika ona he keyu, micha okla hot apehot oiya he keyu, im achi tok.

3. Mihma Moses vt vla cha, Chihowah im anumpa iluppa moma ka, micha nan isht apesa iluppa moma ka okla ha im anoli tok; mihma okla moma kvt anumpa achvfut afalamichit, Anumpa moma Chihowah yut achi tuk mak o e yumihcha chi hoke, achi tok.

4. Mihma Moses vt Chihowah im anumpa moma ka holissochi tok, atuk osh onnahinli fehna wakaya cha, nunih nutaka ya alta, micha Islael apehlichi auahtuklo vlhpisa ho tonik auahtuklo ikbi tok.

5. Mihmvt Islael im vlla vhleha hatak himithoa ya pila na, yummak ash osh hukmit ishtaiokpvchi, micha iti nanaya yosh isht aiokpvichi aiena wak vbit onochit Chihowah ya im issa tok.

6. Mihma Moses vt issish a iklunna ho ishi ch, ampoa mahaia uni tok, mihmvt issish a iklunna alta yash o fimmi tok.

7. Mihmut nan itim apesa holisso ha eshi cha, okla hut hakklo ho itim anumpuli: tok; mihma okla hut, Chihowah yut achi tuk, moma kak o e yumihcha chi hoke, micha il im antia chi hoke, achi tok.

8. Mihma Moses vt issish ash eshi cha, okla ha o fiimmi 
mvt, Yakeh, nan itim apesa issish, Chihowah yvt anumpa iluppa puta ka isht hvchitim apesa tuk mak oke, achi tok.

9. Yohmi ma Moses, micha Alon, Natab, micha Abihu, micha Islael im asononchi pokoli untuklo aienvt oiya tok.

10. Micha Islael in Chitokaka ya pisa tok; micha iyi nuta ya saffai tuli yosh a patulhpo ha tok, micha shohkauali kvt shutik fehna kak o chohmi tok.

11. Mihma Islael im vlla vhleha $\underline{i}$ holitopa vhleha ha ibbak ik onocho tok: yummak kia Chitokaka ya pisa mut, imput nan ishko tok.

12. Mihma Chihowah yut Moses a, Nunih ha oiyut is sum vlashke, micha yvmmak o ish antashke, mikma nanvlhpisa, micha anumpa vlhpisa holissochi li tuk vt tuli patussa chima lashke; yumohmi ka ish im abvchashke, im achi tok.

13. Mihma Moses micha in tishu Choshua vt wakaya tok; mihma Moses vt Chitokaka i nunih ha oiya tok.

14. Micha asunonchi vhleha hạ, Ashvt hush pi hoyo tuk o, anonti e chim vlashke; mikma yakeh, Alon, micha Hu vt hush apehushke, hatak kuna kia nana a yumihcha he vt im ashakmvt, yummak o im vlashke, im achi tok.

15. Mihmut Moses vt nunih ha oiya tok, mihma hoshonti vt nunih ha ompoholmo tok.

16. Mihma Chihowah im a holitopa vt Sinai nunih ha on talaia ma, hoshonti hut ompoholmo na, nitak hannali tok; atuk osh nitak isht untuklo ma Moses a hoshonti iklunna ai i howa tok.

17. Mihma Chihowah im a holitopa pisa ka Islael im vlla vhleha hut pisa ho luak nahukmit tahli ak o chohmi hosh nunih paknaka talaia tok.

18. Mihma Moses vt ivt hoshonti iklunna ka ibachukowvt nunih ha oiya tok; micha Moses vt nunih ha ahanta na, nitak pokoli ushta micha ninak pokoli ushta tok. 


\section{CHAPTA XXV.}

MIHMA Chihowah yot Moses a im anumpulit,

2. Islael im vlla vhleha ha ish im anumpuli na, nan im issa isht vm vlashke; hatak moma chukvsh aiokpanchit bohli ka nan im issa ya is sum eshashke.

3. Micha nan im issa iluppak o hosh im ai ishashke, toli holisso lakna, micha tvli hvta, micha asonak lakna,

4. Micha okchumali, micha humakbi, micha tishepa, micha linen lapushki, micha issi kosoma hishi,

5. Micha chukfi nakni hakshup humma, micha bacha hakshup, micha shittim iti,

6. Pula ha chị ka bila, bila ishtahama ya chi ka, micha nabalama ya chi ka na balama puta,

7. Oniks tuli, micha efot, micha ikkishi alata, ontaloha chi ka tuli ak ashke.

8. Micha vlhtipo holitopa vm ikbi na, takla ahanta lashke.

9. VIlhtipo holba ya, micha ishit toksuli chi pisachi le tuk, moma ka iakaiya hosh, yohmi fehna ho hobachit ish ikbashke.

10. Mikma shittim iti yo itombi holitopa ya ikbashke, falaia kvt kubit tuklo micha iklunna, micha auahta kvt kubit achvfa cha iklunna, micha chaha kut kubit achvfa cha iklunnashke.

11. Micha anuka, micha kucha aiena ka tvli holisso lakna ahli yo ish lapalechashke; micha tvli holisso lakna afohoma isht apakfoyupa chi ka ish ai ikbashke.

12. Micha tvli holisso lakna chvnaha bilelit akmochit chukbi ushta ka ish atakalichashke; micha in tunnvp achvfa ka chunaha tuklo hosh takohlashke; mikma in tunnup achvfa ka chunaha tuklo hosh takohlashke.

13. Micha shittim iti yo tvli ish ikbi cha, toli lakna lapalachit ish tahlashke.

14. Micha tvli ya chvnaha yash itombi holitopa in tunnup bika ya ish apittashke; yumohmi ho itombi holitopa ya ishit sholashke. 
15. Tubi vt itombi chunaha ya aivlhta hi oke; kocha wiha he keyu hoke.

16. Micha itombi holitopa ya nan atokowa chima la chi ka ish fohkashke.

17. Micha tvli lakna bano ho im ai i kanvt aiombinili ya isht ish ikbashke: falaia kut kubit tuklo cha iklunna aienakmot, auahta kvt kubit achvfa cha, iklvnna aienashke.

18. Micha tuli holisso lakna yo Chilubim tuklo ho isht ish ikbashke; bovt toba ho ish ikbi cha, im ai i kanut aiombinili vhli tuklo ka ish hiohlechashke.

19. Micha chelub achvfa ka vhli achvfa ka ish hilechikmut, chelub achvfa ya vhli achufa ya ish hilechashke: im ai i kanvt aiombinli vhli tuklo fehna kak o chelubim ish ikbashke.

20. Mikma chelubim vt sanahchi ya ashatublichit vba wielit, sanahchi ya im ai i kanvt aiombinili ya isht ai ompohomashke; mikma nashuka vt iti sanalashke, im ai i kanvt aiombinili ya chelubim nashuka vt pit talaiashke.

21. Micha im ai i kanvt aiombinili ya itombi holitopa paknaka ish ontalalashke, micha itombi holitopa ya nan atokowa chima la chi ka ish fohkashke.

22. Mikma im ai i kanvt aiombinili paknaka chelubim tuklo kvt nanvlhpisa afohka itombi holitopa paknaka a hieli ka itin takla ya yumma chi ai afamut nana moma anumpa vlhpisa ha Islael im vlla vhleha imma ho chima la chikmvt chitim anumpule lachi hoke.

23. Shittim iti yo patussa ish ikbashke, falaia kvt kubit tuklo, auahta kvt kubit achvfa, chaha kvt kubit achvfa cha, iklunna hashke.

24. Micha tuli holisso lakna bano ho ish lapalechashke, micha tuli holisso lakna yo vhli i fullota ikbit ish apakfoyublashke.

25. Micha im aivhli apakfoyupa kvt ibbak pvtha achvfa ho ish ikbi cha, vhli $\underline{i}$ fullota yash tuli lakna yo $\underline{\mathrm{i}}$ fullota apakfoyupa ish ikbashke.

26. Micha tuli holisso lakna chunaha ushta ho ish ikbi cha, chukbi ushta iyi ushta ai o hikia ka ish talohlichashke. 
27. Uhhli yo bilika yo chunaha hvt takohlikma, tubi vt shamulli ho patussa yash ishit sholashke.

28. Micha tubi shittim iti yo ish ikbi cha, tuli holisso lakna ish lapalechashke, yumohmi ho patussa ya ishit sholashke. 29. Micha im ampo, im ishtimpa, im isht ompoholmo, micha nan isht impuchi nana isht tosholi aiena a puta ka ish ikbashke, toli lakna bano ho ish ikbashke.

30. Micha sa tikba ya puska otuni a patussa ya is sum on talali na a biliashke.

31. Micha tuli holisso lakna bano ho pula aiohikia ish ikbashke : bovt toba ho pvla aiohikia ish ikbashke, im vpi,

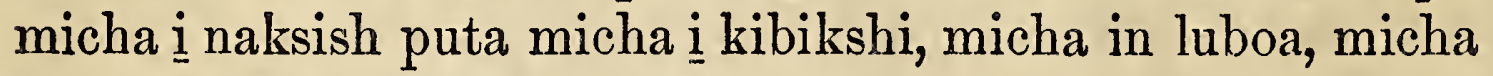
i napakanli yummak in lashke.

32. Mikma tahlaklaka ya naksish tuchina hosh akohchashke, pula aiohikia in tunnup achufa ka naksish tuchina hosh akohchakma, pula aio hikia in tunnep achvfa ya naksish tuchina hosh akohchashke.

33. Naksish achvfa ka almont holbvt toba hosh kibikshi tuchina, luboa achvfa, pakanli achvfashke, micha naksish achufa ya almont holbut toba kibikshi tuchina, micha luboa achvfa, micha napakanli achufashke; micha pula aiohikia naksish kohcha hannali ka yohmashke.

34. Micha pvla aiohikia yạ $\underline{\mathbf{i}}$ kibikshi vt almont holbvt toba ushta hosh in luboa, micha i napakanli aiena hi oke.

35. Mikma yummak inli ka naksish tuklo nutaka ya lumbo achufa, micha yummak inli ka naksish tuklo nutaka ya lumbo achvfa, micha yummak inli ka naksish nutaka ya lumbo achufa, pula aiohikia naksish hannali akohcha ya vlhpisashke.

36. In lumboa, micha i naksish puta kvt yummak inlashke; moma kvt tuli holisso lakna bano hosh bovt tobashke.

37. Micha pula aivlhto untuklo ka ish ikbashke ; mikma okla pulali na, itikba ya tohwikela hi oke.

38. Mikma ishit kiselichi, micha pula wishakchi tuptua aivlhto vt tvli holisso lakna banashke.

39. Tuli holisso lakna talent achvfa ho yumma, nan aivlhto iluppa puta ka aieninchit ikbashke. 
40. Mikma ahah ish ahni cha, holbvt toba he ya nunih paknaka a chi pisachi le tuk a hobachit ish ikbashke.

\section{CHAPTA XXVI.}

T ICHA vlhtipo ya nantunna vba takali pokoli hosh linen 1 tapuski itapuna yosh okchvmali, micha hummaii, micha tishepa ish ikbashke: toksuli imponna yosh ikbi yo chelubim ish ikbashke.

2. Nantunna vba takali achvfa kvt falaia kvt kubit pokoli tuklo akucha untuchinashke, mikma auata kut nantunna vba takali achvfa kvt kubit ushtashke; micha nantunna vba takali moma kvt vlhpisa aiachvfashke.

3. Nantunna vba takali tahlapi kvt itvchakvchashke, mikma nantunna vba takali inla tahlapi kvt itvchakvchashke.

4. Nantunna uba takali achufa itvchaka vhli ya ahlopulli okchumali ya ish ikbashke; micha nantunna vba takali inla atukla yut itvchaka vhli fehna ka yohmi akinli ho ish ikbashke.

5. Nantunna uba takali achvfa ka ahlopulli pokoli tahlapi ho ish ikbashke, micha nantunna vba takali atukla yot, itvchaka yot vhli ha ahlopulli pokoli tahlapi ho ish ikbi na, ahlopullit iti halvllashke.

6. Micha tvli holisso lakna yo ishtakamvssa pokoli tahlapi ho ish ikbashke, micha nantunna vba takali ya ishtakamussa ak $\mathrm{o}$ isht ish itvchakalashke; mikma vlhtipo achvfashke.

7. Micha issi kosoma hishi yo nantunna vbatakali ish ikbi na, vlhtipo ya ompoholmo yashke; nantunna vbatakali auahchvfa họ ish ikbashke.

8. Nạntunna vbatakali achvfa falaia kvt kubit pokoli tuchinashike ; micha nantunna vbatakali achufa auata kvt kubit ushta hashke, micha nantunna vbatakali auahchvfa kvt vlhpisa kvt ai achvfashke.

9. Mikmut nantunna vbatakali tahlapi ka ila ish itachakalashke; mikmut nantunna vba takali hanali ka ila ish ita 
chakali cha, nantunna vbatakali ishit hannali ka vlhtipo itikba ya ish a pohlomashke.

10. Micha nantunna vbatakali achvfa itachahkvehi ya vhli fehna vhli ya ahlopulli pokoli tahlapi, micha nantunna vbatakali atukla ai itachaka ya ablopulli pokoli tahlapi ho ish ikbashke.

11. Micha asonak lakna yo isht akamvssa pokoli tahlapi ho ish ikbi cha, ahlopulli ya ish apitta cha, ish itachakli na, vlhtipo vt achvfashke.

12. Mikma nantunna vbatakali atampa vlhtipo ompoholino nantunna vbatakali iklunna kvt vlhtipo ya ont atampvt asha ka pit takalashke.

13. Mikma vlhtipo ya nantunna vbatakali afalaiaka atampa kvt in tunnup achufa ka kubit achvfa ho, micha intunnup achvfa ka kubit achvfa ho ont vhlashke; vlhtipo ya ompoholmot ont atamput in tunnvp ilvppa, micha in tunnup yumma takalashke.

14. Micha vlhtipo ya ompoholmo ya chị ka, chukfi nakni hakshup humma yo, micha paknaka isht ompoholmo ya chị ka bacha hakshup o ish ikbashke.

15. Micha vlhtipo ya shittim iti busha hiohli ho ish im ikbashke.

16. Iti busha achvfa kvt falaia kvt kubit pokolikma, auata kvt iti busha achvfa kvt kubit achvfa cha, iklvnna aienashke.

17. Iti busha achvfakma ai ishit shumohli vt tuklo cha, a shưmohli ya itvlhpisashke; yưmohmi bieka ho vlhtipo im iti busha moma ka ish ikbashke.

18. Vlhtipo ya oka mahli in tunnup, oka mahli pilla ho iti busha pokoli tuklo ho ish ikbashke.

19. Micha iti busha pokoli tuklo nutaka ya a shamvlli ya tuli huta pokoli ushta ho ish ikbashke; ititi. besha achvfa nutaka ya ishit shamvlli tuklo ka a shamulili kvt tutulashke; mikma iti busha inla nutaka ya ishit shamulli iń tụklo ka ashamulli tuklo hashke.

20. Mikma vlhtipo in tunnup atukla falummi in tunnưp a iti busha pokoli tuklashke.

21. Mikma ashamvlli vt $\underline{\mathrm{i}}$ pokoli ushtashke; iti busha 
achvfa nuta ka ya ashamvlli tuklo; micha iti busha inla nuta ka ya ashamulli tuklashke.

22. Mikma vlhtipo ya in tunnup hushi aiokatula pilla ho iti busha hannali ho ish ikbashke.

23. Micha vlhtipo in tunnup tuklo chukbi ya iti busha tuklo ho ish ikbashke.

24. Micha nutaka yvt itvchakashke, micha paknaka ya itachakvt chunaha achufa ho shamvllashke, itatuklot yakohmashke; chukbi in tuklashke.

25. Micha iti busha untuchinashke; mikma ashamvlli tvli huta yut ashamvlli im auah hannalashke; iti busha achufa nutaka ya ashamvlli tuklo, micha iti busha inla nutaka ya ashamvili tuklashke.

26. Micha im okhowatkvchi ya shittim iti yo ish ikbashke, vlhtipo in tunnup achvfa im iti busha ya tahlapashke.

27. Mikma vlhtipo in tunnup achvfa iti busha ya okhowatkvchi tahlapashke; mikma vlhtipo in tunnvp achvfa hvshi ai okatula pilla in tunnup tuklo iti busha ya okhowatkvchi tahlapashke.

28. Mikma itibusha iklunna okhowatkvchi vt vhli ka vttut iakmut ont vhli ka onashke.

29. Micha iti busha ya tuli holisso lakna ish lapalechi cha, okhowatkvchi aivlbiha chi ka tuli holisso lakna chunaha ish ikbashke, micha okhowatkvchi ya tuli lakna ish lapalechashke.

30. Micha kaniohmit vlhtipo vt toba chi ho nunih ha a chipesvchi tuk mak o ish hilechashke.

31. Micha isht ompoholmo ya linen tapuski itapuna, okchumali, hummaii, micha tishepa aiena toksuli imponna ikbi yo ish ikbashke, chelubim a tohashke.

32. Micha shittim iti tonik ushta hosh tvli lakna alapali ho ish atalsalehastrke; tvli hota ashamulli ushta ka toli lakna atakali dit as áshke.

33. Mitcha isht ompoholmo ya isht akamussa nutaka ish takalichashke; yumohmi ho nan vlhpisa afohka itombi holitopo ya ompoholmo kash anukaka isht chukowvt ish takalichashke, mikma ompoholmo yut aholitopa, micha holitopa i shahli ya chim iti filummichashke. 
34. Micha nanvlhpisa afohka itombi holitopa moma i shahli anukaka talaia ka im ai i kana ai ombinili ya ish ontalalashke.

35. Micha iti patussa ompoholmo kocha ish talalashke, micha pula ai $\underline{o}$ hikia ya vlhtipo lapalika oka mahli pilla in tunnup iti patussa ichapa ka ak $\mathrm{o}$ ish hilechashke; micha iti patussa ya falummi in tunnup ak $\underline{o}$ ish hilechashke.

36. Micha vlhtipo okhissa takali ya linen tapuski itapuna hosh okchumali, hummaii, micha tishepa chufak ushi isht vlhtaha ho ish ikbashke.

37. Micha atakali ya shittim iti tonik tahlapi ho ish ikbi cha, tuli holisso lakna ish lapalechashke; mikma atakali vt tvli lakna yashke; micha asonak lakna vt tvli lakna yashke, micha asonak lakna ashamulli tahlapi ho bilelit ish im akmochashke.

\section{CHAPTA XXVII.}

MICHA shittim iti yo alta falaia kvt kubit tahlapi, mi11 cha auata kvt kubit tahlapi itilaui banot fullota ho ish ikbashke, mihma chaha kvt kubit tuchinashke.

2. Micha chukbi ushta kạ, lapish ushta ho ish ikbashke, lapish vt yummakinlashke; micha asonak lakna ya ish lapalechashke.

3. Micha $\underline{i}$ hituk chubi aivlhta chị ka im ampmahaia, micha hituk chubi im isht piha, micha im ampushi, micha nipi im isht yichiffi, micha luak im ai vlhto mahaia aiena $\mathrm{ka}$ ish ikbashke, nan aivlhto moma ka asonak lakna yo ish ikbashke.

4. Micha asonak lakna tunna ya ish im ikbashke; micha tunna vt chukbi ushta ka asonak lakna chunaha ushta ho ish ikbashke.

5. Micha nuta alta aivhli ka ish bohlashke: yohmi na, tunna vt alta iklunna ya ont vhlashke.

6. Micha alta ya shittim iti tubi yo tubi ish im ikbashke, micha asonak lakna ish lapalechashke.

7. Mikma tubi vt chvnaha ya aivlhtashke, micha ishit 
shola chị ho, tubi vt alta ya in tunnup tuklo ka shumohlashke.

8. Itombi vt chiluk a asha ho iti busha yo isht ish ikbashke; nunih ha a chi pesuchi tuk mak o yohmi ho okla ikbashke.

9. Micha vlhtipo i wanuta ya ish ikbashke, oka mahli in tunnup oka mahli pilla wanuta ya na takohli vt linen tapuski ita puna yosh in tunnup achufa ka falaia kvt kubit tahlepa achvfashke.

10. Mikma in tonik pokoli tuklo, micha ashamulli pokoli tnklo kvt asonak lakna yashke: tonik in chanakbi, micha afohoma aiena kvt tuli hvta yashke.

11. Mikma falummi in tunnup ak kia falaia ka na takali vt falaia kut kubit tahlepa achvfakma, tonik pokoli tuklo, micha ashamvlli pokoli tuklo kvt asonak lakna yashke; to nik i na chanakbi afohoma aiena kvt tuli hvta yashke.

12. Mikma hushi ai oka tula in tunnop wanuta auata natakali vt kubit pokoli tahlapashke, in tonik vt pokolikma ashamulli vt pokolashke.

13. Micha hvshi ak ochaka in tunnup hushi akochaka pilla, ho wanuta auata kvt kubit pokoli tahlapashke.

14. Okhissa in tunnup achufa natakali vt kubit auah tahlapashke, in tonik vt tuchinakma, ashamvlli vt tuchinashke.

15. Mikma in tunnup achufa ya natakali vt kvbit auah tahlapashke, in tonik vt "tuchinakma, ashamvlli vt tuchinashke.

16. Mikma wanuta okhissa ya natakali vt linen tapuski itapuna okchumali, hummaii, micha tishepa chofak ushi isht vlhtaha hosh kvbit pokoli tuklashke, mikma in tonik vt ushtakma, ashamulli vt ushtashke.

17. Tonik moma wanuta apakfoyupa kvt toli hvta isht afohomashke, ína chanakbi vt tuli hota yokma, ashamulli vt asonak lakna yashke.

18. Wanuta falaia kvt kubit tahlepa achufakma, auata kvt pokoli tahlapi bikashke; mikma chaha kvt kubit tahlapi hosh linen tapuski itapuna yashke, micha ashamulli vt asonak takna yashke. 
19. Vlhtipo ya nan aivlhto moma, nan isht apilesa, micha isht a honvhla moma, micha wanuta ya isht a honvhla moma aiasha kvt asonak lakna yashke.

20. Micha Islael im vlla vhleha ha ish im apesa na, bila pưla hut lúa na, bilia chi ka alif bila ahli bout pula hưchin toba ya isht chim vlashke.

21. Okla moma im vlhtipo isht ompoholmo kocha yummut ishtatokowa itikba ya Alon, micha ushi vhleha aiena kut hielit opiaka, micha onnahinli aiena Chihowah $\underline{i}$ tikba talaia chị ka apihisa hi oke, im isht atiaka bilia ka nan vlhpisa bilia yosh in toyula cha, Islael im vlla vhleha ha i yumohmashke.

\section{CHAPTA. XXVIII.}

MICHA Alon chitibapishi, micha ushi vhleha aiena ka, 11 Islael im vlla vhleha i filvmmichit Alon, micha $\mathrm{Na}$ tab, micha Abihu, Eliesa, micha Ihlama, Alon ushi vhleha aiena ka ish eshi na, naholitopa isht ai vtta ya isht vm ahashwashke.

2. Micha chitibapishi Alon a nafohka holitopa, micha isht aiokli ya ish im ikbashke.

3. Micha hatak chukvush hopoyuksa moma im anukfila hopoyuksa chukush alotoli li tuk $\underline{a}$ ish im anumpuli na Alon a hullochi na, na holitopa isht aivtta isht vm ahanta chi ka Alon a nafohka im ikbashke.

4. Nafohka iluppa puta kak o ikbashke, ikkishi alata, micha efot, micha nafohka holitopa, micha i fullota asha nafohka, micha iahlipa, micha vskofvchi, aiena hashke; micha chitibapishi Alon, micha ushi whleha aiena ka, nafohka holitopa im ikbi na, naholitompa isht aivtta ya isht vm ahashwashke.

5. Micha tvli holisso lakna micha okchumali, micha hummaii, micha tishepa micha linen tapuski itapuna aiena ho ishashke.

6. Micha efot a tuli holisso lakna, okchumali; hummaii, 
tishepa, micha linen tapuski yo ishtatoba toksuli imponna yo ikbashke.

7. Tahchi abana tuklo cha, aivhli tuklo ka ai itachakashke, yohmi hosh itachakashke.

8. Mikma vskofvchi inla efot a atakali kvt yummakinli isht vlhtaha ya chohmit tvli lakna, okchumali, micha hummaii, micha tishepa, micha linen tapuski itapuna yashke.

9. Micha oniks toli tuklo ho ish eshi cha, Islael im vlla vhleha hohchifo ka in chunlit ish lapalechashke.

10. Hohchifo hannali ka tuli achufa ka ish takalichikmut, hohchifo inla ka aivtta tok vlhpisa ho tvli achvfa ya ish takalichashke.

11. Tuli in chunli yosh ikbi yo ishtin chunli in chuwa holba ho Islael im vlla vhleha hohchifo aieninchit tvli tuklo ka ish in chunlashke, tuli lakna ashamvlli asha yo ish ontalohlashke.

12. Micha efot tahchi ak o tvli tuklo kash ish ontalohlashke; Islael vt tvịi nan isht ithaiyana na bilia yashke; mikma nan isht ithaiyana he mak $\underline{o}$, Alon vt Chihowah itikba ya tahchi vbanalit hikiashke.

13. Micha tuli holisso lakna ashamvlli ya ish ikbashke.

14. Micha ont vhli ka tvli lakna bano itachahkvchi tuklo ho ish ikbashke, i poshota vlhtaha ho ish ikbi cha, i poshota itachahkuchi yash ashamulli ya ish takalichashke.

15. Tuli imponna yosh ikbi yo nan isht apesa ikkishi alata ish ikbashke, efot toba ak o holba ho ish ikbashke; tvli holisso lakna, nan okchumali, hummaii, micha tishepa, micha lineu tapuski itapana yo isht ish ikbashke.

16. Pohloma hosh tahchaka ushta kvt iti laui banashke, falaia kvt span achvfakma, auata kvt span achvfashke.

17. Micha tuli ontula tuli itiakaiya ushta ho ish ontalalashke, itiakaiya ummona satis, topas, micha kabunkel, ilvppak osh itiakaiya vmmona hashke.

18. Mikma itiakaiya atukla kvt emelal, micha saffai, micha taimont ashke.

19. Mikma itiakaiya ont atuchina kut likua, micha akat, micha amihlist ashke. 
20. Mikma itiakaiya ont aiushta kvt belil, micha oniks, micha chashpa ashke, tvli lakna afohka ai ontulashke.

21. Mikma tvli vt Islael im vlla vhleha hohchifo lapohli hashke, hohchifo ha, vlhpisvot auahtuklo hosh isht in chuwa in chuwa ka chohmi hashke, moyuma kvt apehlichi auahtuklo vlhpisa ho hohchifo hosh lapohli hashke.

22. Micha tvli holisso lakna bano ho im poshota toba yo ikkishi alata ont vhli ka itachakvchi ish ikbashke.

23. Micha tuli holisso lakna chunaha tuklo ikkishi alata ya ish ikbashke, mikmut chunaha tuklo ya ikkishi alata ont vhli tuklo ka ish atakolichashke.

24. Micha tvli holisso lakna itapakshuna itvchakvchi tuklo ka ikkishi alata ont vhli ka tuli holisso lakna chvnaha tuklo ya ish atakohlichashke:

25. Micha itapakshuna itachakuchi tuklo kash wishakchi tuklo ka ashamvlli tuklo ya ish a kullochi cha, itikba takali efot in tahchi abana ya ish abanalashke.

26. Micha tuli holisso lakna chunaha tuklo ho ish ikbashke; micha ikkishi alata chukbi tuklo aivhli yumma efot anukaka takali okhowataka ya ish takohlichashke.

27. Micha tvli holisso lakna chunaha tuklo inla ya ish ikbi cha, efot aka pilla okhowata tuklo itikba takali pilla efot im uskofuchi pisa inla ya paknaka isht itachaka ya in chapaka ak o ish talohlichashke.

28. Mikma ikkishi alata ya chunaha ya efot in chunaha ak o sita okchvmali yo isht ish itatakchichi na, efot im vskofuchi pisa inla ya paknaka takalashke, micha ikkishi alata vt efot a ik $\underline{i}$ mokofo kashke.

29. Mikma Alon vt Islael im vlla vhleha hohchifo hvt afoyuka ho aholitopa ya chukowakmvt Chihowah yvt isht ithaiyana bilia chi ka nanisht apesa ikkishi alata ya chukush alatali hosh iba chukowashke.

30. Micha nanisht apesa ikkishi alata ya Ulim micha Hlummim a ish fohkashke; atukma Alon vt chukowvt Chihowah itikba onakmvt chukvish a onochit shaiyalashke, micha Islael im vlla vhleha nan im aivlhpiesa ya Alon vt chúkush onochit shaiyalit Chihowah itikba hikia na biliashke. 
31. Micha efot nafohka falaia holitopa ya okchwmali bieka ho ish ikbashkc.

32. Mikma paknaka iklunna ya chiluk vt hikiashke, mikma chiluk a nantunna yosh $\underline{i}$ fullotvt apakfoyupa kvt tonvp ilefohka ak o chohmi na ik hlilafo kashke.

33. Micha aka pilla vhli ya pomkilanit okchvmali, micha hummaii, micha tishepa yo vhli fullota ish ikbashke, mikma tvlula toli holisso lakna itin takla ya takohlit fullotashke.

34. Nafohka falaia holitopa aivhli fullota ka tulula tvli holisso lakna, mikma pomkilanit, tvlula tvli holisso lakna, mikma pomkilanit osh takalit iashke.

35. Mikma Alon vt nan isht im vtta ma onvtoyulashke: atukma aholitopa ya chukowvt Chihowah itikba ya o nakma, atuk osh kohchakma, ohonla haklo na ik illo kashke.

36. Micha tuli holisso lakna bano patussa ish ikbi cha, isht in chuwa in chüwa ya chohmi ho in chunlit CHIHOwaH IM I HоLITOPA ноке, achit ish takalichashke.

37. Mikma sita okchumali ya ish lapalechi na iahlipa ha ontalaiashke, iahlipa itikba ak o ontalaiashke.

38. Mikma Alon noshkobo ha on talaia na, na habenvchi holitopa moma Islael im vlla vhleha hvt hullochi tuk, naholitopa aiashvchi puta ka Alon vt shaiyalashke: micha Alon noshkobo ha on talaia na, biliashke, yumohmi ho Chihowah yut aiokpuchashke.

39. Micha linen tapuski nafohka aiokli ho achunlit ish ikbashke, micha iahlipa ha linen tapuski o ish ikbashke, micha chufakushi isht vlhtaha ho vskofvchi a ish ikbashke.

40. Micha Alon ushi vhleha ha ilefoka lumbo ish im ikbashke, micha ishit holitopa ya fvchi ish im ikbikmvt shapo ya ish im ikbashke.

41. Micha chitibapishi Alon, micha ushi vhleha aiena ka ish fohkvchechashke, micha bila isht ish ahvmmashke, micha $\underline{\mathrm{i}}$.hullochit ish kashofashke; yumohmi na naholitompa isht aivtta ya isht vm ahashwashke.

42. Micha isht il ompohoma chi ka linen obalafoka ya ish im ikbashke, hvtip a vttut iakmvt iyubi ont vhlashke.

43. Mikma Alon micha ushi vhleha aiena kvt okla moma 
im vlhtipo ant chukowakmvt, keyukmvt aholitopa nan isht ai vtta chi hosh alta ya bilika onakmvt, foyukashke; yumohmi hosh nan ashvchi onvtoyulvt ik illo kashke. I nan vlhpisa bilia yosh im isht atiaka yvt iakaya aiena kvt in toyulashke.

\section{CHAPTA XXIX.}

TCHA nana iluppak o ish $\underline{i}$ yumihchi hosh na holitomI pa isht ai vtta ya isht vm aiashwa chi ka ish hullochashke; wak hobvk himmita achvfa, micha chukfi nakni tuklo ik aiono kut iksho yo ish eshi cha,

2. Micha puska ik shatummo, micha pvska lumbo ik shatummo bila yummi, micha puska tapuski ikshatummo bila ahama aiena ka onush bota yo ish ikbashke.

3. Mikmvt kishi achvfa ho ish fohki cha, kishi a vlhto ho wak hobvk, micha chukfi nakni tuklo ka aieninchit isht ish lashke.

4. Micha Alon, micha ushi vhleha aiena ka okla moma im vlhtipo okhissa itikba ya jsht ish ona cha, oka isht ish achefashke.

5. Micha ilefohka ya ish eshi cha, nafohka lumbo, micha nafoka holitopa falaia efot, micha efot, micha ikkishi alata ya ish fohkechi cha, vskofuchi inla fehna efot a ish vskofvchechashke.

6. Micha iahlipa ha noshkobo ha "ish on talalashke, micha bita holitopa ya iahlipa ha ish on talalashke.

7 Yumohmikmut bila isht ahummi ya ish eshi cha, noshkobo ha on hlatublit ish ahummashke:

8. Micha ushi vhleha ha isht ishla cha, ilefoka lumbo ish fohkvchechashke.

9. Micha, Alon, micha ushi vhleha aienà ka vskofuchi ish vskofvchechashke micha shapo ish shapolechashke; mikma naholitompa isht ai vtta yvt immi ha he; nan vlhpisa biliashke; micha Alon micha ushi vhleha aiena ka ish hullochashke.

10. Micha wak hobok himmita ya okla moma im vlhtipo itikba isht vla he ya ish apesashle; mikma Alon, micha 
ushi vhleha aiena kut wak hobuk noshkobo ya ibbak isht ombitepashke.

11. Mikma wak hobvk ash okla moma im vlhtipo okhissa bilika Chihowah itikba ya ish aivbashke.

12. Micha wak hobuk im issish a ish eshi cha, alta lapish a chibbak ushi isht ish lapalichashke; micha issish a mominchit alta akishtvla lapali ka ish hlatublashke.

13. Micha anukaka ompoholmo nia moma, michá takoba nia yvmmut salakha ya onvtoyulaka, micha haiyichi tuklo, micha nia yut onvtoyula aiena ka ish eshi cha, alta ya onochit ish hukmashke.

14. Amba wak hobvk ash nipi, micha hakshup, micha 1 yulhki aiena ka binah kohcha ya luak isht ish hukmashke : nan ashuchi im issa yoke.

15. Micha chukfi nakni achufa ho ish eshashke, mikma Alon, micha ushi vhleha aiena kvt chukfi nakni noshkobo ha ibbak a isht ombitepashke.

16. Mihma chukfi nakni yash ishbashke, micha im issish

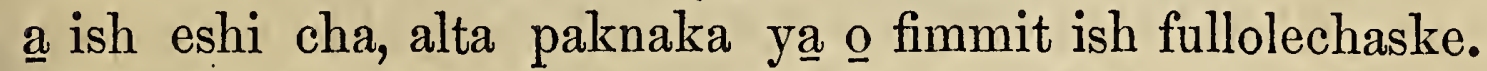

17. Micha chukfi nakni yash bushlit tushahlichit ish tahli cha, anukaka micha iyi aiena ka ish achefa cha, ai itim aiyuka li ka ish ita chakli cha, noshkobo ya ish achakalashke.

18. Micha chukfi nakni yash moma alta onochit ish hukmashke; hukmit Chihowah im issa yoke ; luak a onochit Chihowah im issa na balama yoke.

19. Mikmut chukfi nakni achvfa ya ish eshashke, mikma Alon, micha ushi vhleha hot chukfi nakni yash noshkobo ha ibbak isht ombitepashke.

20. Yohmikma chukfi nakni yash ishbashke, micha im issish a ish eshiknivt Alon haksobish ishtimpakimma vhli, micha ushi ứhleha haksobish ishtimpakimma vhli, micha ibbak ishtimpakimma ibbak ishki, micha iyi ishtimpakimma iyishki aiena ka ish lapalechashke, micha issish a alta ya o fimmit ish lapalechaske.

21. Micha issish alta on asha ka, micha ishtahummi bila ya ish eshi cha, Alon micha i nafohka, Alon ushi vhleha, micha ushi vhleha $\underline{\mathbf{i}}$ naföhka aiena $\mathbf{k} \underline{a}$ ish $\underline{0}$ fimmashke, 
mikma Alon, micha $\underline{i}$ nafohka, micha ushi vhleha, micha ushi vhleha i nafohka aiena kut hullochi yashke.

22. Micha chukfi nakni yash nia, micha hvtip, micha anukaka ya ompoholmo nia, micha takoba nia, yummut salvkha onvtoyula ka, micha haiyihchi tuklo micha nia yvt assha, micha fullup ishtimpakimma aiena ka ish ishashke ; chukfi nakni nahullochi yoke.

23. Micha puska lumbo chito achvfa, micha pvska lumbo iskitini bila yummi achufa, micha puska tapuski achvfa aiena ka puska ik shatummo kishi vlhtot Chihowah itikba talaia aiena ka kohchit ish eshashke.

24. Micha moma ka Alon ibbak, micha ushi vhleha ibbak ish fohki cha, Chihowah itikba ya $\underline{\underline{i}}$ walit im issa ya ish walashke.

25. Mikmvt ibbak a kohchit ish im ai eshi cha, hukmit im issa chi ka , Chihowah itikba na balama ya chi ho, alta onochit ish hukmashke, luak onochit Chihowah im issa yoke.

26. Micha Alon hullochi chukfi nakni ikkishi ha ish eshi cha, Chihowah itikba ya wali ya chị ho ish walashke, mikma chimmi hashke.

27. Micha Alon, micha ushi uhleha aiena ka immi ha he, ishit chukfi nakni wali tuk, micha vba pit wali tuk, walit im issa ikkishi, micha vba pit walit fulup a ish hullochashke.

28. Mikma Islael im vlla vhleha hak osh ima ho, Alon micha ushi vhleha immi ha he, nan vlhpisa bilia yashke; vba pit walit im issa yoka, micha Islael im vlla vhleha hut iti nanaiyvchi im issa, vbit isht aiokpvechi, vba pit walit im issa, vba pit walit Chihowah im issa yoke.

29. Mikma Alon i nafohka holitopa yut ushi vhleha iakaiya kvt immi cha, foyukut bila ahama he, micha foyuka ho hullocha hi oke.

30. Micha ushi yosh vlhtobvt naholitompa isht vtta toba kvt okla moma im vlhtipo ya chukowvt aholitopa ya nan isht ai ahantakmot nitak untuklo foyukashke.

31. Atuk o hullochi chukfi nakni ya ish eshi cha nipi aholitopa ya ish ahonashke. 
32. Mikma Alon, micha ushi vhleha hvt chukfi nakni yash nipi ya micha puska kishi vlhto tuk a okla moma im vlhtipo okhissa talaia ka aieshit aieninchit ai upa hi oke.

33. Micha hullochi, micha kashofa chi nana puta ka isht atobbi tuk ak o ai vpashke: amba okla inla yvt ai vpa he keyu, holitopa hoka.

34. Micha hullochi tuk nipi, keyukmut pvska nana kia, atampa hosh asha na onnahinlikma, yohmikma atampa tuk ash luak ishit ish hukma hi oke, vpa he keyu, holitopa hoka.

35. Micha nana moma chim apesa li tuk mak o, Alon micha ushi vhleha ha ish $\underline{\mathrm{i}}$ yumihchashke, nitak untuklo ho hullochi hosh isht ish ahantashke.

36. Micha nitak aiyukali ho wak hobvk a nanashvchi ishit vlhtoba ho ish im issashke, micha nan ishit atobbi alta ish im ikbikmvt, alta ya ish kashoffaske, micha kashoffit bila ish ahummashke.

37. Nitak untuklo ho alta ya atobbit ish kashoffashke, mikma alta vt holitopa $\underline{\mathbf{i}}$ shahli yashke; nana hosh alta ya halelikmot holitopa hi oke.

38. Yohmi ka iluppak o alta ya onochit ish im issa hi oke: onna hosh onnakma chukfushi afummi vmmona tuklo ho ish im ihisa na, bilia hi oke.

39. Chukfushi yash achvfa ka onnahinlikma ish im issa tukmut, achufa ya opiakma ish im issashke.

40. Micha chukfushi achvfa ka bota tohbi kvshkoa ishit pokoli ho boa na bila toba hin achvfa kvshkoa ai ushta ho ittaiyomi, micha nanishko im issa ya okapaki hin achvfa kushkoa aiushta ho ish im issashke.

41. Micha chukfushi achvfa ya na-balama, micha luak ishit hukmit Chihowah im issa mak o onnahinli ya ilhpak im issa yash, micha ishko im issa ya tuk mak o chohmichit ish yumihchashke.

42. Ilvpput hvchim ai itishali moma ka hukmit im issa bilia yo Chihowah itikba okla moma im vlhtipo okhissa ya a yumohma hi oke; yummak o a hvchi itafamvt, yummak $\underline{0}$ a chim anumpule la hi oke.

43. Micha yummak o Islael im vlla vhleha ha ai itafama 
la hi oke, mikma vlhtipo hvt vm ai isht aholitopa ak $\underline{o}$ ishit kashofa hi oke.

44. Micha okla moma im vlhtipo, micha alta aiena ka kashofi la hi oke; micha Alon, micha ushi vhleha hak kia naholitopa isht ai vtta ya isht vm a a hashwa chị ka hullochi la chi hoke.

45. Micha Islael im vlla vhleha ha itin takla ya ahanta lish in Chitokaka sia ha chi hoke.

46. Mikma vno ak osh in Chitokaka Chihowah sia, atuk osh itin takla ahanta la chi hosh Echip yakni ya pehlichit kohcha li tok a ithaiyana chi hoke. In Chitokaka Chihowah sia hoke.

\section{CHAPTA XXX.}

ICHA na balama ai onochit hukma chị ka alta ish 11 ikbashke, shittim iti yo ish ikbashke.

2 Falaia kut kubit achufashke, mikma auata kvt kubit achvfashke iti laui bat fullotashke; mikma chaha kvt kubit tuklo hashke, lupish vt yummak inlashke.

3. Micha paknaka, micha naksika apakfoyupa ka, micha lupish aiena ka tvli lakna bano ho ish lapalechashke, micha tvli holisso lakna i fullotvt apakfoyupa ish ikbashke.

4. Micha $\underline{i}$ fullota nutaka chukbi tuklo ha tuli lakna chvnaha tuklo ho ish ikbashke, in tunnup tuklo ka ish takohli chashke, mikma tubi vt shamohli na ishit shohla hi oke.

5. Micha shittim iti yo tubi ish ikbashke, micha tuli lakna ish lapalechashke.

6. Micha nantvnna vba takali yvmmut nan vlhpisa afohka itombi holitopa bilika takali ka itikba im ai isht i kana om binili, yummvt nan vlhpisa afohka paknaka talaia ka itikba ish talalashke, yummak o achi afama la hi oke.

7. Mikma Alon vt onnahinli aiyukali ka nabalama onochit hukma hi oke, pvla puta ka ai iskiachikmut nabalama onochit hukma hi oke.

8. Micha Alon vt opiakma pula ha palalikmvt, nabalama 
onochit hukma hi oke, chim ai itishahli moyuma ka Chihowah itikba ya na balama bilia hi oke.

9. Nabalama inla, micha vbit hukmit im issa, micha im ilhpak im issa aiena ka onochit ish im issa he keyu hoke; micha nan ishko im issa aiena ka ish on hlatubla he keyu hoke.

10. Mikma Alon vt afưmmi achvfakma himonna ho nanashvchi im issa issish isht atobbi ya atobbit lupish a onocha hi oke; chim ai itishali moyuma ka afummi achufakma onochit atohomba hi oke: Chihowah ya $\underline{\mathrm{i}}$ holitopa kvt moma i shahli hoke, achi tok.

11. Mihma Chihowah yvt Moses a im anumpulit,

12. Islael im vlla vhleha ha noshkobo holhtina ka ish eshikma hatak moyuma $k \underline{a}$ ish hotihnakma imi shilombish vlhtoba ya Chihowah imashke; yumohmi ho ish hotihnakma ililli okpulo itin takla ya iksho kashke.

13. Iluppak o imashke, holhtina ka iba holhtina tuk moma kut vlhtipo holitopa shekel im vlhpisa vlhpisa ho, shekel iklunna ho ima hi oke; shekel achufa kvt kelah pokoli tuklo hoke, shekel iklunna kak o Chihowah ya im issa ya hi oke.

14. Afưmmi pokoli tuklo vttut vba maya holhtina ka iba holhtina tuk moma kut Chihowah im issa ya ima hi oke.

15. Hvchimi shilombish isht vlhtoba nan im issa ya Chihowah hush imakmut, nan in laua yut shekel iklunna ka ont ia ho ima ke keyukma, ilbusha yut ola takla ho ima he keyu hoke.

16. Mikma Islael im vlla vhleha ha nan isht atobbi tvli holisso ya ish eshi cha, okla moma nan im vlhtipo nan isht ai ahanta isht ish apesashke; yumohmi ho Islael im vlla vhleha ha Chihowah itikba ai isht ithaiyana he, micha hvchimi shilombish isht vlhtoba yoke, achi tok.

7. Mikmut Chihowah yut Moses a im anumpulit,

18. Aiachefa yo asonak lakna ampo chito, micha iyi vt asonak lakna ak inli ho ish ikbashke, micha okla moma im vlhtipo, micha alta itin takla ish hilechi cha oka ish vnashke. 
19. Yvmmak o Alon micha ushi vhleha aiena kvt ibbak, micha iyi aiena ka ai achefa hi oke.

20. Okla moma im vlhtipo ya chukowakmvt, keyukmut luak ishit hukmit Chihowah im issa ya hukma chi hosh alta ya bilika ona aienakmvt ik illo $\mathrm{ka} \mathrm{hi}$ oke.

21. Yummohmit ibbak, micha iyi aiena ka achefa cha ik illo kashke; micha nan vlhpisa vlhpesa bilia yosh, Alon, micha ushi vhleha im ai iti shali moma kvt in toyulashke, achi tok.

22. Micha Chihowah yut Moses a im anummulit,

23. Nabalama i shahli puta ma ahli shekel tahlepa tahlapi, sinnamon balama vt yumma iklunna shekel tahlepa tuklo cha pokoli tahlapi, kalamus balama vt shekel tahlepa tuklo cha pokoli tahlapi,

24. Micha kassia vt shekel tahlepa tahlapi vlhtipo holitopa ai vlhpisa shekel ashke, micha alif bila yvt hin achufa ho ish ishashke.

25. Micha nanishtahama holitopa bila, ikhịsh ikbi ithaiyana hosh ikbi yo nan ahama ish ikbashke; isht ahama holitopa bila hi oke.

26. Micha okla moma im vlhtipo ya micha nanulhpisa afoyuka itombi holitopa,

27. Micha aiimpa, micha nan im ai vlhto moma, micha pula aiohikia, micha nan im aivlhto, nabalama ahukmi alta,

28. Micha ai onochit hukmit im issa alta, micha nan im aivlhto moma, micha aiachefa ampo chito, micha jyi aiena ka isht ish ahummashke.

29. Micha ish kashofi na holitopa kvt moma $\underline{i}$ shahli yashke, nana hosh halelikmvt holitopa hi oke.

30. Micha Alon, micha ushi vhleha aiena ka ahvmmit ish hullochashke, mikma na holitompa isht aivtta ya isht vm aiahashwashke.

31. Micha Islael im vlla vhleha ish im anumpulit, hvchim ai itishali moma ka nan isht ahama bila iluppot a holitop chi hoke.

32. Hatak nipi okuno o fohopa he keyu, micha itaioma ya hobachit inla ya ish ikba he keyu hoke, holitopa hoke, hvchi holitopa hi oke. 
33. Kuna hosh yưmma chohmi ka itaiomi, keyukmut okla inla yo onochikmvt, im okla achvfa ya ai $\underline{\mathrm{i}}$ filvmmit fiopa tupa hi oke ish achashke, achi tok.

34. Mihmvt Chihowah yvt Moses a, Nabalama istakti, oneka, micha kalbanum, ish ishashke; nabalama iluppa puta ka falakinsen bano yo, moma weki kvt iti laui hashke.

35. Micha ikhish ikbi champullichi ak o hobachit nabalama vlhpesut itaioma kashofa, micha holitopa yo ish ikbashke.

36. Micha kaniohmi ka bolit ish lapushkichi cha, kaniohmi ka okla moma im vlhtipo anukaka isht atokowa itikba a chi afama la chị ka ish talalashke, chị holitopa $\underline{i}$ shahli ya chi hoke.

37. Micha nabalama ish ikba chi kut itaioma, yvmma chohmi ka chimmi ish ikba he keyu, Chihowah immi hosh chi holitopa hi oke.

38. Kuna hosh yumma chohmi ka huwa chi hosh ikbikmot im okla achvfa ya ai i filvmmit fiopa topa hioke, im achi tok.

\section{CHAPTA XXXI.}

IHMVT Chihowah yut Moses a im anumpulit,

1 2. Yakih, Chutah apehlichi aiachvfa $\mathrm{Hu}$ nshi, Uli yo ushi Besaleel a atokolit hochifo lishke,

3. Micha hopoyuksvt, micha nana akostininchit, micha nanithanvt micha na toksvli ilaiyukali moma imponnot.

4. Natoksvli inla tuli holisso lakna, micha tuli hvta, micha asonak lakna toksula he,

5. Micha toli tihlit on talohla he, micha iti ya chakonlichi na toksuli ilaiyukali moma apesvehit imponna chị ho chükvsh Chitokaka imi shilombish a alotoli lishke.

6. Micha vno vt, yakeh vno vt Tan apehlichi aiachvfa Ahisamak ushi Aholiab a aieninchit ima lishke; chim apesa li tuk moma yumicha chị kạ, chụkvsh hopoyuksa puta ka chukush $\underline{i}$ hopoyuksa moma nukfoki li tuk oke.

7. Orla moma im vlhtipo, micha nan vlhpisa a foyuka 
itombi holitopa, micha ai im isht i kana ai om binili, yvmmot paknaka talaia ka micha vlhtipo i nan chukushpa moma,

8. Micha aiimpa, micha $\underline{\underline{i}}$ nachukushịpa, micha pvla ai $\underline{o}$ hikia kashofa, i nachukushpa aiena moma, micha nabalama alta,

9. Micha aionochit hukmit im issa alta $\underline{i}$ nachukushpa aiena moma, micha ampo chito, micha iyi,

10. Nan isht vtta $\underline{i}$ nafohka, micha naholitompa isht vtta Alon a i nafohka holitopa, micha ushi whleha hvt na holitompa isht ai vtta ya isht ashakmvt i nafohka,

11. Micha isht ahama bila, micha aholitopa nabalama aie-

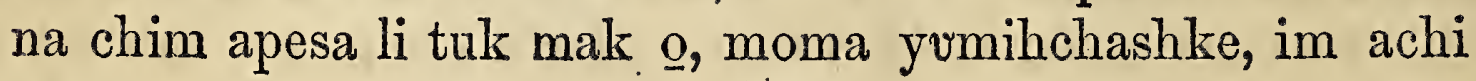
tok.

12. Micha Chihowah yut Moses a im anumpulit,

13. Islael im vlla vhleha ha , A nitak hullo ya a hlit hush holitoblashke; hvchim ai iti shali moma ka nan ishtatakowa yosh hupim itin takla ya itoyula chị hoke; yưmohmina, Chihowah sia hvchi kashofi li ka hush ithaiyana chị hoke.

14. Yvmohmi hoka nitak hullo yo hush holitoblashke, hvchi holitopa hoka, kuna hosh kobvffikmut illi pulla hi oke; kuna hosh nitak yumma na toksuli nana kia yumihchikmvt, shilombish yummut im okla ha ai i filummit fiopa tupa hi oke.

15. Nitak hannali ho natoksuli ya yumihcha hinla hoke, Amba isht untuklo hokvto nitak hullo afoha, Chihowah $\mathrm{i}$ holitopa hoke; kuna hosh nitak hullo ya natoksvli nana kia yumihchikmut illi pulla hi oke.

16. Yvmohmi hoka Islael im vlla vhleha ind ai itishali moma kut nan itim apesa bilia ya nitak hullo ja boljoblit, nitak hullo ya ithaiyana hi oke.

17. Nan isht atakowa yosh vno, micha Isläel im vila ville ha pim itin takla ya itoyula na bilia hi oke; nitak hannali họ Chihowah yvt vba shntik, micha yakni aiena ka ikbi mut; nitak isht untuklo ka foha cha, im achukma tok oka, ish im achashke, im achi tok.

18. Atuk osh Moses a Sinai nunih paknaka ai im anumpuli tuk vt ont tahli mot, nan vlhpisa patvssa tuklo tvli patussa Chitokaka ibbak ushi yosh ai o holissochi yo ima tok. 


\section{CHAPTA XXXII.}

\section{CIAPTA XXXII.}

IIHMA Moses vt nưnih ha akowa kvt ahchibali na, okI la hut pisa mut, okla hash osh Alon a im itunaha cha, Wakaya cha, pi tikba ia chi ka naholbut toba ya pim ikbashke, Echip yakni a pehlichit pi kohchi tok hatak Moses iluppak okvno, nana kaniohmi tuk a kil ithano hokvt, im achi tok.

2. Mihma Alon vt, Tuli holisso lakna haksobish takali, hvchi tekchi vhleha micha hvchiso vhleha, micha hvchiso tek vhleha aiena haksobish takohmaya ka tuptulit isht hus sum vlashke, im achi tok.

3. Mihma okla moma kut tuli holisso lakna haksobish takali haksobish takohmaya tuk a tuptuli cha, Alon a isht im ona tok.

4. Mihma ibbak a im eshi cha, bilvt akmot, wakushi ikbi ha yot, ishit pilesa ishit tihlit ikbi tok: mihma, Ilvppa puta kak osh hvchi na holbüt toba yosh Echip yakni ya apehlichit hvchi kohchi tuk mak oke, Islael ma, okla achi tok.

5. Atuk o Alon vt pisa mut, itikba ya alta ikbi tok; micha anumpa bohlit, Onnaha yut Chihowah impvchi yoke, achi tok.

6. Atuk osh onna kvt onnahinli fehna moma ho, okla wakaya cha, hukmit im issa ya im issa mut, itin nanaiyvchi im issa ya isht vla tok, mihmot okla hot binilit impvt nan ishko tul gsh: wakayut ihlauvlli tok.

7. Magda Cinhowah yut Moses a, Iah, akowah, chim okla Echipyakn a pehlichit ish kohchi kash osh il okpuni hoke. . Im apesa li tuk hina ya chekosi fehna ho kohchit fullọtushke, bilut akmot wak ushi toba ikbi cha, aiokpvchit nan vbit im issa cha, Ilvppa puta kak osh hvchi naholbut toba vhleha hosh Echip yakni hvchi pehlichit kohchi tuk oke, Islael ma, achishke, achi tok.

9. Mihmut Chihowah yut Moses a, okla iluppa pihisa li tuk oke; mikma yakeh, ikunla kvllo okla hoke.

10. Yumohmi hoka inta, ik asha is sum ahni na, vm ai $\underline{\mathbf{i}}$ 
nukkilli vt $\underline{\mathrm{i}}$ sanalit libbashke, micha yưmmak o lushummi lashke, micha chishno ak o okla chinto ho chikbi lashke, im achi tok.

11. Mihma Moses vt in Chitokaka Chihowah ya im ilbvshvt, Chihowah ma, nanta katiohmi ho chim ai i nukkilli vt chim okla hạ, nan isht aiahli chinto, micha ibbak kvllo hosh pehlichit Echip yakni ya isht ish kohcha tuk ut $\underline{\mathbf{i}}$ sanalit libbi cho?

12. Nanta katiohmi ho Echip okla hut nan ik achukmo yumihchit nunih fohka ai ubit tahlit yakni paknaka ai ishit kanchit mosholicha chi hosh pehlichit kohcha tuk oke, acha chi cho? chim ai i nukkilli libbi kvt ish shanaiya cha, nan ik achukmo chim okla i sanali iluppa ish issashke.

13. Eblaham Aisak, micha Islael chin tishu vhleha, yummak oka chishno ak inli ile hochifot anumpa kvllo anumpulit, chim isht atiaka apaknvchit vba shutik fochik a chohmichi la chi hoke, micha yakni isht anumpuli li tuk iloppa chim isht atiaka ya ima likma immi bilia hi oke, ish im achi tok vt ish ithaiyanashke, achi tok.

14. Mihma Chihowah yot im okla ha $\underline{\mathrm{i}}$ sanalit nanuk achukmo yumihcha chi hosh anukfilli tuk vt issa tok.

15. Mihma Moses vt filema mut, nunih ha akowa tok, mihma nanvlhpisa ai o holisso patussa tuklo kvt ibbak a foka tok; patussa yut tahlaklakka a holisso ha tok; in tunnvp achvfa ka a holisso mvt, in tunnưp achufa ka a holisso ha tok.

16. Micha patussa yut Chitokaka yosh ikbi ha tok, micha holisso ha Chitokaka yosh holissochit patussa ya in chunli ha tok.

17. Atuk o Choshua vt okla shakapa ka haklo mut, Moses $\underline{a}$, Binah ha tunup shakapa ho haklo lishke, im achi tok.

18. Mihma Im aiya hosh shakapa ano keyu, micha ilap ak o im aiya ho paya hoh keyu, amba taloa hosh shakapa ka haklo lishke, achi tok.

19. Atuk o yakohmi tok, binah ha bilika ona mut, wakushi yash, micha hihla aiena ka pisa tok; mihmot Moses im ai i nukkilli vt libbi mut, nunih nuta ya patussa yash ibbak a mokofit aka pila cha, koli tok. 
20. Micha wak ushi okla ikbi kash eshi cha, luak ituchit hukmi mvt, fotohlit pushechi cha, oka ya o fimmi mut Islael im vlla vhleha ha ishkochechi tok.

21. Mihmut Moses vt Alon a okla hut nanta ho chi katiohmichi ho nanashvchi chinto yakohmi ka ish onochi cho: im achi tok.

22. Mihma Alon vt, $\underline{\mathbf{A}}$ shahli im ai i nukkilli vt ik libbo kashke, okla hvt nan ik achukmo amosholi ya ish ithaiyanushke.

23. Pi tikba ia chi ka naholbut toba ya ish pim ikbashke; Echip yakni ya pehlichit pi kohchi tuk, hatak Moses iluppak okuno nana kaniohmi tuk a il ithana kẹyu hoka, vm achi tuk oka.

24. Mihma kuna hosh tuli holisso lakna ishikmvt tvblashke, im achi li tuk oke. Yohmi ma okla vma tuk oke: yohmi na luak pit ituchili ma, wak ushi iluppak osh kohcha tuk oke, achi tok.

25. Atuk o Moses vt okla hut nipi bano na pisa mvt (Alon vt in tunup vhleha ha im i hofahyalit nipi banochi tuk oke.)

26. Yohmi ma Moses vt binah okhissa ya hikia cha, Kvta hosh Chihowah ya ibafoyuka cho ; vno vm vlashke, achi tok, Mihma Lefai ushi vhleha moma kvt im itunaha tok.

27. Mihma Chihowah Islael in Chitokaka vt yak achishke, Hatak moma kvt hvchi bushpo falaia ya hush ilapakshunni cha, binah okhissa puta chukowvt kohchvt hlopullit hatak moma kvt huchitibapishi hạ, micha hatak moma kvt ai itapiha ya, micha hatak moma kvt in chuka a bilika ya hush bashke, achishke, achi tok.

28. Mihma Lefai im vlla vhleha hot Moses im anumpa hatuk mak o, yumihchi tok; mihma nitak yumma akakoha kvt hatak tahlepa sipokni tuchina foka tok.

29. Moses vt himak nitak a hatak moma kvt ilap ushi, micha itibapishi hatuk kia Chihowah im isht ile hullochashke; yumohmi ho himak nitak a nan isht yukpali a horchi onochashke, achi hatuk oka.

30. Atuk o onna ma, yakohmi tok, Moses vt okla hạ, Nan- 
ashuchi chinto ho hush ashvchishke, atuk o himak a oiyut Chihowah ya im ia la chi hoke, na hvsh ashvchi ya atobbi li yoba na (or kashofit atobbi li yoba na) achi tok.

31. Mihmut Moses vt falamvt Chihowah ya im ona cha, Ikikkeh, okla ilupput nan ashvchi chito ho ashvchi cha, tvli holisso lakna naholbut toba ikbishke.

32. Yohmi kia himak a ish ahnikmvt, nanashvchi ya ish i kashofashke, atuk o ish yumihcha he keyukmut, chi holisso ho ish holissochi tuk vt is sa kashofashke, achi tok:

33. Mihma Chihowah yvt Moses a, Kvna hosh a yoshuba tukma, yummak $\underline{a} \underline{a}$ holisso ha a kashofi la chi hoke.

34. Yumohmi hoka himak a iah, micha okla pehlichit, yakni chim anoli li tuk a ish onashke. Yakeh, vm enchil vt hvchi tikba ia chi hoke: yohmi kia im aya li nitak mut, nan ashvchi tuk a im ayvt onochi la hi oke.

35. Atuk osh Chihowah yut okla ha Alon vt ikbi ma, wak ushi okla ikbi tuk pulla kak o nan ik achukmo onochi tok.

\section{CHAPTA XXXII.}

IHMUT Chihowah yut Moses a, Iah, micha chishno, 11 micha chim okla ha Echip yakni ya apehlichit ish kohchi tok aienvt ilvppa ashut ivt, yakni Eblaham, micha Aisak, micha Chekob aiena ka anumpa kvllo im il onochit chim ishtatiaka ima la chi hoke, achi li tok a ish onashke.

2. Mikma enchil a hvchi tikba pila la chi hoke; micha Kananait, Amolait micha Hittait, Pelisait, Hifait, micha Chebusait aiena ka tihlelit kohcha wehli la chi hoke.

3. Pishukchi, micha foebila ayanahanli yakni o hvchisht ona chi hoke: hvchitin takla ayvt ia la he keyu; ikunla kvllo okla, hvchia hoka, hina takla mosholichit huchi kanchi li yoba na, im achi tok.

4. Atuk o okla hot anumpa ik achukmo iluppa haklo motiokla yaiya tok, mihma achufa kia isht ilakshema isht ik shemo tok:

5. Chihowah yot Moses a, Islael im vlla vhleha, Ikunla 
kvilo okla hvchia hoke, yakosi itin takla kia huchi takla ya onvt mosholichit hvchi kanchi la chi hoke : yumohmi hoka himaka nan isht ilakshema ya bush shoelashke, yumohmi ho nana hvchi kaniohmichi la chi kvt ithana lashke, ish im achashke, im achi tuk oke.

6. Mihma Islael im vlla vhleha hvt nan isht ilakshema ya Holeb nunih bilika ya shoeli tok.

7. Mihma Moses vt vlhtipo ya eshi cha, binah ha kohcha hopaki ho hilechi cha, Okla moma im vlhtipoh achi tok. Atuk o yakohmi tok, kvna hosh Chihowah ya hoyokmut, kohchut okla moma im vlhtipo kohcha talaia kak $\underline{\text { o ona be- }}$ ka tok.

8. Atuk o yakohmi tok, Moses vt kohchut vlhtipo ya ona ma, okla hash ot moma kvt wakaya cha, hatak moma kvt i binah aiyukali okhissa ya hiohmayvt, Moses a pit iakaiyvchit pisa na, vlhtipo ya ont chukowa tok.

9. Atuk o yakohmi tok, Moses vt vlhtipo ya ont chukowa makinli ho, hoshonti tonik vt aka mintit vlhtipo okhissa ya ont a hikia tok; mihma Chihowah yvt Moses a itim anumpuli tok.

10. Mihma okla moma kvt hoshonti tonik vt vlhtipo okhissa ont hikia na pisa tok; mihmot okla moma kvt wakaya cha, hatak moma kut I binah okhissa ya ai aiokpvchi tok.

11. Mihma Chihowah yot Moses $\underline{a}$, hatak osh $\underline{\underline{i}}$ kana itim anumpuli ka chohmit nashuka iti sanalit itim anumpuli tok. Mihma anonti falamvt binah ha ont chukowa tok: amba in tishu hatak himita Nun ushi Choshua vto vlhtipo ya kohchưt ik aiyo tok.

12. Mihma Moses ut Chihowah ya yakeh, Okla iluppa isht ish onashke, is sum achishke: yohmi hokak osh kuna hosh ish atokoli na si awant ia chi ka chik sum anoloshke: yohmi hokak osh hohchifo ho ishit chithana li, micha na sa tikba ya vm isht $\underline{i}$ kana ya ish aiahayuchi ak inlishke, ish achi tuk oke.

13. Yvmohmi hoka himak a chim asilhha lishke, chi tikba ya chim isht $\underline{i}$ kana aiahayuchi li hokma, himak a chi 
hina is sum otuninchashke: yohmi na chithaiyana lashke, yohmi na, chi tikba ya chim isht $\underline{i}$ kana ai ahayuchi lashke: micha okla ilupput chim oklushi ya ish anukfillashke, im achi tok.

14. Mihma Uno ak osh huchi awant ia la chị hoke, micha hvchi fohachi la chi hoke, achi tok.

15. Mihma, Si awant ish ia chi keyukmut, iluppa a pishit chik aiyo kashke.

16. Nanta hak $\underline{\text {, }}$ Uno, micha chim okla aiena kut chim isht $\underline{\underline{i}}$ kana ya ahayuchi tuk a iluppak o isht ai akostinincha hinla cho; pi awant ish aya kak a hinla keyu cho? Yohmi hosh vno micha chim okla aiena kvt okla moma yakni paknaka aiasha ka i filumma hi oke, im achi tok.

17. Mihma Chihowah yot Moses a, Ish anumpuli tuk, iluppak kia yumihchi la chi hoke. Um isht $\underline{i}$ kana ya ish ahayuchi tuk okvt, micha chi hohchifo ha ithana li hoke, im achi tok.

18. Mihma Chim asilhha lishke, chim aholitopa ya is sa pesvchashke, achi tok.

19. Mihma, Si ai achukmaka ya mominchit chitikba ya ont iachi la chi hoke, micha chitikba ya Chihowah hohchifo ha ai anoli la chi hoke: micha kưna ho $\underline{i}$ kana la he, ahni likmot, i kana la hi oke; micha kuna ị nukhakklo la hi, ahni likmot, i nukhakllo la hi oke, achi tok.

20. Micha, Sa nashuka ya ish pisa he keyu hoke, hatak nana hosh sa pesa cha, okchaya he keyu hoka, achi tok.

21. Micha Chihowah yvt, Yakeh vm a kanima kvt talayushke, mikma tuli chito paknaka ya ish o hikiashke.

22. Atukma yakohma hi oke, vm aholitopa yvt ant aya takla kạ, tvli chito wakla ya chi fohki la chị hoke: micha ant aya li takla kvt subbak isht chi ompohomo la chi hoke.

23. Atuk osh subbak a ishe likma, vm ashaka ak o ish pisa hi oke, amba sanashuka ya pisa he keyu hoke. 


\section{CHAPTA XXXIV.}

IIHMA Chihowah yut Moses a, Tuli patussa tuklo vm11 mona tuk a chohmi ho ish tuhlashke, mikma tuli patussa yummak o tuli patussa ummona ish koli kash anumpa atakali tuk a ai o holissochi lashke.

2. Mikma onnahinlikma chim vlhtaha mvt, onnahinlikma Sinai nunih ha ish oiya cha, nunih paknaka ya sa tikba ya is sum vlashke.

3. Mikma hatak nana kia chi awant oiya he keyu; micha nunih afullota moma hatak nana kia hikia na, ik peso kashke; mikma nan vlhpoa chipunta, micha nan vlhpoa hochito aiena kvt nunih yumma itikba ya ik ai impo kashke, achi tok.

4. Mihma tuli patussa tuklo vmmona tuk a chohmi ho twhli tok, micha Moses vt onnahinli fehna moma ho, wakaya cha Chihowah yut $\underline{i}$ miha tuk mak $\underline{o}$, tuli patussa tuklo kak osh ibbak foka hosh Sinai nunih ha oiya tok.

5. Mihma Chihowah yut hoshonti afoyuka cha, akowa mot, yvmmak o takkla hikia cha, Chihowah hohchifo ha anohonli tok.

6. Micha Chihowah yot itikba ont ia cha, chitolit, Chihowah, Chitokaka Chihowah nan i nukhakklo, $\underline{\underline{i}}$ kana, i sulaha, micha ai achukma, micha aiahli aiena kvt in lawah.

7. Tahlepa sipokni hoh kia i nukhakklot bilia, ik vlhpieso, micha anumpa kobuffi, micha aiashvchi aiena ka i kashofi, micha anumpa hut onvtola he vlhpesa ya hlakoficha he keyu, iki puta ik ai vlhpeso atuk mak o im vlla vhleha puta ka, micha vlla vhleha hash im vlla vhleha ai iti shali ont atuchina, micha ont ai ushta kia im aya yoke, achi tok.

8. Mihma Moses vt túshpvt noshkobo ha aka pit isht akachunoli cha aiokpuchi tok.

9. Micha Chihowah ma, himak a chim isht ikana ahayuchi li hokma, chim asilhha lishke, an Chihowah vt pi awant iashke (okla ikunla kvllo vhleha hoka), ik pi ai vlhpieso, micha il aiashvchi ya ish pi kashofashke, micha chimmi ikbit ish pi eshashke, achi tok. 
10. Mihma, Yakeh anumpa ahli ikbi lishke, chim okla moma itikba ya na fehna yumma chohmi kut yakni moma ka, micha oklushi nana kia, a yưmohmi chatuk keyu ho yumihchi la chi hoke; mikma okla takla ish ahanta tuk vt moma kut Chihowah na yumihchi ka pisa chị hoke, na huchi yumihchi li ka ishit nukhlakancha ya chi hoke.

11. Himak nitak a chim apesa li ka ish ithaiyanashke. Yakeh, Amolait, micha Kananait, micha Hittait, micha Pilissait, micha Hifait, micha Chibusait aiena ka huchi tikba tihlelit kohcha wehli lishke.

12. Ahah ish ahni cha, yumma ish ia kvt yakni ya okla ai asha ka anumpa chik itim apesokashke; ishit yoshoba yosh hvchitin takla ya aiasha yoba na.

13. Amba im alta puta, $\underline{i}$ naholbvt toba puta $\mathrm{ka}$ ish okpuni cha, im iti lokoli puta ka chanlit ish akvchashke,

14. Naholbut toba chitokaka inla ka ish aiokpvcha he keyu hoka, Chihowah yumma hohchifo kvt Nuktanhla ya tuk osh, Chihowah nuktanhla yoke.

15. Yakni ya okla aiasha ka nan ish itim apesa na, hauit $\underline{i}$ na holbut toba ya iakaiya cha, $\underline{i}$ naholba ya nan vbit isht aiokpvchi, atuk $\underline{o}$ chim anoli na, vbit isht aiokpvchi a ishpa yoba nah.

16. Micha chiso vhleha ha ushetik vhleha ha ishit ish ima na, ushetik vhleha hash osh hauit $\underline{\mathrm{i}}$ naholbvt toba ya iakaiya cha, chiso vhleha ha hauit i naholbut toba iakaiyvchi yoba na.

17. Bilvt akmot naholbvt toba ya chik ikbo kashke.

18. Puska ik shatummo impvchi ya ish holitoblashke. Hushi Abib nitak o chim achi li tuk mak o, nitak untuklo ho puska ik shatummo ish pashke; Abib hushi o Echip a ish kohcha tok okvt.

19. Im oshutto tiwi moma kvt vmmi hoke; micha chi nanulhpoa moma tikba vtta kvt wak okma, chukfi hokma nana kia, nakni okmvt vmmi hoke,

20. Amba issuba nashoba vtta ahpi ano falvmmichit chukfushi isht ish chumpashke; micha falummichit ish chumpa he keyulkmvt, ikunla ya ish kotvffashke; micha 
chiso vlheha vtta ahpi moma ka falummichit ish chumpashke; mikma achvfa kia ibbak bieka hosh sa tikba ya iklo kashke.

21. Nitak hannali ho ish toksvlashke, amba nitak isht untuklo kano ish fohashke; yakni patuffi nitak, micha nawaya nitak a ish fohashke.

22. Micha onush vmo waya vmmona wek puta impvehi ya , micha afvmmi ont taha, na waya hoyo impvehi ya ish holitoblashke.

23. Afummi achufakma hituchina ha nakni yosh hvchim ai asha moma kvt Chitokaka Chihowah Islael in Chitokaka itikba ya hiela hi oke,

24. Oklushi puta ka hvchi tikba kohchit kanchi lish, chim ai vhli a chitolichi la chi hoke, mikma afummi achvfakma hituchina ha chin Chitokaka Chihowah ya itikba ish hikia chị hosh ish iakma, hatak nana kia chị yakni a bunna he keyu hoke,

25. Ubit isht aiokpvchi issish a puska ikshatummo aieninchit is svm issa he keyu hoke, micha vbanvblit ontia impvchi vbit isht aiokpvchi vt asha na onnahinli ya ona he ke keyu hoke,

26. Nawaya vmmona achukma $\underline{\mathrm{i}}$ shahli aya chin Chitokaka Chihowah im aboha isht ish onashke. Issi kosoma ushi ya ishki i pishukchi a isht ish hona he keyu hoke, achi tok.

27. Mihmot Chihowah yut Moses a, Anumpa ilvppa puta ka ish holissochashke; anumpa iluppa puta chohmi kak o, chishno micha Islael aiena ka itim apesa li hoka, im achi tok,

28. Micha yumma nitak pokoli ushta, micha ninak pokoli ushta ho Chihowah takla ahanta tok, puska ya ikpo, micha oka ya ik ishko tok; micha nan vlhpisa anumpa nan vlhpisa pokoli yash tvli patussa ya ai o holissochi tok,

29. Atuk o yakohmi tok, Moses vt Sinai nunih ha nanvlhpisa tuli patussa tuklo kvt Moses ibbak a foka ho akowa mut nunih ha akowa mut, im anumpuli ma, nashuka yot shohmalali ka ik ithano ki tok. 
30. Atuk o Alon, micha Islael im vlla vhleha moma kvt Moses nashuka yut shohmakali ka pisa mut, bilika ona ka nukshopa tok.

31. Mihma Moses vt $\underline{\mathbf{i}}$ howa tok; mihma Alon micha okla moma pehlichi vhleha hut falamvt im ona tok, mihma Moses vt itim anumpuli tok.

32. Atuk osh $\underline{\mathrm{i}}$ himmak ak o Islael im vlla vhleha moma kvt bilika ona tok; mihma nanvlhpisa ha Chihowah yut Sinai nunih ha ai im anumpuli tuk moma ka ima tok.

33. Micha Moses vt im anumpulit ik tahlo kisha kvt, nashuka ya ishtompoholmo ya isht ompohomo tok.

34. Amba Moses vt itim anumpula chi hosh Chihowah itikba ya ona mvt, nashuka isht ompoholmo ya ishi tuk osh kohcha tok; micha kohcha mut, Chihowah i miha tuk mak o Islael im vlla vhleha ha im anumpuli tok.

35. Atuk o Islael im vlla vhleha hut Moses nashuka ya pisa ma, Moses nashuka yot shohmalali tok, mihma Moses vt anonti nashuka ya isht ompoholmo ya isht ompohomo tuk osh im anumpula chi hosh iba chukowa tok.

\section{CHAPTA XXXV.}

MICHA Moses vt Islael im vlla vhleha moma ka itunnali 11 cha, Anumpa iluppa puta kak o hush yumihcha chi ho, Chihowah yot hvchim apesa tuk oke.

2. Nitak hannali ho natoksuli vt vlhtahashke, amba nitak isht untuklo hokvto nitak chi holitopa Chihowah $\underline{\mathrm{i}}$ nitak hullo a foha hi oke: kuna hosh yummak o natoksuli nana kia yvmihchikmot illi pullashke.

3. Nitak hullo ya hush aiasha ahlopulli ka luak nana kia hvchik ikbo kashke, achi tok.

4. Micha Moses vt Islael im vlla vhleha okla moma ka im anumpulit, Nana iluppak o Chihowah yvt huchim apesut,

5. Huch itin takla ya Chihowah nan im issa hush ai ishashke; kvna hosh chukvsh aiokpanchikmvt, Chihowah isht aiokpuchi, tuli holisso lakna, tvli huta, micha asonak lakna, 
6. Micha nan okchamali, micha hummaii, micha tishepa, micha linen tapuski, micha issi kosoma hishi,

7. Micha chukfi nakni hakshup humma, micha bacha hakshup micha shittim iti,

8. Pula ha chi ka bila micha ishtahama bila ya chi ka micha nabalama ya chi ka nabalama,

9. Micha oniks tvli, micha efot micha ikkishi alata ontvla chi ka tuli puta ka isht vlashke.

10. Mikma chukvsh hopoyuksa vhleha hvchi takla aiasha kut vlut Chihowah yvt apesa tuk moma ka yumihchashke.

11. V'lhtipo tabanake, micha im ompoholmo, im ishtakamussa, micha im iti busha, im okhowatkvchi, in tonik, micha im ashamvlli,

12. Itombi holitopa, micha in tubi, ai i kana ai ombinili aiena, micha isht ompoholmo, ompoholmo,

13. Ai impa, micha in tubi, micha nan im aivlhto moma, micha puska otuni,

14. Micha tohwikela chị ka pula ai o hikia, micha i nachukushpa, micha pvla bila aivlhto tohwikela chi ka bila aiena,

15. Micha alta nabalama ai o hukmi, micha in tubi, micha ahama bila, micha nabalama, micha vlhtipo ya achukowa okhissa ya atakali,

16. Alta ai o hukmit isht aiokpvchi, im asonak lakna tunna im aiena in tobi, micha nan im aivlhto moma, ampo mahaia chito, micha iyi,

17. Wanuta ya natakali, in tonik, micha im ashamvlli, micha wanuta okhissa ya in takali,

18. Vlhtipo ya isht akamussut shumohli, micha, wanuta isht akamussut shumohli, micha im asita puta.

19. Aholitopa nan isht ai vtta chi ka nafohkvt isht vtta, naholitompa isht vtta, Alon i nafohka holitopa, micha naholitompa isht ai vtta isht asha chi ka ushi vhleha i nafohka puta ka ikba chi hoke, achi tok oke, im achi tok.

20. Mihma Islael im vlla vihleha okla moma kvt Moses a ai i filummit ia tok.

21. Mihma chukvush vt ayimitvchi tuk puta kvt, micha 
shilombish vt vlhpiesa ahnichechi tuk puta kvt vla tok; micha Chihowah nan im issa ya okla moma im vlhtipo, micha nan isht aiahanta moma, micha nafohka holitopa a toba chi ka isht vla tok.

22. Mihma chukvush aiokpanchi aiynkali kvt hatak, micha ohoyo aiena kvt, vlut, ibbak vlbeha, micha haksobish takali, micha ibbakushi vlbeha, micha shakba vlbeha tvli lakna isht ilakshema moma isht vla tok, mihma im issa tuk aiyukali hatak vt tvli holisso lakna, im issa ya Chihowah im issa tok.

23. Mihma hatak kuna hosh nan okchamali, micha nahummaii, micha na tishepa, micha linen tapuski, micha issi kosoma hishi, micha chukfi nakni hakshup homma, micha bacha hakshup aiena kvt im asha tuk vt isht vla tok.

24. Kuna hosh tuli huta, micha asonak lakna im issa ya im issa aiyukali kut Chihowah nan im issa ya isht vla tok; mikma hatak nana hosh shittim iti nan isht aiasha toba he vt in tonlakmvt isht vla tok.

25. Mikma ohoyo chukkush hopoyuksa moma kvt ibbak ishit shunni cha shunni tuk vt nan okchamali, micha na hummaii, micha natishepa micha linen tapuski aiena ka isht vla tok.

26. Micha chukvsh vt hopoyuksachit ayimitvchi tuk ohoyo moma kut issi kosoma hishi o shunni tok.

27. Mihma pehlichi vhleha hvt efot, micha ikkishi alata ya ontula chị ka oniks tvli, micha tuli puta ka isht vla tok.

28. Micha ahama ya chi ka, micha nabalama chị kạ, nabalama, micha tohwikeli ya chi ka bila aiena ka isht vla tok.

29. Islael im vlla vhleha hut hatak micha ohoyo moma kvt chukvsh vt vlhpesa ahnichichi na, na toksvli ilaiyukali moma kut toba chị ho Chihowah yut Moses a toksuli apesa tuk a, chukvush aiokpanchi hosh Chihowah nana im issa ya isht vla tok.

30. Mihma Moses vt Islael im vlla vhleha hạ, Yakeh, Chihowah yot hochifot Chutah apehlichi aiachvfa $\mathrm{Hu}$ ushi Uli ya ushi Besaleel ahe atokoli tuk oke. 
31. Micha Chitokaka shilombish alotoli na, hopoyuksa nanithana, micha nana kostininchi,' micha natoksuli ilaiyukali moma imponna;

32. Micha natoksvli inla fehna anukfillit, tvli holisso lakna, micha tvli hota, micha asonak lakna toksvli,

33. Micha on taloha chị kvt tvli tvhli, micha iti ya bambakechi natoksuli inla fehna nana kia ikbi ya nukfohki tuk oke.

34. Micha im abvecha he ya, yumma, micha Tan apehlichi aiachvfa Ahisamak, Ảholiab itatuklo ka nukfohki tuk oke.

35. Yummak o natoksuli ilaiyukali moma nan in chunli, micha natoksuli inla fehna imponna, micha alakali, okchamali, micha hummaii, micha tishepa, micha linen tapuski, nantunna, micha natoksuli kaniohmi kia yumihchi, natoksuli inla fehna kia anukfilli aiena ka imponna nukfohki tuk oke.

\section{CHAPTA XXXVI.}

YOHMI ma Besaleel, micha Aholiab, micha hatak chu1 kvsh hopoyuksa puta kvt natoksvli ilaiyukali moma vlhtipo ya isht atoksuli ya chị ho Chihowah yut apesa tuk moma ka yumihcha chi ho, Chihowah yut hopoyuksa, micha nanithanvt toksvli imponna nukfohki tuk vt ikbi tok.

2. Mihma Moses vt Besaleel, micha Aholiab, micha hatak chụkush hopoyuksa aiyukali Chihowah yvt chukvsh $\underline{a}$ hopoyuksa nukfohki tuk, vlut natoksuli ya yumihcha chi ho chukvush vt a ayimmitvchi tuk puta ka hoyo tok.

3. Mihma vlhtipo ya isht a toksvli toba chi ka ishit toba ya Islael im vlla vhleha hot im issut isht vla tuk moma ka Moses a im ai ishi tok, micha onnahinli moma ma chukvsh aiokpanchi hosh nana im issa ya isht im ahanla tok.

4. Mihma hatak hopoyuksa moma vlhtipo ya natoksvli puta aiasha ka toksvli tuk vt nan ikbit toksuli tuk vt issut ia tók.

5. Micha Moses a okla im anumpulit, Okla hut natoksuli 
toba ka Chihowah yvt apesa tuk a atampa fehna ho ishtvlushke, achi tok.

6. Mihma Moses vt apesa na, okla anolit binah ha hlopullichit, Hatak nana kia, micha ohoyo nana kia, vlhtipo toba chi ka natoksuli ikbit iklo kashke, achi tok. Yohmit okla ha im alvmmi na, ishit iklo tok.

7. Nana im asha kvt natoksuli moma ishit toba ha vlhpesa ka ona, micha atampa fehna hoka.

8. Mihma hatak hopoyuksa aiyukali takla asha tuk vt vlhtipo natoksvli a ikbi kut, nan tvnna vba takali pokoli hosh linen tapuski puna okchamali, micha hummaii, micha tishepa aiena ka ikbi tok, natoksvli inla Chelubim puta ka ikbi tok.

9. Takali achvfa kut falaia kut kubit pokoli tuklo akucha untuchinakma, takali achufakmot auata kvt kubit ushta tok; vbatakali moma kvt vlhpisa kvt iti laui ha tok.

10. Takali tahlapi ka itachakli tok; micha takali inla tahlapi ka itachakli tok.

11. Micha takali achvfa vhli ai itvchakvchi vhli ya ahlopulli okchamali ya ikbi tok; micha takali achufa ont vhli fehna atukla itachakvchi a ai ikbi ak inli tok.

12. Takali achvfa ka ahlopulli pokoli tahlapi ho ikbi tok, micha atukla ai itachakvchi takali vhli ha ahlopulli pokoli tahlapi ho ikbi tok; ahlopulli vt ita halvlli tok.

13. Micha tvli holisso lakna isht a kamvssa pokoli tahlapi ho ikbi tok, micha isht a kamvssa ya takali ya ishit itachakli tok. Yohmi ho vlhtipo achufa toba tok.

14. Micha issi kosoma hishi yo takali ya vlhtipo ya im ikbi na, vlhtipo ya ompoholmo tok; takali auahachvfa ho ikbi tok.

15. Takali achufa kvt falaia kvt kubit pokoli tuchinakmvt, takali achvfa kvt auata kvt kubit ushta tok; takali auahachvfa kut vlhpisa kvt iti laui tok.

16. Takali tahlapi ka ila itachakli mvt, takali hannali ka ila itachakli tok.

17. Micha takali ai itachaka ont vhli fehna ya ahlopulli pokoli tahlapi ho ikbi tok, micha takali ai itachaka atukla ka ahlopulli pokoli tahlapi ho ikbi tok. 
18. Micha vlhtipo ya itachakali na, achvfa chi ka asonak lakna isht a kamussa pokoli tahlapi ho ikbi tok.

19. Micha vlhtipo ompoholmo ya chukfi nakni hakshup humma ikbi mut, yưmma paknaka ompoholmo ya bacha hakshup o ikbi tok.

20. Micha vlhtipo ya shittim iti yo iti busha hiohli yo im ikbi tok.

21. Iti busha vt achvfa kvt falaia kvt kubit pokolikmvt, iti busha achufa kvt auata kvt kubit achufa cha iklunna tok.

22. Iti busha achufa kut ishit shamvlli tuklo hosh iti hopaki kvt iti laui tok, yakohmichi hosh iti busha moma ka vlhtipo ya im ikbi tok.

23. Micha vlhtipo ya iti busha im ikbi ka ; oka mahli pilla oka mahli in tunnup a iti busha pokoli tuklo tok.

24. Micha iti busha pokoli tuklo kash nutaka ya tuli huta yosh ashamohli pokoli ushta ho ikbi tok; iti brsha achvfa im isht shamohli in tuklo ka nutaka ya ashamohli tuklokma, iti busha achufa im isht shamohli tuklo ka ashamohli tuklo tok.

25. Micha vlhtipo in tunnvp achufa falummi pilla aivhli ya iti busha pokoli tuklo ikbi tok.

26. Micha tuli huta yosh im ashamohli pokoli ushta ho ikbi tok, iti busha achufa nutaka ya ashamohli tuklokma, iti busha inla nutaka ya ashamohli tuklo tok.

27. Micha vlhtipo hưshi aiokatula pilla in tunnup a iti busha hannali ho ikbi tok.

28. Micha in tunnup tuklo vlhtipo chukbi puta ka iti busha tuklo ho ikbi tok.

29. Micha akishtvla ya chunaha achvfa ho isht ita chakali mut, paknaka ya ita chakali tok; yakohmi hosh chukbi ita tuklo ka yumihchi tok.

30. Mihma itibusha untuchinakma tuli huta yash, ashamulli auah hannali tok; iti busha achufa nutaka ya ashamulli tuklo bieka tok.

31. Micha vlhtipo in tunnup achvfa iti busha ya shittim iti yo im okfoatkvchi tahlapi họ,

32. Micha vlhtipo in tunnup achufa iti busha ya im ok- 
howatkvchi tahlapi; micha vlhtipo in tunnup hushi ai okatula pilla iti busha ya im okhowatkuchi tahlapi ho ikbi tok.

33. Micha iklunna okhowatkvchi iti busha puta kash hlopullechi kvt ant vhli ia kvt ont vhli achvfa ya ona tok.

34. Micha iti busha ya tuli holisso lakna yo lapalichi mut, okhowatkuchi ashamohli ya chi ka tuli holisso lakna chunaha im ikbi mut, okhowatkvchi ya tuli holisso lakna lapohlichi tok.

35. Micha isht ompoholmo ya okehamali, micha hummaii, micha tishepa, micha linen tapuski itapana cha, tunna yo ikbi tok toksuli imponna yosh ikbi ho chelubim atoba yo ikbi tok.

36. Micha shittim iti yo tonik ushta ho im ikbi cha, toli holisso lakna lapalichi tok; isht afacha yut tuli holisso lakna, micha aivlbeha ya tuli hvta yo i bilelit akmochi tok.

37. Micha vlhtipo okhissa in takali ya okchamali, micha hummaii, micha tishepa, micha itapana cha tunna linen tapuski chufak ushi isht atahli yo ikbi tok.

38. Micha tonik tahlapi kash $\underline{i}$ chanakbi aiena ka ikbi tok, micha noshkobo micha im apakfopa aiena ka tuli holisso lakna lapalichi tok; amba ashamohli tahlapi kut o asonak lakna a tok.

\section{CHAPTA XXXVII.}

IHMA Besaleel vt shittim iti yo itombi holitopa ikbi 1 tok; falaia kvt kubit tuklo cha iklvnakma, auata kvt kubit achufa cha iklunakma, chaha kut kubit achưfa cha ikluna aiena tok.

2. Micha troli holisso lakna bano ho anukaka, michra: kohcha aiena lapalichi tok, micha tuli holisso lakna fullota ikbit apakfobli tok.

3. Micha chukbi ushta ka takohlicha chi hosh chunaha tuli holisso lakna ushta ho bilelit akmochi tok; in tunnup achvfa ka chunaha tuklokma, in tunnup achvfa ka chvnaha tuklo tok. 
4. Mihmut shittim iti yo tubi ikbi cha, tuli holisso lakna lapalichi tok.

5. Micha tubi yash itombi holitopa naksika chunaha takohli kash isht sholachi ho shamohli tok.

6. Micha ai i kana aiombinili ya tuli holisso lakna bano ho ikbi tok; falaia kut kubit tuklo cha iklunakmvt, auata kvt kubit achufa ikluna aiena tok.

7. Micha ai i kana aiombinili ont vhli tuklo kạ, Chelubim tuklo tuli holisso lakna yo ikbi tok; mih achvfa ho bolit ikbi tok;

8. Chelub achvfa kvt ont vhli ilvppa o hikiakma, chelub achvfa kvt ont vhli in tunnvp yumma o hikia tok; ai i kana aiombinili aiachvfa ho ont vhli tuklo ka chilubim ikbi tok.

9. Mihma Chelubim vt sanahchi ya ashatublit vba weli cha ai ikana aiombinili ya sanahchi isht ompohomo hosh nashuka iti sanalit hieli tok, Chelubim nashuka yot ai i kana aiombinili pilla pit taloha tok.

10. Mikma shittim iti yo aiimpa ikbi tok, falaia kvt kubit tuklokma, auata kvt kubit achvfakma, chaha kvt kubit achvfa cha ikluna tok.

11. Micha tuli holisso lakna bano ho lapalichi cha, tuli holisso lakna apakfoyupa im ikbi tok.

12. Micha i fullota ya ibbak putha achufa ho im ikbit apakfobli tok; micha $\underline{i}$ fullota yash tuli holisso lakna yo apakfoyupa ikbi tok.

13. Mihmut tuli holisso lakna yo chưnaha ushta ho bilelit akmochi cha, chunaha yash iyi ushta chukbi ushta takoh. lichi tok.

14. İ fullota yo bilika ak o tubi ut shamohli hosh sholachi ho chunaha hut takohli tok.

15: Micha aiimpa ishit sholachi tubi ya shittim iti yo ikbi cha, tvli holisso lakna ya lapalichi tok.

16. Micha nan aivlhto, aiimpa paknaka aiasha, im ampo patussa, micha im ishtimpa, micha im ampo ompoholmo isht ompoholto aiena ka tưli holisso lakna bano ho ikbi tok.

17. Mícha pula aiohika yạ tuli holisso lakna bano ho ikbi tok; bout toba ho pula dịohikia ikbi, im vpi micha i nak- 
sish filamohli, im ampo, micha $\underline{\mathrm{i}}$ bikbina, micha $\underline{\mathrm{i}}$ pakanli vt mih makinli tok.

18. Yohmi ka naksish vt hannali kvt tvhlalaka akohcha tok; pula aiohikia ya naksish tuchina hosh in tunnup a akohcha ma, pula aiohikia ya naksish tuchina hosh akohcha tok.

19. Naksish achvfa ka almont holbut toba hosh, ampo tuchinakmvt Jumbo achvfa, micha napakanli achvfa; mikma naksish achvfa ka, ampo tuchinakmvt, almont holbvt toba hosh, lumbo achvfa, micha napakanli achvfa tok; yohmi kut pula aiohikia ya naksish vt akohchvt ia hannali kvt hlopulli tok.

20. Mihma pvla aiohikia ya ampo ushta, almont holkvt toba, micha in luboa, micha $\underline{\mathrm{i}}$ napakanli aiena kvt asha tok.

21. Yohmi ka yummak ash inli naksish tuklo, nuta ya lumbo achufa, yvmmak ash inli naksish tuklo nuta ya lumbo achvfa, yummak ash inli naksish tuklo nuta ya lumbo achufa, naksish hannali kohchut ia ka vlhpisa tok;

22. In lumbo, micha i filamohlichi vt ai achvfa hatok; moma kvt tuli holisso lakna achvfa bano hosh bovt toba hatok.

23. Micha $\underline{i}$ bila aivlhto untuklo ka. micha pvla im isht tuptohli, micha pula tuptoha im aivlhto aiena tuli holisso lakna bano ho isht ikbi tok.

24. Tuli holisso lakna bano weki talent achufa ho ikbi tok; micha im ai vlhto moma ka ikbi tok.

25. Nabalama ahukmi alta ya shittim iti yo ikbi tok; falaia kvt kubit achvfakma, auata kvt kubit achvfa tok, apatvssa ushta hatok; micha chaha kvt kubit tuklo hatok, in lupish vt yummak inli hatok.

26. Micha paknaka ya , micha apatussa apakfoyupa, micha lapish aiena ka tvli holisso lakna bano ho lapalichi tok; micha toli holisso lakna yo $\underline{i}$ fullota apakfoyublit ikbi tok.

27. Micha i fullota nutaka ya iti tupa ashamohli na, shohlachi ka tuli holisso lakna chunaha tuklo ho iti chablit ont achukbi tuklo ka takohlichi tok. 
28. Micha iti tupa ya shittim iti yo ikbi cha, tuli holisso lakna lapalichi tok.

29. Micha ishtahama bila holitopa, micha nabalama shobulli a hli ho nabalama yo, ikhịsh atahli mak o ikbi tok.

\section{CHAPTA XXXVIII!!}

ICHA shittim iti yo aionochit hukmit im issa alta ya 1 ikbi tok, falaia kvt kubit tahlapi mvt, auata kvt kubit tahiapi hatok, okhowataha ushta ha tok, micha chaha kvt hubit tuchina tok.

2. Micha chukbi ushta ka in lapish $\underline{a}$ ikbi tok, lapish vt ai achufa hatok, micha tuli asonak lakna yo lapalichi tok.

3. Micha alta ya nan im aivlhto moma, shuti, micha tobaksi isht piha, micha ampmahaia, micha nipi ishit yichiffi, micha luak aivlhto ampo aiena ka ikbi tok, nan aivlhto moma ka asonak lakna yo ikbi tok.

4. Micha alta ya asonak lakna tunna yosh ai apakfoyupa nutaka ya bilika vttut ia hosh iklunna ka ona ikbi tok.

5. Micha asonak lakna tunna aivhli ushta ka iti tupa ai vlbeha chị ka asonak lakna chunaha ushta ho bilelit im ikbi tok.

6. Micha iti tvpa ya shittim iti yo ikbi cha, asonak lakna yo lapalichi tok.

7. Micha vba takalicha chị ka alta okfowataka bilika yo bohlit chunaha yash pit apitta tok, alta yvt chiluk asha yosh iti busha yo isht ikbi tok.

8. Micha nan ai vlhto chito asonak lakna yo, micha iyi vt asonak lakna yosh ohoyo itunaha hvt okla moma im vlhtipo okhissa ya ai itunaha chatuk vt apisa yo isht ikbi tok.

9. Micha wanuta ya ikbi tok; okamahli in tunnup oka- . mahli pilla ho wanuta ya in takohli vt punvt tunna linen tapuski yosh kubit tahlepa achvfa tok.

10. In tonik vt pokoli tnklo cha, ashamohli asonak lakna yot pokoli tuklo tok; tonik im atakali vt micha im isht ita halvlli vt tuli huta atok. 
11. Micha falummi in tunnzp a takali vt kubit tahlepa achufa ma, in tonik vt pokoli tuklo chă, im a shamohli asonak lakna vt pokoli tuklo tok; tonik im atakali, micha im isht ita halvlli vt tvli hota atok.

12. Micha hushi aiokatula in tunnup a takali vt pokoli tahlapi ma, in tonik vt pokoli cha, im ashamohli vt pokoli tok; tonik im atakali, micha im isht ita halvlli vt tuli hvta atok.

13. Micha hushi" akohchaka in tunnvp, hushi akohcha pilla ho kubit pokoli tahlapi tok.

14. Holihta okhissa in tunnvp achufa ka vba takali vt kubit auahtahlapi tok; in tonik vt tuchina cha, im ashamohli vt tuchina tok.

15. Micha wanuta okhissa in tvnnup achvfa ilvppa in tonnvp, micha yvmma in tvinnup, atakali vt kubit auahtahlapi tok, in tonik vt tuchina cha, im ashamohli vt tuchina tok.

16. Wanuta apakfoyupa fullota takali moma kvt punvt tunna linen tapuski atok.

17. Mihma tonik im ashamohli puta kvt asonak lakna atok; tonik im atakali, micha im isht ita halvlli vt tvli hvta atok; micha 1 noshkobo lapali kvt tvli hvta atok, wanuta tonik moma kvt toli hvta yosh isht itahvllit taha tok.

18. Micha wanuta holihta okhissa takali vt chufak ushi isht vlhtaha hosh, okchamali, micha hummaii, micha tishepa yosh punvt tunna linen tapuski atok; micha falaia kvt kubit pokoli tuklo cha, auata yưmma chaha kvt kubit tahlapi wanuta takali ya chohmi tok.

19. Micha in tonilk vt ushta, micha im ashamohli vt asonak lakna yosh ushta tok; im atakali vt tuli hvta, micha $\underline{\mathbf{i}}$ nóshkobo puta lapali, micha im isht ita halvlli puta kut tvli huta atok.

20. Micha vlhtipo ya , micha wanuta apakfoyupa ka isht ahonvhla moma kvt asonak lakna atok.

21. Na holitompa isht vtta Alon ushi Ihlama vt pehlichi ho, Lefai vhleha hut nan isht aiasha chị ho, Moses vt apesa tuk mak $\mathrm{o}$, isht atokowa vlhtipo pulla holhtina tuk vt vlhtipo moma ka iluppak oke. 
22. Mihma Chihowah yut Moses a im apesa tuk mak $\underline{o}$, Chutah apehlichi aiachvfa $\mathrm{Hu}$ ushi, Uli ya ushi Besaleel vt moma ka ikbi tok.

23. Micha Ahisimak ushi Aholiab Tan apehlichi aiachufa nan in chunli, micha natoksuli imponna, micha i fullota okchamali, micha hummaii micha tishepa, punnut tunna linen tapviski isht vtta vlhtaha yosh iba foka tok.

24. Micha aholitopa natoksvli moma isht vlhtaha tuk, tvli holisso lakna moma kvt im issa, tuli holisso lakna fehna kvt talent pokoli tuklo akucha chakali cha, shekel tahlepa untuklo cha, pokoli tuchina kvt vlhtipo aholitopa shekel a vlhpisa hatok.

25. Micha tvli hota ya okla moma im aiishit holhtina tuk a talent tahlepa achvfa cha, shekel tahlepa sipokni achvfa cha tahlepa untuklo, pokoli untuklo akucha tahlapi kut vlhtipo aholitopa shekel a vlhpisa hatok.

26. Hatak moma kut afummi pokoli tuklo vhlit vba maya hosh holhtina ia chatuk vt hatak tahlepa sipokni tahlepa hannali, micha tahlepa sipokni tuchina, tahlepa tahlapi cha, pokoli tahlapi kut bekah achvfa, yummut shekel ikluna hosh vlhtipo aholitopa shekel a vlhpisa hatok.

27. Micha tuli hota talent tahlepa achvfa tuk ak o bilelit akmochit, vlhtipo aholitopa im ashamohli, micha isht ompoholmo im ashamohli aiena ka ikbi tok, talent tahlepa achufa tuk a ashamohli tahlepa achvfa tuk, talent achvfa tuk $\underline{\text { a }}$ ashamohli achvfut ia tok.

28. Micha shekel tahlepa sipokni achvfa tahlepa untuklo cha, pokoli untuklo akuhcha tahlapi tuk a, tonik im atakali a ikbi mut, $\underline{\mathrm{i}}$ noshkobo ya lapalichi cha, ita takchichi tok.

29. Mikma asonak lakna im issa vt, talent pokoli untuklo, micha shekel tahlepa sipokni tuklo cha tahlepa ushta tok. 30. Micha yummak o okla moma im vlhtipo okhissa afohka isht ikbi mvt, asonak lakna alta, micha asonak lakna tonna, micha alta nan im aivlhto moma ka

31. Micha wanuta apakfoyupa ka im ashamohli, micha wanuta holihta okhissa im ashamohli, micha vlhtipo im isht 
afacha moma micha wanuta apakfoyupa ka isht bonvhla moma ka isht ikbi tok.

\section{CHAPTA XXXIX.}

MICHA aholitopa atoksvla chị ka nafohka fohkvt toksvI li, okchamali hummaii, micha tishepa ya isht ikbi tok; micha Chihowah yot Moses a $\underline{i}$ miha tuk mak $\underline{\text {, }}$ Alon a nafohka holitopa ya im ikbi tok.

2. Micha efot vt okchamali, micha hummaii, micha tishepa, micha punnut tunna linen osh tuli holisso lakna ishta toba yo ikbi tok.

3. Micha tvli holisso lakna ya lvllit tapushichi cha, chuhli mot, okchamali, micha hummaii, micha tishepa, micha pvnvt tunna linen ash toksuli imponna hosh isht shemvchechit shemvchit tahli tok.

4. Isht itachaka chi ka tahchi abana ikbi cha, takchaka tuklo kak o isht itachaka tok.

5. Mikma efot inla im vskofvchi vt atakanli tuk vt yummut aivlhtaha tuk akinli ho chohmi tok; Chihowah yut Moses a $\underline{i}$ miha tuk mak o tvli holisso lakna isht vlhtahvt, okchamali, micha hummaii, micha tishepa, punvt tunna linen atok.

6. Micha oniks tvli ikbi mut in chuwa yvt chuwa chatuk holbvchi hosh chunlit, Islael im vlla vhleha hohchifo lapohli ho afohka yut tuli holisso lakna yo ontalohli tok.

7. Micha Chihowah yut Moses a $\underline{i}$ miha tuk mak $\underline{0}$, Islael im vlla vhleha hot tvli ishit ithaiyana chị ho efot in tahchvbana ya on talali tok.

8. Micha efot vlhtaha ka chohmi hosh toksuli imponna yosh ikbi yo ikkishi alata ikbi tok; tuli holisso lakna vlhtaha hosh, okchamali, micha hummaii, micha tishepa, micha pvnut tunna linen $\underline{\mathrm{g}} \mathrm{ikbi}$ tok.

9. Iti laui bano fullota hatuk; ikkishi alata vt pohloma ha ikbi tok; pohloma hosh falaia kvt span achvfakma, auata kvt span achufa tok. 
10. Mihmut tuli iti akaiya ushta ho on talohli tok; iti akaiya vmmona kut sativs, topas, micha kabunkel atok; iluppak osh iti akaiya vmmona hatok.

11. Mikma iti akaiya atukla kut emilal, safai, micha taiamont ak atok.

12. Mikma iti akaiya atuchina kut liku, akat, micha amahlist ak atok.

13. Mikma iti akaiya ont ai ushta kvt belil, oniks, micha chaspa atok, ai on taloha on talohvt tuli holisso lakna yo on talohli tok.

14. Micha tvli vt Islael im vlla vhleha ha vlhpisa hosh auahtuklo hohchifo ho vlhpisa tok, in chụwa ya holbvt chụwa hosh hohchifo aiyukali lapalit apehlichi auahtuklo tuk a vlhpisa tok.

15. Micha tuli holisso lakna bano ho itapanut ishit toba ho ikkishi alata takchaka ya itatakuli takohlichi tok.

16. Mihmvt aiontaloha tuklo ka tvli holisso lakna yo ikbi mvt, tvli holisso lakna chunaha tuklo ho ikbi cha, ikkishi alata takchaka tuklo ka chvnaha tuklo ya takohlichi tok.

17. Mihmvt tvli holisso lakna itatakvli itapunut itatakvli yash ikkishi alata takchaka ya chvnaha tuklo kash apitta tok.

18. Micha itapunvt itatakvli tuklo kash wishakchi ya aiontaloha tuklo kash atakchichi mvt, efot itikba tahchibana ya ontakohlichi tok. $: \because$

19. Micha tuli holisso lakna chunaha tuklo ho ikbi cha, ikkishi alata takchaka tuklo vhli efot anukaka pilla et apota ha takohlichi tok,

20. Micha tuli holisso lakna chvnaha inla tuklo ka $\mathrm{jkbi}$ cha, efot vhli tuklo nutaka tikba pilla ai ita talakchi ka ichapaka, efot isht talakchi vskofuchi inla paknaka yak o takalichi tok.

21. Micha Chihowah yut Moses a im achi tuk mak $\underline{0}$, ikkishi alata yash in chunaha yak $\underline{0}$, efot in chunaha ya sita okchamali ya pit isht atakchichi tok; yumohmi ho efot isht talakchi vskofvehi pisa inla ya paknaka yokma, ikkishi alata yut efot a ik i makofo ka chi hatuk. 
22. Micha tunnut toba yosh okchamali bieka ho efot nafohka falaia ya ikbi tok.

23. Mihma nafohka falaia iklunna ya tưnnup nafohka chiluk o chohmi hosh chiluk vt hikiakma, chiluk a $\underline{\mathrm{i}}$ fullotot taiyaha kvt ik hlilafó ka chi hatok,

24. Micha nafohka falaia wishakchi ya pamkilanit okchamali, hummaii, micha tishepa itapanvt tunna linen aiena ho ai ikbi tok.

25. Micha tuli holisso lakna bano ho tvlula yo ikbi cha, nafoka falaia wishakchi ya pamkilanit vt takohli itin takla tulula ya takohlichi mvt, pamkilanit itin taklvt apakfoyupa ka takohlichi tok.

26. Chihowah yut Moses a $\underline{\mathrm{i}}$ miha tuk mak $\underline{\mathrm{o}}$, fohkvt nan isht vtta ya chị ho nafohka falaia wishakchi ya tvlula, mikmot pamkilanit tulula mikmvt pamkilanit takohlit apakfoyupa tok.

27. Micha tunnut toba yo linen tapuski nafohka ya Alon micha ushi vhleha hạ,

28. Micha linen tapuski iahlipa, micha linen tapuski noshkobo ishit shema, aiokli, micha punvt tunna linen linen obalafohka,

29. Micha punvt tunna linen tapuski uskofuchi, micha okchamali, micha hummaii, micha tishepa chofak ushi isht vlhtaha ho Chihowah yvt Moses a i miha tuk mak o ikbi tok.

30. Micha tuli holisso lakna bano patussa holitopa bita ho ikbi cha in chüwa holbut chüwa ho, aio . holissochit CHinoWAH I HOLITOPA, achi ho lapalichi tok.

31. Micha Chihowah yot Moses $\underline{a} \underline{i}$ miha tuk mak $\underline{\underline{o}}$, sita okchamali ishit takchi cha, chahvchit iahlipa ha atakchichi tok.

32. Yakohmi hosh okla moma im ai vtta vlhtipo natoksuli moma kut vlhtaha tok ; mihma Islael im vlla vhleha hvt yumihchit, Chihowah yut Moses a $\underline{i}$ miha tuk mak o moma ka okla yumihchi tok.

33. Micha vlhtipo ai vtta, micha i nachukushpa moma, im isht a kamussa, im iti busha, im okhowata, micha in tonik, micha im ashumohli, 
34. Micha isht ompoholmo chukfi nakni hakshup humma, micha isht ompoholmo bacha hakshup, micha vlhtipot ompoholmo,

35. Nanvlhpisa afohka itombi, micha im iti tuptua, micha aiombinilit i nukhaklo,

36. Aiimpa, micha nan im aivlhto moma, micha pvska otuni,

37. Mih bano pula aiohikia bila ai im aivlhto, bila aivlbto iti akaiyut hiohla chi fehna ka , micha in aivlhto moma, micha pvla lua chi ka bila,

38. Micha tuli holisso lakna alta, micha bila ishtahama, micha nabalama micha vlhtipo okhissa ya takali,

39. Asonak lakna alta, micha asonak lakna tunna, im iti tuptua, micha nan im aivlhto moma, ampo chito, micha iyi,

40. Wanuta atakali, in tonilk, micha im ashamohli, micha wanuta holihta okhissa atakali im asita, micha im ishtafacha, micha okla moma im aivtta vlhitipo atoksuli nan aivlhto moma,

41. Aholitopa ya nan isht aivtta chikmut fohkvt nan isht vtta, micha naholitopa isht aivtta ya isht vtta chikmvt fohka he ya naholitompa isht vtta Alon inafohka holitopa, micha ushi vhleha i nafohka;

42. Chihowah yut Moses a miha tuk moma mak 0 , yumohmit Islael im vlla vhlể hut natoksuli moma ka yưmihchi tok,

43. Mihma Moses vt natoksuli moma ka pisa tok; mihma yakeh, Chịhowah yot miha tuk mak o okla yvmihchi tuk atok, mihma Moses vt yukpali anumpa onochi tok.

\section{CHAPTA XL.}

MIHMA Chihowah yout Moses a im anumpulit, I 2. Okla moma im aivtta vlhtipo ya hushi ummona, micha nitak vmmona ka ish hilechashke.

3. Micha nanvlhpisa afohka itombi holitopa yummak o 
ish fohkashke, "micha isht ompoholmo ya itombi holitopa yash isht.ish ompohomashke.

4. Micha aiimpa ya isht ish chukowa cha, nana achukmut taloha chi ka achukmalit ish ontalalashke; micha pvla aiohikia ya isht ish chukowa cha, bila aivlhto ya ish pulalashke.

5. Micha tvli holisso lakna alta nabalama ahukmi ya nanvlhpisa afohka itombi holitopa itikba ish talalashke, micha vlhtipo ya okhissa takali ya ish atakalichashke.

6. Micha okla moma im aivtta vlhtipo okhissa itikba ya ai onochit hukmit im issa alta ya ish hilechashke.

7. Micha okla moma im aivtta, micha alta iti takla ya ampo chito ya ish talali cha, oka ish vnashke.

8. Micha apakfoyulit wanuta ya ish talali cha, wanuta holihta okhissa natakali ya ish takalichashke.

9. Micha ishtahama bila ya ish eshi cha, vlhtipo ya micha anukaka, nana moma aiasha ka, micha nan im aivlhto aiena moma ka ish ahvmmi cha, ish hullochashke, mikma hullashke;

10. Micha aionchit hukmit im issa alta, micha im aivlhto moma ka ish ahvmmashke, micha alta ya ish hullochashke. micha alta hullo kvt moma i shahlashke.

11. Micha ampo chito, micha iyi aiena ka ish ahummi cha, ish hullochashke.

12. Mikmut Alon, micha zashi vhleha aiena ka okla moma im vlhtipo okhissa itikba ya isht ish ona cha, oka isht ish achefashke.

13. Micha Alon a nafohka holitopa ish fohkuchechaske; micha ish ahvmmi cha, ish hullochashke; yumohmi ho naholitompa isht aivtta ya isht vm ai vttashke.

14. Micha ushi vhleha ha isht ishla cha, nafohka ish fohkvchechashke.

15. Micha iki ya ish ahummi tuk vt chohmichit ish ahvmmashke; yohmikma naholitopa isht aivtta ya isht um ai ashwashke, ahama kvt im aiitishali mahaya ka naholitopa isht vtta bilia ya chi pulla hoka. 
16. Yummak o Moses vt yumihchi tok, Chihowah yut $\underline{i}$ miha moma tuk mak o yumihchi tok.

17. Atuk o yakohmi tok, hushi vmmona afummi atukla, hushi vt nitak vmmona ma, vlhtipo vt vlhtaha tok.

18. Mihma Moses vt vlhtipo ya hilechi cha, im ashamohli a ita kvllochi mut, iti busha ya hiohlechi cha, okhowatkvchi a okfowvilichi cha tonik a hiohlichi tok.

19. Mihmut Chihowah yut Moses a $\underline{i}$ miha tuk mak $\underline{0}$ vlhtipo yash ompoholmo ya auatulit ompohomo tok; micha ompoholmo yash, paknaka ya ompohomo tok.

20. Micha nanvlhpisa ya eshi cha, itombi holitopa ya fohki mut, iti tuptua ya itombi holitopa ya apitta tok; micha itombi holitopa paknaka ya aiombinili i nukhạklo ya ontalali tok.

21. Mihmut Chihowah yut Moses a i miha tuk mak $\underline{\mathbf{o}}$, itombi holitopa ya vlhtipo ya isht chukowa cha, isht ompoholmo ya hilechi cha, nan vlhpisa afohka, itombi holitopa ya ompohomo tok.

22. Micha aiimpa 'ya okla moma im aiutta ya anukaka vlhtipo ya falummi in tunnup ompoholmo ya kohcha yo hilechi tok.

23. Micha Chihowah yưt Moses a $\underline{i}$ miha tuk mak $\underline{o}$, puska ya Chihowah itikba ya achukmalit talali tok.

24. Micha pvla ai@hikia ya okla moma im aivtta anukaka aiimpa ichapaka vlhtipo ya im okamahli in tunnup a hilechi tok;

25. Micha Chihowah yut Moses a $\underline{i}$ miha tuk mak $\underline{o}$, pula ha Chihowah itikba a palali tok.

26. Micha tuli holisso lakna alta ya okla moma im aivtta isht ompoholmo itikba ak o hilechi tok."

27. Micha Chihowah yut Moses a $\underline{i}$ miha tuk mak o nabalama aionochit hukmi tok.

28. Micha vlhtipo okhissa takali hilechi tok.

29. Micha Chihowah yvt Moses a $\underline{\mathbf{i}}$ miha tuk mak $\underline{\mathbf{o}}$, aionochit hukmit im issa alta, okla moma im aivtta vlhtipo okhissa ya bilika hilechi cha, hukmit im issa, micha ilhpak im issa onochit im issa tok. 
30. Micha ampo chito ya okla moma im aiutta, micha alta itin takla hilechi cha, ai ibbachefa chi hosh oka uni tok.

31. Micha Moses, micha Alon, micha ushi vhleha aiena ibbak, micha iyi aiena ka yummak aiachefa tok.

32. Chihowah yut Moses a $\underline{i}$ miha tuk mak $\underline{0}$, okla moma im ai vtta chukowakmvt, micha alta ya bilika onakmvt, ibbachefa beka tok.

33. Micha vlhtipo, micha alta apakfoyupa ka wanuta ya hilechi mut, wanuta okhissa takali ya hilechi tok. Yvmohmi hosh Moses vt natoksuli a atahli tok.

34. Yohmi ma hoshonti vt okla moma im aivtta ya ompoholmo ma, Chihowah im aholitopa vt vlhtipo ya alotoa tok.

35. Mihma okla moma im aivtta ya hoshonti vt ompoholmo hatuk $\underline{\mathrm{o}}$, micha Chihowah im aholitopa vt vlhtipo $\underline{\mathbf{a}}$ alotoa hatuk o Moses vt chukowa he keyu tok.

36. Atuk o vlhtipo paknaka ya hoshonti hash ai ishit vba ishtia ma, Islael im vlla vhleha hvt anowvt aya moma ka mahaya tok.

37. Amba hoshonti ha vba isht ik aiyokma, ik aiyo tuk osh, nitak nana ka vba ishtiak mak o beka tok.

38. Islael in chukachvfa moma kvt nowvt ayvt ont hlopulli moma ka pisa ho Chihowah $\underline{i}$ hoshonti hut nitak ma, vlhtipo ya onvtoyula tuk osh, ninak ma, luak osh onvitoyula beka tok oke. 


\section{LEFITIKUS HOLISSO.}




\section{MOSES I HOLISSO ATUKLA KUT}

\section{LEFITIKU S HOHCHIFO HOKE.}

\section{$\therefore \quad \therefore$ CHAPTA I.}

IIHMA Chihowah yut Moses a $\underline{i}$ howa cha okla moma 1 im vlhtipo ya et irn anumpulit,

2. Islael im vlla vbleha ha im anumpulit, Hatak hush kanima hosh nan im issa ya Chihowah isht hush im onakmut, na hush im issa, vlhpoa hochito ai ishit wak, micha vlhpoa chipunta ya isht hush im onashke.

3. Mikmut nan im issa yut vlhpoa hochito ai ishit na hukmit im issa hokmvt nakni yosh ai okpuloka iksho ho im issashke, ilap ahni hosh Chihowah itikba okla moma im vlhtipo okhissa yak o ai im issashke.

4. Micha na hukmit im issa noshkobo ha, ibbak isht om bitepashke. Micha im aiokpvichi na, isht im vlhtoba hi oke.

5. Mikma wak hobuk ash Chihowah itikba ai uba hi oke; mihmana holitopa isht'asha Alon ushi vhleha hvt issish a isht vla cha, issish ash alta, yummut okla moma im vlhtipo okhissa bilika hikia ka o fimmit fullohlichashke.

6. Micha na hukmit im issa ya hlufi cha bushlit tahla hi oké.

7. Mikma na holitompa isht vtta Alon ushi vhleha hvt alta ya luak onochi cha achukmalit luak a iti onochashke.

8. Mikma na holitompa isht asha Alon ushi vhleha hut, tuptua, noshkobo micha nia aiena ka luak vt alta on asha iti on asha ka paknaka ya achukmalit onochashke.

9. Amba ikfoka, iyi aiena kuno, oka isht achifa hi oke: mihma na hukmit im issa Chihowah $\underline{i}$ balama achukma lua yo naholitopa isht vtta yut mominchit alta onochit hukma hi oke. 
10. Micha nan im issa kut nan vlhpoa chipunta chukfulhpoa, keyukmut issi kosoma yo hukmit im issak mut, nakni yosh ai okpuloka kvt iksho ho isht vlashke.

11. Micha Chihowah itikba alta ya falummi in tunnup $\underline{0}$ ai vbashke; micha na holitompa isht asha Alon ushi vhleha hut issish $\underline{a}$ alta ya $\underline{o}$ fimmit fullohlichashke.

12. Micha noshkobo micha nia aiena ka bushlit tahlashke. Mikma na holitopa isht vtta yut alta ya luak on asha na iti vt $\underline{\text { a asha }}$ ka achukmalit onochashke.

13. Amba ikfoka micha iyi aiena kuno oka isht achifashke, micha na holitompa isht vtta yut mominchit isht vla cha alta ya onochit hukmashke; na hukmit im issa luak ishit hukmit im issa Chihowah ya nan $\underline{i}$ balama achnkma yoke.

14. Micha na hukmit im issa ya Chihowah ya nan im issa kvt, hushi yokma, nan im issa yut pvchi yushoba, keyukmut puchushi yo isht vlashke.

15. Mikma na holitopa isht vtta yut alta bilika isht ona cha, noshkobo ha shưnnit tubli cha alta ya onochit hukmashke; micha im issish a shunnit alta lapalika a hlatublashke.

16. Micha impuffakchi ya naliteha aieninchit eshi cha alta lapalika $\underline{i}$ hvshi akochaka in tonnup, hituk chubbi aiasha bilika pilashke.

17. Micha sanahchi vt asha moma ho pahlvllashke; yohmi kia ik ita hokofo kashke; mikma na holitopa isht vtta yvt alta ya luak on asha ka mikma iti on asha ka onochit hukmashke; na hukmit im issa luak ishit hukmit Chihowah nan i balama achukma yoke.

\section{CHAPTA II.}

MICHA kuna hosh ilhpak im issa Chihowah ya im issak1 mut nan im issa kvt bota tohbi lipehbi ya hi oke; micha bila ya on hlatublikmut filakinsen onochashke.

2. Mikmut na holitompa isht asha Alon üshi vhleha ha isht im onashke, mikma naholitompa isht vte yut bota. 
tohbi micha bila, micha filakinsen moma aiena ibbak alota achvfa ho eshi cha isht ithana yo alta onochit hukmashke. Chihowah nan i balama achukma yo luak ishit hukmit im. issa yashke.

3. Mikma ilhpak im issa atampa hokvto Alon micha ushi whleha immi hashke; luak ishit hukmit Chihowah im issa nana holitopa moma i shahli hoke.

4. Apaluska a nuna yo ilkpak im issa ya isht ishla hokmut, bota tohbi lipehbi puska lumbo ik shatvmmo bila yummi yashke, keyu hosh puska lapushki ik shatummo yokmut bila isht ahamashke.

5. Micha ilhpak ish im issa yut ampo mahaia a nuna yokmut bota tohbi lipehbi toba hosh ik shatummo bila yummi yashke.

6. Ish bushullichi cha bila ish on hlatublashke: ilhpak im issa yolke.

7. Micha ilhpak ish im issa yut ampo vlwusha a nuna yokmut bota tohbi lipehbi bila yưmmi yashke.

8. Illipak im issa yut iluppa chohmi puta kak $\mathrm{o}$ atoba $\mathrm{ka}$ Chihowah isht ish im onashke; atuk o na holitompa isht vtta ya imakma yưmmak osh alta a hikia ka isht onaslke.

9. Micha na holitompa isht vtta yot ilhpak im issa yash ai ishit isht ithana yo ikbit alta onochit hukmashke; luak ishit hukmit im issa Chihowah nan $\underline{i}$ balama achukma yoke.

10. Micha ilhpak im issa atampa kvt Alon, micha ushi vhleha immi ha hi oke; luak ishit hukmit Chihowah im issa . ya nana holitopa moma i shahli hoke.

11. Thpak im issa nana kia Chihowah hush im issa kvt shatummi ya he keyu hoke; luak ishit hukmit Chihowah im issa nana kia shatvmmi, keyukmot foi bila nana kia hush hukma he keyu hoke.

12. Nawaya ummona im issa hokuno, Chihowah hush im issa hi oke. Yohmi kia na balama yo alta ai on lua he keyu hoke.

13. Miça ilhpak hvsh im issa puta kvt hvpi ha hvsh yưmmichashke; ilhpak im issa ya Chihowah nan itim apesa $\therefore \rightarrow$ 
hupi ik ono kvt iksho kashke; nana hvsh im issa moma kvt hupi aieninchit hush im issashke.

14. Micha ilhpak im issa na chi waya ummona ya Chihowah ya ish im issakmut ilhpak im issa na chi waya vmmona ya onush hiloha luak ishit shilvt onush noshkobo alota chilohka yashke.

15. Micha bila ish on hlatubli cha filakinsen ish onochashke; ilhpak im issa yoke.

16. Mikma na holitopa isht vtta yot isht ithana yo onush chilohka kashapa, micha bila kashapa, micha filakinsen moma aiena ka hukmashke; luak ishit hukmit Chihowah im issa yoke.

\section{CHAPTA III.}

ICHA nan im issa yut itin nanaiyuchit im issa yokmvt, 1 vlhpoa hochito ai ishit nakni yokma, tek okmá, nana ho im issakmut, ai okpuloka iksho ho Chihowah itikba ai im issashke.

2. Nan im issa noshkoba ha ibbak isht ombitepa cha okla ritoma im vlhtipo im okhissa yak o ai vbashke: mikma na holitompa isht asha Alon ushi vhleha hut issish $\underline{a}$ alta $\underline{\mathbf{o}}$ fimmit fullohlechashke.

3. Micha itin nanaiyuchit im issa illichit im issa yo luak ishit hukmit Chihowah im issa yo im issashke: ikfoka ompohomo nia, micha ikfoka ompoholmo nia momashke.

4. Micha haiyihchi tuklo micha nia yut on asha yvmmut ikfeksa bilika asha micha svlakha i hlapa, micha haiyihchi aiena ka ishashke.

5. Micha Alon ushi vhleha hot yumma alta onochit hukmit isht aiokpvchi vt iti luak on asha pakna on asha. ka a luacha hi oke, luak isht hukmit nan im issa yut Chihowah $\mathbf{i}$ balama achukma yoke.

6. Micha nan im issa yut itin nanaiyuchit im issa illichit im issa yut vlhpoba hochito ai ishit nakni, keyukmut tek okmá nana hokma, aiokpuloka iksho ho im issashke. 
7. Chukfushi yo im issa hokmut Chihowah itikba ai im issashke.

8. Micha nan im issa tuk vt noshkobo ha ibbak isht ombitepa cha okla moma im vlhtipo itikba ai vbashke : mikma Alon ushi vhleha hvt issish a alta $\underline{o}$ fimmit apakfoyublashke.

9. Micha illichit im issa itin nanaiyuchit im issa luak ishit hukmit Chihowah im issa yo im issashke: i nia micha hasimbish vt moma, nvli foni ya ont tikelichit tublashke: micha ikfoka ompohomo nia micha ikfoka nia moma,

10. Micha haiyihchi tuklo, micha nia yummut ikfeksa bilika âsha kă micha svlakha ya paknaka i hlapa haiyihchi aiena ka ishashke.

11. Mikma na holitompa isht vtta yot alta onochit hukmashke : ilhpak im issa im ilhpak o luak ishit hukmit Chihowah im issa yoke.

12. Micha nan im issa yut issi kosoma yokma, Chihowah itikba ai im issashke.

13. Micha noshkobo ha ibbak isht ombitepa cha okla moma im vlhtipo itikba ai vbashke: mikma Alon ushi vhleha hvt issish a alta o fimmit apakfoyublashke.

14. Micha nan im issa yot luak ishit hukmit Chihowah im issa yo im issashke: ikfoka ompoholmo nia micha ikfoka nia moma ashaka.

15. Micha haiyihchi tuklo, micha nia yummut ikfeksa bilika asha ka , micha sulakha paknaka i hlapa micha haiyihchi aiena ka ishashke.

16. Mikma na holitompa isht vtta yot alta onochit hukmashke; ilhpak luak ishit hukmit im issa na balama achukma yoke; nia moma kvt Chihowah immi hoke.

17. Nia micha issish aiena ka hvchik po ka he vt, hvchim ai unchuloli fullota ka nan vlhpisa bilia yosh itoyula hi oke, im achi tok. 


\section{CHAPTA IV.}

MICHA Chihowah yut Moses a im anumpulit,

2. Islael im vlla vhleha ha im anumpulit, Hatak osh ik ithano hosh nana ashvchit Chihowah $\underline{i}$ nan vlhpisa, yummak oka yumihcha he vlhpesa tuk keyu hosh yumma kanima kia kobvffikmut.

3. Na holitompa isht vtta nan ahama tuk osh okla nan ashvchi ya yumihchit ashvchikmvt, yohmikmvt nan ashuchi ya ashuchi tuk vt nan ashuchi im issa ya wak hobvk himita ai okpuloka iksho ho Chihowah isht im onashke.

4. Micha wak himita ya Chihowah itikba okla moma im vlhtipo okhissa ya isht onashke; micha wak hobvk noshkobo ya ibbak isht ombitepashke: micha Chihowah itikba ya wak hobvk ash ai vbashke.

5. Mikma na holitompa isht vtta nan ahama tuk ot wak hobvk ash im issish a ai eshi cha,okla moma im vilhtipo ya isht onashke.

6. Micha na holitompa isht vtta yut ibbak ushi ya issish ash oka hilechi cha Chihowah itikba a holitopa natakali itikba ya issish a untuklo ha fimmashke.

7. Micha na holitompa isht vtta yvt issish kanohmi na balama achukma a hukmi alta Chihowah itikba okla moma im vlhtipo anukkaka hikia ka lapish $\underline{a}$ on lapalichashke; micha wak hobuk im issish moma ka ai onochit hukmit im issa alta, yummut okla moma im vlhtipo okhissa hikia ka akishtvla ya pit hlatublashke.

8. Micha nan ashvchi im issa ya chi ka, wak hobvk ash $\underline{i}$ nia ya moma ishashke; ikfoka ya nia yvt ompoholmo ka, micha nia moma ikfoka ai asha ka,

9- Micha haiyihchi tuklo, micha nia yummut ikfeksa bilika asha ka , micha sulakha paknaka $\underline{i}$ hlapa, haiyihchi aiena ka ishashke.

10. Itin nanaiyvchit im issa illichit im issa wak hobvk a ai ishi tuk a chohmichit ishashke; micha na holitompa isht vtta yot ai onochit hukmit im issa alta ya onochit hukmashke. 
11. Micha wak hobvk ash hakshup, micha nipi moma noshkobo aiena, micha iyi aiena, micha ikfoka micha yvlhki aiena.

12. Wak hobvk ash mominchit bina kocha a kashofa, hituk chubbi ahlatapa ya isht ona cha iti onochit luak ishit hukmashke; hituk chubbi a hlatapa yak o a luashke.

13. Micha Islael okla moma kvt ik ithano hosh nana ashvchi hokma, mihma okla moma kvt in luma tuk, mihmvt Chihowah $\underline{i}$ nan vlhpisa ya yoshubit, nana kanohmi yummak oka yumohma he vlhpesa tuk keyu hosh yumohmi tuk;

14. Nan ashuchi ya ashvchi tuk vt akostininchikmvt okla hut nan ashuchi tuk vt wak hobvk himita yo im issa cha okla moma im vihtipo itikba isht onashke.

15. Mikma okla moma im asunonchi vhleha hut wak hobuk ash Chihowah itikba ya ibbak isht ombitepashke : mikma wak hobvk vt Chihowah itikba ai illashke.

16. Mikma na holitompa isht vtta nan ahama tuk vt wak hobvk ash im issish a okla moma im vlhtipo ya isht onashke:

17. Micha na holitompa isht vtta yot issish kaniohmi ka ibbak ushi oka hilechi cha Chihowah itikba a holitopa natakali itikba fehna ka untuklo ha fimmashke.

18. Micha issish ash kaniohmi ka alta yvmmut Chihowah itikba okla moma im vlhtipo anukaka hikia lapish a isht $\underline{0}$ fimmashke: Micha issish ash mominchit ai onochit hukmit im issa alta yvmmott okla moma im vlhtipo okhissa hikia akishtula ya pit hlatublashke.

19. Micha i nia moma ka im aieshit alta onochit hukmashke.

20. Micha wak hobvk a nanashvchi im issa wak hobvk $\underline{a}$ yumihchi tuk a chohmichit ilvppak kia yumihchashke, mikma na holitompa isht vtta yot i kashofikma i kashofashke.

21. Mikma wak hobvk ash bina kocha isht ona cha wak vmmona hukmi tuk vt chohmichit hukmashke; okla moma nan ashuchi im issa yoke.

22. Hatak pehlichi yosh nan ashvchi, ik ithano hosh Chitokaka in Chihowah i nan vlhpisa achufona ya kobvffit 
nana ka a yumihcha he vlhpesa keyu yumihchi tuk ash nan ashvchikma;

23. Nan ashvchi ya ashvchi tuk vt ont nukfoyukakmvt nan im issa yut issi kosoma ushi nakni yosh ai okpuloka iksho ho isht vlashke.

24. Micha issi kosoma noshkobo ha ibbak isht umbitepa cha Chihowah itikba hukmit im issa ai vbi ak o ai vbashke: nan ashvchi im issa yoke.

25. Mikma na holitompa isht vtta yvt nan ashuchi im issa issish a ibbak ushi isht eshi cha aionochit hukmit im issa alta lapish a $\underline{o}$ lapalichashke : micha im issish $\underline{\text { a aiono- }}$ chit hukmit im issa alta akisht vla ya ahlatublashke.

26. Micha itin nanaiyvchit im issa illichit im issa nia ya chohmichit nia moma ka alta onochit hukmashke: nan ashuchi tuk a na holitompa isht vtta yut $\underline{i}$ kashoffikma $\underline{i}$ kashofashke.

27. Micha okla hut kanima hosh nana ka ikithano hosh nan ashvchi tuk nana ka ayumihcha he vlhpesa keyu ho yumihchi cha Chihowah $\underline{i}$ nan vlhpisa ha kobvffi tuk ash im ai ahlikmvt;

28. Keyukmvt nan ashvchi ashvchi tuk vt ithaiyanakmvt, nanashvchi ya ashvchi tuk vt issi kosoma ushi tek ash ai okpuloka iksho ho nan im issa isht onashke.

29. Micha nan ashuchi im issa noshkoba ha ibbak isht umbitepa cha hukmit ai im issa ak o nan ashvchi im issa yo ai vbashke.

30. Mikma na holitompa isht vtta yut issish a ibbak ushi isht eshi cha ai onochit hukmit im issa alta lapish $\underline{a} \underline{o}$ lapalichashke: micha issish a mominehit alta akishtvla ya a hlatublashke.

31. Micha itin nanaiyvchit im issa illichit im issa nia ishi chatuk a chohmichit nia moma ka ishashke: micha Chihowah i balama achukma ya chi ka na holitompa isht vtta yut alta onochit hukmashke: micha na holitompa isht vtta yvt i kashoffikma i kashofashke.

32. Micha nan ashvchi im issa yo chukfushi isht onakmut tek ash ai okpuloka iksho ho isht onashke. 
33. Micha nan ashvchi im issa noshkobo ha ibbak isht umbitepa cha nan ashvchi im issa mak o hukmit im issa ai vbi ya ai vbashke.

34. Mikma naholitompa isht vtta yvt nan ashvchi im issa issish a ibbak ushi isht eshi cha ai o hukmit im issa alta lapish a o lapalichashke : micha issish a mominchit alta akishtula ya ahlatublashke.

35. Micha itín nanaiyvchit im issa illichit im issa chukfushi nia ishi tuk a chohmichit nia moma ka ishashke: micha na holitompa isht vtta yvt luak ishit hukmit Chihowah im issa mak o yumihchit alta onochit hukmashke : micha nanashvchi yumihchi tuk a na holitompa isht vtta yut $\underline{i}$ kashoffikma i kashofashke.

\section{CHA P T A 。}

IICHA shilombish nan ashvchikmvt anumpa kvllo 1 anumpuli ka haklo, na pisa yosh pisakmá, keyukmưt akostininchikma akaniohmikia, ik anolokmvt nan ashvchi vt onvtonlashke.

2. Keyukmut 'shilombish osh nana liteha yo potoli, poa liteha illi okmá keyukmut vlhpoa liteha illi okmá, keyukmá na balahanli liteha yokmá nana hatuk oh kia, atuk osh ik ithano hatukmvt, yummak kia liteha cha nan ashvchi vt onvtonla hi oke.

3. Keyukmvt hatak liteha yo haleli tukma, liteha nana ho isht litehvt liteha hatukma, atuk osh ik ithano tukmvt ithanakmvt nanashvchi vt onvtonla hi oke.

4. Keyukmvt shilombish osh nana ik achukmo micha nana achukma nana hoh kia, anumpa kvllo il onochit ila piti vlbi o achit yumihcha chi ikithano hosh miha hokmvt, atuk osh akostininchikmut anumpa yummak ash achvfa kanima ka nan ashvchi im aiahla hi oke.

5. Atukma yakohma hi oke: iluppa achufa kanima ka nan ashvchi tuk vt im ai ahhlikmut, nana yumma ashvchi tuk vt il ai anolashke.

6. Mikmut nan ashuchi ya ashvchi tuk vt yoshobvt nan 
im issa ya vlhpoa shipunta tek $\underline{o}$ ai ishit chukfushi, keyukmot issi kosomushi yo issi kosoma ai ishit nan ashuchi im issa ya chị ho Chihowah isht im onashke: mikma nanashvchi tuk a naholitompa isht vtta yut i kashofashke.

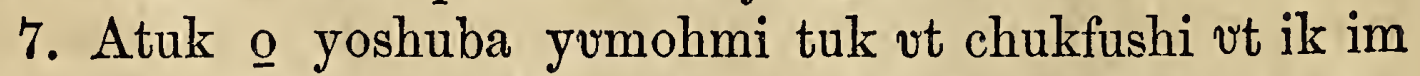
iksho mvt, pvchi yoshoba tuklo, keyukmvt pvchushi tuklo ho Chihowah isht im onashke: achvfa hvt nan ashvchi im issa yokma, achvfa kvt hukmit im issa yashke.

8. Micha na holitompa isht vtta ya isht im onashke; mikma yummak osh tikba nan ashvchi im issa yak o im issut noshkobo ha shưnit tublashke; yohmi kia ita pahlulla. he keyu hoke.

9. Micha nan ashvchi im issa issish a alta lapalika o fimmi cha, issish vt asha moma ka alta akishtula ya shunnit hlatublashke; nan ashvchi im issa yoke.

10. Micha atukla ka ayumihchi chatuk a yumihchit hukmit fim issa yo im issashke : nan ashvchi ya ashvchi tuk a na holitompa isht vtta yut i kashoffikma i kashofashke.

11. Amba pvchi yoshaba tuklo, keyukmvt pvchushi tuklo kvt ik im ikshokmvt, nan ashvchi tuk vt nan ashvchi im issa im issa kut bota tohbi lipehbi efah kushkoa isht pokoli ho isht onashke; bila yummicha he keyu, micha filakinsen onocha he keyu hoke; nan ashvchi im issa yoka.

12. Yohmikmut na holitompa isht vtta isht im ona hi oke, mikma na holitompa isht vtta yut ibbak alota achufa ho ishi cha isht ithaiyana mak o alta onochit hukma hi oke; luak ishit hukmit im issa yot ayumihchit Chihowah im issa chatuk mak o yumihcha hi oke : nan ashvchi im issa yoke.

13. Mikma nanashuchi achưfa iluppa kanima ho ashuchi tukma naholitompa isht vtta yut $\underline{\underline{i}}$ kashoffikma $\underline{\mathbf{i}}$ kashofashke; mikma atampa kvt ilhpak im issa chohmi naholitompa isht vtta immi hashke, ish im achashke, im achi tok.

14. Micha Chihowah yot Moses a im anumpulit,

15. Shilombish osh na yoshoba yumihchi, micha ik ithano hosh Chihowah i naholitopa ya im ashvchi tuk, yohmikmot nayoshuba tuk vt na yoshoba im issa yo chukfushi nakni ai okpuloka iksho ho, ish hotihna ho tuli huta shekel ka- 
nohmi, aholitopa shekel vlhpisa mak o Chihowah isht im onashke.

16. Micha na holitopa okpuni tuk ut achukmalashke, micha kushkoa ishit tahlapi ho apotali cha naholitompa isht vtta ya imashke; mikma naholitompa isht vtta yut nanashvchi im issa yash chukfi nakni yash isht $\underline{\underline{i}}$ kashoffikma $\underline{\mathrm{i}}$ kashofashke.

17. Micha shilombish osh nan ashvchit Chihowah $\underline{i}$ nan vlhpisa hut olubi tuk iluppa kanima kia yvmihchikmvt ik ithano hatuk oh kia nan ashvchi vt im ai a hli cha nanashechi vt onvtuyula hi oke.

18. Na yoshoba im issa ya vlhpoa chipunta ai ishit chukfi nakni ai okpuloka iksho ho ish $\underline{\mathrm{i}}$ hotehna tuk a aieninchit na holitompa isht vtta isht im onashke, mikma naholitompa isht vtta yut nan ik ithano hosh ashuchi tuk a na yoshoba im issa yash isht $\underline{\underline{i}}$ kashoffikma $\underline{\mathrm{i}}$. kashofashke.

19. Na yoshoba im issa yoke; Chihowah 1 yoshoba tuk o ahli hoke, im achi tok.

\section{CHAPTA VI.}

ICHA Chihowah yvt Moses a im anumpulit, 1 2. Shilombish osh nan ashuchit Chihowah i yoshoba ya yumihchit ịsha chi ho nana ibbak fohki tuk, keyukmot itapela yatuk o in chuka a bilika hatak o $\underline{i}$ holabi, keyukmot nana ho afoa hosh im eshi, keyukmvt in chuka a bilika hatak o haksichi tuk;

3. Keyukmut nana kunia tuk o ahayuchi tuk osh yumma holabi, micha holabi hosh anumpa kvllo anumpuli, ilvppa moma kanima ho hatak vt yumihchi cha ai ashvchikmvt,

4. Yohmikmvt yakohma hi oke, nan ashvchi tuk osh nan ashvchi kvt im aiahli hokvt, nana im afoa hosh im eshi tuk vt i falumminchashke; keyukmut haksichi hosh ishi tuk, keyukmot im isha chi ho ibbak fohki tuk, keyukmut nana kvnia ahayuchi tuk,

5. Keyukmut nana moma holabi hosh anumpa kullo 18 
anumpuli tuk osh yoshoba tuk vt nan im issa nita kmvt nana

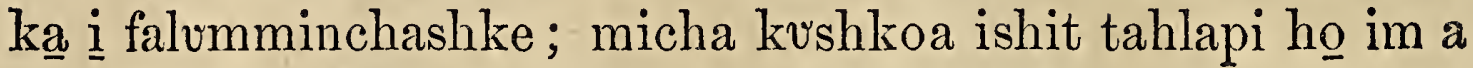
lapalichi cha kuna ho immi hatuk a imashke.

6. Micha na yoshoba im issa chi ho nan vlhpoa chipunta ai ishit, chukfi nakni ai okpuloka iksho ho ish ị hotehna tuk a aieninchit, nayoshoba tuk vt im issa Chihowah isht im onvt naholitompa isht vtta ya isht im onashke.

7. Mikma naholitompa isht vtta yot Chihowah itikba ai i kashoffa hi oke; micha yoshobvt nana moma yumihchi tuk vt i kashofashke, achi tok.

8. Mihma Chihowah yut Moses a im anumpulit,

9. Alon micha ushi vhleha ha ish $\underline{\mathrm{i}}$ mihvt, Ilvppvt hukmit im issa $\underline{i}$ nan vlhpisa hoke. Alta ya onvtuyulut luhuwa na, ninak vt ont hlopullit onna, micha alta ya luak vt a chieyvt luhuwa yoka, hukmit im issa yoke.

10. Micha na holitompa isht vtta yvt $\underline{i}$ linen na fohka ya ishit fohkashke, michá i linen obala fohka ya nipi ya ishit fohkashke; micha luak vt hukmit tahli tuk hituk chubbi, micha hukmit im issa aiena alta on asha ka eshi cha alta apotoa kak o áshachashke.

11. Mikmvt i nafohka ya shufi cha nafohka inla ho ishit fohka cha, hituk chubbi bina kocha akashofa ho isht onashke.

12. Mikmà alta ya luak on asha kvt a luhuwa hi oke; mosholicha he keyu hoke; mikma na holitompa isht vtta yot onnahinli momakna it onochit hukmi cha hukmit im issa ya achukmalit onochi beka hi oke ; micha itin nanaiyuchit im issa nia ya hukma hi oke.

13. Luak vt alta on chieyvt luhuwa na bilia hi oke, moshola he keyu hoke.

14. Micha iluppak osh hukmit im issa nan vlhpisa hoke; Alon ushi vhleha hak osh Chihowah itikba alta aiasha ya ai im issa hi oke.

15. Micha ilhpak im issa bota tohbi, micha bila on asha tuk aiena, micha filakinsen ilhpak im issa on asha tuk moma aiena ka ibbak alota achvfa ho eshi cha na balama achukma ka isht ithaiyana mak $\underline{\text { alta }}$ onochit Chihowah ya $\underline{\mathrm{i}}$ hukma hi oke. 
16. Micha atampa yano Alon micha ushi vhleha aiena hosh upa hi oke; pvska ik shatvmmo aiena ho aholitopa ak o ai vpa hi oke; okla moma im vlhtipo i wanuta ya ai vpa hi oke.

17. Isht shatummi aienvt puska he keyu hoke. Luak ishit hukmit im issa ya $\underline{i}$ kashapa mak o ima li tuk oke. Yvmmut nanashvchi im issa, micha nayoshoba im issa chihyuhmit holitopa kvt moma i shahli hoke.

18. Alon im vlla vhleha ibafoka nakni moma kut ai vpa hi oke. Luak ishit hukmit Chihowah im issa yut huchim ai unchuloli ha nan vlhpisa bilia ya chị hoke; potoli aiyukali kvt holitopa hi oke, im achi tok.

19. Micha Chihowah yut Moses a im anumpulit,

20. Iluppak osh Alon micha ushi vhleha im issa yosh nan ahama nitak fehnakmut Chihowah im issa hi oke; ilhpak im issa bilia mak osh boia tohbi lipehbi efah kushkoa ont ishit pokoli ho iklưna ka onnahinlikma im issa hokmut, opiakma iklunna ka im issashke.

21. Ampo mahaia vlhto ho bila ishit yummicha hi oke; atuk osh paluskakma isht ish chukowa hi oke; micha ilhpak im issa paluskvt tushahli ya na balama achukma mak o Chihowah ish im issa hi oke.

22. Mikma ushi vhleha naholitompa isht vtta yvt vlhtobvt nan ahama tuk vt im issa hi oke. Chihowah i nan vlhpisa bilia hoke: momut lua hi oke.

23. Ilhpak im issa na holitompa isht vtta ilap aiyuka hokut momut lua hi oke; vpa he keyu hoke, im achi tok.

24. Mihma Chihowah yout Moses a im anumpulit,

25. Alon micha ushi vhleha im anumpulit, Iluppak osh nanashvchi im issa nan vlhpisa yoke; hukmit im issa ai vbi ak o nan ashuvchi im issa ya Chihowah itikba ai vbashke; holitopa i shahli hoke.

26. Nan ashvchi ho im issa tuk, naholitompa isht vtta ak osh vpashke. Okla moma im vilhtipo i wanuta aholitopa ak o ai vpa hi oke.

27. Nana hosh nipi yash halelikmvt holitopa hi oke; micha yumma issish vt nafohka nana ho ai o fimimpa tuk ma, ai $\underline{o}$ fimimpa tuk a aholitopa yak $\mathrm{o}$ ish ai achifashke. 
28. Amba lukfi shuti ahonni tuk ano kola hi oke, micha asonak lakna yo ahonni tuk muno, kasholichi cha, oka isht' achifa hi oke.

29. Naholitómpa isht asha nakni moma kak osh vpa hi oke; holitopa i shahli hoke.

30. Mikma nan ashvchi im issa nana kia itin nanaiyvcha chi hosh issish a okla moma im vlhtipo aholitopa ya isht chukowa tuk a vpa he keyu hoke; luak ishit lua hi oke.

\section{CHAPTA VII.}

MICHA iluppak osh yoshoba im issa nan vlhpisa hoke: 1 Lolitopa i shahli hoke.

2. Hukmit im issa ai ubi ak o yoshoba im issa ya ai vba hi oke; mikma issish a alta o fimmit apakfobla hi oke.

3. Micha nia moma ka im issa hi oke: hasimbish micha nia yut ikfoka ompoholmo aiena.

4. Micha haiyihchi tuklo, micha nia on asha yummut ikeksa bilika asha, micha i hlapa yvmmvt sulakha paknaka asha haiyihchi aiena $k \underline{a}$ ishashke.

5. Micha na holitompa isht vtta yut luak ishit hukmit Chihowah im issa mak o alta onochit hukma hi oke; yoshoba im issa hoke.

6. Naholitompa isht vtta nakni moma kvt vpashke; a holitopa ak o ai vpashke, holitopa i shahli hoke.

7. Nanashuchi im issa yut kaniohmi hatuk a yoshoba im issa yot yohmi akinli hoke, nan vlhpisa im achufa hoke? isht atobbichi tuk naholitompa isht vtta yak osh isha hi oke.

8. Hatak kuna hosh hukmit im issa ya naholitompa isht vtta yut hukmit im issa ya im issa tuk naholitompa isht vtta fehna kak osh hakshup a il ai ishashke.

9. Apaluska ya apaluska tuk ilhpak im issa moma, micha ai vlwusha, micha ampo mahaia ya a nunanchi tuk moma aiena kvt naholitompa isht $v$ tta yot im issa tuk mih fehna kak o immi hashke.

10. Micha bila aiyoma tuk micha shila, ilhpak im issa 
moma ka Alon ushi vhleha moma kvt itilaui bieka hosh ishashke.

11. Micha iluppak osh itin nanaiyvchit im issa, vbit im issa Chihowah im issa hi ya nan vlhpisa hoke.

12. Yakoke, ahnit isht aiokpvchi yo im issakmvt, yak oke ahnit isht aiokpvchi ya puska ik shatummo bila aiyoma yo, micha puska lapushki bila ahama, micha bota tohbi lipehbi puska bila aiyoma aienvt vlwusha ho im issashke.

13. Puska yumma im issakmut nan im issa ya puska shatummi, micha yakoke ahnit vbit im issa, itin nanaiyvchit im issa aieninchit im issashke.

14. Micha isht aiokpvchi ya moma kash achvfa ka Chihowah $\underline{\mathbf{i}}$ weli yo im issa hi oke; mikma itin nanaiyvchit im issa issish a fimmi tuk naholitompa isht vtta kak o immi ha chi hoke.

15. Mikma yakoke, ahnit isht aiokpvchi vbit im issa itin nanaiyuchit im issa yash nipi ya im issa nitak mihmakinli ho vpashke; asha na onna he keyu hoke.

16. Amba anumpa il onochi tok ash im issa, keyukmot ilap ahnit im issa yokmuno, vbit im issa yut im issa mih nitak akinli ka vpashke, atukmut onna yak kia a asha tuk a vpashke.

17. Amba vbit im issa nipi vt asha na nitak ont atuchina tuk vt, luak ishit lua hi oke.

18. Micha vbit im issa itin nanaiyuchi im issa nipi yash nitạk ont atuchina ka ikpa nana kia im aiokpvcha he keyu, micha im issa tuk vt isht im vlhpisa he keyu hoke; nan ishit yuwala hi oke; shilombish kuna hosh vpakmot ilap nan ashvchi onvtonla hi oke.

19. Micha nipi yosh nana liteha ya haleli tuk a, vpa he keyu, luak ishit hukma hi oke, micha nipi yokvno, kashofa moma kvt vpa hi oke.

20. Amba shilombish nana hosh im a liteha yut onvtonla hosh, vbit im issa itin nanaiyvchi im issa Chihowah immi ho vpakmvt, shilombish yummak kia im okla ha ai i filummit fiopa tupa hi oke.

21. Micha shilombish kuna hosh nana liteha, hatak liteha, 
keyukmut nampoa liteha, keyukmvt nan isht yuwala liteha nana kia potoli cha, vbit im issa itin nanaiyvchi im issa Chihowah immi ha vpakmvt, shilombish yumma fehna kvt im okla im ai itin takla ya $\underline{1}$ filummit fiopa topa hi oke; ish im achashke, im achi tok.

22. Mihma Chihowah yut Moses a im anumpulit,

23. Islael im vlla vhleha ha im anumpulit, Wak nia, keyukmot chukfi, keyukmut issi kosoma nia nana kia hvshpa he keyu hoke.

24. Micha nampoa ilap inlit illi tuk nia, micha nampoa nana hosh niblit vbi tuk nia ya , nana kaniohmicha hinla, yohmi kia ik kaniohmi nana kia hushpa he keyu hoke.

25. Kuna hoh kia nampoa nia, yummak oka hatak vt luak ishit hukmit Chihowah im issa chatuk a vpakmvt, vpa tuk shilombish fehna kvt im okla im ai itin takla i filvmmit fiopa tupa hi oke.

26. Micha hush aiasha ya kanima kia hushi okma keyukmot nampoa issish nana kia hvshpa he keyu hoke.

27. Shilombish kvna hosh issish kaniohmi kia vpakmut shilombish yumma fehna kut im okla im ai itin takla i filummit fiopa tupa hi oke, ish im achashke, im achi tok.

28. Mihma Chihowah yut Moses a im anumpulit,

29. Islael im vlla vhleha ha im anumpulit, Kvna hosh vbit im issa, micha itin nanaiyvchi im issa ya Chihowah im issakmut vbit im issa, micha itin nanaiyvchi im issa ya Chihowah hak o isht im onashke.

30. Luak ishit hukmit Chihowah im issa ya ilap ibbak akinli hosh isht ona hi oke, nia micha ikkishi aieninchit isht vlashke; yoḥmi na Chihowah itikba fahfuli mak o fahfulashke.

31. Mikma nia ya naholitompa isht vtta yut alta onochit hukmashke; amba ikkishi huto Alon micha ushi vhleha hak o immi hashke.

32. Micha ubit im issa itin nanaiyvchi im issa ya wahlit im issa mak $\underline{0}$ fulup isht impak imma ya naholitompa isht vtta yak $\underline{0}$ ish imashke.

33. Alon ushi vhleha hạ kanima hosh itin nanaiyvchi im issa 
issish micha nia aiena ka im issa hokvt mihi yosh fulup isht impak imma ya immi mak osh ishashke.

34. Islael im vlla vhleha im ai itin takla ya nan vlhpisa bilia mak $\underline{o}$ vbit im issa, micha itin nanaiyvchi im issa ya ai ishit fahfuli ikkishi micha wahli fulup a Islael im vlla vhleha im ai ishit naholitompa isht vtta, Alon micha ushi vhleha ha ima li tuk oke, ish im achashke, im achi tok.

35. Ilvppak osh Alon nan ahama tuk vt, micha ushi whleha nan ahama tuk vt, luak ishit hukmit Chihowah im issa yut naholitompa isht vtta ya Chihowah nan isht im vtta chi ho atokoli nitak ma immi toba tok oke.

36. Yummak oka im ai unchuloli hlopulli ka nan vlhpisa bilia mak o Chihowah yut nan ahummi nitak mut Islael im vlla vhleha hot ima he im apesa tok oke.

37. Iluppak osh hukmit im issa, micha ilhpak im issa, micha nanashuchi im issa, micha yoshoba im issa, micha hullochi im issa, micha vbit im issa itin nanaiyvchi im issa aiena nan vlhpisa mak $\underline{\text {; }}$

38. Chihowah yut Moses a Sainai nunih hạ, Islael im vlla vhleha hvt Chihowah ya nan im issa chị ho $\underline{i}$ miha nitak mot Sainai yakni haiaka ya ai im apesa tok mak oke.

\section{CHAPTA VIII.}

TIHMA Chihowah yut Moses a im anumpulit,

12 . Alon micha ushi vhleha aieninchit, micha nafohka ya micha isht ahama bila aieninchit, micha nanashuchi im issa mak o wak hobvk, micha chukfi nakni tuklo, micha puska ik shatummo kishi achvfa aiena ho ish ishashke.

3. Micha okla moma hoyot okla moma im vlhtipo okhissa ya ish ai itunahlashke, im achi tok.

4. Mihma Chihowah yvt ai i miha tuk mak o, Moses vt yumihchi tok; mihma okla hut okla moma im vlhtipo okhissa ya ai itunaha tok.

5. Mihma Moses vt okla moma ka, Iluppak osh jumohma chị ho Chihowah yut miha tok oke, im achi tok. 
6. Mihmot Moses vt Alon micha ushi vhleha isht vla cha oka isht achifa tok.

7. Micha nafohka ya fohkachechi mvt vskofvchi ya uskofachechi cha nafohka holitompa fohkachechi mvt efot a fohkachechi tok; micha vskofvchi inla fehna efot atakali yash isht vskofvehechi tok.

8. Mihmut ikkishi alata yash $\underline{i}$ nuchechi tok, micha ikkishi alata yash Ulim micha Hlvmmim aiena ka lapalichi tok.

9. Micha iahlipa hash noshkobo ha on talali tok, micha iahlipa ha ibitakla fehna ka tuli lakna patussa bitah holitopa yo lapalichi tok. Chihowah yut Moses a $\underline{1}$ miha tuk mak $\underline{0}$ yohmi tok.

10. Micha Moses vt isht ahama bila ya eshi cha, vlhtipo micha nana anukkaka asha tuk moma ka ahummi cha hullochi tok.

11. Micha alta ya untuklo ha isht $\underline{0}$ fimmi tok, micha alta, mikma nan im ai vlhto moma, ampo mahaia chito, iyi aiena ka hullocha chi hosh ahvmmi tok.

12. Micha Moses vt hullocha chi hosh isht ahama bila fa Alon noshkobo ha on hlatubli tok.

13. Micha Chihowah yvt Moses $\underline{a} \underline{i}$ miba tuk mak $\underline{o}$ Moses vt Alon ushi vhleha ha isht vla cha nafohka fohkvchechi mut, uskofvchi ya vskofuchechi cha shapo ta shapolechi tok.

14. Micha nanashvchi im issa mak 0 wak hobuk a isht. vla tok ; mihma Alon micha ushi vhleha hut nan ashuchi im issa mak o wak hobuk noshkobo ha ibbak ish $\frac{}{2}$ umbitepa tok.

15. Mihma vbi tok; mihma Moses vt issish a eshi cha itịn nanaiyvcha chi mak osh alta lapish a íbak ushi isht o fimmit fullohlechi cha, alta ya kashoffi mut, hullochit issish ash alta akishtula ya a hlatubli tok.

16. Micha ikfoka nia yvt on asha tik moma, micha sv-

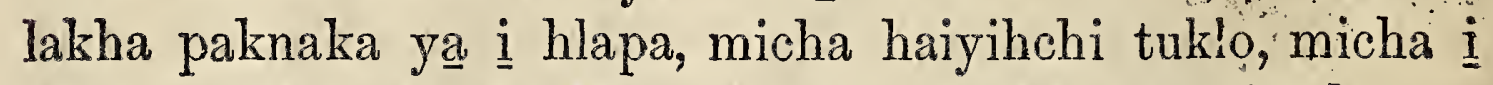
nia moma ka eshi cha Moses vt alta onochit hukmi tok.

17. Amba wak hobvk ash, micha hakshup, micha nipi micha $\underline{i}$ yulhki aiena kvno, Chihowah yut Moses a $\underline{\mathrm{i}}$ miha tuk mak o binah ba kocha ya luak ishit a hukmi tok. 
18. Micha hukmit im issa mak o chukfi nakni a isht vla tok; mihma Alon micha ushi vhleha hvt chukfi nakni yash noshkobo ha ibbak isht umbitepa tok.

19. Mihma vbi tok: mihma Moses vt issish a alta ya isht o fimmit fullohlechi tok.

20. Micha chukfi nakni yash bushlit tahli tok; micha Moses vt noshkobo, micha tushahli, micha nia aiena ka hukmi tok.

21. Micha takoba, micha iyi aiena ka oka isht achifa tok; micha Moses vt chukfi nakni yash mominchit alta onochit hukmi tok, na balama achukma mak o vbit im issvt hukmi, micha luak ishit hukmit Chihowah im issa tok; Chihowah yut Moses $\underline{a}$ i miha tuk mak o yumohmi tok.

22. Micha chukfi nakni achvfa, hullochi chukfi nakni ya isht vla tok; micha Alon micha ushi vhleha hvt chukfi nakni noshkobo ha ibbak isht umbitepa tok.

23. Mihma ubi tok; micha Moses vt issish a eshi cha Alon haksobish wishakchi, micha isht impakimma ibbak is hki, micha isht impakimma iyi ishki aiena ka o lapalichi tok.

24. Micha Alon ushi vhleha ha isht vla cha Moses vt issish ash isht impakimma haksobish wishakchi, micha isht impakimma ibbak ishki, micha isht impakimma iyi ishki aiena ka o lapalichi tok; micha Moses vt issish ash alta ya isht o fimmit fullohlechi tok.

25. Micha nia, micha hasimbish, micha anukaka nia asha tuk a, micha sulakha paknaka i hlapa micha haiyihchi tuklo, micha i nia, micha fulup isht impakimma aicna ka ishi tok.

26. Mieha puska ik shatummo kishi vihto, Chihowah itikba talaia tuk a puska ik shatummo achvfa ai ishit, micha puska bila yummi, micha puska tapuski achufa aiena ka eshi cha, nia yash onochi mut, fulup isht impakimma ya onochi tok.

27. Mihmut moma ka Alon ibbak a fohki cha, ushi vhleha ibbak fohki mvt, fahfulit im issa mak o Chihowah itikba fahfuli tok.

28. Mihma Moses vt ibbak a akohchit im eshi cha ai $\underline{Q}$ 
hukmi alta ya onochit hukmi tok, na balama achukma hullochi ya tok; luak ishit hukmit Chihowah im issa yoke.

29. Micha Moses vt ikkishi ha eshi cha fahfulit im issa mak o Chihowah itikba fahfuli tok, yummvt hullochi chukfi nakni ya Moses ak o immi hatuk oka, Chihowah yut Moses a $\underline{\mathrm{i}}$ miha tuk mak o yumohmi tok.

30. Micha Moses vt isht ahama bila ya micha issish vt alta on asha tuk a eshi cha, Alon micha $\underline{\mathrm{i}}$ nafohka ya $\underline{\mathrm{o}}$ fimmi mut, micha ushi vhleha, micha ushi vhleha $\underline{i}$ nafohka ya o fimmi tok; micha Alon micha i nafohka, micha ushi whleha micha ushi vhleha $\underline{\mathrm{i}}$ nafohka ya aieninchit hullochi tok.

31. Micha Moses vt Alon a micha ushi vhleha ha Okla moma im vlhtipo okhissa yak o nipi ya hush a honashke, micha yummak $\underline{0}$, Alon micha ushi vhleha aienvt ai upashke, achi li tok mak o, yummak o hullochi puska kishi alhto aieninchit hushpashke.

32. Micha nipi, micha puska aiena kut a asha moma ka luak ishit hush hukmashke.

33. Micha okla moma im vlhtipo okhissa ya nitak untuklo itin takla ka hvchi hullochi nitak vt ont ik taho kisha kạ, hvchik kohcho kashke nitak untuklo ho hvchi hullocha chị hoke.

34. Huchi kashoffa chi hosh himak nitak a yumihchi tuk vt yohma chi Chihowah yut miha tuk oke.

35. Yumohmi hoka nitak micha ninak moma ka okla moma im vlhtipo anukaka hush asha na nitak untuklashke, micha Chihowah yut atokolit miha tuk a hush holitoblashke; yohmi hosh ik huchillo kashke; yummak o a miha tuk oka; im achi tok.

36. Yvmohmi ma Chihowah yut Moses a tohnot $\underline{\mathrm{i}}$ miha tuk mak o, Alon micha ushi vhleha aiena kvt nana moma ka yumihchi tok. 


\section{CHAPTA IX.}

UMOHMI ma nitak ont isht untuchina ma, yakohmi 1 tok; Moses vt Alon micha ushi vhleha, micha Islael asunonchi vhleha aiena ka pit hoyo tok.

2. Mihmut Alon a, Nan ashvchi im issa chi mak o wak ushi himita ya, micha nahukmit im issa chị ho, chukfi nakni aiokpulo iksho ho ish ieshi cha Chihowah itikba ya ish im issashke.

3. Mikmvt Islael im vlla vhleha ha im anumpulit, nan

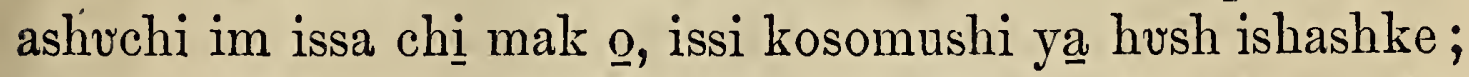
micha nahukmit aiokpưcha chi ho, micha wak ushi, micha chukfulhpoa ushi aiokpulo iksho ho afummi vmmona bika ya hush ieshashke.

4. Yvmohmikmvt itin nanaiyvchi im issa ya Chihowah itikba ya vbit isht ai okpvechi ya wak hobvk, micha chukfi nakni a nan ilhpak im issa bila itaiyuma ka aieninchashke; himak nitak a Chihowah yut auet huchim otvna chi hoka, ish achashke, achi tok.

5. Mikma Moses vt nana im apesa tuk ak o okla moma in tabanekel itikba ya isht aivla tok; mihma okla moma kvt bilika vla cha Chihowah itikba ya ita hieli tok.

6. Yohmi ma Moses vt nana hush yumihcha he Chihowah yut huchim apesa tok a iluppak oke; yohmikma Chihowah nan isht im a holitopa yut hvchim a haiaka hi oke, achi tok.

7. Mihmut Moses vt Alon a, Alta ya ish ona cha, nan chim ashuchi im issa, micha chin nahukmit im issa mak $\underline{o}$ ish im issa hi oke; yohmi cha chishno inli, micha okla aiena pulla tuk mak $\underline{0}$, nan isht a kashofa ish im ikba hi oke; yumohmi cha okla puta nan im issa ya ish ema cha, Chihowah yot achi tuk mak $\underline{o}$, nan isht $\underline{\underline{i}}$ kashofa ka ish im ikba hi oke, im achi tok.

8. Yohmi ka Alon vt alta ya ona cha nan ashuchi im issa wak ushi ilap immi atuk mak o vbi tok.

9. Mihma Alon ushi vhleha hut issish atuk a isht im ona tok; mihma yummvt ibbak ushi a issish ash a oklubichi tuk 
osh alta lupish puta ka on lapalichi cha, alta akishtvla ya issish a vhlatubli tok.

10. Amba nia, micha haiyihchi, micha nanashvchi im issa sulakha paknaka onhlepa, yumma Chihowah yut Moses a im

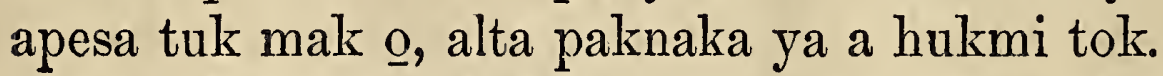

11. Micha nipi, hakshup aiena ka vlbina akohcha ka hukmi tok.

12. Micha na hukmit im issa ya vbi tok; mihma Alon ushi vhleha hut issish atuk a ima tok, mihma yummut alta ai apakfopa ai o fimmi tok.

13. Yumohmi cha na hukmit im issa im ita kvshkoa, micha noshkobo aiena ka ima tok; mihma alta paknaka ya ai o hukmi tok.

14. Micha takba foka, iyi puta aiena ka achefut tahli cha nahukmit im issa alta ai onochi cha hukmi tok.

15. Micha okla puta nan im issa ya isht vla cha issi kosoma, yummut okla puta nan ashyychi im issa yoka vbi cha vmmona chohmi tuk $\underline{o}$, nan ashvchi im issa atukmak $\underline{o}$ chohmichit im issa tok oke.

16. Micha na hukmit im issa isht vla cha ayohmi chatuk vlhpesa ho im issa tok.

17. Yumohmi cha nan ilhpak im issa ya isht vla cha ibbak alota achufa ho ishit alta paknaka onochit onnahinli nahukmit im issa ya aieninchit hukmi tok.

18. Okla moma nan im itin nanaiyvchi vbit isht aiokpvcha he a wak hobvk, chukfi nakni aiena ka vbi tok; mihma Alon ushi vhleha hvt issish a ishit ema na, alta paknaka aiapakfoblit $\underline{o}$ fimmi tok.

19. Wak hobvk micha chukfi nakni nia, hasimbish, haiyihchi, takba foka isht ompoholmo, sulakha on hlepa ya ima tok.

20. Mihma nia ya ikkishi onochi tok; mihma nia yash alta paknaka ya ai o hukmi tok.

21. Mihma Alon vt ikkishi micha fulup isht impakimma ita tuklo na wali im issa chi mak $\underline{o}$, Moses vt apesa tuk

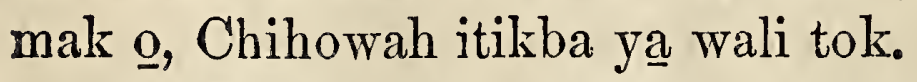

22. Mikmut Alon vt ilap ibbak a okla ha vba pit $\underline{i}$ weli 
cha yukpali tok; nan ashvchi im issa, na hukmit im issa, micha itin nanaiya im issa imihisisut tahli mut ai $\underline{\underline{i}}$ filvmmit akowa tok.

23. Yohmi ma Moses micha Alon ita tuklot okla moma in tabanekel a iba chukowa tuk osh kohcha mut, okla hash yukpali tok.

24. Yvmohmi ma luak osh Chihowah itikba ya vttut kucha cha nahukmi im issa micha nia vt alta onvtoyula ka hukmi tok; yummak o okla hut pisa mut shakapa cha nashuka ya aka isht hlipkaiyachi tok.

\section{CHAPTA X.}

AUK o Alon ushi Netab micha Abihu vt nabalama a A hukmi ishi bikka cha luak a fohki mut nabalama ya onochi cha, yummak oka i miha tuk keyu kạ luak inla Chihowah ya im issa tok.

2. Mihma luak vt Chihowah ya a minti hosh kohchvt ivt hukmit tahli na Chihowah itikba ai illi tok.

3. Yohmi ma Moses vt Alon a, Ilvppak o Chihowah yut anumpulit, 1 bilika vla ka a holitopa la chi hoke. Micha okla moma itikba a holitopa la chi hoke, achi tuk mak oke, im achi tok. Mihma Alon vt nan ik acho ki tok.

4. Mihma Moses vt Alon imoshi Ussiel ushi Mishael micha Elsafan a hoyo cha, Bilika hushla cha hvchitibapishi vhleha ha okla moma im vlhtipo itikba ai ishit binah ha kocha isht hush onashke, im achi tok.

5. Yohmi ho Moses vt achi tuk mak $\underline{0}$, bilika ona cha $\underline{i}$ nafohka ya foka ho binah ha kocha isht ona tok.

6. Mihma Moses vt Alon, micha ushi Eliesa micha Ihlama, Huchi noshkobo o hlipa ya huvchik shuffo kashke, micha huvchị nafohka ya hơchik hlilvffo kashke; hveh illi ná, micha im ai i nukkilli vt okla moma ka onvtuyula ná: amba hvchitibapishi vhleha Islael in chukachvfa moyuma kvt luak a Chihowah yot oti tuk ak o chitoli hosh yaiyashke.

7. Micha okla moma im vlhtipo okhissa ya $\underline{i}$ filvmmit hv- 
chik ayo kashke, huvch illi ná; nan isht ahama Chihowah i bila yut huchi onvtonla hokvt, im achi tok. Mihma Moses im anumpa hatuk mak o okla yumohmi tok.

8. Mihma Chihowah yvt Alon a im anumpulit,

9. Chishno micha chiso vhleha hush aiena kut okla moma im vlhtipo ya hvsh chukowakmvt oka paki, micha nan ishko kvllo aiena ka hvchik ishko kashke, hvch illi ná, ilupput hvchi ai unchuloli hlopulli ka nan vlhpisa bilia ya chi hoke.

10. Yvmohmikma naholitopa, micha nan ik holitopo, micha nan ik kashofo, micha nana kashofa ya hvsh itim inluchashke.

11. Yumohmi hosh Chihowah yut Moses a tohnot ai im anumpohonli tok nan vlhpisa moma ka Islael im vlla vhleha ha hush im abahanchashke; im achi tok.

12. Mihma Moses ut Alon micha ushi okchaya moma tuk Eliesa micha Ihlama aiena $k \underline{a}$ in anumpulit, Ilhpak im issa luak ishit hukmit Chihowah im issa asha moma tuk a hush eshi cha isht shatummi ut iksho ho alta bilika hush ai vpashke: holitopa i shahli yoke.

13. Ilvpput vbit luak ishit hukmit Chihowah im issa puta ak o ai ishit chimmi, micha chiso vhleha immi hatuk okvt, a holitopa ak o hush ai vpashke, yummak o a miha tuk mak oka.

14. Micha fahfuli ikkishi micha wahli fulup a akashofa ho chishno, micha chiso whleha, micha chisotek vhleha aienvt hush ai vpashke, itin nanaiyvchi im issa vbit im issa Islael im vlla vhleha hut ima tuk akohcha kvt chimmi micha chiso vhlehar aienvt immi hatuk okvt.

15. Falfulit ikkishi im issa ya Chihowah itikba fahfula chị kvt, wahli fulup, micha fahfuli ikkishi hạ luak ishit hukmit im issa nia ya aieninchit isht vlashke; yohmi ka Chihowah yvt miha tuk mak o yummvt chimmi micha chiso vhleha immi nan vlhpisa bilia ya chi hoke, im achi tok?

16. Atuk o Moses vt nan ashuchi im issa issi kosoma ya achunanchit hoyo ma, Yakeh, lua tuk atok: mihma Afon ushi vhleha okchaya moma tuk, Eliesa micha Thlama ya 1 nukhobela cha, 
17. Nanta katiohmi ho nanashvchi im issa, aholitopa ya hvch ik ai apo tuk oh cho? holitopa i shahli hatuk, micha okla moma nan ashvchi ya Chihowah itikba hush a shaiyala chị, micha isht hosh i kashoffa chi aiena ho Chitokaka yut hvech ima hatuk oka.

18. Yakeh, issish a aholitopa anukaka isht ikloshke hvchi miha li tnk mak o aholitopa ya hush ai upa muhla he tuk oke, im achi tok.

19. Mihma Alon vt Moses a, Yakeh, himak nitak nan ashvchi im issa, micha hukmit im issa aiena ka Chihowah itikba ai im issa tuk oke; atuk o nana yakohmi kak osh si onvtulushke: atnk osh himak nitak a nan ashvchi im issa vpa li tuk ma, Chihowah yut aiokpucha he tuk oh cho? im achi tok.

20. Atuk o Moses vt yumma haklo mvt, im vlhpesa tok.

\section{CHAPTA XI.}

\section{IHMA Chihowah yut Moses micha Alon itatuklo ka im 11 anumpulit,}

2. Islael im vlla vhleha ha im anumpulit, nampoa yakni paknaka aiasha moma ka, nampoa iluppa puta kak o hush pa hi oke.

3. Nampoa puta ka nana hosh iyakchush vt iti filummi, iyi vt chulakto cha, hushuk vpa tuk vt falvmminchit kohchit hopasa ak o hushpa hi oke.

4. Yohmi kia ilvppa puta kak o hushưk upa tuk vt falvmminchit kohchi cha hopasa yoh kia, michaiyakchush chulakto yoh kia, hushpa he keyu hoke. Kamel a chohmi ka hushuk vpa tuk vt kohchi cha hopasa kia, iyakchush a chuhluff keyu hokvt, hvchin liteha hoke.

5. Micha chukfi hushuk vpa tuk vt falumminchit kohchit hopasa kia iyakchush a ik chuhluffo hokvt hvchin liteha hoke. 6. Micha puta kvta hvshnk a vpa tuk vt falvmminchit kohchit hopasa kia iyakchush a ik chublvffo hokvt hvchin liteha hoke. 
7. Micha shukha, yvmmvt iyakchush chuhlvffi kia hvshuk vpa tuk vt falvmminchit kohchit hopasa keyu hokvt huchin liteha hoke.

8. Nipi ya hvshpa he keyu, micha haknip illi ya hush potola he keyu hoke, hvchin liteha hoke.

9. Oka aiasha ka iluppa puta moma kak o hushpa hi oke, nana hosh oka, micha okhuta, micha okhina ai itunowa yosh isht okyohli micha hakshup fachula im asha ya hushpa hi oke.

10. Micha isht okyohli micha hakshup fachula ik im iksho moma okhvta micha okhina aiasha, oka ai itunowa moma, nana okchaya nana hoh kia oka aiasha kvt hvchi yuwala ya hi oke.

11. Isht hvchi yuwala he mak oke; nipi ya hvsh pa he keyu, amba nipi illi yot isht hvchi yuwala hi oke.

12. Nana hosh isht okyohli micha hakshup fachula ik im iksho hosh, oka aiashakmvt mihi yosh isht hvchi yuwala hi oke.

13. Micha hushi puta ka iluppak osh isht hưchi yuwala hi oke, vpa he keyu; ishit yuwala hoke, Ussi micha ossiflech (okhvta ussi) micha osple, (uيssi nuni vpa)

14. Micha fulta, micha kait im aiachvfa,

15. Fula chito moma im ai achufa,

16. Micha opah : micha oksup-ibafohli, micha kuku micha biakak im ai aohvfa.

17. Micha opah iskitini, micha kamolant micha opah chito.

18. Micha okak, micha chilantakoba micha kia-ússi.

19. Micha stok micha itikushayaiiya (hushwak) im ai achvfa, micha lapwin, micha halambisha aiena ka hushpa he keyu hoke.

20. Hushi puta balvlli iyi ushta kash ishit nohowa moma kvt isht hvchi yuwala hi oke.

21. Yohmi kia naheli balvlli, iyi ushta ishit nohowa iyi paknaka ya iyi holhki vt ashvt yakni ya isht on tullit aya yokma moma ka hushpa hi oke.

22. Iluppa puta kak o hush ai upa hi oke. Hawa ilap 
aiachvfa, micha hawa yushmilali ilap ai achvfa micha shushi lapitta ilap ai achufa, micha hatvffo ilap ai achvfa aiena kak oke.

23. Amba naheli balahanli inla iyi im ushta moma kvt nan isht hvebi yuwala ya chi hoke.

24. Micha iluppa puta kak o isht hvchi liteha hi oke. Kvna hosh yumma illi ya halelikmut litiha na, ont opia hi oke.

25. Micha kuna hosh illi yumma kaniohmi ho sholi hokmut i nafohka ya achifa cha litiha na, ont opia hi oke.

26. Nampoa hot iyakchush iti filvmmichi, micha iyi pahlata keyu hosh hushuk vpa tuk vt falumminchit kohchit hopasa keyu puta haknip illi yut hvchin liteha hoke.

27. Micha nampoa iyi ushta ishit nohowa ilaiyuka puta, nampoa nana hosh iyi puta ishit nohowa hokmut yvmma puta kvt hvchin liteha hoke: Kuna hosh haknip illi haleli hokmut litiha na, ont opia hi oke.

28. Micha illi yumma sholi tuk mut i nafohka ya achifa cha litiha na, ont opia hi oke; hvchin liteha yoke.

29. Iloppa puta aiena kak osh na balali yakni om balahanli vt huchin liteha hi oke: weasel, micha pinti micha luksi ilap ai achvfa,

30. Micha fellet, 'micha kamelion, micha halanchilawa, micha snail, micha yulhkun aiena kak a hi oke.

31. Iluppa puta balahanli puta kvt hvchin liteha hoke. Kuna hosh illi tuk a halelikmut litiha na, ont opia hi oke.

32. Micha nana ho iti nan ai vlhto, keyukmut nafohka, keyukmot na hakshup, keyukmvt bahta, nan aivlhto nana kia, nan ai atahli chatuk o illi yosh onvtulakma, liteha hi oke; oka okvchi cha litiha na ont opia hi oke; yohmi hosh kashofa hi oke.

33. Micha lukfi tunna puta nan ai vlhto nana kia, nana hosh alhto tuk o okatulakma liteha hi oke; mikma hvsh kola hi oke.

34. Ilhpak moma upa he vihpesa ho oka yumma chohmi. hosh on hlatapakma yummak atuk vt liteha hi oke; micha 
nan ishko nana ho ishka he vlhpesa hosh nan aivlhto puta yumma chohmi ho vlhtokmut liteha hi oke.

35. Micha nana puta kanima ho illi yash kaniohmi hosh onvtulakma liteha hi oke; apalvska, keyukmut shuti vt. baivllit ataloha, kovt kinahla hi oke, liteha yokvt, micha hvchin liteha hi oke.

36. Yohmi kia kvli, keyukmvt kvli hofobi yosh oka lawa achukma hokmuto kashofa hi oke; amba yưmma illi ya haleli holkvto liteha hi oke.

37. Micha illi yash kaniohmi hosh napehna hollokcha chin tuk a onvtulakma kashofa hi oke.

38. Amba napehna ya oka nana hosh o hlatapa tuk o illi yash kaniohmi hosh onvtulakma hvchin liteha hi oke.

39. Nampoa nana hosh hushpa hinla tuk osh illikma kvna hosh illi yumma halelikmvt litiha na, ont opia hi oke.

40. Micha kuna hosh illi yash vpakmvt $\underline{i}$ nafohka ya achifa cha litiha na, ont opia hi oke; micha kuna hosh illi yash sholi tuk oh kia i nafohka ya, achifa cha litiha na, ont opia hi oke.

41. Micha na balali puta yakni paknaka ya o o balahanli vt nan ishit yuwala ya hi oke; vpa he keyu hoke.

42. Nana hosh ikfoka ishit nohowa, keyukmot iyi ushta ho ishit nohowa, keyukmut na balali yakni om balahanli ya iyí laua kvt i shahlikma, yummak oka hush pa he keyu, nan isht yuwala yoka.

43. Na balulli balahanli chatuk nana kia, ishit yuwala isht hush il ikba he keyu, micha yummak oka isht hush ile litehli cha isht hvchi liteha he keyu hoke

44. Chitokaka hvehi Chihowah sia hoka, yumohmi hoka hvsh ile hullochi cha hvchi holitopashke, holitopa sia hoka ; micha na balulli yakni o balahanli chatuk nana kia isht hush ile litehla he keyu hoke.

45. Huchin Chitokaka sia ha chi hosh Echip yakni ya apehlichit hvchi kohchi tuk Chihowah sia hoka; yvmohmi hoka hvchi holitopashke; sa holitopa hoka.

46. Iluppak osh nampoa, micha hushi, micha okchaya 
puta oka ya atâya, micha nana puta yakni ya om balahanli aiena nan vlhpisa hoke.

47. Ik kashofo, micha kashofa, micha nampoa upa he vlhpesa ka, micha nampoa vpa he vlhpesa keyu ya ishit itim inluchi yoke; hush im achashke, im achi tok oke.

\section{CHAPTA XII.}

ICHA Chihowah yot Moses a im anumpulit, I1 2. Islael im vlla vhleha ha im anumpulit, Ohoyo hot chakali cha vlla nakni yo eshikmut nitak untuklo ho ik kashofo ka hi oke, liteha kvt ila filummi chatuk nitak itin takla mak o ik kashofo ka hi oke.

3. Atuk $\underline{o}$ nitak ont isht untuchinakma vila yot hakshup in trpashke.

- 4. Yomohmi ka ohoyo hash ot issish onvtoyulut ile kashofi kvt nitak pokoli tuchina akucha tuchina hi oke; ile kashofi nitak vt ont ik taho kisha ho nana hullo nana kia potola he keyu, micha vlhtipo holitopa ont chukowa he keyu hoke.

5. Amba vila tek o eshi hokmuto nitak hullo tuklo ho ik kashofot ile filvmmicha hi oke; micha issish onvtoyulvt ile. kashofi kut nitak pokoli hannali akucha hannala hi oke.

6. Micha vlla nakni, keyukmvt vlla tek atuk o ile kashofi nitak vt ont tahakma, hukmit im issa yo chukfushi afummi ummona, michạ nanashuchi im issa yo puchushi, keyukmvt pvchi yoshoba aiena ho okla moma im vlhtipo okhissa isht onvt naholitompa isht vtta ya isht im ona hi oke.

7. Mikma yummak osh Chihowah itikba ya ai im issa cha 1 kashoffa hi oke. Mikma issish i yanali tuk vt kashofa hi oke. Iluppot nakni, keyukmut tek o eshi tuk vt i nan vilhpisa hoke.

8. Yohmi ka chukfushi ya isht vla he keyukmut pvchi yoshoba tuklo, keyukmut puchushi tuklo ho isht vlaşhke; achvfa kvt hukmit im issa yokma, achvfa kut nanashvchi 
im issa ya hi oke, mikma naholitompa isht vtta yvt i kashoffikma, kashofa hi oke; ish im achashke, im achi tok.

\section{CHAPTA XIII.}

1 ICHA Chihowah yot Moses micha Alon a im anumpu-

2. Hatak nipi hakshup a shatali, hlachowa, keyukmut tohbi hosh talaia, micha nipi hakshup a lapali kvt ililli leplosi vbi chohmikma, naholitompa isht vtta Alon, keyukmut ushi vhleha naholitompa isht asha achvfa isht im ona hi oke.

3. Mikma naholitompa isht vtta yut nipi hakshup ililli onvtonla ka pisashke; atuk o ililli a lapali ka hishi tohbit isht ia, micha ililli pisa ka kolukbi kvt nipi hakshup inla ka i shahlikmut ililli leplosi ýoke: mikma naholitompa isht vtta yut pesa cha ik kashofo, achashke.

4. Nipi hakshup tohbi chohmi tuk vt tohbikma, micha kolukbi kvt hakshup a i shahli keyu, micha hishi vt tohbi keyu, yohmikma ililli ishi tuk ash naholitompa isht vtta yot nitak untuklo ho aboha fohkit im okhishta hi oke.

5. Atukmut nitak isht untuklokma naholitompa isht vtta yot pisa hi oke : atuk $\mathrm{o}$ yakeh! ililli vt pisa ka ont talaia cha jlilli vt hakshup a chitot isht ik aiyokma, naholitompa isht vtta yot himakma nitak untuklo ho aboha fohkit im okhishta hi oke.

6. Atuk mut naholitompa isht vtta yvt nitak ont isht untuklokma anonti pisa hi oke : atuk o yakeh! ililli hvt lusbi choyuhmi cha ililli hot hakshup a chitot isht ik aiyokma, naholitompa isht vtta yut kashofah, acha hi oke: peh hlachowa yoke;'mikma i nafohka ya achifa cha kashofa hi oke.

7. Amba kashofa chị ho naholitompa isht vtta yut pisa ha ya hlachowa yvt hakshup a chito fehnvt isht iakma, naholitompa isht vtta yvt anonti pisa hi oke.

8. Atuk osh nabolitompa isht vtta yvt pisakma yakeh! ililli hut hakshup a chitot isht ia ho pisakmut naholitompa isht vtta yut ik kashofo, acha hi oke, leplosi yoke. 
9. Ililli leplosi vt hatak a onvtoyulakma naholitompa isht vtta ya isht im ona hi oke.

10. Mikma naholitompa isht vtta yvt pisa hi oke, micha yakeh! shatali yot hakshup a tohbi, micha hishi a tohbichi, micha shatali yot okchushbut homma ho pisakmut,

11. Leplosi sipokni yosh nipi hakshup a onvtoyula hoke; mihma naholitompa isht vtta yut ik kashofo, acha hi oke; micha ik im okhishto kashke, ik kashofo hoka.

12. Micha leplosi yut kohchut nipi ya onvtoyulut mahayakma, micha ililli onvtola tuk ash nipi moma ka ompoholmot noshkobo vttut ia hosh iyi ya ont vhlichikma, naholitompa isht vtta yut kanima yo a pesakmvt,

13. Yohmikmut naholitompa isht vtta yut anukfillashke; atuk o yakeh! leplosi yut nipi ya mominchikma ililli onvtola tuk ash kashofa, achashke, momot tohbit taha hoke; kashofa hoke.

14. Yohmi kia nipi okchaki yut haiakakma ik kashofo ka hi oke.

15. Mikma naholitompa isht vtta yvt nipi okchaki a pisa cha, ik kashofo, acha hi oke, nipi okchaki yut ik kashofo yoka; leplosi yoke.

16. Keyukmut nipi okchaki yut anonti inlut isht ia cha tohbi tobakma, naholitompa isht vtta ya im ona hi oke.

17. Mikma naholitompa isht utta yut pisa hi oke; mikma yakeh! ililli yot tohbit tahakma naholitompa isht vtta yvt ililli onvtola kash kashofa, acha hi oke : kashofa hoke:

18. Micha nipi yumma hakshup a hichi ut talaia tuk osh vtta hoke.

19. Micha hichi atalaia tohbi shatali, keyukmut tohbi iskitinesi hosh talaia, tohbi micha homma chohmi hosh talaia tuk o naholitompa isht vtta ya pisachi;

20. Atuk o naholitompa isht vtta yut pisakma, Yakeh! Kolukbi kut hakshup a ishahli, micha hishi vt tohbit tahakma, naholitompa isht vtta yvt ik kashofo, achashke; ililli leplosi yosh hichi ya a kuhla hoke.

21. Amba naholitompa isht vtta yut pisakma, yakeh! hishi tohbi kvt iksho, micha kolukbi kvt nipi hakshup inla 
ya ik i shahlokma, amba lusbi choyuhmi; yohmikma naholitompa isht vtta yot aboha fohkit im okhishta na, nitak untukla hi oke.

22. Amba nipi hakshup $\underline{a}$ chitot isht $\underline{i}$ shahlit mahayakma, naholitompa isht vtta yut ik kashofo, acha hi oke, ililli hoke.

23. Amba tohbi talaia tuk vt atalaia tuk a talaia cha chitot isht ik aiyokma, hichi lua yoke; mikma naholitompa isht vtta yot kashofa, acha hi oke.

24. Keyukmvt hakshup a lushpvt hukmi fehna, nipi okehushba homma lua ya tohbi iskitini homma chohmi, keyukmvt tohbi hosh talaiakma;

25. Yohmikma naholitompa isht vtta yut pisa hi oke; mikma yakeh! tohbi iskitini talaia ho hishi vt tohbit isht ia, micha pisa ka kolukbi kut nipi a $\underline{\text { i }}$ shahlikma, leplosi yosh lua ka atobut kohcha hoke, yumohmi hoka naholitompa isht vtta yut ik kashofo, acha hi oke, ililli leplosi yoke.

26. Amba naholitompa isht vtta yut pisa ma, yakeh! tohbi iskitini ka hishi tohbi kvt iksho, micha kolukbi kvt nipi inla ya ik 1 shahlokma, amba lusa chohmi; yohmikma naholitompa isht vtta yut nitak untuklo ho aboha fohkit im okhishtashke.

27. Atuk osh nitak ont isht untuklokma naholitompa isht vtta yut pisa hi oke; yohmikma nipi ya chitot isht $\underline{i}$ shahlit isht iakma naholitompa isht vtta yvt ik kashofo, acha hi oke; ililli leplosi yoke.

28. Micha tohbi iskitini talaia kvt mihinli talaia cha, nipi ya chitot isht ik aiyo, amba lusa chohmikma lua shatali yoke; na holitompa isht vtta yvt kashofa, acha hi oke; luvt homma yoke:

29. Hatak osh keyukmvt ohoyo hosh ililli hut noshkobo keyukmvt nutakhish a onvtolakma,

30. Yohmikma naholitompa isht vtta yvt ililli ha pisa hi oke; mikma yakeh! pisa ka kolukbi kvt nipi ya $\underline{i}$ shahlikma, micha hishi lakna tapuski chohmi hosh asha, yohmikma naholitompa isht vtta yut ik kashofo, acha hi oke; hla- 
chowa shila, leplosi fehna hosh noshkobo, keyukmut nutakhish onvtoyula hoke.

31. Micha naholitompa isht vtta yut ililli hlachowa ya pisa ma, yakeh! pisa ka kolukbi kvt nipi ya ik i shahlo, micha hishi lusa yvt iksho, yohmikma naholitompa isht vtta yut ililli hlachowa onvtonla tuk a nitak untuklo ho aboha fohkit im okhishta hi oke.

32. Atuk osh nitak ont isht untuklokma naholitompa isht vtta yut ililli yash pisa hi oke; mikma yakeh! ililli hlachowa yot chitot isht ik aiyo, micha hishi lakna kvt iksho, micha pisa ka hlachowa hut kolukbi kvt hakshup a ik shahlokma,

33. I shafa hi oke; yohmi kia hlachowa yano shafa he keyu hoke; mikma naholitompa isht vtta yut hlachowa ya himakma nitak untuklo ho aboha fohkit im okhishta hi oke.

34. Atuk osh nitak ont isht untuklokma naholitompa isht vtta yut hlachowa yash pisa hi oke; mikma yakeh! hlachowa yut nipi ya chitot isht ik aiyo; micha pisa ka kolukbi kvt ik $\underline{\mathrm{i}}$ shahlo, yohmikma naholitompa isht vtta yvt kashofa, acha hi oke; mikma i nafohka ya achifa cha kashofa hi oke.

35. Amba kashofa ha ya hlachowa yut nipi ya chito fehnut isht ia,

36. Yohmikma naholitompa isht vtta yut pisashke; mikkma yakeh! hlachowa yut nipi ya chitot isht iakma, naholitompa isht vtta yot hishi lakna hoya he keyu, ik kashofo hoke.

37. Amba hlachowa yash osh pisa ka mih achufa talaia, mikma hishi lusa yot yomma ai offokma, hlachowa yot hlakofi, kashofa hoke, mikma naholitompa isht vtta yvt kashofa, acha hi oke.

38. Micha hatak osh keyukmut ohoyo hosh nipi hakshup a tohbi taloha, tohbi shohkauwanli taloha,

39. Yohmikna naholitompa isht vtta yut pisa hi oke; mikma yakeh! nipi a tohbi taloha kut lusa chohmikmut, chikchiki talaia yosh nipi ya toba hoke; kashofa hoke.

40. Micha hatak osh pashi yưt noshkobo ha achilofa cha yushmilali, yohmi kia kashofa hoke. 
41. Micha kuna hosh noshkobo ha nashuka pilla hosh pashi yvt i chilofakmot ibitakla yushmilali hoke, yohmi kia kashofa hoke.

42. Micha noshkobo yushmilali, keyukmut ibitakla yushmilali ya hlachowa tohbi homma chohmit talaiakmvt; noshkobo yushmilali keyukmut ibitakla yushmilali ya leplosi tobut kohcha hoke.

43. Yohmikma naholitompa isht vtta yvt pisa hi oke; mikma yakeh! noshkobo yushmilali ya keyukmut ibitakla yushmilali hlachowa shatali kvt tohbi homma chohmi leplosi yưt nipi ya a haiaka chatuk a chohmikmvt,

44. Hatak leplosi onvtonla, ik kashofo hoke; naholitompa isht vtta yot ik kashofo kvmohmi, acha hi oke; ililli hut noshkobo hak o onvtola hoke.

45. Mikma ililli onvtonla tuk leplosi vbi yash osh i nafohka yot hlilafa cha noshkobo bieka hi oke, micha itivlbi vba ya ompohomo cha payvt, Ik kashofo, Ik kashofo, ahan cha hi oke.

46. Ililli hvt onvtoyula nitak moyuma ka liteha hi oke; ik kashofo hokvt ilap banot ahanta hi oke; aitta yut binah ha kocha ya hi oke.

47. Micha nafobka ya ililli leplosi vt onvtonla, chukfi hishi yokma, linen okma,

48. Linen osh keyukmot chukfi hishi yosh vpi tvnna yokma, keyukmot ishit tunna yokma, nahakshup okma, keyukmut nahakshup osh nana toba yokma,

49. Ililli hut okchamali chohmi, keyukmut homma chohmi hosh nafohka ya, keyukmot nahakshup, keyukmut vpi tunna, keyukmot ishit tvnna, keyukmot nahakshup nana ho onvtonlakmvt, ililli leplosi yoke; atuk o naholitompa isht vtta ya pisacha hi oke.

50. Mikma naholitompa isht vtta yut ililli ha pisa cha ililli onvtonla tuk a im okhishta na nitak untukla hi oke.

51. Atuk osh nitak ont isht untuklokma nan ililli hash pisa hi oke; nafohka ya vpi tunna ak okmá; keyukmot ishit tunna ak okmá, keẙukmvt nahaksup ak okmá, keyukmvt nana hosh nahakshup ishit toba ak okmá, nana ho ililli hvt 
chitot isht iakma, ililli hot leplosi chilita yoke; ik kashofo hoke.

52. Yohmi hoka chukfi hishi yosh, keyukmut linen osh vpi tunna, keyukmot ishit tunna, keyukmot nahakshup keyukmvt nahakshup ishit toba yosh ililli hot onvtonla nafohka yumma hukma hi oke : leplosi chilita yoka, luak ishit luashke.

53. Atuk o naholitompa isht vtta yut pisakma, yakeh! nafohka ya upi ponola yokma, keyukmut ishit tunna yokma keyukmvt nahakshup nana ho ishit toba ya ililli hvt chitot isht ik aiyokma,

54. Yohmikma naholitompa isht vtta yut ililli onvtonla nana kash achifa chi ka i miha cha, himakma nitak untuklo ho im okhishta hi oke.

55. Atukma ahchifa ha ya naholitompa isht vtta yot pisa hi oke, mikma yakeh! ililli pisa kvt inlut isht ik aiyo, micha ililli hut chitot isht ik aiyokmvt ik kashofo hoke; luak ishit ish luacha hi oke; noshkobo, keyukmut ibitakla yushmilali hokmá, nana kia anukaka chilita yoke.

56. Micha naholitompa isht vtta yvt pisakma yakeh! ahchifa ha ya ililli hut lusa chohmikma, nafohka yash, keyukmot na hakshup ash, keyukmot vpi yash, keyukmut ishit tonna yash hliluffit kohcha hi oke.

57. Atuk o anonti nafohka yash, keyukmut vpi yash, keyukmot ishit tonna yash, keyukmot nahakshup osh natoba yash haiakakmut ililli vlmoli yoke: ililli onutonla yumma luak isht ish hukma hi oke.

58. Micha nafohka ya upi hokmá keyukmut ishit tunna yokmá; keyukmut nahakshup osh nana kia toba tuk okmá nana ish achifa tuk ash ililli hvt kuniakma, atukla ma vlhchifa cha kashofa hi oke.

59. Nafohka yut chukfi hishi, keyukmut linen osh vpi yokmá; keyukmut ishit tưnna yokmá, keyukmut nahakshup isht atoba nana ho ililli hut onvtoyula na, kashofa, acha he; keyukmvt ik kashofo, acha hi yạ, nan vlhpisa hut ilvppak oke, im achi tok. 


\section{CHAPTA XIV.}

MICHA Chihowah yot Moses a im anumpulit,

IL 2. Iluppak osh leplosi vbi vt kashofa he nitak a nan vlhpisa hoke ; naholitompa isht vtta ya isht im ona hi oke.

3. Mikma naholitompa isht vtta yut binah ha kohchvt ia hi oke; micha naholitompa isht vtta yvt. pisa hi oke; mikma yakeh! lepa hatak leplosi vbi vt ililli leplosi onvtonla tuk vt hlakofikma,

4. Yohmikma kashofa chi kash. hushi kashofa okchaya tuklo ho, micha chuahla iti, micha tishepa, micha hissop aiena ho im esha hi ya naholitompa isht vtta yut miha hi oke.

5. Micha hushi yash achvfa hosh lukfi tvnna nan aivlhto fohka hosh oka kapussa paknaka ai illa hi yạ, mihashke.

6. Hushi okchaya yano, yumma, micha chuahla iti micha tishepa micha hissop aiena kash eshi cha yumma micha hushi okchaya yash, oka kapussa paknaka ai illi tuk ash issish a akvcha hi oke.

7. Micha kashofa chị kash untuklo ha o fimma hi oke, micha kashofa, achi cha hushi okchaya oktak ashahbi ho hikacha hi oke.

8. Micha kashofa chi kvt i nafohka ya achifa cha pashi moma ka sháfikmvt oka ya ilachefa cha kashofa hi oke; atuk osh yohmi ha yosh binah ha onashke; micha nitak untuklo. ho binah ha kocha ya ahantashke.

9. Yohmi kia nitak ont isht untuklokma noshkobo pashi ya mominchit micha nutakhish, micha im osana aiena mominchit shâfashke; peh i pashi mominchit shâfashke; michă i nafohka ya achifashke; micha nipi ya oka isht achifa cha kashofa hi oke.

10. Micha nitak isht untuchinakma chukfushi nakni tuklo aiokpulo kvt iksho, micha chukfushi tek afvmmi ummona achufa hosh aiokpuloka iksho, micha hukmit im issa mak o bota tohbi lipehbi vlhpisa ishit pokoli tuchina hosh bila aiyoma ho micha bila ampushi achufa aiena ho isha hi oke. 
11. Mikma kashoffa chin tuk naholitompa isht vtta yut hatak kashofa chi kash, micha nana yumma puta kash Chihowah itikba okla moma im vlhtipo okhissa ya isht onashke.

12. Micha na holitompa isht vtta yut chukfushi nakni achvfa ka ishi cha na yoshobat im issa ya im issa chị, micha bila ampushi achưfa ka eshi cha fahfulit im issa mak o Chihowah itikba ya fahfulashke.

13. Micha chukfushi yash nan ashvchi im issa micha hukmit im issa aivba he aholitopa ak o ai vba hi oke; nan ashuchi im issa yot naholitompa isht vtta immi hatuk a yummak inli chohmit nayoshobat im issa yak kia immi hoke ; holitopa $\underline{\mathrm{i}}$ shahli hoke.

14. Micha naholitompa isht vtta yut vbanublit im issa issish a eshi cha naholitompa isht vtta yut kashofa chi kash haksobish isht impakimma wishakchi, micha ibbak isht impakimma ibbak ishki, micha iyi isht impakimma iyishki aiena ka lapalicha hi oke.

15. Micha naholitompa isht vtta yvt bila ampushi achvfa kash kanohmi ka eshi cha tosholit ilap ibbak vlhfubekimma ibbak puta ya ona hi oke.

16. Micha naholitompa isht vtta yut ibbak ushi isht impakimma ya bila yut ibbak vlhfubekimma alhto kash okvchi cha bila yash Chihowah itikba ya untuklo ha ibbak ushi ishit fimma hi okc.

17. Micha bila yut ibbak alhto moma tuk a naholitompa isht otta yot kashofa chi kash isht impakimma haksobish wishakchi, micha ibbak isht impakimmalibbak ishki, micha yyi isht impakimma iyishki aiena ka yoshobut im issa issish alapanli ka onocha hi oke.

18. Micha bila yash atampa kut naholitompa isht vtta ibbak alhto ka kashofa chi kash noshkobo ha on hlatubla hi oke, micha naholitompa isht vtta yut Chihowah itikba im a kashoffa hi oke.

19. Micha naholitompa isht vtta yvt nan ashvchi im issa im issa cha ik kashofo tuk vt kashofa chin tuk ash i kashofa hi oke, micha $\underline{i}_{\text {himmak }}$ h hukmit im issa ya vba hi oke. 
20. Micha naholitompa isht vtta yvt hukmit im issa micha ilhpak im issa ya alta ya onochit im issa hi oke, micha naholitompa isht vtta yut i kashoffikma i kashofa hi oke.

21. Mikma hatak ilbusha yatuk osh yohmi ka ahayucha he keyukmvt vbanvblit im issa mak o chukfushi achvfa, micha ilhpak im issa mak $\mathrm{o}$ bota tohbi lipehbi kushkoa ishit pokoli achvfa bila aiyoma, micha bila ampushi achvfa aiena fahfula chi, micha isht im atobba chi $\mathrm{ka}$ isha hi oke.

22. Micha puchi yoshoba tuklo, keyukmvt puchushi tuklo ahayucha hinla kvt achvfa kvt; nanashvchi im issa, micha achvfa kut hukmit im issa ya chi aiena hi oke.

23. Micha nitak isht untuchinakma i kashofa chi hosh Chihowah itikba okla moma im vlhtipo okhissa ya isht onvt naholitompa isht vtta ya isht im ona hi oke.

24. Mikma naholitompa isht vtta yut chukfushi vbanvblit im issa yash, micha bila ampushi achufa kash eshi cha Chihowah itikba fahfulit im issa mak o fahfula hi oke.

25. Micha vbanublit im. issa chukfushi yash vbi cha naholitompa isht vtta yvt vbanvblit im issa yash issish kaniohmi ka eshi cha kashofa chi kash haksobish isht impakimma wishakchi, micha ibbak isht impakimma ibbak ishki, micha iyi isht impakimma iyishki aiena ka lapalechashke.

26. Micha bila yash kaniohmi ka naholitompa isht vtta yut tosholit ilap vlhfubekimma ibbak puta ya ona hi oke.

27. Micha naholitompa isht vtta yut ibbak vlhfubekimma bila allito kash kaniohmi ka ibbak ushi isht impakimma ya untuklo ha Chihowah itikba isht fimma hi oke.

28. Mikmot naholitompa isht vtta yot bila ibbak alhto tuk vt kaniohmi ka kashofa chin tuk ash haksobish isht impakimma wishakchi, micha ibbak isht impakimma ibbakushi, micha iyi isht impakimma jyishki aiena, vbanvblit im issa issish alapanli ka lapalecha hi oke.

29. Micha bila yvt ibbak a alhto moma tuk a Chihowah itikba im a kashoffa chi hosh i kashofa chi kash nashkobo ha on hlatubla hi oke.

30. Micha pvchi yoshoba yash, keyukmot puchushi yash ahayucha hinla kvt achufa $\mathrm{ka}$ im issa hi oke. 
31. Ahayucha hinla tuk ash $\underline{o}$ achvfa ka nanashvchi im issa mak o micha achvfa ka hukmit im issa mak o ilhpak im issa aieninchit im issa hi oke. Micha naholitompa isht vtta yot i kashoffa chin tuk a Chihowah itikba ya $\underline{\underline{i}}$ kashoffa hi oke.

32. Iluppak osh ililli leplosi onvtonla isht kashofa he ya ibbak vt ahayucha he keyu ya $\underline{\text { i }}$ nan vlhpisa hoke, im achi tok.

33. Mihma Chihowah yut Moses micha Alon a im anumpulit,

34. Kenan vt hvchi yakni ya chi ho hvchima li ka hush onakma, atuk o hưchị yakni ya chuka yo ililli leplosi ya onochi likma,

35. Chuka yash immi hut naholitopa isht vtta ya ont im anolit, chuka ya ililli hut onvtonla, vm ahobushke, achikma, 36. Yohmikma naholitompa isht vtta yut ililli pisauchi kvt aboha ya ik chukowo kisha, anukaka asha moma ka toshoht tahla chị ka $\underline{i}$ miha hi oke; yumohmi ho aboha asha tuk moma kvt ik kashofo ik tobo kashke; atuk $\underline{\underline{i}} \underline{\mathrm{i}}$ himmak a ya naholitompa isht vtta yvt aboha ya pisa chi hosh chukowa hi oke.

37. Micha ililli ha pisa hi oke; mikma yakeh! ililli hot aboha itabana ya kolukbit okchamali chohmit, keyukmüt hamma chohmit buskvehi hosh pisa ka kolukbi kut itabana ya $\underline{\text { i shahlikma, }}$

38. Yohmikma naholitopa isht vtta yvt aboha ya kohchut aboha okhissa ya ona chá nitak untuklo ho okhishtut hilecha hi oke.

39. Atuk osh nitak isht untuklokma naholitompa isht vtta yut vla cha, anonti pisa hi oke; mikma yakeh! ililli hvt aboha itabana ya chitot isht iakma,

40. Yohmikma naholitompa isht vtta yut ililli onvtonla tuli

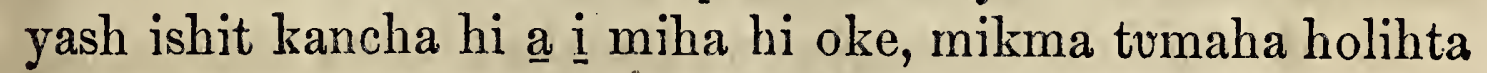
kohcha ik a kashofo yo ont kancha hi oke.

41. Micha aboha anukkaka fullota ka shafichi cha ná pushi shafa kash tumaha holihta kohcha ik a kashofo yo ont hlatubla hi oke. 
42. Micha tuli inla eshi cha, tuli yummak ash atobbichit bohla hi oke; micha yưmmuska jnla yo eșhi cha aboha ya isht apolusla hi oke.

43. Atuk o ililli hut anonti vla cha, tvli ya ishit kanchi ha yạ, micha aboha ya shạf ha ya micha aboha yut vlhpolosa ha ya aboha ya kohchakma,

44. Yohmikma naholitompa isht vtta yvt ona cha, pisa hi oke; mikma yakeh! ililli hut aboha ya chitot isht iakmvt, leplosi chilita yosh aboha ya onvtola, ik kashofo hoke.

45. Micha aboha yash tuli, micha iti, micha aboha in lukfi yummuska moma aiena ka tiubla hi oke, micha tumaha holihta ya kohcha ik akashofo yo isht ona hi oke.

46. Mikma kuna hosh okhishtut hikia moma ho chukowa tuk mot ik kashofo ho ont opia hi oke.

47. Mikma kuna hosh aboha yash itola tukmut i nafohka ya achifa hi oke; mikma kuna hosh aboha yash ai impa tukmut i nafohka ya achifa hi oke.

48. Mikma naholitopa isht vtta yvt ont chukowa cha pisa ma, yakeh! aboha yut vlhpolusa ha ya illilli hut chitot isht ik aiyokma, naholitompa isht vtta yut aboha yut kashofah, achashke, ililli hut hlakofi hoka.

49. Micha aboha ya kashofa chi kvt hushi tuklo, micha chuahla iti, micha tishepa, micha hissop aiena ho isha hi oke.

50. Micha hushi yash achufa ka lukfi tunna fohkit oka kapussa paknaka yo uba hi oke.

51. Mikmvt chuahla iti, micha hissop, micha tishepa micha hushi okchaya yash aiena ka eshi cha hushi illi yash issish, micha oka kapussa yash okvchi cha untuklo ha aboha ya o fimma hi oke.

52. Micha aboha ya hushi issish, micha oka kapussa, micha hushi okchaya, micha chuahla ịti, micha hissop, micha tishepa aiena ho ishit kashoffa hi oke.

53. Amba hushi okchaya vno tumaha holihta kohcha oktak a shahbi yo isht onvt hikachi cha aboha ya $\underline{\mathrm{i}}$ kashoffa hi oke, mikma kashofa hi oke.

54. Ilvppak osh ililli ilaiyukali leplosi, micha hlachowa a puta. 
55. Micha nafohka, micha aboha in leplosi, 56. Micha shatali, micha hlachowa, micha shohkaualit talaia aiena kvt i nanvlhpisa hoke.

57. Ik kashofokma, micha kashofakma im vlbuchi yoke. Iluppak osh leplosi nan vlhpisa hoke, im achi tok.

\section{CHAPTA XV.}

\section{ICHA Chihowah yut Moses micha Alon a im anum- 1 pulit,}

2. Islael im vlla vhleha im anumpvlit Hatak nana hosh nipi a yanvlli ya ishikmvt i yanulli ak atuk mak o ik kashofo hoke.

3. Micha im a yanulli ka iluppak osh ik im a kashofo ya hi oke; im a yanvili vt nipi ya a yanvlli hokma, keyukmut im a yanvili ut nipi ya issa hokma nana kia ik im a kashofo yoke.

4. Yanvlli ya ishi yosh topa ai onvtola chatuk puta kvt ik kashofo hoke; micha nana ho om binili tuk moma kut ik kashofo ya hi oke.

5. Mikma kuna hosh in topa ha halelikmut $\underline{\mathrm{i}}$ nafohka ya achifa cha oka yupa hi oke; micha ik kashofo ho ont opia hi oke.

6. Mikma yanvlli ishi hosh on asha tuk a kuna hosh on asha tuk mut i nafohka ya achifa cha, oka yupa hi oke, micha ik kashofo ho ont opia hi oke.

7. Micha yanulli isshi ho kuna hosh nipi ya potoli tukkmut i nafohka ya achifa cha, oka yupa hi oke: micha ik kashofo ho ont opia hi oke.

8. Micha yanulli ishi yosh kashofa yo ontofa, yohmikma i nafohka ya achifa cha, oka yupa hi oke; micha ik kashofo ho ont opia hi oke.

9. Micha yanvlli yo ishi yosh issuba om patulhpo nana ho om binilikma ik kashofo ka hi oke.

10. Micha nana ho im patali tuk o kuna hosh halelikmut ik kashofo họ ont opia hi oke; atuk o kuna hosh nana 
yumma kaniohmi ka sholi tuk mut i nafohka ya achifa cha, oka yupa hi oke; micha ik kashofo ho ont opia hi oke.

11. Micha yanvlli ishi hosh kvna ho potoli tuk ma (micha ibbak a oka isht ik achifo tukmvt) $\underline{\mathbf{i}}$ nafohka ya achifa cha, oka yupa hi oke; micha ik kashofo ho ont opia hi oke.

12. Micha yanvlli ishi hosh lukfi tưnna nan aivlhto yo potoli tukma koa hi oke, micha iti nan aivlhto moma kvt oka isht achifa hi oke.

13. Atuk osh yanulli ishi tuk osh $\underline{i}$ yanvlli vt $\underline{i}$ kashofakmot, kashofa chị kvt nitak untuklo ho im ile hotehnvt $\underline{i}$ nafohka ya achifa cha, oka yanvlli yo yupa hi oke, micha kashofa hi oke.

14. Micha nitak ont isht untuchinakma puchi yoshoba tuklo, keyukmot puchushi tuklo ho eshi cha, Chihowah itikba onvt okla moma im vithtipo okhissa ya ona cha naholitompa isht vtta ya ima hi oke.

15. Mikma naholitompa isht vtta yvta chufa ka nan ashvchi im issa mak $\underline{0}$ micha achufa ka hukmit im issa mak $\underline{0}$ im issa hi oke; micha naholitompa isht vtta yut i yanulli ya Chihowah itikba im ai i kashofa hi oke.

16. Itapotakma isht ai unchuloli vt hatak nana hosh kohchakmvt nipi moma ka oka isht achifa cha ik kashofo ho ont opia hi oke.

17. Micha nafohka puta micha na hakshup puta ka itapotakma isht ai unchuloli hvt on asha kvt oka isht achifa cha, ik kashofo ho ont opia hi oke.

18. Ohoyo ak kia yummak oka hatak vt itapotakma isht ai unchuloli hut on asha hosh iba tushkikma ita tuklot oka yupa hi oke, micha ik kashofo ho ont opia hi oke.

19. Micha ohoyo hosh yanvlli eshi cha haknip a yanvlli vt issish okma, nitak untuklo ila filvmmicha hi oke, mikma kuna hosh halelikmut ik kashofo ho ont opia hi oke.

20. Mikmä ila filummit ahanta hosh nana onvtola tuk moma kvt ik kashofo ka hi oke, micha nana on asha tuk moma kvt ik káshofo ka hi oke.

21. Mikma kuna hosh in topa ha halelikmut i nafohka ya 
achifa cha, oka yupa hi oke, micha ik kashofo ho ont opia hi oke.

22. Mikma kuna hosh nana ai on asha tnk a halelikmut i nafohka ya achifa chs, oka yupa hi oke; micha ik kashofo ho ont opia hi oke.

23. Micha in topa hạ, keyukmut nana ho on asha tuk halelikmut ik kashofo ho ont opia hi oke.

24. Micha hatak nana hosh kaniohmit iba tushki tuk osh issish vt on ashakmut nitak untuklo ho ik kashofo ka hi oke; micha topa ai itola tuk moma kut ik kashofo ka hi oke.

25. Micha ohoyo hut issish $\underset{1}{i}$ yanulli kvt ila filvmmit ahanta nitak a nitak laua hosh atapakma, keyukmut yanvlli kut ila filvmmit ahanta nitak a ont iakma, ik im a kashofo yanulli nitak moma kut ila filummi nitak ak inli ho chohmashke; ik kashofo ka hi oke.

26. I yanulli nitak moma hosh topa moma ai onvtola yot ila filummi topa yak o in chohma hi oke, micha nana ho on asha tukmut ik kashofo kvt ila filvmmit ahanta kvt ik kashofo yatuk ak o chohma hi oke.

27. Atuk o kuna hosh nana yumma puta ka halelikmut ik kashofo ka hi oke; micha $\underline{i}$ nafohka ya achifa cha oka yupa hi oke, micha ik kashofo ho ont opia bi oke.

28. Amba $\underline{i}$ yanvlli tuk vt $\underline{i}$ kashofakma nitak untuklo ho il im ahotehna hi oke; atuk i himmak a yo kashofa hi oke. 29. Atuk osh nitak ont isht untuchinakma pvchiyoshoba tuklo, keyukmut puchushi tuklo eshi cha naholitompa isht vtta okla moma im vlhtipo okhissa yak $\underline{o}$ isht im ona hi oke.

30. Mikma naholitompa isht vtta yut achufa ka nanashvchi im issa mak $\underline{o}$, micha achvfa ka hukmit im issa mak o im issa hi oke ; micha naholitompa isht vtta yut Chihowah itikba ik i kashofo yanvlli im a kashofa hi oke.

31. Yakohmichi hosh Islael im vlla vhleha ha ik im a kashofo ya hush $\underline{\mathrm{i}}$ filummicha hi oke; yumohmi ho vm vlhtipo yut im ai itin takla hikia ka litehli cha ik kashofot ik illo ka hi oke. 
32. Iluppak osh yanvlli ya ishi, micha isht ai unchuloli hut kohcha tuk mut ishit liteha tuk.

33. Micha ohoyo hvt hullo isht abeka, mikma hatak vt micha ohoyo hut yanvlli ishi, micha ik kashofo yo iba tvshki tuk aiena ka 1 nan vlhpisa hoke, hvsh im achashke, im achi tok.

\section{CHAPTA XVI.}

MICHA Alon ushi tuklo hosh Chihowah itikba nan im 1 issa cha illi, illi ha ya , Chihowah yut Moses a im anumpuli tok.

2. Micha Chihowah yvt Moses a, Chitibapishi Alon a ish im anumpulashke. Yvmohmi ho aholitopa natakali anukaka aiombinilit i nukhakklo itikba, yummut itombi holitopa ontalaia ka nitak moma ik ono kashke. Yvmohmi hosh ik illo kashke; hoshonti anukaka yo ai ombinilit i nukhạklo paknaka ahaiaka la chi hoke.

3. Yakohmi hosh Alon vt aholitopa ka ya ona hi oke; nan ashuchi im issa mak $\underline{o}$, wak hobvk himita, micha hukmit im issa mak o, chukfi nakni jesha hi oke.

4. Linen nafohka holitopa foyuka cha, obalafoka linen vt nipi alataiya hi oke, micha linen vskofvchi ya vskofvchikmvt, linen iahlipa ha ishit shema hi oke; iluppa puta kvt nafohka holitopa hoke. Yvmohmi hoka nipi ya oka isht achifa cha ishit fohka hi oke.

5. Micha nan ashuchi im issa mak o issikosoma ya issikosomushi tuklo ho aiishi, micha hukmit im issa mak $\underline{0}$, chukfi nakni achufa ho Islael im vlla vhleha okla moma ka im esha hi oke.

6. Mikmut nanashvchi im issa mak o Alon vt $\underline{i}$ wak hobuk o yummut ilap akinli mak o im issa cha, ilap, micha in chuka achvfa ya $\underline{i}$ kashoffa hi oke.

7. Micha issikosoma tuklo kash eshi cha, Chihowah itikba isht onvt okla moma im vlhtipo okhissa ya isht ona hi oke.

8. Micha Alon vt issikosoma tuklo ho nashoeli onocha 
hi oke; nashocli achvfa kut Chihowah ak o immi hokma, nashoeli achufa kut issikosoma okchaya immi ha hi oke.

9. Micha Alon vt issikosoma Chihowah $\underline{\mathrm{i}}$ nashoeli vt onvtola kásh isht onvt nanashvchi im issa mak $\underline{\text { im issa }}$ hi .oke.

10. Amba issikosoma okchaya ya chi mak o nashoeli vt onvtola tuk a isht i kashofa chi hosh, micha issikosoma okchaya chi mak o yakni haiaka chufficha chi kash okchaya ho Chihowah itikba isht ona hi oke.

11. Micha Alon vt nanashvchi im issa wak hobak ash yommut ilap atuk mak $\underline{Q}$ isht ona cha, ilap micha in chuka achvfa ya $\underline{i}$ kashoffa hi oke, micha ilap akinli nanashvchi im issa mak o wak hobak ash vba hi oke.

12. Micha alta yut Chihowah itikba hikia ka tobaksi ai eshi chá, nabalama aivlhto ya alotoli cha, bout lapushki yo nabalama ya ibbak alotcli cha, natakali anukka ya isht onashke.

13. Micha na balama ya Chihowah itikba luak ai onocha hi oke; yumohmi ho shobulli hoshonti kvt ai ombinilit i nukhaklo yummut isht atokowa ontalaia ka ompoholma hi oke; yomohmi ho ik illo ka hi oke.

14. Micha wak hobvk ash im issish a ai ombinilit $\underline{\underline{i}}$ nukhakklo hushi akochaka pilla ka ibbak ushi isht $\varrho$ fimma hi oke; micha ai ombinilit $\underline{i}$ nukhaklo itikba ya issish ash untuklo ha ibbak ushi isht fimma hi oke.

15. Yohmikmut nanashvchi im issa issikosoma yummut okla hatuk mak o vbi cha, im issish a natakali anukaka isht onashke; micha issish yummakma wak hobvk im issish ishit yumihchi tuk vt chohmicha hi oke, micha ai ombinilit i nukhakklo paknaka o fimmit micha ai ombinilit i nukhakklo itikba fimma hi oke.

16. Micha Islael im vlla vhleha hut ik kashofo kak o micha nanashvchi moma ka yumihchi tuk ak o aholitopa ya $\underline{\mathrm{i}}$ kashoffa hi $\mathrm{i}_{\mathrm{o}}$ oke; micha ik a kashofo im itin takla ya okla moma im vlhtipo yut hikia tuk a yumihcha hi oke.

17. Micha okla moma im vlhtipo aholitopa ya kashofa chi hosh chukowakmut, ilap micha in chuka achvfa, micha Is- 
lael okla moma aiena ka kashoffi cha, ik kohcho kịsha kạ, hatak nana kia iksho ka hi oke.

18. Micha kohchut Chihowah itikba alta hikia ka ona cha, yumma kashoffa hi oke; micha wak hobvk issish micha issikosoma issish a ishi cha, alta lapish a lapalichit fullolecha hi oke.

19. Micha issish a untuklo ha ibbak ushi isht o fimmi cha, Islael im vlla vhleha ik a kashofo yatuk a kashoffit hullocha hi oke.

20. Atuk osh aholitopa, micha okla moma im vlhtipo, micha alta aiena ka kashoffit tahlikmvt issikosoma okchaya yash isht ona hi oke.

21. Micha Alon vt ibbak a ita tuklonchit issikosoma okchaya noshkobo ha isht umbitepa cha, Islael in vlla vhleha ik aivlhpieso, micha nanashvchi moma aiyoshciba chatuk moma ka anolit issikosoma noshkobo onochi cha, hatak ai vlhpesa ho ibbak fohkit yakni haiaka pit pila hi oke.

22. Mikma issikosoma yot ai vlhpesa keyu moma shaiyalit yakni okla a binili keyu isht ona hi oke; mikma issikosoma ya yakni haiaka ya ont chufficha hi oke.

23. Mikma Alon vt okla moma im vlhtipo ya ont chukowa cha, aholitopa chukowa chikmvt linen nafohka ishit fohka tuk vt shufi cha yvmmak inli ho bohla hi oke.

24. Micha nipi ya aholitopa ya oka isht ai achifa mvt, $\underline{\mathbf{i}}$ nafohka ishit fohka cha, ilap hukmit im issa, micha okla hukmit im issa aiena ka im issa cha, ilap, micha okla aiena ka i kashoffa hi oke.

25. Micha nan ashvchi im issa nia ya alta onochit a hukma hi oke.

26. Mikma issikosoma okchaya mak $\underline{\text { issikosoma chuffi- }}$ chi tuk vt i nafohka ya achifa cha oka yupi ha yosh binah ha ont chukowa hi oke.

27. Micha nanashvchi im issa mak o wak hobvk micha nanashuchi im issa mak o issikosoma, yvmma im issish $\underline{0}$ aholitopa ya kashoffa chi hosh isht chukowa kash, achofut binah ha kohcha isht ona hi oke; micha hakshup, micha nipi, micha i yulhki aiena ka luak itvchit hukma hi oke. 
28. Micha hukmi tuk vt i nafohka ya achefakmut oka yupi ha yosh binah ha ont chukowa hi oke.

29. Micha iluppak osh hvehi nan vlhpisa bilia ya chi hoke. Hushi isht untuklo, hushi talali tuk nitak ont ishit pokolikma, hvchi yakni ai achufa hokma, keyukmut okla inla yosh hvchi takla anta tuk okma, nana kia aienvt huchi shilombish a hush ilbushali cha natoksuli nana kia hush yumihcha he keyu hoke.

30. Nitak yummak o naholitompa isht vtta yut Chihowah itikba ya na hush aiashvchi moma a hvchi kasoffi na hvchi kashofa chị ho hrchi kashoffa hi oke.

31. Nitak hollo afoha hvchi nitak a hi oke: mikma nan vlhpisa bilia ya hvchi shilombish a huvsh ilbushala hi oke.

32. Micha naholitompa isht vtta yut naholitompa isht ai vtta ya iki ya vlhtobvt isht utta chị ho nana ahummi tuk, micha hullochi mihi yosh i kashoffa hi oke, micha linen nafohka, nafohka holitopa fehna ka foyuhka hi oke.

33. Micha aboha holitopa ya $\underline{i}$ kashoffa hi oke, micha okla moma im vlhtipo, micha alta aiena ka $\underline{i}$ kashoffa hi oke; micha naholitompa isht asha ya , micha okla moma ka i kashoffa hi oke.

34. Micha iluppak osh afummikma himmona ha Islael im vlla vhleha nanashvchi moma ka $\underline{\underline{i}}$ kashofa he vt hvchi nan vlhpisa bilia ya chị hoke, ish im achashke, achi tok. Mihma Chibowah yot Moses a ai i miha tuk mak o yumihchi tok.

\section{CHAPTA XVII.}

ICHA Chihowah yot Moses a im anumpulit, 17 2. Alon, micha ushi vhleha, micha Islael im vila vhleha moma aiena im anumpulit, Nana iluppak o Chihowah yot anumpulit,

3. Hatak kvna hosh Islael in chuka achvfa ya ai achvfa yosh wak hobvk, keyukmut chukfushi, keyukmvt issikosoma ya binah ha ai vbi, keyukmvt binah ha kocha ai vbi.

4. Atuk osh okla moma im vlhtipo okhissa isht onvt Chi- 
howah im issa mak o Chihowah im vlhtipo itikba ya ont ik in ai issokmvt hatak yvmma issish vt onvtola hi oke; issish hlatubli tuk oke, micha hatak yummut im okla im ai itin takla ya fiopa trpa hi oke.

5. Yvmohmi ho Islael im vlla vhleha hưt vbit im issa ya oktak ashahbi ai im issa Chihowah ya isht im onvt okla moma im vlhtipo okhissa ya isht onvt, naholitompa isht vtta ya isht im ona cha itin nanaiyvchi im issa mak o Chihowah im issa hi oke.

6. Mikma na holitompa isht vtta yvt issish a Chihowah im alta okla moma im vlhtipo okhissa hikia ka o fimma hi oke, micha Chihowah $\underline{i}$ balama achukma mak o nia ya hukma hi oke.

7. Micha himak pilla ma nan isht ahullo okpulo puta, yummak o haui tobvt iakaiya tuk vt vbit im issa he keyu hoke : Iluppak osh im ai itishali hlopulli ka i nan vlhpisa bilia ya chi hoke.

8. Mikma Islael in chuka achvfa ya , keyukmut okla inla yosh hvchi takla ahanta yosh hatak nana hosh hukmit im issa; keyukmvt vbit im issa yo im issa,

9. Micha Chihowah im issa chi kut okla moma im vlhtipo okhissa ya isht ik onokmvt, hatak yvmmut im okla im ai itin takla ya fiopa tvpa hi oke.

10. Micha hatak nana hosh Islael in chuka achufa ya ai achufa hokma, keyukmvt okla inla yosh hvchim aitin takla ahanta hokma, nana hosh issish kaniohmi nana kia upakma; issish vpa tuk hatak shilombish yumma sa nashuka ya isht a sanalichi la chị, micha im okla im ai itin takla ya fiopa tubli la hi oke.

11. Nipi aiokchaya yut issish ak oka; atuk o hvchimi shilombish isht a kashofa chị ho alta onochit hvch ima li tuk oke, issish ak okvt hatak shilombish a i kashoffi hoka.

12. Yvmohmi hatuk o Islael im vlla vhleha ha Hatak hush achvfa kia, issish a hushpa he keyu, michẩ okla inla yush huchitin takla ahanta kvt issish vpa he keyu, achi li tuk oke.

13. Micha hatak kuna hosh Islael in chuka achvfa ya 
ai achufa yosh, keyukmut okla inla hvchitin takla ahanta yosh owvtta tuk osh nampoa, keyukmut vba hushi kia vpa hinla vlhpesa ka ishikmut, issish a aka hlatubli cha, lukfi pushi isht ompohoma hi oke.

14. Na nipi moma isht ai okchaya yoka issish ak osh yumma isht ai okchaya yoke: Yvmohmi hatuk o Islael im vlla vhleha hă, Nipi kaniohmi nana kia issish a hvshpa he keyushke, achi li tnk oke. Na nipi moma isht ai okchaya yvt issish ak oka, kvna hosh vpakmot fiopa tupa hi oke.

15. Micha hatak nana hosh (hvchi yakni aiachvfa yokmá, keyukmut okla inla yokma nana hosh) ilap akinli hosh illi, keyukmut nana hosh upi tuk o vpa tukmvt $\underline{\mathrm{i}}$ nafohka ya achifa mot, oka yupi cha ik kashofo ho ont opia hi oke: yohmi hosh kashofa hi oke.

16. Amba ik achifo, micha ik yupokmvt nanashvchi vt onvtoyula hi oke, ish im achashke, achi tok.

\section{CHAPTA XVIII.}

M

ICHA Chihowah yut Moses a im anumpulit, osh Chitokaka huchin Chihowah sia hoke, ish im achashke.

3. Echip yakni aiasha, yumma hush aiasha tuk, yummut nana a kaniohmi ya hobachit hush yvmihcha he keyushke; micha Kenan yakni huch isht ona la chi yumma asha kvt nana yưmohmi ya hobachit hush yưmohma he keyu hoke, micha $\underline{i}$ nan vlhpisa ka im antivt hush nohowa he keyu.

4. V'm aivlhpesa micha nan isht apesali, puta ka hush yumihchi cha, a nan vlhpisa ha holitoblit hush foyuka hi oke; Chitokaka hvchin Chihowah sia hoke.

5. Yumohmi hoka a nanvlhpisa, micha a nanvlhpisa aivlhpisa ak o hush yumihcha hi oke; yummak o hatak vt yvmihchi hokmak osh aiokchaya hi oke: Chihowah sia hoke.

6. Hưsh achufa kia i kanohmi hạ, nipi bano ho i wakumma chi hosh bilika hvch ik ono kashke; Chihowah sia hoke. 
7. Chịki nipi bano ya , keyukmut chishki nipi bano ya chiki wakummo kashke; chishki yoka, nipi bano ya ish $\underline{\underline{i}}$ wakumma he keyu hoke.

8. Chiki tekchi nipi bano ya ishi wakumma he, keyu hoke; chiki nipi bano yoke.

9. Chin tek chịki ya ushetik, keyukmot chishki ya ushetik, chuka ak inli ho keyukmvt yakni inla ai itola tok okma nana kia nipi bano ya ish $\underline{\mathrm{i}}$ wakumma be keyu holke.

10. Chiso ha ushetik, keyukmut chiso tek a ushetik nipi bano ya ish i wakumma he keyu hoke: yummak osh chi nipi bano ash inli hoka.

11. Chiki tekchi ushetik, chiki vt tobachi tuk (chin tek oka) nipi bano ya ishi wakvmma he keyu hoke.

12. Chiki in tek nipi bano ya ishi walumma he keyu hoke. Yvmmut chiki ya i kanohmi fehna ohoyo hoke.

13. Chishki itibapishi nipi bano ya ishi wakumma he keyu, chịshki ya $\underline{\mathrm{i}}$ kanohmi fehna ohoyo hoka.

14. Chiki itibapishi ha nipi bano ya ishi wakumma he keyutekchi ya ish apota he keyu: chi hukni yoka.

15. Chipok nipi bano ya ishi wakumma he keyu, chiso ha tekchi yoke; nipi bano ya ishi wakumma he keyu hoke.

16. Chitibapishi tekchi nipi bano ya ishi wakvmma he keyu, chitibapishi nipi bano yoke.

17. Ohoyo ha micha ushetik a nipi bano ya ishi wakvmma he keyu; michá ushi ya ushetik, keyukmot ushetik a ushetik a ishit nipi bano ya ish $\underline{\underline{i}}$ wakumma he keyu, $\underline{\mathrm{i}}$ kanomi fehna ohoyo hoka: na haksi yoke.

18. Micha itibapishi hvt okchaya moma ka ohoyo ishit itaienvchit nipi bano ya wakummit chukvsh a ish okpuna he keyu hoke.

19. Micha ohoyo hut ik kashofo ho naksika binilechi tuk a nipi bano ya $\underline{\mathrm{i}}$ wakumma chi hosh bilika ish ona he keyu hoke.

20. Micha chin chuka a bilika hatak tekchi ya apotut isht ish ile litehla he keyu hoke.

21. Micha chim isht atiaka ya luak hlopullichit Molek a 
ish im onacha he keyu; micha chin Chitokaka hohchifo ha chik ahobalecho kashke; Chihowah sia hoke.

22. Hatak bika ya ohoyo chohmichit chik iba trshko kashke; nan isht yuwala yoke.

23. Micha nan vlhpoa nana kia iba tushkit isht ish ile litehla he keyu; micha ohoyo kuna kia nan vlhpoa ya tushka chi hosh itikba hikia he keya hoke; itaiyokoma hoke.

24. Nana iluppa puta nana kia isht huchik ile litehla kashke; iluppa puta kak o oklushi puta huchi tikba akocha wehli li tuk vt isht ile litehli tuk oka.

25. Mikma yakni vt liteha hoke; yumohmi kak o nan ashuchi tuk ak o im aya hi oke; miluma yakni oh kia okla hạ hoetut kohchi hoke.

26. Yumohmi hoka, a nanvlhpisa puta, micha nan vlhpesa apihissali puta ka hưsh yumihchi cha nan isht yuwala iluppa kanima kia huchik yumihcho kashke; hưchim ai olkla akinli, keyukmut okla inla yosh hvchi takla ahanta kanima kia huchik yumihcho kashke.

27. Nan isht yuwala iluppa moma kak o yakni ya hatak vhleha hvchi tikba aiasha tuk vt yumihchi ho yakni vt liteha tuk oke.

28. Yvmohmi ho oklushi puta hvchi tikba aiasha tuk a hoetut kohchi tuk vt yakni vt huchishno ak kia hoctvt ik hvchi kohcho kashke.

29. Nan isht yuwala iluppa puta kanima ka kuna hosh yumihchikmvt yumma yumihchi tuk shilombish vt im okla im ai itin takla ya akohchvt fiopa tupa hi oke.

30. Yumohmi hoka a nanulhpisa ya hush holitobli hosh nan isht yuwala puta hvchi tikba aiasha tuk vt yumihchi tok achufa nana kia huchik yumihcho kashke, micha yummak o isht hvchik ile litehlo kashke: Chitokaka hvchin . Chihowah sia hoke, ish im achashke, im achi tok. 


\section{CHAPTA XIX.}

ICHA Chihowah yut Moses a im anumpulit,
2. Islael im vlla vhleha okla moma ka im anumpulit, Nan ashuchi ik huchim iksho kashke: Chitokaka hvchin Chihowah sia hut nanashuchi ik sum iksho hoka.

3. Hatak hvsh puyuta kvt hvchishki, micha hvchiki aiena ka hvsh $\underline{\mathrm{i}}$ nukwia cha, a nitak hollo ya hvsh holitoblashke : Chitokaka hvchin Chihowah sia hoke.

- 4. Fullotut na holbut toba ya huchik im ono kashke; micha bilut akmot na holbut toba huch ik ikbo kashke: Chitokaka hvchin Chihowah sia hoke.

5. Micha vbit in issa ya itin nanaiyuchi im issa yo Chitokaka hush im issakmut, huchishno hvchim anukfila hosh hush im issa hi oke.

6. Hush im issa i nitak akinli, micha im onna aiena kak o hushpa hi oke; atuk o kaniohmi kut nitak ont atuchina ka ashakmvt luak ishit hukma hi oke.

7. Micha nitak ont atuchina ka kaniohmi ka vpakma nan isht yuwala yoke; aiokpvecha he keyu hoke.

8. Yumohmi hoka kuna hosh vpakmvt nanashvchi tuk vt onvtonla hi oke; Chihowah i nahullochi ya ik ahobalo hoka; micha shilombish yummut. im okla im aiitin takla akohchut fiopa tupa hi oke.

9. Micha hvchi yakni nana awaya hushmokmut, hvchim osapa chukbi puta ka vmohmichit hushma he keyu hoke; micha hush ai vmo yot vlbullit hush ai vma he keyu hoke.

10. Pakupi hvchim ahollokchi a hush vlbulla he keyu; micha pakvpi hvchim ahollokchi yash pạki ya mominchit hush ai vma he keyu hoke; ilbusha micha okla inla aiena ka hush im ashacha hi oke; Chitokaka hvchin Chihowah sia hoke.

11. Hush hukopa he keyu, micha hush haksicha he keyu, micha hush iti holaba he keyu hoke.

12. Micha a hli keyu ka sa hochifot anumpa kvllo hush 
anumpula he keyu; micha chin Chitokaka hohchifo ha ik ahobalot chik hochifo kashke: Chihowah sia hoke.

13. Chin chuka bilika hatak a ish haksicha he keyu; micha ish wehpula he keyu; hatak ilhtono vt asitubi tuk vt chin tonla na ik onno kashke.

14. Ik haponaklo ya anumpa okpulo isht chik mihacho kashkc, micha niskin lupa ya isht ibetubli itikba chik bohlo hosh, amba chin Chihowah ya ish $\underline{\mathrm{i}}$ nukwiyashke: Chitokaka sia hoke.

15. Nanapesa ka ai vlhpesa keyu ya ish yumihcha he keyu; hatak ilbvsha nipi ya ish holitobla he keyu; micha hatak holitopa annoa ya ish holitobla he keyu hoke: amba aivlhpiesa ho chin chuka bilika hatak a ish im apesa hi oke.

16. Chim okla itin takla ya nan anoli yosh chuka abaiyut ish aya he keyu; micha chin chuka bilika hatak im issish a i sanalit ish hikia he keyu hoke: Chitokaka sia hoke.

17. Chitibapishi ha chi chukvush a ish $\underline{i}$ nukkilla he keyu hoke; ik kaniohmi kia chin chuka a bilika hatak a ish im anumpula hi oke, micha nanashvchi vt ik onvtonla; ish ahna. he keyushke.

18. Chim okla vlla vhleha ha hưsh il aiokha, he keyu; micha hush $\underline{i}$ potunna he keyu; amba chin chuka a bilika hatak a hush holitobli kvt chishno akinli ish chohmichashke: Chitokaka sia hoke.

19. A nanulhpisa ha hush holitoblashke. Chi nan ulhpoa ya ilaiyukalichit ish itim ai ikbicha he keyu hoke: Chim osapa ya na nihi itaiyoma yo ish ahokcha he keyu hoke: micha linen micha chukfi hishi itaiyoma nafohka ya ish foyuka he keyu hoke.

20. Micha kuna hosh yuka ohoyo yosh hatak im vlhpoa, micha falumminchit chumpa tok keyu, micha yuka issa ima tok keyu ho iba tushki tuk ma fuma hi oke; yuka issa keyu yatuk oka illit okla he keyu hoke.

21. Mikma vbanubli im issa ya Chihowah isht im onvt, vbanublit im issa mak $\underline{o}$, chukfi nakni yo okla moma im vlhtipo okhissa ya isht ona hi oke

22. Mikma na holitompa isht vtta yut nanashvchi yumihchi 
kash chukfi nakni yash o Chihowah itikba ya isht im ại i kashoffa hi oke; mikma nanashvchi yumihchi tuk vt $\underline{\mathrm{i}}$ ka. shofa hi oke.

23. Atuk o yakni ya hush onakmvt ilhpak achị ho iti puta hish hokchi tukmvt wayakma, hakshup ik tapo hvsh itapesa hi oke: afummi tuchina kak osh hakshup ik tupo yo hvchi chohma hi oke: ai vpa he keyu hoke.

24. Amba afummi ont ai ushta hokma waya moma kvt holitopa ho Chihowah isht holitoblicha hi oke.

25. Atuk osh afummi ont ishit tahlapi kak o waya ka hush ai vpa hi oke: yumohmi ho huchị wahaya hi oke: hvchin Chitokaka Chihowah sia hoke.

26. Nana hoh kia issish aieninchit hushpa he keyu hoke; micha nan isht yushpakummi ya hush yumihcha he keyu, micha nitak hush apestikela he keyu hoke.

27. Huchi noshkobo chukbi pashi ya vmot hvchik apakfoblicho kashke, micha hvchi nutakhish chukbi ya hvchik okpano kashke.

28. Illi ak atuk o huchi nipi ya hush ile bushla he keyu, micha in chuwa ya hush ile yumihcha he keyu hoke: Chihowah sia hoke.

29. Chisotck a haui ikbicha chi hosh chik kancho kashke, yumohmi ho yakni vt haui toba bieka, mikmvt yakni vt nahaksi ho isht alotowa hi oke.

30. A nitak hullo ya hush holitobli cha vm aboha holitopa ya hush holitoblashke: Chihowah sia hoke.

31. Shilombish okpulo ai itim achvfa ya hvchik ahnincho kashke; micha isht ahullo, yumma isht hvchi liteha chi ka hvchik im asilhho kashke: hvchin Chitokaka Chihowah sia hoke.

32. Hatak noshkobo tohbi yo itikba ya ish wakaiyashke; micha hatak sipokni nashuka ya ish holitoblashke; micha chin Chitokaka ya ish i nukwi yashke: Chihowah sia hoke.

33. Micha okla inla yosh hvchi takla hvchi yakni ahanta tuk a apistikelit chukush hush okpuna he keyu hoke.

34. Amba okla inla yosh huchi takla ahanta kvt bvchim ai itin takla ai vtta tuk akinli ho hvchin chohmashke, mikma 
hvch i holitopa kvt hvchishno akinli hvchin chohmashke; okla inla yosh Echip yakni ya hush ai asha tuk oka; hvchin Chitokaka Chihowah sia hoke.

35. Nan apesa, isht vlhpisa, ishit weki, keyukmut ai vlhpisa yoh kia, ik ai vlhpeso nana ho hvsh yumihcha he keyu hoke.

36. Aweki aivlhpesa, micha isht weki aivlhpesa, efah aivlhpesa, micha hin aivlhpesa hosh hvchim asha hi oke. Huchin Chitokaka Chihowah sia hosh Echip yakni ya apehlichit hvchi kohchi li tok oke.

37. Yvmohmi hoka a nanvlhpisa, micha vm aivlhpesa yumihchi ak o ithanut hush yumihchashke Chihowah: sia hoke, ish im achashke, achi tok.

\section{CHAPTA XX.}

ICHA Chihowah yut Moses a im anumpulit,

1 2. Anonti Islael im vlla vhleha ha im anumpulit, Kuna hosh Islael im vlla vhleha hosh, keyukmut okla inla Islael takla vtta yosh im isht atiaka yo Molek a imakmvt illi pulla hi oke, yakni yash okla hvt toli ishit bola hi cke.

3. Mikma hatak yưmma sa nashuka ya isht $\underline{\mathbf{i}}$ sanali lish im okla ai itin takla ya a kohchit ishit kanchi la hi oke; vm aboha holitopa ya litehla chi hosh micha sa hohchifo holitopa ik ahobalo ka chị hosh im isht atiaka yo Molek a ima hoka.

4. Micha yakni ya okla hvt hatak vt im ishtatia ka ya Molek a ima ho pisa kia kaniohmit nishkin luhmi cha ikbo tuk ma,

5. Yumohmikma hatak yumma, micha in chuka achufa ya a, micha Molek itị haua chi hosh hauit iakaiya moma ka, sa nashuka isht $\underline{\mathrm{i}}$ sanali lish, im okla im ai itin takla ya kohchit fiopa tubli la hi oke.

6. Micha shilombish kuna hosh fullotut shilombish okpulo itim aiachufa micha isht a hullo ya iakaiyut hauit iakaiyak- 
ma, shilombish yumma sa nashuka isht $\underline{\underline{i}}$ sanali lish, im okla im ai itin takla ya kohchit fiopa tubli la hi oke.

7. Yvmohmi hoka, hush ile hullochi cha hvchi holitopashke; hvchin Chitokaka Chihowah sia hoke.

- 8. Atuk o a nanvlhpisa ha hush holitobli cha hush yumihchashke. Chihowah sia hosh hvchi hullochi li hoke.

9. Kuna hosh ikki, keyukmut ishki ya kallakshichit anumpulikmvt illi pulia hi oke; îki, keyukmvt ishlii ya kallakshichit anumpuli hoka; yohmi ka im issish vt ilap ak inli ka onvtoyula hi oke.

10. Micha kvna hosh hatak tekchi yo itin lumalka tuk, chuka a bilika hatak tekchi itin lumaka yash itin lumaka hatak, micha itin lumaka ohoyo aiena kvt illi pulla hi oke.

11. Micha hatak vt ịki tekchi ya iba tushki tuk vt ikki nipi bano ya wakummi tuk oka, ita tukklot illi pulla hi oke; im issish vt ilap ak o onvtonla hi oke.

12. Micha hatak osh ipok a iba tushkikmvt ita tukklot illi pulla hi oke; itaiyokomachi hokvt; im issish vt ilap akinli hosh onvtoyula hi oke.

13. Micha hatak vt hatak bika iba tushkit ohoyo iba tushki ka chohmikmvt, ita tukkiot nan isht yuwala ya yumihchi hokvt, illi pulla hi oke; im issish vt ilap akinli hosh onvtola hi oke.

14. Micha hatak osh ohoyo ha micha ishki ya aieninchit ishikmot haksi yoke; yumma micha ita tukklo ka aienvt luak ishit lua hi oke: yumohmi ho huchitin takla ya haksi kvt iksho kashke.

15. Micha hatak osh nan vlhpoa iba tushkikmot illi pulla hi oke; mikma nanulhpoa ya hushbi pulla hi oke.

16. Micha ohoyo hosh nanvlhpoa bilika onvt nanvlhpoa in tushkikma, ohoyo hash micha nanvlhpoa aieninchit hvsh ba hi oke: illit okla pulla hi oke; im issish vt onvtuyula hi oke.

17. Micha hatak vt in tek a iki ushetik a, keyukmvt ishki ushetik a ishi cha nipi bano ka pisa, micha ilap a nipi bano ka pisakma nahaksi yoke: im okla itikba ya fiopa tuput okla 
hi oke: in tek nipi bano ya wakummi tuk oka, nanashvchi vt onvtola hi oke.

18. Micha hatak osh ohoyo abeka yo iba tushki cha nipi bano ya wakummikmvt, im a yanvlli ya pisa hoka, mikma ohoyo hvt im issish a yanvlli wakummi hoka ita tuklot im okla im ai itin takla issut fiopa tuput okla hi oke.

19. Micha chishki itibapishi micha chịki in tek nipi bano ya ish wakumma he keyu ; $\mathrm{i}$ kanomi fehna ya $\underline{\mathrm{i}}$ wakummi hoka: nanashvchi vt onvtonla hi oke.

20. Micha hatak osh imoshi tekchi ya iba tuslikikmut imoshi nipi bano ya wakummi hoka, nanashvchi vt onvtonla hi oke; vlla ik im iksho hosh illit okla hi oke.

21. Micha hatak osh itibapishi tekchi ya ishikma, nan ikkashofo yoke: itibapishi nipi bano ya wakvmmi hoke, vlla ik im iksho hosh illit okla hi oke.

22: Yvmohmi hoka, a nanvlhpisa moma, micha vm aivlhpesa yưmihchi aiena ka hush holitobli cha, hush yumihchashke; yumohmi hosh yakni hush aiasha chi ka huvchisht ona la chi kut hoetvt ik hvchi kohcho kashke.

23. Hvchi tikba a kanchi li tok oklushi vt nana akaniohmi ya hush aya he keyu hoke, nana iluppa puta kak o yvmihchi chatuk, yumohmi hatuk o shitilema li tuk oke.

24. Yumohmi tuk kia I yakni ya hush aiasha hi oke, hvchim achi li hatuk, atuk osh yakni pishukchi micha foibila aiyanahanli ya hush aiasha chi ho huchima la chi hoke; hrvehin Chitokaka Chihowah sia hosh okla inla ya hvch iti filumminchi li tuk oke.

25. Yvmohmi hoka, nanvlhpoa kashofa, micha ik kashofo, micha hushi ik kashofo, micha kashofa puta ka hush itim inlucha hi oke; yohmi ka nanvlhpoa, keyukmvt hushi, keyukmot nan okchaya kaniohmi yosh yakni om balahanli, yummak o jk kashofo apesut hvchi filvmmichi li tuk a hvchi shilombish a ishit yuwala yo ishit hvchi ikbo kashke.

26. Micha hus sum i holitopa hi oke; Chihowah sia hut sa"holitopa hosh vmmi hvchia ha chị ho okla inla ya ile hvchi filummichi li tuk oke.

27. Micha hatak osh, keyukmvt ohoyo hosh shilombish 
okpulo ai itibai achvfa, keyukmut isht a hullo vt illi pulla hi oke; toli yo ishit bola hi oke; im isssish vt ilap ak o onvtonla hi oke, ish im achashke, achi tok.

\section{CHAPTA XXI.}

ICHA Chihowah yut Moses a im anumpulit, Na holi11 tompa isht asha Alon ushi vhleha ha im anumpulit, Kanima kia im okla ai illi ya ishit liteha he keyu.

2. Amba i kanomi hosh $\underline{i}$ kanomi fehna, ishki, micha iki micha ushi, micha ushetik micha itibapishi,

3. Micha in tek osh hatak haleli keyu, $\underline{\mathrm{i}}$ kanomi fehna yosh i hatak iksho chatuk, yummak okuno liteha hinla hoke.

4. Yohmi kia im okla im ai itin takla ya hatak i shahli yatuk okvt, ile litehlit ik ile holitoblo kashke.

5. Noshkobo ha yushmilali ikba he keyu, micha nutakhish chukbika ya shafa he keyu, micha nipi ya ile bushla he keyu hoke.

6. In Chitokaka ya i holitopa cha, i Chitokaka hohchifo ha ik ahobalecho kashke; luak ishit hukmit Chihowah in issa, micha in Chitokaka i puska yo im issa hokvt; yumohmi hokut holitopa hi oke.

7. Haui yă, keyukmut ik ahobo ohoyo ba ik esho kashke, micha i hatak vt kanchi tok ohoyo ha isha he keyu hoke; in Chitokaka i holitopa hoka.

8. Yvmohmi hoka chin Chitokaka i puska im issa hoka ish hullocha hi oke; chi holitopa hi oke; hvchi hullochili tuk Chihowah sia hot sa holitopa hoka.

9. Micha naholitompa isht vtta ushetik osh hauit anta cha ik ilahobalo tuk mut, iki ya ik ahobalo hoke: luak ishit lua hi oke.

10. Mikma itibapishi vhleha im ai itin takla ya naholitompa isht vtta $\underline{i}$ shahli, noshkobo ha isht ahama bila yut on hlatapa tok, micha nafohka ya fohka chi ho hullochi tok vt noshkobo ompohomo ya shufa he keyu, micha i nafohka ya hlilvffa he keyu hoke. 
11. Micha haknip illi ya iba chukowa he keyu, micha ikki, keyukmot ishki yatuk ok mak o ile litehla he keyu hoke.

12. Micha aboha holitopa ya kohcha he keyu, micha in Chitokaka im aboha holitopa ya ik ahobalo ka he keyu hoke ; in Chitokaka isht im ahama bila isht a holitopa yut onvtonla hokvt; Chihowah sia hoke.

13. Micha hatak ik halelo yo ohoyo ha isha hi oke.

14. I hatak illi, keyukmvt i hatak vt kanchi, keyukmut ik ahobo, keyukmut haui aiena ka isha he keyu, amba ilap. im okla akinli hatak haleli keyu ho ohoyo isha hi oke.

15. Micha im okla im ai itin takla ya im isht atiaka ya ik ahobalecho kashke: Chihowah sia hosh hullochi li hoke, ish im achashke, im achi tok.

16. Mihma Chihowah yot Moses a im anumpulit,

17. Alon a im anumpulit, Kuna hosh chim isht atiaka yosh im ai iti shali ha anta kvt ik im ai ono vt ashakmvt in Chitokaka i puska ya im issa chi hosh bilika ik ono kashke, ai vla he kevu;

18. Hatak nishkin lupa, keyukmut iyimomokpulo, keyukmut ibechulo latussa, keyukmo nana hoh kia isht atampa,

19. Keyukmot hatak osh iyi kobafa, keyukmot ibbak Kobafa,

20. Keyukmut nuli kofona, keyukmut hofanti ik ono, keyukmot nishkin okpulo, keyukmot nutak balakchi hlachowa, keyukmut hlachowa ik vtto, keyukmut i nihi kuhla hatak nana kia ai okpuloka im asha kuto bilika ona he keyu hoke.

21. Hatak nana hosh naholitompa isht vtta Alon isht atiaka yosh ai okpuloka im asha kvt luak ishit hukmit Chihowah im issa ya bilika onvt im issa he keyu hoke: aiokpu-: loka im asha hoke; bilika onvt in Chitokaka i puska ya im issa he keyu hoke.

22. In Chitokaka i puska holitopa i shahli, micha holitopa aiena ka upa hi oke.

23. Aiokpuloka im asha hokvt, peh chukiowvt natakali ya onvt alta ya bilika ona he keyu hoke; yumohmi hosh vm 
aboha holitopa ya ik ahobalo kashke: Chihowah sia hosh hullochi li hoka, ish im achashke, achi tok.

24. Mihma Moses vt Alon micha ushi vhleha, micha Islael im vlla vhleha moma ka im anoli tok.

\section{CHA PTA XXII.}

\section{MICHA Chihowah yvt Moses a im anumpulit,}

11 2. Alon micha ushi vhleha ha aiena ka ish im anumpuli na, Islael im vlla vhleha nan im aholitopa ya ai i filummi cha, nana a hullochi tuk a sa hohchifo ha isht ik ahobalo kashke:"Chihowah sia hoke.

3. Hưchim isht atia moma ka kuna hosh huchim ai iti shali ha anta kut ik im akashofo vt onvtonla hosh nana holitopa Islael im vlla vhleha hut Chihowah ya $\underline{i}$ hullochi cha tuk a bilika onakmut sa tikba ai i filvmmit fiopa tupa hi oke; Chihowah sia hoke.

4. Hatak nana hosh Alon ushi yosh lepa, keyukmvt i yanạhanli vt ik kashofo kisha na, naholitopa ya ai vpa he keyu hoke. Micha kuna hosh nan illi isht ik kashofo, keyukmut im isht ai unchuloli vt $\underline{i}$ kohcha tuk o halelikmvt,

5. Keyukmot kuna hosh na balvlli nana ho ik kashofo ishit toba tuk, keyukmot hatak osh ik a kashofo kaniohmi ho im aiesha hinla aiena ho haleli tuk,

6. Shilombish osh iluppa chohmi kanima ho haleli tuk mvt, ik kashofo ho ont opia cha, nipi ya oka isht ik achifo. kisha kut naholitopa ya ai upa he keyu hoke.

7. Atuk osh hvshi vt okatulakma kashofa hi oke, micha im ilhpak atuk okvt $\underline{\mathrm{i}}$ himmak a yano naholitopa ya ai vpa hi oke.

8. Ilap akinlit illi tuk, micha nampoa hosh vbi tuk a vput, isht ile litehla he keyu hoke, Chihowah sia hoke.

9. Yvmohmi hoka, a nan vlhpisa ya holitoblashke, ik ahobalot nan ashvchi isht onvtoyula cha, illi nah: Chihowah sia hosh hullochi lishke.

10. Okla inla nana hosh naholitopa ya upa he keyu hoke: 
naholitompa isht vtta i nowvt ayạ, micha tishu ilhtono áiena kvt naholitopa ya vpa he keyu.

11. Amba naholitompa isht vtta yvt shilombish $\underline{0} \underline{i}$ tvli holisso ishit chumpa tuk vt ai upa hi oke: micha in chuka ya ai vtta tok aiena kvt im ilhpak a okla ai vpa hi oke.

12. Naholitompa isht vtta ushetik vt okla inla yo auwayakmut naholitopa im issa ya ai vpa he keyu hoke.

13. Amba naholitompa isht vtta ushetik vt $\underline{i}$ hatak illi, keyukmvt kanchi tuk o vlla ik im iksho yosh falamvt iki in chuka onakmot himita tok o chohmit iki im ilhpak a vpa hi oke; yohmi kia okla inla nana hosh vpa he keyu hoke.

14. Michathatak vt ik ithano hosh naholitopa ya vpakmvt, kashapa ont ishit tahlapi ya ibafoka cha, naholitopa aieninchit naholitompa isht vtta ya ima hi oke.

15. Micha Islael im vlla vhleha hut naholitopa Chihowah im issa tuk a ik ahobalo kashke.

16. Micha naholitopa ya vpa cha, vbanvbli nan ashvchi vt ik onvtonlá, ik ahno kashke: Chihowah sia hosh hullochi li hoka, ish im achashke, achi tok.

17. Micha Chihowah yvt Moses a im anumpulit,

18. Alon, micha ushi vhleha ha micha Islael im vlla vhleha moma aiena ka im anumpulit, Islael in chuka achvfa yạ, keyukmut okla inla yosh Islael a asha kvt hatak kvna hosh anumpa kvllo il onochi tuk moma isht aiokpvehi im issakmvt, micha hukmit im issa nana ho ilap ahnit im issa ya Chihowah im issakmut:

19. Huchishno, hvsh ahni hosh nakni yosh aiokpuloka iksho wak, keyukma chukfi kia micha issi kosoma aiena ho hush im issashke.

1*. 20. Amba nana hosh aiokpuloka im ashakma hush im issa he keyu hoke : huchim aiokpvcha he keyu hoka.

21. Micha kvna hosh anumpa kvllo il onochi tuk vt aiahlicha chi hosh itin naniyuchi im issa mak o vbit im issa, keyukmvt ilap ahni hosh, wak okmá keyukmut chukfi yokmá nana im issa mak o Chihowah im issakmut aiokpvcha chi ka ik ai ono vt iksho ka hi oke.

22. Niskin lupa, keyukmvt kobafa, keyukmvt imomokpu- 
lo, keyukmot shatali im asha, keyukmvt nutak balakchi, keyukmot hlachowa aiena ka Chihowah hush im issa he keyu, micha alta onochit luak ishit hukmit Chihowah hush im issa he keyu hoke.

23. Wak hobvk osh, keyukmut” chukfushi yosh i nana ho isht atapa, keyukmut isht ik ono ya ilap ahnit ish im issa hinla hoke; amba anumpa kvllo il onochi atuk ano aiokpucha he keyu hoke.

24. Litowa keyukmut kuhla, keyukmut kobafa, keyukmut busha aiena ka Chihowah hush im issa he keyu hoke; micha huchi yakni ya hush ai im issa he keyu hoke.

25. Micha okla inla ibbak foyuka ho iluppa kanima ka huchin Chitokaka i puska ya hush im issa he keyu hoke; im a liteha yot foka, micha aiokpuloka yut foka hoka, hvch im aiokpvcha he keyu hoke; ish im achashke, achi tok.

26. Micha Chihowah yut Moses a im anumpulit,

27. Wak hobvk, keyukmvt chukfi, keyukmvt issi kosoma yo isht ona hokma, nitak untuklo ho ishki ya im anta ha hi oke; nitak isht untuchina ai vhlit ia holkwno luak ishit hukmit Chihowah im issa yo aiokpvecha hi oke.

28. Micha wak tek okma, chukfitek okma nana kia nitak achâfa it aieninchit hushba he keyu hoke.

29. Micha yakoke ahnit ima, vbit im issa yo Chihowah ya hush im issakmut, hvchishno bush aiahni hosh hush im issashke.

30. Mih nitak akinli ho vput taha hi oke, hush ashanchi na ont onna he keyu: Chihowah sia hoke.

31. Yumohmi hoka vm anumpa vlhpisa ha hush holitobli cha, hush yomihcha hi oke; Chihowah sia hoke.

32. Micha sa hohchifo holitopa ka hvch ik ahobalo ka he keyu; amba Islael im vlla vhleha im ai itin takla ya sa holitopa hi oke; Chihowah sia hosh hvchi hullochi lishke.

33. Huchin Chihowah sia ha chi hosh Echip yakni ya pehlichit huchi kohchi li tok oke: Chihowah sia hoke; im achi tok. 


\section{CHAPTA XXIII.}

ICHA Chihowah yot Moses a im anumpulit,

11 2. Islael im vlla vhleha ha im anumpulit, Chihowah impvchi yummak oka itunaha holitopa achit hush anola chi, iluppa puta kak osh impuchi li yoke.

3. Nitak hannali ho natoksuli vt vlhtaha hi oke: amba nitak isht untuklo kvto nitak hullo afoha aiitunaha holitopa yoke: yvmmak oka natoksvli nana kia hush yumihcha he keyu hoke; hush aiasha moma ka Chihowah $\underline{i}$ nitak hollo - ya hi oke.

4. Iluppa puta kak osh Chihowah impuchi itunaha holitopa yo ont ai vlhpiesakma hvsh anola hi oke.

5. Hushi ummona nitak ont isht auahhushta ont opia kak osh Chihowah im vbanublit ont ia yoke.

6. Micha hushi mih makinli nitak ont isht auahtahlapi kak osh puska ik shatummo impvchi Chihowah isht im asha yoke: nitak untuklo ho puska ik shatummo hưshpa hi oke.

7. Nitak vmmona kak o itunaha holitopa hvchim asha hi oke: tishu atoksuli nana kia hush yumihcha he keyu hoke.

8. Amba nitak untuklo ho luak ishit hukmit im issa mak o Chihowah hush im issa hi oke: nitak isht untuklo kvt itunaha holitopa hoke, tishu atoksvli ya hush yumihcha he keyu hoke, ish im achashke, im achi tok.

9. Micha Chihowah yut Moses a im anumpulit,

10. Islael im vlla vhleba ha im anumpulit, Yakni huch ima li ka hush ona cha, nawaya hushmokmut, waya vmmona hushmo tuk vt sita achvfa ho naholitompa isht vtta ya isht hushim ona hi oke.

11. Mikma huchim aiokpancha chị ho Chihowah itikba ya sita yash hush fahfula hi oke : nitak hullo tuk onnakma naholitompa isht vtta yvt fahfula hi oke.

12. Micha sita ha hvsh fahfuli nitak mvt, hukmit Chihowah im issa mak o chukfushi nakni aiokpuloka iksho afummi vmmona hush im issa hi oke. 
13. Micha balama achukma mak o luak ishit hukmit Chihowah im issa ilhpak im issa yvt bota tohbi lipehbi kushkoa ishit pokoli tuklo hosh bila aiyoma hi oke; micha ishkot im issa oka paki hin kushkoa isht ai ushta ha hi oke.

14. Micha puska, micha tanchi vlwusha, micha tanchi hiluha noshkobo aiena ka huvchin Chitokaka ya nan im issa ya isht hush im ona mih nitak ona keyu ka hvshpa he keyu hoke; hush aiasha moma hvchim ai itishali hlopulli ka nan vlhpisa bilia ya hi oke.

15. Mieha nitak hollo ha ya onnakma sita ha fahfulit im issa yo ont isht hush chukowa tuk nitak vttut ia ho 'hush hohtenashke; nitak hullo untuklo hosh tahashke.

16. Nitak hullo ont isht untuklo ha yo onna ont vhli ho nitak pokoli tahlapi ho hush hotehnashke; micha ilhpak im issa himona yo Chihowah hush im issashke.

17. Kushkoa ishit pokoli tuklo atoba, fahfuli puska lumbo tuklo ho hvsh aiasha ya ishit kohchvt isht hvsh onashke; bota tohbi lipehbi yosh ishit shatummi aiyomvt paluska ya hi oke, Chihowah ya i nawaya vmmona yoke.

18. Micha puska ya chukfushi aiokpuloka iksho afummi ummóna untuklo, micha wak hobvk himita achvfa, micha chukfi nakni tuklo aieninchit hvsh im issa hi oke; hukmit Chihowah im issa mak osh ilhpak im issa micha ishkot im issa aiena Chihowah $\underline{\mathrm{i}}$ balama achukma luak ishit hukmit im issa ya hi oke.

19. Yohmikmvt nan ashvchi im issa mak o issi kosomushi achufa ho issi kosoma yo ai ishit hushba hi oke, micha itin nanaiyvchi im issa vbi mak o chukfushi afummi vmmona tuklo ho hushba hi oke.

20. Mikma naholitompa isht vtta yot fahfulit im issa mak o waya vmmona puska ya chukfushi tuklo kash aieninchit Chihowah itikba afahfula hi oke: Chíhowah i hullochi yosh naholitopa isht vtta immi ha hi oke.

21. Micha mih nitak ak inli ho hush anoli na itunaha hvchi holitopa yashke: yummak oka tishu atoksvli hush yumihcha he keyu. Hush ai asha moma huchim ai itishali a hlopulli ka nan vlhpisa bilia ya hi oke. 
22. Atuk osh hvchi yakni nan awaya ya hush ai vmokmut hvsh bushlikmvt hvchim osapa chukbi puta ka kashofit hush isha he keyu, micha na hvchi waya vlbullit hush aiowa he keyu hoke; hatak ilbvsha micha okla inla aiena kak $\underline{o}$ hush im ashancha hi oke: huchin Chitokaka Chihowah sia hoke, ish im achashke achi tok.

23. Micha Chihowah yot Moses a im anumpulit,

24. Islael im vlla vhleha im anumpulit, Hushi isht untuklo hushi yumma nitak vmmona ma nitak hullo isht ithaiyana hinla isht pufa olahanchi, itunaha holitopa hvchim asha hi oke.

25. Yvmmak oka tishu a toksuli ya huvsh yumihcha he keyu hoke; amba luak ishit hukmit im issa ya Chihowah hush im issa hi oke, ish im achashke, achi tok.

26. Micha Chihowah yot Moses a im anumpulit,

27. Micha hvshi isht untuklo ilvppa i kashoffi nitak vt aiasha hi oke: itunaha hvchi holitopa hi oke, micha huchimi shilombish a hvsh ilbvshali cha luak ishit hukmit Chihowah im issa ya hush im issashke.

28. Micha nitak yumma na toksuli nana kia hush yumihcha he keyu hoke: huchin Chitokaka Chihowah itikba ya hvchi kashoffit na kashoffi nitak oka.

29. Nitak yumma shilombish nana hosh ilbusha he keyukmut, shilombish yummut im okla im ai itin takla kohchut fiopa tupa hi oke.

30. Micha nitak yumma shilombish nana hosh natoksuli nana kia yumihchikma shilombish yumma im okla im ai itin takla ai ishit kanchi la hi oke.

31. Na toksvli nana kia hvsh yvmihcha he keyu hoke; hvsh ai asha moma buchim ai itishali hlopulli ka nan vlhpisa bilia ya hi oke.

32. Afoha hvchi nitak hollo ya hi oke, mikma huchimi shilombish a hush ilbushala hi oke; hushi vt nitak ont ishit chakkali vt opia, opia ka vttvt ia hosh opia ka ont vhli kak o huchi nitak hollo ya hush holitobla hi oke, im achi tok. . 33. Micha Chihowah yvt Moses a im anumpulit, 34. Islael im vlla vhleha im anumpulit, Hvshi isht untuk. 
lo iluppa nitak ont isht auahtahlapi kak o nitak untuklo ho Chihowah ya vlhtipo im ai impucha hi oke.

35. Nitak vmmona ka itunaha holitopa asha hi oke, yummak oka yuka toksuli nana kia hush yumihcha he keyu hoke.

36. Nitak untuklo ho luak ishit hukmit im issa ya Chihowah hush im issa hi oke: nitak ont isht untuchina ka itunaha huchi holitopa asha hi oke, micha luak ishit hukmit Chihowah hush im issa hi oke: itunaha holitopa yoke; yuka natoksuli nana kia hush yumihcha he keyu hoke.

37. Chihowah impvchi iluppa puta kak o luak ishit hukmit im issa Chihowah im issa ya pak im issa, vbit im issa, micha ishkot im issa aiena nana iluppa puta ka nitak im aivlhpiesakma itunaha holitopa achit hush anola hi oke.

38. Micha Chihowah i nitak hollo puta, micha na huchị hvlbina, micha anumpa kvllo hush ilonochi moma, micha ilap ahnit hush im issa, Chihowah hush ima aiena hi oke.

39. Micha hushi ont isht untuklo nitak ont isht auah tahlapikma, yakni ya awaya tuk a hoiyvt hvchim vlhtahakmut nitak untuklo ho impvchi yo Chihowah isht hv'sh im aiasha hi oke, nitak vmmona kvt nitak hollo yatukma, nitak ont isht untuchina kvt nitak hollo ya hi oke.

40. Micha nitak vmmona ka iti hochukma naksish, pam iti naksish, micha iti hishi chito naksish micha bok ushi ontvlaka itokowisha aiena hvsh ishashke; micha nitak untuklo ho hvchin Chitokaka Chihowah itikba ya hvchi yukpashke.

41. Micha afummi achvfakma nitak untuklo impuchi yo Chihowah isht hush im aiasha hi oke. Hvchim ai itishali ha nan vlhpisa bilia ya hi oke; hushi isht untuklokma isht hush aiasha hi oke.

42. Chishako hush ai asha na nitak untukla hi oke; Islael osh vtta tok moma kvt chishako aiasha hi oke.

43. Yumohmi ho Echip yakni ya pehliechit kohchi li mut, Islael im vlla vhleha ha chishako ashachit isht aya li tok a hvchim ai itishali hvt ithaiyana hi oke, hvchin Chitokaka Chihowah sia, hoke, ish im achashke, achi tok. 
44. Mihma Moses vt Islael im vlla vbleha ha Chihowah impvchi puta ka im anoli tok.

\section{CHAPTA XXIV.}

IHMA Chihowah yvt Moses a im anumpulit,

I. Islael im.vlla vhleha ha ish i miha na, pvla ha chị ka pula bila ai vlhto vt luhưwa na bilia chị ka, alif bila boa. ahli yo isht chim onashke.

3. Nan isht atokowa in takali ya kocha okla moma im vlhtipo anukaka Chihowah itikba yak o opia vttut ia hosh onnahinli ont vhli ha Alon vt ai im apihisa biliashke; hvchim ai itishali ha nan vlhpisa bilia ya hi oke.

4. Pvla ai o hikia kashofa pula bila aivlhto on taloha ka Chihowah itikba talohonli na bilia he, apesashke.

5. Micha bota tohbi lipehbi yo ish ishi cha puska lumbo. auahtuklo ho ish puskashke: puska achvfakma kushkoa ishit pokoli tuklo kashke.

6. Micha itiakaiya tuklo ho ish talohlashke, itiakaiya achvfakma hannali hosh ai impa kashofa Chihowah itikba. hikia ka on talohashke.

7. Micha itiakaiya aiyukali ka filakinsen bano yo ish on ashachashke: yưmohmi ho luak ishit hukmit Chihowah im issa isht ithana bilia yosh puska ya on ashashke.

8. Nitak hollo moma ka nan itim vlhpisa bila yo Islael im vlla vhleha im ai ishi tuk mak o Chihowah itikba achukmvt talohli na biliashke.

9. Mikma Alon, micha ushi vhleha ha immi ha hi oke; micha aholitopa ak o ai vpa hi oke; nan vlhpisa bilia ho luak ishit hukmit Chihowah im issa yosh $\mathrm{i}$ holitopa fehna hoka, ish im achashke, achi tok.

10. Atuk o iki vt Echip hatak o Islael ohoyo ushi yosh. Islael im vlla vhleha ha takla kohchvt aya tok; atuk o Islael ohoyo ushi ilupput bina ha Islael hatak o itachowa tok.

11. Mihma Islael ohoyo ushi vt CHrHowaH hohchifo ha ik ahobalot anumpulit kallakshichit anumpuli tok; mikma 
Moses a isht im vla tuk, mihma (ishki vt Tan apehlichi ai achvfa Tibli ya ushetik osh hohchifo kvt Shilomilh atok.)

12. Mihma Chihowah im anukfila hovt im otvna chi ho, ai atoni fohki tok.

13. Atuk o Chihowah yot Moses a im anumpulit,

14. Kallakshichit anumpuli tuk a binah ha kocha isht ish onashke; mikma haklo tuk moma kvt ibbak a noshkobo onochashke, mikma okla moma luvt tuli ishit bolashke.

15. Micha Islael im vlla vhleha hạ im anumpulit, kuna hosh in Chitokaka ya kalakshichit anumpulikmot nan ashvchi vt onvtonlashke.

16. Mikma kuna hosh Chihowah hohchifo ha ik ahobalot anumpulikmot illi pulla hi oke, mikma okla moma kvt tvli ishit boli pulla hi oke; okla inla yưt yakni ya ai vtta tok akinli chohmit CHно waн hohchifo ha ik ahobalot anumpulikmvt illi pulla hi oke.

17. Micha kuna hosh hatak bika vbikmvt illi pulla hi oke.

18. Micha kuna hosh nan vlhpoa yo vbikmvt achukmala hi oke, nan vlhpoa yokma nan vlhpoa ya hi oke.

19. Micha hatak vt chuka abilika vtta ya im okpvnikma, yvmihchi tuk a cholımichit ilap ma yumihcha hi oke.

20. Kinuffi hokma kinafa, nishkin okma nishkin, nuti yokma nuti; hatak o kaniohmichit im okpunikma, anonti yohmi ho ilap a yumihcha hi oke.

21. Micha kvna hosh nan vlhpoa vbikmvt atobbashke, micha kvna hosh hatak a vbikmvt illa hi oke.

22. Okla inla huchi yakni ya ai vtta tuk a itilauet nan vlhpisa huchim achufa hi oke, hvchin Chitokaka Chihowah sia hoka, ish im achashke, achi tok.

23. Mihma kalakshichit anumpuli tuk ash binah ha kocha isht ona cha tvli ishit bola chị ho Moses vt Islael im vlla vhleha ha im anumpuli tok, mikma Chihowah yut Moses a i miha tuk mak o Islael im vlla vhleha hvt yumihchi tok. 


\section{CHAPTA XXV.}

ICHA Chihowah yot Moses a Sinai nunih ha ai im
anumpulit,

2. Islael im vlla vhleha ha im anumpulit, Yakni hvch ima la chi kạ, hush onakma, yohmikma yakni ut Chihowah i nitak holitopa ya $\underline{\mathrm{i}}$ holitobla hi oke.

3. Afummi hannali ho chim osapa ya ish hokchikmut, afummi hannali ho chi pakvpi ahollokchi ya naksish vmot vni ya ishma hi oke.

4. Amba afummi isht untuklo hokvto yakni afoha nitak hollo ya hi oke, Chihowah i nitak hollo ya hi oke; chim osapa ya ish ahokcha he keyu, micha chi pakupi a hollokchi ya naksish a ishma he keyu hoke.

5. Ish ai vmo ya ilap akinli hosh awaya ishma he keyu, micha chi pakvpi a hollokchi vt naksish ik vlmo ya paki ya ishma he keyu, yakni ut afoha he afvmmi yoka.

6. Micha yakni ya $\underline{i}$ nitak hollo ya ilhpak chim asha hi oke; chishno, micha chin tishu, micha chin tishu ohoyo, micha tishu chim ilhtono, micha okla inla yosh chi awant anta aiena,

7. Micha chi wak, micha nan. vlhpoa chị yakni aiasha aiena ka awaya moma kvt ilhpak vt im aiasha hi oke.

8. Micha afummi nitak hollo untuklo ho afummi untuklo bat untuklo ho ish hohtenashke; mikma afummi nitak hollo untuklo kvt chim afummi "pokoli ushta akucha chakkali hashke.

9. Yohmikma hushi ont isht untuklo kvt nitak ont ishit pokolikma, chubili isht púfa ish olachechashke, $\underline{i}$ kashoffi nitak ma hvchi yakni moma ka olachechit hush hlopullecha hi oke.

10. Micha afummi ont ishit pokoli tahlapi ya hush hullocha hi oke, micha yakni moma hlopullechit okla aiasha moma ka yuka issachit anumpa hưsh i bohlashke: hvchị chubili ya hi oke, mikma hatak hush moma kvt i yakni aiyukali, 
micha hatak hush moma kvt in chuka aiyukali hush ona hi oke.

11. Afummi ishit pokoli tahlap yummvt hvchi chubili ya hi oke: nana hush hokcha he keyu, micha ilap akinli offo ya hushma he keyu, micha pakvpi naksish vlmo tuk keyu ya paki hush ai vma he keyu hoke.

12. Chubili yoka; hvchi holitopa hi oke: osapa awaya ak o hushpa hi oke.

13. Chubili afummi iluppa hatak puta kvt ilap i yakni. aiyukali ka falamot hush ona hi oke.

14. Micha chuka abilika hatak a nana kia ish $\underline{\mathbf{i}}$ kanchikmot, keyukmvt nana kia chuka a bilika ish in chumpakmvt hush itilbushala he keyu hoke.

15. Chubili ha ya afummi holhtina vlhpisa ho chin chuka abilika ya ish in chumpa hi oke, mikma nawaya afummi holhtina vlhpisa ho yummak osh chi kancha hi oke.

16. Afưmmi laua ka vlhpisa hosh aivlli ka $\underline{i}$ shahlechit isht ish ia hi oke, micha afummi iklauo ka vlhpisa ho aivlli $\mathrm{k} \underline{a}$ ish ola takluchashke; nawaya ya afưmi holhtina y $\underline{\mathbf{a}}$ vlhpisa ho chị kanchi hoka.

17. Yvmohmi hoka, hush itilbushala he keyu hoke, amba chin Chitokaka ish i nukwiya hi oke, hvchin Chitokaka Chihowah sia hoke.

18. Yvmohmi hoka a nan vlhpisa ka hush yvmihcha hi oke, micha vm aivlhpiesa yumihchi ya hvsh holitoblashke, mikmut achukma hosh yakni ya hvsh aiasha hi oke.

19. Mikma yakni vt na waya ka waya hi oke, micha imput huchi kaiya cha yummak o achukma hosh hush aiasha hi oke.

20. Atuk o afummi isht untuklo ka nana ho hokchit na waya ka emachi keyu ho kvt nanta hak o e pa chị cho? hush achi hokma,

21. Yohmikma afummi ont ishit hannalikma ishit yukpali li ya apesut hvchi onochi la hi oke, mikma afummi tuchina waya yo huchi waya hi oke.

22. Atuk o afummi ont isht untuchinakma nana hush hokcha hi oke, micha nawaya sipokni ak o hushpa na, 
afummi ont ishit chakkala hi oke; yumma i na waya kvt iklo kisha ka sipokni ak o hoshpa hi oke.

23. Yakni vt kvnivt bilia he keyu hoke; yakni ut vmmi hoka, okla inla, micha nowvt aya huchia hosh hush aiasha hoka.

24. Yohmi ka yakni hush aiasha moma ka yakni falumminchit chumpa ka vlhpesa hush ahna hi oke.

25. Hush itibapishi hut ilbushut isht ia cha, i yakni kaniohmi ka kanchi tuk o, i kanomi hosh falvmminchit chumput vlakmvt itibapishi hvt kanchi tuk a falumminchit chumpashke.

26. Mikma hatak vt falvmminchit im a chumpa hinla kvt ik im ikshokmvt, amba ilap vto falumminchit chumpa hinla,

27. Yuhmikma a kunia tok afummi ha hohtena cha atampa ka $\underline{i}$ kanchi tok $\underline{\text { a }} \mathbf{i}$ falvmminchashke yumohmi ho falamut i yakni ya onashke.

28. Amba i falvmmincha he keyukma, na kunia tuk vt chumpa tuk ash ibbak foka na chubili afummi ka ona hi oke; atuk osh chubili ya mokofa hi oke, mikma i yakni ya falamut ona hi oke.

29. Micha hatak vt tvmaha holihta ya chuka in talaia tuk osh kanchi tuk mut, kunia ha ya afummi achufa fehna itintakla ho falvmminchit chumpa hinla hoke, afummi achvfa vlhpesakma falumminchit chumpashke.

30. Micha afummi achvfa vlhpesa ka falvmminchit ik chumpokma, tumaha holihta chuka talaia tuk vt chumpa tok ash kullot im ai itishali a hlopulli ka immit bilia yo tobashke; chubili ma kohcha he keyu hoke.

31. Amba chuka alokoli yo chuka taloha holihtvt apakfoyupa keyu hokvto yakni haiaka osapa ya chohma hi oke, falumminchit chumpa hinlakmvt yumohma hi oke, mikmvt chubili ma kohcha hi oke.

32. Yohmi kia Lefait vhleha in tumaha holihta ya micha i yakni tumaha a holihta chuka puta ka nitak nana ho falummnchit chumpakmot Lefait vhleha hvt yumihcha hi oke.

33. Micha hatak osh Lefait a in chumpa tuk ma chuka 
kunia tuk a micha i yakni tumaha holihta aiena kvt chubili ma kohcha hi oke; Islael im vlla vhleha im ai iti takla ya Lefait, vt in tumaha holihta chuka puta kak osh $\underline{\mathrm{i}}$ yakni yoka.

34. Amba in tumaha holihta abilika fullota yakni haiaka hokvto kunia he keyu, i yakni bilia yoka.

35. Micha chitibapishi hvt chiba fokvt ilbushvt kunia cha, nana bunnakma ish apelvchashke, ahli okla inla micha nowvt aya yoh kia, yumohmi ho chi takla ahantashke.

36. Micha chelichi micha $\underline{\underline{i}}$ shahlit isht ia aiena ka chik im esho kashke; amba chin Chitokaka ya ish i nukshopashke; yumohmi ho chitibapishi hot okchaya cha chi takla ahantashke.

37. Chelichi ho chin tuli holisso ya ish ima he keyu, micha $\underline{\underline{i}}$ shahlit ibafohka chị ho chim ilhpak o ish $\underline{\mathrm{i}}$ pota he keyu hoke.

38. Hvchin Chitokaka Chihowah sia hosh, Kenan yakni ya huch ima lish, hvchin Chitokaka sia chi hosh Echip yakni ya a hvchi pehlichit kohchi li tok oke.

39. Micha chitibapishi hvt chi bilika ahanta kvt ilbvshvt kuniakma micha chi kanchikma; yuka chohmichit ish toksvlecha he keyu ;

40. Amba tishu ilhtono micha nowvt aya chohmit chin toksulit chi takla ahanta na, chubili afummi ha ont vhla hi oke.

41. Atuk osh yohmikma ilap, micha im vlla vhleha aiena itapihut chi filvmmit falamvt ilap in chuka achvfa micha iki vhleha i yakni ya ona hi oke.

42. An tishu vhleha hosh Echip yakni pehlichit kohchi li tok oka, hatak yuka chohmichit kancha he keyu hoke.

43. Kvllot ish toksvlecha he keyu, amba chin Chitokaka ya ish i nukshopa hi oke.

44. Hatak yuka micha ohoyo yuka chim aiasha kvt oklushi Chihowah ik ithano chi afolublit aiasha kak a hi oke; yohmi kak o hatak yuka micha ohoyo yuka aiena ka hush a chumpa hi oke. 
pokma, chubili afummi hano, ilap micha im vlla vhleha aiena kvt apiha ho kohcha hi oke,

55. Islael im vlla vhleha hvt vno ak $\underline{0}$ an tishu vhleha hoka, an tishu vhleha hosh Echip yakni ya pehlichit kohchi li tok oke: huchin Chitokaka Chihowah sia hoke.

\section{CHAPTA XXVI.}

A holbut toba, micha na holbut toba in chuwa hush ikba 1 he keyu, micha na holbut toba ikbit hush hilecha he keyu, micha toli na holbut toba nana kia hush im aka chunola chi kvt chi yakni a hush hilecha he keyu hoke, hvchin Chitokaka Chihowah sia hoka.

2. A nitak hollo ya hush holitobli cha vm aboha holitompa ya hush holitobla hi oke, Chihowah sia hoke.

3. A nan vlhpisa hush ai itunowa cha vm anumpa vlhpisa ya ieshit hush yumihchikma,

4. Yohmikma ont aivlhpiesakma, umba ka hvoch inima la hi oke, mikma yakni vt na waya ha hvchi waya hi oke; mikma kowi iti hikia kvt vnit huchi waya hi oke.

5. Mikma hush nihechi kut pạki vmo ya ona he, micha paki vmo yut hokcha he vlhpesa ona hi oke; mikma puska uput hvchi kaiya cha, achukma hosh huchi yakni ya hush aiasha hi oke.

6. Mikma yakni ya iti nanaiya ima la hi oke, yohmikma hush tushkikma nana hosh huchi nukshobla he keyu, amba nampoa okpulo ya yakni ya kohchit ishit kanchi la hi oke; mikma bushpo falaia yut huchi yakni ya hlopulla he keyu hoke.

7. Mikma hvchin tunup a hush tihleli na, yvmmak osh huchi tikba ya bushpo falaia ishit akakoha hi oke.

8. Mikma hush tahlapikmut tahlepa achufa kia hush tiblela he, micha tahlepa hush achufakmut tahlepa sipokni pokoli kia hush tiblela hi oke, mikma hvchin tunvp vt huchi tikba ya bushpo falaia ishit akakoha hi oke.

9. Hvch ithaiyana lish bvchi wayachit hvchi apaknvchi 
la hi oke, micha vm anumpa kvllochit na hvchitim apesa la hi oke.

10. Mikma ilhpak sipokni ak o hushpa tuk osh himona ak atuk pulla kak $\mathrm{o}$ isht hush kohcha hi oke.

11. Mikma an tabenakel a hvchim ai itin takla ya hilechi la hi oke micha vm ishilombish vt hvchi yuwala he keyu hoke.

12. Micha huch im ai itin takla ya a nowa la hi oke, micha hvchin Chihowah sia hokma, vm okla hvchia ha hi oke.

13. Hvchin Chitokaka Chihowah sia hosh yumma i yuka hatak hvchia ha he keyu mak o, Echip yakni ya pehlichit hvchi kohchi li tuk oke, micha ikonla abana ishit talakchi puta ka tvptulit apissulechit hvchi nowvchi li hatuk oke.

14. Yohmi kia hus sa haponakla he keyukma, micha vm anumpa iluppa moma hush yumihcha he keyukma,

15. Micha a nan vlhpisa ha hush shitelemakma, keyukmut huchimi shilombish vt vm aivlhpesa yưmihchi ya yuwala cha a nan vlhpisa moma ka hush yumihcha he keyukma, amba na hvch itim apesa li ya hush kobvffikma,

16. Uno ak kia iluppa huchi yumibchi la hi oke; vno fehna kut komonta, tahut isht ia, yunha mikma niskin elli yo micha chukvush nukhakklochi yo onochit hvchim apesa la hi oke, mikma peh pilla ho huchi napehna ya hush hokcha hi oke; huchin tunvp ak osh vpa hi oka.

17. Mikma sa nashuka ya isht hvchi sanalichi likma, hvchin tunup itikba ya hvch illa hi oke; hvchi nukkilli puta kvt hvchi pehlicha hi oke, mikma kuna hosh huchi hlioli keyu kia hush tihlahaya hi oke.

18. Micha nana iluppa moma ka hưs sa haponakla he keyukma, na hush ashuchi pulla kak o, himakma napalummi untuklo ha isht huchi onochi la hi oke.

19. Micha nan isht hvsh a kvllo isht ilefehuvchi ya kobuffi la hi oke; micha huchi shutik a tuli chohmichi likmvt, hvchi yakni ya asonak lakna ya hvchin chohmichi la hi oke.

20. Mikma pilla ho huchim i hlampko hut taha chị hoke; 
hvohi yakni yơt nawaya ya huchi waya he keyu hoke, micha yakni ya iti puta kut uni ya waya he keyu hoke.

21. Micha hưs sum ilapunla cha hưs sa haponakla he keyukma, na hush ashvchi atukmak o ililli untuklo ho himakma hvchi onochi la hi oke.

22. Micha nampoa okpulo aiena hvchim itin takla et tihleli likma yummut hvchim vlla vhleha hạ wehpuli cha, hvchi nan vihpoa ya vbit tahla hi oke, micha hvchi kanamosechi likma, huchi hina puta kvt aksha hi oke.

23. Atuk osh iluppa puta ka isht huchi kostininchi la he keyukma, amba hvs sum ilapunlakma,

24. Yohmikma vno ak kia hvchim ilapunla lish, na hvsh ashvchi pulla ka untuklo ha na palvmmi huvchi onochi la hi oke.

25. Micha a nan vlhpisa ha $\underline{i}$ sanali tuk a ilaiokha chị ka bushpo falaia ya hvchi onochi la hi oke; mikma hvchin tvmaha holihta anuka itahobvt hush itunahakma, ililli hvchi onochi la hi oke, mikma tunup ibbak hush fohka hi oke.

26. Mikma ishit hlampko hvehi puska ya kobvffi likma, ohoyo pokoli hosh huchi puska ya a paluska achvfa ho anunachi cha hvchi puska ya wekichi hosh anonti hvch ima hi oke; mikma hushpa kia ik huchi fihopo ka hi oke.

27. Micha iluppa moma kvt yumohmi kia hus sa haponakla he keyu, amba hưs sum ilapunlakma,

28. Yohmikma vno ak kia nukhobela hosh hvchim ilapunlvt aya la chi hoke; micha vno, vno fehna kut na hush ashvchi tuk a untuklo ha napalvmmi hvchi onochi la hi oke.

29. Mikma hvchiso vhleha nipi ya hushpa cha huchiso tek vhleha nipi ya hushpa hi oke.

30. Micha hvch im achaha puta ka jshit kanchi lish na holba hvchin toba puta ka chanlit akakohli likmvt hvchi haknip illi ya hvchi na holbvt toba puta ka on ashachi la hi oke, micha vmi shilombish vt hvchi yuwala hi oke.

31. Micha huchin tvmaha hohlita puta ka chukilissvehi lish, hvchim vlhtipo holitompa puta ka chukilissuchi likmvt, na huchi balama ya huwa la he keyu hoke.

: 32. Micha yakni ya a chukilissa isht ona la chị hoke, 
mikma hvchin tunvp vhleha yumma ai ahanta kut okokko! ahna chi hoke.

33. Micha oklushi Chihowah ik ithano puta ka im itin takla hvchi fimmi lish bushpo falaia shuffit hvchiakaiyachi la hi oke; mikma hvchi yakni vt chukilisșakma, huchin tumaha holihta puta kvt ai okpuloka hi oke.

34. Yohmikma huchin tunup $\underline{i}$ yakni a hvsh aiasha na yakni vt chukilissa momakmot $\underline{\underline{i}}$ nitak hollo ya a yukpa hi oke; yohmi pulla hokmuno yakni ut foha cha, i nitak hollo ya a yukpa hi oke.

35. Chukilissut talaia momakmuto foha hi oke; hush on asha ma hvchi nitak hollo ya ik foho tuk okvt.

36. Micha okchaya momvt in tunup i yakni asha ka chụkvsh iilli fohki la hi oke, mikma iti hishi fatahvchit ola yvt tihlela hi oke; mikma bushpo falaia i yihlepa chohmit yihlepa hi oke, micha kvna hosh hlioli keyu kia akakoha hi oke.

37. Micha kvna hosh hlioli keyu kia bushpo falaia itikba yo chohmit ito kahut akakoha hi oke, mikmot kvllot huchin tunup itikba hush hiela he keyu hoke.

38. Micha oklushi Chihowah ik ithano im itin takla ai illit huchi taha hi oke, mikma hucchin tunup i yakni vt huchi tahla hi oke.

39. Micha hvchi okchąya kvto hvchin tunup i yakni ai ashvt nan ashvchi ishit hlipushit, micha ilki nan ashvchi ya ishit hlipushit ia hi oke.

40. Yohmi kia ilap nan ashvchi, micha iki vhleha nan ashuchi, yoshoba aiena a yoshoba tuk, micha vm ilapunla tuk,

41. Micha vno ak kia im ilapunla li tuk, micha in tunup $\underline{\mathbf{i}}$ yakni ya isht ona li tuk aiena ka $v m$ il aionohonlikma, micha chukvsh hakshup ik tapo vt akanlosi cha, nan ashvchi pulla hosh na palvmmi onvtula tuk vt vlhpesa ahnikma,

42. Yohmikma Chekob a nan itim apesali tok vt ithaiyana la hi oke, micha Aisak a nan itim apesali tok vt ithaiyana la hi oke, micha Ebleham a nan itim apesali tok aiena kut ithaiyana, la hi oke, micha yakni ya ithaiyan a la hi oke. 43. Micha yakni yaiissakma, yưmmut iksho ho chukilis- 
sut talaiakmut, $\underline{\mathrm{i}}$ nitak hollo ya a yukpa hi oke : micha nan ashvchit na palummi onvtula tuk vt vlhpesa ahna hi oke; vm aivlhpesa yumihchi ya shitilema tuk okvt, micha imi shilombish vt a nan vlhpisa puta ka shitilema tuk okvt.

44. Amba yumma moma ka ik yohmi kia in tunup i yakni ashakma kanchi la he keyu, micha shitilemvt nan itim apesali vt kobvffit kanchit vmohmichi la he keyu; in Chitokaka Chihowah sia hokvt.

45. Amba yummak atuk pulla mak $\mathrm{o}$ in tikba vhleha ha yummak oka in Chitokaka sia ha chi hosh, oklushi Chiho- wah ik ithano vhleha hvt pisa ho, Echip yakni ya pehlichit kohchit nan itim apesali tok vt, ithaiyana la hi oke, Chihowah sia hoke, ish im achashke, achi tok.

46. Nana vlhpisa ilvppa puta, micha ai vlhpesa yumihchi, micha nanvlhpisa iluppa puta kak o Chihowah yot Moses a . tohnot Sinai nunih ha Islael im vlla vhleha ha ai itim apesa tok oke.

\section{CHAPTA XXVII.}

MIHMA Chihowah yut Moses a im anumpulit,

1 2. Islael im vlla vhleha ha im anumpulit, Hatak nan ahlichi anumpa ha atokolit ilonochikmvt, ish im apesa ho hatak vt Chihowah immi ha hi oke.

3. Ish im apesa kut nakni yosh afummi pokoli tuklo vttut ia hosh afummi pokoli hannali ont vhli ha hi oke, ish im apesa kut vlhtipo holitopa shekel ak o iakaiya hosh tuli huta shekel pokoli tahlapa hi oke.

4. Micha ohoyo hokmuno shekel pokoli tuchina ho ish im apesa hi oke.

5. Micha afummi tahlapi ho vttut ia hosh afummi pokoli tuklo ont vhli hokma nakni yokma, shekel pokoli tuklo, ohoyo hokma shekel pokoli ho ish im apesa hi oke.

6. Micha hushi achufa ho vttut ia hosh afummi tahlapi ont vhli kano nakni yokma, tvli hota shekel tahlapi ish im apesa he, micha oboyo hokma tuli huta shekel tuchina ho ish im apesa hi oke. 
7. Micha afummi pokoli hannali vttut vba maya ka nakni yokma, shekel auahtahlapi ho, micha ohoyo hokma, shekel pokoli ho ish im apesa hi oke.

8. Yohmi kia ilbusha hatuk osh ish im apesa tuk a ik onanchokmvt na holitompa isht vtta itikba ya ilotunicha he, mikma na holitompa isht vtta yvt vlhtoba onocha hi oke ; anumpa ahlichi ilonochi ash osh yumihcha hinla vlhpisa ho vlhtoba onachashke.

9. Micha nan vlhpoa yo hatak vt Chihowah im issut isht im ona, yohmikma hatak kuna hosh yumma chohmi ho Chihowah im issakma, moma kut holitopa hi oke.

10. Inlvcha he keyu, micha okpulo yatuk a achukma yo, keyukmvt achukma yatuk a okpulo yo atobbicha he keyu hoke, micha nan vlhpoa yatuk o nan vlhpoa yo atobbiechikma yumma micha vlhtoba kash aiena kvt holitopa hi oke.

11. Micha nan vlhpoa liteha nana ho yummak oka vbit im issa mak o Chihowah im issa keyu, yohmikmut nan vlhpoa ya naholitompa isht vtta ya isht im ona hi oke.

12. Mikma naholitompa isht vtta yut achukma hokmá, okpulo yokmá nana kia vlhtoba onocha hi oke; naholitompa isht vtta chia hvt vlhtoba ish onochi tuk mak o yvmohma hi oke.

13. Yohmi kia falumminchit chumpa chi pulla hokmvt, ish im apesa tuk a ishit tahlapi ho apotola hi oke.

14. Micha hatak osh in chuka ya Chihowah $\underline{\mathrm{i}}$ holitopa chi ho hullochikma naholitompa isht vtta yut achukma rhokmá okpulo yokmá nana kia vlhtoba onocha hi oke, naholitompa isht vtta yut vlhtoba onochi tuk mak o kvllot hikia hi oke.

15. Atuk o hullochi tuk ash osh in chuka falumminchit chumpa chikma vlhtoba ish apesa tuk a tuli holisso ishit tahlapi ho apotoli cha immi hashke.

16. Micha hatak vt im osapa kashapa ka Chihowah ya hullochikma, napehna ya vlhpisa ho ish im apesa hi oke: bali pehna homa achufa kvt tuli huta shekel pokoli tahlapi vlhpisa hi oke. 
17. Chubili ak o vttut ia ho im osapa ya hullochi tuk ma, ish im apesa tuk ash vlhpisa hosh hikia hi oke.

18. Amba chubili $\underline{i}$ himmak o im osapa ya hullochi tuk ma, naholitompa isht vtta yvt afummi takanli moma chubili afummi ont vhli kak o vlhpisa ho tvli holisso i hohtena hi oke, mikma ish im apesa tuk ash isht ola takla hi oke.

19. Atuk o osapa ya hullochi tuk ash osh kaniohmit falumminchit chumpa hinlakmut ish im apesa tuk ash, toli holisso ishit tahlapi ka ibafohkikmvt kvllot immi ha hi oke. 20. Micha osapa ha falvmminchit chumpa he keyukmvt, keyukmvt osapa ya hatak inla yo $\underline{i}$ kanchi tukmvt himmakma falumminchit chumpa he keyu hoke.

-21: Amba osapa yvt chubili ya .kohchakmvt Chihowah holitopa hullochi osapa ya hi oke; yummut naholitompa isht vtta immi ha hi oke.

22. Micha hatak osh osapa chumpa tuk osh yvmmvt i yakni yatuk keyu ho Chihowah ya $\underline{\mathrm{i}}$ hullochi tuk ma,

23. Yohmikma naholitompa isht vtta yut ish im apesa tuk vlhpisa ho chubili afummi ont vhli ho $\mathrm{i}$ hohtenashke; mikma nitak yumma ish im apesa tuk nana holitompa mak o Chihowah ya imashke.

24. Chubili afummi ya in chumpa tuk ash osapa yut falamut 1 yakni atuk ak o im onashke.

25. Micha vlhtipo holitopa shekel ak o iakaiya hosh ish im apesa bieka hi oke, kelah pokoli tuklokmut shekel achufa hi oke.

26. Vlhpoa ushi vttahpi ak illa hosh yummuto Chihowah im vttahpi ya hi vlhpisa hoke, hatak nana hosh hullocha he keyu hoke, wak hobvk okmá, chukfi nana kia, Chihowah immi hoke.

27. Micha nan vlhpoa liteha yokma, ish im apesa tuk a iakaiya hosh falumminchit chumpa cha, ishit tahlapi ibafohka hi oke; keyukmvt falvmminchit ik chụmpokma, ish im apesa tuk a iakaiya hosh kunia hi oke.

28. Yohmi kia nana hullochi tuk nana im asha moma ka ai ishit, hatak o keyukmvt, nan vlhpoa yo keyukmvt, osapa aiena kanima ho hullochi tukma kvnia he keyu, micha 
falumminchit' chumpa he keyu hoke: nana hullochi puta kvt Chihowah i holitompa i shahli hoke.

29. Nana hullochi achvfa kia hatak vt hullochi tuk a falvmminchit chumpa he keyu hoke, amba illi pulla hi oke. 30. Micha yakni ya nana ishit pokoli puta, yakni nana awaya puta, keyukmut iti vni puta kut Chihowah immi hoke, Chihowah i holitopa hoke.

31. Yohmi ka hatak osh im ishit pokoli falumminchit chumpa pullakmvt ishit tahlapi ka apotola hi oke.

32. Micha nan vlhpoa hochito, keyukmut chipunta aiena ishit pokoli ak okmvt kanima hosh fuli nuta ont ia ak a hi osh, ishit pokoli ak osh Chihowah i holitopa hi oke.

33. Achukma hokmá, okpulo yokmá nana ka hoya he keyu, micha inla atobbicha he keyu, inla atobbichi pulla hokma, yumma micha vlhtoba kash aiena kvt i holitopa ha hi oke, falumminehit chumpa he keyu hoke, ish im achashke, im achi tok,

34. Nan vlhpisa iluppa puta kak o Islael im vlla vhleha i nan vlhpisa ha chi ka Sinai nưnih ha Chihowah yut Moses a ai im apesa tok oke. 



\section{NUMBAS HOLISSO.}

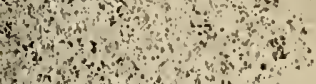

anton 



\section{MOSES I HOLISSO AIUSHTA KVT}

\section{NUMBAS HOHCHIFO HOKE.}

\section{CHAPTA I.}

IHMA Chihowah yvt yakni haiaka Sinai ya Echip yakni I ya a kohchvt minti tok afúmmi atukla, hushi atrikla kvt nitak vmmona ma, okla moma im vlhtipo ya Moses a et. ai im anumpulit,

2. Islael im vlla vhleha moma holhtina ka in chuka achufa jakaiya ho iki in chuka achvfa iakaiya ho nakni puta noshkobo vlhpisa hohchifo holltina ka hesh ishashke.

3. Afummi pokoli tuklo ai vhlit vba maya kohchut tunup ia hinla puta, Islael ai asha ka, chishno micha Alon itatuklot tushka pehlichi aiyukali ho hvsh hotehnashke.

4. Mikma apehlichi aiyukali ka hatak achvfa iki vhleha. in chuka achvfa $\underline{i}$ noshkoboka aiyukali vt hvch ibafoka hi oke.

5. Hatak hohchifo iluppa puta kak osh hvchi apehvt hiela chị hoke. Luben apehlichi ya , Sheteu ushi Elisü; -

6. Simeon apehlichi ya , Sulishattai ushi Shelumiel,

7. Chutah apehlichi ya , Amminatab ushi Nahshon,

8. Issaka apehlichi ya , Sua ushi Nehlanecl,

9. Sebultun apehlichi a, Helon ushi Eliab,

10. Chosef im vila vhleha; Eflaim apehlichi, Ammihut ushi Elishama; Manasseh apehlichi a Petahsu ushi Kamaliel,

11. Benchamin apehlichi a , Kiteoni ushi Abitan,

12. Tan apehlichi a Amishattai ushi Ahiesa,

13. Asha apehlichi a Oklan ushi Pakiel,

14. Kat apehlichi a Teuel ushi Eliasaf,

15. Naftali apehlichi a Enan ushi Ahila, 
16. Iluppa puta kak osh okla moma ka ai annohowa ilki vhleha apchlichi yo i holitopa Islael a tahlepa sipokni puta i noshkoboka yatok.

17. Mihma Moses micha Alon itatuklo kut hatak iluppa puta hohchifo hosh takali tuk o ishi tok.

18. Micha okla moma ka hvshi atukla nitak vmmona ma itunahli tok, mihma in chuka achvfa iakaiyvt iki vhleha in chuka vlhpisvt hohchifo holhtina yatuk a noshkobo holhtina afummi pokoli tuklo ai uhlit ưba maya ka okla anoli tok.

19. Mihma Chihowah yvt Moses a im apesa tuk mak $\underline{o}$, yohmit Sinai yakni haiaka ya a hohtena tok.

20. Mihma Islael ushi akni fehna Luben im vlla vhleha ai unchuloli iki vhleha in chuka achvfa yvt chuka achvfa vlhpisut nakni puta afummi pokoli tnklo ai vhli vttut vba maya kohchvt tunup itibit ia hinla moma kvt noshkobo holhtina hosh hohchifo holhtina tok.

21. Luben apehlichi yosh holhtina kvt tahlepa sipokni pokoli ushta cha tahlepa tahlapi tok.

22. Simeon im vlla vhleha ai unchuloli vt iki vhleha in chuka achvfa vt, in chuka achvfa vlhpisut nakni puta afummi pokoli tuklo vttut vba maya kohchut tunvp itibit ia hinla puta kvt noshkobo holhtina hosh hohchifo holhtina tok.

23. Simeon apehlichi holhtina kvt tahlepa sipokni pokoli tahlapi akucha chakkali cha tahlepa tuchina tok.

24. Kat im vlla vhleha ai unchuloli hot iki vhleha in chuka achvfa yvt chuka achvfa vlhpisut afummi pokoli tuklo ai vhli vttut vba maya kohchut turup itibit ia hinla puta kvt hohchifo holhtina tok.

25. Kat apehlichi yumma holhtina kvt tahlepa sipokni pokoli ushta akucha tahlapi, tahlepa hannali cha pokoli tahlapi tok.

26. Chutah im vlla vhleha ai unchuloli hvt iki vhleha in chuka achvfa yvt chuka achvfa vlhpisvt afummi pokoli tnklo ai vhli vttut vba maya kohchvt tunvp itibit ia hinla puta kvt hohchifo holhtina tok.

27. Chutah apehlichi yummut holhtina kvt tahlepa sipokni pokoli untuklo akucha ushta cha tahlepa hannali tok. 
28. Issaka im vlla vhleha ai unchuloli hut iki vhleha in chuka achvfa vt chuka achufa vlhpisa hosh afummi pokoli tuklo ai vhli vttut vba maya kohchvt tunvp itibit ia hinla puta kvt hohchifo holhtina tọk.

29. Issaka apehlichi yummut holhtina kvt, tehlepa sipokni pokoli tahlapi akucha ushta cha tahlepa ushta tok.

30. Sebulun im vlla vhleha ai unchuloli hvt iki vhleha in chuka achvfa vt chuka achvfa vlhpisvt afummi pokoli tuklo ai vhli vttut vba maya kohchut tunvp itibit ia hinla puta kvt hohchifo holhtina tok.

31. Sebulun apehlichi yvmmvt holhtina kvt tahlepa sipokni pokoli tahlapi akucha untuklo cha tahlepa ushta tok.

32. Chosef im vlla vhleha yvmmvt Eflaim im vlla vhleha ai unchuloli hot iki vhleha in chuka achvfa yvt chuka achvfa vlhpisa hosh afummi pokoli tuklo ai vhli vttut vba maya kohchut tunup itibit ia hinla puta kut hohchifo holhtina tok.

33. Eflaim apehlichi yummut holhtina kvt tahlepa sipokni pokoli ushta cha tahlepa tahlapi tok.

34. Manasseh im vlla vhleha ai unchuloli hvt iki vhleha in chuka achvfa yot chuka achvfa vlhpisa hosh afvmmi pokoli tuklo ai vhli vttut vba maya kohchvt tunvp itibit ia hinla puta kvt hohchifo holhtina tok.

35. Manasseh apehlichi yummvt holhtina kvt tahlepa sipokni pokoli tuchina akucha tuklo cha tahlepa tuklo tok.

36. Benchamin im vlla vhleha ai unchuloli hot iki in chuka achvfa yvt chuka achvfa vlhpisa hosh afvmmi pokoli tuklo ai vhli vttut vba maya kohchvt tunvp itibit ia hinla puta kvt hohchifot holhtina tok.

37. Benchamin apehlichi yummut holhtina kvt tahlepa sipokni pokoli tuchina akucha tahlapi cha tahlepa ushta tok.

38. Tan im vlla vhleha ai unchuloli hvt iki vhleha in chuka achvfa yvt, chuka achvfa vlhpisvt afummi pokoli tuklo ai vhlit vba maya kohchvt tunup itibit ia hinla puta kvt hohchifot holhtina tok.

39. Tan apehlichi yummot holhtina kvt tahlepa sipokni pokoli hannali akucha tuklo cha tahlepa untuklo tok. 
40. Asha im vlla vhleha ai unchuloli hvt iki in chuka achufa, chuka achvfa vlhpisvt afummi pokoli tuklo ai vhlit vba maya kohchut tunvp itibit ia hinla puta kvt hohchifot holhtina tok.

41. Asha apehlichi yummut holhtina kvt tahlepa sipokni pokoli ushta akucha achvfa cha tahlepa tahlapi tok.

42. Naftali im vlla vhleha ai unchuloli hut ilki vhleha in chuka achufa yot chuka achvfa vlhpisut afommi pokoli tuklo ai vhlit vba maya kohchot tunvp itibit ia hinla puta kvt hohchifot holbtina tok.

43. Naftali apehlichi yummut holhtina kut tahlepa sipokni pokoli tahlapi akucha tuchina cha tahlepa ushta tok.

44. Ilvppa puta kak osh, Moses micha Alon itatuklo kvt hohtena ma holhtina tok; Islael i holitopa hatak auah tukló : iki vhleha in chuka achvfakma achvfut ia tok.

45. Yohmi ho Islael im vlla vhleha moma iki vhleha in chuka achufa vlhpisa hosh afummi pokoli tuklo ai vhli vttut vba maya kohchut tunvp itibit ia hinla puta kvt holhtina tok.

46. Holhtina kvt moma kvt tahlepa sipokni tahlepa hannali cha tahlepa sipokni tuchina tahlepa tahlapi cha pokoli tahlapi aiena tok.

47. Amba Lefait vhleha hvt iki vhleha apehlichi kvt takla ik holhtino tok.

48. Chihowah yut Moses a im anumpulit,

49. Lefai apehlichi illa ho ish hohtena he keyu, micha Islael a takla holhtina ka ish isha he keyu;

50. Amba Lefait vhleha isht atokowa vlhtipo, micha nan ai vlhto moma yumma foyuka, micha nana hosh ai immi putakma, apistikli ya chi ka ish atokolashke, mikma vlhtipo yạ, micha nanaivlhto moma aiena ka shoyula chi hoke: micha nana isht im ahashwa, micha vlhtipo holitopa ya abinachit afolubla hi oke.

51. Vlhtipo holitopa yvt ia chilkma, Lefait vhleha hak osh akvcha hi oke; micha vlhtipo holitopa yot hikia chikma, Lefait vhleha hak osh hilecha hi oke; atuk o hatak nan ik ithano yosh bilika ona hokvt illa hi oke.

52. Mikma Islael im vila vhleha hokvt ilap ini vlhtipo 
puta ka i binah aiyukali a hilecha hi oke; micha hatak aiyukali kvt $\underline{i}$ na huta shali abinanchit okla moma hlopulla hi oke.

53. Amba Lefait vhleha hokuto nan isht atokowa vlhtipo holitopa hilechit apakfoyuput binancha hi oke: yvmohmi ho im ai i nukkilli vt Islael im vlla vhleha ha ik onvtulo kashke: mikma Lefait vhleha hak osh nan isht atokowa vlhtipo holitopa ya ieshit atonna hi oke, im achi tok.

54. Mihma Islael im vila vhleha hut Chihowah yut Moses a im apesa tuk mak o, moma yumihchi tok.

\section{CHAPTA II.}

IHMA Chihowah yvt Moses micha Alon a im anumpuI lit,

2. Islael im vlla vhleha hatak puta kvt ilap $\underline{i}$ nahvta shali, ㅌki in chuka achvfa $\underline{\underline{i}}$ nahuta aiyukali kak o abinancha hi oke: okla moma im vlhtipo holitopa i hopakechit afolublit a binancha hi oke.

3. Mikma i hushi akochaka pilla hak o Chutah $\underline{\mathrm{i}}$ nalivta shali ai achvfa kvt okla lana moyuma kvt abinancha hi oke; mikma Amminatab ushi Nahshon ak osh. Chutah im vlla vhleha hạ $\underline{\underline{i}}$ kvpituni ha chi hoke.

4. Mihma in tushka vhleha holltina kvt tahlepa sipokni pokoli untuklo akucha ushta cha tahlepa hannali tok.

5. Mikma yumma pit iakaiyut binanchi kuto Issaka apeh. lichi kak a hi oke: mikma Sua ushi Nehlaneel ak osh Issaka im vlla vhleha ha $\underline{\underline{i}}$ kvpituni ha chi hoke.

6. Mihma in tushka vhleha hot holhtina kut tahlepa sipokni pokoli tahlapĩ akucha ushta cha tahlepa ushta aiena tok.

7. Yohmi ma Sebulun apehlichi kak a tok; mihma Helon ushi Eliab ak osh Sebulun im vlla vhleha ha $\underline{\mathrm{i}}$ kupituni ha chi hoke.

8. Mihma in tushka vhleha hut holltina kut tahlepa sipokni pokoli tahlapi akucha untuklo cha tahlepa ushta aiena tok. 
9. Chutah i binah aiasha holhtina moma kvt okla moyuma kvt tahlepa sipokni achufa cha tahlepa sipokni pokoli untuchina tahlepa sipokni hannali micha tahlepa ushta aiena tok, ilvppa puta kak osh tikba iashke.

10. Oka mahli in tunnup a Luben in tushka vhleha mak osh a binanchi ya i nahvta shali a hikia chị hoke: mikma Luben im vlla vhleha $\underline{i}$ kvpituni vt Sheteu ushi Elisu ak a hi oke.

11. Mihma in tushka vhleha micha a holhtina kvt tahlepa sipokni pokoli ushta akucha hannali cha tahlepa tahlapi tok.

12. Mikma apontut binanchi kvt Simeon apehlichi ak a chi hoke; mikma Simeon im vlla vhleha i kupituni ut Sulishattai ushi Shelumiel ak a chi hoke.

13. Mikma in tưshka vhleha micha holhtina kvt tahlepa sipokni pokoli tahlapi akucha chakkali cha tahlepa tuchina tok.

14. Yvmmakma Kat apehlichi ak a chị hoke, mikma Kat ushi vhleha 1 kupituni vt Leuel ushi Eliasaf ak a chị hoke.

15. Mihma in tushka vhleha micha holhtina tuk ut tahlepa sipokni pokoli ushta akucha tahlapi micha tahlepa hannali cha pokoli tahlapi tok.

16. Luben $\underline{\mathrm{i}}$ binah ha aiashvt holhtina tuk vt okla moma kvt tahlepa sipokni tahlepa achvfa tahlepa sipokni pokoli tahlapi akucha achvfa, micha tahlepa ushta cha pokoli tahlapi tok: micha iluppa puta kak osh aya atukla yosh wakaya hi oke.

17. Yohmikma okla moma im vlhtipo holitopa yvt wakayvt Lefait vhleha i binah, binah iklunna aiasha tuk aienvt ia chi hoke; kaniohmit binachi tuk vt moma hosh ahikia aiyukali, micha i nahvta shali aiyukali hielit iashke.

18. Hushi ai okatula in tunnvp a Eflaim in tushka vhleha mak osh $\underline{i}$ binah nahuta shali vt hikia hi oke : mikma Eflaim im vlla vhleha ha $\underline{\mathrm{i}}$ kupituni vt Ammihut ushi Elishama ak a chi hoke.

19. Mihma in tushka vhleha, micha holhtina tuk vt tahle. pa sipokni pokoli ushta cha tahlepa tahlapi tok. 
20. Mikma apotoa kut Manasseh apehlichi ak a chi hoke, mikma Manasseh im vlla vhleha ha $\underline{\underline{i}}$ kvpetuni vt Petahsu ushi Kamaliel ak a chi hoke.

21. Micha in tushka vhleha, micha holhtina tuk vt tahlepa sipokni pokoli tuchina akucha tuklo cha tahlepa tuklo tok.

22. Yohmikma Benchamin apehlichi ak a chi hoke; mikma Benchamin im vlla vhleha i kvpituni vt Kiteoni ushi Abitan ak a chi hoke.

23. Micha in tushka vhleha, micha holhtina tuk vt tahlepa sipokni pokoli tuchina akucha tahlapi cha tahlepa ushta tok.

24. Eflaim i binah hut holhtina moma, okla moma kvt tahlepa sipokni tahlepa achvfa, tahlepa sipokni untuchina cha tahlepa achufa tok; micha aya atuchina kak o hielit ia chi hoke.

25. Tan $\underline{i}$ binah nahvta shali vt falummi in tunnup im ok la aiasha bilika hikia chi hoke : mikma Tan im vlla vhleha i kvpituni vt Ammishattai ushi Ahiesa yak a chi hoke.

26. Mihma in tvshka vhleha, micha holhtina kvt tahlepa sipokni pokoli hannali akucha tuklo cha tahlepa untuklo tok.

27. Mikma apotout binachi kut Asha apehlichi ak a chi hoke, mikma Asha im vlla vhleha i kupituni vt Oklan ushi Pakiel ak a chi hoke.

28. Mihma in tvshka vhleha, micha holhtina tuk vt tahlepa sipokni pokoli ushta akucha achvfa cha tahlepa tahlapi tok.

29. Yummakma Naftali apehlichi ak a chi hoke; micha Naftali im vlla vhleha i kupituni vt Enan ushi Ahila ak a chi hoke.

30. Mihma in tushka vhleha, micha holhtina tuk vt tahlepa sipokni pokoli tahlapi akucha tuchina cha tahlepa ushta tok.

31. Tan i binah aiashvt holhtina moma kvt tahlepa sipokni tahlepa achvfa tahlepa sipokni pokoli tahlapi akucha untuklo cha tahlepa hannali tok: yummuto i nahvta ieshit ashaka fehna aya chi hoke. 
32. Islael im vila vhleha ilvppa puta kak osh iki in chuka achufa vlhpisa hosh holhtina tok. Okla moma binah puta ka aiashvt holhtina tuk moma kvt tahlepa sipokni tahlepa hannali, tahlepa sipokni tuchina cha tahlepa tahlapi pokoli tahlapit aiena tok.

33. Amba Chihowah yut Moses $\underline{a}$ im apesa tuk mak $\underline{o}$, Lefait vhleha hvto Islael im vlla vhleha takkla ik holhtina tok.

34. Mihma Chihowah yut Moses a im apesa tuk mak $\underline{\underline{o}}$, moma Islael im vlla vhleha hvt yvmihchi tok; yohmi hosh iki vhleha in chúka achufa vlhpisa hosh in chuka achufa aiyukali i nahvta shali abinachi, micha yohmi hosh wakayut ia beka tok.

\section{CHAPTA III.}

JUUPPA puta aiena kak osh, Chihowah yut Sinai nunih 1 ha Moses a im anumpuli nitak, Alon micha Moses a anoli holisso yoke.

2. Mihma hohchifo iluppa puta kak osh Alon ushi vhleha hatok. Natab vt vtta vmmona, micha Abihu, Eleasa micha Ihlama ak' atok.

3. Hohchifo iluppa puta kak osh Alon ushi vhleha yosh naholitompa isht asha ya chi bila ahvmmit yummot naholitompa isht asha vlhtoka isht ai ahashwa chị ho hullochi tok oke.

4. Atuk o Natab micha Abihu vt yakni haiaka Sinai ya Chihowah itikba ya luak inla yo im issa cha illi tok, mihmvt vlla ik im iksho ki tok; mihma Eleasa micha Ihlama itatuklo kvt iki Alon itikba ya naholitompa isht vtta vlhtoka ya isht ai ahashwa tok.

5. Mihma Chihowah yvt Moses a im anumpulit,

6. Lefai apehlichi yo bilika isht ishla cha, naholitompa isht vtta Alon itikba ya isht ish onashke; yohmi na yummak o nana isht im ahantashke.

7. Micha nan isht im ai vtta ya yumihinchit, micha okla moma nan isht im ai vita, okla moma im vlhtipo holitopa 
itikba ya a yumihinchit, vlhtipo holitopa isht ai ahanta ya yumihinchashke.

8. Micha okla moma im vlhtipo holitopa nan isht toksvli puta ka ieshit Islael im vlla vhleha nan isht im aivtta yumihinchit vlhtipo nan isht aiahanta ya yumihinchaske.

9. Lefait vhleha hạ, Alon micha ushi vhleha hak o ish imashke : Islael im vlla vhleha ha akohchit mominchit ima li hatuk oke.

10. Mikma Alon micha ushi vhleha ha ish atokoli na, naholitompa isht vtta vlhtoka ya isht ahashwa chi hoke : mikma hatak inla yosh bilika vlakmvt illi pulla chi hoke, achi tok.

11. Micha Chihowah yut Moses a im anumpulit,

12. Micha vno vt yakeh ! vno vt Islael im vlla vhleha ha vttahpi ishki im oshuto tiwi puta ya atobbichit Islael im vlla vhleha ha akohchit Lefait vhleha hak o ishe lishke; yohmi hoka Lefait vhleha hvt vmmi ha chi hoke.

13. Uttahpi moma kvt vmmi hokvt; Echip yakni ya vttahpi moma ka vbit tahli li tok nitak mut Islael a hatak, micha nanvlhpoa aiena vttahpi puta ka hullochit il ai ishi il tok okvt; ummi ha chi hoke. Chihowah sia hoke, achi tok.

14. Micha Chihowah yut yakni haiaka Sinai ya Moses a im anumpulit,

15. Lefai im vlla vhleha ha iki in chuka achvfa ahohchifot iakaiyvt ilap im iksa ibafohkvt ish hohtenashke: nakni puta hushi achvfa vttut vba maya kak o ish hohtenashke, achi tok.

16. Mihma Moses vt Chihowah yvt im apesa tuk im anumpa hatuk mak o hohtena tok.

17. Mihma Lefai ushi vhleha hohchifo hut iluppak atok; Keshom micha Kohalh micha Melali ak atok.

18. Mikma Keshom ushi vhleha ahohchifot in chuka achufa yvt Libni micha Shimei iluppak atok.

19. Mikma Kohalh ushi vhleha in chuka achvfa yvt Amlam micha Iseha, Heblon micha Ussiel, iluppak atok.

20. Mikma Melali ushi vhleha in chuka achufa yut Mahli 
micha Mushi ak atok; Iluppa puta kak osh iki vhleha in chuka achvfa vlhpisa hosh Lefait vhleha in chuka achvfa hatok.

21. Keshom a chuka achifa Libnait, micha chuka achvfa Shimait ak atok, Keshomait vhleha hot chuka achvfa ilvppak atok.

22. Yvmmut nakni moma holhtina holhtina tuk vt hushi achvfa ai vhli vttut vba maya holhtina mvt, tahlepa sipokni untuklo cha tahlepa tahlapi tok.

23. Keshomait vhleha chuka achvfa vt hvshi ai okvtula pilla vlhtipo holitopa ashaka yak o binacha hi oke.

24. Keshomait vhleha iki in chuka achvfa $\underline{i}$ shahli vt Lael ushi Eliasaf ak a hi oke.

25. Micha Keshom ushi vhleha hvt okla moma im vlhtipo ya nan isht aiahanta yvt, vlhtipo holitopa, micha vlhtipo, yumma isht ompoholmo, micha okla.moma im vlhtipo okhissa a takali,

26. Micha wanuta natakali puta, micha wanuta okhissa yummut vlhtipo holitopa micha alta bilika fullota natakali, micha yumma im isht talakchi puta, micha nan isht aiahanta puta iesha chi kak oke.

27. Mikma Kohalh a chuka achufa Amlamait micha chuka achvfa Isekalait, micha chuka achufa Heblonait micha chuka achufa Ussielait: iluppa puta kak osh Kohalhait chuka achvfa puta hatok.

28. Nakni moma hushi achufa ai vhlit vba mayvt holhtina kvt tahlepa sipokni untuchina cha tahlepa hannali hosh vlhtipo holitopa ya atonit ieshi tok.

29. Kohalh ushi vhleha in chuka achvfa puta kvt oka mahli pilla vlhtipo apotoa ho binacha hi oke.

30. Kohalhait vhleha chuka chufa puta ka iki in chuka achvfa i shahli vt Ussiel ushi Elisafan ak achi hoke.

31. Micha nana aiatoni ieshi kvt itombi holitopa, micha ai impa, micha pula ai o hikia, micha alta puta, micha nan isht toksuli nan aivlhto vlhtipo holitopa aiasha, micha natakali, micha nan isht toksuli puta kak a hi oke.

32. Mikma naholitompa isht vtta Alon ushi Eleasa yak 
osh Lefait vhleha $\underline{\mathbf{i}}$ shahli yạ $\underline{\mathbf{i}}$ shahli yạ chị hoke, micha vlhtipo holitopa apesuchi vhleha yo apistikela chị hoke.

33. Melali ya Mahlait in chuka achuta, micha Mushait in chuka achufa ak atok. Iluppak osh Melali in chuka achufa ya tok.

34. Mihma hushi achvfa vttvt vba mayvt nakni moma holhtina mak osh holhtina tuk vt tahlepa sipokni hannali, cha tahlepa tuklo tok.

35. Mikma îki in chuka achvfa Melali in chuka achvfa ya Abihail ushi Suliel ak osh i shahli ya chi hoke. Iluppa puta

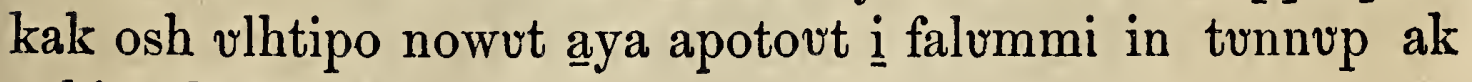
o binacha hi oke.

36. Melali ushi vhleha nana apistikelit ieshi vto chuka nowvt aya im iti shima, micha im okhowatakvchi, micha in tonik, micha ai o hikia, micha nana aivlhto moma, micha nana isht toksuli moma,

37. Micha tonik puta wanuta apakfoyupa micha ai o hikia moma, micha isht ishtuffena puta, micha isht talakchi puta aiena kak a chị hoke.

38. Amba chuka nowvt aya itikba hushi akochaka pilla okla moma im vlhtipo hvshi akocha pilla ho binachi hokvto; Moses micha Alon micha ushi vhleha aiena kak achi hoke, yummut vlhtipo holitopa ya atonit ieshit Islael im vlla vhleha ha pehlicha chị hoke, hatak inla yosh bilika vla hokvto illi pulla chi hoke.

39. Lefait vhleha holhtina moma, Chihowah yvt apesa na chuka achvfa puta Moses micha Alon ita tuklo kvt hohtena tuk a nakni moma hushi achvfa ai vhli vttut vba maya kvt tahlepa sipokni pokoli tuklo akucha tuklo tok.

4.0. Mihma Chihowah yvt Moses a, Islael im vlla vhleha nakni vttahpi moma hushi achvfa ai vhli vttvt vba maya ka ish hohtena cha hohchifo holhtena ka ish ishashke.

41. Micha Islael im vlla vhleha ai iti takla ya tikba vtta ak oh chatuk a atobbichit Lefait vhleha hak o is sum isha chi hoke, (Chihowah sia hoke,) micha Islael im vlla vhleha i wak ai iti takla tikba cheli ak oh chatuk a atobbichit Lefait vhleha $\underline{i}$ wak ak a chi hoke, im achi tok. 
42. Mihma Chihowah yut im apesa tuk mak $\underline{\text {, }}$ Moses vt Islael im vlla vhleha tikba vtta moma ka hohtena tok.

43. Mihma nakni tikba vtta moma hushi achvfa ai uhlit vba maya hohchifot holhtina holhtina tuk vt tahlepa sipokni pokoli tuklo akucha tuklo cha tahlepa tuklo pokoli untuklo akucha tuchina tok.

44. Mihma Chihowah yot Moses a im anumpulit,

45. Islael im vlla vhleha tikba vtta moma ak oh chatuk $\underline{\text { a }}$ atobbichit Lefait vhleha hak $\underline{\underline{0}}$, micha $\underline{\underline{i}}$ wak ak oh chatuk a atobbichit Lefait vhleha $\underline{i}$ wak ak 0 ish ieshashke; mihma Lefait vhleha hak osh vmmi hà chị hoke: Chihowah sia hoke.

46. Micha falumminchit chumpa chi Islael im vlla vhleha tikba vtta ai achufa tahlepa tuklo cha pokoli untuklo akucha tuchina yummvt Lefait vhleha i shahli kâ,

47. Noshkobo vlhpisa hô shekel tahlapi bieka ho ish isha chi hoke, vlhtipo holitopa ai vlhpisa shekel vlhpisa ho ish isha chi hoke, shekel vt kelah pokoli tuklo hoke.

48. Micha vlhkocha falvmminchit chumpa he tuk $\mathrm{o}$ ishit chumpa tvli holisso yash Alon micha ushi vhleha hak o ish imashke, achi tok.

49. Mihma Moses vt falvmminchit ishit chumpa tvli holisso yash Lefait vhleha hvt falvmminchit chumpa tuk vlhkocha yash im ishi tok.

50. Islael im vila vhleha tikba vtta hak o tvli holisso im ai eshi tok; vlhtipo holitopa ai vlhpisa shekel iakaya hosh shekel tahlepa sipokni achvfa cha tahlepa tuchina pokoli hannali akucha tahlapi tok.

51. Mihma Chihowah im anumpa mak o Chihowah yvt Moses a im apesa hatuk mak o falumminchit chumpa tuk vlhtoba kash ishi cha, Alon micha ushi vhleha aiena ka ima tok.

\section{CHAPTA IV.}

MHMA Chihowah hot Moses micha Alon a im anum-
pulit, 
2. Kohalh ushi vhleha Lefai ushi vhleha ai iti takla aiasha ka iki vhleha in chuka achufa vlhpisa, micha in chuka achvfa vlhpisa ho holhtina ka ish ishashke.

3. Afummi pokoli tuchina ai vhlit vba mayut afummi pokoli tahlapi ont ai vhlit okla moma $\underline{i}$ binah nana a toksuli isht ai ahanta chị hosh okla ho ibafohka moma kak ashke.

4. Ilvppak osh Kohalh ushi vhleha nana isht aiahashwa ya chi hoke, okla moma $\underline{i}$ binah anukaka, naholitopa moma i shahli ak achị hoke.

5. Atuk osh binah hut tikba iakma, Alon micha ushi vhleha aiena kut vla cha, isht ompoholmo on hlipia ya akuchi cha, nan isht atokowa afohka itombi holitopa ya isht ompohomashke.

6. Micha bacha hakshup isht ompoholmo yo isht om patali cha nantunna okchamali bieka ho om patali cha in tubi a apitta chi hoke.

7. Micha puska otuni im ai impa paknaka ya nantunna okchamali yo isht ompohomo cha, ampo, micha isht impa puta, micha ampo puta micha isht ompoholmo aiena ompohoma chi hosh on ashacha chi hoke; mikma puska talaya bilia yot on aiasha chi hoke.

8. Micha nantunna tishepa ya isht ompohoma cha, yưmma bacha hakshup isht ompoholmo yo isht ompohomokmut in tubi a apitta chi hoke.

9. Micha pvla ai o hikia, micha pula i bila ai vlhto, micha in tobaksi isht kiseli, micha i pula tuptua ai vlhto, micha bila ai vlhto puta im isht ai ahanta chatuk"a nantunna okchamali ho ishi cha isht ompohoma chi hoke.

10. Micha yumma, micha nan ai vlhto moma ka bacha hakshup sht ompoholmo anukaka ashachi cha, iti fabussa ya on talala chi hoke.

11. Micha tuli holisso lakna alta ya nantunna okchamali yo om patali cha, bacha hakshup isht ompoholmo yo isht ompohomokmut, in tubi ya apitta chi hoke.

12. Micha nan isht pilesa puta vlhtipo holitopa ya ishit atoksvli chatuk moma ka eshi cha nantunna okchamali ho 
apitta cha, bacha hakshup isht ompoholmo cha iti fabussa ya on talala chi hoke.

13. Micha hituk chubbi alta ya on asha ka ishit kanchi cha nantunna humma ho om patala chị hoke.

14. Micha nan aivlhto puta, isht toksvli chatuk puta, na balama a hukmi, micha nipi isht okhawi, micha luak isht pehli, micha ampo mahaia chipunta, micha alta $\underline{i}$ nan aivlhto puta aiena ka on ashacha chi hoke, micha bacha hakshup isht ompoholmo yo isht ompohoma chị hoke, micha in tubi apitta chi hoke.

15. Atuk o Alon micha ushi vhleha aiena kvt vlhtipo holitopa, micha vlhtipo holitopa $\underline{i}$ nan ai vlhto puta aiena ka ompohomot ont im vlhtaiyaha na, binah hvt iakma, yohmikma Kohalh ushi vhleha hut shala chi hosh vla chi hoke; amba nana holitopa ya potola he keyu, yohmikmot illa chi hoke. Iluppa puta kak osh okla moma i binah aiasha kvt Kohalh ushi vhleha i shapoh ha chi hoke.

16. Mihma naholitompa isht vtta Alon ushi Eleasa nan vlhtoka kut, pula ha chi bila, micha na balama champuli, micha nitak moma ilhpak im issa, micha ahummi bila, micha binah moma, micha vlhtipo holitopa micha nana aiasha moma, micha aivlhto aiasha aiena ka apistikela chi hoke, achi tơk.

17. Mihma Chihowah yot Moses, micha Alon a im anumpulit,

18. Kohalhait vhleha in chukachvfa puta apehlichi ka Lefait vhleha ai ishit tvblit hveh ik kancho kashke.

19. Amba iluppak o ish i yumihchi na, naholitompa moma i shahli bilika onakmvt okchaya cha ik illo kashke; Alon micha ushi vhleha aiena kut iba chukowa cha, isht ai vtta, micha i shapoh alyuka ka im anola chi hoke.

20. Yohmi kia naholitopa puta kvt ompoholmo takla ka chukowvt pisa he keyu, yohmikmvt illa hi oke, achi tok.

21. Mihma Chihowah yut Moses micha Alon a im anumpulit,

22. Keshon ushi vhleha ha iki vhleha in chuka achvfa puta hlopullit chukachvfa vlhpisa ho holhtina ka ish ishashke. 
23. Afummi pokoli tuchina ai vhlit vba mayvt afummi pokoli tahlapi ont vhli kak o ish hohtenashke; nan isht ahanta chi hosh okla moma $\underline{\mathrm{i}}$ binah atoksuli isht ahanta chi hosh chukowa moma kak a chi hoke.

24. Nan isht ahanta iluppak osh Keshonait in chukachvfa puta isht ahanta, micha i shapoh ha chi hoke.

25. Micha binah nantunna takali puta micha okla moma i binah, micha im isht ompoholmo, micha bacha hakshup isht ompoholmo paknaka ompoholmo tuk, micha okla moma i binah okhissa ya in takali,

26. Micha wanuta ya in takali puta, micha wanuta $\underline{i}$ ho-

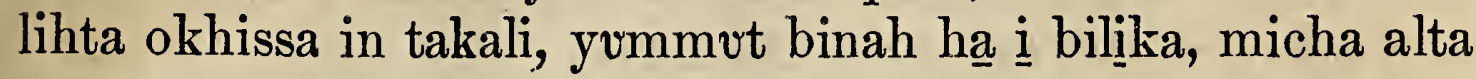
ya bilika apakfoyupa, micha im isht talakchi, micha isht pilesa 1 moma, michä nan im ikbi tuk moma kak a hi oke, yvmohmi hosh isht ahantashke.

27. Alon micha ushi vhleha aiena kak osh Keshonait ushi vhleha nan im isht aiahanta he moma, i shapoh moma micha im isht aiahanta he moma aiena kot im vlhpisashke i shapoh, nan isht aiahanta moma hush im apesa hi oke.

28. Tluppak osh Keshonait ushi vhleha in chuka achufa puta okla moma i binah ha isht aiahanta ya chi hoke; mihma naholitompa isht vita Alon ushi Ihlama ak osh pehlicha chil hoke:

29. Melali ushi vhleha hano iki vhleha in chuka achvfa, in chuka achufa ulhpisa ho ish hohtenashke:

30. A fumm pokoli tuchina ai vhlit vba mayut afummi pokoli tahlapi ont uhli nan isht aiahanta okla moma i binah atoksuli isht aiahanta chi hosh iba chukowa ya ish hohtenashke.

31. Mikma okla moma i binah nan im isht aiahanta moma I sthepoh aiena kvt illuppak achi hoke; binah im iti busha, micha im iti okhowat kvchi micha in tonik, micha ai o hikia im puta,

32. Micha wanuta apakfoyupa tonik puta, micha im ai o hikia puta, micha im isht ahonahla puta, micha im isht. talakchi puta, nan im isht pilesa puta; micha nan im isht aia- 
hanta puta aiena kak achi hoke; micha $\underline{\mathbf{i}}$ shapoh ieshi nan isht pilesa puta ka hochifo hosh ish hohtena chi hoke.

33. Nan isht ahanta iluppak o naholitompa isht vtta Alon ushi Thlama yut pehlichi ho Melali ushi vhleha in chuka achufa puta kut okla moma i binah nan im isht aiahanta moma ka isht ahashwa chi hoke, achi tok oke.

34. Mihma Moses micha Alon micha okla moma 1 shahli aiena kvt Kohalhait a in chuka achvfa vlhpisa ho micha iki vhleha in chuka achufa vlhpisa ho hohtena tok.

35. Afummi pokoli tuchina ai vhlit vba mayut afummi pokoli tahlapi ont vhli isht aiahanta okla moma i binah atoksuli iba chukowa tuk puta kak a tok.

36. Mihma in chuka achvfa vlhpisa ho holhtina kvt tahlepa sipokni tuklo cha tahlepa untuklo pokoli tahlapi tok.

37. Ilvppa puta kak osh Kohalhait vhleha in chuka achvfa puta, okla moma i binah atoksvli isht aiahanta vlhpesa, Chihowah hut Moses a tohnvt apesa na, Moses micha Alon ita tuklo kvt hohtena na holhtina tok.

38. Mikma Keshon ushi vhleha in chuka achufa puta hlopullit iki in chuka achvfa vlhpisa hosh,

39. Afummi pokoli tuchina ai vhlit vba mayvt afummi pokoli tahlapi ka ont vhli nan isht aiahanta okla moma i binah atoksuli iba chukowa puta,

40. In chuka achvfa puta hlopullit iki in chuka achufa vlhpisa hosh holhtina tuk vt tahlepa sipokni tuklo cha tahlepa hannali pokoli tuchina tok,

41. Ilvppa puta kak osh Keshon ushi vhleha in chuka achvfa puta okla moma i binah ha nan isht aiahanta hịla moma kạ, Chihowah hot apesa tuk mak $\underline{\text {, Moses micha }}$ Alon ita tuklo kvt hohtena na holhtina tok.

42. Mihma Melali ushi vhleha in chuka achufa puta holhtina kvt chuka achvfa puta hlopullit iki vhlêha in chuka achvfa vlhpisa hosh,

43. Afummi pokoli tuchina ai vhlit vba mayut afummi pokoli tahlapi ont vhli nan isht ahanta okla moma i binah atoksvli iba chukowa puta, 
44. In chuka achvfa puta vlhpisa hosh holhtina tuk vt tahlepa sipokni tuchina cha tahlepa tuklo tok.

45. Ilvppa puta kak osh Melali ushi vhleha in chuka achvfa puta, Chihowah hvt Moses a tọhnot apesa tuk mak $\underline{o}$, Moses micha Alon ita tuklo kvt hohtina na holhtina tok,

46. Lefait vhleha moma ka Moses micha Alon micha Islael i shahli vhleha aiena kvt in chuka achvfa puta vlhpisa, micha iki vhleha in chuka achufa puta vlhpissa ho hohtena na holhtina tok.

47. Afummi pokoli tuchina ai vhlit vba mayut afummi pokoli tahlapi ont vhli moma hosh nan isht aiahanta isht vtta chị, micha okla moma i binah shapoh ai asha ka isht vtta chi hosh vla tuk vt,

48. Holhtina tuk vt tahlepa sipokni untuchina cha tahlepa tahlapi pokoli untuchina tok.

49. Chihowah hvt Moses a tohnot apihisa tuk mak $\underline{o}$, isht aiahanta aiyukali vlhpisa hosh micha $\underline{\mathbf{i}}$ shapoh aiyukali vlhpisa hosh holhtina tok, yakohmi hosh Chihowah yut Moses a im apesa tuk mak o holhtina tok.

\section{CHAPTA V.}

MIHMA Chihowah hot Moses $\underline{a}$ im anumpulit, 12. Islael im vlla vhleha hvt lepa puta, micha hayihchchi yunahanli puta, micha kuna hosh illi isht liteha tuk puta aiena ka binah ha akochicha chi ka ish im apesashke.

3. Nakni micha tek aiena hush kohchashke; binah kohchit kocha ya isht hưsh onashke; yumohmi hosh $\underline{\underline{i}}$ binah ai iklunna ajahanta li ka ik litehlo kashke, achi tok,

4. Mihma Islael im vlla vhleha hot yumihchi tok; micha binah ha kohchit kocha jsht ona tok; Chihowah yut Moses

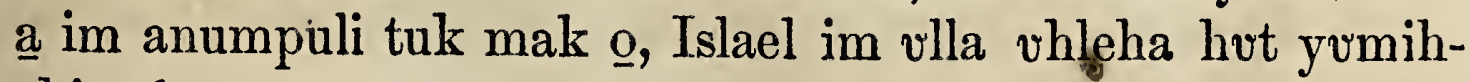
chi tok.

5. Mihma Chihowah hvt Moses a im anumpulit,

6. Islael im vlla vhleha ha ish im anumpulashke, Hatak okma, ohoyo hokma nana hosh nanashvchi hatak ayumoh- 
mi chatuk a yumohmit Chihowah $\underline{1}$ yashoba yumihchi cha hatak yvmmvt onvtoyulakma.

7. Yvmohmikma nana ashvchi yumohmi tuk vt okla il ai anola hi oke, micha $\underline{1}$ yoshoba tuk a nana hatuk o $\underline{\mathrm{i}}$ falumminchi cha ishit tahlapi ho im apotola hi oke.

8. Amba $\underline{i}$ yoshoba tuk vt $\underline{i}$ falummincha he vlhpesa $\underline{i}$ kanomi vt iksho hokmuno, yoshoba tuk vt Chihowah yak o $\underline{i}$ falumminchit naholitompa isht vtta ya imashke, nan ashvchi tuk vt isht $\underline{i}$ kashofa chi kvt isht vlhtoba chukfi nakni aiena ho ima hi oke.

9. Micha nan im issa puta Islael im vlla vhleha i nana holitopa puta moma naholitompa isht vtta isht im vla kut immi ha hi oke.

10. Micha hatak puta i nahullochi puta kvt immi ha chi hoke; hatak nana hosh nana ho naholitompa isht vtta ya imakma, immi ha chi hoke; achi tok.

11. Mihma Chihowah yut Moses a im anumpulit,

19. Islael im vlla vhleha im anumpulit, Hatak kvna kia tekchi yosh naksika onvt nayoshoba i yoshobakma,

13. Yohmikma hatak vt iba tushkit itin lumakakma, $\underline{i}$ hatak vt ik peso, yohmi na ik otano, mihma liteha, mihma i sanalit atokola he vt iksho, micha yumohmi ka ik peso,

14. Mikma hatak ash chukvsh nuktvhla yvt onvtoyula cha, tekchi yash $\underline{i}$ nuktvhla, mikma ohoyo hot litehakma, keyukmut chukvush nuktvhla yvt onvtuyula cha tekchi yash $\underline{\underline{i}}$ nuktuhla kia ohoyo hvt liteha keyu,

15. Yohmikma hatak ash osh tekchi ya naholitompa isht vtta isht im ona he, mikmvt ohoyo hash nan im issa bali pushi efah ishit pokoli ho isht im vlashke; bila on hlatubla he keyu, micha filakinsen onocha he keyu; yummut nuktvhla nan im issa, isht ithaiyana nan im issa yosh nan ashvchi ithunanchi yoka.

16. Mikma naholitompa isht vtta yvt bilika isht vlvt Chihowah itikba binilecha hi oke.

17. Micha naholitompa isht vtta yvt oka hullochi ya lukfi ampo vnit isha hi oke, micha lukfi pushi vlhtipo holitopa asha ka ishi cha, oka yash una hi oke. 
18. Micha naholitompa isht vtta yvt ohoyo hash Chihowah itikba binilechit, ohoyo noshkobo ompoholmo im ai ishi cha isht ithaiyana nan im issa yash, yummot nvktuhla nan im issa yash ibbak fohka hi oke, micha naholitompa isht vtta yut oka homi, nan ik achukmo tobachi yut ibbak fohka chi hoke.

19. Micha naholitompa isht vtta yvt anumpa kvllo anumpulit, ohoyo hash atokolit $\underline{\mathrm{i}}$ mihut, Hatak nana kia chiba tushki tuk keyu, micha naksika onvt chi hatak ak keyu, amba inla yo na liteha iti yumohmi ya chik yumohmo tuk mut oka homi ilvppa nan ik achukmo tobachi ya nan isht ik chi kaniohmo kashke.

20. Amba naksika onvt inla ho chi hatak ak keyu ho ish im ona tuk osh chi litehakma, micha chi hatak keyu, hatak nana hosh chiba tushki tuk ma,

21. Yohmikma naholitompa isht vtta yash osh ohoyo hash anumpa kvllo anumpulit atokolit i miha hi oke, micha naholitompa isht vtta yvt ohoyo hash, Chihowah yvt chiyubi ya shuachikmut micha chikfoka ya shatublichikmvt chim okla ai ititakla ya nan ik achukmo onvtonla, anumpa kullo onvtonla Chihowah yut chikbashke.

22. Mikma nan ik achukmo tobachi oka ilupput chikfoka ya shatublicha chị hosh micha chiyubi ya shuacha chi hosh chi takoba ya chukowa chị hoke, im acha hi oke. Mikma ohoyo hash ot Amen, Amen, acha hi oke.

23. Mikma naholitompa isht vtta yvt nan ik achukmo iluppa puta ka holisso ho aholissochi cha oka homi yash ishit kashoffa hi oke.

24. Micha ohoyo hash nan ikachukmo tobachi oka homi ya ishkochecha hi oke, mihma nan ikachukmo tobachi oka yvt chukowakmvt homa hi oke.

25. Yohmikma naholitompa isht vtta yvt nuktuhla nan im issa ohoyo ibbak foyuka kash ishi cha nan im issa yash Chihowah itikba ya wali cha alta onöchit im issa hi oke.

26. Mihma naholitompa isht vtta yvt nan isht ithaiyana nan im issa yash, ibbak alota achvfalit ishi cha, alta onochit 
hukma hi oke, yumihchi ha yosh oka yash ohoyo hash $\underline{0}$ ishkochecha hi oke.

27. Atuk o oka ya ishkochechikma, yohmikma yakohma hi oke, liteha tuk okma, micha $\underline{\mathrm{i}}$ hatak $\underline{\mathrm{a}} \mathrm{i}$ yoshoba tuk okma nan ik achukmo tobachi oka yash osh chukowakmut homa hi oke, micha ikfoka yvt shatublikma iyubi vt shua hi oke, mikmvt ohoyo hash osh im okla ai iti takla ya nan ikachukmo onvtuyula yosh ahanta hi oke.

28. Amba ohoyo hash osh liteha hatuk keyu, amba kashofa hatuk okmvt yohmikmut nan ik $\underline{\mathrm{i}}$ kaniohmo ka he, micha vlla shala hi oke.

29. Ohoyo hut i hatak ak keyu inla ho im onvt naksika ona cha litehakma nuktuhla nan vlhpisa iluppak a hi oke.

30. Keyukmut chukvsh nuktuhla vt onvtoyula cha tekchi ya i nuktuhlakmvt, Chihowah itikba binilecha na, naholitompa isht vtta yvt nan vlhpisa iluppa moma ka onocha hi oke.

31. Yohmikma hatak ash osh nan ashvchi ik onvtoyulo ho, ohoyo ilvppak osh nan ashvchi vt onvtoyula hi oke, ish im achashke, im achi tok.

\section{CHAPTA VI.}

- M IHMA Chihowah yout Moses a im anumpulit,

I 2. Islael im vlla vhleha ha im anumpulit, Hatak okmá, ohoyo hokmá, nana hosh ila filummichit Chihowah ya il im issa chị hosh Nasalait anumpa il onochi ya anumpa il onochikmut,

3. Oka paki micha nan ishko kvllo ya ila filummichi cha, oka paki hauwushko, micha nan ishko kullo hauwushko ya ik ishko ka hi oke, micha oka paki okchi nana kia ik ishko kashke, micha paki nuna, keyukmot paki shila yoh kia ikpo kashke.

4. Ile filummichit" ahanta nitak moma ka nana hosh pakvepi atohomba, nihi vttut hakshup ont vhli atoba ka vpa he keyu hoke. 
5. Ile filvmmichit anumpa ilonochit ahanta nitak moma ka nutakhish isht vlmo nana kia noshkobo ha ik onvtoyulo ka hi oke, ile filvmmichi Chihowah ya im il issa tok nitak vt ik taho kisha ka holitopa cha noshkobo pashi ya apoacha hi oke.

6. Ile filvmmichit Chihowah ya im il issa nitak tạkla kvt nipi illi ya bilika ik ono ka hi oke.

7. Iki atuk oh kia, keynkmvt ishki atuk oh kia, itibapishi atuk oh kia, keyukmot in tek atuk osh illi kia, isht ile litehla he keyu hoke, Chitokaka ya im ile hullochi tuk vt noshkobo ha onvtoyula hoke.

8. Ile filummichit ahanta nitak moma hokvt Chihowah ya i holitopa hoke.

9. Atuk o yakosi itin takla fehna ho bilika ho hatak vt illi na ile hullochi noshkobo onvtonla kash litehli tukmvt ile kashoffi nitak akinli hosh noshkobo hạ shâfa hi oke, nitak isht untuklokma shafa hi oke.

10. Atuk osh nitak isht untuchinakma pvchi yoshoba tuklo, Keyukmut puchi himita tukloho na holitompa isht vtta isht im onvt okla moma i binah okhissa ya isht ona hi oke.

11. Mikma naholitompa isht vtta yvt achvfa ka nan ashvchi im issa yo im issakmut, achvfa ya hukmit im issa yo im issa cha $\underline{i}$ kạshoffashke, illi yo ishit yoshoba hoka, micha nitak yummakinli ho noshkobo ha hullocha hi oke.

12. Mikma ile filummichit ahanta he nitak puta ka Chihowah ya $\mathrm{i}$ hullocha hi oke; micha yoshoba tuk im issa ya chukfi ushi afưmmi vmmona yo isht ona hi oke; Yohmi kia nitak tikba hatuk vto kvnia chi hoke, ile filvmmichi tuk vt liteha hatuk oka, achi tok.

13. Micha iluppak osh Nasalait $\underline{\mathrm{i}}$ nanvlhpisa hoke; ile filvmmichi nitak vt ont tahakma, okla moma i binah okhissa ya isht vla hi oke.

14. Mikma Chihowah nan im issut hukmit im issa ya chukfi ushi nakni afummi vmmona aiokpuloka iksho achvfa micha nanashvchi im issa yo chukfi ushi tek afummi vmmona ai okpuloka kvt iksho, achvfa micha iti nanaiyvchit im issa yo chukfi nakni aiokpuloka kvt iksho achvfa ho, 
15. Micha puska ik shatummo kishi achufa, bota tohbi lipehbi pvska lumbo bila itaiyoma, micha pvska ikshatummo tapuski bila ahama, micha ilhpak im issa, micha nan ishkot im issa aiena ho im issa hi oke.

16. Mikma naholitompa isht vtta yvt Chihowah itikba isht vla cha, nanashvchi im issa, micha hukmit im issa ya im issa hi oke.

17. Micha chukfi nakni ya itin nanaiyvchi im issa vbit im

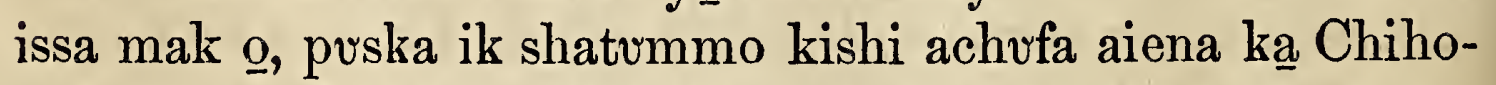
wah im issa hi oke. Naholitompa isht vtta yot ilhpak im issa micha nan ishko im issa ak kia im issa hi oke.

18. Mikma Nasalait ash osh ile filvmmichi noshkobo ha okla moma i binah okhissa ya a shafa hi oke, mikmvt ile filvmmichi noshkobo pashi yash luak yummvt itin nanaiyvchi im issa vbit isht aiokpvchi nutaka asha tuk a itvcha hi oke.

19. Mikma naholitompa isht vtta yvt chukfi nakni yash fullup honni ya , micha puska ikshatummo lumbo achufa họ, kishi ha akohchit, micha puska ik shatummo tapuski achufa aiena ho, ishi cha ile filummichi pashi vt vlmo ha yo ibbak fohka hi oke.

20. Micha naholitompa isht vtta yvt walit im issa yo Chihowah itikba ya walashke, iluppak okvt ikkishi wali, micha fullup wali aiena kvt naholitompa isht vtta ya $\underline{\mathbf{i}}$ hullocha hi oke, mikma i himmakmuno Nasalait ash ot oka paki ishka hinla hoke.

21. Ilvppak osh Nasalait yummut anumpa ilonochi tuk $\mathbf{i}$ nan vlhpisa hoke, micha ile filummichit Chihowah nan im issa, micha ibbak vt ishi tuk aiena hoke; anumpa ha il onochi tuk mak ash yakohmit ile filummichi nan vlhpisa iakaiyut yumihchi pulla hi oke, ish im achashke im achi tok:

22. Mihma Chihowah yut Moses a im anumpulit,

23. Alon micha ushi vhleha ha im anumpulit, yakohmichi hosh Islael im vlla vhleha ha yukpali anumpa ha hush onochashke.

24. Chihowah yut hvchi yukpalashke, micha hvchi halanlashke. 
25. Chihowah yvt i nashuka ya hvchi on tohwikelichashke; micha hvchi kanashke.

26. Chihowah yut $\underline{i}$ holhponayo hanta hvchi onochi cha a nuktaiyala hvch imashke, hush im achashke, ish achashke, im achi tok.

27. Micha sa hohchifo hạ Islael im vlla vhleha ha onocha hi oke, naikma yukpali la hi oke, achi tok.

\section{CHAPTA VII.}

AUK o yakohmi tok, Moses vt vlhtipo hạ hilechit taiA yahli cha, nan ahvmmit hullochit, micha nan isht pilesa aiasha moma, micha alta, micha nan aivlhto aiasha moma aiena ka nan ahummit bullochi tok, nitak akinli ka.

2. Islael $\mathrm{i}$ holitopa vhleha iki vhleha in chuka achvfa $\mathrm{i}$ noshkoboka, yummut apehlichi puta ka $\underline{\mathrm{i}}$ holitopa vhleha, micha holhtina tuk a pehlichi yatuk osh, im issa tok.

3. Micha nan im issa yot itichantlli ompoholmo hannali, micha wak toksuli auaktuklo ho Chihowah itikba ya isht vla tok, i holitopa tuklokma itichanvlli achvfa, mikma aiyukali ka wak achufa bicka tok, micha vlhtipo itikba isht vla tok.

4. Mihma Chihowah yut Moses a im anumṕulit,

5. Ish im ai ishashke, mikma okla moma $\underline{\underline{i}}$ binah ha isht in toksulashke, micha Lefait vhleha hak o hatak vt isht aiahanta aiyukali vlhpisa ho ish imashke.

6. Mihma Moses vt itichanvlli micha wak aiena kash ishi cha Lefait vhleha ha ima tok.

7. Itichanulli tuklo micha wak ushta aiena ka Keshon ushi vhleha hak o isht aiahanta vlhpisa mak o ina tok.

8. Micha itichanvlli ushta micha wak untuchina aiena ka Melali ushi vhleha hak o isht aiahanta vlhpisa mak o ima na, naholitompa isht vtta Alon ushi Ihlama ibbak foyuka tok.

9. Amba Kohalh ushi vhleha hano ik emo, vlhtipo holitopa ak 0 isht aiahanta ak osh immi hosh tahchi onochit shoyula chi hatuk oka. 
10. Mihma holitopa vhleha hut alta ya hullochit nan ahvmmi nitak ma nan im issa tok; holitopa vhleha hut nan im issa ya alta itikba ya ai im issa tok.

11. Mihma Chihowah yot Moses $\underline{a}, \underline{I}$ nan im issa ya alta

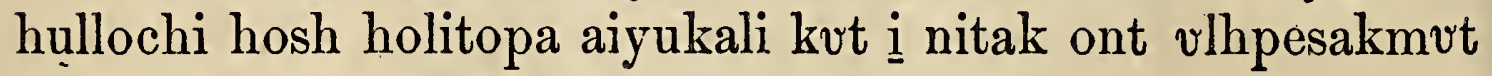
im issashke, im achi tok.

12. Atuk o nitak vmmona i nan im issa im issa kvit Chutah apehlichi ai achufa Amminatab ushi Nahshon ak atok.

13. Micha i nan im issa yut tuli huta ampo chito achufa, weki kvt shekel tahlepa achufa cha pokoli tuchina, tvli hvta ampo achufa shekel pokoli untuklo vlhtipo holitopa i shekel vlhpisa hatok; ita tuklo kvt bota tohbi lipehbi bila itaiyoma ilhpak im issa ya chi hosh alota bika tok.

14. Isht impa achvfa tvli holisso lakna shekel pokoli yosh na balama alota,

15. Hukmit im issa ya chi ka wak hobvk himita achufa, chukfi vlhpoa nakni achvfa, chukfi vlhpoa ushi afummi vmmona achufa;

16. Nan ashvchi im issa yut issi kosoma ushi achvfa,

17. Micha isht itinnanaiyuchi im issa ya chi ka wak hobak tuklo, chukfi nakni tahlapi, issi kosoma nakni tahlapi, chukfi ushi afummi ummona tahlapi aiena tok. Ilvppak puta kak osh Amminatab ushi Nahshon nan im issa yatok.

18. Nitak ătukla ka Sua ushi Nehlaneel Issaka ai achvfa hi olitopa ak osh im issa tok.

19. Micha i nan im issa yut tvli huta ampo chito achufa, weki kvt shekel tahlepa achufa cha pokoli tuchina, tvli huta ampo achufa shekel pokoli untuklo vlhtipo holitopa i shekel vlhpisa hatok ; ita tuklo kut bota tohbi lipehbi bila itaiyoma, ilhpak im issa ya chi hosh alota bika tok.

20. Isht impa achvfa tuli holisso lakna shekel pokoli hosh na balama alota;

21. Hukmit im issa ya chị ka wak hobvk himita achufa, chukfi nakni achvfa, chukfi ushi afummi vmmona achvfa;

22. Nan ashvchi im issa yut issi kosoma ushi achufa;

23. Micha isht itinnanaiyuchi im issa ya chi ka wak hobvk tuklo, chukfi nakni tahlapi, issi kosoma nakni tahlapi, chukfi 
ushi afummi ummona tahlapi aiena tok. Iluppa puta kak osh Sua ushi Nehlaneel nan im issa ya tok.

24. Nitak atuchina ka Helon ushi Eliab Sebulon im vlla vhleha holitopa ak osh im issa tok.

25. Micha i nan im issa yut tuli hvta ampo chito achufa, weki kut shekel tahlepa achufa cha pokoli tuchina, tuli huta ampo achufa shekel pokoli untuklo, vlhtipo holitopa i shekel vlhpisa hatok; ita tuklo kut bota tohbi lipehbi bila itaiyoma ilhpak im issa ya chi hosh alota bika tok.

26. Isht impa achufa tuli holisso lakna shekel pokoli hosh na balama alota;

27. Hukmit im issa ya chi ka wak hobuk himita achvfa, chukfi nakni achvfa, chukfi ushi afummi vmmona achvfa;

28. Nan ashvchi im issa yut issi kosoma ushi achvfa;

29. Micha isht itin nanaiyvchi im issa ya chi ka, wak hobvk tuklo, chukfi nakni tahlapi issi kosoma nakni tahlapi, chukfi ushi afummi vmmona tahlapi aiena tok. Ilvppak osh Helon ushi Eliab nan im issa ya tok.

30. Nitak ont ai ushta ma, Sheteu ushi Elisu, Luben im vlla vhleha i holitopa ak osh im issa tok.

31. Micha $\underline{1}$ nan im issa yut tuli huta ampo chito achufa, weki kvt shekel tahlepa achvfa cha pokoli tuchina tvli huta ampo achufa, shekel pokoli untuklo, vlhtipo holitopa i shekel vlhpisa hatok; ita tuklo kvt bota tohbi lipehbi bila itaiyoma ilhpak im issa ya chi hosh alota bika tok.

32. Isht impa achufa tuli holisso lakna shekel pokoli hosh na balama alota;

33. Hukmit im issa ya chị ka wak hobvk thimita achufa, chukfi nakni achvfa, chukfi ushi afummi vmmona achvfa,

34. Nan ashuchi im issa yut issi kosoma ushi achvfa; 35. Micha isht itin nanaiyvchi im issa ya chi ka, wak hobvk tuklo, chukfi nakni tahlapi, issi kosoma nakni tahlapi, chukfi ushi afummi vmmona tahlapi aiena tok, Ilvppak osh Sheteu ushi Elisu nan im issa ya tok.

36. Nitak ont ishit tahlapi ma Sulishattai ushi Shelumiel, Simeon im vlla vhleha 1 holitopa ak osh im issa tok.

37. Micha i nan im issa yut tuli huta ampo chito achufa, 
weki kvt shekel tahlepa achvfa cha pokoli tuchina, tvli hota ampo achufa shekel pokoli untuklo, vlhtipo holitopa i shekel vlhpisa hatok; ita tuklo kut bota tohbi lipehbi bila itaiyoma ilhpak im issa ya chi hosh alota bika tok.

38. Isht impa achufa tuli holisso lakna shekel pokoli hosh na balama alota;

39. Hukmit im issa ya chi ka wak hobvk himita achvfa chukfi nakni achvfa, chukfi ushi afummi vmmona achvfa,

40. Nan ashuchi im issa yut issi kosoma ushi achvfa,

41. Micha isht itin nanaiyvchi im issa ya chị ka, wak hobvk tuklo, chukfi nakni tahlapi, issi kosoma nakni tahlapi, chukfi ushi afummi vmmona tahlapi aiena tok. Iluppak osh Sulishattai ushi Shelumiel nan im issa ya tok.

42. Nitak ont ishit hannali ma Teuel ushi Eliasaf, Kat im vlla vhleha $\underline{i}$ holitopa ak osh im issa tok.

43. Micha $\underline{i}$ nan im issa yut tvli hvta ampo chito achvfa, weki kvt shekel tahlepa achvfa cha pokoli tuchina, tvli hvta ampo achufa shekel pokoli untuklo vlhtipo holitopa $\underline{i}$ shekel vlhpisa hatok; ita tuklo kvt bota tohbi lipehbi bila itaiyoma, ilhpak im issa ya chị hosh alota bika tok.

44. Isht impa achufa tuli holisso lakna shekel pokoli hosh na balama alota.

45. Hukmit im issa ya chi ka wak hobvk himita achvfa, chukfi nakni achvfa, chukfi ushi afummi vmmona achvfr;

46. Nan ashvchi im issa yvt issi kosoma ushi achufa;

47. Micha isht itin nanaiyvchi im issa ya chi ka , wak hobvk tuklo, chukfi nakni tahlapi, issi kosoma nakni tahlapi, chukfi ushi afummi ummona tahlapi aiena tok. Iluppak osh Teuel ushi Eliasaf nan im issa ya tok.

48. Nitak ont ishit untuklo ma Ammihut ushi Elishama Eflaim im vlla vhleha $\underline{i}$ holitopa ak osh im issa tok.

49. Micha i nan im issa yvt tuli hvta ampo chito achufa, weki kut shekel tahlepa achvfa cha pokoli tuchina, tuli huta ampo achvfa shekel pokoli untuklo, vlhtipo holitopa i shekel vlhpisa hatok; ita tuklo kvt bota tohbi lipehbi bila itaiyoma, ilhpak im issa ya chi hosh, alota bika tok. 
50. Isht impa achvfa tvli holisso lakna, shekel pokoli hosh na balama alota.

51. Hukmit im issa ya chi ka wak hobuk achufa, chukfi nakni achvfa, chukfi ushi afummi vmmona achvfa;

52. Nan ashvchi im issa yut issi kosoma ushi achufa;

53. Micha isht itin nanaiyuchi im issa ya chị ka, wak hobuk tuklo, chukfi nakni tahlapi, issi kosoma nakni tahlapi, chukfi ushi afummi vmmona tahlapi aiena tok. Ilvppak osh Ammihut ushi Elishama nan im issa ya tok.

54. Nitak ont ishit untuchina ma Petashu ushi Kamaliel, Manasseh im vlla vhleha $\underline{i}$ holitopa ak osh im issa tok.

55. Micha i nan im issa yut tuli huta ampo chito achufa, weki kvt shekel tahlepa achvfa cha pokoli tuchina, tuli hvta ampo achvfa, shekel pokoli untuklo, vlhtipo holitopa $i$ shekel vlhpisa ha tok: ita tuklo kvt bota tohbi lipehbi bila itaiyoma, ilhpak im issa ya chi hosh alota bika tok.

56. Isht impa achvfa tvli holisso lakna shekel pokoli hosh na balama alota;

57. Hukmit im issa ya chi ka wak hobuk himita achufa, chukfi nakni achvfa, chukfushi afummi vmmona achvfa;

58. Nan ashuchi im issa yut issi kosoma ushi achvfa;

59. Micha isht itin nanaiyvchi im issa ya chi ka wak hobvk tuklo, chukfi nakni tahlapi, issi kosoma nakni tahlapi, chukfushi afummi ummona tahlapi aiena tok. Iluppak osh Petashu ushi Kamaliel nan im issa ya tok.

60. Nitak ont ishit chakkali ma Kiteoni ushi Abitan, Benchamin im vlla vhleha i holitopa ak osh im issa tok.

61. Yohmi kut i nan im issa yut tuli huta ampo chito achufa, weki kvt shekel tahlepa achvfa cha pokoli tuchina, tvli huta ampo achvfa shekel pokoli untuklo, vlhtipo holitopa $\underline{i}$ shekel vlhpisa ha tok; ita tuklo kut bota tohbi lipehbi bila itaiyoma ilhpak im issa ya chi hosh alota bika tok.

62. Isht impa achufa teli holisso lakna shekel pokoli hosh nabalama alota;

63. Hukmit im issa ya chị ka wak hobvk himita achvfa, chukfi nakni achvfa, chukfushi afummi vmmona achvfa;

64. Nan ashuchi im issa yut issi kosoma ushi achufa; 
65. Micha isht itin nanaiyvchi im issa ya chi ka, wak hobvk tuklo, chukfi nakni tahlapi, issi kosoma nakni tahlapi, chukfushi afummi vmmona tahlapi aiena tok. Ilvppak osh Kiteoni ushi Abitan nan im issa ya tok.

66. Nitak ont ishit pokoli ma Ammishattai ushi Ahiesa, Tan im vlla vhleha $\underline{i}$ holitopa ak osh im issa tok.

67. Yohmi kut i nan im issa yut tuli huta ampo chito achvfa, weki kvt shekel tahlepa achvfa cha pokoli tuchina, tvli hvta ampo achvfa shekel pokoli untuklo, vlhtipo holitopa i shekel vlhpisa ha tok; ita tuklo kvt bota tohbi lipehbi bila itaiyoma ilhpak im issa ya chi hosh alota bika tok.

68. Isht impa achvfa tvli holisso lakna shekel pokoli hosh nabalama alota;

69. Hukmit im issa ya chi ka wak hobvk himita achvfa chukfi nakni achvfa, chukfushi afummi ummona achvfa;

70. Nan ashvchi im issa yut issi kosoma ushi achvfa;

71. Micha isht itin nanaiyvchi im issa ya chi ka, wak hobvk tuklo, chukfi nakni tahlapi; issi kosoma nakni tahlapi, chukfushi afommi vmmona tahlapi aiena tok. Ilvppak osh Ammishattai ushi Ahiesa nan im issa ya tok:

72. Nitak ont ishit auachvfa ho Oklan ushi Pachiel, Asha im vlla vhleha i holitopa ak osh im issa tok.

73. Yohmi kvt i nan im issa yut tuli hvta ampo chito achvfa weki kvt shekel tahlepa achvfa cha pokoli tuchina, tvli huta ampo achvfa shekel pokoli untuklo, vlhtipo holitopa i shekel vlhpisa hatok; ita tuklo kvt bota tohbi lipehbi bila itaiyoma ilhpak im issa ya chi hosh alota bika tok.

74. Isht impa achufa tuli holisso lakna shekel pokoli hosh nabalama alota;

75. Hukmit im issa ya chi ka wak hobvk himita achufa chukfi nakni achvfa, chukfushi afummi vmmona achvfa ;

76. Nan ashvchi im issa yot issi kosoma ushi achvfa;

77. Micha isht itin nanaiyuchi im issa ya chi kâ, wak hobok tuklo, chukfi nakni tahlapi, issi kosoma nakni tahlapi, chukfushi afummi vmmona tahlapi aiena tok. Iluppak osh Oklan ushi Pachiel nan im jssa ya tok. 
78. Nitak ont ishit auahtuklo ho Enan ushi Ahila, Naftali im vlla vhleha i holitopa ak osh im issa tok.

79. Micha I nan im issa yut tuli huta ampo chito achvfa weki kvt shekel tahlepa achvfa cha pokoli tuchina, tuli huta ampo achvfa shekel pokoli untuklo, vlhtipo holitopa i shekel vlhpisa hatok; ita tuklo kvt bota tohbi lipehbi bila itaiyoma ilhpak im issa chi hosh alota bika tok.

80. Isht impa achufa toli holisso lakna shekel pokoli hosh nabalama alota;

81. Hukmit im issa ya chi ka, wak hobuk himita achvfa, chukfi nakni acvhfa, chukfushi afummi ummona achufa;

82. Nan ashvchi im issa yut issi kosoma ushi achufa;

83. Micha isht itin nanaiyuchi im issa ya chi ka, wak hobuk tuklo, chukfi nakni tahlapi, issi kosoma nakni tahlapi, chukfushi afummi ummona tahlapi aiena tok. Iluppak osh Enan ushi Ahila nan im issa ya tok.

84. Ilvppak osh Islael holitopa vhleha nan ahvmmit alta ya (ahama nitak ma) hullochi mak atok oke, tuli huta ampo chito auah tuklo, micha tvli huta ampo anah tuklo, tvli holisso lakna isht impa auahtuklo.

85. Tuli hvta ampo chito vt shekel tahlepa achufa cha pokoli tuchina bieka tok, micha ampo vt pokoli untuklo bieka tok; tuli huta nan aivlhto moma kvt vlhtipo holitopa shekel vlhpisa hosh shekel tahlepa sipokni tuklo cha tahlepa ushta tok.

86. Tuli holisso lakna isht impa na balama alota yash vto auahtuklo hosh vlhtipo holitopa shekel vlhpisut shekel pokoli bieka tok, tvli holisso lakna isht impa moma kvt shekel tahlepa achufa cha pokoli tuklo tok.

87. Hukmit im issa wak moma kut wak hobvk auahtuklo, chukfi nakni auahtuklo, chukfushi afummi ummona auahtuklo, ilhpak im issa aiena; micha nan ashvchi im issa issi kosoma ushi vt anahtuklo tok.

88. Micha isht itin nanaiyuchi vbit im issa wak hobvk moma kut pokoli tuklo akucha ushta, chukfi nakni pokoli hannali, issi kosoma nakni pokoli hannali, chukfushi afummi ummona pokoli hannali tok. Iluppak osh alta nan ahama ha yo hullochi mak atok. 
89. Atuk o Moses vt itim anumpula chi hosh okla moma $\underline{i}$ binah ha ont chukowa mvt, achvfa hosh ai $\underline{i}$ nukhaklo nan itim apesa afohka itombi holitopa paknaka ompoholmo chelubim tuklo ka iti takla et ai im anumpohonli na haklo tok, mihma im anumpuli tok.

\section{CHAPTA VIII。}

$\mathbf{M}$ ICHA Chihowah yot Moses a im anumpulit, 2. Alon a im anumpulit, Pvla ha ish pvlalikrna, pula untuklo kut pula ai o hikia ichapaka ya on tohwikelashke; ish im achashke, im achi tok.

3. Mihma Alon vt yumihchi tok; Chihowah yut Moses a im apesa tuk mak o, pula ai o hikia ichapakă ya pula ha puläli tok.

4. Pula ai o hikia vlhtaha ilupput tuli holisso lakna lulla atok, vpi ont vhli, micha ai in chuwa ont vhli kvt tvli holisso lakna lvllut vlhtaha ya tok; holbvt toba Chihowah yvt Moses

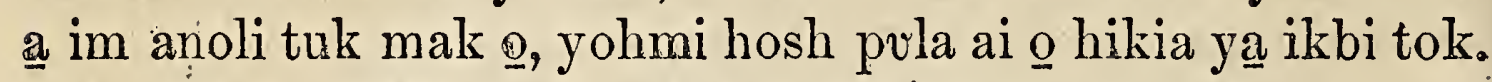

5. Mihma Chihowah yot Moses a im anumpulit,

6. Lefait vhleha ha Islael im vlla vhleha i filvmmichit ish ishi cha ish kashoffashke.

- 7. Micha ish kashoffi kvt ish yakohmichashke, Oka ishit kashofa ish o fimmashke, mihma nipi ya moma shafashke, micha i nafohka ya achefashle, yumohmi hosh ile kashoffashike.

8. Yohmikmut wak hobuk himita, micha ilhpak im issa, yummut bota tohbi lepehbi bila aiyoma aiena ishashke, mihma wak hobuk himita inla nan ashvchi im issa yo ish ishashke.

9. Micha Lefait vhleha ha okla moma i binah itikba ya isht ish onashke; micha Islael im vlla vhleha moma ka hoyot ish itunahlashke.

10 Micha Lefait vhleha ha pehlichit Chihowah itikba ya isht ish onashke: mihma Islael im vlla vhleha hut tiefait: vbleha ha ibbak onochashke. 
11. Mihma Alon vt Islael im vlla vhleha nan im issa mak $\varrho$, Lefait vhleha hạ, yummut Chihowah nan isht im aiahanta isht ahanta chi ho Chihowah itikba ya im issa hi oke.

12. Mihma Lefait vhleha hut wak hobvk himita puta kash noshkobo ha ibbak onochashke, mihma achvfa ka nan ashvchi im issakmut achvfa kano hukmit im issut, Chihowah ish im issvt Lefait vhleha ha ish $\underline{\underline{i}}$ kashoffashke.

13. Micha Lefait vhleha ha Alon micha ushi vhleha itikba ish hilechi cha, Chihowah nan im issa yo ish im issashke.

14. Yakohmichi hosh Lefait vhleha ha Islael im vlla whleha ish ai i filummichashke; mikma Lefait vhleha hvt ómmi ha chi hoke.

15. Atukma $\underline{i}$ himmak mak $\underline{0}$, Lefait vhleha hvt ${ }^{\circ}$ iba chukowut okla moma i binah nan ai isht ahanta ya yumihcha hi oke: mihma ish kashoffi cha nan im issa yo ish im issashle.

16. Islael im vlla vhleha ai iti takla ka aiokluhut vmmi toba họka; im oshvto puta tiwi vmmona chatuk Islael im vlla vhleha vttahpi moma chatuk a atobbichit il ai ishi li tuk oke.

17. Islael im vila vhleha vtta ahpi moma kvt, hatak micha nan vlhpoa aiena kvt vmmi hoka. Echip yakni ya vttahpi ya issot kanchi li nitak mut hullochit il ai eshi li tok oke.

18. Micha Lefait vhleha hak o Islael ịm vlla vhleha vttahpi moma tuk a atobbichit ishi lishke.

19. Micha Lefait vhleha hvt orka moma $\underline{\mathrm{i}}$ binah nan a toksuli isht ahantut Islael im vllavihleha i kashoffa chi ho Islael ina vlla vhleha ai i filvmmichit, Alon micha ushi vhleTha aiena ka na hulbina ohmi ho ima li tuk oke. Yvmohmi ho Islael in vlla vhleha hut vlhtipo holitopa bilika onakma, Islael in vlla vhleha à ịti takla ya ililli okpulo vt iksho kashke, achi tok.

20. Mihma Moses micha Alon micha Islael im vlla vhleha okla moma kvt dejeait vhleha ha $\underline{i}$ yumihehi tok. Lefait vhilehardulla ka Chihowah yvit Moses a im apihisa tuk mak o, yohmi he Islael im vlla vhleha hvt i yumihchi tok. 
.21: Mihma Lefait vhleha hut kashofa cha i nafohka ya achéfa tok: mihma Alon vt nan im issa yo, Chihowah itikba im issa tok; micha Alon vt kashofa chi hosh $\underline{i}$ kashoffi tok.

22. Atuk o $\underline{i}$ himmak ak o Lefait vhleha hot Alon micha ushi vhleha aiena itikba ya okla moma i binah a toksvli yumihcha chi hosh iba chukowa tok; Lefait vhleha pulla ka Chihowah yut Moses a im apesa tuk mak o, okla hut $\underline{\mathbf{i}}$ yumihchi tok.

23. Mihma Chihowah yot Moses a im anumpulit,

24. Iluppak osh Lefait uhleha immi mak oke; afummi pokoli tuklo akucha tahlapi kak o vhlit vba maya kak oș iba chukowvt okla moma i binah a toksuli ya , isht aiahantashke.

25. Micha afummi pokoli tahlapi ont vhli kak osh atoksuli isht ahanta kut issa cha himmakma isht ik ahanto kashke ;

26. Yohmi kia itibapishi vhleha takla okla moma i binah isht aiahantut apistikeli cha na toksvli hano ik yumihcho ka hi oke. Apistikeli yummak okưno Lefait vhleha ha ish $\underline{\mathrm{i}}$ yumihchashke, achi tok.

\section{CHAPTA IX.}

W IHMA Chihowah yut Moses a yakni haiaka Sinai ya hushi vmmona ho im anumpulit,

2. Islael im vila vhleha hut vbanublit ont ia vlhpisa vt ont vlhpesakma holitoblashke.

3. Himak hvshi nitak ont isht auahushta kvt opiakma vlhpisa hut ont vlhpesakma, hush holitoblashke; nan vlhpisa

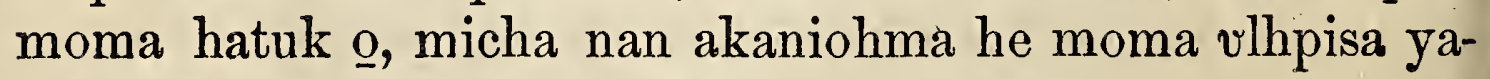
tuk mak $\underline{o}$, hvsh holitoblashke, im achi tok.

4. Mihma Moses vt Islael im vlla vhleha hut vbanublit ont ia ya holitoblicha chi ho im anumpulit tok.

5. Mihma hushi vmmona nitak ont isht auahushta opia ma, yakni haiaka Sinai ya vbanublit ont ia ya isht aiasha 
tok; Chihowah yut Moses a im apesa tuk moma kak o, Isläel im vlla vhleha hut yumihchi tok.

6. Atuk o hatak kaniohmi kvt hatak illi nipi ishit liteha tuk osh, nitak yumma vbanublit ont ia isht asha he keyu tok; micha nitak yumma Moses itikba micha Alon itikba ona tok.

7. Micha hatak yummak ash osh, Hatak illi nipi yo ishit pi litehvshke, nanta katiohmi kak o pi katvbli na Islael im vlla vhleha ai itin takla ya Chihowah nan im issa ya vlhpisa vt ont ai vlhpiesakma kil im isso ka chị cho? im achi tok.

8. Mihma Moses vt, Lumvt hush hinlashke, mikma huchishno pulla ka Chihowah ak osh nan apesa he, haklo lashke, im achi tok.

9. Mihma Chihowah yut Moses a im anumpulit,

10. Islael im vlla vhleha ha im anumpulit, Hatak chi kanima hosh, keyukmvt hvchi aionchuloli yosh nipi illi yo ishit litehakma, keyukmot yakni hopaki nowvt aya tuk okma, nana kia, vbanublit ont ia ya Chihowah isht im aiasha hi oke.

11. Hushi atukla nitak ont isht auahushta opiakma isht ai asha cha puska ik shatummo, micha haiyukpulo homi aiena it ai impvcha hi oke.

12. Ilatombut bohli na ik onno kashke; micha foni nana kia ik kobafo kashke, vbanublit ont ia nan ṿlhpisa moma hatuk mak o isht aiashashke.

13. Amba hatak vt liteha keyn, micha nowvt aya keyu hosh vbanublit ont ia ya isht ik ahantokmvt, yvmmak okvt im okla ai itin takla ya fiopa tupashke, Chihowah nan im issa ya vlhpisa tok vt ont vlhpiesakma; isht iklo hokvt, hatak yummut ilap nan ashuchi vt onvtoyulashke.

14. Okla inla yosh hvchi takla ahanta tuk osh, vbanvblit ont ia ya Chihowah isht im ahanta he ahnikmvt, vbanvblit

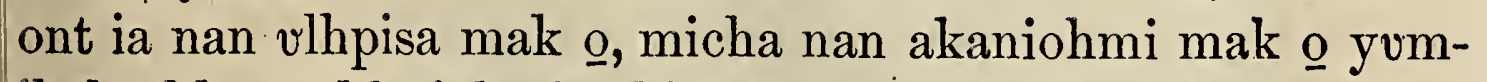
ihchashke; okla inla, hvchi yakni yash ai vtta tuk aiena kvt nan vlhpisa hvchim achvfashke, ish im achashke, im achi tok.

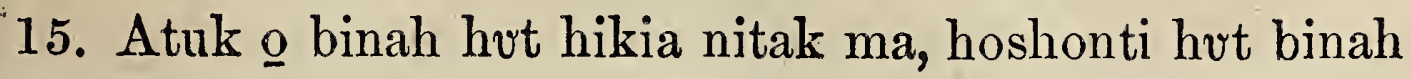


yummut isht atokowa binah ha onvtoyula tok, atuk osh opia ma luak holba hosh binah ha onvtoyula na onnahinli tok.

16. Yvmohmi na bilia beka tok, hoshonti hosh nitak ma onvtoyula tuk osh ninak ma luak ahoba hosh onvtoyula tok.

17. Atuk o hoshonti hosh binah paknaka ai ishit vba isht iakma, Islael im vlla vhleha hvt nowvt ia beka tok; atuk osh kanima ho hoshonti hot hikiakma, Islael in vlla vhleha hut yummak o binachi beka tok,

18. Chihowah hut im apesa ho Islael im vlla vhleha hvt nowvt ia beka tok; atuk osh Chihowah hut im apesa ho binachi beka tok; hoshonti hvt binah onvtola takla im vlhtipo fohvt asha beka tok.

19. Atuk o hoshonti hut hopaki micha nitak laua ho binah paknaka onvtoyulakma, Islael im vlla vhleha hvt Chihowah nan isht im ahanta ka holitobli cha ik ayo beka tok.

20. Atuk o yakohmi beka tok, nitak kanohmi takla ho hoshonti hut binah ha onvtoyulakma, Chihowah im anumpa

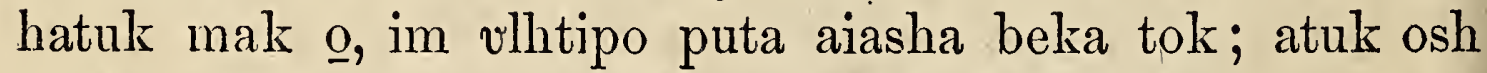
Chihowah im anumpa mak o nowvit ia beka tok.

21. Atuk osh yakohmi beka tok, hoshonti het talaia na opiaka vttvt onnahinli ont vhlikma, atuk osh onnahinlikma hoshonti ha vba isht iakma nowvt ia beka tok; nitak olkmá, ninak okmá nana ho vba isht iakma nowvt ia beka tok.

22. Keyukmot nitak tuklo hokmá, keyukmut hvshi achvfa hokma, keyukmvt afummi achvfa hokma, nana ho hoshonti hut binah paknaka talaia cha on talaia momakma, Islael im vlla vhleha hvt im vlhtipo aiasha cha, nowvt ik aiyo tok; amba vba isht iakma nowvt ia beka tok.

23. Chihowah im anumpa pulla ho im vlhtipo fohvt ai asha tok, anonti Chihowah im anumpa pulla ho nowvt ia beka tok; Chihowah nan isht im ahanta ha holitoblit Chihowah yvt Moses a nan im apesut tohno tok a ieshi beka tok. 


\section{CHAPTA $\mathrm{X}$.}

MIHMA Chihowah yvt Moses a im anumpulit,

I 2. Tuli huta isht puffa tuklo ho ish ikbashke; ita chakuchi keyu hosh ish ikbashke: yummak o okla moma isht ish $\underline{i}$ howashke, micha binah puta kut ia chi isht ish im apesashke.

3. Atuk o ita tuklot isht olachikma okla moma kut okla moma i binah okhissa ya chim ai itunahashke.

4. Atuk osh isht pufa achufa bano ho olachikma, holitopa vhleha yưmut Islael tahlepa sipokni puta $\underline{i}$ noshkoboka vt chim ai itunahashke.

5. Nuktahla he ish olachikma, binah puta hushi akochaka in tonnup asha kvt tikba iashke.

6. Atukla ma nuktahla he ish olachikma, binah puta oka mahli pilla in tunnup aiasha kvt, nowvt iashke; nowvt ia chi puta ka nuktạhla hi ya okla olahanchashke.

7. Amba okla moma kutlitunaha chi hokma ish olachi hoh kia, nuktahla hano chik olacho kashke.

8. Mikma naholitompa isht asha Alon ushi vhleha hak osh isht pufa ya olachashke; micha chim ai itishali puta hlopulli ka chi nan vlhpisa bilia ha chi hoke.

9. Atuk osh chi yakni yạ, huchin tunup vt hưch ilbushali na, in tanampit hush iakmut nuktahla he isht pufa hush olachashke ; yohmikma hvchin Chitokaka itikba ya hvch ithana hi oke, mikma hvchin tunup a hvchi hlakoficha hi oke.

10. Micha hvchi yukpa nitak, micha nitak hvchị holitopa, micha huchi hushi isht ia vmmona puta ka, na hukmit im issa, micha isht itin nanaiyachi im issut vbi puta ka paknaka isht pufa ${ }_{\text {hush }}$ ai olachashke : yohmi na yummut isht ithana bilia yosh Chihowah itikba itoyulashke, Chitokaka hvchin Chihowah sia hoke, achi tok.

11. Atuk o yakohmi tok, afummi ont atuklahvshi atukla kvt nitak ishit pokoli tuklo ma, hoshonti ha nan isht atokowa binah paknaka ya ai ishit vba isht ia tok.

12. Yohmi ma Islael im vlla vhleha hvt Sinai yakni haia- 
ka isht ia tok; atuk o Pelan yakni haiaka yak o hoshonti hut ont talaia tok.

13. Micha Chihowah hut Moses a tohno tuk mak o, tikba ia tok:

14. Tikba fehna ya Chutah im vlla vhleha i binah na hvta shali ak osh okla vlhpisa mak osh ia tok; mikma in tushka moma pehlichi vt Amminatab ushi Nahshon ak atok.

15. Mikma Issaka im vlla vhleha apehlichi tvshka moma pehlichi vt Sua ushi Nehlaneel ak atok.

16. Micha Sebulon im vlla vhleha apehlichi okla ha pehlichi vt Helon ushi Eliab ak atok.

17. Mihma binah ha akvehi ma, Keshon ushi vhleha, micha Melali ushi vhleha aiena kak osh binah ha sholi cha iakaigut ia tok.

18. Mihma Luben i binah na huta shali ak osh okla ha vlhpisa mak osh wakayvt ia tok, mihma Sheteu ushi Elisu ak osh in tushka vhleha ha pehlichi ak atok.

19. Mikma Simeon im vlla vbleha apehlichi tushka vhleha pehlichi vt Sulishattai ushi Shelumiel ak atok.

20. Mikma Kat im vlla vhleha apehlichi tushka vhleha pehlichi vt Teuel ushi Eliasaf ak atok.

21. Mihma Kohalhait vhleha hut vlhtipo holitopa yo shaiyalit wakayut ia tok; mikma ik ono kissha ho inlaka yvt binah ha hilechi tok.

22. Mihma Eflaim im vlla vhleha i binah nahvta shali okla ha vlhpisa mak osh wakayut ia tok; milhma in tushka vhleha ha pehlichi kut Ammihut ushi Elishama ak atok.

23. Mikma Manasseh im vlla vhleha apehlichi tushka vhleha pehlichi vt Petashu ushi Kamaliel ak atok.

24. Mikma Benchamin im vlla vhleha apehlichi tvshka vhleha pehlichi vt Kiteoni ushi Abitan ak atok.

25. Mikma Tan im vlla vhleha i binah nahvta shali vt yummak osh tushka vhleha puta i binah puta moma hlopulli ka ashaka yatuk osh wakayut ia tok; mihma in tushka vhleha pehlichi kut Ammishattai ushi Ahiesa ak atok.

26. Mikma Asha im vlla vhleha apehlichi tushka vhleha pehlichi vt Oklan ushi Pachiel ak atok. 
27. Mikma Naftali im vlla vhleha apehlichi tushka vhleha pehlichi vt Enan ushi Ahila ak atok.

28. Yakohmi hosh Islael im vlla vhleha hut okla puta ka vlhpisa hosh wakaiyut iakmut itunowa beka tok.

29. Mihma Moses vt Lekuel ushi Hobab Mitianait Moses ipochi yash, Yakni yumma Chihowah yut chima lashke, achi tok a ia hosh il itunowa; pi awant ish iakma, nana achukma ka e chi yumihchashke; Chihowah yut Islael pulla ka achukma ho animpuli tuk oka, im achi tok.

30. Mihma, Ia la he keyu, amba ivt a yakni akinli.micha a kanomi ak o im ona la chi hoke i im achi tok.

31. Mihma, Chik pisso kashke, chim asilhha lishke; yakni haiaka yo binachit il itunowa chị ka ish ithana hokvt, pi nishkin a he tuk a chishno ak ashke.

32. Mikma yakohmi hi oke, pi awant ish iakma ahli, yakohma hi oke; nana achukma nana ho Chihowah yut piyvmihchikma, yummak inli ho e chi yumihcha chi hoke, achi tok.

33. Mihma Chihowah $\underline{\underline{i}}$ nunih ha issut nitak tuchina anowa okla ona tok; mihma Chihowah nan itim apesa afohka itômbi holitopa ak osh nitak tuchina anowa yumma im afoha hoyot im ahayucha chi hosh tikba ia tok.

34. Mihma binah kohchut ia nitak ma, Chihowah $\underline{\mathrm{i}}$ hoshonti het onvtoyula beka tok.

35. Atuk o yakohmi tok, itombi holitopa yut wakayvt iakma, Moses vt Wakaya, Chihowah ma, mikma chin tunvp puta kut ita fimimpashke; micha chi nukkilli vhleha hot chi tikba yihlepashke, achi tok.

36. A tuk osh talaiakma, Islael tahlepa sipokni laua ka falamvt ish im vlashke, Chihowah ma, achi tok.

\section{CHAPTA XI.}

A TUK o okla hvt na mihvt anumpuli ma, Chihowal yvt A ik im vlhpieso tok; micha Chihowah yot haklo tok; mihmot anukhobela yvt libbi tok, mihma Chihowah in luak vt luachit binah vhli puta aiasha tuk a hukmit tahli tok. 
2. Mihma okla hut Moses a i pahaya tok; mihma Moses vt Chihowah im asilhha ma luak vt mosholi tok.

3. Mihma yakni yumma Tabelah hochifo tok, Chihowah in luak vt ai itin takla ka hukmi tuk oka.

4. Mihma okla laua itaiyokoma takla asha tuk vt pushlivnot wakaya tok; micha Islael im vlla vhleha hot anonti yaiya mvt, kuta hosh nipi ya pipeta chi cho?

5. Echip a nuni, kukvmba, micha shukshi puta, micha leek, micha shachuna puta, micha kalik puta laua ho epa fehna beka tok vt il ithaiyanushke.

6. Yohmi tok kia himak ano pim ishilombish vt shilvt kunivshke; nana kut iksho kumohmi, manna iluppak illa pi nishkin itikba aiashushke, achi tok.

7. Mihma manna yet kolianta nihi ak o chohmi hosh pisa hokvto tellium ak o holba tok.

8. Mihma okla kohchut itunowut aiowa cha afotoha a fotohli, keyukmut kiti a hosi cha, ampmahaia fohkit puska cha puska lumbo ikbi beka tok; mikma vput pisa ka bila himona chohmi beka tok.

9. Michá ninak o fichak vt binah ha onvtolakma manna et onvtola beka tok.

10. Mihma Moses vt okla hut in chuka achvfa puta hlopulli kvt $\underline{i}$ binah okhissa ya , hatak moma kvt yahaya na haklo tok; mihma Chihowah im ai i nukkilli vt libbi fehna tok; Moses ak kia ik im vlhpieso tok.

11. Mihmá Moses vt Chihowah ya , nanta katiohmi ho chin tishu ha ish ilbushali cho? nanta katiohmi ho chim isht i kana ak chi ahayucho ho, okla illuppa moma na weki is si onochi cho?

12. Okla iluppa moma ka shali li tuk oh cho? vno ak osh tobachi li tuk $\underline{0}$, iki yosh vlla pishi ya shoyuli chohmit, chi shakba fohkit shoyulit iki vhleha anumpa ilonochit yakni im issa li tok a isht ona, is sum acha hinla cho?

13. Okla iluppa moma ka katima ak o nipi ai isht ima la hinla eho? a yaiyut, Nipi ish pima na epashke, ahanchi hoka,

14. Úno ak illa hoșh okla iluppa moma ka shali la hekeyushke, a weki fehna hokvt. 
15. Micha iluppa $\mathrm{o}$ is sa yumihcha chi hokmut vmohmichit is subashke; na E kana chi tikba ai ahayuchi likma chim asilhha lishke, mikma a sulbusha kvt ak peso kashke, im achi tok.

16. Mihma Chihowah yut Moses a Islael im asunonchi pokoli untuklo, yumma im asunonchi, micha nan vlhtoka ya ish ithana kvt, vm itunahlit okla moma $\underline{i}$ binah isht ish ona na chi apehvt yưmma hielashke.

17. Mikma akowa lish yummak o a chim anumpule lashke; micha shilombish chi onvtoyula ka ishi lish yummak o onochi lashke; mikma okla pehlichị weki ka chiba shaiyalashke, mikma chishno ak bano hosh chik shaiyalo kashke.

18. Micha onnaha takla ka hvsh ile hullochashke; atuk osh nipi ya hushpa chi hoke; hush yahayut kuta hosh nipi ya pipeta chi cho? Echip ano pim aiachukma tok oka; hush achi ho Chihowah yut haklo tuk oka; yumohmi hoka, Chihowah yut nipi huch ima na, hush pa chi hoke,

19. Nitak achufa hô hush pa he keyu, michá nitak tuklo keyu, micha nitak tahlapi keyu, micha nitak pokoli keyu, micha nitak pokoli tuklo keyu,

20. Amba hushi achufa ont hlopulli hok kia hush ahampakma, polaka huch ibishakni a kohchakmvt nan isht huchi yuwala ya chị hoke; Chihowah huchi takla ahanta ka hush shitilema cha, itikba ya ayaiyut, Nanta katiohmi ho Echip a e kohcha tuk oh cho? hush achi hokvt, ish im achashke, achi tok.

21. Mihma Moses vt okla takla aya li, hatak aka aya yvt tahlepa sipokni bat tahlepa hannali hoke; mihma nipi ima likma, hushi achvfa hlopulli kia vpashke, ish achishke

22. Vlhpaa chipunta aya kanohmi kia, micha vlhpoa hochito aya kanohmi kia im vbi na fihopa chi cho? ak cho? okhuta nuni aiasha moma hoyot im itunahlit fihobla hinla cho? achi tok.

23. Mihma Chihowah yut Moses a Chihowah ibbak vt ikfalaiot taha hoh cho? um anumpa hut chim aiahlikmá keyukmá ṇanakmá ish pisa chi hoke, im achi tok.

24. Mihma Moses vt kohchvt ia cha, Chihowah im anum- 
pa hash okla ha im anoli tok; micha okla im asunonchi pokoli untuklo kash hoyo cha binah ha afolublichit hiohlechi tok.

25. Mihma Chihowah yut hoshonti ishit akowa cha, im anumpuli tok, micha shilombish onvtoyula tuk a ishi cha, im asunonchi pokoli untuklo kash onochi tok, atuk o yakohmi tok; shilombish vt onvtoyula ma tikbanli nana anolit Chihowah ya aiokpahanchi cha ik isso tok.

26. Yohmi kia hatak tuklo kvt achvfa hohchifo kvt Eltat, mikma achvfa hohchifo Metat osh binah ha ahashwa moma tok; mihma shilombish vt yummak o onvtoyula tok, yvmmak okvt holisso ka ibafoka tuk kia, kohchvt binah ha ik ono tuk atok, binah ahashwa hak inli kia tikbanli nana anolit Chihowah ya aiokpvchi tok.

27. Mihma hatak himita yosh malelit ivt Moses a im anolit, Eltat micha Metat ak kia binah ha ashwvt tikbanli nana anolit Chihowah ya aiokpvchishke, achi tok.

28. Mihma Nvn ushi Choshua Moses in tishu hot hatak himithoa im asha achufa yvt afalaminchit 1 shahli Moses ma! ish im olvbashke achi tok.

29. Mihma Moses vt, Uno ak atuk mak o ish potunno cho? Chihowah yot vlhpesa ahni na, Chihowah im okla moma kvt tikbanli nana anolit Chihowah ya aiokpvehi hokbano, micha Chihowah hut shilombish a onochi hokbano, im achi tok.

30. Mihma Moses micha Islael im asvnonchi aiena kvt binah ha ona tok.

31. Atuk o Chihowah hvt mahli ha kohchichi na, kofi ha okhvta ha ai isht vlvt okla i binah iluppa in tunnup nitak achvfa anowa, mihmot in tunnvp a nitak achvfa anowa chohmi ho ai eshit binah ha apakfoyupa cha yakni paknaka ya , kubit tuklo chohmi ho aiasha tok.

32. Mihma okla hut nitak yưmmut hlopulli ka, micha ninak yummvt hlopulli ka , micha nitak atukla kvt hlopulli ka hielit aiowa tok; moma ik alauwo ho aiowa kvt homa pokoli ho aiowa tok micha binah ha a mominchit apakfoyuplit patali tok. 
33. Atuk o nipi ash o noti ya ishit kiseli moma ik hopaso kisha ho Chihowah im ai i nukkilli vt okla ha $\underline{\text { i sanalit }}$ libbi tok; micha Chihowah yut okla ha illilli okpulo fehna ho isht isso tok.

34. Mihma yakni yumma Kiblolh hattaafah hochifo tok; yummak o pushkuno tuk okla hut ahollohpi tok oka.

35. Mihma okla hvt Kiblolh hattaafah ya ashut ia hosh Haselolh a ona tok: micha Haselolh ak o aiasha tok.

\section{CHAPTA XII.}

A TUK o Miliam micha Alon ita tuklo kvt Moses vt EhliA opian ohoyo yumma itauwaya tuk ak o na mihachit anumpuli tok. Ehliopian ohoyo ho itauwaya tok atuk oka.

2. Micha Chihowah yut Moses ak illa ho tohnot anumpuli tuk oh cho? pishno ak kia pi tohnot anumpuli tuk ak inli keyu cho? achi tok. Mihma Chihowah yot haklo tok.

3. Yohmi ka hatak Moses vt chukvsh yohbi kut hatak moma yakni paknaka aiasha ka $\underline{i}$ shahli tok.

4. Mihma Chihowah yut yakosi itin takla ho Moses micha Alon micha Miliam aiena ka im anumpulit, Hush tuchina kvt kohchvt okla moma i binah ha hvshlashke. Mihma yumma tuchina kut kohcha tok.

5. Mihma Chihowah yut hoshonti tonik ibafokut akowa cha, binah holitopa okhissa ya hikia cha, Alon micha Miliam a $\underline{\mathrm{i}}$ howa tok ; mihma ita tuklot kohcha tok.

6. Mihma Himak a vm anumpa ha hvsh haklashke; hveh itin takla ya hopaii vt ahantakma, Chihowah sia hvt nan otuni yo im ile haiakuchi lashke, micha holhpokna ho itim anumpule lashke.

7. Yohmi kia an tishu Moses vt yohmi keyu, yummak okvt an chuka ya nana aiasha moma ka aiahli hoke.

8. Yummak okuno itih bika itim anumpohonli chohmit itim anumpule la chi hoke: micha anumpa luma ha chi Keyou hoke; mikma Chihowah holba pisa chi hoke; yohmi 
ka nantihmi ho an tishu Moses a na mihachit anumpuli ka ik hvchi nukwio tuk oh cho? im achi tok.

9. Mihma Chihowah im ai $\underline{\mathrm{i}}$ nukkilli vt $\underline{\mathrm{i}}$ sanalit libbi tok; mihmut ia tok.

10. Mihma hoshonti hvt binah holitopa yash issut ia tok; mihma yakeh, Miliam vt leplosi vbit tohbi kvt okti chiyuhmi tok; mihma Alon vt Miliam a pisa ma, yakeh, leplosi vbi tok.

11. Mihma Alon vt Moses a Ikkikkeh! a shahli ma! chim asilhha lishke, im anukfila iksho pi yumohmi tuk a micha nanashuchi a pi yumohmi tuk, nan ashvchi ya chik pi onocho kashke.

12. Illi tuk osh ishki im oshuto ya kohcha mut nipi vt iklunna kut taha yo ik chohmo kashke, im achi tok.

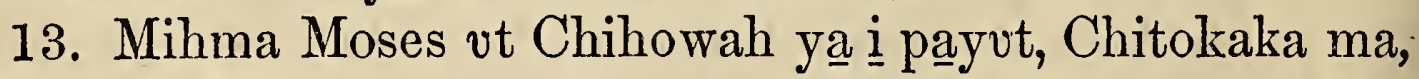
himak a ish hlakoffichashke, chim asilhha lishke, achi.tok.

14. Mihma Chihowah yvt Moses a Iki vt nashuka ya on tofa illa tuk okma, nitak untuklo ho hofahya hinla keyu cho? nitak untuklo ho binah ha kohchichashke, yohmi ha yosh anonti falumminchit ishashke, achi tok.

15. Mihma Miliam vt nitak untuklo ho binah ha kocha ahanta tok; mihma Miliam a falumminchit isht iklo kisha ka okla hut nowvt ik aiyo tok.

16. Atuk osh $\underline{\mathbf{i}}$ himak ak $\underline{\mathrm{o}}$, okla hut Haselolh asha tuk vt kanullit Palan yakni haiaka ak o abinanchi tok.

\section{CHAPTA XIII.}

MIHMA Chihowah hvt Moses a im anumpulit,

1 2. Hatak pit ish tihleli na, Kenan yakni ya yưmma Islael im vlla vhleha ima li tok a, pisashle; ilki vhleha apehlichi aiyukali ka hatak achufa aiachvfa pehlichi yo ish pilashke, achi tok.

3. Mihma Moses vt Chihowah ak osh anumpuli ho yakni haiaka Palan a a tihleli tok; hatak yumma puta kvt Islael im vlla vhleha ha i noshkoboka bieka tok. 
4. Micha iluppa puta kak osh hohchifo: Luben apehlichika Sakka ushi Shammua ak atok.

5. Simeon apehlichi ka Holi ushi Shafat ak atok.

6. Chutah apehlichi Chefunneh ushi Kaleb ak atok.

7. Issaka apehlichi Chosef ushi Ikal ak atok.

8. Eflaim apehlichi, Nun ushi Oshea ak atok.

9. Benchamin apehlichi Lafu ushi Palti ak atok.

10. Sabulon apehlichi Soti ushi Kattiel ak atok.

11. Chosef apehlichi yummut Manasseh apehlichi Susi ushi Katti ak atok.

12. Tan apehlichi Kemalli ushi Ammiel ak atok.

13. Asha apehlichi, Mikael ushi Selhu ak atok.

14. Naftali apehlichi Fofsi ushi Nahbi ak atok.

15. Kat apehlichi Maki ushi Keuel ak atok.

16. Hatak hohchifo iluppa puta kak osh yakni ya pisut hlopulla chị ho Moses vt tihleli tok. Micha Nun ushi Oshea vt Chehoshua hochifo tok.

17. Micha Moses vt Kenan yakni pisa chị ka pit tihlelit Oka mahli imma iluppak o iut nunih ha ont hush chukowashke :

18. Micha yakni vt kaniohmi ka hush pisashke, micha okla hot aiasha aiena kạ, kvllo hokmá, ik kvllo hokmá, iklauo hokmá, laua hokmá nana ka ;

19. Micha yakni vt kaniohmi ho aiasha ka achukma hokmá, keyukmvt okpulo yokmá; micha tumaha holihta puta ho aiashakma, vlhtipo yokmá, keyukmut holihta kvllo yo aiasha hokmá:

20. Micha yakni vt kaniohmi hokmá, nia hokmá, keyukmot, chunna hokmá, iti vt a sha hokmá keyukmá, nana hvsh pisashke; Micha hvchi nakni achukma cha yakni ya nana a waya ka isht hushlashke, im achi tok. Yohmi ka nitak yummak okvt paki waya vmmona nitak atuk atok.

21. Yohmi ho ia cha yakni yash ahopokoyot Sin yakni haiaka vttut hatak vt Hamalh pit ai itvnowvt Lehob a ont whlichi tok.

22. Micha oka mahli imma oiyut ia cha Heblon a vla tok: yummak oka Ahiman, Sheshai micha Talmai aiena 
Anak im vlla vhleha hut asha tok. Yohmi ka Heblon vt Soan Echip talaia yut ik tobo kisha afummi untuklo mak o, toba tok atok.

23. Atuk osh Eshkol bokushi ya ona mut, yưmma pạki lokoli achvfa takali ho naksish achvfa ho chanlit akvchi cha tuklo hosh tubi takalichit sholi tok; micha pomeklanit, micha fik aiena ka ai isht vla tok.

24. Islael im vlla vhleha hut yakni yumma paki lokoli achvfa achanlit akvchi tuk ak osh bokushi Eshkol hochifo tok.

25. Atuk osh nitak pokoli ushta kvt ont taha ma, yakni ya a hopokoyvt itvnowa tuk vt falamvt vla tok.

26. Micha ivt Moses micha Alon micha Islael im vlla vhleha okla moma kvt Palan yakni haiaka Katesh ak o asha tuk o. im ona tok; micha anumpa falama isht im ona cha, yakni yash nana a waya ka okla moma ka $\underline{i}$ haiakvchi tok.

27. Micha im anolit, Pit ish pi pila tuk yakni ya il ona tuk, mibma pishukchi micha foi bila aiena a yanahanli kot aiahlishke; yohmi ka iluppak osh nan awaya mak oke.

28. Yohmi kia okla yakni ya aiasha kvt kvlloshke, mikma tumaha holihta puta kvt aholihta hosh chito fehnvshke, micha Anak im vlla uhleha hut yumma asha na, e pisa tuk oke.

29. Amalekait vhleha hvt oka mahli yakni ak o aiashushke; mikma Hittait vhleha micha Chebusait vhleha, micha Amolait vhleha aiena kvt nunih foka ak o aiasha hoke; mikma Kenanait vhleha hvt okhota bilika micha Chatan aivhli aiena kak o aiasha hoke, achi tok.

30. Mihma Kaleb vt okla hut Moses itikba aiasha ka nuktalali cha, Yakosi iti takla ivt iloh ishashke, il imaiya he vlhpesa fehna hokvt, achi ok.

31. Amba apihut ia tuk vto, Okla ha itibit il ia he pi vlhpesa keyushke, kvllo kvt pi shahli hoka.

32. Micha yakni ya ahopokoyo tuk vt ik achukmo ho Islael a anumpa isht im vlvt; Yakni yash il a hopokoyo kut okla ha tahli yakni chohmishke ; micha yumma aiasha na e písa ka hatak chaha fehnushke. 
33. Micha yumma hatak hofaloha Anak ushi vhleha, yummut hatak hofaloha vhleha a kohcha tok a e pisa tuk oke: mikmut pishno ile pisa kut hatofo ak o pi chohmi tuk; mikma yưmmut pi pesa ka yummak inli il in chohmi tuk oke: achi tok.

\section{CHAPTA XIV.} IHMA okla moma kvt chitolit yaiya tok; micha ninak
yvmma okla yaiya tok.

2. Micha Islael im vlla vhleha moma kvt Moses micha Alon ita tuklo ka na mihachit anumpuli tok; micha okla moma kvt, Chihowah yvt vlhpesa ahni na Echip yakni ak inli ho a pilli tok okbano; keyukmut Chihowah yvt vlhpesa ahni na, yakni haiaka a pilli tuk okbano;

3. Nanta katiohmi ho bushpo falaia ishit pi akakoha na, pi tekchi micha pim vlla aiena kvt nawehpoa toba chi $\mathrm{k}$, Chihowah yut pi pehlichit yakni iluppa pishit vla tuk oh cho? Echip yakni falamut il ona ka achukma kvt pim $\underline{i}$ shahla hinla keyu cho? ahanchi tok.

4. Micha ilap bika.itim achit, $\mathrm{P} \underline{i}$ kupituni iloh ikbi cha, falamut Echip a il onashke, itim achi tok.

5. Yohmi ma Moses micha Alon ita tuklo kvt Islael im vlla vhleha okla moma itunahut aiasha tuk a itikba ya, nashuka aka ishit hlipkaiyuchi tok.

6. Mihma Nun ushi Choshua micha Chifunneh ushi Kaleb ita tuklo yummut yakni yash ahopokoyo aiena tuk osh i nafohka ya hliluffi tok.

7. Micha Islael im vlla vhleha moma ka im anumpulit, yakni yash yumma hopokoyut a hlopulli tuk vt yakni achukma atampushke.

8. Chihowah yvt pi ahninchi hokmvt, yohmikmut yakni iluppa pisht ona cha pima chi hoke; yakni pishukchi micha foi bila ayanahanli yoke.

9. Peh, Chihowah ya i yoshobvt hvchik i filvmmo kashke, micha okla yakni ai asha ka hvehik i nukshopo kashkc; yummut pi puska yoka; yumma ai atukko yut i kunia hoke, 
mikma Chihowah yvt piba foyuka hoke; hvchik i nukshopo kashke, im achi tok.

10. Yohmi kia okla moma kut tuli ishit bola chị ka miha tok. Mihma Islael im vlla vhleha itikba ya Chihowah im aholitopa yvt okla moma i binah holitopa ya ahaiaka tok.

11. Mihma Chihowah yot Moses a katiohmi foka ho okla ilupput sa nukowahancha chi cho? micha katiohmi fokak mak o nan isht atokowa puta im iti takla ai otunihinchi li kak o a yimma chi cho?

12. Ililli okpulo isht issot kanchi lish, ai isha he vlhpesa im ishi lashke, micha chishno ak o oklushi chito micha kvilo yumma i shahli ka chi ai ikbi lashke, achi tok.

13. Mihma Moses vt Chihowah ya , Yohmikma Echip okla hot hakla chi hoke (nan isht chim ai ahli pulla hosh okla iluppa im ai iti takla ya akohchit isht ishla tuk oka;)

14. Micha yakni iluppa okla aiasha ka im anola chi hoke: Chihowah chia hut okla iluppa ish iba foyuka ka haklo tuk, Chihowah ya nashuka bika, iti pisa tuk, chi hoshonti hut o. wakayvt hikia tuk, nitak ma hoshonti tonik, atuk osh ninak ma luak tonik o isht ish pehlichi ka hakklo tok oka.

15. Himak a okla ilvppa moma ka hatak achufa chiyuhmichit vbit ish tahlikma, oklushi puta chi annoa ka hahalklo tuk vt anumpulit,

16. Chihowah yut yakni ya ima he mihut anumpa kullo il onochi tuk vt pehlichit ishit chukowa he tuk vt, nanihma he keyu hatuk osh, yakni haiaka ai vbit tahlishke, acha hi oke.

17. Yohmi ka himak a chim asilhha lishke, ish anumpulit,

18. Chihowah yot nuktaiyala na hopaki, micha isht $\underline{i}$ nukhakklo vt chinto hosh, nan ashvchi micha yoshoba ya $\underline{\mathrm{i}} \mathrm{ka}-$ shoffi, micha nan ashvchi im ahli ya hlakoffichi keyu, micha iki vhleha nan ashvchi tuk a im vlla vhleha ai iti shali ont atuchina, micha ont ai ushta hoh kia, nana palvmmi ish onocha he ish miha tok mak osh, an Chihowah nan isht im aiahli vt chitashke.

19. Nan isht ish i nukhakklo chito hatuk mak osh, micha okla iluppa ish i kashofi na Echip ashut mintit himak a ant 
aivhli hatuk mak osh okla nan ashvchi ya ish $\underline{i}$ kashoffashke, chim asilhha lishke, achi tok.

20. Mihma Chihowah yvt, chim anumpa hatuk mak o i kashoffi lishke.

21. Yohmi kia si okchaya pulla ka yakni moma kvt Chihowah isht im aholitopa alotowa pulla chi hoke.

22. Hatak yumma puta moma kut vm isht aholitopa, micha nana afehna puta Echip a micha yakni haiaka ya a yumihchi ka pihisa tuk osh ilvpput ont ishit pokoli mak $\mathbf{0}$, sasht imomaka pisa cha vm anumpa ha ik haklo tuk okvto,

23. Iki vhleha anumpa kullo il onochit im issa li tok yakni ya pisa he keyu; micha sa nukowvchi tuk puta hokvto pisa chi keyu pulla hoke.

24. Amba an tishu Kaleb yummak okvto shilombish inla ieshi cha siakaiya ahli tuk oka, yummak okuno yakni a achukowvt aya tuk a isht ona la hi oke; mihma im isht atiaka yot abinila hi oke,

25. (Yohmi ka Amalekait micha Kenanait vhleha aiena kvt okfa ha aiasha tok.) Onnaha ma fullolvt yakni haiaka okhuta humma pilla hak o hush iashke, achi tok.

26. Mihma Chihowah yot Moses micha Alon a im anumpuilit,

27. Katiohmi foka ho okla ik achukmo iluppot a sanalit namihachit anumpuli ka, peh ik ashah im ahni la chi cho? Islael im vlla vhleha na miha anumpuli puta yummot a sanalit na miha ka haklo lishke.

28. Sa okchaya pulla kvt anumpulit sa haksobish a hesh haklochi pulla mak o hvohi yumihchi la chi hoke, Chihowah yut achishke, hush im achashke.

29. Hưchi haknip vt yakni haiaka iluppa akakoha chi hoke; mikma a sanalit na miha tuk, hvchi holhtina tuk moma, afummi pokoli tuklo vttut vba mayut huchi holhtina tuk moma mak osh.

30. Yakni yumma hvchi ashachi la he, anumpa kvllo il onochi li tok a hush chukowa chi keyu pulla hoke, CheTunneh ushi Kaleb micha Nun ushi Choshua ak illa chi hoke.

31. Amba huchim vila vhleha na wehpoa ya chi, hush 
achi tuk, yummak okvno ishit chukowa likma, yakni hush shitilema tuk a ithana chi hoke.

32. Amba huchishno ak okvto, hvchi haknip illi vt yakni haiaka iluppa akakoha chi hoke.

33. Mikma hvchim vlla vhleha hot afvmmi pokoli ushta ho yakni haiaka ya ai itunowvt hush haui puta kvt vt onvtoyula na hvchi haknip vt yakni haiaka ya ataha chi hoke.

34. Nitak kaniohmi ho yakni ya hvsh a hopokoyo tuk; yummut nitak pokoli ushta tuk a vlhpisut (nitak achufa kvt afummi achvfut ia hosh) afvmmi pokoli ushta ho na hvsh ashvchi vt hvchi onvtoyula chi hoke; mikma nana im issa li tok vt kobvffi li ka hvsh ithaiyana chi hoke.

35. Chihowah sia hut okla ik achukmo ilvpput iti hoyot a sanali tuk a moma ka $\underline{i}$ yomihchi li pulla chi hoke; yakni haiaka iluppak o ataha chi hoke, micha yummak o okla ai illa chi hoke, achi li hatuk oke, im achi tok.

36. Atuk o yakni ya ahopokoya chi ho Moses vt pila tuk hatak vhleha, yummvt falamvt vla mvt, yakni ya kalakshi ànumpa onochit isht vla cha okla moma ka $\underline{\mathrm{i}}$ sanalechit na miha ka anumpolechi tuk vt,

37. Yakni ya anumpa ik achukmo onochit isht vla tuk pulla kvto Chihowah itikba ililli okpulo isht illit taha tok.

38. Amba Nvn ushi Choshua, micha Chefunneh ushi Kaleb yưmmut yakni ya ahopokoyut ia ka ai achufa tuk vt okchaya tok.

39. Mihma anumpa iluppa puta ka Moses vt Islael im vlla vhleha moma ka im anoli tok; mihma okla moma kvt yaiya fehna tok.

40. Atuk osh onnahinli fehna moma ho okla hvt wakaya cha nunih oiyut paknaka ona mut, Yakeh, ilvppa e hielishke, micha yakni Chihowah yut pim issa tok a il ia chi hoke, pi yoshoba tuk okvt, achi tok.

41. Mihma Moses vt, Nanta katiohmi ho himaka Chihowah im anumpa hvsh kobvffi cho? yohmi kia nana kvt im ahlia he keyu hoke.

42. Hvchik aiyo kashke, Chihowah yut hvchiba foka ke- 
yu hoka; yohmi hosh hvchin tunup itikba hvch ik akakoho kashke.

43. Amalekait micha Kenanait aiena kvt hvchi tikba yumma asha hoka; mikma bushpo falaia ishit hush akakoha chi hoke; Chihowah ya hush i filummi hatuk oka, yohmi hoka Chihowah yut huchiba foka he keyu hoke, achi tok.

44. Yohmi kia ilapunlvt ivt nunih paknaka ya ona tok; yohmi kia Chihowah nan itim 'apesa afohka itombi holitopa micha Moses aiena kvto binah ha ik kohcho tok.

45. Yohmi ma Amalekait micha Kenanait aiena nưnih yumma aiasha tuk vt akowvt mintit fahama cha imaiyvt yuhlit Holmah ya ont vhlichi tok.

\section{CHA PTA XV.}

MIHMA Chihowah yut Moses a im anumpulit,

2. Islael im vlla vhleha ha im anumpulit, Hvsh aiasha chi yakni huch ima li tok a hush onakmut,

3. Micha il onochi tok osh yumihchi hosh hukmit im issa, keyukmvt ubit im issa, keyukmvt ilap ahnit nan im issa, keyokmvt vlhpoa hochito, keyukmut vlhpoa chipunta ai ishit Chihowah ya na balama im ikbit impvechi holitopa isht asha hosh luak ishit hukmit Chihowah ya im issakmvt:

4. Yohmi hosh Chihowah nan im issa im issakma ilhpak im issa bota tohbi kushkoa ishit pokoli, bila hin kushkoa ai ushta itaiyoma ho isht vlashke.

5. Micha oka paki hin kushkoa ai ushta chukfulhpoa ushi achufa ho hukmit im issa, keyukmut vbit im issa hokmá aienint ish atahlashke.

6. Keyukmut chukfi nakni yokma ilhpak im issa yo bota tohbi kushkoa ishit pokoli tuklo ho bila yut hin kushkoa atuchina itaiyoma ho ish atahlashke.

7. Micha nan ishko im issa ya Chihowah nan $\underline{i}$ balama yo oka paki hin kushkoa isht atuchina ho ish im issashke.

8. Micha anumpa il onochi tuk osh yumihchi hosh huk- 
mit im issa, keyukmvt vbit isht aiokpvchi, keyukmvt itin nanaiyvchit im issa yo wak hobvk im atahlikmvt,

9. Yohmit wak hobvk isht vlakmut ilhpak im issa bota tohbi kushkoa ishit pokoli tuchina, bila hin iklunna itaiyoma ho isht vlashke.

10. Micha nan ishko im issa Chihowah $\underline{\underline{i}}$ balama yo luak ishit hukmit im issa ya oka paki hin iklunna ho isht ishlashke.

11. Yakohmichi hosh wak hobvk achvfakma, keyukmot chukfi nakni achvfakma, keyukmvt chukfulhpoa ushi achvfakma, keyukmut issi kosoma ushi achvfakma hush yumihchashke.

12. Holhtina kaniohmi ho hvsh atahli tuk mvt, holhtina hatuk mak o yohmi bieka ho hush yumihcha chi hoke.

13. Yakni ya aivtta tuk moma kvt nan im issa ya Chihowah $\underline{\mathrm{i}}$ balama luak ishit hvkmit im issakmvt ilvppa puta kak o chohmichit iakaiya hosh yumihcha hi oke.

14. Micha okla inla yosh hvchi takla ahanta hosh, keyukmot hvch isht atiaka puta ka kvna hosh ahanta cha nan im issa ya Chihowah $\underline{\mathrm{i}}$ balama luak ishit hukmit im issakmvt, hush yumihchi kak o yummak kia yumihcha hi oke.

15. Okla hvchia, micha okla inla huchi takla ahanta hush aiena kvt hvchị nan vlhpisa achvfa yosh hvch isht atiaka puta ka nan vlhpisa bilia ya chi hoke; hush kaniohmi ka okla inla ak kia Chihowah itikba ya yưmohma he ak inli hoke.

16. Hvchishno micha okla inla hvchi takla ahanta aiena kut nan vlhpisa achvfa, micha nana akaniohmi hvchim achvfa hi oke, achi tok.

17. Mihma Chihowah yvt Moses a im anumpulit,

18. Islael im vlla vhleha ha im anumpulit, Yakni hvch isht ona la chi yumma bush onakmut,

19. Yohmikmut yakohma hi oke, yakni yash puska ya hushpakmut $\underline{i}$ walit im issa ya Chihowah hush im issa hi oke.

20. I walit im issa ya onush bota yummuska vmmona puska lumbo hush im issa hi oke: a nihechi ya ai i walit hush im issa chatuk vt yomihchi hosh hush $\underline{i}$ wala hi oke.

21. Huch isht atiaka puta ka onush bota yummusska ummona ya i walit im issa yo Chihowah hưsh im issa hi oke. 
22. Atuk o hvsh ashvchi, micha nan vlhpisa ilvppa puta ka Chihowah yvt Moses a tohnot anumpuli tuk isht huvchik ahanto tok mvt,

23. Chihowah yut Moses a im apesa tok nitak vttut huchim isht atiaka ai itin takla himaka pilla mahaya ka Chihowah yut Moses a tohnot apihisa tok fehna ka ik yumohmo tuk mut,

24. Yvmohmikmvt yakohmashkc, okla moma kvt ik ithano nan ik ithano hosh nana kia yumihchikma, okla moma kvt wak hobvk himita achvfa ho hukmit im issa Chihowah i balama, micha ilhpak im issa, micha nan ishko im issa, ai yumihchi mak $\underline{o}$, micha nan ashvchi im issa yo issi kosoma ushi achvfa aiena ho im issashke.

25. Mikma naholitompa isht vtta yut Islael im vlla vhleha okla moma ka isht $\underline{\mathrm{i}}$ kashoffi na $\underline{\mathrm{i}}$ kashofa hi oke; nana ik ithano yoka: mikma nan im issa vbit im issut luak ishit hukmi ya Chihowah isht ima hi oke, micha nana ik ithano tuk vt nan ashuchi im issa ya Chihowah itikba isht ona hi oke,

26. Mikma Islael im vlla vhleha okla moma, micha okla inla apehvt aiasha tuk aiena kvt $\underline{i}$ kashofa hi oke, okla moma kvt nana ik ithano hatuk oka.

27. Atuk o hatak nana hosh ik ithano hosh yoshobakmut nan ashuchi im issa yo issi kosoma tek afummi vmmona isht onashke.

28. Mikma naholitompa isht vtta yvt hatak nan ik ithano hosh ashvchi kash nan ik ithano hosh Chihowah itikba ai ashuchi a $\underline{i}$ kashoffashke, mikma $\underline{i}$ kashofa hi oke.

29. Nan ik ithano hosh ashvchi ka Islael im vlla vhleha takla ai vtta tuk, micha okla inla yosh takla ahanta tuk aiena nan vlhpisa huchim achufashke.

30. Amba hatak nana hosh, peh ilapunla hosh nana akaniohmikmvt, yakni yash inli ai vtta tuk okma keyukmvt okla inla yokmá nana kia, yummak okvt Chihowah ishit yopoma hoke: mikmut hatak yuvmmut im okla ai iti takla ya fiopa tupa hi oke.

31. Chihowah im anumpa ha shitilema cha im anumpa 
vlhpisa ha kobvffi hokvt, hatak yumma im ilhfiopak vt tuput vmohma chi hoke; nan ashvchi vt ilap ak inli ho onvtoyula chi hoke, achi tok.

32. Atuk o Islael im vlla vhleha hut yakni haiaka asha moma mvt, hatak vt nitak hullo nitak o iti tuptua aiowa hosh anta na pisa tok.

33. Micha iti tuptua aiowa hosh anta na pisa tok vt Moses micha Alon micha okla moma aiena ka isht im ona tok.

34. Mihma kaniohma he miha kvt iksho hatuk o ai atoni okla fohki tok.

35. Mihma Chihowah yvt Moses a, Hatak okvt illi pullashke :-binah kocha yo okla moma kvt tvli isht a bolashke, im achi tok.

36. Mihma Chihowah yut achi tuk mak o, okla moma kvt binah ha kocha isht ona cha tuli ishit boli na illi tok.

37. Mihma Chihowah yut Moses a im anumpulit,

38. Islael im vlla vhleha ha ish im anumpuli na, im isht

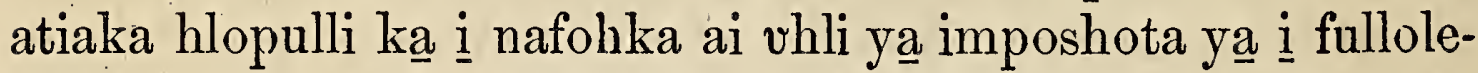
chashke ; micha $\underline{\mathrm{i}}$ nafohka im poshota ya sita lapushki okchamali lapalechashke,

39. Mikma huchim poshota yosh hush pihisakmvt Chihowah im anumpa moma ka ithaiyanvt hrsh yvmihcha chi hoke; mikmvt huchi chukvush akinli, micha hvchi nishkin akinli iakaiyvt hauit hush itvnowa beka tuk vt hvchik yvmohmo kashke.

40. Yumohmi ho hush ithaiyana cha vm anumpa ha moma hush yumihchi cha Chitokaka ya hush i holitopashke.

41. Uno ak osh hvchin Chihowah Chitokaka sia hosh hvchin Chitokaka sia ha chi hosh Echip yakni ya a hvchi pehlichit kohchi li tok oke, Chihowah hvchin Chitokaka sia hoke, ish im achashke, achi tok.

\section{CHAPTA XVI.}

IHMA Kolah, yvmmvt Isha ushi, yummot Kohalh ushi, 1 yvmmut Lefai ushi atok, micha Eliab ushi, vhleha 
Tahlan micha Abilam, micha Pelelh ushi On, Leuben ushi vhleha hvt hatak kaniohmi ka ishi tok.

2. Micha Islael im vlla vhleha aiachufa yummut okla moma itunahut chieya ka $\underline{i}$ holitopa, hatak ithana tahlepa tuklo cha pokoli tahlapi hatak holitoput annoa ai itapeha hosh, Moses itikba ya wakayvt hielit

3. Moses micha Alon a $\underline{i}$ sanalit iti hoyot itunaha cha, Atablit hush il onochishke, okla moma kut holitopa hoka, moma kvt, mikmvt Chihowah yvt ibafoka hoka. Yohmi ka nanta katihmi ho Chihowah im okla ha $\underline{\mathrm{i}}$ shahlechit hush ile hilechi cho? achi tok.

4. Atuk o Moses vt haklo mot nashuka ishit aka hlipia tok.

5. Micha Kolah micha im apeha tuk moma ka im anumpulit, Onnaha fehnak mak o kuna hosh immi hokma micha kvna hosh holitopa hokma Chihowah yot otunincha chi hoke; micha ilap a bilika isht ona chi hoke; ilaput atokoli tuk pulla kak osh bilika isht ona chi hoke.

6. Ilvppak o hush yumihchashke. Kolah micha hush ai itapeha moma kvt, na balama a hukmi hush ishashke.

7. Micha onnaha ma Chihowah itikba ya luak hush fohki cha na balama hush fohkashke; atukma yakohma chi hoke, Chihowah yut hatak nana ho atokolikma, yummak osh holitopa chi hoke. Lefai ushi vhleha hvchia hut, atablit hush ilonochishke, achi tok.

8. Mihma Moses vt Kolah ya Lefai ushi vhleha ma! hush haklashke, hvchim asillha lishke.

9. Islael in Chitokaka yut Islael okla moma ka hvchi i filumminchit Chihowah $\underline{i}$ binah nan isht hush ai ahanta chi ho, micha okla moma nan isht hush im ahanta chi hosh itikba hush hiela chi ho ilap i bilika hvch isht ona tuk a nan isht ik hvchim ahobo cho?

10. Micha chishno, micha Lefai ushi vhleha, chitibapishi vhleha moma aiena ka bilika hvchi hvchisht ona tuk oke, micha naholitompa isht ai vtta aiena hvchi bunna cho?

11. Yvmma pulla kak osh chishno micha ish ai itvpeha aiena kut Chihowah ya i sanalit iti hoyut hush itunahushke; 
micha Alon ak osh kuta hatuk o yưmma i sanalit namihachit hvsh anumpuli cho? im achi tok.

12. Micha Moses vt Eliab ushi tuklo Tahlan micha Abilam a pit hoyot anumpa i pila tuk o yummak ash osh, Il ona he keyu, achi tok.

13. Micha yakni pishukchi micha foibila aiena a yanahanli ya a pehlichit ish pi kohchi kvt, yakni haiaka ai vbit ish pi tahla chin tuk vt nana iskitini ho amba pi shahli, pi miko ish il ikbi cho?

14. Micha pishukchi, micha foi bila ayanahanli yakni isht chik pimoshle, micha osapa micha pakupi ahollokchi ibai asha he a chik pimo kishushke; hatak iluppa puta nishkin a ish kocha wehla hinla cho? il ona he keyu hoke, achi tok.

15. Mihma Moses ut nukowa fehna cha, Chihowah ma! nana chim ihisa ka chik aiokpacho kashke: issuba nashoba achufa kia im ishi li tuk keyu hoke, micha achvfa kia okpuni li tuk keyu hoko, achi tok.

16. Micha Moses vt Kolah ya , Chishno micha ish aiitupeha aiena kut Chihowah itikba, chishno micha yumma, micha Alon aienvt onnakma hush onashke.

17. Micha hatak hush moma kut na balama im aivlhto aiyukali ka na balama fohkit hatak moma kvt na balama im ai vlhto aiyukali na balama ai vlhto aiyukali na balama ai vlhto tahlepa tuklo cha pokoli tahlapi ho ieshit Chihowah itikba isht hush onashke; Chishno micha Alon hush aiena na balama ai vlhto hush ieshi biekashke, im achi tok,

18. Mihma hatak puta kvt na balama im aivlhto luak fohki cha na balama onochit ieshi cha Moses micha Alon a apehut okla moma i tabenakel okhissa ya hieli tok.

19. Mihma Kolah vt okla moma ka im itunahlit okla moma i tabenakel okhissa ya isht ona tok, mihma Chihowah im aholitopa yut okla moma ka $\underline{i}$ haiaka tok.

20. Mihma Chihowah yot Moses micha Alon a im anumpulit,

21. Okla iluppa hrsh il i filưmmichashke, Yohmikma yakosi itin takla vbit lushummi lashke, achi tok.

22. Mihma nashuka ishit hlipkaiyachi cha Chitokaka ma! 
Nipi moma imi shilombish in Chitokaka ma! hatak achufa hosh yoshoba na okla moma ka ish i nukkilla chị cho? achi tok.

23. Mihma Chihowah, yut Moses a im anumpulit,

24. Okla moma ka in anumpulit, Kolah. Tahlan micha Abilam $\underline{i}$ binah apakfoyupa ka issut hush wakayashke, ish im achaskike, im achi tok.

25. Mihma Moses vt wakaya cha Tahlan micha Abilam a im ona tok, mihma Islael im asunonchi vhleha hut iakaiya tok,

26. Micha okla moma ka im anumpulit, Hatak haksi puta iluppa i binah ha issut hush iashke, huchim asilhha lishke; micha nana immi puta ka hush potoli na, keyu hosh nan ashvchi moma ka takkla illit hvchi taiyaha nah, achi tok.

27. Yvmohmi ma, Kolah, Tahlan micha Abilam i binah apakfoyuput fullota tuk vt wakayvt i filvmmi tok, mihma Tahlan micha Abilam vt tekchi vhleha, micha ushi vhleha, micha im vlla chipunta vhleha aiena kvt kohchut i binah okhissa ya ont hieli tok.

28. Mihma Moses vt, Iluppak o na toksuli iluppa puta yumihchi la chi ho Chihowah yut sa tohno tuk a, ishit hush ithaiyana chi hoke; vno ak inli vm anukfila hosh yvmihchi li tuk keju hoka.

29. Hatak iluppa puta kut hatak moma illi chatuk mak $\underline{Q}$ illikma, keyukmot hatak móma im akaniohmi chatuk mak o i yumohmikma, yohmikma Chihowah yut sa tohno tuk keyu ka chi hoke.

30. Amba Chihowah hut nana himona ho ikbi na, yakni yash osh itakpashali cha, yumma micha nana im aiasha moma ka ishit nanubli na, okchaya hosh illi aiasha ka pit tamoakma, hatak iluppa puta kut Chihowah ya nukowvehi tuk a hush ithaiyana chi hoke.

31. Atuk o yakohmi tok, anumpa iluppa moma anumpuli kut ont tahli ma, yakni vt nutaka fehna ita puhlata tok.

32. Micha yakni vt itakpashali cha yumma micha in chnka puta micha hatak moma Kolah apeha tuk, micha im ilayak moma ka ishit nanubli tok. 
33. Yvmma micha nana im aiasha tuk moma kut okchaya moma hosh illi aiasha ka pit tumoa na, yakni vt itafamvt ompohomo tok, mihma okla moma ka $\underline{i}$ filummit illit taha tok.

34. Mihma Islael moma apakfoyuput aiasha tuk vt yvmmut yaiya kak o yihlepa tok. Pishno ak kia yakni vt ishit pi nanvbli mak, achi hatuk okvt.

35. Mihma luak vt Chihowah ya akohchvt minti cha hatak tahlepa tuklo cha pokoli tahlapi, na balama im issa kash o hukmit tahli tok.

36. Mihma Chihowah yut Moses a im auumpulit,

37. Naholitompa isht vtta Alon ushi Eleasa ya ish im anumpuli na, na balama ai vlhto puta ka alua ya akohchashke, micha luak a yumma pit ish fimmashke, hullochi tuk oka.

38. Ilap imi shilombish akinli i yoshoba vhleha iluppa na balama im aivlhto ya alta ompoholmo ya chi ho patussulit okla puthuchashke; Chihowah itikba ai im issa tuk oka, hullochi hatuk oke, mihma Islael im vlla vhleha ha nan isht atokowa ya chi hoke, ish im achashke, im achi tok.

39. Yvmohmi ma naholitompa ishit vtta Eleasa yvt asonak lakna na balama ai vlhto luvt taha tuk vt ai im issa kash ishi tok, mihma patussa putha alta ompoholmo toba tok.

40. Okla inla Alon isht atiaka keyu kvt bilika onvt Chihowah itikba na balama ik im isso pulla hosh Kolah micha ai itupeha yatuk a ik chohmo ka hi vt Chihowah vt Moses a tohnot im achi tuk mak $\underline{o}$ isht ithaiýana bilia hi atok.

41. Yohmi tuk kia onna ma Islael im vlla vhleha moma kut Moses a $\underline{i}$ sanalit micha Alon a $\underline{i}$ sanalit na mihachit Chihowah im okla ha vbit hush tahlishke, achi tok.

42. Atuk o yakohmi tok, okla moma. kut itunahut Moses

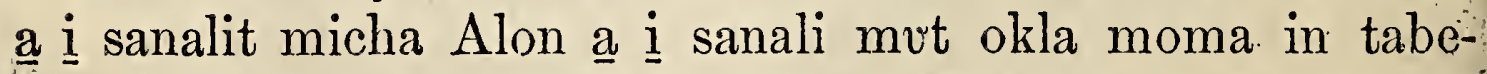
nakel a pit hopokoyo tok mihma Yakeh! hoshonti hvt ompohomo tok, micha Chihowah im aholitopa yut haiaka tok.

43. Mihma Moses micha Alon vt okla moma in tabenakel a itikba ona tok. 
44. Mihma Chihowah yot Moses a im anumpulit,

45. Okla moma iluppa wakayut ish $\underline{i}$ filummashke mikma yakosi iti takla chohmi ho vbit lushummi lashke, achi tok. Mihma nashuka ya aka ishit hlipkaiyachi tok,

46. Mihma Moses vt Alon a nabalama ai vlhto ishi cha alta ya luak ai ishit fohk it nabalama onochi cha tushpvt ivt okla moma ya im onvt ish $\underline{\underline{i}}$ kashoffashke; im ai $\underline{i}$ nukkilli vt Chihowah ya a kohchvt ia hoka, ililli okpulo vt isht ivshke, im achi tok.

47. Mihma Moses vt im apesa tuk mak o, Alon vt ishi cha malelit okla moma yash iklunna ona tok; mihma yakeh! ililli okpulo vt okla ai iti takla ya ai ishit wakaya tuk atok; mihma nabalama onochi cha okla ha $\underline{i}$ kashoffi tok.

48. Micha illi micha okchạya aiena iti takla ya hikia tok, mihma ililli okpulo vt yokopa tok.

49. Yohmi ka Kolah nan im akaniohmi isht illi tuk vto asha na, ililli okpulo isht illi kut tahlepa sipokni auahushta cha tahlepa untuklo hosh illi tok.

50. Mihma Alon vt falamvt Moses a im onvt okla moma in tabenakel a ona tok: mihma ililli okpulo vt yokopa tok.

\section{CHAPTA XVII.}

IHMA Chihowah yut Moses a im anumpulit, I1 2. Islael im vlla vhleha ha ish im anumpuli cha, iki vhleha in chuka achvfa vlhpisa ho tubi achufa bieka ho im ai ishit, iki vhleha in chuka achufa vlhpisa ho holitopa puta ka tvbi auahtuklo ho ish ishashke, micha hatak hohchifo aiyukali ka in tubi ya ish atakalichashke.

3. Micha Alon hohchifo ha Lefai in tubi ak o ish a takalichashke; iki vhleha in chuka achufa $\underline{1}$ noshkoboka yvt tubi im achufut ia chi hoke.

4. Micha okla moma in tabenakel anukaka a chi afama la chi, isht atokowa itikba ak 0 ish ashachashke.

5. Atukma yakohma hi oke, hatak mihi yo atokoli la chi in tubi vt pakanla chi hoke; micha Islael im vlla vhleha hvt 
namihachit anumpuli ishit hvchi sanalit na mihachi ka 1ssuchi li a kunia chi hoke, achi tok.

6. Mihma Moses vt Islael im vlla vhleha ha im anumpuli ma, i holitopa vhleha puta kut tubi achufa bieka ho ima tok, holitopa achufa kvt achvfa bano tok, iki vhleha chuka achufa vlhpisa hosh tubi auahtuklo tok, mihma Alon in tubi vt in tubi puta ka ibafoyuka tok.

7. Mihma Moses vt tubi puta kash Chihowah itikba nan isht atokowa tabenakel ak $\mathrm{o}$ ashachi tok.

8. Atuk o onna ma yakohmi tok, Moses vt nan isht atokowa tabenakel a ont chukowa tok; mihma yakeh! Lefai in chuka achufa ya Alon in tubi ak osh bokobli tuk atok, micha bokoblit kohcha mut pakanli cha almont o waya tok.

9. Mihma Moses vt tubi puta Chihowah itikba aiasha tuk ishit kohchvt Islael im vlla vhleha ha isht im ona tok: mihma okla pisa cha hatak puta kvt in tubi aiyukali ka ishi tok.

10. Mihma Chihowah yut Moses a I filvmmit $\underline{\underline{i}}$ sanali vhleha isht atokowa yosh itoyula chi ho Alon in tubi ya anonti isht ish la cha nan isht atokowa itikba ya ish bohlashke; micha na mibachit anumpuli ya a kanvllechit isht ish kanchashke, mihma ik illo kashke, im achi tok.

11. Mihma Moses vt yumihchi tok, Chihowah yut im achi tuk mak $\underline{0}$, yumihchi tok.

12. Mihma Islael im vlla vhleha hut Moses a yakeh! pillishke, ilbushut pillishke, moma ilbushut pillishke,

13. Kuna hosh Chihowah in tabenakel a bilika kanima onakmut illa chị hoke; illit ont pi taha chị cho? im achi tok.

\section{CHAPTA XVIII.}

TIHMA Chihowah yvt Alon a, Chishno micha chiso I vbleha, micha in chuka achufa chi apeha kvt vlhtipo holitopa nan ai ashvehi vt hvchi onvtoyula chị hoke; micha chishno micha chiso vhleha aiena kvt naholitompa isht hush ai vtta ya ai ashvehi kvt hvechi onvtoyula chi hoke.

2. Micha chitibapishi vhieha apehlichi ai achvfa chiki 
apehlichi yak kia chi apehvchit isht ish lashke, mikma chibafoyuka cha nan isht chim ahantashke; amba chishno micha chiso vhleha aienvt chi apeha hokvto nan isht atokowa tabenakel itikba ak o nan isht hưsh ai ahashwa chi hoke.

3. Mikma nan isht ish ahanta ya ieshi cha, tabenakel ai atoni moma ka ieshashke; peh nan aivlhto holitopa aiasha micha alta bilika aiena ka iklo kashke. Yohmi hosh yumma micha hvchishno aiena kvt ik hvch illo kashke.

4. Micha okla chibafoyukashke, micha okla moma in tabenakel ai atoni ; tabenakel nan ai isht ahanta puta ka ieshashke, mikma okla inla yokvt hvchi bilika ona he keyu hoke.

5. Mikma vlhtipo holitopa ai atoni, micha alta ai atoni aiena ka hush iesha chị hoke; yưmohmi ho himakma ai $\underline{\underline{\mathbf{i}}}$ nukkilli vt Islael im vlla vhleha ha ik onvtoyulo kashke.

6. Mikma vno, yakeh! vno vt hvchitibapishi Lefait vhleha, Islael im vlla vhleha ha $\underline{\mathrm{i}}$ filummichit ishi li tuk: oke; Chihowah immi hosh okla moma in tabenakel nan isht ai ahanta chi ho na hulbina yo chohmit hvchima lishke.

7. Yvmohmi hoka chishno micha chiso vhleha hush ai itapeha hokvto, alta isht ahalaya nana puta, micha im ompoholmo anukaka aiena isht hush ai ahanta chi hosh naholitompa isht ai vtta ya hush ieshashke, naholitompa isht hush ai vtta ya na hulbina nan isht vtta yo hvchima li hatuk oke : Yohmi ka okla inla yosh bilika onakmut illi pulla hi oke, achi tok.

8. Mihma Chihowah yot Alon a im anumpulit, Yakeh! Islael im vlla vhleha $\underline{i}$ nahullochi puta, $\underline{i}$ walit im issa puta atoni ya chima lishke : bila ahamut chi vlhtoka yatuk mak o chima lishke, micha nan vlhpisa kullo bilia mak o chiso vhleha ima lishke.

9. Naholitompa i shahli luak a ilatomba tuk iluppak osh chimmi ha chi hoke, im isht aiokpvchi puta, ilhpak im issa puta, micha nan ashuchi im issa puta, micha yoshoba im issa puta, yumma vm ihissa tuk puta kut chishno micha chiso vhleha aiena kvt hvchi holitopa moma i shahli ya chi hoke.

10. Aholitopa $\underline{\mathrm{i}}$ shahli ak $\mathrm{o}$ hush ai vpa chi hoke, nakni puta kak osh upa chi hoke, hvchi holitopa ya chi hoke. 
11. Micha iluppak osh chimmi ha chi hoke, Islael im vlla vhleha na hulbina $\underline{\mathrm{i}}$ walit im issa, micha $\underline{\mathrm{i}}$ fahfolit im issa moma aiena ak oke; nana vlhpisa bilia yo chishno, micha chiso vhleha, micha chiso tek vhleha chibafoka aiena ka chima lishke; chin chuka ya kashofa aiasha puta kvt vpa chi hoke.

12. Micha bila achukma i shahli moma, micha oka paki achukma i shahli moma, micha onush aiena nawaya vmmona, Chihowah im issa tuk a yumma puta chima lishke.

13. Micha yakni ya nana hosh tikkba waya na Chihowah ya isht im onakma, chimmi ha chị hoke, kashofa hosh chin chuka ahanta moma kvt ai upa chi hoke.

14. Nana puta kvt Islael a ahullochi kvt chimmi ha chị hoke.

15. Nana moma nipi puta ai achvfa hosh ushvto ya tiwi na Chihowah ya isht im vla puta, hatak okmá, nana vlhpoba yokmá nana kia chimmi ha chi hoke : Yohmi kia hatak osh vtta ummona yokuno falumminchit ish chumpa pulla chi hoke; micha nan vlhpoa ik kashofo vtta ummona aiena kvno falumminchit ish chumpa hi oke.

16. Falvmminchit chumpa chi hokvto hvshi achvfa vhli kak $\underline{0}$ ish apesa mak $\underline{o}$, tvli holisso shekel tahlapi, vlhtipo holitopa shekel iakaiya hosh, yummut kelah pokoli tuklo ho'ka, falumminchit ish chumpa hi oke.

17. Amba wak chelahpi, keyukmut chukfi chelahpi keyukmvt issi kosoma chelahpi aiena hokvno, falvmminchit ish chumpa he keyu hoke, holitopa hoka, im issish a alta ya ish o fimmi cha, Chihowah $\underline{i}$ balama ya chi ho, luak ishit hukmit im issa yo $\underline{i}$ nia ya luak isht ish hukmashke.

18. Micha ikkishi fahfuli, micha fulup isht impakimma yưt chimmi ka yummak inli chohmit $\underline{i}$ nipi vt chimmi ha chi hoke.

19. Pit 1 walit im issa holitopa Islael im vlla vhleha hut Chihowah ya im issa tuk moma ka chishno, micha chiso vhleha, micha chiso tek uhleha chibafoka aiena kạ, nan ulhpisa bilia yo chima lishke; Chihowah itikba hupi nan 
itim apesa kvt chishno, micha chim isht atiaka aiena bilia ya chi hoke, achi tok.

20. Micha Chihowah yvt Alon a im anumpulit, Chishno vto i yakni ya ish isha he keyu hoke, micha ibatakklut chi kashapa ish isha he keyu hoke; vno ak osh Islael im vlla vhleha ai itin takla ya chi kashapa, chimmi sia hoke.

21. Micha yakeh! Lefai im vlla vhleha hot nan isht ai ahanta isht ahashwa tuk okla moma i tabenakel isht ai ahanta tuk vt ai isha he vlhpesa yut Islael a ishit pokoli moma kak o ima li hoke.

22. Micha himak a pilla hoka Islael im vila vhleha hot okla moma in tabenakel a bilika vla he keyu, yohmit nan ashvchi shaiyali cha illi yoba nah.

23. Amba Lefait vhleha ak osh okla moma in tabenakel a nan ai isht ahanta chi hoke: micha yvmmak osh ilap nan ashvchi ya shaiyala chị hoke, chim isht atiaka hlopulli puta ka Islael im vlla vhleha ai itin takla ka ai isha he vlhpesa yvt ik im iksho nan vlhpisa bilia ya chi hoke.

24. Amba Islael im vlla vhleha nan im isht $\underline{i}$ pokoli, yumma $\underline{i}$ walit im issa yo Chihowah im ihisa yak $\underline{o}$, ai isha he vlhpesa mak o Lefait vhleha ima li hoke. Yohmi kak o Islael im vlla vhleha ai itin takla ya ai isha he vlhpesa yut ik im iksho ka chi hoke, im achi li tuk oke, achi tok.

25. Micha Chihowah yvt Moses a im anumpulit,

26. Yakohmichit Lefait vhleha ha im anumpulit, Hush ai isha he vlhpesa yo hvch ima li tuk a Islael im vlla vhleha ha ishit pokoli ya hush im ai ishikmvt ishit pokoli kash ishit pokoli ka walit im issa yo Chihowah ya hvsh im issashke.

27. Mikma i walit ish im issa iluppak okvt a nihechi $\underset{\underline{i}}{\mathbf{i}}$. patulhpo onush asha tuk, micha paki a okbushli alota atuk chim itilaua hi oke.

28. Huchishno ak kia yakohmichit Islael im vlla vhleha hush im ai ishi tuk, ishit hvchi pokoli moma kvt walit im issa yo Chihowah hush im issashke, micha walit Chihowah im issa yash, naholitompa isht vtta Alon a hush ima hi oke. 29. Na huchi hulbina moma ka akohchit walit Chihowah 
im issa achukma i shahli moma hullochi kashapa ka akohchit hush im issashke.

30. Yvmohmi hoka achukma i shahli ka akohchit hvsh walikma, yohmikma yummut anihechi patulhpo onush awaya asha tuk, micha paki ai okbushli awaya tuk ak inli, Lefait vhleha im itilaua hi oke.

31. Mikma kanima moma ka hvchishno micha hvchin chuka achvfa hush aiena kvt hvsh ai vpa hi oke; okla moma in tabenakel nana isht hush ai ahashwa tuk huchim vlhtoba mak oke.

32. Micha achukma i shahli ka ai ishit hrsh walikma, yummak atuk mak $\underline{\underline{o}}$, nan ashvchi ya hush shaiyala he keyu hoke; micha Islael im vlla vhleha $\underline{i}$ naholitompa puta $\mathrm{k}$ a hush litehla he keyu hoke, hush yohmi cha hvch illi nah, ish im achashke, im achi tok.

\section{CHAPTA XIX.}

MIHMA Chihowah yot Moses micha Alon a im anumpulit, 1 2. Ilvppak osh nan vlhpisa Chihowah yot anumpulit, Islael im vlla vhleha ha ish im anumpuli na, wak tek himita homma, inlvt takali iksho, ik ai ono kvt iksho, yummak oka. ikunla abana yot onvtoyula tuk keyu ho isht chim vlashke.

- 3. Mikma naholitompa isht vtta Eleasa ya ish ima na, bi nah ha kocha isht onakma, achvfut nashuka itikba ya ai vbashke.

4. Mihma Eleasa naholitompa isht vtta yut im issish a ibbak ushi isht ishi cha im issish a okla moma in tabenakely itikba fehna untuklo ha a fimmashke.

5. Mihma p sa ho achufut wak tek himita yash hukma hi oke; hakshup micha nipi micha im isssish, i yulhki aiena ka hukma hi oke.

6. Mihma naholitompa isht vtta yvt chuahla iti, hissop, micha natishepa aiena ka ishi cha, wak tek himita lua ka iklunna pit pila hi oke.

7. Yohmikma naholitompa isht vtta yvt $\underline{i}$ nafohla ya 
achefa cha nipi ya oka isht achifashke, atuk osh $\underline{i}$ himmak a yo binah ha ont chukowashke, micha naholitompa isht vtta yot liteha na opiashke.

8. Mikma hukmi tuk vt $\underline{i}$ nafohka ya oka isht achefashke, micha nipi ya oka isht achefashke, micha liteha na opiashke.

9. Mikma hatak kashofa hosh wak tek limita hituk chubbi ya aiowa cha binah puta ka kocha akashofa ho ashachashke; atuk o Islael im vlla vhleha okla moma kvt ila filvmminchi oka yosh im aiasha hi oke, nanashvchi ishit kashofa yoke.

10. Mikma wak tek himita hituk chubbi aiowa tuk vt $\underline{\mathrm{i}}$ nafohka achifa cha liteha na, ont opia hi oke; micha Islael ini vlla vhleha, micha okla inla yosh takla ahanta aiena kvt i nan vlhpisa bilia hi oke.

11. Hatak kuna kia illi nipi yo potolikmut nitak untuklo ho liteha hi oke.

12. Nitak ont isht atuchinakma isht ile kashoffashke, micha nitak ont isht untuklokma kashofa chi hoke; amba nitak ont isht atuchina ka ik ile kashoffokmvt nitak ont ishit untuklo ka kashofa he keyu hoke.

13. Kuna hosh hatak kuna illi nipi illi tuk o potoli tuk osh, ik ile kashoffokmvt Chihowah in tabenakel a litehlishle. $x$ nikmut shilombish yummot Islael a $\underline{i}$ filummit fiopa tupa hi oke, le filummichi oka yut ik o fimmo tok okvt, liteha hi oke, in a liteha kvt onvtonla moma hoke.

14. Hatak osh vlhtipo ai illikma nan vlhpisa hut iluppak oke; bina hash ont chukowa tuk moma, micha binah aiasha tuk aiena kut nitak untuklo ho liteha hi oke.

15. Mikma nan ai vlhto tiwa tuk puta on hlipia isht ompoholmo tuk keyu kut liteha hi oke.

16. Mikma kuna hosh achufut ashahbika yo bushpo falaia isht illi tuk o, keyukmvt illi nipi, keyukmvt hatak foni, keyukmvt ahollohpi yo potoll tuk mut nitak untuklo ho liteha hi oke.

17. Mikma hatak liteha ka, nan ashvchi ishit kashofa, wak tek himita yash hituk chubbi ya ishi cha ai vlhto itibanashke. 
18. Mikma hatak kashofa yosh hissop ishi cha oka yash okvchi cha binah hash, micha nan ai vlhto puta moma micha hatak yumma aiasha tuk, micha foni, keyukmut vbi na illi tuk, keyukmvt illi, keyukmot ahollohpi potoli tuk $\underline{a} \underline{\mathbf{o}}$ fimmashke.

19. Micha hatak kashofa yvt ik kashofo yosh nitak ont isht atuchina, micha ont isht untuklo ka o fimma hi oke; mikma nitak ont isht untuklokma ile kashoff cha $\underline{i}$ nafohka ya achifakmvt oka yupi cha opiakma kashofa hi oke.

20. Amba liteha hoh kia hatak yummut ile kashofa he keyukmvt shilombish yummut okla moma ka ai i filummit fiopa tupa hi oke; Chihowah im vlhtipo holitopa ya litehli tuk okvt, ile filumminchi oka yut o fimimpa tuk keyu hoka; liteha hoke.

21. Ile filummichi oka $\underline{a}$ fimmi tuk vt $\underline{i}$ nafohka ya achifa he, micha ile filummichi oka ya potoli tuk vt liteha na ont opia hi vt hvchi nan vlhpisa bilia hi oke.

22. Micha nana ho hatak liteha kut potolikma liteha hi oke, micha shilombish vt potolikmvt liteha na ont opia hi oke.

\section{CHAPTA XX.}

YOHMI ma Islael im vlla vhleha okla moma kvt hushi 1 vmmona ho Sin yakni haiaka ya ona tok; mihma okla hut Katesh ak $\underline{o}$ aiasha tok; atuk $\underline{0}$ Miliam vt yummak $\underline{\mathrm{g}}$ ai illi cha yummak o a hollohpi tok.

2. Atuk o okla moma kut oka ik im iksho tok, mihmvt Moses micha Alon a 1 sanalit iti hoyot itahoba tok.

3. Micha Moses $\underline{a} \underline{i}$ nukowvt anumpulit, Chihowah hot vlhpesa ahni na pitibapishi vhleha hot Chihowah itikba illit taha ka takla pilli tuk okbano.

4. Micha nanta katiohmi ho Chihowah im okla hut yakni haiaka iluppa pishno, micha pi wak aiena kvt pi ai illa he a pisht ish la tuk oh cho?

5. Micha nanta katiohmi ho ik ai achukmo iluppa pisht ishla chi kvt Echip a ish pi kohchechi tok oh cho? nanihi, 
micha fik, micha pakvpi, micha pomeklanit aiasha keyushke; micha oka ishka hi vt ikshoshke, achi tok.

6. Mihma Moses micha Alon vt okla ha itikba ai i filvmmit okla moma in tabenakel okhissa ya ona cha, nashuka ishit hlipkaiyachi tok. Mihma Chihowah im aholitopa yot i haiaka tok.

7. Mihma Chihowah yut Moses a im anumpulit,

8. Tubi ya ishi cha okla hoyot ish itunahlashke, chishno micha chitibapishi Alon ita tuklo kvt, micha nishkin itikba tuli chito ya hush im ai anumpulashke; mikma im oka ya kohchicha chi hoke; mihma tvli chito ak o akohchit oka ish imashke; yohmi hosh okla moma, micha i nan vlhpoba aiena ka nan ishko hush imashke, im achi tok.

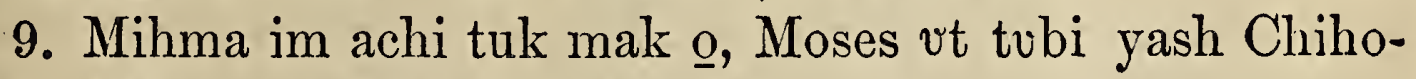
wah itikba itoyula tuk a ishi tok.

10. Atuk osh Moses micha Alon vt okla moma ka hoyot tuli chito yash itikba ya ai itunahli tok, micha Ila filummit i sanali vhleha hvchi ma! ho haklo, twli chito iluppa oka e hvehim akohcha chi cho? im achi tok.

11. Micha Moses vt ibbak a vba pit weli cha tvli chito ya in tubi yash hitukla ha isht isso tok; mihma oka laua fehna hosh et kohcha tok, mihma okla moma, micha i nan vlhpoa aiena kvt ishko tok.

12. Mihma Chihowah yut Moses micha Alon a im anumpulit, Hựchik' sa yimmot Islael im vlla vhleha itikba ya hvchik sa holitoblo tuk okvt, yumohmi tuk okvt okla iluppa pehlichit yakni ima li tok a isht hush chukowa chị keyu hoke, im achi tok.

13. Iluppak osh Melibah oka mak oke, Islael im vlla vhleha hot Chihowah ya 1 kvllo na isht aholitopa tuk oka.

14. Mihma Moses vt Katesh a vttut anumpa shali vhleha ha ha Etom i miko ya pit i pilvt, Chitibapishi Islael vt yak achishke, nan isht ilbusha pi onvtoyula moma ka ish ithanushke.

15. Piki vhleha hut Echip a ona tok, atuk o Echip $\underline{a}$ il aiasha na hopaki, mihma Echip okla hvt pishno, micha piki vhleha aiena ka pilbushali tok. 
16. Atuk o Chihowah ya il i pahaya ma, pim anumpa - haklo cha, enchil o et pila cha pehlichit Echip a ai ishit pi kohchichi tuk oke; atuk o yakeh! chim ai vhli fehna tvmaha holihta Katesh ak o il ai ashushke.

17. Chi yakni ya ke hlopullih, chim asilhha lishke, osapa puta, micha paki ahollokchi puta ka e hlopulla he keyu; michá kuli hofobi puta ka oka il ai ishka he keyu hoke ; miko ij hina ak o il ja cha isht impakimma, micha vlhfubekimma pit ke fulloto hosh chim ai vhli ya e hlopulla chi hoke, achi tok.

18. Mihma Etom vt, A bilika ont hvsh ia he keyu, yohmikma bưshpo falaia isht huchi afama la chi hoke, achi tok.

19. Mihma Islael im vlla vhleha hvt, Hina ak $\underline{o}$ il ia chi hoke, atuk o vno, micha a nan vlhpoa yvt chim oka ya ishkokma atobbi lashke, peh, nan inla ka ak yumihcho hosh, aka ayut hlopulli lashke, im achi tok.

20. Mihma, Ish hlopulla he keyu, achi tok. Mihmot Etom vt okla laua ho ieshi hosh, micha ibbak kvllo hosh kohchvt afamvt minti tok.

21. Yakohmi hosh Islael vt im ai vhli hlopulla chin tuk a vlhpesa ik im ahno tok, yưmohmi ho Islael vt $\underline{i}$ filummit ia tok.

22. Micha Islael im vlla vhleha hut okla moma kut Katesh a asht ia hosh hunih Ho ya ona tok.

23. Mihma Chihowah yut nunih Ho Etom yakni ai vhli bilika yo Moses micha Alon a im anumpulit,

24. Alon vt im okla ha ont ibafohka chi hoke, oka Melibah ya vm anumpa hạ i sanalit hvsh filvmmi tuk oka, yakni Islael im vlla vhleha ima li tok a chukowa he keyu hoke.

25. Alon micha ushi Eleasa ya ish ishi cha nunih Ho ya oiyvt isht ish onashke.

26. Micha Alon i nafohka ya ish $\underline{\mathrm{i}}$ shufi cha, ushi Eleasa ak $\underline{0}$ ish fohlivchashke; mikma Alon vto im okla hạ ont ibafohka chị hoke, micha yummak o ai illa chị hoke.

27. Mihma Chihowah yut apcsa tuk mak o, Moses vt yumihchi tok; micha okla moma kut pisa ho nunih Ho ya pit oiya tok. 


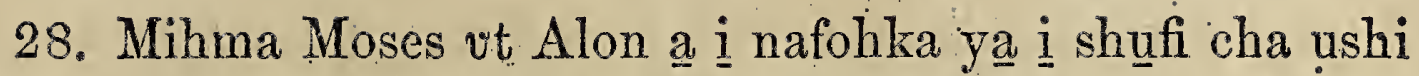
Eleasa ak o fohkvchi tok: mihma Alon vt nunih paknaka" yummak o ai illi tok: mihma Moses micha Eleasa ita tuklo kvt nvnih ha akowa tok.

29. Atuk o okla moma kvt Alon vt illi tuk a ithana mvt, Islael in chuka achufa moma kvt Alon a ishit yahaya na nitak pokoli tuchina tok.

\section{CHAPTA XXI.}

TUK o Kenanait miko Alat oka mahli pilla vtta tuk vt A yakni pisa vhleha ataya tuk ak $\underline{o}$, Islael vt minti ho anoli na haklo tok, mihmut Islael a itibi cha kanohmi ka ynkachi tok.

2. Mihma Islael vt anumpa il onochi Chihowah ya im il onochit, okla iluppa subbak a ish fohki ho muhlikma, yohmikma in tumaha holihta puta moma ka okpunit tahlit vmohmichi lashke, achi tok.

3. Mihma Chihowah yưt Islael im anumpa ha haklo cha, Kenanait vhleha ha ibbak fohki tok; mihma yumma, micha in tumaha holihta puta aiena ka okpunit taiyahli tok; mihma yakni yash Homah hochifo tok.

4. Mihma Etom yakni ya apakfopa chi hosh nunih Ho ya ashut Okhuta Humma pilla hina yo ia tok: mihma okla hot hina pulla kak o chukvush isht ielli tok.

5. Micha okla hvt Chitokaka ya $\underline{\mathrm{i}}$ sanalit, micha Moses a i sanalit anumpulit, Nanta katiohmi ho yakni haiaka ai illit pi taha chị ho Echip a pi pehlichit ish pi kohchi tak oh cho? puska yut iksho, micha oka yvt iksho, micha puska okpulo iluppano pi yuwalut tahushke, achitok.

6. Mihma Chihowah yut sinti luak o okla ai itin takla on tihleli na okla ha kopoli tok, mihma Islael okla laua kut illi tok.

7. Yumohmi ho okla hut Moses a im vla cha, Pi yoshobushke; Chihowah ya micha chishno ya e chi sanalit il anumpuli tuk okvt, Chihowah ya ish im asilhha na, sinti ya pi 
kanvllichit ishit kanchashke, achi tok. Mihma Moses vt okla ha asilhhvt isht anumpuli tok.

8. Mihma Chihowah hvt Moses a Sinti luak o ish ikbi cha, iti fabussa wishakchi ish ontalalashke : atuk o yakohma hi oke, sinti vt kopoli tuk aiyuka hokvt yumma pisakmut okchaya hi oke, achi tok.

9. Mihma Moses vt asonak lakna yo sinti ikbi cha iti fabussa wishakchi on talali tok, atuk o yakohmi tok, sinti vt hatak nana ho kopoli tuk ma, asonak lakna sinti yash pisakmut okchayja beka tok.

10. Atuk osh Islael im vlla vhleha hut tikba ivt Obolh ak o abinanchi tok.

11. Atuk osh Obolh a ashưt ia mvt yakni haiaka Icheabalim, yưmmut hushi akochaka pilla Moab itikba ya talaia kak o a binanchi tok.

12. Yvmma ashvt kanvlli mut Salet okfa ak o a binanchi tok.

13. Yvmma ashvt kanvlli mvt, Anon mishtunnup yvmmvt Amolait im ai vhli et kocha yakni haiaka ak o a binanchi tok. Moab micha Amolait ititakla ya Anon ak osh Moab im aivhli yatuk oke.

14. Yumohmi hatuk o Chihowah itin tanampi tok holisso hạ, Okhvta Humma micha Bokushi Anon.

15. Micha bokushi yanvlli A aivtta pilla ia, micha Moab im aivhli bachaya aiena ka a yumihinchi tok achit a holihiso hoke.

16. Micha yumma ashut ia mut Bee ya ona tok, yummut kuli hofobi yummak o Chihowah yot Moses a ai im anumpulit, Okla hoyot ish itunahli na oka ipeta lashke, achi tok.

17. Yohmi ma Islael vt taloa iluppa taloa tok. Kuli hos fobi ma! ish mitafashke; hush in taloashke.

18. Hatak $\mathrm{i}$ shahli vhleha ak osh kulli; okla holitopa vhleha ak osh nan apesa yak osh miha ho in tubi ishit kulli tok oke. Atuk osh yakni haiaka ashvt ia mvt Mattanah ya ona tok.

19. Atuk osh Mattanah ashvt ia mvt Nahaliel ona tokatuk osh Nahaliel a ashvt ia mut Bemolh ona tok. 
20. Atuk osh okfa talaia Bemolh yummut Moab i yakni ya talaia ka ashut ia mut Piskah paknaka yummut Cheshimon pit talaia ka ona tok.

21. Mihma Islael vt Amolait vhleha $\underline{\mathrm{i}}$ miko Sihon $\underline{a}$ anumpa shali pit i pilut,

22. Chi yakni ya e hlopullashke, fullotot chim osapa puta, micha chi pakupi ahollokchi puta ka e chukowa he keyu ; micha kưli hofobi ya oka il ai ishka he keyu hoke, amba miko i hina ak o ayvt chim aivhli ya ont ivt e hlopullashke, achi tok.

23. Mihma Sihon vt im ai vhli ya Islael vt ik hlopullih, ik im ahno tok; amba Sihon vt im okla moma ka hoyot itunahli cha, yakni haiaka Islael ai itiba chi hosh kohchut ia tok; atuk osh Chahas a vla cha Islael a itibi tok.

24. Mihma Islael vt bushpo falaia halupa ishit fahamot kanchi tok, micha i yakni ya im eshit Anon vttut ia hosh Chabbok ont aivhli, Ammon im vlla vhleha im ai vhli.y isht ona tok. Ammon im vlla vhleha im ai whli vt kvllo fehna hatuk oka.

25. Micha Islael vt tumaha holihta iluppa puta moma ka ishi tok; mihmot Islael vt Amolait in tumaha holihta puta Heshbon aiasha ka, micha chuka im a lokoli moma aiena ka aiasha tok.

26. Heshbon ak osh Amolait okla i miko Sihon yummvt chashpo ka Moab i milko ya itibi cha i yakni moma Anon ont vhli ha ibbak ai ishi tok $\underline{\text { a }}$, in tumaha holihta yatuk oke.

27. Yvmohmi hatuk o nan achi anumpuli kut Heshbon a hush lashke, Sihơ in tumaha holihta yut tobut vlhtahashke.

28. Luak vt Heshbon a kohchvt ia hoka, luak libbi kvt Sihon in tumaha kohchvt ia hoka; Moab talaia A, micha Anon achahaka puta hochitoka vhleha ha hukmit tahlishke.

29. Na palummi chi onvtolushke, Moab ma! okpulokut huchi tahushke, Chemosh okla ma! ushi vhleha micha ushetik vhleha.aiena kvt hlakofi tuk a Amolait $\underline{i}$ miko Sihon yuka ikbit im issushke.

30. E husa tuk oke; Heshbon vt illit taha kvt Tibon a 
ont vhlishke, micha chukilissuchit Nofah yummut Meteba ona ka isht il onvshke, achi hoke.

31. Yakohmi hosh Islael vt Amolait i yakni ya aiasha tok.

32. Mihma Chaasa ya pisa chi ho Moses vt yakni pisa ya pit pila tok, micha in chuka lokoli puta ishi cha Amolait yumma aiasha tuk a tihlelit kocha wehli tok.

33. Mihmut fullotut ivt Bashan pilla ho pit fullota tok; mihma Bashan i miko Ok, yumma micha im okla moma aiena kvt Etlei ya ai itiba chi hosh i sanalit kohehvt ia tok.

34. Mihma Chihowah yut Moses a Ish i nukshopa nah, yumma micha im okla moma, micha 1 yakni aiena ka chibbak fohki li hoke; mikma Amolait î miko Sihon Heshbon ai vtta yatuk a ish $\underline{i}$ yumihchi tok vt chohmichit ish yumih cha chi. hoke, im achi tok.

35. Yvmohmi hosh yumma, micha ushi vhleha, micha im okla moma ka issot kanchi na, okchayut im anta kvt iksho tok, micha i yakni ishit abinilj tok.

\section{CHAPTA XXII.}

IHMA Islael im vlla vhleha hvt tikba ia mut, Moab N yakni patali chatan ola in tunnup Cheliko bilika ak $\underline{\mathbf{c}}$ abinanchi tok.

2. Mihma Sippo ushi Balak vt Islael ut Amolait a kaniohmichi tok moma ka pisa tok.

3. Mihma okla hut laua fehna hatuk oka, Moab vt i nukshopa fehna tok; mihma Islael im vlla vhleha pulla kak $\underline{o}$ Moab vt komunta tok.

4. Micha Moab vt Mitian im asunonchi vhleha ha Himak a okla iluppot pi afolublit äsha tuk moma ka, wak osh osapa hushuk asha ho holakshit ishi chatuk ak o chohmit holakshit pisha chị hoke, im achi tok. Micha Sippo ushi Balak vt nitak yumma Moabait okla i miko yatok.

5. Yvmohmi hatuk osh Beo ushi Balaam, Pehlo yummut im okla im vlla vhleha i yakni okhina bilika talaia ka anta tuk o hoya chi hosh, anumpa shali pit i pilut, Yakeh! Okla 
hosh Echip a akohchut mintit vlushke; Yakeh! yakni paknaka ya ompohomoshke, micha sa chapaka yak o okla ai ashushize.

6. Yvmohmi hoka, minti cha himak a okla illuppa anumpa kalakshichi onochit ant is sa mihashke, a kvllo kvt atupa hoke: imaiy flkma issot e kanchikma, tihlelit yakni ya kocha wehli li yoba ná; kuna ho na yukpali anumpa ish onochikma, na yukpa ka, micha kuna ho anumpa kalakshichi ish onochikma kalakshi ka ithaiyana li hoke.

7. Mihma Moab im asunonchi vhleha, micha Mitian im asvnonchi vhleha aiena kvt isht ahullo na habenvchi vt. ibbak foyuka hosh ilhkoli tok; atuk osh Balaam a im vla mot, Balak im anumpa hatuk a im anumpuli tok.

8. Mihma Himak ninak a iluppak $\underline{o}$ hvchi nusashke, atuk o Chitokaka yot kaniohmit vm anumpulikma, anonti isht huchim vla la chi hoke; mihma Moab holitopa vhleha hvt Balaam a takla asha tok.

9. Mihma Chitokaka yut Balaam a im vla cha, Hatak nanta hosh ilvppa chi apehvt aiasha cho? im achi tok.

10. Mihma Balaam vt Chitokaka ya Moab i miko Sippo ushi Balak vt et sa hoyot,

11. Yakeh! okla hot Echip a a minti hosh vla cha yakni paknaka ya ompohomoshke; himak a ishla cha anumpa kalakshichi onochit ant is sa mihakma, im aiya lish tiblelit kocha weli li yoba na; achishke, im achi tok.

12. Mihma Chihowah hut Balaam a, Awant ish ia he keyu; anumpa kalakshichi ish onocha he keyu; holitopa hoka, im achi tok.

13. Atuk o onnahinli ma Balaam vt wakaya cha Balak $\underline{\mathbf{i}}$ holitopa vhleha hạ, Hvchi yakni ya ont hush chukowashke; huchi awant ia la he ya Chihowah yut v́m olvbi hoka, im achi tok.

14. Mibma Moab holitopa vhleha' hot wakaya cha Balak a im ona mut, Balaam vt pi awant minti ik bunnoshke, achi tok.

15. Mihma Balak vt anonti holitopa vhleha laua i shahli, micha holitopa kvt i shahli ho pila tok.

16. Mihma Balaam a im vla cha, Sippo ushi Balak vit 
yak achishke, Nana kia is sum vla chị ka ik chi ataklummo kashke, chim asilhha lishke.

17. Holitopa chinto fehna ho chi hilechi la hi oka, micha nana ho is sa mihakma yumihchi la chi ; yumohmi hoka, minti cha okla iluppa kalakshichi, ant vm onochih, chim asilhha lishke, achishke, im achi tok.

18. Mihma Balaam vt afalamichit, Balak in tishu vhleha hạ, Balak vt tvli hvta, micha tvli holisso lakna ya im aboha alota achvfa ho.ik suma chi hok mak o, Chitokaka an Chihowah im anumpa ha ont ivt, ola takla, micha i shahli hoh kia yomihchi la he keyu hoke.

19. Yvmohmi hoka himak a ashut himak ninak ak kia iluppa hvchi nusashke, hvchim asilbha lishke; Yohmi na himakma Chihowah hut nana ho vm acha hinla ka akostininchi lashke, im achi tok.

20. Atuk o ninak o Chitokaka yvt Balaam a im vla cha, Hatak vhleha hash osh chi hoyot aya tuk ma, ish wakaya cha, awant ish iashke; yohmi kia anumpa chim achi la chi yummak o, ish yumohma chi hoke, im achi tok.

21. Atuk o onnahinli ma Balaam vt wakaya cha, im issuba nashoba ya om patali mut Moab holitopa vhleha ha awant ia tok.

22. Mihma ia kak o Chitokaka im ai i anukkilli vt libbi tok: mihma Chihowah im enchel vt in tanampi hosh hina ya i sanalit hikia tok. Mihma im issuba nashoba ya om binilit aya ma, in tishu tuklo kut takla aya tok.

23. Mihma issuba nashoba yvt Chihowah im enchel vt hina takla hikia mut, bushpo falaia shufa ibbak foyuka hosh hikia na pisa tok; micha issuba nashoba yut fullotvt hina ya kohchvt osapa ont chukowa tok; mihma Balaam vt hina ya pit fohkvchi hosh issuba nashoba ya isso tok:

24. Yohmi kia Chihowah im enchel vt pakvipi a hollokchi hina ya hikia ma, holihta chaha yvt ilvppa bachaya ma, anonti holihta chaha yot yumma bachaya tok.

25. Atuk o issuba nashoba yut. Chihowah im enchel pisa mut holihta kvllo ont nuhli cha Balaam iyi ya holihta kullo ya akatanlechit litoli tok, mihma anonti isso tok. 
26. Mihma Chihowah im enchel vt mishema ona cha ai atukonofa, isht impakimma ia he, micha ulhfubekimma ia he kia, iksho ho a hikia tok.

27. Atuk o issuba nashoba yut Chihowah im enchel a pisa mut Balaam nutaka itula tok; mihma Balaam im ai $\underline{\mathbf{i}}$ nukkilli vt libbi mut issuba nashoba yash tubi isht isso tok.

28. Mihma Chihowah yot issuba nashoba im itih ha wakummi na, Balaam a, Nanta chi katiohmichi li ho iluppa ont isht atuchina mak $\underline{0}$ issusso cho? im achi tok.

29. Mihma Balaam vt issuba nashoba yash, Isht is sa yopula hoka; bushpo falaia yut subbak a foka tuk okbano, ahni lishke, himak a chibe la he tuk okvt, im achi tok.

30. Mihma issuba nashoba yash osh Balaam a Chim issuba nashoba sia ho chimmi sia hatok ititakla himak nitak ant ai vhli ka is si ombinili chatuk keyu cho? himonna kia chi ayumohmi li cha tuk oh cho? im achi tok, Mihma, Keyu, achi tok.

31. Yohmi ma Chihowah yut Balaam nishkin fatummi na, Chihowah im enchel vt bushpo falaia shufa ibbak afoyuka hosh hina ya hikia ho pisa tok: mihmvt noshkoba ha akachunolechit nashuka ya aka ishit hlipia tok.

32. Mihma Chihowah im enchel vt, Nanta katiohmi ho ilupput ont isht atuchina mak o chim issuba nashoba ya ish isso cho? Yakeh! sä tikba ya ish ataya kut ai vlhpesa keyu kak o, chi sanali la chi hosh kohchut ia li tuk oke.

33. Mihma issuba nashoba yut sa pisa cha, iluppot ont isht atuchina mak o a shanaiya tuk oke; ik sa shanaiyo tuk okma, himak a chohmi ka chibe li pulla cha, yummuno okchalinchi la he tuk oke, im achi tok.

34. Mihma Balaam vt Chihowah im enchel a sai yoshoba tuk oke, a sanalit sa tikba hina ya ish hikia ka ak ithano tuk okvt; yumohmi hoka, himak a ik chim vlhpesokma anonti falamut ia lashke, im achi tok.

35. Mihma Chihowah im enchel vt Balaam a Hatak ash awant ish iașke; yohmi kia, peh, anumpa chim anumpuli li illa kak o ish anumpula chi hoke, im achi tok; yohmi ma Balaam vt Balak i holitopa vhleha ha awant ia tok. 
36. Atuk $\underline{\mathrm{o}}$, Balaam vt vla na Balak vt haklo mut afamut ivt, Moab in tumaha holihta yummut Anon vhli yummut ont vhli fehna hatuk o ona tok.

37. Mihma Balak ut Balaam a, Ahli hosh pit chi pilut chi hoyo li tuk keyu cho? nanta katiohmi ho chik sum alo tuk oh cho? chi holitoblichi la he vt si vlhpesa mvhli keyu cho? im achi tok.

38. Mihma Balaam vt Balak a, Yakeh! chim vla lishke; nan isht im aiahli nana ho ishi li kak osh nana achi la hinla cho? anumpa nana ho Chihowah yot sutih ha fohkikma yommak $\underline{0}$ anumpuli la chi hoke, achi tok.

39. Mihma Balaam vt Balak a awant ia ma Kichalh-husolh a onvi okla tok.

40. Mihma Balak vt wak micha chukfi aiena ka im issa mut, Balaam micha holitopa vhleha apeha aiena ka pit i pila tok.

41. Atuk osh ont onna ma yakohmi tok, Balak vt Balaam a ilaueli cha Baal achahaka puta isht ona tok, yummak o hikia cha okla hash aiasha ont vhli fehna ka pisa chị hatok.

\section{CHAPTA XXIII.}

IHMA Balaam vt Balak a, Ilvppa alta untuklo ho is II sum ikbashke, micha wak untuklo, micha chukfi untuklo aiena ho is sum atahlashke, im achi tok.

2. Mihma Balak vt Balaam vt anumpuli tnk mak o yvmihchi tok; mihma Balak micha Balaam ita tuklot alta puta wak hobvk achufa, micha chukfi nakni achvfa aiyukali ho onochit im issa tok.

3. Mihma Balaam vt Balak a, Na hukmit ish im issa iluppa bilika ish hikia na, ia lashke: Chihowah yvt si afamut vlà hinla yoba kah; atuk o nana ho vm otuninchikma chim anoli lashke; im achi tok. Mihmvt achaha ka yo ona tok.

4. Mihma Chitokaka yvt Balaarn a afama tok; mikma Alta untuklo ho atahli lishke, micha alta puta ka wak hobvk 
achufa micha chukfi nakni achufa bieka ho onochit im issa lishke, achi tok.

5. Mihma Chihowah yut Balaam itih ha anumpa fohki mut, Falamvt Balak a im ona cha yakohmi hosh ish im anumpulashke, im achi tok.

6. Mihma falamot im ona tok, mihma, yakeh! hukmit isht aiokpvchi ya bilika, yumma, micha Moab i holitopa whleha moma aienut hieli tok.

7. Mihma nan isht vlhpisa ya ishi mut, Moab i miko Balak vt Alam ont ai ishit hvshi akochaka nunih foka ya akohchit susht vla cha, Minti cha, Chekob a anumpa kallakshichit ant vm onochí, micha Islael ant a pafi ; achishke.

8. Katiohmichi kak osh Chitokaka yvt kallakshichi tuk keyu ka, anumpa kallakshichi onochi la hinla cho? micha Chihowah yvt nan ik achukmo im anukfilli tuk keyukma, katiohmichi kak osh nan ikachukmo im anukfilli la hinla cho?

9. Tuli hochito paknaka hikiut pisa li, micha nunih puta hikiut pisa li hokvt, Yakeh! okla hash osh ila aiasha chị hoke, micha oklushi puta ka iba holhtina he keyu hoke.

10. Kuta hosh Chekob lukfi pushi ya koa ont isht aiushta hoh kia hohtina hinla cho? Aivlhpiesa illi kak o chohmit sullashke, micha vm ai isht aiopikmvt yummak o chohmashke, achi tok.

11. Mihma Balak vt Balaam a, Nanta mak o is si yumihchi cho? an tunup vhleha ha anumpa kallakshichi ish ono-

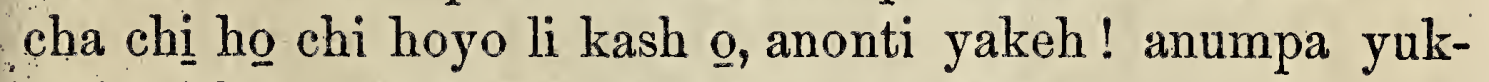
pali a ish onochi kak biekushke, im achi tok.

12. Mihma afalaminchit, Ahah! ahni lish Chihowah yot sutih ha fohki tuk ak o anumpuli la he vlhpesa keyu cho? im achi tok.

13. Mihma, Balak vt, Mintih, chim asilhha lishke, inla ho si awant ish ona cha yommak 0 ish a pisashke, ont vhli fehna kak illa ho ish pisa chi, moma ish pisa he keyu hoke, micha yummak o hikivt anumpa kallakshichi is sum onochashke, achi tök.

14. Mihmut Sofim osapa Piskah tabokaka ya isht ona cha 
alta untuklo ho ikbi mvt, alta achvfa ka wak hobvk achvfa micha chukfi nakni achufa bieka ho onochit im issa tok.

15. Mihma Balak a, Chihowah ya afamut yumma ia li takla ka hukmit ish im issa iluppa bilika ish hikiashke, im achi tok.

16. Mihma Chihowah yut Balaam a afama cha itih ha anumpa fohkit, Anonti Balak a ish im ona cha yak ish im achashke, im achi tok.

17. Atuk o im vla ma, Yakeh! hukmit im issa bilika hikia ma, Moab holitopa vhleha hut apeha tok. Mihma Balak vt Chihowah hot nanta ho anumpuli cho? achi tok.

18. Mihma nan isht vlhpisa ishi cha, Balak ma, wakaya cha haklo, Sippo ushi chia hut is sa haponaklashke.

19. Chitokaka yut hatak atuk osh holaba he keyu hoke; micha hatak ushi atuk osh im anukfila inlut nukhakla he keyu hokc: miha tuk vt ik yumihcho ka hinla cho? anumpuli tuk vt ik ahlecho ka hinla cho?

20. Yakeh! yukpali la chi ho anumpa uma tuk oka: micha yukpali tuk oke, atuk o inlvchi la he keyu koke.

21. Chekob a nanashvchi asha ik peso tuk, micha Islael a nan ai vlhpiesa keyu ka ik peso tuk oke; Chihowah in Chito. kaka yut ibafohka hoke; micha im itin takla ya miko shakapa hosh takali hoke.

22. Chitokaka ak osh Echip a pehlichit kohchi tok, imi hlampko hvt lapish achvfa ak o chohmi hoke.

23. Chekob a i sanalit ishit yushpakvmma he vt iksho hoke; micha Islael a i sanalit isht ahulla he ot iksho hoke: himak a chohmi ka Chekob a micha Islael a Chitokaka yut nan atahli hoke, acha hi oke.

24. Yakeh! okla hut koi chito chohmit wakaya hi oke, micha koi chito himita chohmit ile tanicha hi oke; nawehpoa ya ik ai upo kisha kvt, micha illi tuk im issish a ik ai ishko kisha kut tushka he keyu hoke.

25. Mihma Balak vt Balaam a , Anumpa kallakshichi chik onocho pullakmvt, anonti anumpa yukpali chik onocho pullashke, achi tok.

26. Yohmi kia Balaam vt afalamichit Balak a chim ano- 
lit, Chihowah yut nana anumpuli yưmma moma ka yựmihchi la chi hoke, achi li tuk keyu cho? achi tok.

27. Mihma Balak vt Balaam a Mintih, chim asilhha lishke, inla ho chisht ona lashke; Chitokaka yut vlhpesa ahni na, yumma hikivt anumpa kallakshichi is sum onochi yoba na, achi tok.

28. Mihmvt Balak vt Balaam a Peo paknaka Cheshimon pit talaia isht ona tok.

29. Mihma Balaam vt Balak a alta untuklo ho iluppa is sum ai ikbi cha, wak hobuk untuklo, micha chukfi nakni untuklo iluppa is sum atahlashke, im achi tok.

30. Mihma Balak vt Balaam vt achi tuk mak $\underline{0}$, yumihchi tok, micha alta puta ka wak hobuk achufa micha chukfi nakni achvfa bieka onochit im issa tok.

\section{CHAPTA XXIV.}

AUK o Balaam vt Chihowah yut Islael a yukpali kak A osh im aivlhpiesa, pisa mvt, ivt yushpakummi tikba hoyo beka tuk a ik chohmo hosh, amba nashuka ya yakni haiaka pilla pit talali tok.

2. Atuk osh Balaam vt akachakali mvt Islael vt apehlichika ulhpisa i binah puta aiasha na pisa tok, mihma Chihowah im ishilombish vt onvtola tok.

3. Mihmut nan isht im vlhpisa ya ishi cha, Beo ushi Balaam vt micha hatak nishkin i fatuma yosh achishke.

4. Chitokaka im anumpa ha haklo tuk, micha nan im aiahli i nan otvini ya pisa tuk, holhpokunna kia nishkin vt fatuma tuk vt achishke.

5. Chi binah puta kut aiyukpushke, Chekob ma! micha chim vlhtipo puta kvt, Islael ma!

6. Okfa hosh pataya ka chohmishke, osapushi yosh okhina bilika talaia ka chohmishke, aloes iti yo Chihowah yut hokchi tuk ak o chohmishke, micha chuahla iti puta hosh oka apotovt hieli kak o chohmishke.

7. Im ishtochi puta ka oka ya a kohchit hlatubla hi oke, 
mikma im ishtatiaka yut oka laua ho aiasha hi oke: micha i miko vt chaha kut Akak a i shahla hi oke, micha im apehlichika yut chaha hi oke.

8. Chitokaka yut Echip a pehlichit kohchi tuk oke ; imi hlampko hvt lapish achvfa yak o holba hoke, oklushi in tunup puta ka tahla hi oke, micha foni ya kobahlichit tahli cha im iti naki ya ishit nuhlit hlopullecha hi oke.

9. Hlipia hoke, koi chohmit micha koi chito chohmit tushki hoke, kuta hosh ilhkolecha hinla cho? kuna hosh anumpa yukpali chi onochikmvt, yukpa hoke : mikma kuna hoșh anumpa ka llakshichi chi onochikmot kalakshi hoke, achi tok.

10. Mihma Balak im ai i nukkilli vt Balaam a $\underline{\mathrm{i}}$ sanalit libbi mvt, ibbak a isht itai issochi tok: micha Balak vt Balaam a, An tunvp a anumpa kallakshichi is sum onochá chị ho chi hoyo li tuk, mihma yakeh! iluppa ont isht atuchina mak o anumpa yukpali ish onochi biekvshke.

11. Yvmohmi hokvt, himak a malelit, ish ai vtta ya oná; holitopa fehna chikbi la he ahni li tuk oke; yohmi tuk kia yakeh! Chihowah yut a holitopa ya chi katublishke, in achi tok.

12. Mihma Balaam vt Balak a chim anumpa shali vhleha et is sam pila túk ak kia im anumpulit,

13. Balak vt tuli huta, micha tuli holisso lakna $\mathrm{ya}$ aboha alota ho ik suma chi hoh kia, vno vm anukfila akinli kvt, achukma hokmá, keyukmot ika chukmo hoh kia yưmohmit Chihowah im anumpa ha ont ia la he keyu hoke. amba Chihowah yak osh nana achilkma, yommak $Q$ yumihchi la chi hoke, achi li tuk keyu cho?

14. Atuk osh yakeh! himak a vm okla aiasha ka ona la chi ; inta! yohmi hoka, nitak ont ishtaiyopikma, okla ilvpput. chim okla ha nana akaniohmicha he ya, chim anoli lashke, achi tok.

15. Mihmut nan isht vlhpisa ya ishi.cha, Beo ushi Balaam vt, achi, micha hatak nishkin $\mathrm{i}$ fatuma yut achishke.

16. Chitokaka im anumpa ha haklo tuk, mịtia Chaha i shahli ai ithuna ka ithana tuk, micha nan im aiahli im otuni 
ya pisa tuk, holhpokunna hoh kia nishkin fatuma tuk vt achishke.

17. Pisa la hi oke, yohmi kia himak ak keyu: Pisa la hi oke, yohmi kia olanlosa he keyu hoke: Chekob a fochik vt a kohcha hi oke, micha Islael a miko in tubi ut a kohcha hi oke, micha Moab in chukbika puta ka issot kancha hi oke, micha Shelh im vlla vhleha moma ka tahla hi oke.

18. Mikma Etom vt i yakni ya hi oke; Sei ak kia in tunup puta $\underline{\mathrm{i}}$ yakni ya hi oke, mikma Islael vt chilita hi oke.

19. Mikma apehlichika im asha chi kvt Chekob a akohcha hi oke, micha tumaha holihta aiasha tuk atampaka vbit tahla hi.oke, achi tok.

20. Atuk osh Amalek a pisa mut, nan isht vlhpisa ya ishi cha, Amalek ak osh oklushi puta ka tịka tuk oke, yohmi tuk kia ont im ishtaiyopi hokvto illit ai okpulokvt taha hi oke, achi tok.

21. Atuk osh Kenait a pisa mot, nan isht vlhpisa ya ishit, Ish aiahanta yut kvlloshke, micha chim vlhpichik a tuli chito yo ish ai ikbishke.

22 Yohmi kia Kenait vt tahovt ia hi oke, atuk o Assha vt yilkachit chisht ia hi oke, achi tok.

23. Mihmut nan isht vlhpisa ya ishi cha, Ikikkeh! Chitokaka yut iluppa yumihchikma kvta hosh okchayvt ahanta hinla cho?

24. Micha peni hochito vt Chittim ai vhli minti kvt vla cha Assha ya ilbushala hi oke, micha Eba ya ilbushalikma, yummut ai okpuloka vt taha hi oke, achi tok.

25. Mihmvt Balaam vt wakaya cha falamut ivt ai vtta ya ona tok, mihma Balak ak kia im atiya ya ia tok.

\section{CHAPTA XXV.}

MIHMA Islael vt Shittim ak $\underline{a}$ ahanta tok, mihma okla II hut Moab ushetik vhleha ha iti hauit isht ia tok.

2. Mihma i naholbut toba puta ka nan vbit isht aiokpv- 
chi mut okla hash hoyo tok; mihma okla hash osh impa cha i naholbut toba puta ka im akachunoli tok.

3. Mihma Islael vt Baal-pio ya ibafohka tok; mihma Chi-

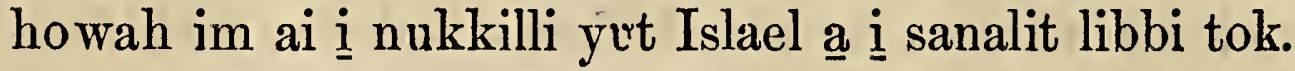

4. Micha Chihowah yut Moses a okla hash noshkobo pnta moma ish ishi cha hushi ya ichapaka Chihowah itikba ya iti a ish atakohlechashke, Yumohmi ho Chihowah im ai i nukkilli libbi kut Islael a i filummashke, im achi tok.

5. Mihma Moses vt Islael nan im apesa vhleha hạ, Baalpio ya ibafohka tuk hatak huchim asha aiyukali kut hush bashke, im achi tok.

6. Atuk o yakeh! Islael im vlla vhleha aiachvfa hosh vlut Mitianait ohoyo ho itibapishi vhleha aiasha ka micha okla moma aiena kvt okla moma in tabenakel okhissa itikba yaiya hosh aiasha tuk osh pisa ho isht ula tok.

7. Atuk o naholitompa isht vtta Alon ushi Eleasa ya ushi Finehas vt pisa mut okla moma takla binili tuk vt tuli halupa isht husa ishi cha wakaya tok.

8. Micha Islael hatak a iakaiyvt ivt binah ha ont chukowa cha Islael hatak ash bahlit hlopullechi mot, ohoyo hash ikfoka ya bahlit hlopullechi tok; Yvmohmi ho ililli okpulo vt Islael im vlla vhleha itin takla ya yokopa tok.

9. Mihma ililli okpulo isht illi kvt tahlepa sipokni pokoli tuklo akucha ushta tok.

10. Mihma Chihowah yut Moses a im anumpulit,"

11. Naholitompa isht vtta Alon ushi Eleasa ushi Finehas vt vno pulla kak o amosholi cha vm ai i nukkilli ya Islael im vlla vhleha im itin takla ya kanvllichi na, sa nuktahla kak osh Islael im vlla vhleha vbit ak tahloshke.

12. Yumohmi hoka, yakeh! iti nanaiya nan itim apesa li ya ima lishke.

13. Atuk o naholitompa isht aiahanta bilia fehna ka yumma, micha im isht atiaka aiena kvt iesha hi oke; in Chitokaka ya im amosholi cha Islael im vlla vhleha ha im atobbi tuk okvt, ish achashke, im achi tok.

14. Mitianait ohoyo pulla ho Islaelait vt awant illi tuk hohchifo kvt Salu ushi Simli atok, yummut Simeonait vhle- 
ha im itin takla ya chuka achvfa $\underline{\mathrm{i}}$ shahli $\underline{\mathrm{i}}$ holitopa yatok.

15. Mikma Mitianait ohoyo illi tuk vt Su ushetik osh hohchifo kut Kosbi atok, yummut okla i noshkoboka yosh Mitian a chuka achvfa ya $\underline{i}$ shahli yatok.

16. Mihma Chihowah yut Moses a im anumpulit,

17. Mitianait vhleha ha ilbvshalechit hvsh fahamashke;

18. Nan isht haksichi in tek Mitian i holitopa ushetik Kosbi pulla ho, micha Pio pulla ka hvochi haksechit hvch ilbushali tuk oka, yummut Pio pulla kak o ililli okpulo vt itonla nitak ma illi tuk oka, achi tok.

\section{CHAPTA XXVI.}

AUK osh ililli okpulo vt assha ha yạ, yakohmi tok, ChiA howah yot Moses micha naholitompa isht vtta Alon ushi Eleasa ya im anumpulit,

2. Islael im vlla vhleha okla moma afummi pokoli tuklo vhli vba mayvt iki vhleha in chuka achvfa hlopullit Islael aiasha kvt tunup itibit ia hinla moma holhtina ka hush ishashke, im achi tok.

3. Mihma:Moses micha naholitompa isht vtta Eleasa ita tuklo kett Chatan a Moab yakni patali Cheliko bilika ya ai im anumpulit,

4. Afưmmi pokoli tuklo vhli vba maya holhtina ka , Chihowah yvt Moses micha Islael im vlla vhleha Echip yakni

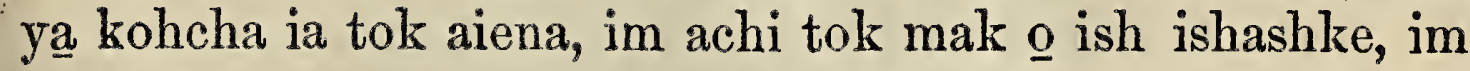
achi tok.

5. Islael ushi akni Luben: Luben im vlla vhleha; Hanok, yvmmak o Hanokait in chuka achvfa akohcha tok: Pallu, Palluait chuka achvfa :

6. Heslon a, Heslonait chuka achvfa: Kamai ya chuka achvfa:

7. Ilvppa pnta kak osh Lubenait chuka achvfa vhleha hoke, atuk osh holhtina kvt tahlepa sipokni pokoli ushta aku- 
cha tuchina mıcna tanlepa untuklo cha pokoli tuchina aiena tok:

8. Mikma Pallu ushi; Eliab.

9. Mikma Eliab ushi vhleha; Nemuel micha Tahlan micha Abilam atok. Tahlan micha Abilam ilupput Kolah ai itvpeha kut Chihowah kvllot i sanali ma, ibafokvt Moses micha Alon ita tuklo ka kvllot i sanalit okla moma im ai annohowa tok mak oke.

10. Mihma yakni vt itakpashali cha Kolah ya ai itapeha ho ishit nanubli tok, ai itapeha yumma, yummvt illi ma luak vt hatak tahlepa tuklo cha pokoli tahlapi aiena ho hushmit tahli tok; yohmi mut yummut nan isht atokowa yo toba tok.

11. Yohmi kia Kolah im vlla vhleha hvt ik illo tok.

12. Simeon ushi vhleha hvt in chuka achvfa vlhpisa hatok: Nemuel a, Nemuelait chuka achvfa: Chamin a, Chaminait chuka achvfa: Chakin a, Chakinait chuka achvfa :

13. Selah ya Sahait chuka achvfa: Shaul a, Shaulait chuka achufa yatok.

14. Iluppa puta kak osh Simeonait chuka achufa vhleha hoke: tahlepa sipokni pokoli tuklo akucha tuklo cha tahlepa tuklo tok.

15. Kat im vlla vhleha in chuka achufa puta vlhpisa hosh, Sefon a , Sefonait chuka achufa ak atok: Hakki ya Hakkait chuka achufa ak atok: Shuni ya, Shunait chuka achvfa ak atok:

16. Osni yạ, Osnait chuka achufa ak atok: Eli ya, Elait chuka achufa ak atok:

17. Alot $\underline{a}$, Alotait chuka achvfa ak atok: Aleli ya , Alelait chuka achvfa ak atok.

18. Ilvppa puta kak osh Kat im vlla vhleha in chúka achvfa hatok; micha holhtina trk mak osh tahlepa sipokni pokoli ushta cha tahlepa tahlapi tok.

19. Chutah im vlla vhleha hut El micha Onan ak atok, micha El micha Onan ita tuklo kut Kenaan yakni ya ai illi tok.

20. Mihma Chutah im vlla vhleha in chuka achvfa vlhpisa hosh: Shelah yạ, Shelanait chuka achvfa ak atok: Fales 
a, Falesait chuka achvfa ak a tok: Selah yạ, Salait chuka achufa ak atok.

21. Mihma Fales ushi vhleha: Heslon a, Heslonait chuka achvfa ak atok: Hamul a, Hamulait chuka achvfa ak atok:

22. Ilvppa puta kak osh Chutah im vlla vhleha; chuka achvfa puta hatok, micha holhtina mak osh tehlepa sipokni pokoli untuklo akucha hannali cha tahlepa tahlapi tok.

23. Issaka ushi vhleha chuka achvfa vlhpisa; Tola ya Tolait chuka achufa ak atok: Pua ya Punait chuka achıfa ak atok:

24. Chashob a, Chashobait chuka achvfa ak atok: Shimlon a, Shimlonait chuka achufa ak atok.

25: Iluppa puta kak osh Issaka im vlla vhleha chuka achufa hatok; micha holhtina mak osh tahlepa sipokni pokoli hannali akucha ushta cha tahlepa tuchina tok.

26. Sebulon ushi vhleha chuka achvfa vlhpisa: Selet a, Satait chuka achefa ak atok: Elon a Elonait chuka achufa ak atok: Chahleel a, Chahleelait chuka achvfa ak atok.

27. Ilvppa puta kak osh Sebulonait chuka achvfa hatok; micha holhtina mak osh tahlepa sipokni pokoli hannali cha tahlepa tahlapi tok.

28. Chosef ushi vhleha chuka achufa vlhpisa hosh Manasseh micha Eflaim ak atok:

29. Manasseh ushi vhleha: Makil a, Makilait chuka achufa ak atok: micha Makil vt Kileat a tobachi tok: Kileat a Kileatait chuka achvfa akohcha tok.

30. Kileat ushi vhleha hvt iluppak atok: Cheesa ya Cheesalait chuka achvfa: Helek a, Helekait chuka achvfa :

31. Micha Asliel a Aslielait chuka achvfa: Shekem a, Shekemait chuka achufa ak atok.

32. Micha Shemita ya , Shemitait chuka achufa: micha Hefe ya , Hefalait chuka achufa ak atok.

33. Mihma Hefe ushi Selofehat vt ushi ik im iksho, amba ushetik vt im asha.tok: micha Selofehat ushetik vhleha hohchifo kvt Mahlah, micha Noah, Hoklah, Milkah micha Tisah ak atok:

34. Iluppa puta kak osh Manasseh chuka achufa hatok; 
micha holhtina mak osh tahlepa sipokni pokoli tahlapi akucha. tnklo cha tahlepa untuklo tok.

35. Ilvppak osh Eflaim ushi vhleha chuka achvfa vlhpisa hatök: Shuhlelah yạ, Shuhlalhait chuka achvfa: Beka ya Baklait chuka achvfa: Tahan a, Tahanait chuka achvfa ak atok:

36. Mihma Shuhlelah ushi vhleha hvt iluppak atok, Elan a Elanait chuka achvfa ak atok.

37. Ilvppak osh Eflaim ushi vhleha chuka achvfa vlhpisvt holhtina mak osh tahlepa sipokni pokoli tuchina akucha tuklo cha tahlepa tahlapi tok: Iluppa puta kak osh Chosef ushi vhleha chuka achvfa vlhpisa hatok:

38. Benchamin ushi vhleha chuka achvfa vlhpisa; Bela yạ, Belait chuka achvfa: Ashbel a, Ashbelait chuka achvfa : Ahilam a, Ahilamait chuka achvfa:

39. Shufam à, Shufamait chuka achvfa: Hufam a Hufamait chuka achufa ak atok.

40. Mihma Bela ushi vhleha hut At micha Naaman ak atok: At a Atait chuka achvfa: Naaman a Naamanait chu$\mathrm{ka}$ achvfa ak atok.

41. Iluppa puta kak osh Benchamin ushi vhleha chuka achufa vlhpisa hatok: micha yumma holhtina mak osh tahlepa sipokni pokoli ushta akucha tahlapi cha tahlepa hannali tok.

42. Ilvppak osh Tan ushi vhleha chuka achvfa vlhpisa: Shuham a Shuhamait chuka achufa ak atok. Ilvppak osh Tan in chuka achvfa puta, chuka achvfa vlhpisa hatok.

43. Shuhainait chuka achvfa moma holhtina mak osh tahlepa sipokni pokoli hannali akucha ushta cha tahlepa ushta tok.

44. Asha im vlla vhleha chuka achufa vlhpisa: Chimna yạ, Chimnait chuka achufa: Chesui yạ, Chesuait chuka achvfa: Beliah yạ, Belait chuka achvfa ak atok.

45. Beliah ushi vhleha: Heba ya , Hebalait chuka achvfa : Malkiel a, Malkielait chuka achvfa ak atok.

46. Mihma Asha ushetik vt hohchifo kvt Selah ak atok.

47. Ilvppa puta kak osh Asha ushi vhleha chuka achvfa 
hatok, micha yumma holhtina mak osh tahlepa sipokni pokoli tahlapi akucha tuchina micha tahlepa ushta tok. : . .

48. Naftali ushi vhleha chuka achvfa vhlpisa: Chahseel a, Chahseelait chuka achvfa : Kuni ya Kunait chuka achufá : 49. Chesa ya , Chesalait chuka achufa : Shillem a . Shillemait chuka achufa ak atok.

50. Iluppa puta kak osh Naftali in chuka achufa chuka achufa vhleha vlhpisa hatok, micha yumma aholhtina kvt tahlepa sipokni pokoli ushta akucha tahlapi micha tahlepa ushta tok.

51. Iluppa puta kak osh Islael im vlla vhleha hosh holhtina mut tahlepa sipokni bat tahlepa hannali cha tahlepa sipokni achufa, micha tahlepa untuklo pokoli tuchina aiena tok.

52. Mihma Chihowah yut Moses a im anumpulit,

53. Iluppa puta kak osh hohchifo holhtina ka vlhpisa i yakni ya chi hosh yakni yash osh i kushkoashke."

54. Laua ka i yakni ya ishahlechit ish imashke, micha ik lauo ka i yakni ik lawo hush imashke, 1 yakni aiyukali. kut holhtina tuk ak o vlhpisa hö ish imashke.

55. Yohmi kia nashoeli hosh yakni ya itikushkoashke, iki vhleha apehlichi hohchifo kak o ai ishashke.

56. Laua micha ik lauo aiena kvt na shoeli hosh yakni ya itakushkoashke.

57. Atuk o chuka achvfa puta vlhpisa hosh Lefait vhleha hut holhtina iluppa puta kak atok, Keshon a, Keshonait chuka achvfa ak atok: Kohalh a, Kohalhait chuka achvfa ak atok: Melali ya Melalait chuka achvfa ak atok.

58. Iluppa puta kak osh Lefait chuka achvfa vhleha hatok: Lebnait chuka achvfa, Heblonait chuka achufa, Mahlait chuka achvfa, Mushait chuka achvfa, Kolahlait chuka achufa ak atok. Atuk o Kohalh vt Amlam a tobachi tok.

59. Mihma Amlam tekchi vt hohchifo kut Chokebet osh Lefai ushetik, ishki vt Lefai ya Echip im ai eshi tok. Atuk osh Alon micha Moses micha in tek Miliam aiena ka Amlam a im eshi tok. 
60. Atuk $\dot{0}$ Alon vt Natab micha Abihu, Eleasa micha Ihlama aiena kvt im vtta tok.

61. Atuk o Natab micha Abihu ita tuklo kvt, luak inla Chihowah itikba ai im issa mut illi tok.

62. Micha holhtina mvt nakni bieka hosh hvshi achvfa vba maya kvt tahlepa sipokni pokoli tuklo akucha tuchina. Islael im vlla vhleha ha takla yalni ik emo hatok. Islael im vlla vhleha ha takla hollitina tok keyu hokvt. .

63. Iluppa puta kak osh Moses micha naholitompa isht vtta Eleasa ita tuklo kut Islael im vlla vhleha Chatan a Moab yakni patali Cheliko bilika a holltina tok, holhtina tok mak oke.

64. Yohmi kia Moses micha naholitompa isht vtta Alon itatuklo kvt Islael im vlla uhleha lia yakni haiaka Sinai ya a hohtena tok a, hatak achufa kia iluppa puta ka itin takla ya iksho tok.

65. Chihowah yut, Yakni haiaka ai illit taha pulla chi hoke, achi tok oka. Milima hatak achufa kia hlakofi kvt iksho tok. Chefunneh ushi Kaleb micha Nun ushi Choshua ak illa tok...

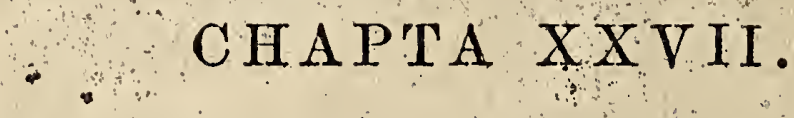

YOHMI ma Selofehat yummut Hefe ushi, mikma yum1 mvt Kileat ushi, mihma yummut Maki ushi, mihma yummut Manasseh chuka achvfa Manasseh ushi, mihma yummut Chosef ushi atok yumma ushetik vhleha hut ona tok, micha ushetik vhleha hohchifo kvt iluppak atok, Mahlah, Noah micha Hoklah micha Milkah micha Tisah atok.

2. Atuk osh Moses, micha naholitompa isht vtta Eleasa, micha holitopa vhleha micha oḱla moma aiena itikba, okla moma in tabenakel okhissa bilikk hieli cha,

3. Piki ut yakni haiaka ai illi tok oke, micha Kolah ai

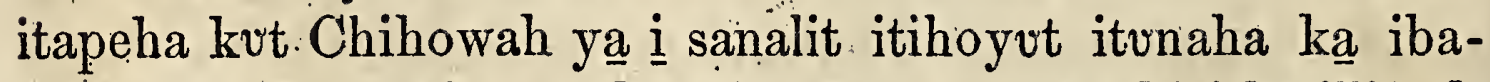
fohka tok keyushke : yohmi kia ilap nanashuchi isht illi tok oke; micha ushi ik im iksho tok oke.

4. Nanta katiohmi ho ushi iksho hatuk ak osh piki hoh- 
chifo kvt in chuka achvfa ai itin takla ya kunia hinla cho? Yvmohmi hoka, piki itibapishi vhleha takla yakni ish pimashke, achi tok.

5. Mihma Moses vt nan isht im akaniohmi ka Chihowah itikba isht ona tok.

6. Mihma Chihowah yot Moses a im anumpulit,

7. Selofehat ushetik vhleha hut vlhpesa ho anumpuli hoke : iki itibapishi uhleha takla yakni ai eshi ya ish ima pullashke: micha ik 1 yakni yash tosholit ish im onachashke.

8. Micha Islael im vlla vhleha im anumpulit, Hatak vt ushi iksho hosh illikma i yakni yash tosholit ushetik ak o. hush im onachașhike.

9. Atuk o úshetik vt ikshokma i yakni yash itibapishi vhleha hak o hosh imashke.

10. Atuk o itibapishi vt ik im ikshokma i yakni yash iki itibapishi vhleha hak o hush imashke.

11. Atuk o ili vt itibapishi uhleha hut ikshokma i yakni yash in chuka achofa ai achufa 1 kanomi pit i bilika yak o hush ima na ieshashke, mikma yummak osh Islael im vlla vhleha, Chihowah yut Moses a im apesa tuk mak vlhpisa ai apihisashke, im achi tok,

12. Micha Chihowah yut Moses a, Nunih Abalim iloppa oiya cha Islael im vlla vhleha yakni ima li tok a pisah.

13. Atuk osh ish pisg ha mut chishno ak kia chítibapishi -Alon vt iba holhtina chohmit chim okla ha ish"ibà holhtina chi hoke.

-14. Chukushmi Sin ash okla moma kut itachowa ma, nishkin itikba ya oka yumma is si aholitobla he tuk vt ilafilummit vm anumpa ha ish i sanali tuk oka, yummvt Melibah oka Katesh talaia yakni haiaka Sin ak atok, im achi tok.

15. Mihma Moses vt Chihowah ha im anumpulit,

16. Chihowah yut nipi puta moma imi shilombish in Chitokaka yut okla moma pehliechi hatak atokoli na,

17. Yummak osh itikba kohchvt ihiya, milha yummak osh itikba ont chukohowa, micha yvmmak osh pehlichit kohoncha, micha yummak osh pehlichit chukowvt ahantashke, 28 
yumolimi ho Chihowah im okla moma yut chukfi yosh apistikeli iksho yo ik chohmo kashke, im achi tok.

18. Mihma Chihowah yut Moses a im anumpulit, Nvn ushi Choshua hatak shilombish nukfoka yummak o ish ishi cha chibbak ish onochashke.

19. Micha Eleasa naholitompa isht vtta itikba, micha okla moma itikba ya ish hilechashke; micha yummak osh pisa ho, anumpa ish imashke.

20. Micha chim aholitopa kaniohmi ka ish onochashke; yumohmi ho Islael im vlla vhleha okla moma kut im antia achukmashke.

21. Micha naholitompa isht vtta Eleasa itikba hikia na yummak osh Chihowah itikba Ulim ai isht apesa yak o isht im asilhhvcha hi oke: im anumpa ho kohchut ia hi oke, micha im anumpa ho ont chukowa hi oke, yumma micha Islael im vlla vhleha apiha aiena kvt okla moma puta kvt yvmohma hi oke, im achi tok.

22. Mihma Moses vt Chihowah yưt im achi tuk mak o yumihchi tok: micha Choshua ya ishi cha naholitompa isht vtta Eleasa itikba, micha okla moma itikba ya hilechi tok.

23. Micha ibbak a onochi mut anumpa ima tok, Chihowah yưt Moses a tohnut im apesa tuk mak o yumihchi tok.

\section{CHA P T A X V VII.}

IHMA Chihowah yot Moses a im anumpulit,

11 2. Islael im vlla vhleha ha im anumpulit, Nan vm issa micha am puska vbit isht aiokpvchi Juak isht hullukmi, a nabalama ya ithaiyanut ont ai vlhpiesakma vm issashke, ish im apesashke.

3. Micha luak ishit hukmit im issa iluppak o Chihowah ya hush im issa hi oke; Chukfi ushi tuklo ho afummi vmmona hosh, ik aiono takali kvt iksho hukmit im issa bilia yo onna hosh onnakma hush yumihinchashke.

4. Chukfi ushi achofa kash onnahinli ish im issa tukmut, chukíushi achvfa ya ont opiakma, ish im issa hi oke: 
5. Micha ilhpak im issa yo bota tohbi lipehbi efah isht pokoli, bovt bila toba hin kushkoa ai ushta kvt itaioma hi oke.

6. Chihowah i nabalama vbit luak ishit hullukmit im issa, hukmit im ihisa bilia yosh Sinai nunih ha ai vlhpisa tok mak oke.

7. Micha nan ishko im issa ya chukfi ushi achvfakma hin kushkoa ai ushta hi oke: nan ishko im issa ya aholitopa ak o oka paki kullo ya Chihowah ish im afohoblicha hi oke.

8. Mikma chukfi ushi achufa yano, opiakma ish im issashke : onnahinli ilhpak.im issa mak $\underline{o}$, micha nan ishko

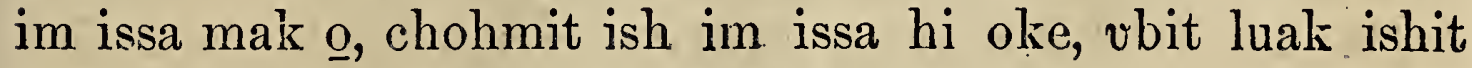
hukmit im issa Chihowah $\underline{i}$ balama ya hi oke.

9. Nitak hullokma chukfi ushi tuklo afummi vmmona ik ai ono takali kut iksho, micha bota tohbi ishit pokoli kushkoa tuklo, bila itaioma ho ilhpak im issa mak o micha nan ishko im issa aiena hi oke.

10. Hukmit im issa bilia, micha nan ishko im issa aiena kvit ashakma, iluppak osh nitak hullo moma hukmit im issa yoke.

11. Micha hushi puta isht huchim ia vmmona ka hukmit im issa ya Chihowah hush im issa hi oke ; wak hobvk himita tuklo, micha chukfi nakni achvfa, chukfi ushi untuklo afummi vmmona ik ai ono takali kvt iksho aiena yashke.

12. Micha wak hobuk achufakma ilhpak im issa mak o bota tohbi ishit pokoli kishkoa tuchina bila aioma yashke; micha chukfi nakni achvfakma ilhpak im issa mak o bota tohbi ishit pokoli kushkoa tuklo bila aioma yashke.

13. Micha chukfi ushi achvfakma, ilhpak im issa mak $\underline{o}$ bota tohbi kushkoa ishit pokoli bano ho bila aioma, nabalama hukmit im issa, micha vbit hukmit Chihowah im issa ya hi oke.

14. Micha ishkot im issa yvt wak hobuk achvfakma, oka paki hin iklunna; micha chulfi nakni achufakma, hin kvshkoa atuchina, micha chukfi ushi achvfakma, hin kvshkoa isht ai ushta ya hi oke. Ilvppak osh afummi hlopulli, hoshi puta kut hushi talalikma, hukmit im issa yoke.

15. Hukmit im issa bilia, micha nan iksho im issa aiena 
kvt ashakma, nan ashvchi im.issa mak o issi kosoma ai ishit issi kosomushi achvfa ho Chihowah im issa hi oke.

16. Micha hvshi vmmona nitak ont isht auahhushta kvt Chihowah im vbanvblit ont ia yoke.

17. Atukma hushi iloppa nitak ont ishit anahtahlapik mak o, impuchi yoke: nitak untuklo ho puska ik shatummo vpa hi oke.

18. Nitak vmmona hokuno itunaha holitopa asha hi oke; yummak oka tishu atoksvli nana kia huchik yumihcho ka hi oke.

19. Amba Chihowah ya hukmit im issa ya vbit luak ishit hukmit im issa ya hush im issa hi oke; wak hobvk himita tuklo, micha chukfi nakni achufa micha chnkfi ushi afummi vmmona untuklo aiena hi oke; yummut ik ai ono takali kvt ik hvchim iksho ka hi oke.

20. Micha im ilhpak im issa yut bota tohbi yosh bila itaioma hi oke; wak hobuk achvfakma ishit pokoli kvshkoa tuchina, micha chukfi nakni achufakma ishit pokoli kvshkoa tuklo ho hush im issa hi oke.

21. Chukfi ushi achvfakma, kushkoa ishit pokoli biekvt chukfi ushi untuklo hlopulla hi oke.

22. Micha nan ashuchi im issa ya issi kosoma achufa ho ishit huchi kashofa ya hi oke.

23. Onnahinli moma hukmit im issa, yummot hukmit im issa bilia yot ashakma, iivppa puta hush im ihisa hi oke.

24. Iluppak o yohmi hosh nitak untuklo hlopulli ka onnakma hush im issa hi oke, vbit luak ishit hukmit aiokpvehi nipi, Chihowah $\underline{\mathrm{i}}$ balama ak a hi oke: hukmit im issa bilia, micha nan ishko im issa aiena kut ashakma, yumma aiena im issa hi oke.

25. Atuk osh nitak ont isht untuklokma itunaha holitopa huchim asha hi oke; tishu a toksvli nana kia hush yumihcha he keyu hoke.

26. Micha nan awaya ummona nitak, ilhpak im issa himona Chihowah isht hush im onakmvt, nitak hullo puta kvt ont huchin taha ha yvt, itunaha holitopa hvchim asha hi oke : tishu atoksuli nana kia hush yumihcha he keyu hoke. 
27. Amba Chihowah i balama yvt hukmit im issa hush im issa hi oke: wak hobuk himita tuklo, micha chukfi nakni achvfa, chnkfi ushi afommi vmmona untukla hi oke.

28. Micha ilhpak im issa yot bota tòhbi bila itaioma yosh wak hobvk achufakma ishit pokoli kushkoa tuchina, chukfi nakni achvfakma ishit pokoli kushkoa tuklo,

29. Chukfi ushi achvfakma kushkoa ont ishit pokoli biekvt chukfi ushi untuklo ho hlopulla hi oke.

30. Micha isht hvchi kashofi vt issi kosoma ya ushi achvfa hi oke.

31. Hukmit im ihisa na bilia. micha ilhpak im issa (yvmmut ik ai ono takali kvt ik hvchim iksho ka hi oke) micha nan ishko im issa aiena kut aiasha ma, yumma puta ka hush im issa hi oke.

\section{CHAPTA XXIX.}

A TUK osh hưshi ont isht untuklo, hushi nitak vmmoA nakma, itvnaha holitopa hvchim asha hi oke: tishu atoksvli nana kia hush yumihcha he keyu : isht pufa olachi hvchi nitak oke.

2. Micha Chihowah $\underline{i}$ balama yo hnkmit im issa hush im issa hi oke: wak hobvk himita achvfa, chukfi nakni achvfa, micha chukfi ushi afummi vmmona untuklo ha hi oke.

3. Mikmvt ilhpak im issa yot bota tohbi bila itaioma hosh, wak hobuk achvfakma ishit pokoli kushkoa tuchina, micha chukfi nakni aøhvfakma, ishit pokoli kvshkoa tuklo,

4. Chukfushi untuklo kvt chukfushi achvfakma ishit pokoli kushkoa achufa bat hlopulla hi oke,

5. Micha nan ashuchi im issa ishit hvchi kashofa chi kvt issi kosoma ya ushi achufa hi oke,

6. Chihowah i balama vbit im isset luak ishit hukmit im issa, hushi yomma, nitak moma luak ishit hukmit im issa, micha ilhpak im issa, micha nan ishko im issa ayumohmi chatuk mak o yumihcha hi oke.

7. Micha hushi isht untuklo ilupput nitak ont ishit pokolikma itunaha holitopa huvchim asha hi oke; micha huchi 
shilombish a hush ilbushala hi oke; micha yvmma natoksuli nana kia hush yumihcha he keyu hoke;

8. Amba Chihowah $\underline{i}$ balama yo hukmit im issa ya hush im issa hi oke; wak hobvk himita achufa, chukfi nakni achvfa, micha chukfi ushi afummi vmmona untuklo yosh, inlut a takali kvt iksho ka hi oke.

9. Micha ilhpak im issa yut bota tohbi bila itaioma yosh wak hobvk achufakma ishit pokoli kushkoa tuchina, chukfis; nakni achufakma ishit pokoli kushkoa tuklo,

10. Chukfushi untuklo kvt chukfi ushi achvfakma ishit pokoli kushkoa achufa bat hlopulla hi oke,

11. Nan ashvchi ishit kashofa, micha hukmit im ihisa na bilia, micha ilhpak im issa, micha nan ishko im issa aiena kvt a sha na, nan ashuchi im issa yo issi kosoma ya ushi achufa ho im issa hi oke.

12. Atuk osh hushi isht untuklo kvt nitak ont isht auah tahlapikma itvnaha holitopa hvchim asha hi oke; tishu atoksuli nana kia hush yumihcha he keyu, micha Chihowah impvchi isht hush im aiasha nitak untuklo ha hi oke.

13. Chihowah i nabalama yo hukmit im issa, vbit luak ishit hukmit im issa hush im issa hi oke; wak hobuk himita auahtuchina, chukfi nakni tuklo, micha chukfi ushi afummi vmmona auahushta, inlut a takali kut iksho ho hush im issa hi oke,

14. Mikma ilhpak im issa yvt bota tohbi bila itaioma yo wak hobvk auahtuchina kash wak hobvk achufakma ishit pokoli kushkoa tuchina biekakma, chtkfi nakni tuklo kash chukfi nakni achvfakma ishit pokoli kushkoa tuklo bika,

15. Mikma chukfushi auahushta kvt chukfushi achofakma ishit pokoli kushkoa achvfa bieka hi oke,

16. Hukmit im ihisa bilia, ilhpak im issa, micha nan ishko im issa aiena kvt aiashakma, nan ashvchi im issa yo issi kosoma ya ushi achufa ho hush im issa hi oke,

17. Atuk osh nitak atuklakma, wak hobvk himita auahtuklo, chukfi nakni tuklo, micha chukfushi afummi vmmona auahushta inlut a takali kut keyu ho hush im issa hi oke.

18. Micha ilhpak im issa, micha nan ishko im issa yvt 
wak hobvk puta, chukfi nakni puta, chukfushi puta aiena holhtina vlhpisa ho ayumohmi chatuk mak o hush im issa hi oke.

19. Hukmit im ihisa na bilia, micha ilhpak im issa, micha nan ishko im issa aiena kvt aiashakma, nanashvchi im issa yo issi kosoma ya ushi achufa ho hưsh im issa hi oke.

20. Atuk osh nitak ont isht atuchinakma wak hobvk auahachvfa, chukfi nakni tuklo, chukfushi afummi vmmona auahushta ik ai ono kvt iksho hashke,

21. Mikma ilhpak im issa, micha nan ishko im issa puta kut wak hobvk puta, chukfi nakni puta, micha chukfushi puta aiena holhtina ka vlhpisa ho ayumohmi chatuk mak a - hi oke.

22. Micha hukmit im ihisa bilia, micha ilhpak im issa, micha nan ishko im issa aiena kvt ashakma, nan ashvchi im issa yo issi kosoma achufa hashke,

23. Micha nitak ont isht ai ushtakma, wak hobuk pokoli chukfi nakni tuklo, micha chukfushi afummi ummona auah ushta ik ai ono kut iksho yashke.

24. Micha ilhpak im issa, micha nan ishko im issa yut, wak hobvk puta, chnkfi nakni puta, chukfushi puta aiena holhtina ka vlhpisa ho ayumohmi chatuk mak o i yumihchashke:

25. Micha hukmit im ihisa na bilia, micha ilhpak im issa, micha nan ishko im issa aiena kvt ashakma, nan ashvchi im issa yo issi kosoma ya ushi achufa yashke.

26. Micha nitak ont ishit tahlapikma wak hobvk chakkali, chukfi nakni tuklo, micha chukfushi afummi ummona auahista ik ai ono kvt iksho yashke.

27. Mikma ilhpak im issa, micha nan ishko im issa puta 4 - kut wak hobuk puta, chukfi nakni puta, chukfushi puta aiena kut holhtina ka vlhpisa ho ayumohmi chatuk mak o i yumihchashke.

28. Micha hukmit im ihisa na bilia micha ilhpak im issa micha nan ishko im issa aiena kvt ashakma, nan ashvchi im * issa yo issi kosoma achofa yashke.

29. Micha nitak ont ishit hannalikma, wak hobvk untu- 
china, chukfi nakni tuklo, micha chukfushi afummi vmmona auah ushta ik ai ono kvt iksho yashke.

30. Micha ilhpak im issa, micha nan ishko im issa puta kvt, wak hobvk puta, chukfi nakni puta, micha chukfushi puta aiena kut holhtina ka vlhpisa ho ayvmohmi chatuk mak o i yumihchashke:

31. Micha hukmit im ihisa na bilia, micha ilhpak im issa micha nan ishko im issa aiena kut ashakma, nan ashuchi im issa yo issi kosoma achvfa yashke.

32. Micha nitak ont isht untuklokma, wak hobvk untuklo, chukfi nakni tuklo, micha chukfushi afummi ummona auahushta ik ai ono kvt iksho yashke:

33. Micha ilhpak im issa, micha nan ishko im issa puta kvt, wak hobvk puta, chukfi nakni puta, micha chukfushi puta aiena holhtina ka vlhpisa ho a yumihchi chatuk mak o i yomihchashke:

34. Micha hukmit im ihisa na bilia, micha ilhpak im issa, micha nan ishko im issa aiena kvt ashakma, nan ashvchi im issa yo issi kosoma achvfa yashke :

35. Micha nitak ont isht untuchinakma itunaha holitopa huchim asha hi oke; nitak yvmma tishu atoksuli nana kia livsh yomihcha he keyu.

36. Amba Chihowah nan $\underline{i}$ balama yo vbit luak ishit hukmit im issa, wak hobvk achvfa, chukfi nakni achvfa, chukfushi afummi ummona untuklo ik ai ono kut iksho aiena ho hukmit im issa yo hush im issa hi oke.

37. Ilhpak im issa, micha nan ishko im issa puta kut, wak hobuk puta, chukfi nakni, micha chukfushi puta aietia holh-. tina ka vlhpisa ho ayvmohmi chatuk mak o i yumihelashike:\%

38. Micha hukmit im ihisa na bilia, micha ilhpale im issa: micha nan ishko im issa aiena kut ashakma, nan ashvehi jm issa yo issi kosoma achvfa hashke.

39. Anumpa hush il onochi puta, micha jlap ahnit nana 6 hush im issa puta, luak ishit hukmit nana hush im issa puta, micha ilhpak hush im issa puta, micha nan ishko hush im issa puta, micha itinnanaiyvchit nana hush im issa puta aiena. hot asshakma, impuchi huchim vlhpisa puta ka iluppa puta 


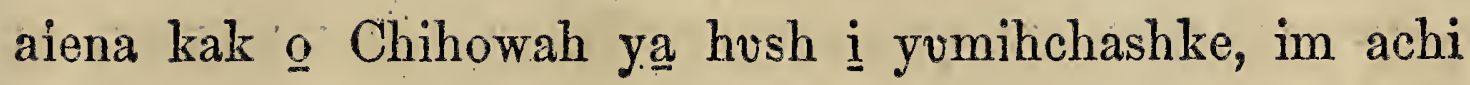
tok.

40. Mihma Chihowah yut Moses a im apesa tuk mak o, moma ka Moses vt Islael im vlla vhleha ha im anoli tok.

\section{CHAPTA XXX.}

1 IHMA Islael im vlla vhleha puta ka, Moses vt apehli1 chi i noshkoboka puta ka isht im anumpulit, Iluppak o Chiliowah yut apesushke.

2. Hatak vt anumpa il onochit Chihowah im il onochi tukmot, keyukmvt anumpa kullo anumpulit imishilombish a -anumpa ho isht ile takchi tuk mut, im anumpa ha kobvffa he keyu, itih ha akohcha tuk moma kut yumihcha hi oke.

3. Ohoyo hot himita yosh ilki in chuka ahantut anumpa il onochi cha, Chihowah im il onochit anumpa isht ile takchi mut,

4. Mikma anumpa il onochi, micha anumpa isht ile takchi yo imishilombish a ishit takchi ka, iki vt haklo, mihmvt ikr yash osh nana ik im achokma; yohmikma anumpa il onochi tuk moma kut aiahla hi oke; michaanumpa isht ile takchi imishilombish ishit takchi puta kvt aiahla hi oke.

5. Amba iki vt haklo nitak akinli hosh vlhpèsa ik im ahno tukma, anumpa il onochi, micha anumpa moma isht iletakchi imishilombish a ishit takchi tnk vtik aiahlo ka hi oke, mihma iki ak osh vlhpesa ik im ahno hatuk o, Chiho. wah yot i kashoffa hi oke.

6. Mieha $i$ hatak vt asha tuk osh anumpa il onochi ma, itih ha nana kia kohchit im ishilombish a ishit takchi ma.

7. Yohmi ma i hatak vt haklo, mihmvt haklo nitak vt nana ik im achokma : yohmikma anumpa il onochi tuk vt aiahla hi oke, micha anumpa isht ile takchi im ishilombish a ishit takchi tuk vt aiahla hi oke.

8. Amba i hatak vt haklo nitak vt vlhpesa ik im ahno tuk not, yohmikmut annmpa il onochi il onochi tuk a, micha itih ha kohchichit imishilombish anumpa isht ile takchi tuk 
a ik ahlecho kashke, mikma Chihowah yut i kashoffa hi oke.

9. Amba ohoyo vlhtakla, micha ohoyo $\underline{i}$ hatak vt $\underline{i}$ kunia hokvto anumpa il onochi ilap imishilombish ishit takchi tuk vt moma kut ahlit takala hi oke.

10. Mikma i hatak in chuka anta hosh anumpa il onochi, keyukmot anumpa isht ile takchi imishilombish. a anumpa kullo ishit takchi tuk ma,

11. Yohmi ma $\underline{i}$ hatak vt haklo kia nana ik acho, micha vlhpesa ik im ahno: yohmikrna anumpa il onochi tuk moma kvt aiahla hi oke; micha anumpa isht ile takchi imishilombish ishit takchi tuk vt aiahla hi oke.

12. Amba $\underline{i}$ hatak vt haklo nitak vt im akshochit kanchikma, yohmikma nana hosh anumpa il onochi tuk, keyukmot imishilombish a anumpa ishit takchi itih ha kohcha tuk vt ik ahlo ka hi oke; i hatak vt im akshochi hoke; mikma Chihowah yut $\underline{i}$ kashoffa hi oke.

13. Anumpa il onochi puta, micha anumpa kullo isht iletakchi imishilombish isht ilbushali puta ka, i hatak vt a hlecha hinla, keyukmut i hatak vt akshocha hinla hoke.

14. Amba i hatak vt onna hosh onna ka nan ik ächo kash osh imomakut; yohmikmet anumpa il onochi tuk moma, micha anumpa isht ile takchi onvtola tuk moma ka kullochi hoke; haklo nitak vt nana ik im acho tuk okvt, kvllochi hoke.

15. Amba haklo ha ya tuk osh kaniohmit akshochikmvt, yohmikmvt i nanashvchi ya shaiyala hi oke, im achi tok oke.

16. Nan vlhpisa iluppa puta kak osh hatak micha tekchi ita tuklo iti takla ya itoyula he, micha iki micha ushetik hi-

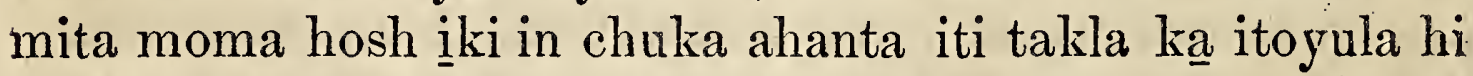
yo Chihowah yut Moses a im apesa tok oke.

\section{CHAPTA XXXI.}

IHMA Chihowah yot Moses a im anumpulit,

2. Islael im vlla vhleha ha Mitianait okla ha ish im 


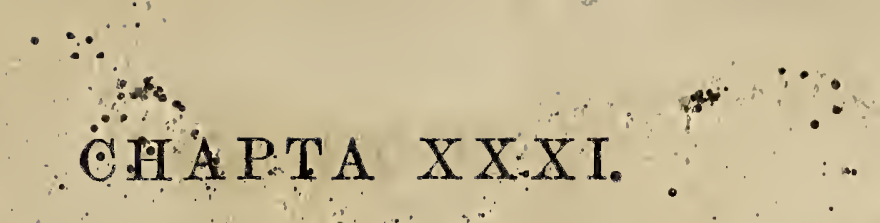

il ai okha pullashke, i himmak $\underline{Q}$ chim okla ha ish iba holhtina hi oke, im achi tok.

3. Mihma Moses vt okla ha im anumpulit, Hvsh kaniohmi kvt tunup hush ia chi kvt na halupa isht hush il atahlashke, micha ivt Mitianait okla ha ont in tanampashke, micha Mitian ak o Chihowah ya im il ai okhashke.

4. Islael apehlichi puta moma hlopulli ka tahlepa sipokni achufa bieka ho, tunup a hush ai i pilashke.

5. Yohmi ho Islael tahlepa sipokni puta ka akohchut apehlichi achvfakma, tahlepa sipokni achufut ia hosh tahlepa sipokni auahtuklo kvt ai i filvmmit na halupa isht im vlhtaha tok.

6. Yohmi ma Moses vt tunup a pila tok, apchlichi achvfakma tahlepa sipokni achvfa ho, yumma micha naholitompa isht vtta Eleasa ushi Finehas aiena kvt naholitopa isht asha, micha olacha chi ho isht púfa yut ibbak foyuka yo tunup a pila tok.

7. Mihma Chihowah yvt Moses a im achi tuk mak o, Mitianait okla ha in tanampi tok; micha nakni moma ka vbit tahli tok.

8. Micha illi tuk vt ashakma, Mitian i miko vhleha ha ubi tok; iluppa puta kak atok, Efi, Lekem, micha Su, micha Hu micha Leba, Mitian i miko tahlapi hatok; 'micha Beo ushi Balaam a bushpo falaia isht vbi tok.

9. Micha Islael im vlla vhleha hut Mitian ohoyo moma, micha im vlla vhleha aiena ka yukachi mvt, na wehpoa yo wak moma, micha vlhpoba chipunta moma, micha im ilayak moma aiena ka ishi tok.

10. Micha in tumaba holihta puta aiasha yatuk moma, micha i holihta moma ka luak ishit hukmit tahli tok.

11. Mihmot hatak, micha nan vlhpoa aiena, na wehpoa moma, micha nan vlhpoa moma ishi tok.

12. Micha yuka vhleha hash micha vlhpoba na wehpoa yash micha nampoa yash, Moses micha naholitompa isht vtta Eleasa micha Islael im vlla vhleha okla moma aiena ka isht im onvt, binah Moab yakni matali ya yvmmut Chatan bilika micha Cheliko bilika talaia ka isht ona tok. 
13. Mihma Moses micha naholitompa isht vtta Eleasa, micha okla moma i holitopa vhleha aiena kvt binah ha kocha afamvt ia tok.

14. Mihma Moses vt tushka chipota i pehlichi vhleha ha micha tahlepa sipokni puta $\underline{1}$ kvpituni vhleha, micha tahlepa puta 1 kupituni vhleha aiena kvt tunup ayvt vla tuk a i nuklibishla tok.

15. Mihmvt Moses vt, Ohoyo moma hush okchalinchi tuk oh cho?

16. Yakeh! iluppa puta kak osh Balaam vt i miha na, Islael im vlla vhleha Pio pulla ka Chihowah ishit $\underline{\mathrm{i}}$ yoshoba tok ; micha ililli okpulo vt Chihowah im okla moma ai ititakla ya itoyula tok.

17. Yumohmi hoka, himak a vlla vhleha nakni moma ka hushbashke, micha ohoyo hut iba tushki cha hatak ithana tuk moma aiena ka hushbashke.

18. Amba ohoyo-vlla moma, iba tushki cha hatak ithana tuk keyu kano, hvchimmi ha chi hosh okchalinchit hush ishashke.

19. Micha bina ha kucha hush aiasha na nitak untuklashke; kuna hosh hatak vbi tukmot, micha kvna hosh illi ya haleli tukmut, hvchishno, micha hvchi yuka aiena kvt, nitak ont isht atuchina, micha nitak ont isht untuklo ka hush ile kashoffashke.

20. Micha huchi nafohka moma, micha nahakshup osh toba, micha issi kosoma hishi yosh toba moma, micha iti yosh nana toba moma ka hush kashoffashke, im achi tok.

21. Mihma naholitompa isht vtta Eleasa vt hatak tunup aya tunup ia tuk a, Iluppak osh nan vlhpisa mak o Chihowah yut Moses a im apesa tuk mak oke.

22. Peh tuli lakna, micha tuli huta, asonak lakna, tuli, asonak hvta micha naki,

23. Nana hoh kia lua he keyukma, luak o hvsh hlopullechikma kashofa hi oke; yohmi kia filummichi oka ya ishit kashofa hi oke: micha lua hinla moma ka oka yak o hush hlopullecha hi oke.

24. Micha nitak ont isht untuklokma hvchi nafohka ya 
hush achefa cha huchi kashofa hi oke ; yohmi ha yosh binah ha ant hush chukowa hi oke, im achi tok.

25. Mihma Chihowah yut Moses $\underline{a}$ im anumpulit,

26. Chishno, micha naholitompa isht vtta Eleasa, micha okla moma iki ishahli vhleha aienvt, hatak, micha nan vlhpoa nawehpoa tuk holhtina ka hush ishashke.

27. Micha na wehpoa kashapa tulilo ita kushuplit, tunup ilonochit tunup ia tuk, micha okla moma ka hush im itakashvblashke.

28. Hatak tưnup aya puta tunư ia tuk a noshkobo atobbi Chihowah immi ya chi ho hush onochashke; hatak puta kash, micha wak puta kash, micha issuba nashoba puta kash, micha chụkfi puta kash aiena ka tahlepa tahlapikma shilombish achufa,

29. Im iklunna hush ai ishi cha, Chitokaka i walit im issa chị ho, naholitompa isht vtta Eleasa ya hush imashke.

30. Mikmut Islael im vlla vhleha im iklunna ya hatak puta kash, micha wak puta kash, micha issuba nashoba puta kash, micha nan vlhpoba chipunta kash micha nan vlhpoba ilaiyuka moma kash pokoli tahlapikma, achufa ho ai ishit Lefait vhleha, yommut Chihowah in tabenakel nan isht ai ahanta ka apistikeli chatuk a hush imashke, im achi tok.

31. Mihma Chihowah yut Moses a im apesa tuk mak o Moses micha naholitompa isht vtta Eleasa ita tuklo kut yvmihchi tok.

32. Mihma nawehpoa tunup aya hatak vhleha hut yukachi tuk atampa mak osh chukfi tahlepa sipolni bat tahlepa hannali, tahlepa sipokni pokoli untuklo, micha tahlepa sipokni tahlapi tok,

33. Micha wak tahlepa sipokni pokoli untuklo akucha tuklo tok,

34. Micha isuba nashoba tahlepa sipokni pokoli hannali akucha achifa tok,

35. Micha iba tvshkit hatak a ithana tuk keyu moma kvt ohoyo tahlepa sipokni pokoli tuchina akucha tuklo tok.

36. Micha iklunna kut, yummut kohchvt tunup ia tuk $\underline{\mathrm{i}}$ kashapa holhtina kut chukfi tahlepa sipokni bat tahlepa tu- 
china, micha tahlepa sipokni pokoli tuchina akucha untuklo, micha tahlepa tahlapi tok.

37. Micha noskobo Chihowah im atobbi vt chukfi tahlepa hannali, micha pokoli untuklo akucha tahlapi tok.

38. Mikma wak vt tahlepa sipokni pokoli tuchina akucha hannali tok; yumma noshkobo Chihowah im atobbi akohcha kvt pokoli untuklo akucha tuklo tok.

39. Mikma issuba nashoba yot tahlepa sipokni pokoli tuchina micha tahlepa tahlapi tok; yumma noshkobo Chihowah im atobbi vt akohcha kvt pokoli hannali akucha achvfa tok.

40. Mikma hatak ohoyo hot tahlepa sipokni auahannali tok; yumma noshkobo Chihowah im atobbi vt akohcha kut ohoyo pokoli tuchina akucha tuklo tok.

41. Mihma Chihowah yut Moses a im apesa tuk mak o Moses vt noshkobo atobbi yash, yvmmut Chihowah i walit im issa yatuk o naholitompa isht vtta Eleasa ya ima tok.

42. Mihma Islael im vlla vhleha im iklunna, yumma Moses vt hatak tanampi tuk ash im ita kashvbli tok.

43. Yohmi ka okla moma ka im iklunna kvt chukfi tahlepa sipokni bat tahlepa tuchina micha tahlepa sipokni pokoli tuchina akucha untuklo, micha tahlepa tahlapi tok,

44. Micha wak tahlepa sipokni pokoli tuchina akucha hannali tok,

45. Micha isuba nashoba tahlepa sipokni pokoli tuchina micha tahlepa tahlapi tok,

46. Micha hatak ohoyo tahlepa sipokni auahhannali tok.

47. Islael im vila vhleha im iklunna ka Moses vt, hatak micha nan vlhpoba aiena pokoli tahlapikma achufa ho ai ishi cha Lefait whleha, yummut Chihowah in tabenakel nan isht aiahanta apistikeli yatuk o Chihowah yut Moses a im apesa tuk mak o ima tok.

48. Mihma okla tahlepa sipokni puta i nan vlhtoka vhleha, micha tahlepa sipokni puta i kupituni vhleha, micha tahlepa puta 1 kupituni vhleha aiena kvt Moses a bilika im ona tok.

49. Micha Moses a, Chin tishu vhleha hvt tunup aya ha- 
tak uhleha e pehlichi tuk aiyukali holhtina ka il ishi ma, hatak achvfa kia iksho kvt ikshoshke.

50. Yumohmi hatuk osh Chihowah isht aiokpvchi isht elushke, pim ishilombish isht atobbi Chihowah itikba il ai ikba chi hosh hatak il aiyukali kvt nana il ishi tuk, tvli lakna isht shema, itatakvli, micha shakba abeha, chvnaha, micha haksobish atakali chunaha, micha tuli lakna inochi aiena ka isht e lushke, im achi tok,

51. Mihma Moses micha naholitompa isht vtta Eleasa ita tuklo kut tuli lakna isht il akshema moma ka im ishi tok.

52. Micha tvii lakna im 'issa, tahlepa sipokni puta i kvpitvni uhleha, micha tahlepa puta $\underline{i}$ kupituni vhleha aiena kut Chihowah im issa tuk moma kvt shekel tahlepa sipokni auahhannali micha tahlepa untuklo pokoli tahlapi tok.

53. Tunup aya hatak puta kvt nawehpoa ya ilap aiyukali kvt ishi tok okvt.

54. Mihma Moses micha naholitompa isht vtta Eleasa itatuklo kvt tvli lakna yash, tahlepa sipokni puta $\underline{\mathbf{i}}$ kupituni vhleha, micha tahlepa puta $\underline{\mathrm{i}}$ kvpituni vhleha ha im ai ishi cha, Islael im vlla vhleha isht ithana bilia hosh Chihowah itikba itoyula chị ho okla moma in tabenakel a ont fohki tok.

\section{CHAPTA XXXII.}

OHMI ma Luben im vlla vhleha, micha Kat im vlla 1 whleha hut wak im apakna. chinto fehna tuk atuk osh Chaasa yakni micha Kiliat yakni ya pisa ma, yakeh, yvmmut wak aiasha ya achukma tok.

2. Yohmi mut Kat im vlla vhleha, micha Luben im vlla vhleha hut Moses micha naholitompa isht vtta Eleasa, micha ckla moma i holitopa vhleha aiena kash im ona cha.

3. Atalolh, micha Tibon, micha Chaasa, micha Nimlah, micha Heshbon, micha Elealah, micha Shebam, micha Nebo, micha Beon.

4. Islael okla moma itikba ya Chihowah yut issot a kan- 
chi tok yakni pulla kut wak i yakni achukma hoke, mihma hvchin tishu vhleha hvt wak im ashushke,

5. Okla achi mut, yumohmi hoka chim isht $\underline{i}$ kana ya chi ai ahaiyuchi tuk ma, yakni iluppak o chin tishu vhleha $\underline{1}$ yakni ya chi ho ibbak fohkashke, micha Chatan a chik pi hlopullecho kashke, im achi tok.

6. Mihma Moses vt Kat im vlla vhleha, micha Luben im vlla vhleha, Hvch itibapishi vhleha hvt tunvp ia na, lumvt iluppa hush chieya chi cho?

7. Micha nanta katiohmi ho Islael im vlla vhleha hvt yakni Chihowah yut ima tok a chukowa he tuk o chukvsh hush illechi cho?

8. Huch iki vhleha hut yakni ya pisa chị ho Katesh-bania vttut pila li ma, yakohmi tok.

9. Eshkol okfa hạ ona cha yakni ya pisa mvt, Islael im vlla vhleha chukvush a illechi na, yakni Chihowah ima tok a ik chukowo tok.

10. Atuk o mih makinli ma Chihowah im ai i nukkilli vt libbi mot, anumpa kvilo anumpulit,

11. Afummi pokoli tuklo ishtivt vba maya hosh Echip yakni ya kohchvt minti tok vt, hatak achvfa kia, anumpa kvllo anumpulit, Ebleham, Aisaak, micha Chekob-a im issa li tok yakni ya pisa chi keyu pulia hoke ; aiokluhut. vm antia tuk keyu hokvt,

12. Kenesait Chefunneh ushi Kaleb, micha Nun ushi Choshua ak illa hi oke; yummak okvto aiokluhut Chihowah im antia tuk okvt, achi tok.

13. Micha Chihowah im ai i nukkilli vt libbi mut yakni haiaka itunowuchi na afummi polioli ushta ma, ai unchuloli yumma moma Chihowah itikba nan ik achukmo yumihchi tuk vt taha tok.

14. Atuk o yakeh! huch iki vhleha vlhtobvt, Chihowah im ai $\underline{i}$ nukkilli vt Islael a $\underline{\underline{i}}$ sanalit libbit isht $\underline{\underline{i}}$ shalit isht ia chi ho hatak nanashvchi ai iti shali hush wakaiyushke.

15. Hush jakaiya tuk vt hush $\underline{i}$ filvmmikma, himakma anonti yakni haiaka ya akancha chi hoke; mikma okla iluppa moma ka ubit hush tahla chị hoke, im achi tok. 
16. Mihma okla hash ot bilika, im ona cha, Pi nan ulhpoa aiasha he chukfi-holihta, micha pim vlla vhleha aiasha he tumaha holihta iloppa il ai ikba chi hoke.

17. Amba pishno vto na halupa pim vlhtaiyaha hosh Islael im vlla vhleha itikba e mahaya cha, aiasha chị ka isht il onashke: mikma pim vlla vhleha hvt yakni ya okla aiasha pulla kak o tumaha aholihta puta ho aiasha chi hoke.

18. Islael im vlla vhleha moma kvt 1 yakni aiyukali kvt ik abinilo kisha hokvno, falamot pin chuka ela he keyu hoke.

19. Chatan tunnup yumma, micha tikba pilla hokuno yakni ya il ibai isha he keyu; pi yakni vt Chatan ola tunnưp hushi akohcha ka pilla kak o pin talaia hokut, im achi tok.

20. Mihma Moses vt, Nana iluppa hvsh yumihchi hokmvt, na halupa huchim vlhtaiyaha cha Chihowah itikba ya tunvp hush iakmut.

21. Micha hush moma kvt na halupa hvehim vlhtaiyaha cha Chatan a hlopullit Chihowah itikba ya hush ia na, in tunup vhleha ha itikba a tihlelit kocha wehlit tahlikma.

22. Mikma yakni vt Chihowah itikba ya ai i nutaka, yohmikma $\underline{i}$ himmak a yo Chihowah itikba ya micha Tslael itikba ya nanashvchi vt onvtola keyu hosh hvsh falamashke, mikma yakni ilupput Chihowah itikba ya huvchi yakni ya chi hoke.

23. Amba hush yumohma he keyukmvt, yakeh! Chihowah ya hush $\underline{i}$ yoshobushke, yohmi ka oh ithana na hvsh ashvchi vt hvchi ahayucha chi hoke. :

24. Huchim vlla tvmaha holihta hush im ikbashke; micha hvchin chukfi ha holihta hush im ikbashke; micha huch itih ha kohcha tuk mak osh hush yumihchashke, im achi tok.

25. Mihma Kat im vila vhleha, micha Luben im vila vhleha hut Moses a im anumpulit, $\underline{\mathbf{A}}$ shahli hvt anumpuli tuk mak o chin tishu vhleha hot yumihcha chi hoke.

26. Pim vlla vhleha, pin tekchi vhleha, pi nanvlhpoa chipunta, micha pi wak moma aiena kvt Kiliat tumaha holihta puta yummak o aiasha chi hoke. 
27. Amba a shahli hvt achi tuk mak $\underline{0}$, chin tishu vhleha hatak moma kvt tanampa chi kvt na halupa im vlhtaha hosh hlopullit Chihowah itikba ya tanampit ia chị hoke, im achi tok.

28. Yohmi ho yưmma pulla ka Moses vt naholitompa isht vtta Eleasa, micha Nvn ushi Choshua, micha Islael im vlla vhleha apehlichi puta iki vhleha $\underline{\mathrm{i}}$ shahli aiena ka im apesa tok.

29. Micha Moses vt, Kat im vlla vhlcha, micha Luben im vlla vhleha aiena hatak puta kvt na halupa im vlhta ha hosh hvchi awant Chatan a hlopullit Chihowah itikha tvnup ia na, yakni vt hvchi tikba ya nutaka hokma; yohmikma ai ahanta chi ka Kiliat yakni ak o hush ima hi oke.

30. Amba nahalupa im vlhtaha hosh hvchi awant hlopulla he keyukma, Kenaan yakni ak o hvch ibai asha hi oke, im achi tok.

31. Mihma Kat im vlla vhleha, micha Luben im vlla vhleha aiena kvt afalaminchit, Chihowah yvt in tishu vhleha ha im achi tuk mak o e yumihcha chi hoke.

32. Na halupa pim vlhtaha hosh hlopullit Chihowah itikba ayut Kenaan yakni ya e chukowa chị hoke; yohmi hosh yakni il isha chi kvt Chatan ola in tunnup ak osh pimmi ha chi hoke, achi tok.

33. Mihma Moses vt Kat im vila vhleha, micha Luben im vlla vhleha, micha Chosef ushi Manasseh apehlichi iklvnna aiena ka , Amolait okla i miko Sihon im apehlichika, micha Bashan i miko Ok im apehlichika, yakni yash, micha im ai vhli tumaha holihta puta ai iti eshi, micha yakni kucha apakfoyupa tumaha holihta puta ka ima tok.

34. Mihma Kat im vlla vhleha hvt Tibon micha Atalolh, micha Aloe,

35. Micha Atlolh, Shofan, micha Chaasa, micha Chokbehah,

36. Micha Belh-nimbah, micha Belh-halan tumaha holihta, micha chukfi i holihta aiena ai ikbi tok.

37. Mihma Luben im vlla vhleha hvt Heshbon micha Elealah micha Kichalh-chealim, 
38. Micha Nebo, micha Baal-meon (hohchifo inla hochifo hosh) micha Shibmah aiena ka ikbi tok: micha tumaha holihta ikbi tuk vt hohchifo inla ima tok.

39. Mihma Manasseh ushi Maki im vlla vhleha hvt Kiliat a ona cha ishi tok; micha Amolait vt yumma asha tuk a im ai ishi tok.

40. Mihma Moses vt Kiliat a Manasseh ushi Maki ya ima na, abinili tok.

41. Mihma Manasseh ushi Chai vt ivt in tumaha ushi puta ka ishi cha Hafolh-Chai hochifo tok.

42. Mihma Nobah hot ivt Kenalh, micha in chuka lokoli aiena ishi cha, ilap hohchifo kak o ahochifochit Nobah hochifo tok.

\section{CHAPTA XXXIII.}

TSLAEL im vlla vhleha Echip yakni ya okla moma kvt 1 Moses micha Alon ita tuklo kvt pehliechi ho kohchvt itunowvt aya tuk vt iluppa puta kak atok.

2. Mihma Chihowah yut im apesa mak o, Moses vt kohchvt nowvt itvnowa tok a holissochi tok: yohmi kvt ilvppa puta kak osh kohchut itunowa kvt ai itunowa tok mak oke.

3. Mihmvt hvshi vmmona, micha hvshi vmmona kvt nitak ont ishit auahtahlapi ma, Lameses a asht ia tok; vbanvblit ont ia yatuk a onna ma, Islael im vlla vhleha hut Echip okla moma kut pisa ho ibbak kullo hosh kohchvt ia tok.

4. Echip okla hut im vlla vttahpi moma, Chihowah yut issot i kanchi na hohpit tahli tuk atuk okvt: micha Chihowah yvt i naholbut toba puta kak kia napalummi onochi tok.

5. Mihma Islael im vlla vhleha hvt Lameses a kanvlli mut Sukkolh a abinachi tok.

6. Atuk osh Sukkolh a kanvlli mvt, Ehlam, yvmmut yakni haiaka vhli talaia ka abinachi tok.

7. Atuk osh Ehlam a kanvlli mot fullotut anonti Pi-hahilolh, yummut Baal-sefon itikba talaia ka ona tok, micha Miktol itikba yak $\mathrm{Q}$ binachi tok. 
8. Atuk osh Pi-hahilolh itikba asht ia mvt okhvta iklunna hlopulli cha yakni haiaka pit chukowvt Ehlam yakni haiaka nitak tuchina a nowa yo ona tok, micha Malah ya binachi tok.

9. Atuk osh Malah ya kanvlli mut Elim a ona tok, mihma Elim a kvli auahtuklo kvt hiohmaya tok, mihma pam iti pokoli untuklo kvt hinli tok, mihma yummak o binachi tok.

10. Atuk osh Elim a asht kanvlli mut okhvta Homma bilika binachi tok.

11. Atuk osh okhvta Homma ya asht kanvlli mut Sin yakni haiaka ya binachi tok.

12. Atuk osh yakni haiaka Sin a asht ia mut Tofkah ya binachi tok.

13. Atuk osh Tofkah ya asht ia mvt, Alush a binachi tok.

14. Atuk osh Alush a asht ia mut, Lefitim, yumma okla oka ishka hi vt iksho ho binachi tok.

15. Atuk osh Liefitim a asht ia mut, yakni haiaka Sinai ya binachi tok.

16. Atuk osh yakni haiaka Sinai ya asht ia mvt, Kiblolhhattaafah ya binachi tok.

17. Atuk osh Kiblolh-hattaafah ya asht ia mut, Haselolh a binachi tok.

18. Atuk osh Haselolh a asht ia mut, Lilhmah ya binachi tok.

19. Atuk osh Lilhmah ya asht ia mut, Limmon-pales a binachi tok.

20. Atuk osh Limmon-pales a asht ia mut, Libnah ya binachi tok.

21. Atuk osh Libnah ya asht ia mut, Lissah ya binachi tok.

22. Atuk osh Lissah ya asht ia mut, Kehelalhah ya binachi tok. .

23. Atuk osh Kehelalhah ya asht ia mut, Shafa nunih hạ binachi tok.

24. Atuk osh Shafa nưnih ha asht ia mvt, Halatah ya binachi tok. 
25. Atuk osh Halatah ya asht ia mıt, Makhelolh a binachi tok.

26. Atuk osh Makhelolh a asht ia mvt, Tahalh a binachi tok.

27. Atuk osh Tahalh a asht ia mot, Talah ya binachi tok.

28. Atuk osh Talah ya asht ia mvt, Milhkah ya binachi tok.

29. Atuk osh Milhkah ya asht ivt, Hashmonah ya binachi tok.

30. Atuk osh Hashmonah asht ivt, Moselolh a binachi tok.

31. Atuk osh Moselolh a asht ivt, Bene-chaakan a binachi tok.

32. Atuk osh Bene-chaakan a asht ivt, Hol-hakitkat a binachi tok.

33. Atuk osh Hol-hakitkat a asht ivt, Chotbahlah ya binachi tok.

34. Atuk osh Chotbahlah ya asht ivt, Eblonah ha binachi tok.

35. Atuk osh Eblonah ha asht ivt, Esion-kaba ya binachi tok.

36. Atuk osh Esion-kaba ya asht ivt, Sin yakni haiaka, yummut Katesh a binachi tok.

37. Atuk osh Katesh a asht kanvlli mvt, Ho nunih Etom yakni vhli ya binachi tok.

38. Mihma naholitompa isht vtta Alon vt Ho nvnih ha Chihowah yut im apesa ho oiya tok, Islael im vila vhleha hot Echip yakni ya kohcha tuk a afummi pokoli ushta ho hushi ont ishit tahlapi nitak vmmona ma yummak $\underline{o}$, ai illi tok.

39. Mihma Alon vt afummi tahlepa achufa cha pokoli tuklo akucha tuchina hosh Ho nunih ha ai illi tok.

40. Atuk o miko Alat Kenaanait, Kenaan yakni oka mahli pilla ahanta kvt, Islael im vlla vhleha hvt minti ka pit haklo tok.

41. Atuk o Ho nưnih ha asht ivt, Salmonah ha binachi tok. 
42. Atuk osh Salmonah ha asht ivt, Punon a binachi tok.

43. Atuk osh Punon a asht ivt, Obolh a binachi tok.

44. Atuk osh Obolh a asht ivt, Iche-abalim Moab im ai vhli ka binachi tok.

45. Atuk osh Tim a asht ivt, Tibon-kat a binachi tok.

46. Atuk osh Tibon-kat a asht ivt, Almon-tiblahlaim a binachi tok.

47. Atuk osh Almon-tiblahlaim a asht kanvlli mut, Nebo itikba Abalim nunih chaha foka yak o binachi tok.

48. Atuk osh Abalim nunih foka ya asht ia mvt, Chatan a Cheliko bilika Moab yakni matali ak o binachi tok.

49. Atuk osh Chatan bilika Belh-chesimolh a ashvt ia mvt, Moab yakni matali Abel-shittim a abinachi tok.

50. Atuk o Chihowah yut Moses a Moab yakni matali Chatan bilika micha Cheliko bilika ya ai im anumpulit,

51. Islael im vlla vhleha im anumpulit, Chatan a hlopullit Kenaan yakni ya ont hush chukowakmvt,

52. Yohmikmut yakni yash okla aiasha tuk a huchi tikba atihlelit hush kohchashke, micha 1 naholbut toba puta moma ka okpunit, bilut akmot naholbut toba puta ka okpunit hush tahlashke, micha im achahaka puta akvchit hush vmohmichashke.

53. Micha okla aiasha tuk a yakni hush im ishi cha hvsh aiasha chị hoke, yakni yạ hush isha chị ho huchima li hatuk oka.

54. Micha yakni ya nashoeli hosh i yakni ya chị ho hưchin chuka achvfa vlhpisa ho hush im ita kvsho lashke: micha laua $\underline{i}$ shahlikma yakni ya $\underline{i}$ shahlechit hesh imashke, micha iklauokma yakni vt iklauo ho hvsh imashke; hatak i yakni aiyukali kvt kanima itulakma yvmmak o i yakni ya chi hoke; hvch iki vhleha apehlichi vlhpisa ho yakni ya hvsh isha chi hoke.

55. Amba yakni yash okla aiasha tuk a hvchi tikba hvchik kocha wehlo tuk ma; atukma yakohma hi oke, hvsh ashanchi tuk vt nahalupa yosh hvchi nishkin a shamvlli ya hi oke, micha kvti chohmit hvchi naksi ya shimowoa ya yakni ya hvsh aiasha ka hvchilbvshala hi oke. 
56. Micha yakohma hi oke, yumma i yumihchi la he aiahni li tuk vt, hưchishno ya huvchị yumihchi la hi oke, ish im achashke, im achi tok.

\section{CHAPTA XXXIV.}

MIHMA Chihowah yot Moses a im anumpulit,

11 2. Islael im vlla vhleha ha im apesut, Kenaan yakni ya ont hvsh chukowakmvt, hush aiasha he vt ilvppak osh Kenaan yakni, micha aivhli fullota yakni hush ai isha chi mak oke,

3. Yohmikma hvchim oka mahli kashapa kvt yakni haiaka Sin ak o vttut ia hosh Etom ai vhli ak o vhlit aya hi oke; micha oka mahli hvchim ai vhli vt okhvta hvpi hvshi akocha ka ont ai vhli fehna ak a hi oke,

4. Atuk osh huchim ai vhli vt oka mahli y.a vttvt fullota kvt Aklabbim atuya onakmut hlopullit Sin a ona hi oke: micha ont akohcha vt oka mahli ha vttut ia hosh Katesh-bania ya onakmut, ont ayut Hasa-atta ona cha, ont ayakmut Asmon a ona hi oke.

5. Atuk osh ai vhli vt Asmon a vttut et fullotakmvt Echip okhina ya ona hi oke, micha akohcha yut okhvta yak a hi oke.

6. Mihma hushi ai okatula ai vhli hokvto okhvta ak inli kak osh huchim ai vhla hi oke : ilvppak osh hushi ai okvtula hvchim ai vhla hi oke.

7. Mihma ilvppak osh falummi huchim ai vhla chi hoke: okhuta chito ya vttut nunih Ho ya ona ka hush apesashke.

8. Ho nunih ha vttut, Hamalh ont achukowa ont vhli ho huchim ai vhli ya hush apesashke, micha ai vhli ont a kohcha kut Setat ak o ona hi oke,

9. Micha ai vhli vt ont ayvt Siflon a ona hi oke, micha ont akohcha kut Hasa-enan ak a hi oke: iluppak osh falummi hvchim ai vhla hi oke.

10. Micha hvshï akochaka hvchim ai vhli ya Hasa-enan a vttut ia hosh Shefam ont vhli ka hush apesashke. 
11. Micha ai vhli vt Shefam vttut okvtahvt ia hosh Ain hushi akochaka in tunnup ayvt Liblah ya onashke ; mihmut ai vhli vt okvtahvt iakmvt okhvta Chinnelolh lapalika hvshi akochaka pilla kak o onashke.

12. Mikmvt ai vhli vt okvtahvt ivt Chatan a ona hi oke: mikma ont akohcha vt okhvta hupi yak a hi oke. Ilvppak osh ai vhlit apakfoyupa aiena kvt hvchi yakni ya chi hoke, ish im achashke, achi tok.

13. Mihma Moses vt Islael im vlla vhleha ha im apesitt, Yakni iluppak o na shoeli hosh hvsh abinila chi ho apehlichi hush chakkali cha iklunna aiena kak o huchima he Chihowah yot apesa tuk oke,

14. Luben apehlichi im vlla vhleha vto iki vhleha in chuka achvfa vlhpisa hosh, micha Kat apehlichi im vlla whleha iki vhleha in chuka achvfa vlhpisa hosh $\underline{1}$ yakni ya ishi tuk oka; mikma Manasseh apehlichi iklunna aiena kvt 1 yakni ishi tuk oka,

15. Apehlichi tuklo, micha apehlichi iklunna kut i yakni ya Chatan ola in tunnưp hushi akochaka pilla, Cheliko bilika hvshi akochaka ak o ai ishi tuk oke, im achi tok.

16. Mihma Chihowah yut Moses a im anumpulit,

17. Hatak iluppa puta hohchifo kak osh yakni ya huchim ita kushkola chi hoke: naholitompa isht vtta Eleasa, micha Non ushi Choshua ita tuklo kak a chị hoke.

18. Micha apehlichi puta ka holitopa achvfa ho hush atokoli na ai isha he vlhpesa vlhpisa ho yalkni yash ita kushkolashke.

19. Micha hatak vhleha hohchifo hvt iluppak oke ; Chutah apehlichika Chefunneh ushi Kaleb ak a hi oke.

20. Simeon im villa vhleha apehlichika Ammihut ushi Shemuel ak a hi oke.

21. Benchamin apehlichika Chislon ushi Elitat ak a hi oke.

22. Mihma Tan apehlichika ai achufa holitopa Chokli ushi Bukki ak a hi oke.

23. Chosef im vila vhleha ai achvfa holitopa Manasseh im vlla vhleha apehlichika Efot ushi Hanniel ak a hi oke. 
24. Mihma Eflaim im vlla vhleha apehlichika aiachvfa holitopa vt Shiftan ushi Kemuel ak a hi oke.

25. Mikma. Sebulon im vlla vhleha apehlichi aiachvfa holitopa vt Panak ushi Elisafan ak a hi oke.

26. Mikma Issaka im vlla vhleha apehlichi aiachvfa holitopa yut Assan ushi Paltiel ak a hi oke.

27. Mihma Asha im vlla vhleha apehlichi aiachvfa holitopa vt Shelomi ushi Ahihvt ak a hi oke.

28. Mihma Naftali im vlla vhleha apehlichi aiachvfa holitopa vt Ammihvt ushi Petahel ak a hi oke.

29. Iluppa puta kak osh Islael im vlla vhleha ha Kenaan yakni ya im ita kvshkola chi họ Chihowah hưt apesa tuk mak oke.

\section{CHAPTA XXXV.}

7 IHMA Chihowah yut Moses a Moab yakni matali ChaI 1 tan bilika, micha Cheliko bilikka ya ai im anumpulit,

2. Islael ị̣ vlla vhleha hovt Lcfait vihleha hut aiasha chi kà ai ishá he vlhpesa yakni ya tumaha holehta puta ka isht ima he, ish im apesashke; micha Lefait whleha ha tumaha holihta puta a bilika fullota ka hush imasshke.

3. Micha tumaha holihta puta aiasha chi kutim ashashke; mikma a bilika puta ka $\underline{i}$ wak, im ilayak micha $\underline{i}$ nan vlhpoa moma aiena kut aiashashke.

4. Micha tumaha holihta puta bilika apakfoyupa Lefait vhleha hush ima chi kvt holihta ya hikivt kocha pilla ia hosh kubit tahlepa sipokni achvfa banot apakfoyupa chị hoke.

5. Mikma tvmaha holihta yumma vittvt kocha ia kvt hvshi akochaka in tunnup, kubit tahlepa sipokni tuklo, micha oka mahli in tunnup vt kubit tahlepa sipokni tuklo, micha hvshi ai okatula in tonnup vt kubit tahlepa sipokni tuklo, micha falummi in tunnup vt kubit tahlepa sipokni tukklo ho hush apesashke: mikma tumaha holihta yvt ai "iklunna hikiashke: iluppak osh in tumaha holihta ya im a bilika a puta yashke. 
6. Micha Lefait vhleha ha tumaha holihta puta hush ima ka tumaha holihta a hlakofi yut hannalashke, yummak oka hatak bika vbi yvt om malela hi yo hvsh im apesashke : micha yưmma tumaha holihta pokoli ushta akucha tuklo ho hush im aienvchashke.

7. Yohmi ho Lefait vhleha ha tumaha holihta hush ima moma kvt pokoli ushta akucha untuchina hi oke, yummak oka abilika fullota aiena ho hush imashke.

8. Micha tumaha holihta puta hush ima kvt Islael im vlla

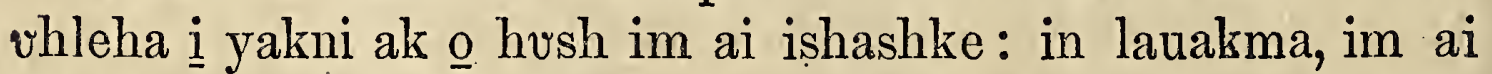
ishit laua ho hosh imashke; amba ik in lauokma im ai ishit ik lawo ho hush imashke: moma kut ai isha he vlhpesa ya ishi kak oșh vlhpisa họ Lefait vhleha ha tưmaha holihta ya imashke, im achi tok.

9. Mihma Chihowah yut Moses a im anumpulit;

10. Islael im vlla vhleha im anumpulit, Chatan a hlopullit Kenaan yakni ya ont hush chukowakmut,

11. Yohmikmut tumaha holihta, yưmmut tumaha holihta ahlakofi ya chi ho hush apesashke; mikma hatak nana hosh ik nuktạhlo hosh kuna ha vbikmvt, yummak o nan vbi vt pit mallelashke.

12. Mikma yummut il aiokha hush im ahlakoffit aiokchaya tumaha holihta puta hashke; yumohmi na hatak ubi vt okla moma itikba hikivt nan ik im vlhpiso kisha kvt ik illo kashke.

13. Micha tvmaha holihta puta hush ima chi kut tumaha holihta hannali kvt hush ahlakofi ya chi hoke.

14. Chatan ola in tưnuvp a tumaha holihta tuchịna ho hush imashke, micha Kenaan yakni ya tumaha holihta tuchina ho hush imashke, yummut ahlakofi tvmaha holihta yashke.

15. Tumaha holihta hannali ilvppak osh Islael im vlla vhleha, micha okla inla, micha nowvt aya tuk osh takla ahanta aiena ka ahlakofi ya chi hoke: yưmohmi ka kuna hosh ik nuktahlo hosh hatak vbikmvt yummak o malelit onashke.

16. Atuk o tuli ishit toksuli yo isht isso na illikma, yummut hatak vbi yoke: hatak vbi vt illi pullashke. 
17. Micha tuli yo pila hosh isso na isht illa he vlhpesa tuk o illikma, hatak obi yoke: hatak vbi vt illi pulla hi oke.

18. Keyukmut iti, ibbak isht itibi yosh isht illa he vlhpesa ho isht isso na illikma, hatak vbi yoke: hatak vbi vt illi pullashke.

19. Issish hlatapa ka il aiokha hot ilap akinli hosh hatak vbi yash vbashke: itafamakmvt vbashke.

20. Amba i nukkilli hosh ishit bahli na illi, keyukmvt in luma tuk osh pit ishit bahli na illi;

21. Keyukmvt i nukkilli hosh ibbak isht isso na, illikma, isso tuk vt illi pullashke; hatak vbi yokvt; issish hlatapa tuk il aiokha yut afamakmut hatak ubi yash vbashke.

22: Amba î.nukkilli keyu hosh yakosi itin takla ho ishit bahli, keyukmot im aịehchi.tuk keyu hosh nana kia pit om pila,

23. Keyukmvt tưli yösh hatak isht illa he vlhpesa ho pisa keyu hosh pit om pila na illikma, mikma in tonup atuk keyukma, micha hotopali bunna hatuk keyukma:

24. Yohmikma okla moma kvt hatak vbi micha im issish hlatapa il aiokha ita tuklo nan vlhpisa iloppak o iakaiya hosh nan im apesashke:

25. Micha okla moma kvt hatak vbi yash issish hlatapa il aiokha ibbak ash akohchit hlakoffichashke, micha okla moma kvt tumaha holihta ai okchaya malelit ai ona tuk, falumminchit fohkashke: mikma yomma ahanta na naholitompa isht vtta moma ishahli bila holitopa ahama tuk vt illashke.

26. Yohmi tuk kia hatak ubi yash osh nitak kaniohmi ho in tumaha holihta aiokchaya malelit ona tuk vt kocha aya hokma,

27. Atuk o issish hlatapa il aiokha yut in tumaha holihta aiokchaya kocha ya ai ahayuchi cha, issish hlatapa il aiokha yut hatak vbi yash vbi kia: issish hlatubli kut im vlhpisa he keyu hoke.

28. In tumaha holihta aiokchaya anukaka ahanta na, naholitompa isht utta moma i shahli vt illi ha yak a hi atuk 
oka: amba naholitompa isht vtta moma $\underline{\underline{i}}$ shahli vt illi hayokmuno, hatak vbi vt falamut i yakni ya ona hi oke.

29. Yvmohmi hoka, ilvppa puta kak osh nan vlhpisa isht apesa yosh hvchi aionchuloli puta hlopulli ka hush aiasha moma ka itoyula chi hoke.

30. Kuna hosh hatak vbikmvt nishkin pisa itih pulla ho hatak vbi vt illa hi oke: amba witness achvfa hosh hatak nana ho $\underline{\mathrm{i}}$ sanalit atokoli na illa he keyu hoke.

31. Micha hatak vbi vt illa he vlhpesa ka im ilhfiopak a nan aivlli hush isha he keyu hoke: amba illi pulla hi oke.

32. Micha malelit tumaha holihta aiokchaya ont chukowa tuk, nan aivlli hush ishi na, naholitompa isht vtta yvt ik illo kisha ho anonti i yakni ai vtta chi hosh vla he keyu hoke.

33. Yvmohmi hosh yakni hush aiasha kut huchik litehlo kashke: issish yummak osh yakni ya litehli hoka: micha yakni vt issish on hlatapa tuk vt kashofa he keyu, amba hlatubli tuk im issish ak o ishit kashofa hi oke.

34. Yumohmi hoka yakni hush aiasha chi, micha ai ahanta li ya hvchik litehlo kashke: Uno, Chitokaka sia hut Islael im vlla vhleha takla ahanta li hoka, ish im achashke, im achi tok.

\section{CHAPTA XXXVI.}

IHMA Chosef ushi vhleha in chuka achvfa puta ka ai1 achvfa Manasseh ushi Maki ushi Kileat im vlla vhleha in chuka achvfa puta iki ishahli vhleha hvt bilika ona cha, Moses, micha holitopa vhleha, Islael im vlla vhleha iki i shahli vhleha itikba anumpulit,

2. Micha a shahli hot Islael im vlla vhleha i yakni ya chi ho, yakni ya na shoeli hosh ima he ya Chihowah yut im apesa tok oke; micha a shahli hot il itibapishi Selofehat $\underline{\mathbf{i}}$ yakni yatuk a ushetik vhleha hak o ima hi a Chihowah yvt apesa tok.

3. Atuk o Islael im vlla vhleha apehlichi inla ushi kanima ho auwayakma, $\underline{1}$ yakni yash piki vhleha $\underline{i}$ yakni ya ai ishi cha, ishi tuk ash apehlichi i yakni ak o ibafohka hi oke: 
yohmi hosh nashoelit il ishi tuk, pi yakni yash pim ai isha hi. oke.

4. Micha Islael im vlla vhleha in chubili vt vlakma, $\underline{\mathbf{i}}$ yakni yash, ishi tuk ash apehlichi i yakni ya ibafohka chi hoke; yumohmi ho $\underline{i}$ yakni yash piki vhleha im apehlichi ha im ai isha hi oke, im achi tok.

5. Mihma Moses vt Chihowah im anumpa hatuk mak o Islael im vlla vhleha ha im apesvt, Chosef ushi vhleha apehlichi hot vlhpesa ho achishle.

6. Nana iluppak o Selofehat ushetị vhleha pulla ka Chihowah hut apesushke, kuna ho vlhpesa ahnikmut auwayashke : yohmi kia iki apehlichi chuka achvfa yak o auwayashke.

7. Yumohmi ho Islael im vlla vhleha $i$ yakni vt kanvllit apehlichi achufa vttut apehlichi inla onvt ik mahayo kashke: Islael im vlla vhleha hvt ilap iki vhleha apehlichi aiyukali kak o ibafoyuka chị hoka.

8. Mihma ushetik aiyukali kvt Islael im vlla vhleha apehlichi kanima ho yakni ai ishi tuk mot, ilap iki apehlichi aiachvfa ya aiauwaya hi oke; yumohmikma Islael im vlla vhleha hatak puta kvt iki vhleha $\underline{i}$ yakni aiyukali ho ai isha hi oke.

9. Micha i yakni vt apehlichi achvfa vttut apehlichi inla onvt mahaya he keyu hoke; amba Islael im vlla vhleha apehlichi aiyukali kut ilap í yakni ak inli ho ibafoyuka hi oke, achi tok.

10. Chihowah yut Moses a im apesa tuk mak $\underline{\underline{a}}$, Selofehat ushetik vhleha hut yumihchi tok.

11. Mahlah, Tisah micha Hoklah micha Milkah micha Noah, Selofehat ushetik vhleha hut iki itibapishi ushi vlleha ho auwaya tok okvt:

12. Micha Chosef ushi Manasseh ushi vhleha chuka achvfa vhleha ai auwaya tok, micha iki in chuka achvfa apehlichi ak $\underline{\text { i }}$ yakni vt ibafoyuka moma tok.

13. Nan vlhpisa, micha ai vlhpiesut im apesut yumihchi

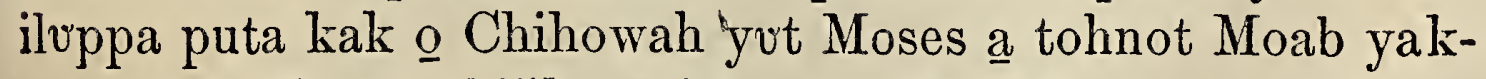
ni matali Chatan bilika, micha Cheliko bilika ya Islael im vlla vhleha ha ai im apesa tok oke. 

TUTEL ONOMI H OLISSO. 



\section{MOSES I HOLISSO ISHT TAHLAPI KUT}

\section{TUTELONOMI HOHCHIF 0 HOKE.}

\section{CHAPTA I.}

ANUPA iluppa puta kak o Moses vt Islael moma ka A Chatan ola in tunvp, yakni patali, yakni haiaka okhvta humma ichapaka ya, Palan micha Tofel, micha Laban, micha Haselolh, micha Tisahab iti takla ya ai im anumpohonli tok.

2. Holeb a vttut Sei nunih ha ont ia hosh Katesh-banea ona ka nitak auahchofa ka anowa yoke.

3. Atuk o yakohmi tok; afummi ont ishit pokoli ushta, hushi ont ishit auahachvfa, nitak vmmona ma, Moses vt Islael im vlla vhleha ha, Chihowah yut nianvlhpisa imma

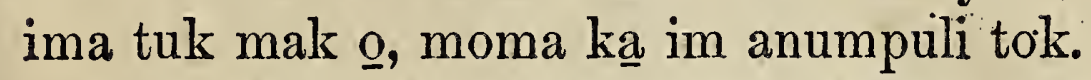

4. Amolait okla i miko Sihon Heshbon ai vtta tok, micha Bashan i miko Ok Etlei talaia Ashtalolh vtta yo vbi ha yvt:

5. Chatan ola in tunnup Moab yakni yạ, Moses vt nan vlhpisa iluppa ai im anumpulit ishtivt,

6. Pin Chitokaka Chihowah yot Holeb a a pim anumpalit, Nvnih iluppa hvsh ahanta tok a hopaki kvt vlhpesushke.

7. Hush fullota cha, itunowut ayvt, Amolait okla i nunih, micha bilika taloha moma, yakni patali, micha nunih foka, micha okfa, micha okamahli, micha okhuta lapalika aiena, Kananait okla i yakni, micha Lebanon aiena ka onvt okhina chito Eufalates okhina ya hush onashke.

8. Yakéh, yakni ya huchi tikba talali lishke; chukowvt yakni ya Chihowah yut huvchiki vhleha Eblaham, micha Aisaak, micha Chekob, micha im ishtiaka iakaiyut mihinti aiena ka anumpa kvllo anumpulit, ima tok a hush ishashke, achi tok oke, achi tok. 
9. Mihmut nitak yumma huchim anumpulit, Uno ak bano kvt hvchi laue la he keyushke, achi li tok.

10. Hvchin Chitokaka Chihowah yvt hvchi apaknvchi tuk oke; atuk o yakeh, hvchi apakna kvt himak nitak a vba fochik puta ka hush chohmishke.

11. Huchiki vhleha in Chitokaka Chihowah yot himak a hush kaniohmi ka laua kvt tahlepa sipokni hoh kia i shahli ha huch ikbashke; yumihcha he hvchi miha tok mak osh, huchi yukpalashke.

12. Katiohmi kak osh vno ak bano kvt nan isht hvsh atakluma, micha na hvchi weki, micha hush itachowa aiena ka shaiyali la hinla cho?

13. Hatak hopoyuksa, nan ithana hvchim apehlichi vt ithana hoka, hush ishi na, hvchi pehlicha he a ikbi lashke, achi li tok.

14. Mikma hvchishno ak osh afalaminchit, Nana ish anumpuli ka e yumihchikmut pim achukma hinlvshke, hus sum achi tok.

15. Yolimi ho huchim apehlichi puta ka $\underline{i}$ shahli vhleha hatak hopoyuksa, ithana vhleha ha ishi lish, hvchi noshkoboka puta ikbit tahlepa sipokni puta i kvpotuni, micha tahlepa puta i kupotuni, micha pokoli tahlapi puta i kupotuni, micha pokoli puta $\underline{i}$ kupotuni hvchim ikbi li tok: micha hvchim apehlichi puta ai itin takla ya nan vlhtoka puta ka ikbi li tok oke.

16. Micha nitak yưmma huvchi nan apesa vhleha ha atokolit $\mathrm{i}$ mihvt, Hvchitibapishi vhleha itin takla, nan iti kaniohmi ya hush im ahaponaklashke; micha hatak puta, micha itibapishi nan iti kaniohmi, micha okla inla takla assha tuk aiena ka ai vlhpiesa ho hush im apesashke.

17. Nan apesa kvt, hatak yohmi hoka, ish ahna he keyu hoke: amba chipunta kak kia hochito ka iti lauechit hvsh i haponaklashke; hatak nashuka ya huvchik i nukshopo kashke; nan im apesa yot Chitokaka immi hoka; atuk osh nana kvt hvchi kvllo fehnakmvt, isht hus svm ala na, vno ak osh haponaklo lashke, im achi li tok. 


\section{CHAPTA I. $^{\circ}$}

18. Micha nitak yumma nana moma hush yumohma chi ka hvchim apesa li tok.

19. Atuk o Holeb a ashvt il ia mvt, yakni haiaka chito, micha palummi, hina takla Amolait okla i nunih ha hush a pisa chash pin Chitokaka Chihowah yvt pim apesa tuk mak o mominchit e hlopulli tok; mihmvt Katesh-banea ya ela tok.

20. Mihma Amolait okla i nunih, yummak oka pin Chitokaka Chihowah yvt pima tuk mak o hushlvshke, hvchim achi li tok.

21. Yakeh, Chin Chitokaka Chihowah yot yakni yash chi tikba talalishke: hvchike vhleha in Chitokaka Chihowah yvt chim achi tuk mak o, ivt ishi: chi nukshopa na, micha chi chukvush vt ik ello kashke, chim achi li tok.

22. Mihma hush moma kut a bilika hushla tok; micha, Hatak ak o pi tikba e pilakma, yummak osh yakni ya pim ahopokoyot tahli cha, hina kanima ho il ia hinla, micha tumaha holihta kanima ho il ona chị ka, anonti anumpa ishit pim vlashke, hush achi tok.

23. Mihma nana hush achi ka vm vlhpesa tok; mihmvt hatak hush auahtuklo, apehlichi achufakma achvfut ia ho ishe li tok.

24. Mihma fullotut nưnih ha ia tok; atuk osh okfa Eshkol a ona cha, ahopokoyot tahli tok.

25. Micha yakni nana awaya ai ishit ibbak a ishit pim vla mot, anonti anumpa isht pim vlvt, Yakni achukma pin Chitokaka Chihowah yot pimushke, achi tok.

26. Yohmi hak inli kia hush ia he keyu tok; amba huchin Chitokaka Chihowah im anumpa ha hvsh kobvffi tok.

27. Mihmvt hvchi bina puta ashvt na mihvt anumpulit, Chihowah yưt pi nukkilli kak osh Echip yâkni ya pi kohchit pisht vla cha, Amolait ibbak a pi fohki na, vbit pi tahla chi ha tuk oke.

28. Katima hak o il ia chi cho? pitibapishi vhleha hvt, Okla hatak hochito, micha chaha kvt pi shahli hoke; tvmaha holihta vt hochito, micha aholihta kvt shutik a ona 
hoke; micha Anakim ushi vhleha ha yvmma il apisa tuk oke, achit pi chukvush a iellichishke, hush achi tok.

29. Yohmi ma, Hush i komunta na, micha yummak o hush i nukshopa na,

30. Hvchin Chitokaka Chihowah ak osh hvchi tikba ivt hush pisa ho, Echip a huchim a yumihchi tok moma ka chohmit hvchim itiba chi hoke.

31. Micha yakni häiaka hush atâya moma ka, hatak osh ushi shoyuli ka chohmit, hvchin Chitokaka Chihowah yut kaniohmit chi shoyuli ho pihisut iluppa hushla tuk oke.

32. Yohmi kia nana iluppa hvchin Chitokaka Chihowah ya hush i yimmi tok keyu hoke.

33. Yvmmak osh hina ya huchi tikba aya tok, micha binah hush ahilecha chi ka hoyot hvchim ahayuchi tok; ninak ma, hvsh atia chị ka hvchim anola chi kvt luak a tok; atuk osh nitak hoshonti ha tok.

34. Mihma Chihowah yut hvchim anumpa ola ka haklo cha, nukhobela mut, anumpa kvllo anumpulit,

35. Hatak iluppa puta aionchuloli haksi iluppa ai achvfa tuk vt achvfa kia iki vhleha ima la he, mihut anumpa kvllo anumpule li tok, yakni achukma iluppa pisa chi keyu pulla hoke.

36. Chefunneh ushi Kaleb ak illa chi hoke: yummut pisa hi oke: yummak osh yakni anowa tuk a ima la hi oke, micha yumma im vlla puta ka ima la hi oke: Chihowah ya aiokluhut im antia tuk oka, achi tok.

37. Micha Chihowah yot huchishno pulla kak o a nukowa chä, yumma ish chukowa he keyu, ak inli.

38. Amba Nun ushi Choshua chi tikba hikia kak osh yumma chukowa chi hoke; ish ai i yimituchashke, yummak osh Islael a abinilicha chi hoke, vm achi tok.

39. Mikma hvchin chipunta vhleha yummak osh wehpoa chi, hush achi tok vto, micha hvchim vlla yvmmvt nitak yummut nan achukma micha ik achukmo aiena ka ik ithano tok, yưmmak okvto yưmma chukowa chi hoke; yumma ima la chị hoke, mikma binila chị hoke. 
40. Amba huchishno vto fullotut yakni haiaka Okhvta humma ak o aiitunowvt hush iashke, achi tok.

41. Yohmi ma afalaminchit hus svm achi mvt, Chihowah ya il i yoshoba tuk oke; pin Chitokaka Chihowah yot nana moma pim achi tuk mak o il ia cha, il itibashke, his sum achi tok, Atuk osh hatak hush moma kut in tunvp na halupa aiyukali hush il apakshunnichi mvt, nunih bokko hvsh ia chi kvt hvchim vlhtaha tok.

42. Mihma Chihowah yvt vm achi mut, Hush ia ná, micha hưsh itibi ná, huchiba foka li keyu hoke; keyukmut hvchin tunup uhleha ha itikba hvchi akakoha na, ish im achashke, vm achi tok.

43. Yohmi ho huchim anumpule li tok; yohmi ma hvsh haponakla he keyu tok; amba Chihowah im anumpa ha $\underline{\mathbf{i}}$ filvmmit hush i sanali cha, ilapunlut nunih foka hushla tok.

44. Mihma Amolait whleha nunih foka y vmma asha tuk vt kohchvt hvchi sanali cha hvchi tihlelit foishki chohmi tok, micha Sei ya huchibit Homah ya hvchishtona tok.

45. Mihma hush falama cha, Chihowah itikba ya hush yahaya tok; yohmi kia Chihowah yvt hvchim anumpa ha hakla he keyu tok; micha hvchi haponakla he keyu tok.

46. Yohmi ho Katesh a hush aiasha na nitak laua kvt, yumma hush aiasha atok mak atok.

\section{$\because \quad$ CHAPTA II.}

VUMOHMI ho e fullota cha, Chihowah yvt vm anum1 puli tuk mak o ; yakni haiaka pilla, Okhvta humma aiitunowvt il ia tok: mihmut nitak laua nunih Sei ya il apakfoyupa tok.

2. Mihma Chibowah yut um anumpulit,

3. Nunih iluppa hush apakfoyupa tok a hopaki kut vlhpesushke : falummi pilla ho hush fullotashke.

4. Micha okla ha im apesut, Hush itibapishi vhleha Esau im vlla vhleha Sei aiasha ka im aivhli ont hush hlopulla chi 
hoke; mikma huchi nukshopa chị hoke; yumohmi hoka, ahah, hvsh il ai ahni fehnashke.

5. Micha hush in tanampi na, i yakni peh iyi putta ahabli achufa chohmi kia, huchima la chi keyu hoka; nứnih Sei ya Esau vt aiasha he a ima li hatuk oka.

6. Ilimpa ya twli holisso ya isht hush in chumpa hosh hush impa chi hoke; micha oka aiena ka tuli holisso ya isht hvsh in chumpa hosh hvsh ishka chi hoke, achi tok.

7. Huchin Chitokáka Chihowah yut chibbak na hush toksuli puta ka na hvchi yukpali tuk oka: yakni haiaka chito iluppa hlopullit hush itunohowa ka ithana hoke; afummi pokoli ushta iluppak ash chin Chitòkaka Chihowah yot chiba foyuka tuk oke: nana ik chim iksho kit iksho tuk oke, achi tok.

. 8. Atuk osh il itibapishi vhleha Esau im vlla vhleha, yvmmot Sei ya asha ka issut ant il ivt, Elalh micha Esion-kaba vttvt aiia, yakni patali hlopulli ya il ona mot, e fullota cha, Moab yakni haiaka hina ya ont il ia tok:

9. Mihma Chihowah yut vm achi mot, Moabait vhleha ha ish ilbvshali na, micha in tanampi hosh ish itibi na: chi yakni ya chị ho, $\underline{\text { I }}$ yakni a chima la he keyu hoka; Lot im vlla vhleha hut i yakni ya chi ho Ah ya ima li tuk oka.

10. Chashpo ma, yummak o Emim vihleha hosh okla chito, micha laua, micha chaha kvt Anakim ak $Q$ ehohmi hosh aiasha tok.

11. Yvmmak kia Anakim ak inli chohmit hatak chaha hohchifo tok; amba Moabait whleha hot Fmim bochifo tok.

12. Holim vhleha hoh kia chashpo ka Sei ya asha beka tok; yohmi tok kia Esau im vlla vhleha ak osh itikba ya ai ishit kanchi ha yut, iakaiya tok; micha Islael vt yakni aiasha Chihowah yut ima tuk a yumihinchi tuk a chohmichi cha vilhtobut aiasha tok.

13. Himak a hush wakaya cha, bok ushi Selet a hush hlopullashke, achi li tok; mihma bok ushi Selet a e hlopulli tok.

14. Mihma Katesh-banea ashot e minti tok, vttut bok ushi Selet a e hlopulli tok, ant vhli ka iti takla kvt, afưmmi pokoli tuchina akucha untuchina tok; polaka hatak 
tunup aya ai iti shali moma kut Chihowah yut anumpa kullo im anumpuli tuk mak $\underline{o}$, okla itin takla ya akohchvt ont taiyaha tok.

15. Chihowah yut okla chito ai iti takla ai ishit kahanchi na, ont moshola chị ka ibbak vt $\underline{\mathrm{i}}$ sanali ho muhli ha tuk oka.

16. Yohmi ho yakohmi tok, hatak tunvp aya moma kut okla ai iti takla ya kohchut ont mosholit illit taiyaha ma,

17. Chihowah yvt vm anumpulit,

18. Himak nitak a Moab im ai vhli Ah ya hush hlopulla chi hoke.

19. Atuk osh Ammon im vlla vhleha ha ichapa ka, bilika ish onakmut ish ilbushala he keyu, micha ish ataklumma he keyu, Ammon im vlla vhleha i yakni a binila he a chima la he keyu hoka; Lot im vlla vhleha i yakni ya chi ho ima li tuk okvt, achi tok.

20. Yummak kia hatak chaha i yakni hohchifo tok; nitak chashpo ka hatak chaha yvt yummak o aiasha tok. Mihma Ammonait vhleha hut Samsummim okla yoke, achi tok.

21. Okla chito, micha laua, micha chaha kvt Anakim ak o chohmi tok; yohmi tuk kia Chihowah yut itikba ya ai ishit kanchi tok; mihma iakaiya cha, ulhtobut aiasha tok.

22. Holim vhleha hash Esau im vlla vhleha hvt Sei aiasha tuk itikba ya ai ishit kanchi ma, yummak inli chohmichi tok; mikma okla iakaiya cha, vlhtobvt aiasha tuk vt himak nitak a ant ai vhli hoke.

23. Mihma Afim vhleha yưmmvt Haselim a aiashvt Assa ont vhli tuk a, Kaftolim yummvt Kafto ashvt kohcha tuk vt ai ishit kanchi cha, vlhtobut aiasha tok.

24. Hush wakaya cha nowvt ivt Anon okhina ya hush hlopullashke, yakeh, Amolait Sihon Heshbon i miko, micha i yakni ya chibbak fohki lishke; abinilit ish wakayashke, itibi yak $\underline{\text { ish }} \underline{\underline{1}}$ kullashke.

25. Himak nitak a oklushi puta shutik nutaka aiasha kvt, chi annowa ka haklo puta ka chi komunta, micha chi nuk- 
shopa onochit ishtia likma, chishno pulla kak o wunihinchi cha, kominhincha chi hoke, achi tok.

26. Mihma anumpa shali vhleha ha Katemolh yakni haiaka ya akohchit Heshbon i miko Sihon a anumpa iti nanaiya ya pit i pilvt.

27. Chi yakni ya ak hlopulli, is sum ahni, hina ak o aya la chị hoke, ishtimpakimma, micha vilhfobekimma pit fullota la he keyu hoke.

p. 28. Upa la chi ka tvli holisso vlhtoba ho ilhpak is svmashke; ishko la chi ho tuli holisso vlhtoba ho oka is sumashke, peh aka hikia hosh chi yakni ya hlopulli lashke.

29. (Esau im vlla vhleha, yummut Sei aiasha kvt, micha Moabait vhleha Ah aiasha kvt si yumihchi tuk oka;) yvmohmi na, Chatan a blopullit pin Chitokaka Chihowah yot yakni pima tok a ona lashke, achi li tok.

30. Yohmi tuk kia Heshbon $\underset{1}{\mathrm{i}}$ miko Sihon vt ant ik hlopulli, ik pim ahno tok; Chin Chitokaka Chilhowah yout imi shilombish a kvllochi tok, micha chukvsh a kullochi tok oka; yummut chibbak a fohka chi kak atok, himak nitak kia yumohmishke.

31. Mihma Chihowah yut, yakeh, Sihon micha i yakni ya chitikba a chimvt isht ia lishke, a binilit ish wakayashke; yohmi cha, i yakni ya ish ishashke, vm achi todk.

32. Yohmi ma Sihon micha im okla moma aiena kut pi sanalit, pitiba chi hosh kohchvt Chahas ak o vlatok.

33. Mihma pin Chitokaka Chihowah yut pi tikba ya pibbak a fohki tok; mihma yumma micha ushí uhleha, micha im okla moma aiena ka issot e kanchi tok.

34. Micha nitak yumma in tumaha holihta puta moma ka il ishi cha, tumaha holihta puta hatak, micha ohoyo, micha vlla aiena ka ishit kanchit il vmohmichi tok; achvfa kia kil apoancho tok.

35. Peh nan vlhpoa ak illa ho, na wehpoa il il aieshi tok; micha tumaha holihta il ishị ha tuk a na wehpoa aiena ak a tok.

36. Aloe yummut Anon okhina ont alaka, micha tvmaha holihta yummut okhina bilika a talaia ka vttut ia hosh Ki- 
leat ont vhli ka, tumaha holihta achvfa kia kvllo kvt pi shahli tok keyushke; pin Chitokaka Chihowah yot mominchit pibbak a fohki tok.

37. Peh Ammon im vlla vhleha i yakni, micha okhina Chabbak atalaia kanima yoh kia, micha nunih foka tumaha holihta puta kạ, micha nana ho pin Chitokaka Chihowah yut pim alummi tuk ma, chik ona tok.

\section{CHA.PTA III.}

YOHMI ma e fullota cha, Bashan hina ia kak o il ia tok 1 mihma Bashan i miko Ok yumma, micha im okla moma aiena kvt pi sanalit pitiba chị hosh, kohchvt Etlei ya vla tok.

2. Mihma Chihowah yut, Ish i nukshopa na, yumma micha im okla moma, micha î yakni ya chibbak fohki la chi hoke; mikma Amolait vhleha, yummvt Heshbon aiasha tuk $\underline{1}$ miko Sihon $a$ ish yumihchi tuk vt ish chohmicha chi hoke, vm achi tok.

3. Yohmi hosh pin Chitokaka Chihowah yut Bashan i miko Ok ak kia micha im okla moma ka pibbak fohki tok; atuk o issot e Kanchi na, i hlakofi kut iksho tok.

4. Micha nitak yomma in tumaha holihta moma ka il ishi tok; - tumaha holihta kil im esho tuk achvfa kia iksho tok, Bashan 1 miko Ok im apehlichika Akob aivhli fullota moma ka tumaha holihta pokoli hannali tok.

5. Tumaha iluppa puta kvt holihta chaha isht aholihta hosh holihta okhissa, micha im okhowatkvchi yokma, tvmaha ushi ik aholihto laua fehna tok.

6. Micha Heshbon $\underline{\mathbf{i}}$ miko Sihon a tok a chohmichit ishit kanchit, il umohmichi tok, tumaha holihta puta hatak, micha ohoyo, micha vlla vhleha aiena ka ishit kanchit il vmohmichi tok.

7. Amba nanulhpoa moma, micha tumaha holihta puta na welhpoa yo il il ai ishi tok.

8. Micha nitak yưmma Amolait okla i milko tuklo ka i 
yakni Chatan ola in tunnup Anon okhina vttut ia hosh nunih Hemon ont vhli ka ibbak a akohchit il im ishi tok;

9. Hemon yumma Sitonian vhleha hut Silion achikma, Amolait vhleha hot Sheni achi chatuk;

10. Yakni patali tumaha holihta moma, micha Kileat moma, micha Bashan moma, Bashan vtta Ok im apehlichika, in tumaha holihta Salkah micha Etlei ont vhli tuk a il ishi tok.

11. Hatak chaha vhleha asha hatuk a Bashan $\underline{i}$ miko $\mathrm{Ok}$ ak bano hosh okchayvt ahanta tok; yakeh, in topa hvt tuli topa hatok; Ammon im vlla vhleha aiasha Labbah hikia keyu cho? hatak 1 kubit vlhpisa hosh falaia kvt kubit chakkali tok, micha auahta kut kubit ushta tok.

12. Mihma yakni iluppa nitak yumma, Aloe yummut Anon okhina bilika talaia ka vttut ia hosh Kileat nunih iklunna vhli kvt in tumaha holihta puta aiena il ishi tuk a Lubenait vhleha, micha Katait vhleha aiena kak o ima li tok.

13. Mikma Kileat atampa ka, micha Bashan moma, yummot Ok im apehlichika ya Manasseh ishtatiaka iklunna ya ima li tok; Akob aivhli moma, Bashan aiena moma, yommvt hatak chaha yakni hohchifo ha tok.

14. Manasseh ushi Chai vt Akob yakni moma Cheshuli micha Maakahli ont vhli ka ishi cha, ilap hohchifo ya ahochifochit Bashan-hafolh-Chai, achi tok, himak nitak a moma hoke.

15. Mihma Kileat a Maki ak o ima li tok.

16. Lubenait vhleha micha .Katait vhleha ha, Kileat a vttut ia hosh Anon okhina ont vhli okfa iklunna, micha aivhli Chabhak okhina yummut Ammon im vlla vhleha im aivbli ya ont vhli;

17. Micha yakni patali, micha Chatan, micha im aivhli Chinnelelh a vttut ia hosh yakni patali okhvta, hupi okhvta, Ashtolh-piskah nunih nuta hushi akochaka imma ont vhli ka ima li tok.

18. Micha nitak yumma hvchim anumpohonli li mut, hvchin Chitokaka Chihowah yut yakni iluppa hush binila chi ka 
huchim vlhtaha hosh, hvchitibapishi vhleha Islael im vlla vhleha ha itikba hush hlopulla chi hoke.

19. Amba hvchi tekchi vhleha, micha hvchim vlla vhleha, micha hvechi nanvlhpoa (nanvlhpoa hvehin laua ka ithana li hoka) aiena kvt hvchin tumaha holihta puta hvchima li tuk a asha na,

20. Chihowah yut huchishno a hvehi yumihchi tuk vt chohmichit, hvehitibapishi vhleha ha fohvchashke: micha yummak kia yakni ya huchin Chitokaka Chihowah yvut Chatan mish-tunnup a ai ima tuk a abinilashke: yohmikma huchima li tuk hvehi yakni a hatak aiyukali kvt falamut hush onashke, hvchim achi li tok.

21. Micha nitak yummak inli ka Choshua ya im anumpulit, Chin Chitokaka Chihowah yut miko tuklo iluppa kaniohmichi tuk moma ka, chi nishkin vt pisushke; apehlichika puta kanima yó ish hlopullikma Chihowah yut yumihcha hi oke.

22. Hush i nukshopa he keyu: Chin Chitokaka Chihowah yummak osh chim itiba chi hoke, chim achi li tok.

23. Micha nitak yumma Chihowah ya im asilhhut,

24. Chitokaka Chihowah ma, a chi chito, micha chibbak akullo ka chin tishu ha im otuninchit ish wakaya tuk oke: Chitokaka nanta hak osh vba ahantakmot, keyukmvt yakni ahanta kut a chi kullo ka yvmohmit, ish a toksvli ya yumihcha hinla hotuma?

25. Chim asilhha lishke, ak hlopulli cha, yakni achukma yummut Chatan mish tunnup talaia ka, nunih aiokli, micha Lebanon yưmma ak pesá, achi li tuk.

26. Yohmi kia Chihowah yot hichishno ak a tuk pulla mak o, a nokowa cha, a haponakla he keyu tok: micha Chihowah yut, Ik chim vlhpesá, himakma nana iluppa isht chik sum anumpulo kashke.

27. Oiyut, Piskah paknaka ya ish onashke, micha hushi aiokatula pilla, micha falummi pilla, micha oka mahli pilla, micha hushi akochaka pilla pit ish hopokoyashke, micha chi nishkin a isht ish pisashke: amba Chatan iloppa ish hlopulla chị keyu hokvt. 
28. Yohmi kia Choshua ya atokolit im anumpulit, aiyimitvchit, ish hlampkochashke; yvmmak osh okla iluppa itikba tanubli cha, yakni ish pisa chị ka a binilicha chị hoke, vm achi tok.

29. Yohmi ho Belhpeo ichapaka okfa hak o il aiasha tok.

\section{CHAPTA IV.}

Y UMOHMI hoka Islael ma, himak a nan vlhpisa, micha 1 aivlhpiesa yumihinchi hvchim abvchi li ka hvsh yumihcha chi kvt, hush haklashke; yumohmi hosh hvchi okchaya cha, hvchiki vhleha in Chitokaka Chihowah yvt yakni chima tok a chukowvt hush a binilashke.

2. Anumpa hvchim apesa li tuk a hvsh ibafohka he keyu, micha hush ola taklvcha he keyu; yumohmi hosh hvchin Chitokaka Chihowah $\underline{i}$ nan vlhpisa puta hvchim apesa li tuk a hush holitoblashke.

3. Baal-peo pulla ho Chihowah yut kaniohmichi tuk a huchi nishkin vt pisa tuk oke; chin Chitokaka Chihowah yut hatak Baal-peo iakaiya tuk moma ka hvch itin takla ya ai ishit kanchi tuk oka.

4. Amba huchin Chitokaka Chihowah ya hush asitia tok vto hvsh moyuma kut himak nitak a hvchi okchayushke.

5. Yakeh, yakni yvmma hush ia kvt, hvsh binilikmvt, hush yumihcha chi ho, an Chitokaka Chihowah yvt vm apesa tuk mak o, chohmit, nan vlhpisa, micha aivlhpiesa yumihchi puta ka huchim abvehi li tuk oke.

6. Yumohmi hoka hush holitobli cha, hvsh yumihchashke; iluppak osh oklushi puta nan vlhpisa iluppa hahaklokmvt, Oklushi chito iluppet okla hopoyuksa, micha nan ithana a hli yole, chi acha he, itikba ya hush apoyuksa, micha na hush ithaiyana hokvt.

7. Oklushi nanta hak osh holitopa kvt yakohmi hotuma? yumma in Chitokaka yut pin Chitokaka Chihowah ya nana moma ka il im asilhha chatuk $\underline{a} \underline{i}$ bilinchi hvt chohmi hotuma? 
8. Micha oklushi nanta hak osh holitopa kvt yakohmi cha, nan vlhpisa, micha aivlhpiesa yvmihchi aiena kvt vlhpesa fehna, nan vlhpisa iluppa moma himak nitak a chi tikba hilechi li ka chohmi kut in tonla hotuma?

9. Peh ahah, hush il ahni cha, hvchimi shilombish a achunanchit hush ieshashke, keyukmut, nana puta hvchi nishkin vt pisa tuk vt huvchim ahaksi ná, micha chi okchaya nitak moma ka huchi chukvush a akohchut kunia na: amba chiso puta, micha chiso ha ushi puta ka ish im abvchashke.

10. Micha Holeb a hvchin Chitokaka Chihowah itikba ya hush hieli tok nitak fehna ka hush ithanashke; Chihowah yut um achi mut, Okla ha is sum ai itunahlashke, mikma vm anumpa ha haklochi lashke, yohmi ho nitak moma okchayvt yakni paknaka aiasha takla kut, a nukwia he osh ithana cha, im vlla vhleha ha im abvchashke, achi tok.

11. Mihma bilika onvt, nunih nutaka ya hưsh hieli tok; mihma nunih hut luak ishit lua hosh shutik iklunna ona ma, okhlili, hoshonti, micha okhlili sukko aiena tok.

12. Milıma Chihowah yvt luak iklunna et a hvchim anumpuli tok; anumpa ola hokvno hush haklo tok, yohmi kia na holba ka huchik peso tok; peh ola kak illa ho hush haklo tok.

13. Micha i nan itim apesa hush yumihcha chị ho hvchim apesa tok, nan vlhpisa pokoli ka hvchim anoli tuk oke; michå tuli patussa tuklo ho ai o holissochi tok.

14. Mihma nitak yumma nan vlhpisa, micha aivlhpesa yumihinchi ya yakni yumma onvt hush a binilikmvt hush yumihcha he yo hvchim abvuchi la chị kạ, Chihowah yut vm apesa tok.

15. Yvmohmi hoka, ahah, hvsh ibai ahni fehnashke; nitak yumma Chihowah yut Holeb a luak iklunna ho et huchim ai anumpuli ma, na holba nana kia hush pisa tok keyu hokvt;

16. Keyukmut hush il okpuni cha, tuhlut na holbut toba ikbit na pisa kaniohmi, nakni, keyukmut, tek nana kia holba; 
17. Nampoa yakni paknaka aiasha holba, hushi sanachi asha helit vba itanowa holba,

18. Nana kia yakni pakna om balahanli holba, nuni nana kia yakni nutaka oka ai itanowa holba ya hvchik bo kashke;

19. Micha keyukmvt, hvchi nishkin a shutik pilla aka chakalichi cha, hushi micha hvshi ninak aya, micha fochik puta, vba nana moma fehna ka hvsh pisakmvt, yummak oka chin Chitokaka Chihowah yvt oklushi puta vba moma nuta ka im ita kashubli tok a, yoshoba cha aiokpvchit hush im antia na.

20. Amba Chihowah yvt huvchishno ya hvch ishi cha, tvli abila Echip fehna ka hvchi kohchi kvt, okla immi ha chi ha

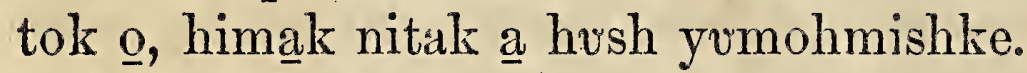

21. Micha Chihowah yut hvchishno ak a tuk pulla mak $\underline{o}$, a nukhobela cha, anumpa kvllo anumpulit, Chatan a ak hlopullo ka he, micha yakni achukma yumma hvchi yakni ya chị ho chin Chitokaka Chihowah yut huchima tok a ak chukowo ka chi miha tok,

22. Amba yakni ilvppak o a sulla chi hoke, Chatan a hlopulli la he keyu hoke; amba hvchishno vto hush hlopulli cha, yakni achukma yumma hush iesha chi hoke.

23. Ahah, hosh il ahnashke; keyukmot nan itim apesa yumma huchin Chitokaka Chihowah yut huch itim apesa tok a hvchim a haksi cha, tuhlut na holbut toba, keyukmvt nana kia holba yumma chin Chitokaka Chihowah yut hvchim olvbi tok a hush ikbi ná,

24. Chin Chitokaka Chihowah yvt luak na hushmit tahli, Chitokaka nuktahahila yoka.

25. Hvchim vlla vhleha hvt, micha vlla vhleha hash im vlla vhleha hot hvchim asha cha, yakni ya hush asha hopakit taiyahakmvt, hush il okpunit tvhlut na holbut toba, keyukmot nana kia holba hush ikbi cha, chin Chitokaka Chihowah itikba ya nana ik achukmo ka yumohmit hush nokowvchikmut;

26. Yakni ya hush isha chị hosh Chatan a hvsh hlopulla chi kvt, chekusi a chukillissut, hush vmohma he ya ; himak nitak a vba yakni, micha yakni aiena kvt hvchi atokola chị 
ho pit hoyo lishke; nitak falaiachit, hush o aiasha he keyu, amba mosholit, hush vmohma hi oke.

27. Mikma Chihowah yvt oklushi puta ka a hvchi ita fimma hi oke : mikma Chihowah ik ithano okla ai iti takla yumma Chihowah yut huch isht aya ka holhtina kvt ik lauo hosh hush aiasha hi oke.

28. Mikmot yumma na holba puta hatak ibbak osh toksuli tuk, iti, micha tvli, na pisa he keyu, micha hakla he keyu, micha impa he keyu. micha huwa he keyu aiena yak o hush im antia hi oke.

29. Yohmi tuk kia yumma ashut chin Chihowah Chitokaka ya ish hoyokmvt, chi chukvvsh a mominchit, micha chi shilombish a mominchit hvsh hoyokmvt, hush ahayucha hi oke.

30. Chilbushakmut nitak ishtaiopi fehna ka nana iluppa puta kut hvchi onvtolakmvt, falamvt chin Chitokaka Chihowah ya ish im ona cha, im anumpa ha ish im antiakma:

31. (Chin Chitokaka Chihowah yut nan i nukhaklo Chitokaka yokvt) chi kancha he keyu hoke, micha ishit chi vmohmicha he keyu hoke, micha hvchịki vhleha ha anumpa kvllo im il onochit nan itim apesa tuk ut im a haksa he keyu hoke.

32. Himak a nitak ant ia tok, yummut chi tikba ya tuk, Chitokaka yut hatak ikbit yakni paknaka ya o hilechi tok, nitak vhlit mihinti ka ish a ponaklashke; micha shutik in tunnup achufa vttut ia hosh in tunnup ont vhli ka ish aponaklashke, nana chito iluppa chiyuhmi kut aiasha tok oh cho? keyukmot nana iluppa chiyuhmi ka haklo tok oh cho?

33. Okla hut himonna kia hush haklo tuk a chohmit Chitokaka yot luak iklunna ho et im anumpohonli na, haklo mot okchaya tuk oh cho?

34. Ak cho? Chitokaka yvt oklushi achufa ho oklushi inla iklunna a kohchit il isha chịkmot im onvt imomaka pisut, nan isht atokowa, micha na fehna, micha tunvp, micha ibbak kvllo hosh, micha shakba ashataput nan isht nukhlakancha chinto, huchin Chitokaka Chihowah yvt hvchi 
nishkin itikba Echip a hvchi yumihchi tok, chiyuhmichi tok oh cho?

35. Hvchishno vto Chihowah ak osh Chitokaka yoke, hvsh ithaiyana chị ho hvchi haiakvchi tok; yvmma inla kvt iksho hoke.

36. Micha hush ithaiyana chị hosh im anumpa ha vba pit a chi haklochi tuk oke: micha yakni paknaka in luak chito chi pesuchi tuk oke; mihma im anumpa luak iklunna minti ka ish haklo tuk oke.

37. Micha hvchiki vhleha ha holitobli ha tuk osh yumma iakaiya im isht atiaka atokoli tok: micha isht im aiahhli kvllo hosh itikba ya Echip a a kohchit chisht aya tok;

38. Oklushi hochito micha kvllo kvt hvchị shahli ho $\underline{\mathrm{i}}$ yakni vt huchimmi ha chi ho hvch isht chukowvchi mut, hvchi tikba tihlelit a kohchi tok a himak nitak a yumohmi hoke.

39. Yumohmi hoka, himak nitak a ish ithaiyanashke, micha chi chukvsh a isht ish anukfillashke; Chihowah ak osh vba yakni, micha nutaka yakni aiena ka yummak osh Chitokaka yoke, inla kut iksho hoke.

40. Yvmohmi hoka i nanvlhpisa bilia, micha im anumpa puta himak nitak a chim anumpohonli li puta ka ish ieshashke: yưmohmi ho chishno micha chim vlla vhleha et chi akaiya aiena kvt chim achukmakvt mahayashke; micha chin Chitokaka Chihowah yvt yakni chima tok a ish aiasha kvt nitak ish falaiachi na, biliashke, achi tok.

41. Yvmohmi ma, Moses vt Chatan ola in tunnup hushi akochaka pilla ha tumaha holihta tuchina ho iti filvmmichi tok;

42. Yummak o hatak bika vbi yưmmut ik nuktahlo hosh chashpo ka i nukkilli chatuk keyu hosh in chuka a bilika hatak o vbikmvt, yưmma malelit ona chi ha tok ; michạ maIelit tumaha holihta puta iluppa achufa ka onakmut okchaya he a tok:

43. Hobchifo kut Bese, yakni haiaka talaia, Lubenait vhleha i yakni patali a talaia; micha Katait vhleha i yakni 
Kiliat talaia Lamolh; micha Manassait vhleha i yakni Bashan talaia, Kolan aiena kak atok.

44. Micha nan vlhpisa iluppak o Moses vt Islael im vlla vhleha ha itikba takalichi tok:

45. Micha nan isht atokowa, micha nan vlhpisa bilia, micha anumpa iluppa puta aiena kak o Moses vt Islael im vlla vhlehá hơt Echip yakni ya kohcha ha ya im anumpuli tok.

46. Moses micha Islael im vlla vhleha aiena kut Echip yakni ya kohcha ha yvt issot kanchi tok, Amolait i miko Sihon, yvmmot Heshbon a aivtta tok, i yakni Belh-peo ichapaka bachaya okfa Chatan ola in tunnup ak a tok:

47. Micha i yakni micha Bashan i miko Ok $\underline{\text { i }}$ yakni aiena a binili tok, Amolait i miko tuklo, yummut Chatan ola in tunnup hushi akochaka pilla ho aiasha tok.

48. Aloe yummut Anon okhina ontalaka bilika talaia ka vttut ia hosh Sion nunih yummot Hemon a ont vhli,

49. Micha Chatan ola in tunnup hushi akochaka pilla yakni patali moma, okhvta yakni patali Piskah kvli puta nutaka ont vhli tok a abinili tok.

\section{CHAPTA V.}

IHMA Moses vt Islael moma ka hoyo cha, Islael ma, 11 nan vlhpisa bilia puta, micha aivlhpiesa yumihinchi puta hush hạklo ho, himak nitak a anumpohonli li ka hush haklashke; yvmohmi hosh hush ithana cha, ieshit hush yvmihinchashke.

2. Pin Chitokaka Chihowah yut pishno ak o Holeb a nan a pitim apihisa tok oke.

3. Chihowah yut piki vhleha iluppa nan itim apihisa tok keyu; amba pishno ya pishno fehna kak a tok $\underline{\underline{a}}$, himak nitak a pi okchaya hosh momut iluppa il aiashushke.

4. Chihowah yot nashuka iti sanali hosh nunih ha luak iklunna et hvchim ai anumpohonlit,

5. (Luak pulla kak o hvchi nukshopa cha, nunih ha hush oiya he keyu ma; vno ak osh nitak yumma Chihowah mi31 
cha, hvchishno ya hvch itin takla hikivt Chihowah im anumpa ha huchim anohonli li tok oke,)

6. Uno ak osh chin Chitokaka Chihowah sia hosh, Echip yakni, micha aboha a yuka chi pehlichit a kohchi li tok oke.

7. Sa tikba ya Chitokaka inla yut ik chim iksho kashke.

8. In chuwwt na holbvt toba, micha nana kia, vba yakni asha, keyukmvt nutaka yakni asha, keyukmut yakni nutaka oka aiasha yumma holba kia chik ik bo kashke.

9. Aka pit ish im akachunola he keyu, micha ish im antia he keyu: Chitokaka chin Chihowah sia hvt, Chitokaka nuktahahla sia hosh, iki vhleha nan ashvchi yash im vlla vhleha hot ai iti shali ont isht atuchina, micha ont isht ai ushta kia a nukkilli ka im aya li hoke.

10. Micha tahlepa sipokni hoh kia sa holitobli cha, vm anumpa ha vm antihiya ya $\underline{i}$ nukhâklo li hoke.

11. Yvmmakfokalechit chin Chitokaka Chihowah hohchifo ka ish hochifa he keyu hoke; hohchifo ha yummakfokalechit hochifo tukma nan ik ashacho, Chihowah yut ahna he keyu hoka.

12. Chitokaka chin Chihowah chim achi tuk mak o, nitak hullo nitak a holitoblit. ish hullochashke.

13. Nitak hannali ho ish toksuli cha, chi na pilesa moma ka ish yumihchashke.

14. Amba nitak isht untuklo hokvto Chitokaka chin Chihowah i nitak 'hullo yoke: yummak okvno chishno, micha chiso, micha chiso tek, micha hatak chin tishu, micha ohoyo chin tishu, micha chi wak toksuli, micha chim issnba nashoba, micha chi nan vlhpoa nana kia, micha hatak ik ithano yosh chi holihta okhissa chi hikia kia na pilesa nana kia yvmihcha he keyu; yumohmi ho hatak chin tishu, micha ohoyo chin tishu aiena kut chishno ak inli chohmit fohashke.

15. Micha chishno yak kia Echip yakni ya tishu chia ha tok, atuk o. Chitokaka chin Chihowah yut ibbak kullo, micha shakba ashatapa hosh yumma chi kohchi tok a ish ithaiyanashke: yumohmi hatuk osh Chitokaka chin Chihowah yut nitak hullo nitak a ish holitobla he a chim apesa tok oke.

16. Chitokaka chin Chihowah yut chim achi tuk mak $\underline{\underline{a}}$, 
chịki, micha chishki ya ish holitoblashke; yumohmi ho yakni Chitokaka chin Chihowah yvt chima tok a ish aiahanta he a nitak vt chin lauashke, micha chim ai achukmakashke.

17. Kuna kia ish bi ná,

18. Micha kuna kia ish itin lumạka ná,

19. Micha ish hukkopa ná,

20. Micha chin chuka a bilika hatak ahanta ka anumpa ahli keyu ka isht chik atokolo kashke.

21. Micha chin chuka a bilika hatak tekchi ya ish ahni na, micha chin chuka a bilika hatak in chuka, im osapa, keyukmvt in tishu hatak, keyukmvt in tishu ohoyo, $\underline{\mathbf{i}}$ wak toksuli, im issuba nashoba, keyukma nana kia chin chuka a bilika hatak immi hokma, huchik im anushkunno kashke, ahanchi tok.

22. Anumpa iluppa puta kak o Chihowah yut chim okla moma ka nunih ha, luak, micha hoshonti, micha okhlili sukko ka ai iklunna chitoli hosh et ai im anumpohonli tok; mihmvt himakma ik achakalo tok, Mihmut tvli patussa tuklo ho ai oholissochi cha, subbak fohki tok.

23. Atuk o yakohmi tok ai okhlilika iklunna ya et ai anumpa na ish haklo mvt, (nunih hvt luak ishit lua tuk oka,) yohmi ma hvchim apehlichika noshkoboka vhleha, micha hvchim asunonchi vhleha aiena kvt a bilika hushla tok.

24. Micha, Yakeh, pin Chitokaka Chihowah yut im aholitopa, micha nan isht im aiahli ataha iksho pi pesvchi hoke; mikma luak iklunna ya im anumpa ha il a hahakklo tuk oke; Chitokaka yut hatak a im anumpuli kia okchaya hinla ka himak nitak a e pisushke.

25. Yvmohmi hoka nanta katiohmi ho himak a pilla hinla cho? luak chito ilupput hukmit pi tahla chi hoke. Himak ma Chitokaka pin Chihowah im anumpa ha e haklokmvt pilla chị hoke.

26. Hatak nipi moma ka kuta hosh Chihowah okchaya yosh luak iklunna et ai anụmpohonli na haklo pishno chohmi mut okchaya chatuk atuma?

27. Chishno ak osh bilika ish ona cha, Chitokaka pin 
Chihowah yut nana achi moma ka ish haklashke; micha Chitokaka pin Chihowah yot nana chim achi moma ka ish pim anumpulashke; mikma e haklo cha, e yumihchashke, hus sum achi tok.

28. Mihma Chihowah yot chim anumpa hos sum anumpuli ka haklo tok; micha Chihowah yvt, Okla iluppot anumpulit chim anumpuli ha haklo lishke : nana moma achi kvt vlhpeśa bano ho achishke.

29. Chukvsh vt yakohmi nukfoyuka tuk okbano, yohmi hosh a nukwia cha, vm anumpa vlhpisa moma ka ieshi na, bilia cha, yumma mihcha im vlla vhleha aiena kvt im ai achukma na, biliakbano ahnit vm achi tok.

30. Ia cha, anonti huchị bina im aiyukali hush onashke, ont ish im achashke,

31. Amba chishno vto a bilika iluppa ish hikiakma, anumpa moma micha nan vlhpisa bilia, aivlhpiesa yumihinchi puta ka chim anumpohonli likma, yummak oka ish im abvchikma aiasha chị ka, yakni ima li tok a aiashvt yumihinchashke, im achi tok.

32. Yvmohmi hoka, Chitokaka hvchin Chihowah yut hvchim apesa tuk mak o hvsh ithaiyana cha, hush yvmininchashke; fullotut naksika isht inppakimma, micha vlhfubekimma pit hush fullota he keyu hoke.

3.3. I hina puta Chitokaka hvchin Chihowah yot huchim apihisa tuk mak o hush ai itanowa cha, hvchi olkchayashke, micha hvchim ai achukmashke, micha yakni hvsh aiasha chị ka, hush aiasha kvt. nitak vt hvchị falaiashke, achi tok.

\section{CHAPTA VI.}

OHMI ka anumpa puta, micha nan vlhpisa bilia puta, 1 micha ai vlhpiesa yumihinchi ilvppa puta huchim vlbvcha he, Chitokaka hvchin Chihowah yvt anumpohonli na, yakni hush aiasha chi hosh hush ia kvt hush a yuminincha chi kak a tok oke.

2. Chitokaka hvchin Chihowah ya ish i nukwia cha, $\mathbf{i}$ 
nan vlhpisa bilia moma ka, micha im anumpa chim anumpohonli li kă, chishno micha chiso, micha chiso ha iso aiena kvt chi okchayvt ish ahanta nitak moma kvt ish ieshi cha, chi nitak vt falaiashke.

3. Yvmohmi hoka Islael ma, ish haiyaklashke, micha ithaiyanvt, ish yumihchashke; yumohmi hosh chiki vhleha ha in Chitokaka Chihowah yvt achit, im issa tok mak o, yakni pishukchi micha foebila ayanahanli ya aiasha apaknvt isht $\underline{\underline{i}}$ shahli fehnut hush mahayashke.

4. Islael ma, hush haklashke, Chitokaka pin Chihowah yvt Chihowah achufa hoke.

5. Yohmi ka Chitokaka chin Chihowah ya chi chukvush a mominchit, micha chi shilombish ma mominchit, micha chi kullo ya mominchit ishit ish holitoblashke,

6. Mikma anumpa iluppa himak nitak a chim anumpohonli li kvt, chi chukvush a foyukashke.

7. Yohmi ka chim vlla vhleha ha achunanchit ish im abvchashke; micha chin chuka ish binilikmot, micha hina takla ish ayakmvt, micha ish itoyulakmvt, micha ish wakayakmvt isht ish anumpohonlashke.

8. Micha nan isht atokowa yo chibbak a isht ish a tak chashke, micha bita hosh chi nishkin itin takla takali ka chin chiyuhmashke.

9. Micha chin chuka tonilk puta ka micha chị holihta okhissa puta ka ish ai o holissochashke.

10. Atukma yakohma hi oke, Chitokaka chin Chihowah yut yakni ya chiki vhleha Eblaham, micha Aisak, micha Chekob aiena ka anumpa il onochit im issa tuk vt chisht onakmvt, tvmaha holihta ho chito, micha hochukma aiokli ish ikbi tok keyu yo,

11. Micha ish alotoli tuk keyu aboha puta nana achukma moma alota, micha ish kulli tuk keyu ho kuli hofobi kula, micha ish hokchi tuk keyu ho, palkupi ahollokchi, micha alif iti puta aiena hvchima na aivpvt chikaiyakmot;

12. Yvmohmikmut ahah, ish ahnashke, keyukmut Chihowah ak osh Echip yakni, micha aboha a yuka pehlichit chi kuchi tuk a chim ahaksa hinla hoke. 
13. Chin Chitokaka Chihowah ya ish i nukwia cha, ish im antiashke, micha yumma hochifot anumpa kullo il onochit ish anumpulashke.

14. Naholbut toba puta, okla hvchi apakfoyupa aiasha ka i naholbut toba puta ka iakaiyut hush ia he keyu hoke.

15. Chin Chitokaka Chihowah yvt Chitokaka nuktahla yosh hvchi takla ahanta hoka; keyukmot, chin Chitokaká Chihowah im ai inukkilli vt chi sanalit libbi na, yakni pakna ya ai okpuloka hvechi taha yoba ná.

16. Massah ya ai imoma ka hush pisa tuk a chohmit, huchin Chitokaka Chihowah ya imomaka chik peso kashke.

17. Hvchin Chitokaka Chihowah im anumpa vlhpisa puta, micha $\underline{i}$ nan isht atokoli puta micha $\underline{i}$ nan vlhpisa bilia aiena chim anumpuli tuk a achunanchi hosh hush ieshashke.

18. Micha Chihowah itikba ya nana ai vlhpiesa micha achukma kak o ish yumihchashke; yohmi hosh hvchim achukmakashke, micha yohmi hosh Chihowah yut chiki vhleha ha anumpa kvllo ilonochit im issa tok yakni achukma ya chukowvt ish abinilashke,

19. Chihowah yvt anumpuli tok mak osh, chin tunvp vhleha moma ka chi tikba akohchit kanchashke.

20. Atuk o nitak inla vla ha ya , chiso hot chim ponaklot, iban isht atokowa micha nan vlhpisa bilia, micha ai vlhpiesa yumihinchi iluppa puta aiena pin Chitokaka Chihowah yut pim apesa tok, nanta ho miha hoh cho? achikma,

21. Yumohmikma, chiso hash, Echip yakni a Falaoh 1 yuka hosh il aiasha tok; atuk o Chihowah yut ibbak kullo hosh Echip a apehlichit pi kohchi tok.

22. Micha Chihowah yot nan isht atokowa, micha na fehna chinto micha palvmmi ho, Echip, micha Falaoh, micha in chuka achufa moma aiena ka e pisa ho onochit otvnihinchi tok.

23. Micha piki vhleha ha anumpa kvllo im ilonohonchi tuk mak osh, pisht vla cha, yakni ya pima chi hosh yumma pi pehliechit pi kohchi tok.

24. Micha Chihowah yot pim apesvt, nan vlhpisa bilia iluppa moma e yumihcha he ishit pim *aiachukmaka na bilia 
chị kvt, pin Chitokaka Chihowah ya il i nukshoyupa he a, achi tok; yohmi hosh pi okchalincha he a tok o himak nitak a ayvmohmi hoke.

25. Mikma iluppa puta moma ka pi miha tok mak o, ithanvt e yumihinchikmvt, pim ai vlhpiesaka ya chi hatok oke, ash im achashke.

\section{CH A P T A VII.}

CHIN Chitokaka Chihowah yut ish a binila chị hosh ish ia ka yakni ya chishtona cha, oklushi laua, Hittait, micha Kikashait, micha Amolait micha Kananait, micha Pelisait, micha Hifait, micha Chebusait, oklushi untuklo hochito, micha kullo kut, chishno ya chị shahli ho chi tikba ya akohchit kanchikmut;

2. Micha chin Chitokaka Chihowah yut chi tikba ya chibhak fohkikma, issot ish kanchi, mosholichit ish kanchit ish vmohmichashke, nan ish itim apesa he keyu, micha ish $\underline{\mathrm{i}}$ nukhâkla he keyu hoke;

3. Micha ish itauwaya he keyu; chiso tek a ushi ya ish i bohla he keyu, micha ushetik a im ai ishit chiso ha ish ima he keyu hoke.

4. Chiso hut si akaiya ya tuk a fullolichi na, na holbut toba puta inla yak o ai okpveha chi hoke; yohmi ho Chihowah im ai i nukkilli vt chi sanalit libbi cha, yakosi itin takla kia ishit chi kancha hi oke.

5. Amba yakohmichi hosh hush yumihcha hi oke: im alta puta hoka okpunit hush tahli cha, im in chuwwt naholbut toba puta ka chanlit hush kinahlichashke, micha im iti lokoli puta hoka chanlit hvsh kinahlichi cha, im i chuwot na holbut toba puta ka luak ishit hukmit hush tahlashke.

6. Chin Chitokaka Chihowah ya im okla holitopa chia hoka; chin Chitokaka Chihowah yut okla moma yakni paknaka aiasha ka $\underline{\underline{i}}$ shahlit immi atokowa chia ha chi ho atokolit chishi tok.

7. Laua kut okla inla ya hush i shahli kak o, Chihowah 
yut hvchihullot hvchi atokoli tok keyu; okla laua kvt okla moma ka hvchik lauwo ke tuk oka.

8. Amba Chihowah yot huchi hullo kak a tok, micha hvchiki vhleha anumpa kvllo im ilonochi tok vt ahlicha he ahni pulla kak osh, ibbak kwllo hosh pehlichit hivehi kohchi tok oke; micha hatak yuka hosh aboha hush ailasha tuk a Echip i miko Falaoh ibbak a kohchit huchi okchalinchi trik oke.

9. Yvmohmi hoka ish ithaiyana, Chihowah chin Chitokaka ak osh Chitokaka, micha Chitokaka aiahlichi vt holitobli cha, im anumpa ha ieshikma, nan itim apesa, micha nan isht i nukhâklo ya ai iti shali tahlepa sipokni hoh kia im iesha hi oke.

10. Micha 1 nukkilli puta ka pisanchi hosh im atobbi cha, ishit kancha hi oke: $\underline{\mathbf{i}}$ nukkilli tuk $\underline{\mathrm{a}} \underline{\underline{\mathrm{i}}}$ svlaha he keyu, pisanchi hosh im atobba hi oke.

11. Yvmohmi holia anumpa vlhpisa puta, nan vlhpisa bilia, ai vihpiesa yumihinchi, himak nitak a chi milia li ka ish ieshi cha, ish yumihinchashlie.

12. Yvmohmi kak o yakohma hi oke, aivlhpiesa yumihinchi ilvppa puta ka haklot hush ieshi cha, hush yumihinchikma, chin Chitokaka Chihowah yot chiki vhleha anumpa kullo im ilonochi tok vt nan itim apesa, micha.rsht i nukhaklo aiena ka hvchim iesha hi oke.

13. Micha chi holitobla hi oke, micha chi yukpala hi oke; micha chi apaknucha hi oke; micha chim oshoto awaya, micha chi yakni awaya, chim onush, micha chini oka paki, micha chị bila, chị wak awaya, micha chi nanvlhpoa chipunta - puta aiena kạ, yakni yo chima he yash chịki vhleha ha anumpa kvllo im ilonochi tok vt a na chi yukpa lashke.

14. Na yukpa kvt okla moma ka ish $\underline{\mathrm{i}}$ shahla hi oke : a huchiti takla ka nakni yokmá, tek okmá, nana kia, micha huchi nan vlhpoa ai iti takla ka ushi iksho vt iksho ka hi oke.

15. Mikma Chihowah yut abeka puta moma ka chim ai ishit kancha hi oke; micha Echip nan ililli okpulo, yumma ish ithana ka chi onocha he keyu hoke; amba chi nukkilli moma kak o onocha hi oke. 
16. Micha okla moma chin Chitokaka Chihowah yut chibbak fohki tuk moma ka mosholichit ish vmohmichashke ; chi nishkin vt $\underline{\mathrm{i}}$ nukhakla he keyu, micha $\underline{\mathrm{i}}$ naholbvt toba puta ka ish im antia he keyu hoke; yummak okvt ishit chi nukpvllichit ya hi oka.

17. Chi ohükvsh anukaka, Oklushi puta iluppa laua kvt a shahli hoka: katiohmi li kak osh kanalichi la hinla cho? ish achikmot,

18. Ish i nukshopa he keyu hoke; amba chin Chitokaka Chihowah yvt Falaoh, micha Echip moma ka i kaniohmichi tok a ish ithaiyana fehna chi hoke;

19. Imomaka pisa puta chi nishkin vt pisa tok, micha nan isht atokowa puta, nan isht a fehna puta, micha ibbak kullo, micha shakba ashatapa hosh chin Chitokaka Chihowah yot ishit ehi pehlichit kohcha tok a ish ithaiyanashke: yumma chi yuhmi hosh chin Chitokaka Chihowah yot okla ish i nukshopa puta ka yumihcha hi oke.

20. Mieha chin Chitokaka Chihowah yut fohkul a i pilakma, hlakoffi tuk, micha chim ile luhmi tuk vt moshola hi oke.

21. Ish i nukshopa he keyu: chin Chitokaka Chihowah yut Chitokaka kullo, micha palummi hosh huchi takla ahanta hoka.

22. Micha chin Chitokaka Chihowah yut oklushi yumma puta ka kanomosichit, micha kanomosichi hosh ishit kancha chi hoke himona achofa tahlit ish mosholicha he keyu, yakni haiaka nampoa hot apaknvt chi sanala hinla hoke.

23. Yohmi kia chin Chitokaka Chihowah yot chibbak fohka chi hoke; micha okpulot taha chinto ho yumihchit ishit kanchi na, ont moshola chi hoke.

24. Micha i miko vhleha ha chibbak fohkikma, shutik nutaka ya hohchifo ha mosholichit ish kancha chi hoke: hatak nana kia chi tikba ya hikia he keyu ho, ish mosholicha hi oke.

25. I na holbvt toba, in chuwvt toba puta ka luak ishit hukmit hush tahlashke: tvli hvta, micha tvli lakna aiena alapali ka ish ahna he keyu, mfcha ish il ai isha he keyu, 
ish yohmi cha, ishit ish yoshoba na; chin Chitokaka Chihowah nan i yuwala yoka.

26. Micha nan i yuwala ya chim aboha isht ish ona he keyu hoke; ish yohmi na kalakshi yvmmak inli ish chohmi na: amba shitilemot ish kaiyanchi cha, yuwalvt ish vmohmicha hi oka, na kalakshi yoka.

\section{CHAPTA VIII.}

NUMPA vlhpisa puia himak nitak a chim anumpoA honli li ka ithaiyanut ish yumihchashke, yumohmi; hosh hvchi okchayashke, micha hvsh apakna cha, Chihowah yut hvchịki vhleha ha anumpa kullo im il onochi tok a yakni yash chukowut hush binilashke.

2. Mikmưt chukkush akanlosecha chi hosh, micha im anumpa hạ ish iesha chị holkmá, keyu hokmá, nana ka chi chukvush foyuka ka akostinincha chi hosh, imomaka chi pesut afummi pokoli ushta iluppa yakni haiaka hina hlopulli moma ka chin Chitokaka Chihowah yot chisht aya tuk a ish ithaiyanashke.

3. Micha chi chukvush akanlusechit chi hohchvfochi mvt, ish ithana tuk keyu ho, micha chiki vhleha hvt ithana tuk keyu ho, manna ya chipihinta tok; yohmi kut puska ak illa ho hatak vt okchaya he keyu, amba Chihowah im anumpa itih ha akohoncha puta kak o hatak vt isht okchaya ka Chihowah yut chithunancha chi ha tok.

4. Afưmmi pokoli ushta iluppa chi na fohka yut chin taha tok keyu, micha chiyi yut shatali tok keyushke.

5. Micha hatak osh ushi ya na hotopa onochi cha tuk a chohmi hosh chin Chitokaka Chihowah yvt na hotopa chi onochi tok a, chi chukvsh isht anukfillit ish pihisashke.

6. Yumohmi hoka chin Chitokaka Chihowah im anumpa vlhpisa puta ish ieshi cha, i hina puta ka anohowa cha, ish $\underline{\underline{i}}$ nukwiashke.

7. Chin Chitokaka Chihowah yvt yakni achukma, yakni 
bok-ushi aiasha, kuli puta, micha oka hofobi puta aiena kut okfa, micha nưnih puta ka atohomba;

8. Yakni onush aiasha, micha bali, micha pakvui, micha fik iti, micha pomkilanit aiasha; yakni alif bila micha foebila aiasha;

9. Yakni. yumma puska ish aivpa kvt chim atikkonofa nana kia ik chim iksho ka he keyu yoke; yakni yumma tuli vt toli yoke; mikma i nunih puta ka asonak lakna ish a kulla hinla ho isht chisht ona hi oke.

10. Ish impa cha, chi kaiyakmot, yumohmikmot, chin Chitokaka Chihowah yot yakni achukma chima tok oka ish holitoblichashke.

11. Ahah, ish ahni cha, chin Chitokaka Chihowah ya im anumpa vlhpisa puta, micha im ai vlhpiesa yumihinchi, micha $\underline{1}$ nanvlhpisa bilia, himak nitak a chim anumpohonli li ka chik ieshot ik chim a hakso kashke.

12. Keyu hosh ish impa cha, chi kaiyakmot, micha aboha hochukma ish ikbi cha, ish aiashakmut,

13. Micha nanvlhpoa chin hochito, micha nanvlhpoa chin chipunta aiena kvt chim apaknakmvt, micha chin tvli hota, micha chin tvli holisso lakna vt chim apaknakmvt, micha nana chim asha tuk moma kvt chim apaknakmot,

14. Yvmohmikmvt chi chukvush ilefehnvehi cha, chin Chitokaka Chihowah yummak osh Echip yakni akohchvt aboha yuka ai ishit chi kohchi tuk, a chim a haksa hi oke.

15. Yummak osh yakni haiaka chito, micha palvmmi, micha sinti chilita, micha shushi nokoa, halambia achi aiasha, micha achahto, oka ik ai ishko ya pehlichit chi hlopullichi tok oke; yummak osh tuli chito tasonak chohmi ya oka ya a chi kohchi tok oke.

16. Yvmmak osh yakni haiaka ya chiki whleha hvt ithana tuk keyu ho manna ya chipeta kvt, chi chukvrsh a akanlosechi, micha imoma ka chi pesa cha, ont chim ishtaiopikma, chim achukmala chi hosh yumihchi ma;

17. Mihma chi chukvush anukaka, Uno sa kvilo, micha subbak kullo hosh ishit holitopa ilvppa isht vma tok oke, ish achi yoba na, 
18. Amba chin Chitokaka Chihowah ya ish ithaiyanashke: yummak osh holitopa ish aiesha chi ka, nan isht aiahli chima kvt, nan itim apesa yummak oka chilki vhleha hạ anumpa kvllo im ilonochi tok a himak nitak a yumohmi hoka ai ahlicha chi kak atok.

19. Atukma yakohma hi oke, chin Chitokaka Chihowah ya peh chim ahaksikmvt, micha na holbvt toba inla puta iakaiyvt ish itvnowakmvt, micha im antivt ish aiokpvchikmvt, ont ish moshola he ya himak nitak a atokolit chim anoli lishke.

20. Oklushi puta ka Chihowah yot chi tikba ai ishit kanchi tok a chohmit ont ish moshola chi hoke; chin Chitokaka Chihowah im anumpa ish im antia he tuk keyu hoke. im achi tok.

\section{CHAPTA IX.}

TSLAEL ma, ish haklashke, himak nitak a oklushi hochi1 to, micha kvllo kvt chi shahli, tumaha holihta hochito, micha a holihta kvt shutik ont vhli ho, chukowvt ish isha chi hosh Chatan a ish hlopullachishke.

2. Okla hochito, micha chaha, Anakim im vlla vhleha yummak oka ish ithana, micha yummak oka, Kvta hosh Anakim im vlla vhleha ha itikba hikia hinla cho? achi kạ ish hakklo chatuk oka.

3. Yvmohmi hoka himak nitak a chin Chitokaka Chihowah yummak osh chi tikba hlopullit ia hoke; luak na hukmit tahli chiyuhmit ishit kancha chi hoke, micha chi tikba ya aka isht ona chi hoke. Yvmohmi hoka Chihowah yut chim achi tuk mak o, tihlelit ish kocha wehli cha, chekosi ishit ish kancha hi oke.

4. Chin Chitokaka Chihowah yut chi tikba ya ishit kanchi ha yạ, chi chúkvsh anukkaka ya anumpulit, Ai vlhpiesa li pulla kak o yakni iluppa a binili la chi ho, Chihowah yot susht vla tuk oke; chik acho kashke; amba oklushi iluppa puta haksi kak o, Chihowah yut chi tikba a tihlelit kocha weli họke. 
5. Ish ai vlhpiesa, micha chi chulkvsh vt ai vlhpiesa kak osh i yakni ya ishit a binilit ish ia keyu; amba oklushi iluppa puta haksi kak o, micha anumpa kvilo ya chịki whleha Eblaham, Aisak, micha Chekob aiena im il onochi tok anumpa ha aiahlicha chi hosh chin Chitokaka Chihowah yvt chi tikba a tihlelit kocha weli hoke.

6. Yvmohmi hoka chin Chitokaka Chihowah yvt yakni achukma iluppa ish a binila chi ho chima kvt, chi ai vlhpiesa kak o keyu ka ish ithaiyanashke; okla ikunla kvllo chia hoka.

7. Yakni haiaka ya chin Chitokaka Chihowah im ai i nukkilli ya ish nokowvchi tok vt, ish ithaiyana cha, ik chim a hakso kashke; Echip yakni ya kohchut ish minti tok, nitak yumma vttut mihintit ilvppa hushla tuk ant vhli ka Chihowah ya isanalit hush i filahummi tuk.oke.

8. Holeb ak kia, Chihowah im ai i nukkilli ya hush nokowvchi tok; yohmi na Chihowah yvt nuklibisha cha, ishit hvehi kancha chị ha tok.

9. Tuli patussa tuklo nan itim apesa toli patussa tuklo Chihowah yut chitim apesa tuk fehna ka ishe la chi hosh, nunih ha oiya li mut; yohmi cha, nunih ha nitak pokoli ushta, micha ninak pokoli ushta ho anta li tok; puska ya ak po, micha oka ya ak ishko tok.

10. Mihma tvli patussa tuklo ka Chitokaka ibbak ushi yosh a holissochi ya, Chihowah yut subbak fohki tok; mihma itunaha chitot aiasha nitak $\underline{0}$, Chihowah yot nunih. ha luak iklunna et ai anumpohonli tuk mak $\underline{\text { e, anumpa hvt }}$ moma hosh ai $\underline{Q}$ holisso tok.

11. Atuk o yakohmi tok, nitak pokoli ushta, micha ninak pokoli ushta kut ont taha ma, Chihowah yot toli patussa tuklo, nan itim apesa tvli patussa fehna kash vma tok.

12. Mihma Chihowah yvt, Wakaya cha tushpvt iluppa vttut ish akowashke; chim okla Echip a pehlichit ish kohcha kash osh ile litehlishke; chekosi i miha li kash hina ya kohchut fullotut naksika ivshke; bilut akmot na holbut toba ikbishke, im achi tok. 
13. Micha Chihowah yvt vm anumpulit, Okla ilvppa pisa lishke; mihma yakeh, okla ikunla kvllo yoke.

14. Is sussa na, ishit kanchi lashke; micha hohchifo ha shutik nutaka ya a kashofit kanchi lashke. Micha chishno ak o oklushi kvllo, micha chito aiena kut yưmma i shahli ho a chikbi lashke, achi tok.

15. Yohmi ho fullota li mut, nưnih ha akowa li tok; mihma nunih hut luak ishit lụhuwwa tok; mihma nana itim apesa tuli patussa tuklo kut subbak tuklo ka alhto tok.

16. Atuk osh pisa li ma, yakeh, chin Chitokaka Chihowah ya i sanalit, yoshobvt, wak ushi bilvt akmot toba hush ikbi tuk a tok: Chihowah yut huvchi miha tuk a chekosi hina ya kohchưt fullotvt naksika ya hush ia tuk a tok.

17. Mihma tuli patussa tuklo subbak alhto kash hush pisa ho huchi tikba ya ishit aka pila lish, kobalichi li tok.

18. Micha vmmona tuk a chohmit Chihowah itikba ya nitak pokoli ushta micha ninak pokoli ushta ho aka itoyula li tok: hvochi yoshoba momá ishit a huchi yoshobvt Chihowah itikba ya hvchi haksi fehnvshke; im ai i nukkilli ya hush nokowuchi ha tuk oka, puska ya ak po, micha oka ya ak ishko tok.

19. (Im ai i nukkilli micha nuklibisha lushpa ho Chihowah yut ishit nuklibisha cha, hvchị sanalit ishit huchi kancha chi kak a sa nukshopa tuk okvt.) Yohmi tuk kia nitak. yummak kia Chihowah yut a haponaklo tok.

20. Micha Chihowah yot Alon ak kia im i nuklibisha fehna cha, ishit kancha chin tok: mihma Alon ak kia nitak yumma isht anumpule li tok.

21. Micha na hush ashvchi, wak ushi hvsh bi tuk ash ishi lish, luak ishit hukmi li mvt, botolit, boshullichit, lipibichit, lukfi pushi chi yumihchi li tok; micha pushi yash bok ushi nưnih et okvtahaka oka hlatubli li tok.

22. Micha Tabelah, micha Massah, micha Kiblolh-hattafah ya ia Chihowah im ai i nukkilli ya hưsh anokowvchi tok.

23. Micha.Chihowah yut Katesh banea ya vttut huvchi pelvt, oh ia cha, yakni huchima li tok a; oh binili, achi tok; 
yohmi ma, chin Chitokaka Chihowah im anumpa hạ i filummit hush i sanali tok; micha hush i yimma he keyukmvt, im anumpa ha hush $\underline{i}$ haponakla he keyu tok.

24. Huch ithana li tok aivhlit mihinti ka Chihowah ya $\underline{\underline{i}}$ sanalit, hvsh i filahvmmi chatuk oke.

25.Yvmohmi ma vmmona kạ, aka itola li tuk a chohmit, Chihowah itikba ya nitak pokoli ushta, micha ninak pokoli ushta ho aka itoyula li tok; Chihowah yut ishit hvchi kancha chi, miha ha tok oka.

26. Yvmohmi ha tuk o Chihowah ya im ilbushut, Chitokaka Chihowah ma, chim okla, micha chimmi, yummak oka a chi holitopa pulla hosh ish okchalinchi tuk; micha chibbak kvllo hosh pehlichit Echip a ish kohcha tuk okvt, ishit chik kancho kashke.

27. Chin tishu vhleha Eblaham, micha Aisak, micha Chekob aiena ka ish ithaiyanashke; okla iluppa chukvush ilapunla, micha a haksi, micha aiashvchi aiena kano chik peso kashke.

28. Yvmohmikma yakni apehlichit ish pi kohchi tok vt, Chihowah yot yakni im issa tok vt isht ona he keyu ha tuk osh, micha i nukkilli ha tuk osh, yakni haiaka ai vbit tahla chi hosh pehlichit kohcha tuk oke, achi yoba na.

29. Yohmi kia chim okla hoke, micha chimmi hosh nan isht chim aiahli kvllo, micha chi shakba kvllo ashatapa hosh pehlichit ish kohchi tuk oke, ahanchi li tok: im achi tok.

\section{CHAPTA $\mathrm{X}$.}

ITAK yumma Chihowah yut, Tuli patussa tuklo vm1 mona kash chohmi ho ish tuhli cha, vm vlut nunih hạ ish oiyashke, micha iti $\underline{0}$ itombi holitopa ya ish ikbashke.

2. Mikma tuli patussa tuklo vmmona ish kobvffi chash, anumpa a foka kash o tvli patussa tuklo ka ai o holissochi lashke; mikma itombi holitopa ya ish fohkashke, vm achi tok.

3. Mihma shittim iti yo itombi holitopa ikbi li mot, tuli 
patussa tuklo ummona kash chohmi ho tuhli lish nưnih ha oiya li mvt, tvli patussa tuklo kvt subbak foyuka tok.

4. Mihma vmmona holisso tuk chohmi, nan vlhpisa pokoli Chihowah yut nunih ha luak iklunna okla itunaha nitak ma, auet hvchim anumpuli tuk vt tuli patussa tuklo ka ai $\underline{o}$ holissochi tok; micha Chihowah yut vma tok.

5. Mihma fullota li mvt, nunih hạ akowa li mut, patússa tuklo kash itombi holitopa ikbi li tuk ash osh follki li tok; atuk o Chihowah yot vm anumpuli tuk mak o yommak o kaiyaha hoke.

6. Mihma Islael im vlla vhleha hvt Chaakan im vlla vhleha hạ immi Beelolh a asht ia mvt, Mosela ya ona tok; yummak o Alon vt illi tok, micha yummak o ahollohpi tok: mihma ushi Eleasa ak osh vlhtobut naholitompa isht ai vtta ya isht ahanta tok.

7. Yvmma asht ia mvt, Kutkotah ya ona tok; atuk osh Kutkotah ya asht ia mvt, okhina puta aiasha yakni Chotbalh a ona tok.

8. Nitak yumma Chihowah yut Lefai apehlichika Chihowah nan itim apesa afohka itombi holitopa shola chi hö, Chihowah itikba ya hieli cha, nan isht im ahanta chị, micha yumma hochifot, anumpa yukpuli isht onohoncha chi ho, filvmmihinchi ho himak nitak a yvmohmi hoke,

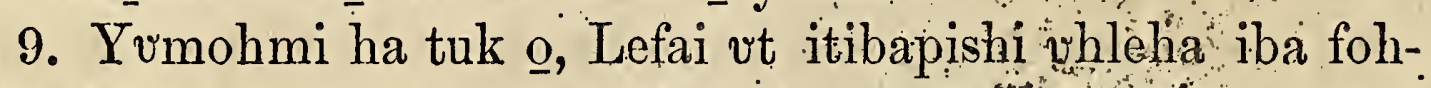

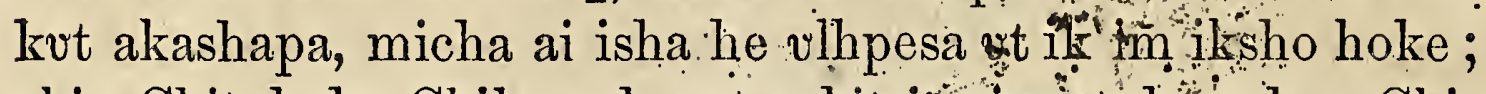

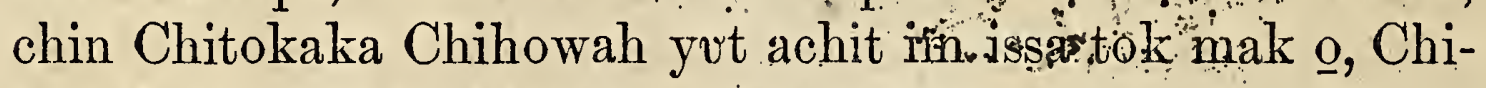
howah ak osh ai ishi vlhpesa immi yoke."

10. Mihma nunih ha vmmona tok a chohmit, nitak pokoli ushta, micha ninak pokoli ushta ho ahanta li tok; mihma Chihowah yut nitak yummak kia a haponaklo cha, Chihowah yot isht ik chi kancho tok.

11. Micha Chihowah yot, Wakaya cha, okla ha itikba ia, yumohmikma ima la chị hosh îki vhleha ha anumpa kvllo im ilonochit, anumpule li tok a iba chukowa cha, yakni ya ish ashke.

12. Yohmi ka himak a Islael ma, nanta ho chin Chitokaka Chihowah yot chim ahoyo cho? amba chin Chitokaka 
Chihowah ya $\underline{i}$ nukwivt, $\underline{i}$ hina moma ka anohowa, micha holitoblit, micha chin Chitokaka Chihowah ya chukvsh ai okluhut, micha chi shilombish ai okluhvt isht ish im antia ho.

13. Chihowah im anumpa, micha $\underline{i}$ nan vlhpisa bilia puta chishno ak inli isht chim ai achukma he $\underline{o}$, himak nitak $\underline{a}$ chi miha li ka holitoblit ish iesha he ak keyu cho?

14. Yakeh; shutik, micha shutik puta $\underline{\mathrm{i}}$ shutik vt, micha yakni al kia nana moma yumma aiasha aiena kvt chin Chitokaka Chihowah ya immi hoke.

15. Peh Chihowah yut chiki vhleha ha holitobli kvt im achụkma tok; micha im ishtiaka pit iakaiya aiena ka okla moma ka im aiyachit atokolit ishi tok $\underline{o}$, himak nitak a yvmohmi holke:

16. Yvmohmi kak o hvchi chukvush tikba hakshup a hush tubli cha, himakma ikunla hvchik kvilo kashke.

17. Hvchin Chitokaka Chihowah yvt na holbut toba puta in Chitokaka, micha Chihowah in Chihowah, Chitokaka holitopa, kvllo, micha palummi yosh yvmmut hatak yohmi hoka, ahni keyu, na hvlbina ishi keyu hoke.

18. Iki illi, micha 1 hatak illi nan im vlhpisa ka im ahlichi kơt, micha okla inla ya ilhpak, micha na fohka ya ima kvt im achukmar loke,

19. Yumollimi hoka okla inla ya shush holitoblashke, Echip yakni ya oklandy 1 osh hush aiasha tok ak inli hokvt.

20. Chin Chitokak Chihowah ya ish i nukwiashke; yummak o ish im antiashke, micha yummak o ish asitiashke, micha yommak o hochifo hosh anumpa kullo ish ilonochashke.

21. Yvmmak osh ishit chi anohowa hi oke, micha chin Chitokaka yoke; yormmak osh na chito, micha palummi puta chi nishkin vt pisa tuk iluppa puta ka chi yumihinchi tuk oke.

22. Chiki whleha hot hatak pokoli untuklo hosh Echip a ai itupehvt ona tok; atuk o himak a chin Chitokaka Chihowah yot chi apaknvchi kvt shutik fochik ak o chi chohmichishke. 


\section{CHAPTA XI.}

VUMOHMI hoka chin Chitokaka Chihowah ya ish 1 holitobli chạ, chi miha tok a, micha $\underline{i}$ nan vlhpisa bilia, micha im ai vlhpiesa yumihinchi, micha im anumpa aiena ka ish ieshi na, biliashke.

2. Micha himak nitak a hush ithaiyanashke; hvchim vlla vhleha ha im anumpule li keyu hoka; yưmmak osh hvchin Chitokaka Chihowah yvt na hotopa onohonchi tuk, im aholitopa, ibbak a kullo, micha shakba ashatapa,

3. Micha i na fehna, micha $\underline{i}$ nan yumihinchi Echip ai iklvnna, Echip i miko Falaoh, micha i yakni moma ka onohonchi tok a ;

4. Micha Echip in tushka chipota, micha im issuba puta micha tunup, im iti chanulli puta ka , kaniohmichi tok $\underline{a}$; hvchi hliolit aya ma, okhvta humma ya oka tamovchi tok, micha Chihowah yut ishit kanchi tok a himak nitak a yumihinchi hoke;

5. Micha yakni haiaka mihintit iluppa hush la ka ant vhli ka hvchi kaniohmichi tuk a

6. Micha Luben ushi Eliab ushi tuklo Tahlan, micha Abilam akaniohmichi tok a yakni 'vt' itakpashali cha, yumma micha in chuka achufa puta, micha 1 bina puta, micha i na cholkushpa puta ishi tuk puta Islael moma ai itin takla ishit nanvbli tok a puta ka ik ithano ki tok, micha ik peso ha tok oka;

7. Yohmi kia nana isht aiahanta chinto fehna moma ka Chihowah yut yumihinchi tuk a hvchi nishkin vt pisvushke.

8. Yumohmi hoka anumpa ha himak nitak a huchim anumpohonli li ka hush ieshashke; yumohmi hosh hush kullo cha, yakni hush isha chi hosh yumma pilla ia kut ont chukowvt hush ishashke.

9. Yvmohmi hosh Chihowah yut hvchiki vhleha, micha im isht atiaka aiena ka ima chi hosh, anumpa kvllo im ilonochi tok a nitak hvchi falaia yakni yash pishukchi, micha foe.bila ayanahanli yakni ya hush ai ahashwashke. 
10. Yakni ya ish isha chi hosh, yumma pilla ish ia kvt, Echip yakni ish a minti tok, yummak oka chi na nihi ish hokchi tuk mvt, haiyukpolo osapushi chohmichit, chiyi ak

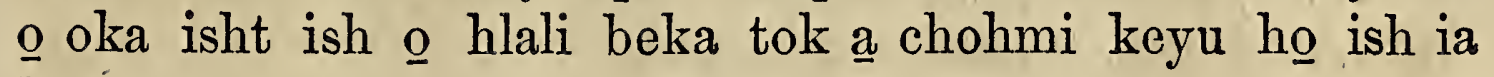
hoka;

11. Amba yakni hvsh isha chi hosh, yvmma hvsh ia kvt, nunih, micha okfa, aiena aiasha yosh shutik vt et ai umba oka ak o ishi hoke.

12. Chin Chitokaka Chihowah yut anukfilli yakni yoke; chin Chitokaka Chihowah i holhponayo yut onvtoyula na, bilia, afummi ishtia vmmona tukmut afummi ont taha ka ont vhli hoke.

13. Atukma yakohma hi oke, achunanchit vm anumpa himak nitak a huchim anumpohonli li ka hush haiyaklo, hvchin Chitokaka Chihowah ya hvsh holitobli, micha hvchi chukush a mominchit, micha hvchimi shilombish ma mominchit hvsh im antiakma,

14. Yumohmikma huchi yakni im umba ont ai vlhpiesakma, vmmona umba, micha ont ishtaiopi umba ya huchima la hi oke; mikma hvchim onush, micha hvchim okapaki, micha huchi bila aiena ka hoyot hush ashacha hi okę.

15. Micha chi nan vlhpoa yut vpa chị ka, yumohmi na, hush impa cha, micha huchi kaiya chi ka huchim osapa puta holka hushuk et huchi pila la hi oke.

16. Ahah, hush ahni na, hvchi chukvesh vt hvchi haksichi na, fullotvt na holbut toba inla puta hưsh im antia cha, hvchik aiokpacho kashke.

17. Yvmohmikma Chihowah im ai $\underline{i}$ nukkilli vt hvchi sanalit libbikmvt, shutik a okshitakma, umba kvt iksho ka he, mikma yakni vt $\underline{i}$ nan waya puta ka ik a wayo ka hi oke: mikma chekosi Chihowah yot yakni hvchima tuk a hush a. mosholi.na.

18. Yvmohmi hoka vm anumpa iluppa puta ka hvchi chukvsh, micha hvchimi shilombish a fohkit hush ieshäshke, micha nan isht atokowa yo huchibbak ishit hush takchi na, hvchi nishkin itin takla ya bita hosh on talaia ka huchin chohmashke. 
19. Micha yumma hvchim aboha hvsh a binilikmvt isht hush anumpohonlashke; micha hina takla hush ayakmvt, hvsh itoyulakmvt, micha hvsh wakayakmvt, hvchim vlla vhleha ha hvsh im abahanchashke.

20. Micha chin chuka in tonik puta kạ, micha chi holihta okhissa puta ka ish ai o holissochashke.

21. Yvmohmi ka hvchi nitak, micha hvchim vlla vhleha hạ i nitak vt, Chihowah yut hvchiki vhleha ha ima chị hosh anumpa kvllo im ilonochi tok a yakni ya huchim ai apakna kvt, shutik nitak vt yakni in takali kak inli ho chiyuhmashke.

22. Anumpa ilvppa puta moma hvchim anumpohonli li ka achunanchit hvsh ieshi cha, yumihchit hvchin Chitokaka Chihowah ya holitoblit, $\underline{\mathrm{i}}$ hina puta moma ka ai itunowa cha, hush im antiakma;

23. Yumohmikma Chihowah yut oklushi iluppa puta moma ka hvchi tikba a tihlelit, kocha welikma, oklushi puta hochito, micha kullo kvt huchịshahi ya hưsh isha chị hoke.

24. Kanima moma huch iyi putta yut anohowa kvt huchimmi ha chi hoke; yakni haiaka, micha Liebanon vttut okhina Euflates okhina vttvt ia hosh okhvta ont vhli fehna ont vhli kak osh huchim ai vhli ya chi hoke.

25. Hatak nana kia huchi tikba ya hikia he keyu ka chị hoke; hvchin Chitokaka Chihowah yvt hvehim achi tuk mak osh, yakni hush anohowa moma ka hvchi nukshopa, micha huchi komunta aiena ka onocha chi hoke.

26. Yakeh, himak nitak a nan isht yukpa, micha nan isht kalakshi ya hvchi tikba takalechi lishke.

27. Nan ishit yukpa, hvchin Chitokaka Chihowah im anumpa himak nitak a hvchim anumpohonli li ka hvsh im antiakmot;

28. Micha ishit kalakshi, hvchin Chitokaka Chihowah im anumpa himak nitak a hvchim anumpohonli li ka hvchik im antio hosh, hina ya kohchvt naksika pit hush fullota cha, na holbut toba inla puta hush ithana tok keyu kvt, iakaiyvt hush iakma.

29. Atukma yakohma hi oke; yakni ya ish isha chi hosh, 
yumma ish ia ka chin Chitokaka Chihowah yot chisht onakma, Kelisim nunih ha anumpa yukpali ish onochikmvt, Ebal ruvnih ha anumpa kalakshichi ish onocha hi oke.

30. Yvmmut Chatan misha tunnup, hushi pit ai okatula pilla, Kananait okla yummot Kilkal ichapaka yakni shahbi asha ya i yakni Moleh yakni patali bilika hieli keyu cho?

31. Chihowah yut huchima tok a yakni ya hvsh isha chi hosh, Chatan a hush hlopulla chi hokvt; micha hush ishikmut hush ai a hashwa chi hoke.

32. Mikmvt nan vlhpisa bilia moma, micha ai vlhpiesa yvmihinchi himak nitak a huchi tikba ya takalichi ka ithaiyanut hush yumihchashke.

\section{CHAPTA XII.}

ANULHPISA bilia, micha aivlhpiesa yumihinchi ilup1 pa puta ka chiki vhleha in Chitokaka Chihowah yut yakni ya ish isha chi ho chima tok a okchayvt yakni paknaka hush ahashwa nitak moma kvt ithaiyanvt hosh yomihchashke.

2. Oklushi puta hush ishi puta kvt, nunih chaha paknaka, micha nunih paknaka, micha iti okchamali nuta puta ka na holbut toba . puta aiokpvchit aiasha tuk moma ka ieshit kanchit hvsh vmohmicha hi oke.

3. Micha im alta puta ka hvsh kinahlicha hi oke, micha in tonik puta ka kobahlichit, im iti lokoli puta ka luak ishit hush hukma hi oke; micha 1 naholbut toba puta in chüwt na holbut toba ya chanlit hush akakohli cha, i hohchifo puta ka yumma ai ishit hush kancha hi oke.

4. Hvchin Chitokaka Chihowah ya hush i yumihcha he keyu hoke.

5. Amba huchin Chitokaka Chihowah ak osh kanima ho hvchim apehlichi puta moma ka ai atokolit, hohchifo hạ a bohlikma, ai ahanta ak o hush hoya chi hoke; micha yummak o ish ona chi hoke,

6. Micha yummak o nahukmit isht hush aiokpvchi, micha 
nan vbit isht hvsh aiokpvchi, micha ishit hvchi pokoli, micha pit $\underline{\mathrm{i}}$ walit isht aiokpvchi chibbak foyuka ka, micha anumpa hush ilonochi, micha ilap ahnit isht hush aiokpvchi, micha hvchi nan vlhpoa hochito, micha hvchi nan vlhpoa chipinta chelahpi puta aiena a puta ka isht hush ona hi oke.

7. Micha yummak o hvchin Chitokaka Chihowah itikba ya hush ai impa hi oke; hvchishno, micha huchin chuka achvfa hush aiena kvt nana moma ka huchibbak onochit ishit hush ahanta na, Chihowah yvt hvchi yukpali tuk hvchi yukpashke.

8. Himak nitak a nana iluppa il akaniohmi kạ, hatak moma kvt ilap anukfilli kvt vlhpesa ahni ka iakaiya ka hvsh yumohma he keyu hoke.

9. Afoha micha aiasha he vlhpesa hvchin Chitokaka Chihowah yut huchima tok a huchik ono kisha hokvt.

10. Amba Chatan a hush hlopulli cha, hvchin Chitokaka . Chihowah yut hvchima yakni ya hush aiashakmvt, hvchin tunup puta apakfoyput aiasha moma ka i hlakofichit hvchi fohvchi na, achukma ho hush aiashakma.

11. Yưmohmikma huchin Chitokaka Chihowah yut y:mmak o $\underline{\mathrm{i}}$ hohchifo aiahanta chi ka atokola chi hoke; yum-

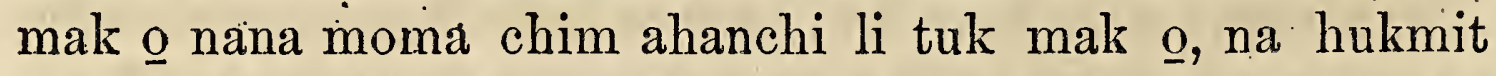
ishit hvsh aiokpvchi, micha nan vbit isht hush aiokpvchi, ishit hvchi pokoli, micha $\underline{\mathbf{i}}$ walit isht aiokpvehi hvchibbak foyuka aiena, micha achukma i shahli moma, anumpa il onochit Chihowah ya hush im ilonochi tuk a puta aiena ka isht hush la hi oke,

12. Mikmvt hvchishno, micha hvchiso vhleha, micha hvchiso tek vhleha, micha hvchin tishu hatak vhleha, micha hvchin tishu ohoyo vhleha, micha Lefait vhleha hvchi holihta okhissa anukaka hikia aiena kvt, $\underline{1}$ kashapa micha aiisha he vlhpesa vt hvchim ai iti takla ya ik im iksho hoka; awant huchin Chitokaka Chihowah itikba ya na hvchi yukpashke.

13. Ahah ish il ahnashke, yumohmi hosh kanima moma ish pisakmvt na hukmit isht ish aiokpvchi ya chik ai im isso kashke: 
14. Amba chim apehlichi puta ka achufa ho kanima ho Chihowah yut atokolikma, yummak o na hukmit isht ish aiokpvchi a ish ai im issa chi hoke, micha yummak o chi miha li tuk moma ka ish ai yưmihcha chi hoke.

15. Yohmi kia nana ho chi chukvush vt bunnakmvt, chi holihta okhissa anukaka moma ka, chin Chitokaka Chihowah yvt nan isht yukpali chima tuk mak $\underline{\text {, ai }}$ vbit nipi ish pashke: ik kashofo micha kashofa aiena kvt issi holba nakni, micha issi nakni chohmi ka ai upa hinla hoke.

16. Peh issish ano hush pa he keyu, oka chohmichit, aka hush hlatubla hi oke.

17. Chim onush isht pokoli, micha chim oka paki ishit pokoli, micha chi bila ishit pokoli, micha chi nan vlhpoa hochito vttahpi, micha chi nanvlhpoa chipunta vttahpi, micha anumpa ilonochi ish ilonochi tok, micha ilap ahnit nan im issa, keyukmvt pit $\underline{i}$ walit im issa chibbak foyuka aiena ka chi holihta okhissa anukaka ya ish aiupa he keyu hoke.

18. Amba chin Chitokaka Chihowah ak osh kanima ho ai atokolikma, chishno, micha chiso, micha chiso tek, micha chin tishu hatak, micha chin tishu ohoyo, micha Lefait chi holihta okhissa anulkaka ahanta aiena kvt, chin Chitokaka Chihowah itikba ish ai impa hi oke; micha nana ho chibbak isht ish ishi moma chin Chitokaka Chihowah itikba na, chi yukpashke.

19. Ahah ish il ahni cha, yakni paknaka ish ahanta moma kvt Lefait vhleha ha chik kancho kashke.

20. Chin Chitokaka Chihowah yut achit chim issa tuk mak osh, chim aivhli ya chitolikma, nipi ya vpa lashke, ish áchikmvt, (chi chukvush vt nipi vpa bvnna ha tuk osh,) nipi nana ho chi chukvsh vt bunnakmut ish pa hinla hoke.

21. Chin Chitokaka Chihowah yvt kanima ho yummak o hohchifo ha a bohla chi hosh atokoli tuk vt chi hopaki fehnakmvt, yohmikmvt, chi nan vlhpoa hochito, micha chi nan vihpo chipunta aiena Chihowah yvt chima tuk a chi miha li tuk mak o, nana ho chi chukvsh vt bunnakmut chị holihta okhissa anukaka yak o ish ai vpashke.

22. Issi holba nakni, micha issi nakni vpa chatuk mak $\underline{o}$, 
chobmichit ish pashke, ik kashofo, micha kashofa aiena kvt iti lanet ai vpa hi oke.

23. Peh issish ano chikpo pullashke; issish ak osh isht okchaya yoke; isht ai okchaya ya nipi aieninchit ish pa he keyu hoke.

24. Ish pa he keyu; oka chohmichit aka ish hlatubla hi oke.

25. Ish pa he keyu hoke; yumohmi hosh Chihowah itikba ya aivlhpiesa ho ish yumohmikmut, chishno, micha. chim vlla vhleha et chi akaiya aiena kut chim achukmashke.

26. Peh na chi hullochi chim asha, micha anumpa ishi lonochi ak illa ho ish ishi cha, Chihowah yvt kanima yo ai atokoli tukma ish ia hi oke.

27. Micha na hukmit isht ish aiokpvehi nipi, micha issish aiena ka chin Chitokaka Chihowah im alta ya onochit ish im issa hi oke: micha nan vbit isht ish aiokpuchi issish vt

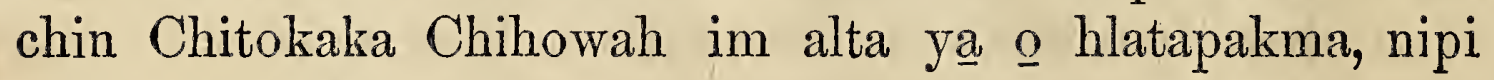
yano ish pa hi oke.

28. Yohmi ahnit ish anukfilli cha, anumpa iluppa moma chi miha li ka ish haklashke; yumohmi hosh chishno, mi-cha chim vlla chi akaiya aiena kvt chin Chitokaka Chihowah itikba ya achukma ho micha ai vlhpiesa ho ish yumihchikmot achukma hosh ish mahaya na biliashke.

29. Ish isha chi hosh ish ia ka chin Chitokaka Chihowah yot olklushi puta kash chi tikba ai ishit kanchi na, ish vlhtoba cha, 1 yakni ya ish ashakmvt;

30. Ahah ish il abnashke, yomohmi hosh chi tikba ai ishit kanchi ha ya iakaiyvt isht ik chi yushobo kashke, micha i naholbut toba puta ka ponaklot, Oklushi iluppa puta kvt i naholbut toba puta ka katiohmit im antia tuk oh cho? uno ak kia yummak o yumihchi la chị hoke, chik achó kashke.

31. Chin Chitokaka Chihowah ya ish yomihcha he keyu; Chihowah yot $\underline{\mathrm{i}}$ yuwala puta moma, micha shitilema ya $\underline{\underline{i}}$ naholbut toba i yumihchi tuk oke; ushi vhleha micha ushetik vhleha aiena ka i naholbut toba luak isht i hukmi fehna tuk oke. 
32. Nana ho hvchim ahanchi li tukma, ithaiyanvt ish yvmihchashke: ish iba fohka he keyu, micha ish ola taklucha he keyu hoke, achi tok.

\section{CHAPTA XIII.}

TUCHIM ai iti takla hopaii yosh, keyukmvt, na holhpo11. kuna ya holhpokuna yosh wakayvt hikia cha, nan isht atokowa, keyukmvt, na fehna yo chị haiakvchikma,

2. Atuk o nan isht atokowa, keyukmvt na fehna ishit chim anumpuli tuk vt ont aiahlikma, yummak oka ish ithana chatuk keyu ka, Naholbvt toba inla iakaiyvt iloh iashke, micha iloh im antiashke, achikma,

3. Hopaii yumma, micha na holhpokuna holhpokuna yumma im anumpa ha ish haponakla he keyu; hvohi chukvsh a mominchit, micha hvchimi shilombish ma mominchi hosh huchin Chitokaka Chihowah ya ishit hush holitoblikma, nanakma, isht akostinincha chi hosh imoma ka hvchi pisa hoke.

4. Huchin Chitokaka Chihowah ya iakaiyut hush itunowashke, micha $\underline{i}$ nukwivt im anumpa ha holitoblit, im anumpa ha hvsh im antivt, in toksahanlit hush asitiashke.

5. Mikma hopaii yummut, micha holhpokuna holhpokuna yummut illashke; huchin Chitokaka Chihowah yvmmak osh Echip yakni ya apehlichit huchi kohchi tok; micha yummak osh aboha ayuka ha a hvehi hlakofichi tok $\underline{a}, \underline{i}$ filvmmichit hvchisht ia chị hosh, hina hush ai itvnowa chi ho hvchin Chitokaka Chihowah yut huchi miha tok a naksika pit huchi tobli bunna hosh annmpuli ha tuk oka. Yษmohmi hosh huchim ai iti takla ya nan ik achukmo ka isht hush kanchashke.

6. Chitibapishi chishki ya ushi yokmá, keyukmut chiso yokmá, keyukmot chisotek okmá, keyukmưt chi tekchi chi apotoa tuk ak okmá, keyukmut chi kana "yosh chimi shilombish ak inli chiyuhmi tuk okmá, nana hosh lumvt chi haksichit, yvmmak oka hvehishno, micha hvchiki vhle- 
ha aiena kut ithana tok keyu kia, Kilia cha, naholbut toba inla,

7. Yvmmvt okla hvchi apakfopvt aiasha, hvchi bilika hokmá, keyukmut hvchị hopaki hokmá, yakni ataha ka in tunnup vttut ia hosh in tunnup ont vhli ka aiasha ka $\underline{\underline{i}}$ na naholbut toba kiloh aiokpvchi, achikma,

8. Vlhpesa, chik im ahno kashke, micha chik i haponaklo kashke: micha chi nishkin vt ik $\underline{i}$ nukhaklo kashke, ish apoacha he keyu, micha ish luhma he keyu hoke.

9. Amba ish bi pulla hi oke: illichi ka tikbanli hosh chishno ak osh chibbak a ish onocha hi oke; mikma i himmak a yo okla moma kut ibbak onocha hi oke.

10. Tuli o isht ish boli na, illashke; chin Chitokaka Chihowah yummak osh Echip yakni, micha aboha a yuka ha apehlichit chi kohchi tok a 1 filvmmichit chi tobli bunna tuk okvt.

11. Mikma Islael moma kvt haklo cha, nukshopakmvt, himmakma yoshuba fehna iluppa chohmi kvt a hvch iti takla ya ik a yưmohmho kashke.

12. Chin tumaha holihta kanima ka yummak oka ish ai asha chi ka chin Chitokaka Chihowah yvt chima tok a ish hạklo ho achi kut,

13. Hatak kaniohmi kut Belial im vlla vhlehia hvt huchi. kohchut ia cha, in tomaha holihta okla hash pehlichit isht ivt, Naholbut toba inla puta hush ithana tuk keyu ya ia cha, kiloh aiokpvchi, achishke, achikma,

14. Yohmikma ish ponaklo cha, silhhit, michá achunanchit ish asilhhvcha hi oke; atuk o, yakeh, na yuwala yumma chohmi huvchi ai iti takla ya yưmihchi tuk vt ahlikma, micha nana kut ai ahli fehnakma;

15. Tumaha holihta yash okla aiasha ka bushpo falaia ishit fahamut, ishit kanchit hush vmohmichi pullashle; micha anukaka nana aiasha moma ka, micha nanvlhpoba yumma aiasha ka bushpo falaia isht hush kanchashke.

16. Micha na wehpoa moma ka tvmaha holihta iti takla ai itunowa iklunna hush itunahli cha, tumaha holihta, micha na wehpoa aiena kash ant isht aiopi moma ka chin Chitoka- 
ka Chihowah itikba ya luak isht hush hukmashke; micha chiffikot chieya na biliashke; anonti ikba he keyu hoke.

17. Mikma nana achufa nana kia kvllakshi yash osh chibbak a foyuka he keyu hoke : yumohmi ho Chihowah yvt chiki vhleha ha anumpa kvllo im il onochi tok mak osh, ai im i nukkilli libbi ka shưnaiachi cha, chi nukhakklot heh chi ahni cha,

18. Chin Chitokaka Chihowah im anumpa ha ish haponaklo cha, im anumpa moma ka himak nitak a chi miha li ka ieshit chin Chitokaka Chihowah itikba ya a aivlhpiesa kak o ish yumihchikma, chi apaknuchashke, achi tok.

\section{CHAPTA XIV.}

UUCHIN Chitokaka Chihowah im vlla huchia hoke; 1 illi pulla kak o hush ile bushla he keyu, micha hvchi nishkin iti takla ya hush yushmilalicha he keyu hoke.

2. Chin Chitokaka Chihowah ya $\underline{\underline{i}}$ holitopa okla chia hokvt; micha oklushi puta yakni páknaka aiasha moma ka $\underline{\mathbf{i}}$ shahlechit im okla hosh inla fehna chia ha chị ho Chihowah yut chi atokoli tok oke.

3. Nana yuwala ya ish pa he keyu hoke.

4. Nampoa iluppa puta ka hưsh pa hi oke: wak, chukfi, micha issi kosoma.

5. Issa nakni, micha issi tek, micha issi humma, micha issi kosoma nukshopa, micha issi antilop achi, micha issi kasel achi, micha issi shammi achi.

6. Micha nampoa moma hosh iyi akchush a iti filummichit pahlvllichi mot, iyi akchush tuklochi cha, hushuk vpa tuk vt, falaminchit kohchit hopasa puta kvt nampoa ai itin takla asha ka yummak o hush pa hi oke.

7. Yohmi kia iluppa puta kak osh hushuk vpa tuk vt kohchit hopasa kia, micha iyi akchush pahlata ya iti filumminchi ak inli kia; yưmmak o hush aivpa he keyu hoke: kamel, micha pvtta kitta, micha chukfi holba, chohmi ka ; yummot hushuk vpa kohchit hopasa kia, iyi akchush a iti 
filummichi keyu hoka; yumohmi hokvt huchin litiha ya hi oke.

8. Mikma shukha yvt iyi akchush a iti filvmmichi hakinli kia, hushuk vpa tuk vt kohchit hopasa keyu hokvt, hvchin liteha ya hi oke; nipi ya hush pa he keyu, micha yưmma nipi illi ya hush potola he keyu hoke.

9. Iluppa puta kak osh oka aiasha ya hush pa hi oke; sanahchi im asha, micha hakshup fachowa im asha aiena kak o hush pa hi oke.

10. Micha nana hosh sanahchi ik im iksho, micha hakshup fachowa ik im iksho ka hush pa he keyu hoke; hvchin litiha ya hi oke.

11. Hushi kashofa puta moma ka hush pashke.

12. Yohmi kia iluppa puta kak o hvsh pa he keyu hoke; ossi micha ossifilachi, micha ospila.

13. Micha kileti, micha kait, micha fulta itiakaiya achufa puta,

14. Micha fula chito ai iti akaiya ai achvfa puta.

15. Micha opa, micha oksupafohli, micha kuku, micha na hokli hak achi, ai iti akaiya achufa,

16. Micha opa iskitini, micha opa chito, micha okak,

17. Micha chilantakoba, micha kie ossi, micha komolant,

18. Micha istak, micha itikushayaiya ai iti akaiya achufa, micha lapwin, micha halambisha,

19. Micha na balahanli hut heli puta kvt hvchin litiha ha chi hoke, vpa he keyu hoke.

20. Amba hushi kashofa puta hokvno hesh pa hi oke,

21. Nana hosh ilap ak inli hosh illi tuk ano hush pa he keyu: okla inla yosh chị holihta okhissa hikia kak o ish ema na, vpa hi oke; keyukmvt okla inla yo ish $\mathrm{i}$ kancha hi oke; chin Chitokaka Chihowah ya alitiha ik im iksho yosh im okla hvchia hokvt. Issi kosoma ushi ya ishki i pishukchi ya isht ish hona he keyu hoke.

22. Osapa yưt chi na nihi ya afummi hosh ont afưmmikma, nawayahanchi ka ishit pokoli ho ahhli hosh ish onohunchashke.

23. Kanima ho hohehifo a bohla chi hosh atokolikma, 
yummak o chim onush, micha chim oka-pạki, micha chị bila ishit pokoli, micha chi nan vlhpoa hochito, micha chi nan vlhpoa chipunta ushi vtta ahpi puta aiena ka chin Chitokaka Chihowah itikba ya ish ai upashke; yumohmi hosh chin Chitokaka Chihowah ya $\underline{i}$ nukwia bilia ka ish ithanashke.

24. Atuk o hina vt chi falaia fehna cha, isht ish ia he keyukmut, micha chin Chitokaka Chihowah yut hohchifo ha yvmmak o a bohla chi hosh atokoli tuk vt chi hopaki fehnakmot, chin Chitokaka Chihowah yot na chi yukpa li mut;

25. Yohmikmvt tuli holisso isht ish itatoba cha, tuli holisso yash takchit, chibbak ish foyuki cha, chin Chitokaka Chihowah yot ai atokoli tuk a ish ona hi oke.

26. Micha chimi shilombish vt nana ho bunnakmvt, wak okma keyukmvt chukfi, keyukmvt okapaki, keyukmvt nan ishko kvllo, keyukmvt chimi shilombish vt nana ho bonnakmvt, yummak o Chihowah chin Chitokaka itikba ya chishno micha chin chuka achvfa aiena kvt na yukpvt imput hush mahaya hi oke.

27. Micha Lefait vt chi holihta okhissa anukaka aiasha ha ish kancha he keyu; yummut chin takkla i kashapa yưt ai isha he ai vlhpiesa yut ik im iksho hoke.

28. Afummi ont a tuchina kvt ont taha ma, afummi yummak inli ho, chi nawaya ishit pokoli moma ka isht ish kohchi cha, chi holihta okhissa anukaka ish ashacha hi oke.

29. Mikma Lefait vt (yummut $\underline{\mathrm{i}}$ kashapa, micha ai isha he vlhpesa yot chiba fokvt ik im iksho hokvt) micha okla inla, michá ilki iksho, micha i hatak illi, chị holihta okhissa anukaka aiasha aiena kvt vla cha, vput fihopashke; yvmohmi ho chin Chitokaka Chihowah yut chibbak na toksahanli moma isht ish ahanta ka chi yukpalashke, achi tok.

\section{CHAPTA XV.}

AUMMI untuklo kut ont taha aiyukalikma, nan i hotofi Al ya ish apesa hi oke. 
2. Micha nan i hotofi yvt yakohma hị okje : nan ahekvchi aiyukali hosh in chuka a bilika hatak a, nana kia im pota tuk vt $\underline{\mathrm{i}}$ hotofa hi oke ; in chuka abilika hatak; jeyukmvt, itibapishi ha $\underline{i}$ hoya he keyu, Chihowah $\underline{i}$ hotofa hohchifo hoka.

3. Okla inla yano ish $\underline{i}$ hoya hi oke; amba chimmi ha tuk a chitibapishi hot ishi ka chibbak vt $\underline{i}$ hotofa hi oke:

4. Ilbusha yut huch itin takla ya iksho hok mak illa hi oke, ish ai isha he vlhpesa yosh ish ai isha chi ho, chin Chitokaka Chihowah yut yakni chima tok a Chihowah yut chi yukpali fehna chi hoke.

5. Peh chin Chitokaka Chihowah im anumpa achukmalit haponaklot, anumpa ilvppa puta moma himak nitak a chim anumpohonli li ka ithaiyanut ish yvmihchilkma.

6. Chin Chitokaka Chihowah yut chim achi tuk mak osh, chi yukpali hoka: mikma oklushi laua ho ish impota hi oke; yohmi kia chishno vto ish pota he keyu, micha oklushi laua ho ish $\underline{\mathrm{i}}$ milka hi oke; amba chishno ano chi mika he keyu.

7. Chitibapishi vhleha aiachvfa, hatak vt ilbusha hosh hvch itin takla chi holihta okhissa moma anukaka chin Chitokaka Chihowah yut yakni chima tuk a ahantakma, chi chukvsh a ish $\underline{i}$ kullocha he keyu, micha chitibapishi ilbusha ya chibbak $\underline{\text { a ish }} \mathrm{i}$ pohloma he keyu hoke.

8. Amba chibbak a auatuchi hosh ish $\underline{\mathrm{i}}$ wakummi cha, nana bunna tuk yumma kaniohmi bunna ka laua kvt im vlhpesa ho ish im pota hi oke.

9. Ahah ish ai ahni na, Afummi isht untuklo afummi ai $\underline{\mathbf{i}}$ hotofa he vt olanlosishke, achi, imanukfila hvt chi chükvsh okpulo ya ik chukowo kashke; micha chi holhponayo hut ik achukmot, chitibapishi hạ 포 sanali cha, nana kia chflk emokma, chị sanalit Chihowah ya im payakma nan ashvchi chị toba na.

10. Ish ima pulla hi oke, micha ish emakmvt, chi chukvsh vt nukhalkla he keyu: nana iluppa pulla kak $\underline{\text { g, nan ish }}$ toksuli puta moma, micha nana moma ho chibbak isht ish ishikma chin Chitokaka Chihowah yot na chi yukpala hi olse. 
11. Yakni yash ilbusha yot ik ai iksho ka he keyu hoke: yưohmi kak o chitibapishi ha ilbusha ya, micha nana ik im iksho yakni yash chim ai asha ka chibbak a auatuchit ish $\underline{\mathrm{i}}$ wakiumma hi oke, chim achi lishke.

12. Atulk o chitibapishi Heblu hatak, keyukmvt Heblu ohoyo hạ chi kanchi tuk o, afummi hannali ho chin toksulikma, afummi ont isht untuklokma, pilla ho chim ai ia he a, ish im ahnashke.

13. Micha pilla ho ish kohchichikmvt, ibbak bieka hosh ik ia, ish im ahna he keju hoke.

14. Laua achukma ho chị nan vlhpoa chipunta, micha chim a nihechi, micha chi paki abusha aiena ka ai ishit ish im atahla hi oke: chin Chitokaka Chihowah yot nan isht chi yukpali tuk a ai ishit ish ima hi oke.

15. Micha hatak yuka hosh Echip yakni ya ish ahanta tuk o, chin Chitokaka Chihowah yvt falvmminchit chi hlakofichi tok $\underline{a}$ ish ithaiyanashke: yumohmi kak o himak nitak a nana iluppa mihut chim ahanchi lishke.

16. Atukma yakohma hi oke, chishno micha chin chuka achvfa holitobli ha tuk osh, micha chi takla ahanta kvt im achukma ha tuk osh, chissot ia la he keyu, chim achikma,

17. Yohmikma chofak ish ishi cha, haksobish a hlopullichit, okhissa ya ish anvhlicha hi oke : micha chin tishu bilia ya hi oke. Micha chin tishu ohoyo ha ish yumihchi ak inlà hi oke.

18. Pilla ho ik ia, ish im ahnikmvt chị kvllo, ahoba he keyu; afummi hannali ho chin toksuli kvt, ilhtono tishu hạ inlohmi tuklo ka holitopa tuk vt chim iti laui tuk oke: micha chin Chitokaka Chihowah yot nana moma ka ish yumihchikma, chi yukpala hi oke.

19. Chị nan vlhpoa hochito, micha chị nan vlhpoa chipunta aiena ka vttahpi nakni puta ka chin Chitokaka Chihowah ya ish $\underline{\mathrm{i}}$ hullocha hi oke : vttahpi chi wak hobvk a isht ish toksvla he keyu, micha chin chukfi vlhpoa vttahpi hishi ya ish ma he keyu hoke.

20. Afummi hosh ont afummikma kanima ho Chihowah

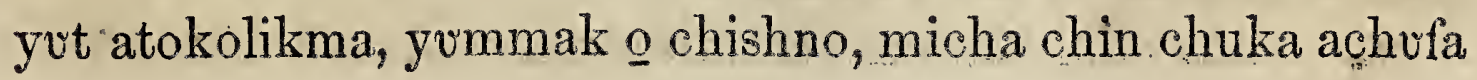


aiena kvt chin Chitokaka Chihowah itikba ya ish ai vpa hi oke.

21. Atuk o ik ai ono kvt takali cha, hanali okpulo, keyukmot nishkin lvpa, keyukmvt ik ai onot.ik achukmo nana hosh takalikma, chin Chitokaka Chihowah ya vbit isht ish ai okpucha he keyu hoke.

22. Chi holihta okhissa anukaka yak o ish ai vpa hi oke: hatak ik kashofo, micha kashofa aiena kvt iti lauit issi, micha lapitta chohmichit ish pa hi oke.

23. Peh issish ano chik po ka hi oke, oka chohmihchit aka ish hlatubla hi oke, achi tok.

\section{CHAPTA XVI.}

TUSHI Abib a ish ithaiyana cha, chin Chitokaka Chiho1 wah ya vbạnublit ontia isht ish im ieshashke: hushi Abib ak o chin Chitokaka Chihọwah yut ninak o Echip a apehlichit chi kohchi tok oke:

2. Chihowah yut kanima ho ai atokolit hohchifo a bohlikma, yummak o chi nan vlhpoa chipunta, micha chi nan vlhpoa hochito aiena ka ai ishit vbanvblit ontia ya ubit chin Chitokaka Chihowah ya isht ish ai okpvehashlke.

3 Puska shatummi ya aieninchit ish pa he keyu ; nitak untuklo ho puska ik shatummo, ilbusha puska pulla ka aieninchit ish pa hi oke; (anukwaya hosh Echip yakni ya kohchut ish minti tok okvt:) yumohmi hosh chi okchaya nitak moma kut Echip yakni ya kohchut ish minti tok nitak a ish ithaiyanashke.

4. Hush aiasha fullota moma ka puska shatummi nana kia ik a haiako ho nitak untuklashke; micha nitak vmmona. opiaka vbit isht ish aiokpvchi nipi vt ninak a itonla na onna he keyu.

5. Chi holihta okhissa kanima kia chin Chitokaka Chihowah yut chima ka anukaka ya vbanvblit oñtia ai vbit isht ish aiokpvcha he keyu hoke.

6. Amba chin Chitokaka Chihowah yak osh kanima ho 
atokolit hohchifo a bohlikma, yummak o opiakma hushi ut okatula ho Echip a a kohchut ish minti tok ont vlhpiesakma, vbanvblit ontia ya isht ish aiokpvcha hi oke.

7. Miknut chin Chitokaka Chihowah yut kanima ho ai atokolikmä ish ai apushli cha, ish pa hi oke: atuk osh onnahinlikma falamvt chi bina ha ish ona hi oke.

8. Nitak hannali ho puska ik shatummo ya ish pa hi oke; atuk osh nitak isht untuklo hokvto chin Chitokaka Chihowah im itunaha holitopa ya hi oke : yummak oka na toksuli nana kia ish yumihcha he keyu.

9. Nitak hullo untuklo ho ish hotihna hi oke; nitak kaniohmi ho onush a ishit busha ya ishit bushlit, isht ish ia mak inli hosh, nitak hullo untuklo ka hotihnvt isht ish iashke.

10. Micha nitak hullo puta impuchi ya chin Chitokaka - Chihowah ya isht ish aiashakmut, chin Chitokaka Chihowah yout na chi yukpali tuk pulla mak o ilap ahnit chibbak foyuka im issa ya aieninchit chln Chitokaka Chihowah ya ish imashke.

11. Micha chin Chitokaka Chihowah itikba ya chishno micha chiso, micha chiso tek, micha chin tishu hatak, micha chin tishu ohoyo, micha Lefait vt chi holihta okhissa anuka aiasha, micha okla inla, micha iki iksho, micha ohoyo vlhtakla hvch itin takla aiasha aiena kvt, kanima ho chin Chitokaka Chihowah yut hohchifo a bohla chi hosh ai atokoli tukma, yumma a yukput ish aiashashke.

12. Micha Echip a hatak yuka hosh ish aiahanta tuk vt ish ithaiyanashke: micha nan vlhpisa bilia ilvppa puta ka ithaiyanvt, ish yumihchashke.

13. Chim onush micha chi paki ya hoyot ish tahli ha yut, tabenakel ai impvchi ya nitak untuklo ho isht ish aiasha hi oke.

14. Micha chim impuchi ya nan isht chi yukpashké, chishno, micha chiso, micha chiso tek, micha chin tishu hatak, micha chin tishu ohoyo, micha Lefait, micha okla inla, micha iki iksho, micha ohoyo vlhtakla chi holihta okhissa anuka aiasha aiena kvt na yukpashke: 
15. Chihowah ak osh kanima ho ai atokolikma, chin Chitokaka Chihowah ya nitak untuklo ho impvchi holitopa isht ish im aiashashke: na chi waya moma, micha chibbak na toksahanli tuk moma ka chin Chitokaka Chihowah yvt na chi yukpala chị hoka; yưmohmi hoka na chi yukpa pulla hi oke.

16. Kanima ho ai atokoli tukma, nakni chim asha moma kut chin Chitokaka Chihowah ya afummi achufakma, hituchina ha itikba ya im itunaha hi oke: prska ik shatummo impvchi, micha nitak hullo puta impvchi, micha tabenakel ai impuchi: micha ibbak bieka hosh Chihowah itikba ya ona he keyu hoke:

17. Chin Chitokaka Chihowah yut nan ishit yukpali chima tuk pulla mak $\underline{\underline{o}}$, hatak puta kvt ai ima he vlhpiesa puta kvt imashke.

18. Chim apehlichi puta hlopulli, chi holihta okhissa chin Chitokaka Chihowah yvt chima tok a nan apesa vhleha, micha nan vlhtoka vhleha aiena ka ish ikbashke; mikma okla ha nan vlhpisa aivlhpiesa ho im apihisa hi oke,

19. Aivlhpiesa vlhpisa ka ish shanaiyacha he keyu: hahatak yohmi hoka, ish ahna he keyu, micha nahulbina ish isha he keyu; na hvlbina hut hopoyuksa nishkin a lvp. lichi hoke; micha aivlhpiesa im anumpa ha shanaiyvchi hoke.

20. Aivlhpiesa bieka kak o ish iakaiya hi oke; yvmohmi hosh chin Chitokaka Chihowah yut yakni chima ka chi okchaya cha ieshit ish aiasha hi oke.

21. Chin Chitokaka Chihowah im alta bilika yumma ish ikba chi kut itilokoli nana kia ish hokcha he keyu.

22. Micha na holbut toba nana kia chin Chitokaka Chihowa yut shitilema ka ish hilecha he keyu, (achi tok.)

\section{CHAPTA XVII.}

W AK hobvk nana kia, keyukmvt chukfi vt ik ai ono, keyukmut pisa ik achukmo aiena vbit, chin Chitoka. 
ka Chihowah ya isht ish aiokpucha he keyu: yummak okvt chin Chitokaka Chihowah ya nan isht $\underline{i}$ yuwala yoka.

2. Huch itin takla chi holihta okhissa, chin Chitokaka Chihowah yưt chihima aiachufona anukaka ya hatak okmá, keyukmut ohoyo hokmá nana hosh chin Chitokaka Chihowa itikba ya nan itim apesa ya vbanubli cha yoshuba yumihchi tuk,

3. Micha ivt na holbvt toba inla, hushi, keyukmut hush ninak aya, keyukmut vba nana puta, yummak oka miha li tuk keyu ho im antia tuk, micha aiokpvchi tuk osh haiakakma;

4. Atuk o chim annoa cha, ish haklo mot, achukmalit ish asilhhvchi tuk, atuk o yakeh, ahli ho micha nana kvt atokowa ka na yuwala yumma chiyuhmi kut Islael a yumohmi tuk achinikma;

5. Yohmikma hatak, keyukmut ohoyo yummut na yoshuba yumma yumihchi kash, hatak yumma, keyukmut ohoyo yưmma pulla ka chi holihta okhissa ya isht ish ona cha, tuli ishit ish boli na, illa hi oke.

6. Witnes tuklo, keyukmut tuchina kanima hosh anoli ho, illa he vlhpesa kvt illashke, amba witnes achufa hosh anoli ho, illa he keyu hole.

7. Uba chi kvt witnes puta ibbak ak osh tikba onvtola hi oke, i himmak a yak o okla moma ibbak vt onvtola hi oke; Yumohmi hosh nana ik achukmo huch itin takla itoyula hash ishit hvsh kancha hi oke.

8. Issish hlatapa tuk o issish hlatapa chi, anumpa iti onochi, micha iti fahama puta, anumpa ishit itachowa puta nana kut chi kullo fehna ho ish im apesa chị, chi holihta okhissa anukaka ya isht chim vlakma, ish wakaya cha, chin Chitokaka Chihowah ak osh ai atokoli tukma yummak o ish onashke.

9. Micha naholitompa isht asha vhleha, Lefait vhleha, micha nan apesa aiena nitak yumma asha tuk a ish im ona cha, ish im asilhha hi oke; mikma yummak osh nan vlhpisa anumpa hoka chim anola hi oke.

10. Micha kanima ho chin Chitokaka Chihowah ak osh 
ai atokoli tuk a asha yosh anumpa chim anoli tuk mak $\underline{0}$, ish yumihcha hi oke; micha nana chim anoli tuk mak $\underline{o}$ moma ka ithaiyanut ish yumihinchashke,

11. Nan vlhpisa anumpa apesut chim abvchi tuk mak $\underline{0}$, micha anumpa vlhpisa chim anoli tuk mak $\underline{o}$ ish yvmihchashke: anumpa vlhpisa chim otuninchi tuk a fullotvt isht impakma, keyukmut vlhfubekimma ish ia he keyu hoke.

12. Mikma hatak kuna hosh ilapunla cha, naholitompa isht vtta yot chin Chitokaka Chihowah itikba ya hikivt, yummak o nan isht ai ahanta ka ik $\underline{i}$ haponaklo, keyukmut nan apesa yo ik $\underline{i}$ haponaklokmut, hatak yummut illi pulla hi oke; micha Islael a nan ik achukmo ya ishit ish kancha hi oke.

13. Mikma okla moma kut haklokmut nukshopa cha, himmakma nana ka ilapunlut ik yumihcho ka hi oke.

14. Chin Chitokaka Chihowah yot yakni chima tok a ish onakmut, ish ishi cha ish aiashakmvt, Oklushi inla puta si apakfoyupa aiasha ka chohmit sa pehlicha chị ka, mikko ikbit binilichi lashke, ish achikmvt,

15. Ik kaniohmi hoh kia kanima ho chin Chitokaka Chihowah ak hosh atokolikma, chi pehliecha chi ka, miko ikbit ish binilicha hi oke, chitibapishi vhleha aiachvfa ho chi pehlihchi miko ikbit ish binilicha hi oke, chitibapishi keyu, okla inla yo chi pehliechi ikbit ish binilicha he keyu hoke.

16. Yohmi kia issuba ha apaknvehit il ai asha he keyu; micha issuba ha apaknveha chi kak osh okla ha falumminchit Echip a pila he keyu hoke; Chihowah yut Himak a pilla ha yumma pilla ka falamot ish ia he keyu chim achi tok oka.

17. Micha chukvsh vt fullotut naksika ik aiyo ka chi, ohoyo ha hullocha hi oke; vttahpi chi wak hobvk $\underline{a}$ isht ish toksula he keyu, micha chin chukfi vlhpoa vttahpi hishi ya ish ma he keyu hoke.

18. Atuk osh yakohma hi oke; im apehliechika miko im ai om binili holitompa ya om binilikmvt, nan vlhpisa ilvpput na holitompa isht vtta Lefait vhleha itikba itoyula ka akohchit hobachit holisso ha a fohkit a holissochashke. 
19. Mikma iba itoyulashke, mihma okchayvt ahanta he nitak moma ka yumma ai itim anumpohonlashke; yumohmi hosh Chitokaka in Chihowah ya $\underline{\mathrm{i}}$ nukwia he, micha nan vlhpisa ilvppa anumpa puta, micha anumpa vlhpisa bilia iluppa puta aiena ka yumihincha chi hosh ithaiya na hi oke.

20. Yvmohmi hosh chukvsh vt vba takalit itibapishi vhleha ha ik i shahlo, micha anumpa hash naksika pit fullotut ishtimpakimma, keyukmut vlbfubekimma ik aiyo kashke: yumohmi hosh Islael ai itin takla ya, yumma micha im vlla vhleha aiena im apehliechika ieshi nitak falaiachashke. (achi tok.)

\section{CHAPTA XVIII.}

AHOLITOMPA isht vtta vhleha, Lefait vhleha, Lefait 1 apehlichi moma aiena kut Islael a takkla akashapa, micha aiisha he vlhpesa ya isha he keyu; luak ishit hukmit, Chihowah im issa, micha im ai isha he vlhpesa aiena kak $\underline{0}$ vpa hi oke.

2. Yvmohmi hokvt itibapishi vhleha itin takla ya yakni ik im iksho ka hi oke, im achi tuk mak osh, Chihowah ak o ai isha he vlhpesa yoke.

3. Micha okla ha im ai ishi kut, wak okmá, keyukmot chukfi hokmá nana ho vbit isht aiokpuchi im issakmvt, naholitompa isht vtta ya fulup, itisupi tuklo micha takoba aiena kak o ima hi oke, mikma iluppak osh naholitompa isht vtta yot immi ha hi oke.

4. Micha onush chi waya vmmona, chim oka-paki, micha chị bila, micha chin chukfi hishi vlmo vmmona aiena ka ish ima hi oke.

5. Chihowah hohchifo pulla ho hikivt nan isht im ahanta chị ho, yưmma, micha ushi vhleha aiena kvt bilia chị ho, chim apehlichi moma ka akohchit chin Chitokaka Chihowah yvt atokoli tuk oke.

6. Yohmi ka Lefait osh Islael a fullota moma holihta okhissa kanima ai vtta tuk osh kohchvt vlakma, micha ka- 
nima ho Chihowah yout ai atokoli tuk a, im anukfila okluhut bunna hosh vlakmvt,

7. Yohmikmut itibapishi vhleha Lefait vhleha hvt Chihowah itikba ya hieli kvt yvmihchi ka chohmit in Chitokaka Chihowah hohchifo pulla ho nan isht ahanta hi oke.

8. In tikba im ilayak kvnia tuk vlbi ka, ishikmvt, vpa chi kut tikba asha, $\underline{i}$ kushkoa chohmi ka isha hi oke.

9. Chin Chitokaka Chihowah yut yakni chima tok a ish onakmvt, oklushi yumma puta a yumihchi nan isht yowala ya hobachit yưmihcha chi ka ish ithuna he keyu.

10. A huchi takla ka, ushi, keyukmvt, ushetik a luak hlopullichi keyukmvt, nan ithana ilahobi keyukmvt, nitak nan isht ithana ilahobi, keyukmvt, atulwvchi, keyukmvt ohoyo isht ahullo,

11. Keyukmut shimohvchi, keyukmot, nana aholhkunna i ponaklo, keyukmvt, nakni nan ishtahullo, keyukmvt, hatak illi itim anumpulit nan anoli nana kia ik ai iksho ka hi oke.

12. Nana iluppa puta yvmihinchi moma ka Chihowah yvt nan ishit yuwala yoke : micha nan ishit yuwala iluppa pulla kak o, Chihowah yut chi tikba ya akocha wehli tuk oka.

13. Chin Chitokaka Chihowah ya ish im ahlashke.

14. Oklushi iluppa ish isha chi puta kut, nitak nan isht ithana ilahobi, micha nan ithanvt anoli ilahobi puta ka $\underline{i}$ haponaklo tuk oka: yohmi kia chishno ano chin Chitokaka Chihowah yot ish yomihcha he ya ik chim ahno tuk oke.

15. Chin Chitokaka Chihowah yut a hvch itin takla hvsh itibapishi vhleha ai achvfa yosh vno chohmi ho, Hopaii chi hilecha chi hoke; yummak $\underline{0}$ ish $\underline{i}$ haponakla hi oke.

16. Holeb a itunahvt ish aiasha nitak mot, chin Chitokaka Chihowah ya ish im ahni moma kvt, An Chitokaka Chihowah itih ha anonti ak haklo kashke, micha himakma luak chito iluppa ak peso kashke, yumohmi hosh ik sullo kashke, ish achi tok.

17. Mihma Chihowah yvt, Anumpuli kvt, vlhpesa ho anumpulishke, vm achi tok.

18. Itibapishi vhleha im itin takla ya , Hopaii chishno chohmi ho i hilechi la hi oke, micha um anumpa ha itih ha 
fohkalikma, im apesa li tuk mak $\underline{\text {, }}$ moma ho im anumpula hi oke;

19. Atukma yakohma hi oke, kuna hosh sa hohchifo pulla ho anumpulikma yưmmut im anumpa hạ hakla he keyukma im a hoyo la hi oke.

20. Amba hopaii vt sa hohchifo pulla ho, yumma anumpula chi im apesa li tuk keyu ho, Anumpule lih mihakmvt, keyukmvt, naholbut toba hohchifo pulla ho anumpulikmot, hopaii yumma fehna kut illa hi oke.

21. Yohmi ka chi chukvush anukaka, Katiohmi kak osh anumpa Chihowah yot anumpuli tuk keyu ka il akostinincha chi cho? ish achikma,

22. Hopaii vt Chihowah hohchifo pulla ka anumpuli tukma, nana kvt ik yumohmo, micha ik aiahlokmut, yummak osh nana Chihowah jot anumpuli tuk keyu; amba hopaii vt ile yimmit anumpuli tuk a hi oke; yumma hush i nukshopa he keyu hoke, (achi tok)

\section{CHAPTA XIX.}

KLUSHI puta ka chin Chitokaka Chihowah yut i yakni ya chima tuk vt ai ishit kanchikma, vlhtobvt in tvmaha holihta puta, micha in chuka puta ish aiashakmot,

2. Ish ai asha chi ho chin Chitokaka Chihowah yut chima ka chi yakni iklunna tumaha holihta tuchina ho ish ilai atokola hi oke.

3. Hina ish ilai ikba hi oke: micha chi yakni aivhli ish aiasha chi ho, chin Chitokaka Chihowah yut chima tuk a kushkoa tuchina ho ish ita kushkola hi oke : yohmikma hatak bịka ubi puta kut yummak o pit malela hi oke.

4. Hatak vbi vt okchaya chi hosh yumma pit maleli kvt yakohma hi oke: Kvna hosh ik akostinincho hosh, yummak oka chashpo ka $\underline{i}$ nukkilli chatuk keyu hosh, chuka a bilika hatak o vbi ;

5. Yohmi kut hatak osh chuka a bilika hatak o ita tuklo cha iti chanla chị hosh iti anukaka ya itonachi mvt, iti chan- 
lit kinvffa chi hosh, iskifa ya ibbak ishit fahama na, noshkobo vt vpi ya shufa cha, chuka a bilika hatak ash onvtola na, illi ; yohmikma tumaha holihta iluppa puta ai achvfa ka malelit ona cha, okchaya hi oke.

6. Issish hlatapa tuk a il aiokha hot chukvush lushpa mo-

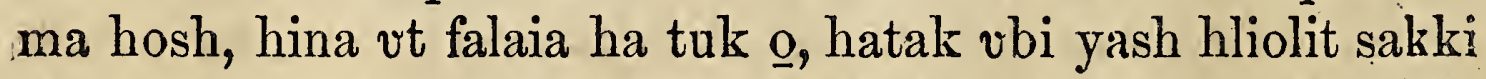
cha, vbi yoba na, chashpo i nukkilli ha tuk keyu hokvt, illa he vlhpesa keyu kia,

7. Yvmohmi hoka tumaha holihta tuchina ho ila filvmmichit ish atokola chi ka chi miha lishke.

8. Atuk o chin Chitokaka Chihowah yvt chiki vileha ha anumpa kvllo il onochit im issa tuk mak osh, chim aivhli ya chitoli cha, chiki vhleha ha im issa tok yakni moma ka chimakma,

9. U'm anumpa puta iluppa himak nitak a chim ahanchi li ka holitoblit ish yumihinchit, chin Chitokaka Chihowah ya ish holitobli cha, $\underline{i}$ hina puta ka ish anohowa na biliakmot: tuchina iluppak ash, himakma tumaha holihta tuchina ho ish il ibafohkashke,

10. Yvmohmi ho chi yakni ya chimmi ha chi ho chin Chitokaka Chihowah yut chima tok a, nan ashuchi keyu. issish vt ik ahlatapo kashke: micha yohmi ho issish vt ik chi onvtolo kashke.

11. Yohmi kia hatak nana hosh chuka a bilika hatak a $\underline{\underline{i}}$ nukkilli cha, im aiehchit itonla tuk osh, $\underline{1}$ sanalit wakaya cha, isht illa he vlhpesa ho fahama na, illi ma; malelit tumaha holihta iluppa puta kash achufa ka onakma:

12. Yohmikma in tvmaha holihta im asunonchi vhleha hut pit hoyot, ont ishtvla cha, issish hlatapa tuk a aiokha ya ibbak fohkashke, yohmi na illashke.

13. Chi nishkin vt ik i nukhaklo kashke; amba nan okpuni keyu issish hlatubli ya Islael a ai ishit ish kanchashke, yơmohmi ho aivlhpesvt chi mạhayashke.

14. Yakni a binilit ish iesha chi chin Chitokaka Chihowah yot chima tok, chị yakni ya chin chuka abilika i yakni vlhpisa, yummot nitak chashpo ma, a hilechi ha tok a ish kanvllicha he keyu. 
15. Witnes achvfa hokvt hatak osh yoshuba tok, keyukmut nan ashuchi tuk nañ ashivchi nana ho ashvchi tuk a $\underline{\mathrm{i}}$ sanalit hikia he keyu; witnes tuklo nana itih ho keyukmot, witnes tuchina nana itih hok mak o nana kvt isht aiahla hi okè.

16. Witnes ik ahlo yosh hatak nana kia i sanalit nan ik achukmo anola chi hosh wakayvt hikiakma;

17. Yohmikma hatak ita chowa kash osh ita tuklo hosh Chihowah itikba, na holitompa isht asha, micha nan apesa vhleha aiena kvt nitak yumma aiasha tuk a itikba hiela hi oke.

18. Mikma nan apesa vhleha hvt achukmalit asilhhucha hi oke: a tuk $\underline{0}$, yakeh, witnes vt witnes ik ahlo yosh anumpa ik ahlo ya itibapishi ha isht i sanalit anoli tukma,

19. Yohmikma itibapishi ha nana yumihcha he im anukfila tuk a ilap ak o chohmit ish yumihcha hi oke: yohmi hosh a huch iti takla ya nan ik achukmo ka isht ish kancha hi oke.

20. Mikma inla aiasha yvt haklo cha, nukshopakmvt, himmakma nan ik achukmo yohmi ka a hưch iti takla ya ik yumihcho kashke.

21. Micha chi nishkin vt ik i nuklakklo kashke: amba im ilhfiopal atukma im ilhfiopak ashke, nishkin atukma nishkin ashke, hoti yatukma noti yashke, ibbak atukma ibbak ashke, iyi yatukma iyi yashke. (achi tok)

\section{CHAPTA XX.}

TANAMPIT kohchut ish ia tuk osh, issuba puta, micha 1 tonup iti chunaha puta, micha okla laua kvt chị shahli ho, ish pisakmvt chik i nukshopo kashke: chin Chitokaka Chihowah (yummak) osh Echip yakni ya apehlichit chi kohchi tok vt chiba foyuka hoka.

2. Atukma yakohma hi oke; itibit bilika hush onakma, naholitompa isht vtta yvt bilika onvt okla ha im anumpula hi oke. 
3. Micha Islael ma, hush haklashke; himak nitak a huchin tunup a hưsh itiba chị hosh bilika hush onashke : hvchi chukush vt ik ello kashke; hvchi nukshopa na, micha hvchi wunihinchi na, micha yumma pulla kak o ik huchi... nukhlakancho kashke.

4. Huchin Chitokaka Chihowah ak osh hvchin tunup a huvchim itibit, huchi okchalincha chị hosh huvchi awant ia hoka im acha hi oke.

5. Mikma nan vlhtoka vhleha hvt okla ha im anumpulit, Hatak nanta hosh chuka himmona ikbi tuk osh ik hullocho kisha cho? falamut in chuka iashke: keyukmut itin tanampi kut illi na, hatak inla hosh hullochi ná.

6. Mikma hatak nanta hosh pakvopi a hollokchi ya hokchi tuk osh waya ka ik aiapo kịsha cho? yưmmak okvt fâlamvt in chuka iashke, keyukmot itin tanampi kvt illi na, hatak inla hosh ai vpa na.

7. Mikma hatak nanta hosh ohoyo itim vlhpoa tuk osh, ik esho kisha tuk oh cho? yummak okvt falamut in chuka iashke, keyukmvt itin tanampi kvt illi na, hatak inla hosh ishi na, achashke.

8. Mikmvt vlhtoka vhleha hut achakalichit okla ha im anumpulit, Hatak nanta hosh nukshopa, micha chukvush elli tho? yummak okut falamvt in chuka iashke, keyukma chukvosh elli ka chohmit itibapishi vhleha hut chukvvsh elli na, acha hi oke.

9. Atukma yakohma hi oke, nan vlhtoka vhleha hvt okla ha im anumpulit ont tahlikmut, okla hạ pehlicha chị ka tushka chipota i kapotuni vhleha okla atokola hi oke.

10. Tumaha holihta ish itiba chi hosh bilika ish onakmut iti nanaiya anumpa ish im anumpula hi oke,

11. Atukma yakohma hi oke, iti nanaiya anumpa falama chima cha, chin tiwikma, yohmikma yakohma hi oke, yvmma aiasha tuk okla moma kut chi nutaka cha, chin toksula hi oke.

12. Amba chiti nanaiya he keyukma, amba chin tanampi pulla kak o ahnikma, abinachit ish apakfoyupa hi oke.

13. Atuk o chin Chitokaka Chihowah yot chibbak foh- 
kikma, nakni yumma moma ka bushpo falaia ishit fahamvt ish kanchashke.

14. Amba ohoyo micha vlla, micha nan vlhpoba, micha nana hosh tumaha holihta ya aiasha kvt moma, na wehpoa puta moma fehna ka ish isha hi oke; chin tunvp vhleha i na wehpoa yummak oka chin Chitokaka Chihowah yut chima tuk oka, ish ai vpa hi oke.

15. Iluppak o tumaha holihta chi hopaki fehna puta oklushi ilvppak inli in tumaha holihta aiachvfa keyu puta ka ish y.umihchashke.

16. Amba okla iluppa in tomaha holihta puta chi yakni ya chi ka, chin Chitokaka Chihowah yut chima tok ano, nana hosh fiopa kvt ashakma ish okchalincha he keyu hoke.

17. Amba ishit kanchit ish vmohmicha hi oke: yummut Hittait, micha Amolait, micha Kananait, micha Pelissait, Hifait, micha Chebusait aiena ka, chin Chitokaka Chihowah yot chi miha tuk mak 0 ish yumihcha hi oke.

18. Yvmohmi hosh nan ashvchi ishit yuwala i naholbut toba $\underline{\mathrm{i}}$ ywmihchi chatuk vt ik hvchik hanancho ho: yohmikmut hvchin Chitokaka Chihowah ya huchik i yoshobo kashke.

19. Tumaha holihta ya abinachit ish aiasha tok hopaki cha, in tanampit ish isha chi kut, iti ya iskifa ishit chanlit ish okpuna he keyu, ish ai vpa hinla hokvt (osapa iti aiasha kut hatak aiokchaya yoka:) abinanchit ish aiasha chanlit akakohlit isht ish i sanala he keyu hoke.

20. Peh iti ai upa chatuk keyu puta ish ithana kak illa hosh okpunit chanlit ish akakohla hi oke: tumaha holihta yosh chin tanampikma, aiatukko holihta ish ikbi cha, ish im aiya hi oke. (achi tok.)

\section{CHAPTA XXI.}

YAKNI ish ai isha chị ho chin Chitokaka Chihowah yut 1 chima tok a achufa hosh illi cha, osapa ai itonlut haiakakma, micha kuna hosh vbi tuk a ik ithanokma, 
2. Yohmikma chim asunonchi vhleha, micha chi nan apesa vhleha aiena kvt kohchvt ia cha, illi itola kash hikivt ia hosh tumaha holihta puta apakfoyupa ka onvt apesa hi oke.

3. Yohmikma yakohma hi oke, hatak illi yash et $\underline{i}$ bilika tumaha holihta ak osh tumaha holihta yumma im asunonchi vhleha fehna kvt wak tek himmita ishit toksvli chatuk keyu, micha ikunla abanvt nana shali chatuk keyu ka ish ashke.

4. Micha tumaha holihta yumma im asunonchi vhleha hot wak tek himmita yash oka akinafa okfa nana awaya keyu, micha ahollokchi keyu yo isht ona cha, wak tek himmita yash ikunla ya okfa yumma a chanlit tublashke.

5. Mikma na holitompa isht asha vhleha, Lefai ushi vhleha hut bilika onvt (yummak osh nan isht im ahanta he, micha hochifot aiokpahancha he, chin Chitokaka Chihowah yvt atokoli tok oka,) yummak ash im anumpa ho anumpa itachowa puta, micha isso tok moma aiena ka nana kvt vlhpesa hi oke.

6. Mikma tumaha holihta hatak illi tuk et $\underline{\underline{i}}$ bilika, yumma im asunonchi vhleha moma kvt okfa yumma wak tek himmita noshkobo atupa tuk ash paknaka ya ibbak ai achefa hi oke.

7. Micha afalaminchit, Issish iluppa pibbak ak osh hlatu-. bli tuk keyu, micha pi nishkin vt pisa tuk keyu.

8. Chihowah ma, chim okla Islael a falumminchit ish chumpa tuk vt ish $\underline{i}$ nukhaklashke, micha chim okla Islael apehlichi a nan okpuni tuk keyu issish hlatapa ya chik onocho kashke, acha hi oke. Mikma issish hlatapa tuk vt i kashofa hi oke.

9. Yvmohmi hosh Chihowah itikba ya ai vlhpiesa ish yumihinchikmvt, nan okpuni tuk keyu issish hlatapa ya ishit ish kanchashke.

10. Hvchin tunvp a in tanampit kohchvt ish ia tuk a, chin Chitokaka Chihowah yot chibbak a fohki na, yukachit ish ishi tuk osh,

11. Atuk osh yuka vhleha ha ohoyo pisa ai okli kvt ibafoyuka na, ish pisa cha, itauwaya chi bunnakmvt, 
12. Yohmikmut ishit chin chuka isht ish ona hi oke; mikma noshkobo ha vmo cha, ibbak ushi akchush a vma hi oke.

13. Mikmvt yuka $\underline{i}$ nafohka y $\underline{a}$ shúfi cha, chin chuka ya ahantut iki, micha ishki ya ishit yahaya na, hvshi achvfa vlhpiesashke; atuk o i himmak a yo ish iba chukowa cha, i hatak ish tobakma, chi tekchi toba hi oke.

14. Atukma yakohma hi oke, chik ahnincho hokmut kanima ho ia bunnakmaik ia, ish im ahna hi oke; amba toli holisso isht ish chumpa he keyu pulla; vlhpoyak nan aivlbi ish ikba he keyu, ish hofahyali hatuk okvt.

15. Hatak osh tekchi in tuklo cha, achufa kvt i holitopa mvt, achufa kano ik ahnincho; atuk o holitopa yash osh, micha ik ahnincho yash osh ita tuklot vlla im ashakma, atuk o ik ahnincho ushi yash osh ushi vtta ahpi yokma,

16. Yohmikma yakohma hi oke, ushi vhleha nana im asha tuk vt imakmvt holitopa ushi atuk a, ik ahnincho ushi ak osh vtta ahpi yo muhli tuk a, tikba vtta ahpi ikba he keyu.

17. Amba ik ahnincho yash ushi ak o nan im asha moma kash osh kvshkoa tuklo ho ima cha, yummak osh vtta ahpi ya anolashke; yummak osh imi hlampko ishtia vmmona yokut, vtta ahpi ai isha he vlhpesa vt immi hoke.

18. Hatak osh ushi vt chukvsh ilapunla, micha anumpa $\underline{\mathbf{i}}$ kobuffi shahli yosh im anta kvt, iki micha ishki im anumpa ha $\underline{i}$ haponakla he keyu, micha fummi kia, im anumpa ha haponakla he keyukma;

19. Yohmikma ịki, micha ishki ita tuklo kvt halvllit ishi cha, aivtta holihta okhissa, micha in tumaha holihta im asunonchi vhleha itikba ya isht im onashke.

20. Micha in tumaha holihta im asunonchi vhleha, Piso iluppot chukvsh ilapunla, micha pim antia he keyu, pim anumpa ha im antia he keyushke, isikopa micha okishko shahli yoke, im acha hi oke.

21. Mikma in tumaha holihta hatak moma kvt tvli ishit boli na illa hi oke: yumohmi hosh ahvchi iti takla nan ik achukmo ka ịshit ish kanchashke, mikma Islael moma kvt haklokmot nukshopashke. 
22. Micha hatak vt jisht illa he vlhpesa ho nan ashvchi yumihchi cha, illa chi vlhpesa na, iti ish in takalichi tukma;

23. Nipi vt iti ya takali na ont onna he key u jk kaniohmi kia nitak yummak inli ho ish hohpi pitilashlke (iti yo atakali hokvto Chitokaka a kallakshi hokä; yvmohmi ho chị yakni ya chi ho chin Clitokaka Chihowah yvt china tok vt ik liteho kashke, (achi tok.) y

\section{CHAPTA XXII.}

CHITIBAPISHI $\underline{i}$ wak toksvli, keyukmvt in chukfi vlhpoa yot kunia na, ish pisakmut, i filvmmit ish luma he keyu ; ik kaniohmi kia falumminchit chitibapishi a isht ish im ona pullashke.

2. Atuk o chitibapishi hot chị bilika keyu, keyukmot chik ithano yohmit, yumohmikmot chin chuka isht ish ona na, chim ahanta tuk o, chitibapishi vt hoyot aya hi oke: mikma falumminchit ish ima hi oke.

3. Im issuba nashoba ya ish yumihchikmvt, $\underline{i}$ nafohka ya ish yumihcha hi oke: micha na kunia puta chitibapishi vt i kunia tuk o ish ahayuchikmut ish yumihchi ak inla hi oke, ish luma he keyu hoke.

4. Chitibapishi im issuba nashoba, keyukmvt $\underline{\mathrm{i}}$ wak toksuli hina takla aka itula tuk osh itonla na, ish pisa mot, i filummit ish luma he keyu : yumma apelvchit anonti ish wakayuchi pulla hi oke.

5. Ohoyo hut hatak immi ha fohka he keyu, mikma hatak ak kia ohoyo i nafohka ya fohka he keyu hoke: yumma yumohmi moma kut chin Chitokaka Chihowah nan isht yuwala yoka.

6. Hina takla iti hikia ho, keyukmvt aka yo hushi im vlhpichik vt chi tikba talaia yobakmá, hofullichi yokmá lubonchi yokmá, nana ho ishki vt hofvlli yash, keyukmvt lubonchi on ashakma, ishki yo ushi aieninchit ish isha he keyu hoke.

7 Amba ik kaniohmi kia ishki ya ish hikvchi cha, ushi 
ya ish isha hi oke: yumohmi hosh chim achukma cha, chị nitak a ish falaiachashke.

8. Chulkarimiona yo ish ikbikmut, chin chuka paknaka ya aholihta ish ikbashke $\therefore$ yumohmi ho hatak nana kia yumma aka pot itula ma, isssish a chin chuka ya isht chik onocho pullashke,

9. Pâki pi chim hollokchi ya na nihi ilaiyukali ho ish ahokcha he keyu: ish yohmi na, chi na nihi ish hokchi tuk waya kvt, micha pakvpi chim ahollokchi a waya kvt ik achukmo nà.

10. Wak toksvili y issuba nashoba itapotolit yakni ish patufficha he keyu holke.

11 Nafohka ilaiyukali chukfi hishi tunna, micha linen chohmi ka himona achvfa ish fohka he keyu hoke.

12. Chim anchi ya ish il anchi ut chukbi ushta ka i poshota ish ikbashke.

13. Hatak kvna hosh ohoyo itauwaya tuk osh iba chukowa mot shitilema,

14. Micha na mihachit isht anumpula he anumpa onochit anumpa ik achukmo ka hohchifo ha onochi cha, Ohoyo ilvppa ishi li tuk osh im ona li mut, ohoyo himita keyu ha pisa lishke, achikma ;

15. Mikma ohoyo hash iki, micha ishki ita tuklo kvt ohoyo himita yut hatak haleli yatuk keyu ishtatokowa ya tumaha holihta im asunonchi vhleha ha isht im onvt holihta okhissa ya isht im onashke.

16. Mikmvt ohoyo himita iki vt im asunonchi vhleha hạ, Sưso tek vt hatak ilvppa tekchi ya chị ho ima li tuk ak $\underline{o}$ shitilemushke.

17. Micha yakeh, na mihachit isht anumpula he anumpa : onochit, chiso tek vt ohoyo himita ya tuk keyu ho pisa lishke, achishke; atuk $\underline{0}$, yohmi kia suso tek vt hatak haleli ya tuk keyu isht otvni ilvppak oke, achashke. Mikmvt tumaha holihta im asvnonchi vhleha itikba ya nantunna yash patalashke.

18. Mikma tumaha holihta yumma im asunonchi vhleha hash osh hatak yummak ash ishi cha na hotopa onocha hi oke. 
19. Micha tvli huta shekel tahlepa achvfa ho i hoyo cha, ohoyo himita yash iki ya imashke: Islael ohoyo himita hatak haleli keyu ho hohchifo ik achukmo onochi hokut; mikma tekchi yashke; okchayut ahanta nitak takla kut kancha he keyu.

20. Amba nana ilupput ahli ha tuk ma, atuk o ohoyo himita vt hatak haleli ya tuk keyu isht atokowa vt ik im ikshokma,

21. Yohmikma ohoyo himita yash iki in chuka okhissa ya isht onakma, in tumaha holihta yash hatak vhleha hvt tuli ishit boli na, illashke; iki in chuka ya haui tobut ahanta cha, Islael i yakni ya nana makali ya yumihchi hokvt: yumohmi hosh nan ik achukmo ka huch itin takla ai ishit ish kanchashke.

22. Hatak vt ohoyo i hatak asha yo iba tushki na, pisakma, ita tuklo hosh illashke; ohoyo iba tushki tuk hatak ash : micha ohoyo hash ita tuklot illashke: yohmi hosh nan ik achukmo ya Islael a ai ishit ish kanchashke.

23. Ohoyo himita hatak haleli keyu hosh hatak im vlhpoa tuk osh tumaha holihta anta na, hatak vt pisa cha, iba tushiki lukma;

24. Yohmikma kohchit tumaha holihta, okhissa yumma. ita tuklo ka ishit hush ona cha, tuli ishit boli na, illashke; ohoyo hut tumaha holihta anta hok akosh ik payo tulk okvt: mikma hatak vt in chuka a bilika hatak tekchi ya hofahyali hokvt: yumohmi hosh nan ik achukmo ka huch iti takla ya ai ishit ish kanchashke.

25. Amba hatak vt ohoyo himita yosh inla im vlhpoa yosh osapa anta na, ahayuchi cha, ai issvt iba tushkikmvt, hatak ash illa hosh illa hi oke.

26. Amba ohoyo himita ano nana ish kaniohmicha he keyu; ohoyo himita vt nan ashvchi isht illa he vlhpesa kvt atokoli keyu hoke: hatak osh in chuka a bilika hatak a $\underline{\mathrm{i}}$ sanali cha, hikia cha, vbi chatuk a nana iluppa yưmmak inli chohmi hoka.

27. Osapa anta na pisa ma, inla im vlhpoa ohoyo himita kash osh paya tuk, mihma hlakoficha he vt iksho tuk oka. 
28. Hatak osh ohoyo himita hatak haleli keyu hosh inla im vlhpoa keyu ho pisa mut, hoklit ishi cha, iba tushki tuk osh haiakakma,

29. Yohmikma iba trshki tuk hatak ash osh ohoyo himita iki ya tuk shekel pokoli tahlapi ho imakma, tekchi ya hi öke, hofahyali tuk okvt, okchayut ahanta kvt kancha he keyu hoke.

30. Hatak okvt ikki tekchi ya isha he keyu, micha iki im anchi a wakumma he keyu hoke. (achi tok)

\section{CHAPTA XXIII。}

TVLI i kvhla, keyukmut im a lımakka tupa yokvt Chihowah 1 im okla moma itunaha ka iba chukowa he keyu hoke. 2. Iki iksho vt Chihowah im okla moma itunaha ka iba chukowa he keyu hoke; ai iti shali ont ishit pokoli ont vhli takla kvt Chihowah im okla moma itunaha ka iba chukowa he keyu hoke.

3. Ammonait, keyukmvt Moabait vt Chihowah im okla moma itunaha ka iba chukowa he keyu; ai iti shali ont ishit pokoli ont vhli takla hokvt Chihowah im okla moma itunaha ka ik iba chukowo bilia hi oke.

4. Echip a kohchut hush minti ma hina takla ya puska micha oka aiena isht ik hvchi afamo tok oka; micha Mesopotamia Péhlu minti Beo ushi Balaam vt kallakshichi anumpa huchi onocha chi ho tohno tok okvt.

5. Yohmi kia chin Chitokaka Chihowah yot Balaam a $\underline{\mathbf{i}}$ haponakla he keyu tok; amba chin Chitokaka Chihowah yot chi holitobli hatuk okvt anumpa hallakshichi yash filimmit na yukpali chim ikbi tok.

6. Chi okchaya nitak moma ka im iti nanaiya, micha nana kut im aiahli achukmut mahaya he a chik i hohoyo ho bilia hi oke.

7. Etomait a ish shitilema he keyu, chitibapishi hoka; Echip okla ha ish shitilema he keyu, hatak ik ithano yosh i yakni a ish aiasha tck okvt. 
8. Yummak o vlla a toba hokvto ai iti shali ont atuchinakmvt, Chihowah im okla moma itunaha ka iba chukowa hi oke.

9. Tushka chipota in tunvp a itibit ish iakma, nana okpulo moma ka chik ahalaiyo kashke.

10. Hatak kuna kia huvch itin takla ya na liteha ninak $\underline{a}$ yohmi yoba cha, litihakmut binah ha kohcha ia hi oke, binah anukaka ya ant ikchukowo ka hi oke.

11. Yohmi tuk kia yakohma hi oke, opivt tahakma, oka isht il achefa hi oke; atuk osh hvshi vt okatulakma anonti binah ha ant chukowashke.

12. Micha binah kohcha ya ai vtta vt chin talaia cha, yommak o kohchvt ish onashke,

13. Micha chi na haluppa ha patussa ish aienchashke: atuk osh yakohma hi oke: kohchvt ish ia cha, ish ile nuktalala chilkmvt, yakni isht ish kullashke; atukmet ish filema cha, chi a kohcha kash ish ompohomashke.

14. Chin Chitokaka Chihowah yvt chi okchalincha chi hosh, micha chi tikba chin tunup a a chim issa chi hosh, huch itin takla chi binah anohowa hoka; yumohmi hoka chi binah hot kashofashke; yumohmi ho naliteha hvt chi a takali na, ik peso hosh chi filummit ik aiyo kashke.

15. Tishu hot impushnayo ya i maleli cha, chim vlakma, falumminchit impushnayo ibbak a ish fohka he keyu.

16. Chi takla ahanta hi oke, hv̛ch itin takla kanima ho atokolikmvt chi holihta okhissa puta achvfa kanima ho ahnikmut, yumma fehna ak a hi oke; ish ilbushala he keyu hoke.

17. Islael ushetik vhleha ha hauit iksho kashke, micha Islael ushi vhleha Sotomait vt iksho kashke.

18. Anumpa il onochi ya haui isht vlhtoba, keyukmvt of isht vlhtoba ya chin Chitokaka Chihowah im aboha ya isht ish ona he keyu; iluppa ita tuklo kvt chin Chitokaka Chihowah ishit yuwala yoka.

19. Chitibapishi nana ish im pota kut chelichi ish $\underline{i}$ hoya he keyu, tvli holisso chelichi, ilhpak chelichi, micha nana hoh kia chelicha chi hosh im pota ya chelichi a ish $\underline{\mathrm{i}}$ hoya he keyu. 
20. Okla inla hano impotut ish chelicha hinla hoke: amba chitibapishi hano impotut ish chelicha he keyu: yumohmi ho yakni ish isha chị hosh ishia kut yumma ish ai ashvt nana ho chibbak isht ish ishi moma ka chin Chitokaka Chihowah yot na chiyukpalashke.

21: Chin Chitokaka Chihowah ya anumpa ilonochi ish im ilonochi tukmot, atobbi kvt ish sulaha he keyu; chin Chitokaka Chihowah yut chi hoyo pulla hi oka, micha na ish ashvchi ya hinla hoke.

22. Amba anumpa ilonochi chik ilonocho tuk okmuno na ish ashvcha he keyu hoke.

23. Nana kut chitih ha a kohcha tuk a ilap ahnit im issa fehna ka , chin Chitokaka Chihowah anumpa ish im ilonochi tuk vt chitih ha mihvt isht ish im issa tuk a holitoblit ish ai ahlicha hi oke.

24. Chin chuka a bilikka i pakvpi ahollokchi ish onakmvt, chishno ish ahni hosh paki vpot chi kaiyashke: yohmi kia nana chim aivlhto ya ish apitta he keyu hoke.

25. Chin chuka abilika im onush hikia ya ish iba chukowakmvt, noshkobo ya chibbak isht ish tvptulashke: yohmi kia chin chuka abilika hatak im onush hikia ya ishit busha y $\underline{a}$ ish $\underline{o}$ wiela he keyu hoke. (achi tok.)

\section{CHAPTA XXIVV.}

TATAK osh ohoyo ishi cha, itauwaya tuk osh, atuk osh 1 yakohmikma, aliteha kaniohmi kia takali ha tuk a ahayuchi cha, ik ahninchokmvt, yohmikmvt iti mokofa holisso yo $\underline{\mathrm{i}}$ holissochit ibbak fohki cha, in chuka ya kohchichashke.

2. Atuk o in chuka ya kohchvt iakmvt, hatak inla tekchi toba hinla ak inli hoke.

3. Atuk o $\underline{i}$ hatak ishtaiopi kash osh shitilema mut, iti mokofa holisso $\underline{i}$ holissochit ibbak fohki cha, in chuka ya kohchit chvffichikma, keyukmvt $\underline{i}$ hatak ishtaiopi vt tekchi ya chi hosh ishi kash osh illikma; 
4. I hatak tikba chvflichi tok vt anonti tekchi ya chị ho liteha ha ya hokuno isha he keyu: yummak okvt Chihowah itikba ya nan ishit yuwala yoka: mikma chi yalki ya chi ho chin Chitokaka Chihowah you chima tok ish yoshoblieha he keyu.

5. Hatak vt tekchi himonna yo ishi tukmut kolidiot tanampit ia he keyu, micha anumpa nana kia vihtokotha he keyu : nana atakluma ikshot afummit in chuka ahanta cha, tekchi ishi tuk vt yukpalashke.

6. Hatak nana kia tanchi afotoha nutaka itola, keyukmut paknaka itola ish atonichit isha he keyu, hatak im illifiopak o isht atonichi ha hinla ishi hokvt.

7. Hatak osh itibapishi vhleha Islael im vlla vhleha aiachufa yo hukoput na kunia ikbi, keyuknut kanchi, yohmikmut hukkopa yummot illashke, micha nana ik achukmo ka a hvch iti takla ya ai ishit ish kanchashke.

8. Leplosi ilitli okpulo ya ahah ish ahnashke, micha naholitompa ișht asha vhleha Lefait vhleha hut nana moma ka hvchim abvehi tuk mak $\underline{0}$, achukmalit ithaiyanvt ish yvmihchashke: yummak ash $\underline{\underline{i}}$ miha li tuk mak $\underline{\mathrm{o}}$ ithaiyanvt ish yumihchashke.

9. Echip a kohchut ish minti ha ya hina takla chin Chitokaka Chihowah yot Miliam a kaniohmichi tok a ish ithai: yanashke.

10. Chitibapishi ha nan ish impota tuk vt isht atonichi ya im aboha ont chukowet ish isha he keyu.

11. Kocha ish hikia na, ish impota, kash hatak ak osh isht atonichi ya kohchvt kocha ya isht chim vla hi oke.

12. Micha hatak ash osh ilbusha yokma, nan isht atonichi yash ishit chi nusa he keyu hoke.

13. Ik kaniohmi kia nan isht atonichi yash hushi vt okatulakma, anonti ish imashke; yumohmi ho im anchi ak inli ho anchit nusa hinla cha, anumpa yukpali chi onuchashke; mikma chin Chitokaka Chihowah itikba ya isht ai vlhpiesa yut chim vlhpisashke,

14. Ilhtohno tishu vhleha hot chitibapishi vhleha immi yosh keyukmut, okla inla immi yosh chi holihta okhissa 
anukaka chi hikia yut nan ik im iksho hosh ilbushakma ish ilbushala he keyu hoke.

15. Nitak ont im vlhpesakma asitubi tuk a ish ima hi oke; micha luvshi vt ont okatula he keyu, ilbusha hatuk osh chukush vt unvtoyula hoka, keyukma chi sanalit Chihowah pit l paharyana, nan ashvchi chin toba na.

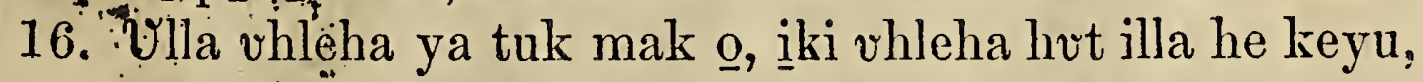

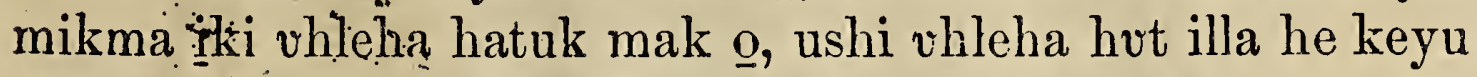
ak inli; hatak puita kvt ilap nan ashvchi tuk mak osh illa he.

17. Okla inla micha vlla vlhtakla iki iksho aiena ka ai vlhpiesa i yumihchi ka ish shanaiyucha he keyu; micha ohoyo vlhtakla i nafohka ya isht atonichi ish im isha he keyu hoke.

18. Amba Echip a hatak yuka hosh hush aiasha tuk $\underline{0}$ chin Chitokaka Chihowah yut falumminchit chumput yummak o chi kohchi tok $\underline{o}$ ish ithaiyanashke; yumohmi ha tuk o nana iluppa ish yumihcha chị ho chi miha lishke.

19. Chim osapa ya na waya bushlit ish akvchi tuk osh, sita achufa ka chim ahaksi na, osapa itonla tukma, hoyot chik aiyo kashke; okla inla vlla iki illi, micha ohoyo vlhtakla aiena kak o immi ha hi oke: yumohmi ho nana moma chibbak vt toksulikma chin Chitokaka Chihowah chi yukpa lashke.

20. Chim alif iti ya ish bolikmvt, anonti naksish a vlbitelit ish bola he keju; okla inla vlla iki illi, micha ohoyo vlhtakla aiena kak o immi ha hi oke.

21. Pakvui chim ahollokchi palki ish aivmokmot, himmakma ish vlbulla he keyu; okla inla vlla ilki illi, micha ohoyo vlhtakla aiena kak o immi ha hi oke.

22. Micha Echip yakni ya hatak yuka hosh hush aiasha tuk vt, hush ithaiyanashke: yumohmi ha tuk osh nana iluppa hush yumihcha he ya huchi miha lishke (achi tok.) 


\section{CHAPTA XXV.}

HATAK vt anumpa itachowa nan apesa vhleha het nan 1 im apesa chị ho, nan ai vlhpisa ya vlakma, aivlhpiesa yano i kashofi cha, yoshuba tuk a anumpa onucha hi oke.

2. Atukma yakohma hi oke, hatak yoshuba tuk vt boa he vlhpesakma, pisa ho ishtakanumi tuk a itvlhpisa ho holhtina kaniohmi kvt aka itola cha, onvtola chi ka nan apesa vt apesashke.

3. Fahama pokoli ushta ho ima hinla; micha vbanupa he keyu; keyukmạ iluppa vbanublichit fahama laua ishit boli hokma, chitibapishi hvt nan isht ik chim ahobo na.

4. Waktoksuli vt onush a nihechikma, ibichilu fohka ish fohka he keyu.

5. Itibapishi vhleha hvt itapihvt asha tuk $\underline{Q}$, achvfa kvt illi mvt, vlla ik im ikshokma illi yash tekchi ya tuk vt naksika hatak inla yo auwaya he keyu; i hatak itibapishi ak osh iba chukowvt itauwaya cha, $\mathrm{i}$ hatak itibapishi nana akaniohma he vlhpesa kak o i yumihchashke.

6. Atulima yakohma hi oke; vtta ahpi ishikma itibapishi hohchifo illi tuk a vlhtobut hikiashke: yumohmi ho hohchifo kvt Islael a jk mosholo kashke.

7. Atuk o hatak ash osh itibapishi tekchi yash ishi ikbunnokma, yohmikma itibapishi tekchi vt holihta okhissa ya onvt im asunonchi vhleha ha im ona cha, $\underline{A}$ hatak itibapishi hut itibapishi hohchifo ha ikbit Islael a ai i hilechi ik bunnoshke; a hatak itibapishi nana akaniohma he vlhpesa ka yumihchi ik bunnoshke, achashke.

8. Yohmikma in tumaha holihta im asunonchi vhleha hut hoyo cha, im anumpulashke; mikma kullot hikia cha, ishi ik sa bunnoshke, achikma,

9. Yohmikma itibapishi tekchi ash osh im asunonchi ai itin takla ho bilika ona cha, iyi shulush honlo tulk a $\underline{\underline{i}}$ shufi cha, nashuka ya on tofashke, micha afalaminchit, Itibapishi 
ha chuka ikbit i hilechi ik bunno yano, hatak a yakohmichashke achashke.

10. Mikma hohchifo kut Islael $\underline{a}$; shulush $\underline{\mathrm{i}}$ shufa in chuka, achashke.

11. Hatak vt ita pakshunvt asha ma, achvfa kash tekchi vt $\underline{\mathrm{i}}$ hatak isso ka ibbak a ahlakoficha chi hosh bilika ona cha, ibbak pit weli mut; obulh takla ya isht ishikma,

12. Yohmikma ibbak a ish tublashke; chi nishkin vt $\underline{\mathrm{i}}$ nukhakkla he keyu.

13. Chi bahta ya ishit weki itaiyukali chito, micha iskitini vt ik chi fohko kashke.

14. Chim aboha ya isht vlhpisa ilaiyukali, chito, micha iskitini aiena kvt chim aiasha keyu kashke.

15. Amba nan isht weki ahli, micha aivlhpiesa yo, micha nan isht vlhpisa ahli, micha aivlhpiesa yosh chim aiashashke, micha yumohmi hosh nitak chi falaivt chin Chitokaka Chihowah yvt yakni chima tok a hush aiashashke.

16. Nana yakohmi ka yumihchi moma kut, micha aivlhpiesa keyu yumihchi moma kut chin Chitokaka Chihowah nan isht yuwala yoka.

17. Echip a kohchut ish minti ma, lina takla ya Amalek vt chi kaniohmichi tok a ish ithaiyanashke.

18. Hina takla yash chi afama cha, olbvl aya ya olbvl aya ik kullo fehna moma ka chi hoyvblit chi kota ma, chi fahama tok; micha Chitokaka ya ik i nukshopo tok.

19. Yvmohmi hoka yakohma hi oke, yakni yash a binilit ish iesha chị ho chin Chitokaka Chihowah yut chima tok $\underline{\text { a }}$ chin tunup puta fullota ka chin Chitokake Chihowah yot chi fohvchikma, shutik nutaka ya Amalek isht ithana ya kashofit ish kanchashke, ik chim ahakso kashke.' (achi tok.)

\section{CHAPTA XXVI。}

TUKMA yakohma hi oke, chị yakni ya chị ho chin ChiA tokaka Chihowah yut yakni chima tok a ish ona cha, yummak o abinilit ish aiashakmut, 
2. Yakni awaya vmmona moma chi yakni chin Chitokaka Chihowah yut chima tok a ish ai ishi cha isht ayvt, kishi ish fohkikmvt, kanima ho chin Chitokaka Chihowah yvt aiatokolit hohchifo ha a bohla he ahnikma, yumma isht ish onashke.

3. Micha naholitompa isht vtta vt nitak yumma anta tuk

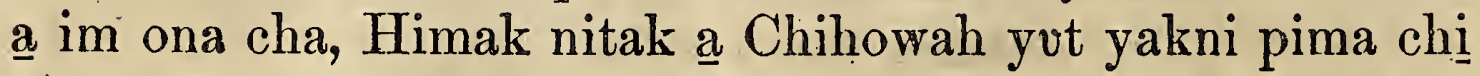
hosh piki vhleha ha anumpa kvllo il im onochi tok a vla li kut, chin Chitokaka Chihowah ya im anohonli lishke, ish im acha hi oke.

4. Mihma naholitompa isht vtta yut kishi chibbak fohka kash ishi cha chin Chitokaka Chihowah im alta itikba ya talala hi oke.

5. Mikma chin Chitokaka Chihowah itikba ya ish anumpulit, Aki vt Silian illa hosi a tok; atuk osh kanomosi hosh aiitvpehut Echip a ona cha, yummak o aiasha tok, micha yummak o aiashut oklushi chito, kvllo, micha laua aiena toba tok.

6. Mihma Echip okla hut na ik achukmo ka pi yumihchit, pilbushali cha, yuka kvllo pi onuchi tok.

7. Mihma piki vhleha in Chitokaka Chihowah ya il i pahaya ma, Chihowah yut pi haponaklo mut, pilbusha ka cha pim atoksuli ka micha pissikkopali ka pihisa tok.

8. Micha Chihowah yot ibbak kullo hosh, micha shakba ashatapa hosh, micha palummi chito hosh micha nan ishtatokowa, micha na fehna yumihinchit pi pehlichit Echip a kohchi tok.

9. Atuk osh iluppa pisht vlushke, micha yakni iluppa, yakni pishukchi, micha foe-bila aiena kvt ayanahanli iluppa fchna ka pimushke.

10. Atuk $\mathrm{Q}$ himak a yakeh, Chihowah ma, is suma tok $\underline{a}$ yakni yash awaya ummona ka isht chim vla lishke, ish acha hi oke; micha chin Chitokaka Chihowah itikba ya ish talalashke, micha chin Chitokaka Chihowah itikba ya ish aiokpvehashke.

11. Micha nana achukma puta chin Chitokaka Chihowah yut chima tok a micha chin chuka achvfa, chishno, micha 
Lefait, micha okla ik ithano chi takla aiasha ka ima tuk a jsht ish aiyukpashke,

12. Afummi ont isht atuchina yummak osh ishit pokoli ishi afummi hoka, na chi waya ishit pokoli moma ka ishit pokoli ya apesut chim vlhtaha cha, chi holihta okhissa anukaka ya imput kaiyut aiasha chi ka Lefait, okla inla, vlla i ki illi, micha ohoyo vlhtakla aiena ka ish emakmut,

13. Yohmikmvt chin Chitokaka Chihowah itikba ya, $\mathrm{Na}$ hullochi yash an chuka ya ishit kohcha lishke, micha chim anumpa moma is sum apihisa tuk mak $\underline{o}$, Lefait, micha okla inla, vlla îki illi, micha ohoyo vlhtakla aiena ka ima lishke; chim anumpa ha ak vbanubloshke, micha ik sumahakoshke,

14. Yaya li moma kvt ak po tuk oke; micha nana achvfa kia na liteha isht ikbi li tuk keyu, micha nana achvfa kia illi ya ima li tuk keyn hoke; amba an Chitokaka Chihowah im anumpa ha haiyaklo li mot, nana is sum apesa tuk moma ká yumihchi li tuk oke.

15. Ish aiahanta holitopa, vba aholitopa ya binilit aka.et ish hopokoyashke, micha chim okla Islael, micha yakni ilvppa piki vhleha ha anumpa kvllo ish in ilonochi tok mak osh, yakni pishukchi micha foe-bila aiena kut ayanahanli ya ish pima tok vt na yukpali ish onochashke, ish achashke.

16. Himak nitak a nanvlhpisa bilia, micha aivlhpiesa ish yumihincha chi ho, chin Chitokaka Chihowah yut chim apesushke; yumohmi hoka chukvsh aiokluhvt, micha chimi shilombish aiokluhvt ieshit ish yomihinchashke.

17. Himak nitak a Chihowah ak osh chin Chitokaka ya chi, micha $\underline{i}$ hina ak $\underline{0}$ ish ai itvnowa chi, micha $\underline{i}$ nanvlhpisa bilia, micha im anumpa micha aivlhpiesa yumihinchi aiena ka ish iesha chí, micha im anumpa ha ish $\underline{\underline{i}}$ haponakla chị, ahli hosh ish mihushke.

18. Mikma mihvt chim issa tok mak osh himak nitak $\underline{a}$, Chihowah yut im okla ila chia ha chị ho ahli hosh mihushke; mikma im anumpa moma ka holitoblit ish ieshikma;

19. Anumpuli tuk mak osh, chi chahvchit, ahninchit isht anumpuli ya micha hohchifo ha, micha holitopvt vmmona ká, oklushi puta moma ka yummak oka ikbi tok vt i shahle- 
chi ya chishno ak o chi ihikbi ha tuk oke; yưmohmi ho chin Chitokaka Chihowah ya okla $\underline{1}$ holitopa chia ha chi ha tok, im ahanchi tok.

\section{CHAPTA XXVII.}

MICHA Moses vt Islael im asunonchi vhleha aienvt okla I ha im anumpulit, Anumpa moma himak nitak a hvchim anumpohonli li ka hush ieshashke.

2. Atuk osh yakohma hi oke, Chatan a vbanublit chin Chitokaka Chihowah yvt yakni chima tok a hvsh ona, nitak mot, toli hochito ish hiohlichi cha, isht vlhpolosa ya isht ish apoluslashke.

3. Micha chiki vhleha in Chitokaka Chihowah yvt mihvt im issa tuk mak osh, chin Chitokaka Chihowah yot yakni chima tok; yummut yakni pishukchi, micha foe-bila aiena kvt ayanahanli ya ish chukowa chi hosh ish vbanvblikmvt, nan vlhpisa iluppa moma anumpa ha ish ai o holissochashike.

4. Yvmohmi hoka yakohma hi oke: Chatan a hush vbanvblikmvt, himak nitak a Ebal nunih ha a hvchi miha li kạ; tvli puta iluppa hush hiohlichi cha, isht vlhpolosa isht ish apoluslashke.

5. Micha yummak o chin Chitokaka Chihowah ya alta tuli alta yo ish im ikbashke; tvli ishit pilesa nana kia ish $\underline{0}$ wiela he keyu,

6. Chin Chitokaka Chihowah ya tuli kaniohmi tuk vt moma yo alta isht ish im ikbashke; micha hukmit im issa onochit chin Chitokaka Chihowah ya ish im issa hi oke,

7. Micha iti nanaiyuchit im issa ish im issakmut, yvmmak o ish impa cha, chin Chitokaka Chihowah itikba ya na yukput ish aiashashke.

8. Micha haiaka achukma fehna ho nan ulhpisa iluppa anumpa moma ka tuli yash ish ai $\underline{o}$ holissochashke, im achi tok.

9. Mihmut Moses micha naholitoblit isht asha, Lefait vhleha aiena kvt Islael moma ka im anumpulit, Ahah ish 
ahni cha ish haklashke, Islael ma, Himak nitak a chin Chitokaka Chihowah ya im okla ish tobushke.

10. Yumohmi hoka chin Chitokaka Chihowah im anum-

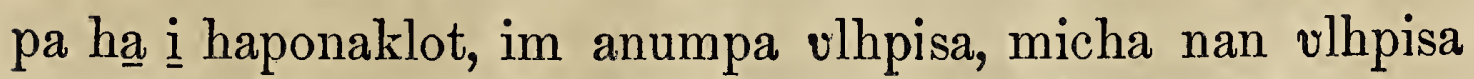
bilia. himak nitak a chị miha li ka ish yumihinchashke, achi tok.

11. Micha Moses vt milh nitak ak inli ho okla atokolit $\underline{\mathrm{i}}$ mihvt,

12. Chatan hush vbanubli tukma iluppa puta, Simeon, Lefai, micha Chuta, micha Issaka, micha Chosif, micha Benchamin aiena kak osh nunih Kelisim paknaka ya hieli cha, okla ha na yukpali anumpa onochashke,

13. Mikma iluppa puta, Lnben, micha Kat, micha Asha, micha Sebulun, Tan, micha Naftali aiena kak osh Ebal nunih paknaka hieli cha, kallakshichi anumpa onochashke,

14. Mihma Lefai vhleha hvt Islacl hatak vhleha moma ka im anumpulit anumpa chitoli hosh,

15. Hatak nana hosh tuhlit naholbvt toba, keyukmut bilut akmot naholbvt toba, Chihowah nan isht yuwala, hatak nan ikbi imponna ibbak osh atahli yo ikbi cha, alumaka hilechikmvt, kallakshashke: mikma okla moma kvt, Amen, achashke;

16. Kuna hosh ilki keyukmvt ishki ya ik ahobalokmut kallakshashke, mikma okla moma kut, Amen, achashke.

17. Kuna hosh in chuka abilika hatak i yakni vlhpisa ya kanvllichikmvt, kallakshashke: mikma okla moma kvt Amen, achashke.

18. Kuna hosh nishkin lupa ya i hina kohchichikmvt kallakshashke : mikma okla moma kvt, Amen, achashke.

19. Kvna hosh okla inla, vlla iki illi, micha ohoyo vlhtakla aiena nan im apesa kvt shanaiyvchikmvt, kallakshashke: mikma okla moma kut, Amen, achashke.

20. Kuna hosh ịki tckchi ya iba tushki kut ịki im anchi ya wakummi hokvt, kallakshashke; mikma okla moma kvt, Amen, achashke.

21. Kuna hosh vlhpoa kaniohmi hoh kia iba tvshkikmvt, kallakshashke : mikma okla moma kvt, Amen, achashke. 
22. Kvna hosh in tek, iki ushetik, keyukmvt ishki ushetik a iba tushkikmut kallakshashke, mikma okla moma kvt, Amen, achashke.

23. Kuna hosh ipochi ohoyo iba tushkikmut kallakshashke: mikma okla moma kvt; Amen, achashkec.

24. Kuna hosh in chuka abilika hatak a luma họsh fahamakmvt kallakshashke: mikma okla moma kut, Amen, achashke.

25. Kuna hosh hatak nan okpuni keyu houba chi họsh nahabenakmut kallakshashke; mikma okla moma kvt, Amen; achaske.

26. Kvna hosh nan vlhpisa iluppa anumpa moma ka iakaiyvt ik yvmihchokmut kallakshashke: mikma okla moma kvt, Amen, achashke. (achi tok)

\section{CHAPTA XXVIII.}

TUKMA yakohma hi oke, chin Chitokaka Chihowah im A anumpa ha achunanchit ish $\underline{\mathrm{i}}$ haponaklo cha, im anumpa moma ka himak nitak a chi miha li ka ithaiyanut ish yumihinchikma, chin Chitokaka Chihowah yvt oklushi moma yakni paknaka ai asha ka $\underline{\text { i }}$ shahlechit a chaha ka chi binilicha hi oke.

2. Mikma chin Chitokaka Chihowah im anumpa ha ish $\underline{\mathrm{i}}$ haponaklokma, na yukpali iluppa puta moma kvt chi sakki cha chi onvtoyula hi oke.

3. Tumaha holihta ya na yukpvt a chi onvtoyula hi oke, osapa na yukpa vt chi onvtoyula hi oke,

4. Chi haknip a toba tuk, micha chi yakni a waya tuk, micha chi nanvlhpoba a waya tuk, micha chi wak tek a waya tuk, micha chin chukfi aya puta aiena kot na yukpa hi oke.

5. Chị kishi micha tạpushi chim a yamuska yot na yukpashke.

6. Ant ish chukowakmut na chi yukpa hi oke, micha ish kohchakmvt na chi yukpa hi oke. 
7. Chin tunvp chi sanalit wakaya vhleha ha chin Chitokaka Chihowah yut chi nashuka itikba akakohvcha hi oke; hina achufa ho minti tuk kia, hina untuklo ho chi tikba yihlepa hi oke.

8. Chiliowah yut chim olhpoyak ai itola chuka, micha nana ho chịbak a isht ish ishikma, na isht yukpali chi onucha hi oke; mieha chin "Chitokaka Chihowah yot yakni a chima tok vt a chi yukpa la hi oke.

9. Chihowah yot anumpa kullo chim ilonohonehi tok mak osh; chin Chitokaka ya im anumpa ish ieshi cha, i hina ya iș itanowakma, im okla i holitopa kullo hosh chikba hi oke. 10. Niikna okla moma yakni paknaka aiasha kvt Chiho-

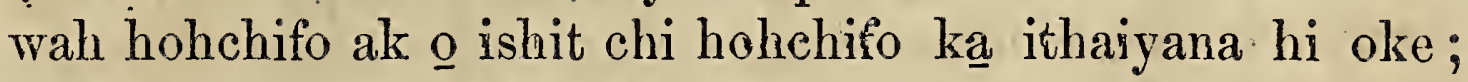
micha chi nukshopa hi oke.

11. Yakni ya chima chi hosh chịki vhleha ha anumpa kvllo im ilonochi tok vt, Chihowah yvt ehim vihpoyak, chi haknip a toba, micha chi nan olhpoba a waya, micha chi yakni a waya aiena ka a chi lauacha hi oke.

12. Chihowah yot im vlhpoyak achukma vba ya chin tiwi cha, ont ai vlhpiesakma, yakni ya chim umbveha he, micha chibbak nan toksahanli tuk moma ka na chi yukpala hi oke; mikma oklushi laua ka ish impota cha, chishno. chik poto ka hi oke.

13. Micha Chihowah yvt noshkobo ak o chikba hi oke, micha hasimbish ak a he keyu: paknaka ak o ish bieka, ish nutaka he keyu hoke, chin Chitokaka Chihowah im anumpa ha himak nitak a chim anumpohonli li ka $\underline{i}$ haponaklot ithaiyanvt ish yumihinchikmvt,

14. Himak nitak a chim anumpohonli li tuk a anumpa kanima kia, i filummit naksi ka ishtimpakimma, keyukmut vlhfubekimma, kanimá kia ivt nakolbut toba iakaiyvt ish aiokpvcha he keya hoke.

15. Amba yakohma hi oke, chin Chitokaka Chihowah im anumpa ha ish $\underline{i}$ haponakla he keyukma, im anumpa moma, micha $\underline{i}$ nan vlhpisa bilia himak nitak a chim anumpohonli li ka ithaiyanut ish yvmihcha he keyukma, na kallakshi iluppa puta kut chi onvtula hi oke, micha chi sakka hi oke. 
16. Tumaha holibta ya a chi kallakssha hi oke, micha osapa ya a chi kallaksha hi oke.

17. Chi kishi hot micha ta pushi chim ayamuska yut kallaksha hi oke.

18. Chi haknip a toba tuk, micha chi yakni a waya tuk, micha chi wak tek a waya tuk, micha chin chukfi ulhpoa aya puta aiena kvt kallaksha hi oke.

19. Ant ish chukowakmvt chi kallaksha hi oke, micha' kohchvt ish iakmvt, chi kallaksha hi oke.

20. Chihowah yut nana moma yumihcha chi ho chibbak isht ish ishikma, nan isht kallakshi, micha ishit chukvsh okpulo, micha nahotupa puta et chi onohonchi na, aiokpulokvt chi taha hi oke, micha tushpvt ont ish moshola hi oke; nana ish akaniohmi kvt okpulo hosh is sa kanchi tuk oka.

21. Chihowah yut ililli okpulo ya chi atakalichikma, yakni ish isha chi kash osh yumma ont a chi taha hi oke.

22. Chihowah yut tahat ishit yunha, micha yunha, micha nipi lua, micha nipi lua atampa, micha bushpo falaia, micha bushshi, micha shummit toshbi aiena ka isht chissa hi oke; mikma chi akaiya na, ont ish moshola hi oke.

23. Mikma chi shutik vt chi noshkobo paknaka talaia kvt asonak lakna ya hi oke; mikma yakni vt chiyi nutaka talaia kut tvli ya hi oke.

24. Chihowah yut chi yakni ai umba ka hituk micha lukfi pushi chohmicha hi oke; vba ya mintit chi onvtohonlakma chi taha hi oke,

25. Chihowah yvt chin tunup vhleha itikba ya a chi akakohvcha hi oke; hina achufa ho ivt ish i sanali tukmut, itikba ya hina untuklo ho ish yihlepa hi oke; mikma apehlichika puta yakni paknaka aiasha moma ka chisht ona hi oke.

26. Micha chi haknip vt uba hushi moma, micha nampoa puta yakni aiasha ka im ilhpak toba hi oke, mikma hatak nana kia mahlolit tihlela he keyu hoke.

27. Chihowah yut Echip hichi a, micha isht asha hla- 
chowa, micha hlachowa fachanli, micha wushko aiena ka isht chissokma chi hlakofa he keyu ka hi oke.

28. Chihowah yut tasimbo, micha nishkin lvpa, micha chukvush okokko, ahni aiena ka isht chissa hi oke.

29. Mikma hushi tubokoli hoh kia posholit jsh hikikia hi oke, nishkin lupa vt aiokhlileka ya na posholit hikikia kak o ish chohmi cha nana ish a kaniohmi kvt chim aiahla he keyu; peh chilbushalit, chi wehpohonli na bilia; mikma hatak nana kia chi okchalincha he keyu hoke.

30. Ohoyo ish itim vlhpoa tukma hatak inla hosh iba tvshka hi oke, chuka ish ikbi tukmot yumma ish ai vtta he keyu, pakvpi ahollokchi ish hokchi tukmut yumma paki a ish ai vma he keyu hoke:

31. Chi wak toksuli ya ish pisa ho chi tikba ai vba he, mikma yumma ish ai vpa he keyu hoke; chim issuba haksobish falaia ya afout chi tikba ai isha hi oke, mikma chi falama he keyu ka hi oke; chin chukfi ha chin tunưp a ima hi oke, hlakoficha he vt ik chim iksho ka hi oke.

32. Chiso vhleha micha chiso tek vhleha aiena ka okla inla ho ima hi oke, mikma chi nishkin vt pisa bunnvt taha hosh, anukfilli na biliat illa hi oke, mikma kvllo kvt chibbak a ik foyuko ka hi oke.

33. Yakni nana a chi waya, micha na ish toksuli tuk moma ka oklushi chik ithano hosh vput tahla hi oke; micha peh ilbusha micha litowvt ish bilia hi oke.

34. Yvmohmi ho chi nishkin vt pisa ka ish pisa kak osh chi tasimba hi oke.

35. Chihowah yut chiyikulaha yạ, micha chiyi ya hichi chi hotopa fehna, micha vtta keyu ho isht chissokma, chiyi putta vttut ia hosh chi noshkobo tabokaka ont vhla hi oke.

36. Chihowah yut oklushi ya chishno, micha chilki vhleha aiena kvt ithana tok keyu ho, chishno micha chi miko chi pehlicha he $\underline{o}$ ish hilechi tuk a aiena ka chisht im ona hi oke; mikma yummak o naholbut toba inla iti, micha tuli aiena ka jsh aiokpvcha hi oke.

37. Chihowah yut oklushi moma itin takla chishtia ka 
yựmak o okokko, isht ahni nana achi, micha isht anumpuli yo ish ita nowa hi oke.

38. Osapa ya pehna laua ho isht ish ia tukmut, kanomosi ho ish hoya hi oke; hawa yut tahla chi hoka.

39. Pakvpi ahollokchi ya ish hokchi cha, ish apoksia cha hi oke; yohmi kia oka paki ya ish ishka he keyu, micha paki ya ish ma he keyu hoke: shushi vt vpa hi oke.

40. Chim ai vhli moma ka alif iti vt chim aiasha hi oke, yohmi kia bila ya isht ish il ai ahumma he keyu hoke: chim alif uni vt chilofa hi oke.

41. Chiso vhleha micha chiso tek vhleha ha ish tobacha hi oke; yohmi kia ish aiyukpa he keyu, yuka tobacha kunia hi oke.

42. Chim iti puta ka micha chi yakni a waya puta ka havia yut tahla hi oke,

43. Okla inla chitin takla ahanta kvt wakayvt chi. shahlit chaha fehna hi oke; mikma akanlosi fehna ish ona hi oke,

44. Chimpota hi oke, mikma ish impota he keyu hoke: noshkobo ya hi oke, mihma chishno vto hasimbish chia ya hi oke

45. Micha na kallakshi iluppa puta moma kut chi onvtula hi oke; micha chiakaiyut chi sahakki na, ont chi taha hi oke; chin Chitokaka Chihowah im anumpa ha haponaklot, im anumpa vlhpisa, micha $\underline{\mathrm{i}}$ nan vlhpisa bilia chi miha tok $\underline{\mathrm{a}}$ chik holitoblo tok oka.

46. Micha nan isht atokowa, micha okokko, isht ahni aiena kvt chishno micha chim isht atiaka aiena ka onvtoyula na bilia hi oke,

47. Nana moma apakna atuk mak $\underline{\mathbf{o}}$, na yukput chukvish yukpa hosh chin Chitokaka Clihowah ya chik im antio tuk okvt,

48. Yumohmi hokvt chin tunup vhleha yummak oka Chihowah yot chị sanali et pila chi ka hohchufo hosh, micha iiukshila hosh, micha nipi bano, micha nana puta moma ka bunna hosh, ish im antia chi hoke; mikma tuli ikunla abana yo chikunla abanalikma ont chi taha hi oke.

49. Chihowah yot oklushi chi sanalichit, yakni hopaki, 
yakni ont a taha ka shinimpa kvt ossi hika choyuhmi, oklushi yumma im anumpa ha ish ithana keyu ho isht vla hi oke.

50. Oklushi pisa chilita hosh, yummak okvt sipokni yoka, ahna he keyu, micha vlla yoh kia i nukhakla he keyu hoke.

51. Micha chi wak awaya, micha chi yakni awaya aiena ka ai ihimpa na, chi taha hi oke; micha yummot onush; oká paki; micha bila, wak tek, micha chin chukfi isht achakvt ia yoh kia ik chim ashancho ho ont chitaha hi oke.

52. Micha chi holihta okhissa puta ka abinanchit fullota na, a chin chaha ka puta, micha holihta chaha puta yummak o ish anukchieto chatuk vt, chị yakni hlopulli moma ka aka itula hi oke; micha chi yakni chin Chitokaka Chihowah yot chima tok hlopulli moma ka chị holihta okhissa puta ka abinancha hi oke.

53. Micha chin tunup puta kvt abinanchit chilbushali na, chi hohchvfokmvt chi haknip atoba ak inli, chiso vhleha, chisotek vhleha nipi chin Chitokaka Chihowah yut chima tok a ish pa hi oke,

54. Yvmohmi ho hatak vt ahah ahnit, ile holitobli hosh chi takla ahanta chatuk kia itibapishi hạ, micha tekchi i holitopa tuk a, micha im vlla vhleha atampa ashachi tuk vt $\underline{i}$ holhponayo ik achukmot pisa hi oke.

55. Yvmohmi cha, im vlla vhleha nipi vpa kvt, yumma kanima kia ipeta he keyu hoke : chi holihta okhissa moma ka chin tunup vt abinachit, afullota cha, chilbushalit abinachit fullota ha tuk o nana kvt ik chim iksho ha tuk okvt.

56. Ohoyo ahah ahnit ile holitobli hosh, chi takla ahanta kvt, ile holitoblit ahah ahni kvt iyi pvtta ya aka ishit hvhla he keyu tuk kia, i hatak $\underline{i}$ holitopa a tuk a, micha ushi a tuk, micha ushetik a tuk,

57. Micha vlla yosh iyi itin takla ya minti tuk, micha im vlla vhleha shoyuli tuk $\underline{\mathbf{i}}$ holhponayo ik achukmot pisa hi oke : micha chin tunup vt chi holihta okhissa puta ka a chilbvshalikma, abinanchit a fullotvt atikkonofikma nana moma bunna hosh lumvt vpa hi oke.

58. Nan ulhpisa iluppa anumpa moma holisso iluppa aho- 
lisso ka ithaiyanvt ish yumihinchi hosh, hohchifo holitopa ị nukwia he vlhpiesa chin Chitokaka Chinowah ilvppa hvchik i nukwiokma;

59. Yohmikma Chihowah yvt nan isht chim ilbusha palummi, chim isht atiaka nan isht $\underline{i}$ palvmmi chinto, micha hopaki ho yvmohmi, micha abeka palummi hopaki ho yvmohmi ya chi onocha hi oke:

60. Micha Echip ililli okpulo ish i nukshopa chatuk a moma chi onocha hi oke; mikma chi atakala hi oke.

61. Micha nan abeka puta, micha napalvmmi puta nan vlhpisa iluppa aholisso ha atakali keyu aiena ka Chihowah yot chi onohonchikma, chi taha hi oke.

62. Mikma apakna kvt vba fochik oka ish chiynhmi tuk oh kia, holhtina kanomosi hosh ish ahomba hi oke; chin Chitokaka Chihowah anumpa ha ish i haponakla he keyu hokvt.

63. Atukma yakohma hi oke, Chihowah yot nana achukma kak o chi yumihchi cha; chi apaknvchi kvt im achukma tuk vt, anonti chi tahlit ont chi mosholichi kvt, im achukma hi oke; mikma yakni yumma ish isha chi hosh ish ia ka ai isht chi kancha hi oke.

64. Micha Chihowah yvt okla moma ka aiyokomvt, yakni a taha ka achvfa vttut ia hosh, in tunnvp ont vhli chi fimma hi oke; micha yummak o naholbut toba inla chishno, micha chiki vhleha aiena kvt ish ithana tuk keyu, iti, micha tvli aiena yo ish im antia hi oke.

65. Micha oklushi yumma puta ai iti takla ya anuktala ya ish ahayucha he keyu; micha chiyi putta yvt afoha pisa he keyu hoke: amba Chihowah yot yummak oka chulvush wonnihinchi micha nishkin elli, micha im anukfila nukhąklo chima hi oke.

66. Mikma chim ilhfiopak a ai isht chi nuktăhlaklakka hi oke, micha nitak, micha ninak moma ka chi nukshopa cha, sai okchaya fẹna chị chik ahno ka hi oke.

67. Chi chukvsh nukshopa ishit chi nukshopakmvt, micha chi nishkin pisa ish pihisakmvt, onnahinlikma, Chihowah yot vlhpesa ahni, Ópiakbano, ish acha hi oke; atuk osh 
opiakma, Chihowah yut vlhpesa ahni, Onnahinlikbano, ish acha hi oke,

68. Mikma Chihowah yot anonti Echip yummak oka hina ya isht chim anumpule li mut, Anonti ish pisa he keyu hoka, achi li tok a peni hochito apittut chisht ona hi oke: mikma yưmmak oka ohoyo yuka micha hatak yuka ikbit chin tunup chi kancha hi oke, mikma hatak nana kia ik chi chumpo ka hi oke; (ähi tok.) :

CHAPA XXIX.

TLUPPA puta kak osh tan itim apesa anumpa ho Holeb L a nan ai itim apesa tok vt asha na, Chihowah yot Moses a tohno na, Islael im villa vhleha ha Moab yakni ya ai itim apesa tok mak okè.

2. Mihma Moses ut Islael moma ka hoyo cha, im anumpulit, Echip yakni chi tikba ya Falaoh, micha in tishu vhleha moma aiena, micha i yakni moma aiena ka Chihowah yot kaniohmichi tok moma ka hush pisa ha tuk oke.

3. Nan isht imomaka pisa hochito, micha nan isht atokowa, micha na fehna hochito yumma aiena ka chi nishkin vt pisa ha tuk oke.

4. Yohmi kia Chihowah yet chukvush nan isht akostininchi, micha nishkin isht pisa, micha haksobish isht haponaklo ya ik hvchimo tok, himak nitak ant vhlishke.

5. Mikma afummi pokoli ushta ho yakni haiaka ya huchi pehlichit aya li tuk oke; micha nafohka hvsh foyuka kvt tahot ishtia keyu, mikma shulush chiyi isht ish holo kvt tahut isht ik aiyo tuk oke.

6. Puska huchik po, micha oka paki, micha nan ishko homi nana kia hush ishko tuk keyushke; yomohmi hosh vno ak osh hvchin Chitokaka Chihowah sia ka hvsh ithaiyana chi hatuk oke

7. Átuk osh iluppa hush la ma, Heshbon i, miko Sihon, micha:Bashan i miko Ok ita tuklo kvt kohchvt pi sanalit pitibi tok; mikma issot e kanchi tok. 
8. Mihmot i yakni ya il ishi cha, Lubenait micha Katait, micha Manasseh apelichi iklvnna aiena kvt i yakni ya chị ho il ima tok.

9. Yvmohmi hoka anumpa nan itim apesa iluppa hush ieshi cha, hush yumihchashike; yumohmi hosh nana hush yumihinchi moma kvt hvchim aiahli hvchim achukmashke.

10. Himak nitak a hvsh moma kvt hvchin Chitokaka Chihowah itikba ya hush hielishke, hvchim apehlichi puta $\underline{\mathrm{i}}$ kupotuni vhleha, huchim asvnonchi vhleha, micha na hvchim vlhtoka vhleha Islael hatak vhleha moma aiena,

11. Huchim vlla vhleha, hvchi tekchi vhleha, micha chim okla inla vhleha hosh chi binah hạ aiasha ya aienvt iti chin chanli vttut ia hosh, oka chim ochi ont vhli ya aienvt hvsh hieli kvt;

12. Chin Chitokaka Chihowah ya nan itim apesa, micha im anumpa kvllo anumpulit, himak nitak a chin Chitokaka Chihowah yot chitim apihisa ka ish itim apesa chi ha tuk oke.

13. Yvmohmi ho himak nitak a nana hvchi miha tok mak o, micha ehiki vhleha Eblaham micha Aisak, micha Chekob aiena ka anumpa kullo im il onochi tok mak osh, kvllochit im okla huch ikbi cha, huchin Chitokaka chi ha tok.

14. Micha huehishno ak bano ho nan itim apesa ilvppa, micha anumpa kullo anumpuli iluppa hvchitim apesa li keyu.

15. Amba himak nitak a pi apehut pin Chitokaka Chihowah itikba hieli, micha himak nitak a pi apehot ik hinlo aiena hoke.

16. Echip yakni ya kaniohmit il aiasha tok, micha oklushi puta kaniohmit hlopullit il iturnowa tok a hvsh ithana.

17. Micha nan isht a yuwala, micha i naholbot toba puta, iti micha tuli, micha tvli hvta, micha tuli lakna aiena kvt im ai itin takla ya aiasha ho hush pisa tok okvt.

18. Keyukma hvch itin takla ya hatak, keyukmut ohoyo, keyukmut chuka achufa, keyukmut apehlichi himak nitak a pin Chitokaka Chihowah ya chukush i filummi cha, oklushi iluppa puta i naholbut toba puta ka ona cha im antia yoba na, keyukma chukvsh homi cha, nan isht yuwala waya chi kvt, akshish hveh itin takla ya itoyula na, 
19. Atuk osh yakohmi na, kallakshichi anumpa iluppa haklokmvt, ilap chukvsh vt na ile yukpalit anumpa il onochit, Sa chukush um anukfila hosh itukshila ya oka haksi akaieninchi hoh kia nana kvt vm achukma chi hoka, achi yoba na:

20. Chihowah yvt apoancha he keyu hoke; amba Chihowah im ai i nukkilli, micha i nuktahla yot hatak yumma i sanalit shobohonla hi oke; mikma kallakshichi anumpa moma holisso iluppa aholisso tuk vt onvtoyula he; mikma Chihowah yut hohchifo ha shutik nutaka ya a kashofit kancha hi oke.

21. Micha Chihowah yot yumma Islael apehlichi puta moma ka ila filummichit, nana ik achukmo ak o isht onvt, nan vlhpisa holisso iluppa nan itim apesa ya kallakshichi anumpa hut takali tuk mak o yumihcha hi oke.

22. Yvmohmi ho hvchim vlla vhleha ai iti shali vt hvch iakaiyvt aiasha he vt, micha okla inla yakni hopaki minti aiena kvt, yakni vt na palummi onvtola tuk, micha abeka ilaiyuka puta Chihowah yut onochi tuk a pisa ka,

23. Mihma yakni yumma moma kvt hituk lakna, micha hupi, micha luhuua, nana ahollokchi keyu, micha nana awaya keyu, micha hushuk nana kia ai offo keyu, okpulot taha kvt, Sotom micha Komolah, Atmah micha Seboim aiena okpulot taha tok mak o, yummak oka Chihowah yut nukhobela hosh, micha im ai i nukkilli vt libbi hosh okpunit tahli tok choyuhmi ka pisa mak osh, acha hi oke.

24. Oklushi moma kak kia, Nanta katiohmi ho Chihowah yut yakni iluppa yumihchi tuk oh cho? nanta katiohmi ho nuklibisha chito lushpa kut yakohmi hoh cho? okla acha hi oke.

25. Yohmikma hatak puta kvt, In Chitokaka. Chihowah yut nan itim apesa i ki vhleha ha Echip yakni ya pehlichit kohcha mvt, itim apesa tok a kobvffit kanchi kak oke,

26. Ivt, naholbut toba inla, naholbut toba puta ithana tuk keyu, micha ima tuk keyu ka im antia cha ai okpuchi tuk oke.

27. Mikma Chihowah im ai i nukkilli ut yakni ilvppa i 
sanalit libbi cha, kallakshichi anumpa moma holisso iluppa a holisso tuk a onohonchi tuk oke.

28. Micha Chihowah yut nuklibisha, micha im ai $\underline{\mathrm{i}}$ nukkilli vt libbi kak osh, micha nukkillit shitilema kak osh tehlit $\underline{\mathrm{i}}$ yakni ya kohchi tuk osh yakni inla yo pit kanchi tok a himak nitak a yomohmi hoke, acha hi oke,

29. Na luma puta hokvt pin Chitokaka Chihowah immi hoke; amba nana ka otunichi tok okvto pishno, micha pim vlla vhleha aiena immi bilia he a tok; yumohmi hosh nan vlhpisa iluppa anumpa moma ka e yumihcha chị ha tok oke. (achi tok.)

\section{CHAPTA XXX.}

TUKMA yakohma hi oke; nana iluppa puta moma na A yukpali, micha nan isht kallakshi chi tikba takalichi li kvt chi onvtoyula cha, oklushi puta itin takla chin Chitokaka Chihowah yut chi tihleli tuk a hieli ont ish anukfilli,

2. Micha chin Chitokaka Chihowah ya falamvt ish im ona cha, chishno, micha chim vlla vhleha aienvt chi chukvsh mominchit, micha chimi shilombish mominchit himak nitak a nana moma chim ahanchi li tuk mak $\underline{\text {, }}$ yomohmit im anumpa ha ish im antiakma;

3. Yohmikma chin Chitokaka Chihowah yot chi nukhaklokmvit, falama cha, chi yuka tuk a chi falumminchi cha, okIushi moma chin Chitokaka Chihowah yvt pit chi fimmi tuk vt, a hoyot chitunala hi oke.

4. Chimmi ha tihlelit shutik a tahaka pilla isht ik ona tuk oh kia, yummak o chin Chitokaka Ghihowah yut ont a hoyot chitunala he, micha yummak ont chisht vla hi oke,

5. Micha chin Chitokaka Chihowah yut, yakni chiki vhleha hut a binili tuk a chisht vla na, ish a binila hi oke ; micha nana achukma chi yumihchikmvt, chi apaknvchi kvt chiki vhleha ha tok a $\underline{i}$ shahlicha hi oke.

6. Mikma chin Chitokaka Chihowah yot chi chukvsh, micha chim vlla vhleha chukvush aiena ka hakshup tubli na, chin Chitokaka Chihowah ya chi chunkvsh mominchit micha 
chimi shilombish a mominchit isht ish holitobli cha chi okchaya hi oke,

7. Mikmut chin Chitokaka Chihowah yut chin tunvp puta micha chi nukkillit chilbushali tuk puta kak o kalakshichi iluppa puta ka onocha hi oke.

8. Mikma ish falama cha, Chihowah im anumpa ha ish im antiakmvt, im anumpa moma himak nitak a chi miha li ka ish yưmihcha hi oke.

9. Mikma chin Chitokaka Chihowah yet na toksuli puta chibbak isht ish ishi ka, chi haknip a toba micha chi wak a toba micha chi yakni a toba aiena ka achukma ho anukfillit, chin lauacha hi oke: Chihowah yut chiki vhleha anukfilli mut, yukpa tok mak osh, anonti achukma ho chi anukfillit yukpa chi hoke,

10. Chin Chitokaka Chihowah ya im anumpa hạ ish $\underline{i}$ haponaklo cha, im anumpa vlhpisa, micha $\underline{\mathbf{i}}$ nan vlhpisa bilia, nan vlhpisa holisso iluppa aholisso tuk a ish ieshikma, micha falamot chi chukvush a mominchit, micha chimi shilombish a mominchi hosh ish im onakma,

11. Anumpa iluppa himak nitak a chim anumpohonli li kut chin luma keyu ; micha chi hopaki keyu hoka;

12. Vba yakni ak o itonla tuk $\underline{0}$, Kvta hosh vba yakni ya pim ia cha, ont isht pim vla na, e haklo cha e yumihcha chi cho? ish acha he o keyu hoka,

13. Micha okhuta mish tunnup ak o itonla tuk o, Kuta hosh okhvta ha pit pi hlopullị cha, ont isht pim vla na, e haklo cha e yumihcha chị cho? ish acha he o keyu hoka,

14. Amba ish yumihcha chi kut anumpa hot chi bilinchi fehnvt, chitih, michạ chi chukvush a foyuka hoke.

15. Yakeh, himak nitak a okchaya micha achukma, illi micha nan ik achukmo aiena ka chi tikba takalichi lishke,

16. Yohmi hosh himak nitak a chin Chitokaka Chihowah ya ish holitobli cha, $\underline{\mathrm{i}}$ hina ya ish ai itunowut, im anumpa, micha $\underline{i}$ nan vlhpisa bilia, micha im aivlhpesa yumihinchi ya ish jeshi cha, chi okchayvt ish apaknvcha he o chi miha lishke: mikma chin Chitokaka Chihowah yot yakni ya ish isha chi hosh yumma pilla ish ia ka a chi yukpala hi oke, 
17. Amba chi chukvsh vt filvmmi, yohmi hosh ish $\underline{\mathrm{i}}$ haponakla he keyu; amba naksika ia cha, naholbvt toba puta aiokpvchi, micha ish im antiakma;

18. Himak nitak a anumpa ahli mak $\underline{0}$ hvch illi pulla he : micha yakni a ish isha chi hosh, Chatan a hlopullit, yumma pilla ish ia kvt huchi nitak a hush falaiacha he keyu ka hvchi miha lishke.

19. Ai okchaya micha ai illi, aiyukpa micha akalakshi aiena ka huchi tikba takalichi li ká, vba micha yakni aiena kvt himak nitak a hvchi atokolit holissocha chi ka hoyo lishke; yumohmi hoka ai okchaya ak o ish atokolashke: yumohmi hosh huchishno micha chim isht atiaka yut okchayashke,

20. Yvmohmi hosh chin Chitokaka Chihowah ya ish holitoblashke, micha yvmohmi hosh im anumpa ha ish im antiashke, micha yumohmi hosh hush asitiashke, (yummak osh chi ai okchaya, micha nitak isht chi falaia yoke;) yumohmi hosh Chihowah yut chiki vhleha Eblaham, Aisak, micha Chekob aiena ka ima chi hosh anumpa kullo im il onochi tok a, yakni ya ish aiashashke, (achi tok.)

\section{CHAPTA XXXI.}

IHMa Moses vt anumpa ilvppa puta kak o Islael mo-
ma ka ont im anumpuli tok.

2. Micha himak nitak a afummi tahlepa cha, pokoli tuklo li hoke; himakma kuhchvt ivt, ant chukowa la he keyu hoke; micha Chihowah yot Chatan ilvpa ish hlopulla he keyu, a miha ha tuk oke;

3. Chin Chitokaka Chihowah ak osh chi tikba ivt hlopulli cha oklushi iluppa puta ka chi tikba ai isht kancha chi hoke, mikma ish isha chi hoke : mikma Chihowah yvt achi tuk mak o, Choshua yummak osh chi tikba ivt hlopulla chi hoke.

4. Mikma Chihowah yvt Amolait i miko tuklo Sinion, micha Ok, micha i yakni aiena yummak oka ishit kanchi tuk vt chohmicha chị hoke. 
5. Micha Chihowah yvt anumpa vlhpisa moma hvchi miha

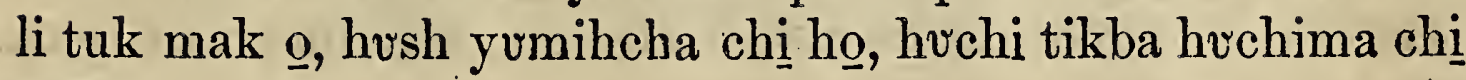
hoke,

6. Huchi kvllashke, micha hvsh aiyimitashke, hvsh i nukshopa na; chin Chitokaka Chihowah yummak osh chi auwant ia hoka, ik chim ai ahlo ka he keyu, micha chi kancha he keyu hoke, achi tok.

7. Mihmot Moses vt Choshua ya hoyo cha, Islael momá kvt pisa ho, Ish kullashke; micha ish aiyimitashke; okla iluppa pehlichit, Chihowah yut iki vhleha ha yakni ya ima chi hosh anumpa kvllo im ilonochi tok a ish onashke; micha ish a binilicha chi hoke,

8. Mikma Chihowah yummak osh chi tikba ia hoke, yummak osh chiba foyuka chi hoke; ik chim ai ahlo ka he keyu, micha chi kancha he keyu hoka; ik chi nukshopo kashke, micha ik chi nukhlakancho kashke, im achi tok,

9. Mihmvt Moses vt nan vlhpisa ilvppa-holissochi cha, Lefai ushi vhleha naholitompa isht asha Chihowah nan itim apesa a fohka itombi holitopa sholi tuk a, micha Islael im asunonchi moma ka ibbak fohki tok.

10. Micha Moses vt $\underline{\mathrm{i}}$ mihvt, Afummi untuklo kvt ont taha aiyukali $\underline{i}$ hotofa afummi ch $\underline{u}$ kvsh nuktaiyala ya tabenakel ai impvchi,

11. Islael-moma kvt chin Chitokaka Chihowah itikba hush hiela chị hosh, kanima ho ai atokoli tuk o, vlakma, Islael moma kut haklo ho nan vlhpisa iluppa itikba ish ai itim anumpula hi oke.

12. Okla, hatak, ohoyo, micha vlla vhleha, micha okla inla yosh chi holihta ok hissa anukaka chim.ahanta aiena ka hoyot ish itunahlashike, yumohmi hosh haklashke, micha yumohmi hosh itbunashke, micha huchin Chitokaka Chihowah ya $\underline{i}$ nukwia cha nan vlhpisa iluppa anumpa moma ka ithaiyanut yumihchashke.

. 13. Mikma im vlla vhleha nana ka ithana tuk keyu kvt, haklo cha, huchin Chitokaka Chihowah ya okchayvt yakni ya hushịisha chi hosh Chatan a hlopullit, yumma pilla hush ia kut. hush aiasha ka ithaiyanvt i nukwiashke, achi tok. 
14. Mihma Chihowah yut Moses a, Yakeh, chilla chi chi nitak vt mihintishke: Choshua ya hoyo cha, okla moma in tabenakel a hush hieli na, anumpa ima lashke, im achi tok. Mihma Moses micha Choshua vt ia cha, okla moma in tabenakel a hieli tok.

15. Mihma Chihowah yut tabenakel a hoshonti tonilk afoyukut haiaka tok: mihma hoshonti tonik vt tabenakel okhissa paknaka hikia tok.'

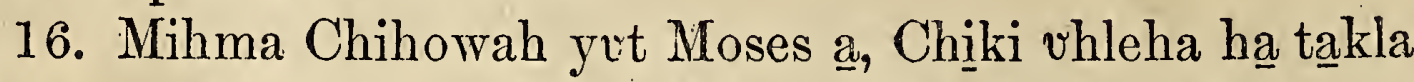
chi nusa chi hoke; mikma okla iluppot, takla aiasha chi hosh, yumma ia kut wakaya cha, hauit yakni yash okla inla i naholbut toba puta ka iakaiyvt itunowa chi hoka; micha sa kanchikmut, nana itim apesa li tuk a kobuffa hi oke.

17. Yohmikma nitak yưmma vm ai i nukkilli vt $\underline{\underline{i}}$ sanalit libba hi oke; micha kanchi likmut, sa nashuka ya in luhmi likma, okpulot taha hi oke; micha nan ik achukmo, micha ishtatakluma laua kvt onvtoyula hi oke; yohmi cha nitak yumma, Nan ik achukmo iluppa puta kvt pin Chitokaka osh pitakla anta keyu kak o pi onvtola keyu cho? acha hi oke,

18. Mikma fullotut naholbut toba inla asitivt nan ik achukmo moma toksuli tuk pulla kak $\underline{\text { }}$. nitak yumma sa nashuka ya luhmi li pulla hi oke.

19. Yumohmi hoka himak a taloa iluppa ish holissochi cha, Islael im vlla vhleha ha ish im abvchashke; itih ho ish fohkashke; yumohmi na, taloa iluppot Islael im vlla vhleha

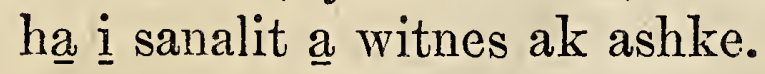

20. Iki vhleha ha anumpa kullo im ilonochi li tuk vt yakni pishukchi, micha foe-bila ayunahanli ya pehlichit isht ona li tuk o; impa tuk osh kaiyvt taha cha, niakmvt; fullotut naholbut toba inla iakaiyvt im antiakmut sa nokowvchi cha, a nan itim apesa ya kobuffa hi oke,

21. Atukma yakohma hi oke, nan ik achukmo, micha aiataklamoa laua hosh onvtolakma, taloa ilupput witnes mak osh $\underline{i}$ sanalit atokolashke; im ishtatia ka itih ha foyuka ka im ahaksa chi keyu hoke; himak a fehna kak kia anumpa kvllo im ilonochi li tuk ut yakni ya isht ak ono kisha ka im 
anukfila hut kaniohmit itunowa ka ithana li hoka, im achi tok.

22. Yưmohmi ma Moses vt mih nitak ak inli ho taloa iluppa holissochi cha, Islael im vlla vihleha ha im abvchi tok.

23. Mihma Nun ushi Choshna ya anumpa imvt, chi kullashke, micha chi aiyimitashke, anumpa kvllo im ilonochi li tok mak o Islael im vlla vhleha ha pehlichit yakni ya isht ish chukowa chi hoke; mikma chibafoyuka la chi hoke, achi tok.

24. Atuk o yakohmi tok, Moses vt nan vlhpisa iluppa anumpa ha holisso ha atakalichit tahli na ont taiyaha ma,

25. Moses vt Lefait vhleha Chihowah nan itim apesa afohka itombi holitopa sholi ya tuk a $\underline{\text { i mihut, }}$

26. Nan vlhpisa holisso iluppa ishi cha, huchin Chitokaka Chihowah nan itim apesa afohka itombi holitopa anukaka hush bohlashke; yưmohmi ho witnes chi sanalit yumma itoyulashke.

27. Ish ile filummichi, micha chikunla kullo ka ithana li hoke; yakeh, himak nitak a si okchayvt hvchi takla anta li moma ka Chihowah ya i filummi hvchi shahli fehnushke; a sulli ha yok mak heno i shahli fehna chi keyu cho?

28. Hvchim apehlichi puta im asvnonchi, micha huchi nan vlhtoka puta ka hvs sum itunahli na, anumpa iluppa puta ka haksobish a haklochi lashke; micha vba, micha yakni vt $\underline{i}$ sanalit holisso takalicha chị ka hoyo lashke.

29. Sulli ha ma il okpunit hush kaiyanchi cha, hina nana hvchi miha li tuk a pit hvsh filema chị : atuk o nitak ont ishtaiopikma, Chihowah itikba ya nan ik achukmo ka yumihinchit huchibbak isht hush toksvlit, hush nokowvchi kak $\underline{o}$ nana ik achukmo kut huchi onvtola chi ka ithana li hoka, im achi tok;

30. Mihmvt Moses vt Islael okla moma ka haklochit taloa iluppa anumpa ha im anumpohonli na ont taiyaha tok. 


\section{CHAPTA XXXII.}

YBA puta ma, hrsh haklashke, mikma anumpule lashke, mikma yakni ma, sutih numpa ha ish haklashke.

2. Um anumpa hot umba chiyuhmit itola chi hoke, micha vm anumpa hvt fichak chiyuhmit chilofa chi hoke, okshimihinchi yosh haiyukpulo waloha onvtola kak o chiyuhmi, micha umba hosh hvshuk onvtola kak o chiyuhmi hoke.

3. Chihowah hohchifo kak o anoli la chi hokvt; pin Chitokaka yo isht a holitopa ya hush imashke.

4. Yvmmak osh tvli chito ak oke; nan isht ai ahanta yvt vlhtaha hoke; nan im a kaniohmi puta kvt aivlhpiesa hoke; Chitokaka ai ahli, micha nan ashvchi iksho: aivlhpiesa, micha vlhpesa yoke.

5. Il okpuni tuk oke; litelit takäli kvt im vlla vhleha liteli yo keyu; ai itishali nan ashvchi, micha shanaiowa vhleha ak oke.

6. Okla im anukfila iksho, micha ik hopoyukso ma; yvmohmi hosh Chihowah ya hush im atobbi cho? chiki yosh chi chumpa tuk keyu cho? chikbi cha kullochit chi hilechi tuk keyu cho?

7. Nitak chashpo a tok a ish ithaiyanashke, micha ai iti shali laua afummi puta ka ish anukfillashke; chiki ya ish $\underline{\mathbf{i}}$ ponaklashke, chi pisucha hinla hoke, mikmut chim asunonchi ma yummak okưt chim anola hinla hoke,

8. Chaha Moma i Shahli vt oklushi puta ka i yakni ya chị ho ita kushkoli tok, Alam ushi vhleha ha ita filummichi muvt, Islael im vlla vhleha holhtina ha tuk mak $\underline{0}$, okla im ai vhli ya apesa tok.

9. Chihowah immi hato im okla hak oke: im ai isha he vlhpesa yut Chekob ak oke.

10. Yakni chokushmi foka yo, micha yakni haiaka achukillissa, nampoa a woha ai ahayuchi tok; fullota hosh ilauelit 
isht aya tok: im abvchi tok, ilap nishkin lumbo ak o im itilauit ieshi tok.

11. Ossi yosh im vlhpichik a koyohlit, ushi ya paknaka hlopohvchit, sanahchi ashatablit ishi sanahchi ya ishit shaiyali chatuk ak o chiyuhmit,

12. Chihowah ak illa hosh ilaueli tok: mihma okla inla i naholbut toba vt ik ibafoyuko tok.

13. Osapa nat awaya ka ai vpa chi kak o, yakni achahaka ya on binflichi tok; micha tuli chito foe-bila yo, micha tasonak tuli chito bila yo a shukvuchi tok.

14. Wak i pishukchi nia, micha chukfi i pishukchi, chukfushi nia aiena, micha Bashan aivlhpoa chukfi nakni, micha issi-kosoma yosh haiyihchi nia aiena, micha onush aiena ho ish impvchi tok: mihma palki okchi a hli issish chohmi ka ish ishko tok.

15. Yohmi tuk kia Cheshulvn vt nivt taha mut, huhli tok: chi nivt tahushke, chi chitot tahushke, nia yosh ishit chi ompoholmoshke: yohmi mut ikbi tok Chitokaka ya kanchi tok, micha aiokchaya toli chito yosh ik ahobalo tok.

16. Okla inla i naholbvt toba ishit nuktahlali mot, na yuwala ya ishit nukowvchi tok.

17. Shilombish okpulo ak o nan vbit isht aiokpvchi tok, Chihowah ak a tok keyu; naholbut toba ithana ya tuk keyu, naholbut toba himona, himona yosh hikia tuk hvchiki uhleha hut i nukshopa tok keyu ya tuk oke.

18. Tuli chito chi tobachi tok ano chik anukfilloshke, micha chikbi tok Chitokaka ano issut ish kanchishke.

19. Atuk o Chihowah yut pisa mut shitilema tok; ushi vhleha micha ushetik vhleha hvt nokowvchi ha tuk oka.

20. Micha sa nashuka ya in luhmi lashke: ishtaiopi kaniohmi na pisa lashke: ai itishali ila filimmi fehna, micha aiyimmi ka iksho vlla vhleha hoka.

21. Chihowah keyu ka ishit sa nuktahlali tuk oke; makali ya ishit sa nokowvchi tuk oke. Atuk o vno vt okla keyu yo ishit nuktahlali la chi hoke; oklushi im anukfila iksho yo ishit nokowuchi la chi hoke.

22. Sa nokowa ma luak ut libbi kut hukmit aiokpuloka 
aka pilla ishtona chi : micha yakni, nan awaya ai ona ka hukmit tahli cha, nunih chaha puta in tola puta ka luak tikelicha chị hoke.

23. Nana ik achukmo ka $\underline{o}$ fohobli la chị hoka, um oski naki ya a tahli la chi hoke.

24. Hochvfo ho ishit lua he, micha naluachi lushpa yo micha okpulot taha homi ho ishitutaha hi oke : micha nampoa noti, micha lukfi pushi sinti in isht illi aiena ka on tihleli lä hi oke.

25: Kocha ya bushpo falaia, micha anukaka ya komunta yosh hatak himita, micha ohoyo himita hatak ik halelo, micha pishi hatak noshkobo tohbi aiena ka vit tahla hi oke.

26. Ita fimmit.achukbeli ya isht ona lashke; ithaiyana ka hatak ai itin takla yaj issuchi lashke, achi li tuk oke.

27. In tunup vhleha nukhobela ha tuk micha $i$ sanali vlfleha hut kaniohmiat int inta fêhna, micha pibbak vt chahushke, Chihowah ak osh luppa moma ka yumihchi tuk keyu hoke, acha he ak $\underline{0}$, ik sa nukwio tuk okmvt.

28. Oklushi ahopoyuksa kvt ik im iksho, micha nana ithana anukfoka keyu hokvt.

29. Hopoyuksa tuk okbano, iluppa ithana tuk okbano, im isht aiopa he vt anukfilli tuk okbano.

30. Katiohmi kak osh achufa kia tahlepa sipokni hoh kia tihlela he tuk keyu? micha tuklokmvt tahlepa sipokni pokoli kia tihlela he tuk keyu? in Toli chito yak osh kanchi tuk keyukma: micha Chihowah ak osh im okshita tuk keyukma?

31. In tuli chito vt pin Tuli chito ya chohmi keyu hoka; pin tunvp vhleha ak inli kvt nan apesa hoka.

32. I pakupi vt Sotom i pakvpi, micha Komolah osapa ak $\ddot{0}$ ai achufa chohmi hoka; $i$ paki ut basunlush paki yoke, in Iokoli vt homi hoke.

$\therefore 33$. Im oka paki vt sinti vt isht illichi, micha asp vt isht illichi isht ilbushali yoke.

34. Ilvpput vm vlhtahvt an toyula keyu cho? micha vm ilayak takla ashanvt itoyula keyu cho?

35. Il aiokhvt hotopali, micha falumminchit im atobbi vt 
vmmi hoke: ont aivlhpesakma iyi yut shakalulla hi oke; nan isht im aiokpuloka chi nitak vt olanlosi hoke: micha nana onvtola chi kut túshpa hoke.

36. Chihowah yưt im okla ha nan im apesa chi hoka; micha isht akvllo vt i kunia, micha im okshilita kvt iksho; micha hlakofi kvt iksho ma, pisa kak osh, in tishu vhleha ha ishit $\underline{\mathrm{i}}$ nukhâkla hi:okẹ:

37. Micha, I naholbơt tôba puta, in tuli chito anukchieto tuk.

38. Yvmmak osh i nan voljit isht aiokpvchi nia vpa tuk, micha ishko im issa oka pâki yo im ishko tuk muto? · Wakaya cha chi apelashke; micha chi okchalinchi yashke, acha hi oke.

39. Himak a pisá, uno, wno fehna kak osh mih sia hoke, yohmi ka naholbut toba yưt sưbo foy uka keyu hoke: vbe li, micha okchalinchi li hoke; hotópali li, micha hlakofiche li hoke; mikma kuna hosh subbak a akohchit hlakoficha he vt iksho hoke.

40. Subbak a uba pit weli lish, Okchạyut ahanta li bilia yoke, achi li hoke.

41. Am bushpo falaia shohkanali ya halupvechi likmvt, micha subbak a nana apesa isht eshi likmvt, an tunvp vhleha hạ il aiokhvt na hotopali onochi la chi hoke: micha a nukkilli puta ka im atobbi la chi hoke.

42. Uัm oski naki ya issish a ishit fihobli la hi oke: mikma a bushpo falaia yut nipi ya tahla hi oke, micha yvmmut in tunư a il ai okha ishtia vmmona ma illi tuk issish, micha yuka tuk ak a hi oke.

43. Oklushi puta ma, im okla takkla hvchi yukpashke: in tishu vhleha im issish atuk a ilai okha chi, micha i sanali vhleha ha ilai okhvt nahotopa onocha chí; micha i yakni micha im okla ha i nukhakla hi oka, achi tok.

44. Mihma Moses micha Nun ushi Choshua vt vla chä; okla hut hạkla ho taloa iluppa anumpa moma kạ anumpohonli tok.

45. Mihmvt Moses vt anumpa iluppa mominchit Islael" moma ka im anumpuli tuk ot ont tahli tok. 
46. Micha anumpa moma himak nitak a hvech iti takla ya ai atokoli li ka hvchi chukvsh a hvsh onochashke, micha hvchim vlla vhleha hut nan vlhpisa ilvppa anumpa moma ka ithanvt yumihcha chi ka hvsh i mihashke.

47. Nan ik ahobo hvehi nana keyu hoka; hvchim ilhfiopak okvt; nana iluppak o yakni hush isha chi hosh Chatan hush hlopullit, yumma hush ia kvt nitak ishit huvehi falaia chi hoke, achi tok.

48. Nitak yummak inli ma, Chihowah yot Moses a im anumpulit,

49. Nunih chaha Abalim iluppa oiyvt Nebo bokko, yvmmut Moab yakni hikia, Cheliko ichapaka ya ish onashke; micha Kenan yakni Islael im vlla whleha hák osh i yakni ya chị ho ima li tuk a ish pisashke.

50. Micha chitibapishi Alon vt Ho nưnih ha ai illi cha, im okla ha ibafohka tok a, chohmit nunih yumma pit ish oiya kut chilli cha chim okla ha ish iba fohka chi hoke.

51. Yakni haiaka Sin Melibah-Katesh oka ya Islael im vlla vhleha ha ai iti takla ya a sanalit ish vbanvbli tok okvt, Islael im vlla vhleha ai iti takla ya chik sa holitoblo tok okvt.

52. Yohmi kia yakni vt chi tikba talaia na ish pisa chi hoke; yohmi kia yakni Islael im vlla vhleha ima li tok a yumma ivt ish ona he keyu hoke, achi tok.

\section{CHAPTA XXXIII.}

WICHA nan isht yukpali Chitokaka im antia hatak MoI ses ut ik illo kisha mut, Islael im vlla vhleha ha isht yukpali tok.

2. Micha Chihowah yut Sinai a minti tok, micha Sei yak $\underline{0}$ i wakaya tok, Palan nunih hak o atohwikelit et a kohcha tok: micha saint vhleha tahlepa sipokni pokoli ho pehlichit vla tok: ibbak isht impakimma ya nan vlhpisa libbi vt vttut ia tok.

3. Ahli, okla ha $\underline{i}$ holitopa tok, imi saint vhleha moma kut chibbak foyuka hoke, micha chiyi ituma chieya tok: moma kvt chim anumpa ha ai isha hi oke. 
4. Moses vt nan vlhpisa pim apesa tok, Chekob okla moma immi ha chi im aivlhpiesa.

5. Micha okla i noshkoboka vhleha, micha Islael im apehlichi puta kvt iti hoyot itunaha ma, Cheshulvn a mikot ahanta tok.

6. Luben vt ik okchaya cha, ik illo kashke; micha hatak vt ik i kanomoso kashke.

7. Mikina iluppak osh Chutah nan isht aiyukpa yoke, micha Chutah vt anumpohonlikmut, Chihowah ma, im anumpa ha ish haklo cha, im okla ha isht ish im onashke; ibbak ak inli kvt im vlhpesashke; apela chia hosh in tunvp vhleha ha ish $\underline{\mathrm{i}}$ hlakofichashke, achi tok.

8. Micha Lefai ano Chin Hlummim, micha chim Ulim ak osh chi holitopa takla yashke, yummak oka Massa ya ai i momaka ish pisa tôk; micha yumma Melibah oka ya ish iti kullo tok.

9. Yummak osh iki, micha ishki ya pisa li tuk keyu, im achi tok: micha itibapishi vhleha ha ik ithano, micha im vlla whleha hak inli kash mih yoh ik acho tok: chi nan vlhpisa ha ithaiyana cha, chi nan itim apesa ieshi ha tuk okvt.

10. Nana chim ai vlhpiesa yưmihinchi ak o Chekob a im abvcha chi, micha chi nan vlhpisa Islael a im abvcha hi oke; na balama ya chi tikba talalikmot, mominchit hukmit isht aiokpvchi ya chim alta ya onocha hi oke.

11. Chihowah ma, im ilayak a ish yukpalashke, micha ibbak toksvli tuk a ish im aiokpvchashke; i sanalit wakayvt hikia, micha i nukkilli puta aiena in chushwa ya nuhlit ish hlopullichị na, anonti ik wakayo kashke, achi tok.

12. Micha Benchamin ano, Chihowah i holitopa vt achukmaka bilikia ai ahanta hi oke, micha CHrHowaH yot nitak moma ompohoma hi oke; mikma tahchi itin takla ya ahanta hi oke, achi tok.

13. Micha Chosef ano, I yakni vto Chihowah yvt yukpali ashke, misha vba nan achukma puta, micha fichak, micha oka hofobi yakni nutaka et a tobohonli kak $\underline{\text {, }}$

14. Micha na waya holitopa puta hushi vt tobahanchi yạ, 36 
micha nana holitopa puta hushi ninak aya vt tobahanchi puta kak $\underline{0}$,

15. Micha chaspo atok nunih chaha puta nan i shahli kak o ; micha nunih hikia na bilia puta nana holitopa aiasha kak $\underline{0}$,

16. Micha yakni ya na holitopa aiasha, mieha isht alotowa aiena kak $\underline{o}$, micha bufaha ya ahanta tok vt aiokpanchi kak o, holitopashke, nan isht yukpali vt Chosef noshkobo hạ, micha itibapishi ha ila filummi tok noshkobo paknaka ya onvtolashke.

17. Im aholitopa yut i wak nakni vttahpi ak o chohmi hoke, micha in lupish vt yunikon lupish ak o chohmi hoke; yummak $\underline{o}$ okla ha ishit toblit yakni atahaka ya isht ona hi oke; micha yummut Eflaim in tahlepa sipokni pokoli yoke, micha yummut Manasseh in tahlepa sip

18. Micha Sebulon ano, Sebulon ma, Kohchvt ish jakmvt chi yukpashke; mikma Issaka yut chi binah antut chí yunkpashke, achi tok.

19. Okla ha i howut nunih ha isht ona hi oke; yưmmak o aivlhpiesa nan vbit isht aiokpuchi ai im issa hi oke; okhvta hochito nan aiapakna, micha naholitopa.luma shinuk aiasha ka shuka hi okvt, achi tok:

20. Mikmvt Kat ano, Kat a chitolichi hokvt, holitopashke; koi chito chiyuhmi hosh ahanta cha, shakba, noshkobo tabokaka aiena ka hlillichi tuk oke.

21. Micha ummona ka yummak o nan vlhpisa ima yut binilichi ha tuk oka, ila tahli tok; micha okla i noshkoboka apehvt vla tok; Chihowah im vlhpiesa, micha Islael a aivlhpiesa i yumihinchi ya aiahlichi tok, achi tok.

22. Mikmot Tan ano, Tan vt Koi chito ushi yoke; Ba shan a vttut tolubla hi oke, achi tok.

23. Mikmet Naftali ano, Naftali ma, im isht ikana thit fihopa, micha Chihowah ishit yukpali isht alotowa yolse; hushi ai okatula, micha oka mahli ya ish ishashke, achi tok.

24. Mikmot Asha ano, Asha yvt vlla vhieha ishit yukpashke; itibapishi vhleha hvt aiokpanchit ishit yukpashke, mikma iyi ya bila yo a hilechashke. 
25. Chi shulush vt tuli, micha asonak lakna yashke; chim i hlampko vt chị nitak puta ka chohmashke.

26. Cheshulvn in Chitokaka yummak okvt chi apela kvt vba aya im aholitopa vba ya chohmi kvt iksho hoke, achi tok.

27. Chitokaka ahanta bilia ak osh ish aiatuko yoke; shakba atala iksho yak osh nuta talaivshke; micha tunup a chi tikba ya kocha pit tobla chi hoke, micha, Ishit kanchi, acha chi hoke.

28. Yohmikina Islael vt ilap bano hosh ai achukmaka ho ahanta chi hoke; Chekob i kuli ak osh yakni onush, micha oka paki ya onvtoyula chi hoke; micha i shutik vt fichak $\underline{0}$ akvcha chi hoke.

29. Islael ma na chị yukpashke, kuta hosh Chitokaka okchalinchi chishno chohmi cho? okla ma, yvmmak osh chin tilehpa chi apela, micha yummak osh bushpo falaia ishit chi a holitopa mak oke : chin tunup vhleha hut chi holabi atuk o pisa chi hoke, mikma im achahaka puta ish ahublicha chi hoke, (achi tok.)

\section{CHAPTA XXXIV.}

IHMA Moses vt Moab yakni patali ya vttut Nebo 1 nunih chaha Piskah paknaka, yummut Cheliko ichapaka ya ona tok; mihma Chihowah yut Kileat yakni moma "Tạn ont vhli ka,

2. Micha Naftali moma, micha Eflaim, micha Manasseh yakni, micha Chutah yakni moma okhuta ont vhli fehna,

: 3. Micha oka-mahli, micha Cheliko okfa yakni patali pam itiotumaha holihta Soa ont vhli ka pisachi tok.

4. Micha Chihowah yvt, Yakni iluppak o Eblaham Aisak, micha Chekob aiena ka anumpa kvllo im ilonochit, chim ishtatiaka ya ima la hi yoke, achi li tok mak oke : chi nishkin ishit chi pisachi lishke, yohmi kia pit hlopullit yumma ish ia he keyu. hoke.

5. Yohmi hosh Chihowah im anumpa hatuk mak $\underline{d}$, 
Chihowah in tishu Moses vt Moab yakni yummak o ai illi tok.

6. Mihma Moab yakni Belhpeo ichapaka okfa ho ahohpi tok; yohmi kia im a hollohpi ya kuna ik ithano ho himak nitak ant ai vhli hoke.

7. Yohmi ka Moses vt illi mvt, afummi tahlepa achvfa cha pokoli tuklo tok; nishkin vt illi keyu, micha nipi kvllo kvt tahvt isht ik aiyo tok.

8. Mihma Islael im vlla vhleha hvt Moab yakni patali Moses a ai ishit yaiya na, nitak pokoli tuchina tok; atuk $\underline{o}$ Moses a ishit nishkin okchi mintit, ishit yaiyvt aiasha tuk nitak vt ont taha tok.

9. Mihma Nvn ushi Choshua hopoyuksa shilombish a alotowa tok; Moses vt ibbak a onochi tuk a tuk oka: mihma Islael im vlla vhleha hut $\underline{i}$ haponaklo cha, Chihowah yut Moses a tohno tok mak $\underline{\mathrm{o}}$, yumihinchi tok.

10. Yohmi ka ola $\underline{i}$ himmak minti ka Moses a chiyuhmi, yummak oka Chihowah yut nashuka iti sanalit ithana tok a chiyuhmi kut hopaii vt wakayvt ik hikiyo chatuk oke.

11. Yohmi kvt nan isht atokowa puta, micha nana afehna puta, yummak oka Echip yakni Falaoh, micha in tishu vhleha moma, micha i yakni moma aiena ka onochit yumihcha chi ka Chihowah yvt tohno tok.

12. Micha ibbak kullo moma yumma, micha napalvmmi chinto yumma, Islael moma kut pisa ho Moses ut haiakuchi tok, (achi tok.) 





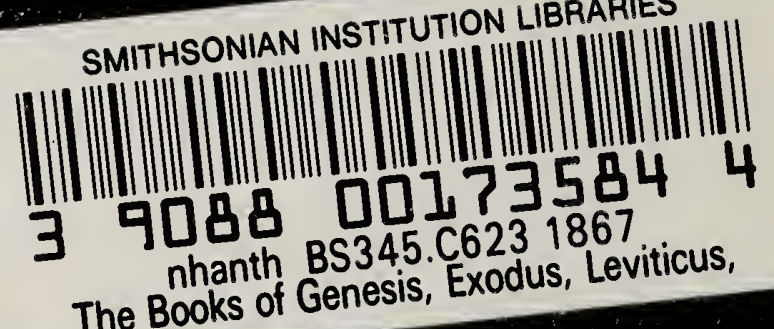

The Books of Genesis, 